\title{
EVALUATING PERFORMANCE OF SOUTHERN ONTARIO BUILDINGS USING SUBMETERING DATA AND WHOLE BUILDING MODELING \\ RESULTS
}

\author{
By \\ Mark Adrian Turcato \\ B.A., McGill University, 2013 \\ A thesis \\ presented to Ryerson University \\ in partial fulfillment of the \\ requirements for the Degree of \\ Master of Applied Science \\ In the Program of \\ Building Science
}

Toronto, Ontario, Canada, 2015

(c) (Mark Turcato) 2015 


\section{Author's Declaration for Electronic Submission of a Thesis}

I hereby declare that I am the sole author of this thesis. This is a true copy of the thesis, including any required final revisions, as accepted by my examiners.

I authorize Ryerson University to lend this thesis to other institutions or individuals for the purpose of scholarly research.

I further authorize Ryerson University to reproduce this thesis by photocopying or by other means, in total or in part, at the request of other institutions or individuals for the purpose of scholarly research.

I understand that my thesis may be made electronically available to the public. 


\title{
Evaluating Performance of Southern Ontario Buildings Using Submetering Data and Whole Building Modeling Results
}

\author{
B.A. McGill University, 2013, Mark Adrian Turcato \\ Ryerson University, Toronto
}

\begin{abstract}
The performance gap, the difference between how a building was intended to perform and its actual performance, poses a challenge to successful high performance design. This research examines the application of submetering data and whole building energy models to evaluate the performance gap in buildings as related to energy consumption, and in specific energy use associated with receptacles and lighting. While difficulties in grappling with large amounts of data persist, results indicate that building management and occupancy issues can offer an explanation for a significant portion of differences between predicted and actual energy use. Experience working with these data sets also suggests that further efforts are required to demonstrate the value of submetering in order to ensure submetering systems are not compromised by the value engineering process.
\end{abstract}




\section{Acknowledgements}

I would like to thank Mark Gorgolewski, Jenn McArthur, and Umberto Berardi for providing insight and guidance during my research.

I would like to thank Arash Ghorayshi, Victor Halder, and Steve Kemp from MMM engineering for cooperating so whole heartedly with allowing my access to the data and their personal knowledge on the projects.

I would like to thank NSERC for providing funding to make this research possible.

I would like to thank all my friends, family and loved ones for supporting me along the way. 


\section{Table Of Contents}

Author's Declaration for Electronic Submission of a Thesis ..................................ii

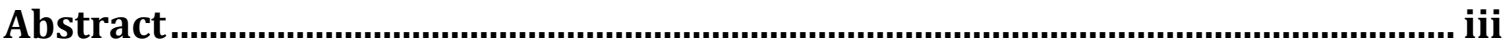

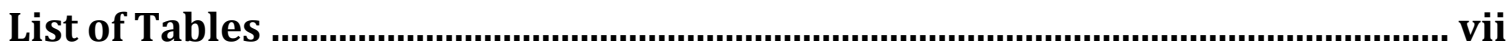

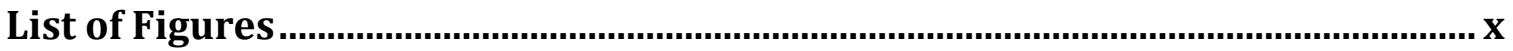

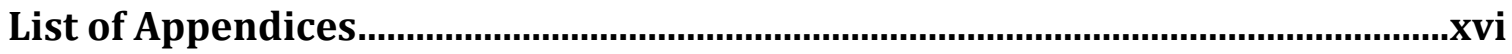

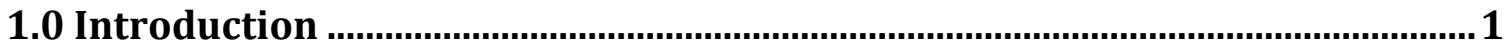

1.1 Research Objectives......................................................................................... 2

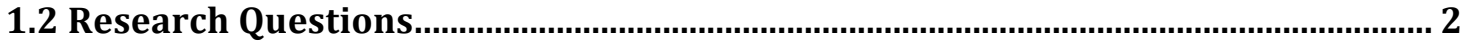

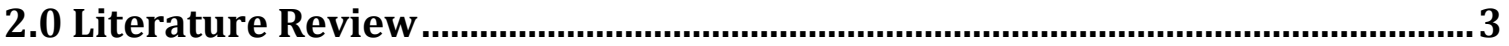

2.1 Building Performance Evaluations .......................................................................... 3

2.2 Whole Building Energy Modeling and Energy Model Calibration ............................ 5

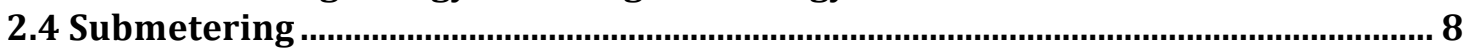

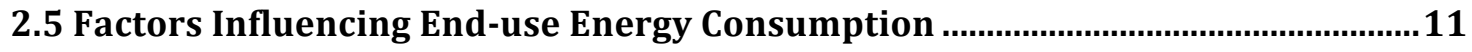

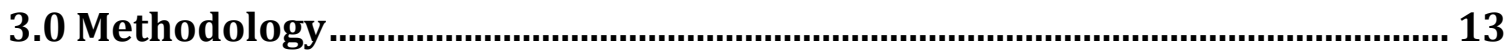

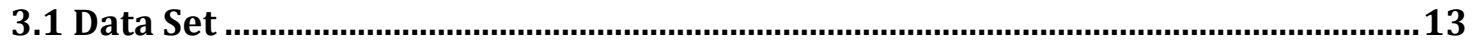

3.2 Building Breakdown ...........................................................................................14

3.3 Data Sources and Availability..........................................................................17

3.4 Errors in Submetering Data ................................................................................22

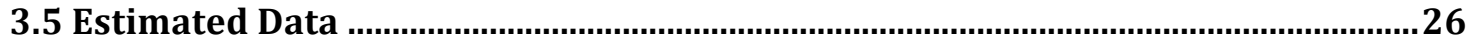

3.6 Graphical Analysis...........................................................................................27

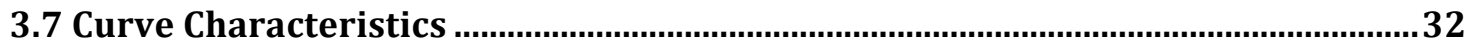

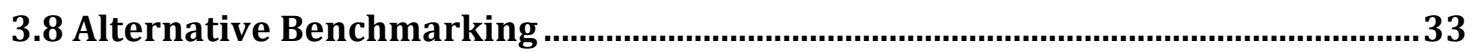

3.9 Error Regression Analysis ..................................................................................

3.10 Daylight Regression Analysis................................................................................35

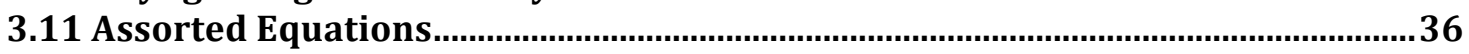

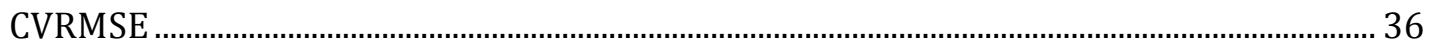

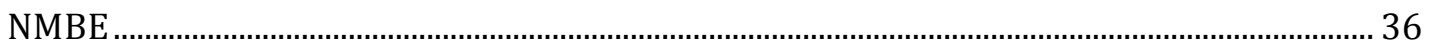

Percent Difference ............................................................................................................................... 37

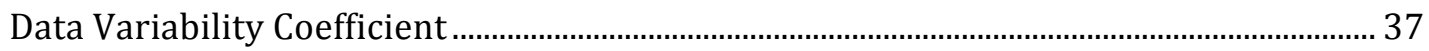

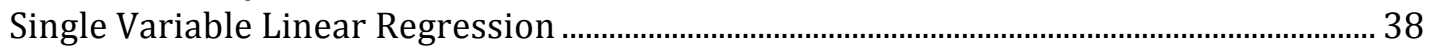

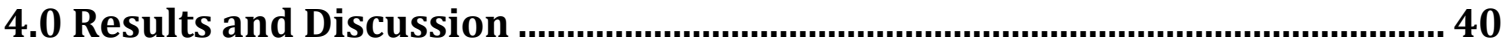

4.1 In-depth Building Profiles ..................................................................................40

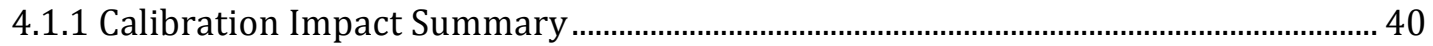

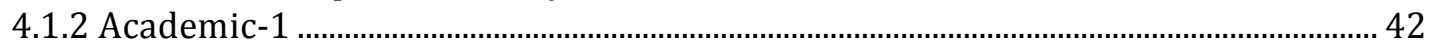

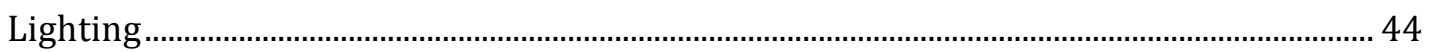

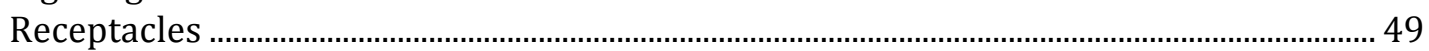

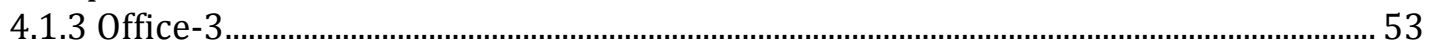

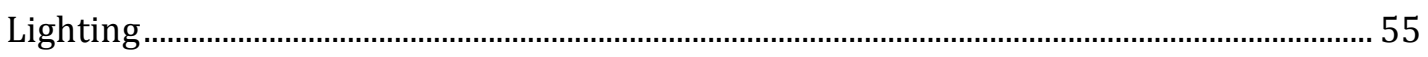

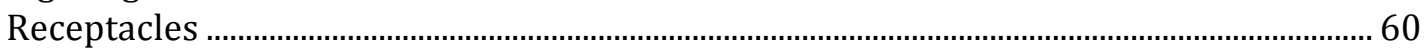

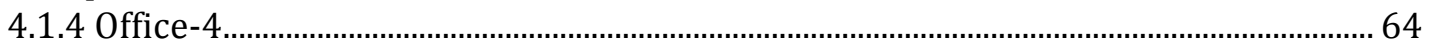

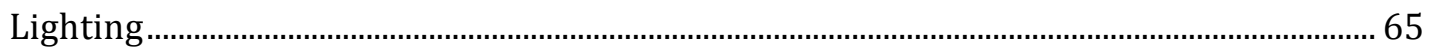

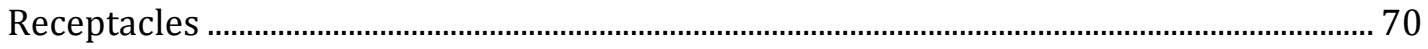




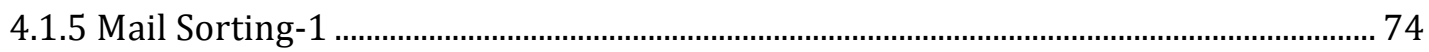

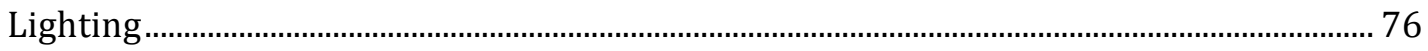

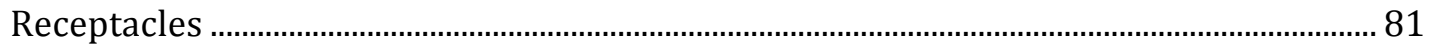

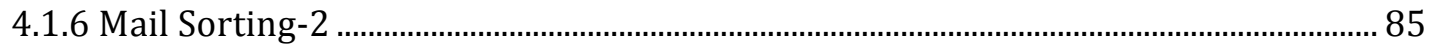

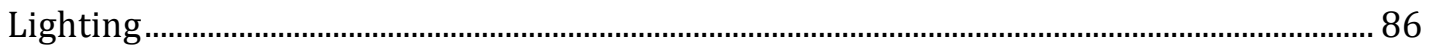

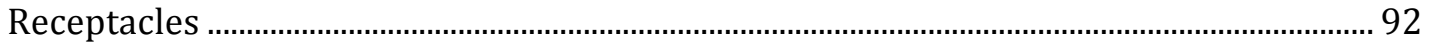

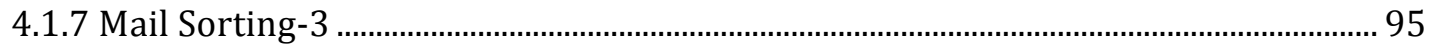

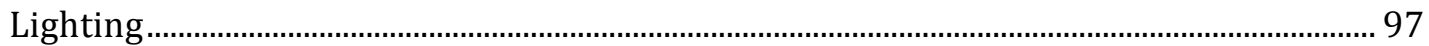

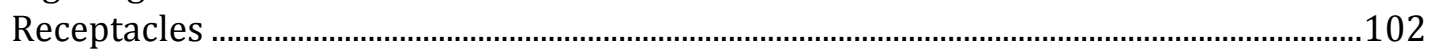

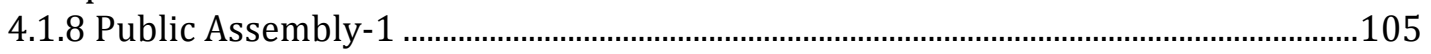

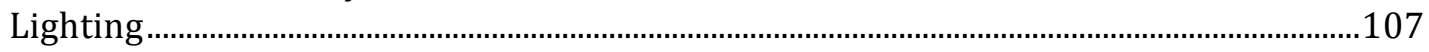

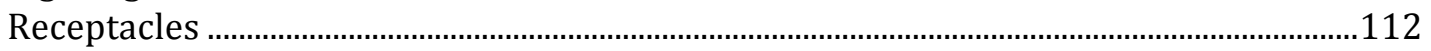

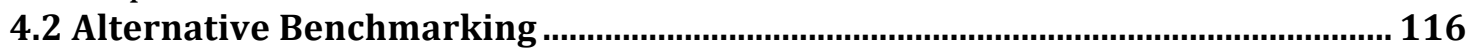

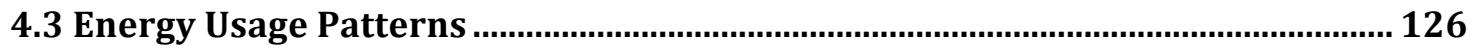

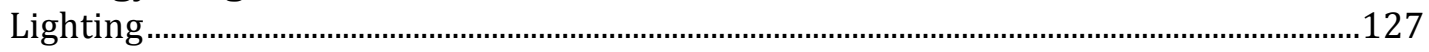

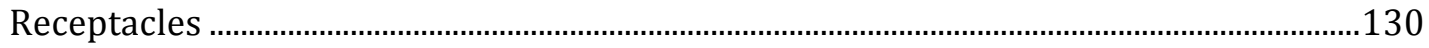

Data Variability- Regression Analysis …………...............................................................133

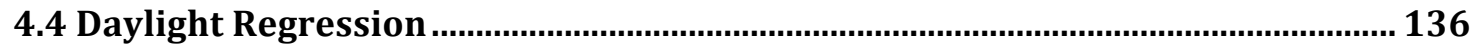

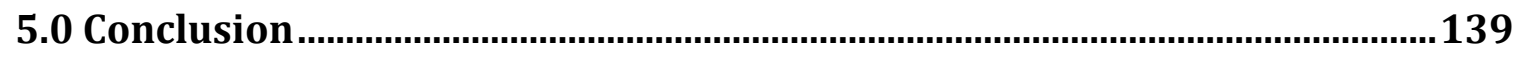

5.1 Data Quality ..........................................................................................................139

5.2 Submetering Practices......................................................................................... 141

5.3 Receptacles and Lighting Performance Gap .......................................................... 143

5.4 Future Work ................................................................................................................. 146 


\section{List of Tables}

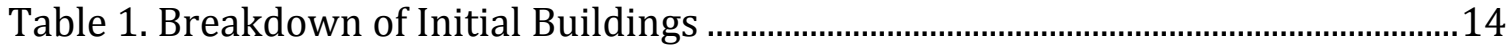

Table 2. Meter Mixing Summary..............................................................................................25

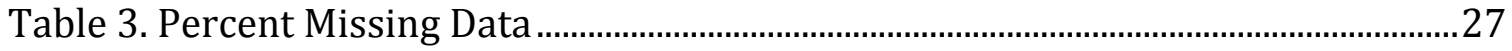

Table 4. Load Profile Characteristics ......................................................................................33

Table 5. Error Tolerance Limits for Calibrated Energy Model Simulations....................33

Table 6. Impact of Energy Model Calibration (Samuelson, Ghorayshi, Reinhart, 2015)

Table 7. Calibrated Energy Model Predictions and Post-processing Energy Consumption Additions ......................................................................................................4

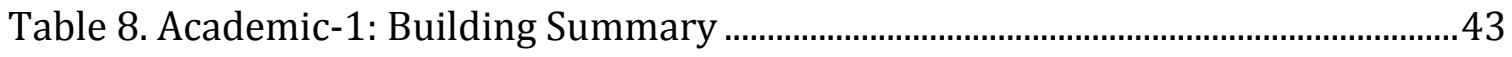

Table 9. Academic-1: Predicted and Actual Energy Use ........................................................43

Table 10. Academic-1: Building Lighting Summary .............................................................44

Table 11. Academic-1: Building Receptacles Summary .....................................................49

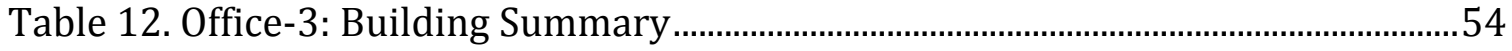

Table 13. Office-3: Predicted and Actual Energy Use..........................................................54

Table 14. Office-3: Building Lighting Summary ……………………………………….....55

Table 15. Office-3: Building Receptacles Summary ……………………………………..60

Table 16. Office-4: Building Summary ..................................................................................65

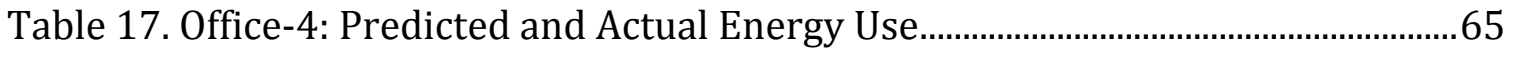

Table 18. Office-4: Building Lighting Summary ................................................................65

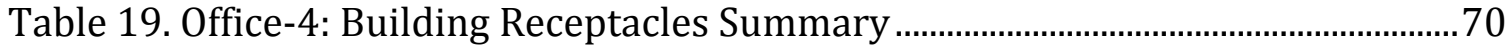

Table 20. Mail Sorting-1: Building Summary ……………………………………………..75

Table 21. Mail Sorting-1: Predicted and Actual Energy Use ...............................................75

Table 22. Mail Sorting-1: Building Lighting Summary …………………………………....76

Table 23. Mail Sorting-1: Building Receptacles Summary ..................................................81

Table 24. Mail Sorting-2: Building Summary....................................................................85 
Table 25. Mail Sorting-2: Predicted and Actual Energy Use …………………………......86

Table 26. Mail Sorting-2: Building Lighting Summary …………………………………....86

Table 27. Mail Sorting-2: Building Receptacles Summary ..................................................92

Table 28. Mail Sorting-3: Building Summary.........................................................................96

Table 29. Mail Sorting-3: Predicted and Actual Energy Use ................................................96

Table 30. Mail Sorting-3: Building Lighting Summary ……………………………….......97

Table 31. Mail Sorting-3: Building Receptacles Summary .................................................102

Table 32. Public Assembley-1: Building Summary ……………………………………...106

Table 33. Public Assembley-1: Predicted and Actual Energy Use ................................... 106

Table 34. Public Assembly-1: Building Lighting Summary …………………………...... 107

Table 35. Public Assembly-1: Building Receptacles Summary ……………………....... 112

Table 36. Average Percent Difference in Relation to Actual Energy Consumption.117

Table 37. Error Evaluation of Total Yearly Building Energy Use....................................120

Table 38. CVRMSE and NMBE for Monthly Total Building Energy Use. ....................... 121

Table 39. Overview Average CVRMSE .............................................................................. 122

Table 40. Overview Average NMBE (calculated with absolute values) ........................122

Table 41. Overview ASHRAE Suggested Error Tolerances (CVRMSE) ..........................122

Table 42. Overview ASHRAE Suggested Error Tolerances (NMBE)..............................122

Table 43. Total to End use Ratio for CVRMSE and NMBE. ..............................................123

Table 44. Overview ASHRAE Suggested Error Tolerances (CVRMSE) with Suggested

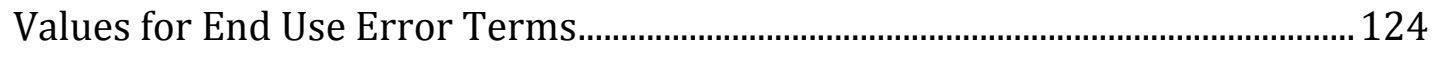

Table 45. Overview ASHRAE Suggested Error Tolerances (NMBE) with Suggested Values for End Use Error Terms.................................................................................. 124

Table 46. Statistical Results for Submetering Comparison to Lighting Schedules... 125

Table 47. Statistical Results for Submetering Comparison to Receptacles Schedules

Table 48. Median Near Base Lighting Loads as Percent of Median Near Peak Lighting Loads 128 
Table 49. Actual Total Lighting Energy Use for Daytime and Nighttime Hours (Predicted Values in Parenthesis)

Table 50. Actual Percent Time Building Operates as Percent of Max Lighting Load

(Predicted Values in Parenthesis).

Table 51. Median Near Base Lighting Loads as Percent of Median Near Peak Lighting Loads 132

Table 52. Actual Total Receptacle Energy Use During Daytime and Nightime Hours

(Predicted Values in Parenthesis) 132

Table 53. Actual Percent Time Building Operates as Percent of Max Receptacle Load (Predicted Values in Parenthesis) 133

Table 54. Results of Daylighting Regression Analysis .................................................. 138

Table 55. Academic-1: Zone and Schedule Description .................................................. 151

Table 56. Office-3: Zone and Schedule Description ............................................................. 152

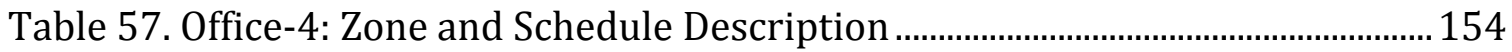

Table 58. Mail Sorting-1: Zone and Schedule Description ............................................... 155

Table 59. Mail Sorting-2: Zone and Schedule Description .............................................. 156

Table 60. Mail Sorting-3: Zone and Schedule Description............................................. 157

Table 61. Zone and Schedule Description...................................................................... 158

Table 62. Receptacles Power Density Summary............................................................... 160

Table 63. Academic-1: Zone and Schedule Description ..................................................... 160

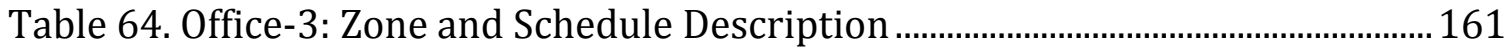

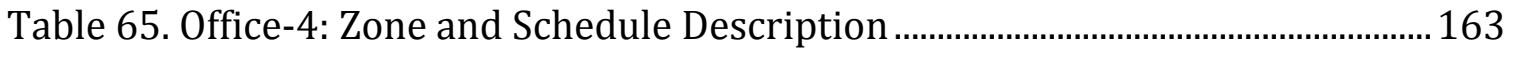

Table 66. Mail Sorting-1: Zone and Schedule Description .............................................. 164

Table 67. Zone and Schedule Description ............................................................................ 165

Table 68. Zone and Schedule Description .............................................................................. 166

Table 69. Public Assembly-1: Zone and Schedule Description ........................................ 167 


\section{List of Figures}

Figure 1. Locations of Buildings in Dataset (blue = buildings chosen for in-depth analysis)

Figure 2. Data Availability (part 1) ………………………………………………………......20

Figure 3. Data Availability (part 2) .........................................................................................21

Figure 4. "Flatlines" in Unmodified Data from Academic-1 ……………………………....23

Figure 5. Metering issues in Unmodified Lighting Data from Mail Sorting-3 ................24

Figure 6. Heating Loads in Unmodified Receptacle Data from Mail Sorting-3 .............26

Figure 7. Weekly Load Profile for Full Data Set (Office-4, Lighting) .................................28

Figure 8. Average, Median and Typical Energy Use Profiles (Office-4, Lighting) ........29

Figure 9. Weekday Energy Load Profile (Office-4, Lighting) ................................................30

Figure 10. Weekend Day Energy Load Profile (Office-4, Lighting) ...................................31

Figure 11. Total Daily Energy Use (Office-4, Lighting) .........................................................32

Figure 12. Academic-1: Full-year 60-Minute Interval Lighting Energy Use Data........45

Figure 13. Academic-1: Average, Median, and Typical Weekly Profiles for Lighting Energy Use ......................................................................................................................46

Figure 14. Academic-1: Average, Median, and Typical Weekday Lighting Profile......46

Figure 15. Academic-1: Average, Median, and Typical Weekend Day Lighting Profile

Figure 16. Academic-1: Full-year Total Daily Lighting Energy Use Data ........................48

Figure 17. Academic-1: Lighting Energy Use Versus Sunshine Hours Regression $\left(\mathrm{r}^{2}=\right.$ 0.45 , slope $=-0.26$ )

Figure 18. Academic-1: Full-year 60-Minute Interval Receptacles Energy Use Data 51

Figure 19. Academic-1: Average, Median, and Typical Weekly Profiles for

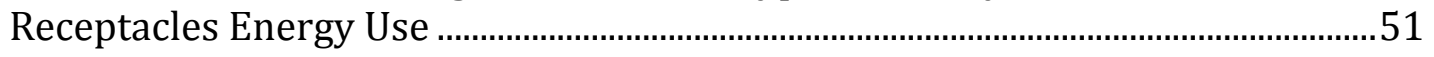

Figure 20. Academic-1: Average, Median, and Typical Weekday Receptacles Profile

Figure 21. Academic-1: Average, Median, and Typical Weekend Day Receptacles Profile 
Figure 22. Academic-1: Full-year Total Daily Receptacles Energy Use Data .................53

Figure 23. Office-3: Full-year 60-Minute Interval Lighting Energy Use Data................56

Figure 24. Office-3: Average, Median, and Typical Weekly Profiles for Lighting

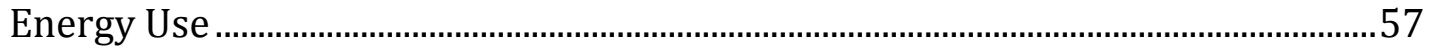

Figure 25. Office-3: Average, Median, and Typical Weekday Lighting Profile ..............57

Figure 26. Office-3: Average, Median, and Typical Weekend Day Lighting Profile.....58

Figure 27. Office-3: Public Assembly-1: Full-year Total Daily Lighting Energy Use Data.

Figure 28. Office-3: Lighting Energy Use Versus Sunshine Hours Regression $\left(\mathrm{r}^{2}=\right.$

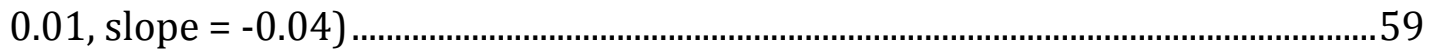

Figure 29. Office-3: Full-year 60-Minute Interval Receptacles Energy Use Data ........61

Figure 30. Office-3: Average, Median, and Typical Weekly Profiles for Receptacles

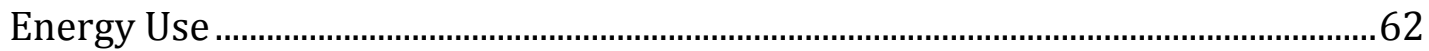

Figure 31. Office-3: Average, Median, and Typical Weekday Receptacles Profile.......62 Figure 32. Office-3: Average, Median, and Typical Weekend Day Receptacles Profile

Figure 33. Office-3: Full-year Total Daily Receptacles Energy Use Data ........................64

Figure 34. Office-4: Full-year 60-Minute Interval Lighting Energy Use Data...............66

Figure 35. Office-4: Average, Median, and Typical Weekly Profiles for Lighting Energy Use

Figure 36. Office-4: Average, Median, and Typical Weekday Lighting Profile 68

Figure 37. Office-4: Average, Median, and Typical Weekend Day Lighting Profile.....68

Figure 38. Office-4: Public Assembly-1: Full-year Total Daily Lighting Energy Use Data.

Figure 39. Office-4: Lighting Energy Use Versus Sunshine Hours Regression $\left(\mathrm{r}^{2}=\right.$ 0.36 , slope $=-0.13$ ) 70

Figure 40. Office-4: Full-year 60-Minute Interval Receptacles Energy Use Data ........71

Figure 41. Office-4: Average, Median, and Typical Weekly Profiles for Receptacles Energy Use .72

Figure 42. Office-4: Average, Median, and Typical Weekday Receptacles Profile.......73 
Figure 43. Office-4: Average, Median, and Typical Weekend Day Receptacles Profile

Figure 44. Office-4: Full-year Total Daily Receptacles Energy Use Data .........................74

Figure 45. Mail Sorting-1: Full-year 60-Minute Interval Lighting Energy Use Data...77

Figure 46. Mail Sorting-1: Average, Median, and Typical Weekly Profiles for Lighting Energy Use. .78

Figure 47. Mail Sorting-1: Average, Median, and Typical Weekday Lighting Profile.78

Figure 48. Mail Sorting-1: Average, Median, and Typical Weekend Day Lighting Profile 79

Figure 49. Mail Sorting-1: Full-year Total Daily Lighting Energy Use Data 79

Figure 50. Mail Sorting-1: Lighting Energy Use Versus Sunshine Hours Regression $\left(r^{2}=0.43\right.$, slope $\left.=0.13\right)$ 80

Figure 51. Mail Sorting-1: Full-year 60-Minute Interval Receptacles Energy Use Data

Figure 52. Mail Sorting-1: Average, Median, and Typical Weekly Profiles for Receptacles Energy Use

Figure 53. Mail Sorting-1: Average, Median, and Typical Weekday Receptacles Profile.

Figure 54. Mail Sorting-1: Average, Median, and Typical Weekend Day Receptacles Profile.

Figure 55. Mail Sorting-1: Full-year Total Daily Receptacles Energy Use Data. .84

Figure 56. Mail Sorting-2: Full-year 60-Minute Interval Lighting Energy Use Data...87

Figure 57. Mail Sorting-2: Average, Median, and Typical Weekly Profiles for Lighting Energy Use.

Figure 58. Mail Sorting-2: Average, Median, and Typical Weekday Lighting Profile.89

Figure 59. Mail Sorting-2: Average, Median, and Typical Weekend Day Lighting Profile .90

Figure 60. Mail Sorting-2: Full-year Total Daily Lighting Energy Use Data 90

Figure 61. Mail Sorting-2: Lighting Energy Use Versus Sunshine Hours Regression $\left(r^{2}=0.00\right.$, slope $\left.=-0.01\right)$

Figure 62. Mail Sorting-2: Full-year 60-Minute Interval Receptacles Energy Use Data 
Figure 63. Mail Sorting-2: Average, Median, and Typical Weekly Profiles for Receptacles Energy Use

Figure 64. Mail Sorting-2: Average, Median, and Typical Weekday Receptacles Profile

Figure 65. Mail Sorting-2: Average, Median, and Typical Weekend Day Receptacles Profile

Figure 66. Mail Sorting-2: Full-year Total Daily Receptacles Energy Use Data. 95

Figure 67. Mail Sorting-3: Full-year 60-Minute Interval Lighting Energy Use Data...98

Figure 68. Mail Sorting-3: Average, Median, and Typical Weekly Profiles for Lighting Energy Use. 99

Figure 69. Mail Sorting-3: Average, Median, and Typical Weekday Lighting Profile.99

Figure 70. Mail Sorting-3: Average, Median, and Typical Weekend Day Lighting Profile 100

Figure 71. Mail Sorting-3: Full-year Total Daily Lighting Energy Use Data 100

Figure 72. Mail Sorting-3: Lighting Energy Use Versus Sunshine Hours Regression $\left(r^{2}=0.42\right.$, slope $\left.=-0.05\right)$ 101

Figure 73. Mail Sorting-3: Full-year 60-Minute Interval Receptacles Energy Use Data

Figure 74. Mail Sorting-3: Average, Median, and Typical Weekly Profiles for Receptacles Energy Use 103

Figure 75. Mail Sorting-3: Average, Median, and Typical Weekday Receptacles Profile

Figure 76. Mail Sorting-3: Average, Median, and Typical Weekend Day Receptacles Profile 104

Figure 77. Mail Sorting-3: Full-year Total Daily Receptacles Energy Use Data......... 105 Figure 78. Public Assembly-1: Full-year 60-Minute Interval Lighting Energy Use Data 108

Figure 79. Public Assembly-1: Average, Median, and Typical Weekly Profiles for Lighting Energy Use.

Figure 80. Public Assembley-1: Average, Median, and Typical Weekday Lighting Profile 109

Figure 81. Public Assembley-1: Average, Median, and Typical Weekend Day Lighting Profile 110 
Figure 82. Public Assembly-1: Full-year Total Daily Lighting Energy Use Data ....... 110

Figure 83. Lighting Energy Use Versus Sunshine Hours Regression $\left(\mathrm{r}^{2}=0.28\right.$, slope = $0.17)$ 111

Figure 84. Public Assembly-1: Full-year 60-Minute Interval Receptacles Energy Use Data. 113

Figure 85. Public Assembly-1: Average, Median, and Typical Weekly Profiles for Receptacles Energy Use

Figure 86. Public Assembley-1: Average, Median, and Typical Weekday Receptacles Profile. 114

Figure 87. Public Assembley-1: Average, Median, and Typical Weekend Day Receptacles Profile.

Figure 88. Public Assembly-1: Full-year Total Daily Receptacles Energy Use Data 116 Figure 89. Actual Versus Predicted (calibrated) Total Building Energy Use ( $n=34)$

Figure 90. Actual Versus Predicted (calibrated) Total Energy Use by Type (natural gas- $n=27$, electricity- $n=34$ ). 118

Figure 91. Actual Versus Predicted Total Energy Use Per End-use Breakdown (heating- $n=8$, cooling- $n=8$, lighting $n=17$, receptacles- $n=12$ ).

Figure 92. High Data Variability Seen in Lighting Load Profile for Public Assembley-1

Figure 93. Low Data Variability Seen in Receptacle Load Profile for Mail Sorting-2

Figure 94. Regression Analysis- Error vs. Data Variability $\left(r^{2}=0.34\right.$, slope $\left.=0.002\right)$

Figure 95. Actual Versus Predicted (calibrated) Total Building Energy Use. 149

Figure 96. Actual versus Predicted (calibrated and uncalibrated) Total Building Energy Use.

Figure 97. Actual Versus Predicted (calibrated) Total Energy Use by Type...... 150

Figure 98. Actual Versus Predicted (calibrated and uncalibrated) Total Energy Use by Type. 150

Figure 99. Lighting Power Density Summary . 151

Figure 100. Academic-1: Hourly Breakdowns for Lighting Schedule A. 152 
Figure 101. Office-3: Hourly Breakdowns for Lighting Schedules A and B................ 153

Figure 102. Office-4: Hourly Breakdowns for Lighting Schedules A and B................. 155

Figure 103. Mail Sorting-1: Hourly Breakdowns for Lighting Schedule A....................156

Figure 104. Mail Sorting-2: Hourly Breakdowns for Lighting Schedule A................... 157

Figure 105. Mail Sorting-3: Hourly Breakdowns for Lighting Schedule A................... 158

Figure 106. Hourly Breakdowns for Lighting Schedules A and B................................. 159

Figure 107. Hourly Breakdowns for Receptacles Schedule A ........................................161

Figure 108. Office-3: Hourly Breakdowns for Receptacles Schedules A and B ......... 162

Figure 109. Office-4: Hourly Breakdowns for Receptacles Schedules A and B ......... 164

Figure 110. Mail Sorting-1: Hourly Breakdowns for Receptacles Schedules A and B

Figure 111. Hourly Breakdowns for Receptacles Schedule A ........................................166

Figure 112. Hourly Breakdowns for Receptacles Schedule A .........................................167

Figure 113. Public Assembly-1: Hourly Breakdowns for Receptacles Schedules A and

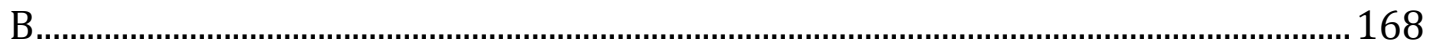

Figure 114. Office-4: Meter Atribution................................................................................ 169

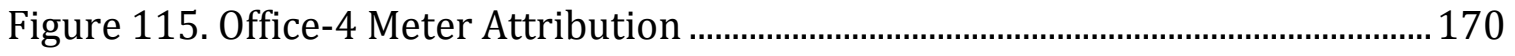

Figure 116. Mail Sorting-1: Lighting Panel, "A" .................................................................. 170

Figure 117. Mail Sorting-1: Receptacles Panel, "B”.............................................................171

Figure 118. Mail Sorting-3: Lighting Panel, "A" ................................................................... 172

Figure 119. Mail Sorting-3: Receptacles Panel, "B"............................................................ 173

Figure 120. Public Assembly-1: Lighting Panel, "L1"........................................................ 174

Figure 121. Public Assembly-1: Lighting Panel, "L2" ....................................................... 174

Figure 122. Public Assembly-1: Plugoad Panel, "P2" ...................................................... 175

Figure 123. Public Assembly-1: Receptacles Panel, "P3" ..................................................176 


\section{List of Appendices}

Appendix A- MMM's Modeling and Calibration Process .......................................148

Appendix B- Lighting Modeling Schedules .......................................................151

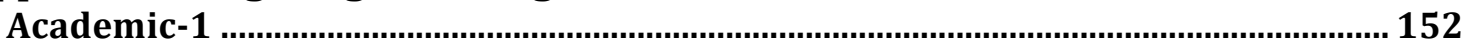

Office-3

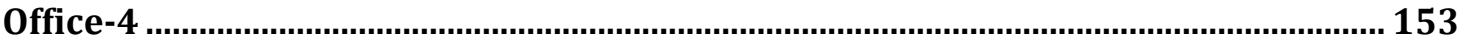

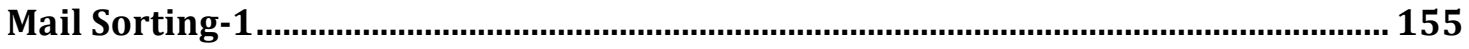

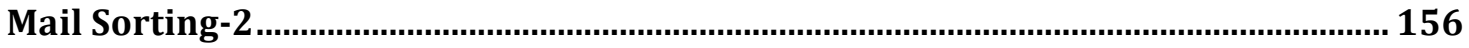

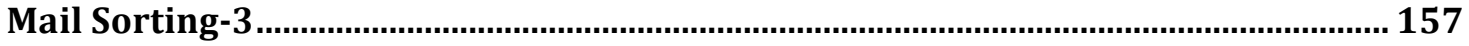

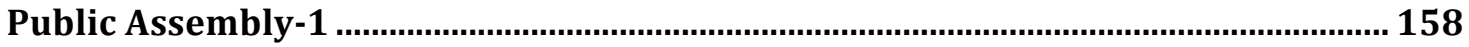

Appendix C- Receptacles Modeling Schedules ...............................................160

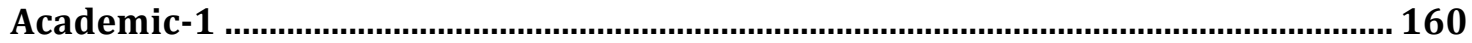

Office-3

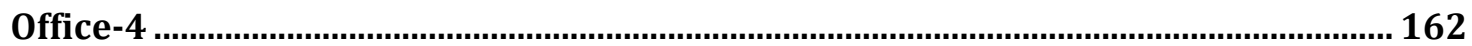

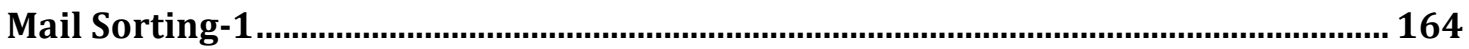

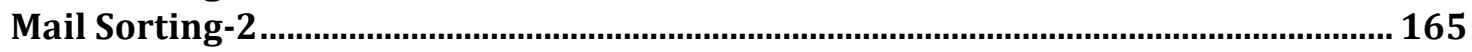

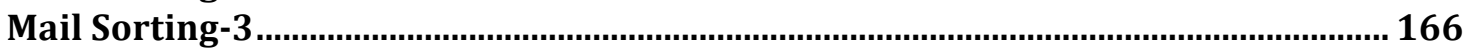

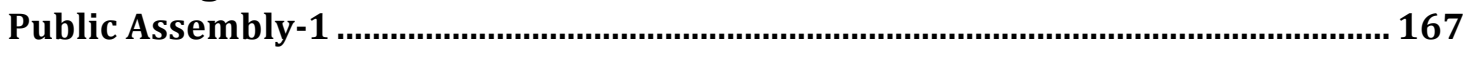

Appendix D- Meter Attribution Documentation ...............................................169

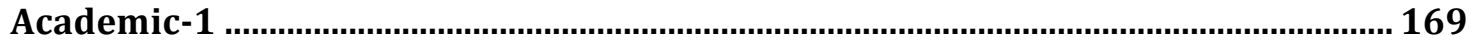

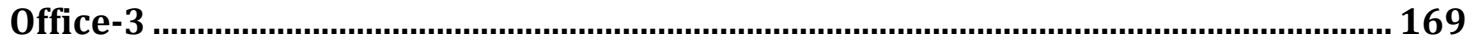

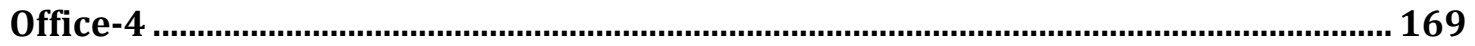

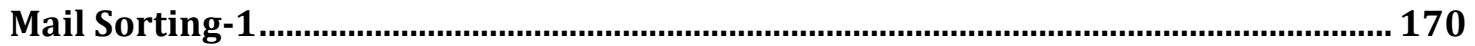

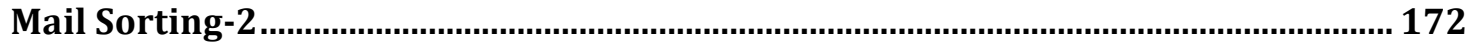

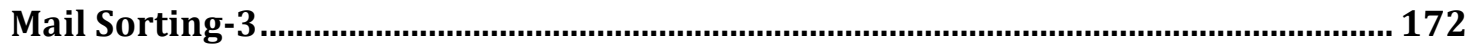

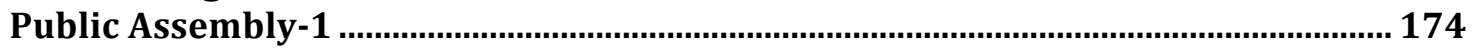

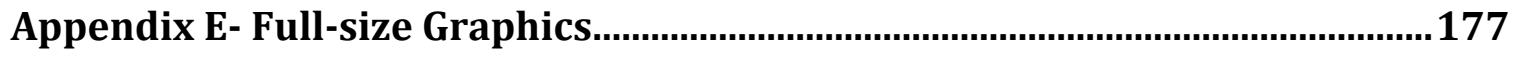




\subsection{Introduction}

The motivation for this research stems from the need to further develop the framework for evaluating buildings after they have been completed and occupied. This goal remains an important step in understanding how specific building technologies and design strategies are performing in our rapidly changing world of building design and construction.

This topic of study is gaining increased traction as research mounts indicating the large health and environmental impacts of buildings. Current estimates state that buildings are responsible for $30 \%$ of Canadian energy use- $40 \%$ in the U.S. (NRCan, 2006)(Lee et al., 2013). As well it is estimated that 50\% of buildings have issues with their building controls and $40 \%$ of buildings have faulty HVAC equipment (Piette, Nordman, \& Greenberg, 1994). The inefficiencies caused by these issues results in significant unnecessary expenditures to businesses. One study suggests that $\$ 18$ billion or more per year could be saved by addressing these shortcomings of building U.S. commercial building stock (Mills et al., 2004). Needless to say, improperly functioning HVAC affects occupant health and comfort. In regards to human costs, in the U.S. alone, health care and absenteeism costs due to poor indoor air quality are tallied at $\$ 10$ billion annually (ASHRAE, 2009).

Methodologies have been developed for improving building performance, such as building performance evaluations/post occupancy evaluations, benchmarking, and building commissioning. These processes have been enriched and deepened by the advances of technologies such as whole building energy modeling and building submetering. Up to this point, these techniques and procedures have remained for the most part distinct. Studies that analyze submetering data rarely have access to calibrated energy models. Building performance evaluations that make comparisons with energy models rarely discuss the calibration process or try to understand what the error levels between predicted and actual data are actually referring to. Studies that calibrate energy models rarely attempt to reconcile difference they measure to any real life phenomenon whether a result of occupancy issues or lack of information available to the modelers. 
This research hopes to explore the synergies between these techniques and technologies in the examination of a data set of Southern Ontario buildings that were modeled and submetered as part of Enermodal Engineering's (now MMM Engineering) measurement and verification (M\&V) program.

\subsection{Research Objectives}

The overall objectives of this thesis include the following:

- Better understand the divergence between energy models and building energy use, and identify the causes for the divergence.

- Better understand the role of calibrated energy modeling in diagnosing building performance issues.

\subsection{Research Questions}

The specific research questions that this thesis will investigate include:

- What is the range of difference between calibrated whole building energy model energy consumption predictions and actual metered building energy consumption (for total energy use as well as for particular end uses)?

- To what extent can remaining difference between calibrated and actual energy use be explained through additional information made available through high resolution (1 hour) end-use submetering?

- What factors affecting lighting and receptacle energy use can be made available through high resolution (1 hour) end-use submetering?

- What methodologies are best for making use of submetering data? 


\subsection{Literature Review}

\subsection{Building Performance Evaluations}

Building performance evaluations (BPE) have been recognized for the past several decades as important tools to understand the performance of buildings, however the process of adoption has been slow to materialize (Preiser, \& Vischer, 2005). Some of the barriers to a more widespread acceptance of BPEs the relative cost and time requirements to complete one, the fear of designers and building owners of critique and bad publicity (or even litigation), as well as the fact that buildings are very complex systems that extend beyond the physical and technical systems, including the psychological and social aspect of individuals expectation for buildings, therefore making study and evaluation challenging (Preiser, \& Vischer, 2005).

The goals of BPEs can be broadly organized into two groups, improving the satisfaction of the buildings occupants, as well as improving the performance of the building in regards to energy consumption and cost of operations. The first group is primarily interested in improving indoor environmental quality towards the end of reducing health impacts of buildings, and the second is primarily interested in improving the energy efficiency of these buildings. These two goals should not however be separated too wholly, as a myriad of mechanical and behavioural issues can effect the desired outcomes on either end.

In examining the energy use of buildings term "performance gap" is used to signify the difference between expected design outcomes and those actually realized. Sometimes this gap is conceptualized as how high performance buildings perform in relation to more traditional building stock (Scofield, 2009; Newsham, Mancini, \& Birt, 2009; Diamond et al., 2006; Fowler \& Rauch, 2008; Turner \& Frankel, 2008; Baylon \& Storm, 2008), but for the most part it is defined in relation to whole building energy models, either design phase (Diamond et al., 2006) or calibrated. Various studies have found that measured electricity demand is between $60-85 \%$ greater than expected (Poirazis et al., 2008), and that on average total 
energy use is approximately $30 \%$ higher than expected, with a large range of values observed (Menezes et al., 2011).

This kind of research has only been made possible in the last several decades due to the widespread availability of personal computers. Traditionally it was difficult to understand a building's energy performance simply because there was no baseline with which to compare a building. While energy use intensity gives provides a certain basis for comparison, no matter how standardized construction has become, every building is essentially unique, with unique contexts occupancies and occupants, and thus direct comparison remains quite tenuous.

In some cases the research regarding the performance gap has only sought to characterize the magnitude of the gap (Scofield, 2009; Newsham, Mancini, \& Birt, 2009; Diamond et al., 2006; Fowler \& Rauch, 2008; Turner \& Frankel, 2008; Baylon \& Storm, 2008), while in others they have taken the logical next step to try and identify solutions for reducing the gap in buildings (InnovateUK, 2014). Furthermore, advances in the availability of metering technology has allowed researchers to move beyond basic utility data to gain a more profound insight into the buildings (Chisholm, 2009; Torcellini et al., 2006). Studies researching the performance gap have made strides to include large numbers of buildings in order to gain broad insight, as of yet this has been less possible for studies that include higher resolution energy use data (ie. submetered and at frequent time intervals).

The literature on performance gaps have identified a number of areas that are likely crucial to address in order to explain and provide solutions for the phenomenon. These areas of concern include: as-built differences, especially in regards to air tightness and thermal bridging; unregulated loads, which on occasion can make up a significant part of a building's energy use (Innovate UK, 2014); operating hours or special functions; occupant density; and building management (Innovate UK, 2014). Post-occupancy evaluation documents that investigate issues of this sort can provide a lot of practical information for informing best practice design of high performance buildings (Innovate UK, 2014; Torcellini et al., 2006).

The need to address this phenomenon has even started to gain traction outside of the research community with leading engineering firms such as Arup 
recognizing the importance of ensuring building performance after construction (Arup, 2013).

\subsection{Whole Building Energy Modeling and Energy Model Calibration}

With the spread of personal computing and energy modeling software it has become possible to approximate a buildings energy use before the building is actually constructed. This has provided a benchmark for building performance that has never before been available. However it is becoming increasingly apparent that design-phase models provide inaccurate estimations, as is well demonstrated in numerous studies (Samuelson, Ghorayshi, \& Reinhart, 2015; Newsham, Mancini, \& Birt, 2009; Ahmad \& Culp, 2006; Torcellini et al., 2006; Norford, Socolow, Hsieh, \& Spadaro, 1994). ASHRAE 189.1 specifically alerts practitioners to this fact (ANSI, ASHRAE, USGBC, \& IES, 2010).

This has led the energy modeling community to primarily focus on producing models specifically for relative comparisons of buildings to standardized baselines. The Ontario Building Code's (OBC) 2006 SB-10 update offers compliance packages that require a full building energy model to compare favorably (i.e. $25 \%$ less than MNECB or 5\% less than ASHRAE 90.1-2010) to a prescriptively designated building in the same software.

This being said there still exists interest in investigating the application of energy models to understanding the energy use of buildings after operation of the building has begun. This generally includes a process called energy model calibration (or sometimes recalibration). Energy model calibration is the process of updating an energy model to reflect actual conditions more closely. This often involves updating the assumptions used in the design phase model for building operating conditions such as magnitude of receptacles, operating hours, and as-built 
design plans, as well as including "unregulated loads" that were not included in original models. ${ }^{1}$

Initial studies on energy model calibration lacked a well-defined and standardized methodology to direct the process. Due to this, some of these studies fell into the trap of using ad-hoc procedures highly dependent on personal judgment. Analysts tended to focus on matching the model to a prescribed acceptance criteria through a process of tuning or even "fudging" data until the desired fit is found between performance data and the given model (Raferty, Keane, \& O’Donnell, 2011; Reddy, 2006). This has been dubbed "results oriented calibration". This has reached an apex in form of research involving parametric analysis or computer learning algorithms to match the energy model to the metered building data as closely as possible (see for example O'Neill and Eisenhower's 2013 paper).

In response to this a number of papers have attempted to articulate an alternative "evidence-based calibration" procedure (Raferty, Keane, \& O’Donnell, 2011). "Evidence-based calibration" have been explicitly accepted in quite a range of calibration studies (Samuelson, Ghorayshi, \& Reinhart, 2014; Im \& Bhandari, 2014). The basic maxim of this procedure is to only ever replace data in a model with data that is known to be more accurate. In the words of Raferty, Keane, and O'Donnell (2011): "This will improve the accuracy of the model by guaranteeing that only verifiable data about the building is used in the calibration process". It follows from this that in calibrating a model the goal is not necessarily to reduce the difference between the model and the actual data. The goal is to reduce the error associated with this value, and thus to make any difference more meaningful.

These papers seem to agree that the remaining downfall is that the process of calibration will remain very time consuming. One study attempts to address this challenge while relying on an evidence-based methodology (Samuelson, Ghorayshi,

\footnotetext{
${ }^{1}$ Unregulated loads are loads that are not accounted for in prescriptive energy modeling compliance packages, thus their magnitude is not regulated by building codes (hence unregulated). These can include, for example, exterior lighting.
} 
\& Reinhart, 2014; Coakley et al., 2012). The study attempts to determine the highest impact calibration steps such that a simplified calibration process can be identified.

On average the two most important steps were identified to be revising receptacles and adding unregulated loads. Surprisingly the addition of more accurate weather data was found to have a smaller impact, in fact in some cases it was shown to actually increase the difference between modeled and actual data. Overall the calibration process reduced the difference between the actual energy use and the modeled energy use from an average of $36 \%$ under-predication to an average $7 \%$ under-prediction.

Energy model calibration relies on several statistical techniques in order to precisely quantify the difference between predicted and actual energy use data sets. These techniques are described in ASHRAE's Guideline 14 (ASHRAE, 2002). Guideline 14 was written in order to standardize the communication of energy and demand savings in commercial transactions. As they point out, energy savings cannot be directly measured. It isn't possible to measure the absence of something. Therefore Guideline 14 describes a methodology for comparing measured energy use post-reduction to the measured energy use pre-reduction normalized to more faithfully represent the conditions that would have occurred had the energy use reduction measure not been enacted.

Out of the various methodologies outlined in Guideline 14, the energy model calibration literature often relies on two statistical terms normalized mean bias error (NMBE) and the coefficient of variation of the root mean square error (CVRMSE). Their formulas will be introduced in Section 3.10 in relation to their direct relevance to this thesis. These terms are introduced in Guideline 14 as describing how well a mathematical model describes variability in the data that it is representing. ASHRAE suggest that models can be considered adequately calibrated when CVRMSE and NMBE meet a certain threshold (p.15, 2002). As a result in many research papers dealing with energy model calibration, both for "results-based calibration" and "evidence-based calibration", the success of the projects are primarily measured in the models adherence to these stipulated limits. This approach fails to attempt any sort of explanation of the observed difference. 
Generally speaking this is due to a lack of available data for which to conduct an analysis.

Another complication within the world of energy modeling, especially in regards comparing energy modeling results with actual energy use, is the differences between various modeling software. Certain software is used specifically in the case for proving code compliance (such as EE4 in Canada) and other software is aimed more towards providing a tool for academic researchers, such as EnergyPlus. Code compliance software is generally not intended to necessarily provide a realistic assessment of actual energy use only to provide a comparison to a benchmark building that represents the building code (Khan, 2007). Furthermore the simpler code compliance software often does not have the ability to include a number of more advanced mechanical systems found in high performance buildings, such as radiative cooling in EE4 (Tian, Love \& Tian, 2009). However with careful use has been found that similar results can be produced through a variety of different software (Tian, Love \& Tian, 2009; Khan, 2007).

\subsection{Submetering}

This process of calibration has been helped along by the advent of more widespread use of energy submetering, which allows a finer detail understanding of energy use within a building. This is sometimes called time resolved building load data (Price, Mathieu, Kiliccote, \& Piette, 2011). The trend is in keeping with larger amounts of data being produced in all areas of life. One recent statistic reports that $90 \%$ of the data generated by the world has been generated over the last two years (SINTEF, 2013).

And while it is seen as highly desirable to use more detailed data in the calibration process (Raferty, Keane, \& O'Donnell, 2011), it is also recognized as often being difficult (Lee, 2013; Brown et al., 2010). Lee suggests that the majority of data generated on building operations, energy consumption, and other activities 
(collected through building management systems, sensors, and meters) is mostly discarded.

As well much of the work on energy model calibration, such as Samuelson, Ghorayshi, and Reinhart, relies on less detailed monthly data. Some studies that do have access to high-resolution submetering data do not attempt to use it to examine broader issues of building performance beyond the standard evaluations (Coakley et al., 2012; Raferty et al., 2011; Torcellini et al., 2006). As it stands it would appear that this is due to the fact that few methodologies have been developed to produce useful insights with a data set of this nature. This being said strategies are being developed in order to make use of detailed energy use data.

One such study, Price, Mathieu, Kiliccote, and Piette (2011), describes a methodology for analyzing submetered data. They suggest a regression analysis method using time of week (a term describing both day of week and time of day, and outdoor weather conditions) resolved to a level of detail matching the energy data. With this hybrid weather normalization technique it is possible to identify which energy use is not attributable to either external weather conditions or normal operating conditions. They also describe a set of definitions in which to describe daily load profiles. These terms allow an important framework for examining energy use. For example plotting "near-peak load", defined as 96.5 percentile of daily load, can show changes in peak energy use over a given period. Terms like these can also profitably be weather normalized with regression analysis in order to gain insight. For example identifiying a morning start-up peak can indicate that the building is entering the morning ramp-up too early. These methods allow an analyst to describe high-level building scale performance issues. However they do not provide a methodology for identifying specific problems. This may still prove to be an important initial step in examining building energy use. In fact researchers have begun to use techniques characterizing building load profiles in order to generate a better basis for the assumptions used in energy models (Cory, Donn, \& Pollard, 2015).

Researchers continue to push the envelope as new types and volumes of data become available. For large long-term data sets, data mining becomes increasingly 
attractive for analyzing patterns of energy (or water) use from submetering data. An example of this is available in Oliver and Peach's 2013 article. Their study was able to identify leaks in domestic water use meters and quantify the percent of water wasted through leaks, which demonstrates an interesting proof of concept however difficult to apply in the case of submeters that are monitoring anything more than a specific set of isolated loads. In other words the leaks were identified because water use is concentrated to a few short periods of the day, which may not be possible, for example, with building energy use.

Technological issues continue to stand in the way of accurate submetering with various issues (such as meter calibration problems, formatting issues, or transmission issues) affecting the ability of researchers to effectively analyze data (Brown et al., 2010). Technological solutions strategies to address these problems are very highly dependent on the particularities of any given situation. Addressing the problems in the data usually requires various types of data cleaning procedures, such as remove excessively large loads (Brown et al., 2010). Brown et al. caution that excessively data cleaning can quickly distort genuine data. On the other hand some very low-tech problems still hamper this genre of research, such as incorrect floor area figures. Furthermore Brown et al. suggest that no estimated data should be used to replace missing data, however no further discussion on this topic is used to support their reasoning on this point. Interestingly the same study, had used data interpolation, as mentioned in a previous version of their article (Wright \& Brown, 2008).

The length of submetering trends varies from study to study, from several weeks (Masosos \& Grobler, 2010), to several years (Brown et al., 2010). The length of the data is relevant to the types of insight that can be gathered, with extended periods of energy consumption data allowing for inter seasonal variations to be observed, and with multi-year energy use data able to provide evidence of long term trends. Brown et al. investigated the multi year trends in receptacle energy use, their study indicated some evidence of a year-to-year increase between 2002 and 2008. They concede that not enough data is available to pinpoint the causes of the increase. 


\subsection{Factors Influencing End-use Energy Consumption}

The ultimate goal of obtaining the submetering data is to produce insights on the way energy use occurs in buildings. The literature investigating this topic has identified roughly speaking three categories of issues affecting energy use, occupancy related, management related, and technological (including both envelope issues and mechanical system issues). The first two, occupant and management issues affecting energy use, are matters of determining the exact extent various behaviours drive energy use, and ultimately how much of that energy use could be considered wasteful, and thus curtailed. The line behind management and occupancy effects is often blurred especially when considering electrical end-use in buildings, such as lighting and receptacle loads. In the case of lighting and receptacles loads some of the wasteful energy use will be a result of management issues (such as purposefully leaving on lighting in the building for security reasons) and occupant behaviour (such as forgetting to turn off the lights). Furthermore it can be confusing because the effects occupants have on energy use can broadly fall into two categories, energy use and waste that is occurring as the occupant is present, and energy use that is occurring when the occupant is not present. Studying the former is more difficult than the later, due to the fact that occupancy values can be hard to obtain especially for building types that do not have stable occupancies, such as academic buildings or other public institutions such as libraries (Bartlett et al., 2014). In at least one study water usage was used as a proxy of occupancy values (Brown et al., 2010), although other more accurate means exist such as using infrared beams to count individuals entering and leaving the building (Gul \& Patidar, 2015). In order to get around this issue it is typical for studies to simply rely on studying energy waste that can be attributed to occupancy issues while the building is unoccupied (Masoso \& Grobler, 2010). Despite these difficulties energy use during occupied periods should be given their due recognition, as several studies have demonstrated that occupancy behaviour also wastes energy during occupied hours (Mahdavi et al., 2008; Lindelof \& Morel, 2006). 
These issues are sometimes conflated. In Masoso and Grobler it is shown that over $50 \%$ of total energy use is used in buildings during unoccupied hours, their immediate conclusion is that overnight energy use is caused by occupancy issues. However no real attempt is made to differentiate between some of the more nuanced issues raised above, such as the difference between occupant and management issues. This is however more fully explored in Webber et al. (2006), they indicate that for receptacle loads in offices (including computers, laptops, monitors, printers, faxes, and copiers) that fully two thirds of the energy use associated with the devices is due to improperly use of power management settings and due to devices being left on when not in use. At the very least, this statistic, that more than half of energy use is during unoccupied hours of building, which has also been found in several other studies (Webber et al., 2006; Staats et al., 2000), does indicate that much room is still left for the optimization of energy performance in buildings.

Despite a consensus that occupancy can have a general impact on buildings the specifics of the situation are open for debate. While studies such as Nguyen and Aiello (2013) have found that occupant presence can add 30\% onto a building's energy use, others such as Gul and Patidar (2015), have found much less impact. In their case, their results suggested that in buildings that rely heavily on building automation systems the impact of occupants can be minimal. When weighing these findings it is also important to know that Gul and Patidar's study focused on a single academic building, which engenders a specific type of use by the occupants. Offices would likely see at different type of usage pattern that may affect the type of measurements examined by Gul and Patidar.

Through analysis of submetering data, Brown et al.'s study (2010) identified four types of wasteful energy use (heating/cooling out of season, heating when building unoccupied, high base load consumption, excessive consumption). The majority of these unfortunately related to space conditioning. However they still provide an indication of the kind of management practices that can lead to excessive energy use, for example $31 \%$ of all the buildings in their study were heated during unoccupied days. 


\subsection{Methodology \\ 3.1 Data Set}

The data for this project was provided by MMM Group (previously Enermodal Engineering), a specialist building services engineering firm. The dataset was built over a period of approximately five years as part of MMMs measurement and verification $(\mathrm{M} \& \mathrm{~V})$ program, and includes calibrated whole building energy models as well as high resolution (often 15 minute intervals) submetering data. The majority of the models were completed using EE4, while a small minority used eQuest. Information regarding MMM's modeling process was gathered through discussions with members of MMM's modeling team, through modeling and calibration documentation, as well as through Samuelson, Ghorayshi, and Reinhart's 2015 paper (Samuelson, Ghorayshi \& Reinhart, 2015), which addresses a separate aspect of the data set used for this research.

The M\&V program provides building owners an opportunity to develop an improved understanding of the energy performance of their buildings and provides them the tools to take corrective action in buildings that are underperforming. As well the energy models were also often used for reasons of code compliance or for meeting the requirements of LEED submittals. The energy models were originally completed as per the requirements of the Performance Compliance for Buildings (NRCan, 1999) protocol. Adherence to this document is necessary to demonstrate compliance with the Model National Energy Code (MNECB) and LEED Canada 1.0. The compliance protocol encourages modelers to use default values for occupancy, receptacle power density, outdoor air ventilation requirements, and operations schedules (among others).

Calibration was conducted on the original models over the year following completion of the building. This process included updating energy model inputs with updated information, updating the typical weather files with actual weather conditions, and adding unregulated loads (such as exterior lights) to the totals generated by the models. Samualson et al. found that adding unregulated loads had the biggest impact on improving the accuracy of the energy model (Samuelson, 
Ghorayshi \& Reinhart, 2015). "Typical” weather data used in the design phase models is made available through a built in EE4 library, originally derived from the Canadian Weather for Energy Calculations (CWEC) database. "Actual" weather data was obtained from the National Climate Data and Information Archive.

Samuelson, Arayshi and Reinhart found that the calibration process reduced under prediction of energy consumption from $36 \%$ to $7 \%$. This research (with a more limited) found that the calibration process on average reduced the under prediction of energy consumption from $23 \%$ to $4 \%$ for total energy, that it on average reduced the under prediction of energy consumption for natural gas energy use from $22 \%$ to $14 \%$, and that it on average reduced the under prediction of energy consumption for lighting energy use from $22 \%$ to $0 \%$. Results of the calibration process are listed in Appendix A.

Energy use monitoring took place during the year after completion and while the buildings were undergoing commissioning. Energy monitoring was started approximately three months after full occupancy of the building.

\subsection{Building Breakdown}

The dataset includes 36 buildings with a range of occupancy types as well as sizes, from $416 \mathrm{~m} 2$ to $114,000 \mathrm{~m} 2$, and from arenas to academic buildings (as listed in Table 1). Figure 1 provides a map to show the relative geographic location of the buidlings in the data set.

Table 1. Breakdown of Initial Buildings

\begin{tabular}{|c|c|c|c|c|}
\hline Identifier & $\begin{array}{l}\text { Area } \\
\left(\mathbf{m}^{2}\right)\end{array}$ & Location & $\begin{array}{l}\text { Period of } \\
\text { Review }\end{array}$ & $\begin{array}{c}\text { Electricity End-use } \\
\text { Submetering* }\end{array}$ \\
\hline Academic-1 & 4,130 & $\begin{array}{l}\text { Hamilton, } \\
\text { ON }\end{array}$ & $\begin{array}{l}\text { Feb } 2011- \\
\text { Jan } 2012\end{array}$ & $\begin{array}{c}\text { Lighting, Receptacles, Cooling, } \\
\text { HVAC, DHW, Café }\end{array}$ \\
\hline Academic-2 & 7,063 & Orillia, ON & $\begin{array}{l}\text { April } 2011- \\
\text { Mar } 2012\end{array}$ & $\begin{array}{c}\text { "Lighting, Receptacles, Cooling } \\
\text { Pumps", Server, Heating } \\
\text { Pumps, Chiller, Ventilation, Lab } \\
\text { Exhaust }\end{array}$ \\
\hline Academic-3 & 9,624 & $\begin{array}{l}\text { Burlington, } \\
\text { ON }\end{array}$ & $\begin{array}{l}\text { Mar } 2011- \\
\text { Feb } 2012\end{array}$ & $\begin{array}{l}\text { Lighting, Café, Elevators, } \\
\text { Emergency Panel, Power } \\
\text { Panels, HVAC }\end{array}$ \\
\hline Academic-4 & 12,600 & $\begin{array}{l}\text { Hamilton, } \\
\text { ON }\end{array}$ & $\begin{array}{l}\text { June } 2010- \\
\text { May } 2011\end{array}$ & $\mathrm{n} / \mathrm{a}$ \\
\hline
\end{tabular}


$\begin{array}{cc}\text { Kitchener, } & \text { Dec } 2008- \\ \text { ON } & \text { Nov } 30\end{array}$

Arena-1 10,250

$\begin{array}{cccc}\text { Arena-2 } & 7,300 & \begin{array}{c}\text { Toronto, ON } \\ \text { July } 2009- \\ \text { June } 2010\end{array} \\ \text { MURB-1 } & 20,033 & \begin{array}{c}\text { Markham, } \\ \text { ON }\end{array} & \begin{array}{c}\text { July } 2011- \\ \text { July } 2012\end{array}\end{array}$

MURB-2 18,013 Markham, Sep $2010-$ ON Aug 2011

\begin{tabular}{|c|c|c|c|}
\hline MURB-3 & 11,971 & London, ON & $\begin{array}{l}\text { Aug } 2007- \\
\text { July } 2008\end{array}$ \\
\hline MURB-4 & 5,204 & $\begin{array}{c}\text { Chatham, } \\
\text { ON }\end{array}$ & $\begin{array}{c}\text { Jan } 2007- \\
\text { Mar } 2008\end{array}$ \\
\hline
\end{tabular}

\begin{tabular}{|c|c|c|c|}
\hline MURB-5 & 9,250 & Toronto, ON & $\begin{array}{c}\text { Dec } 2010- \\
\text { Nov } 2011\end{array}$ \\
\hline MURB- & 16,568 & $\begin{array}{c}\text { Brampton, } \\
\text { ON }\end{array}$ & $\begin{array}{c}\text { Dec } 2010- \\
\text { Nov } 2011\end{array}$ \\
\hline
\end{tabular}

MURB-7 45,700 Markham, Jan $2010-$ ON Dec 2010

\begin{tabular}{|c|c|c|c|}
\hline Office-1 & 2,154 & $\begin{array}{c}\text { Kitchener, } \\
\text { ON }\end{array}$ & $\begin{array}{c}\text { Sep } 2009- \\
\text { Nov } 2010\end{array}$ \\
\hline Office-2 & 27,000 & $\begin{array}{c}\text { Mississauga, } \\
\text { ON }\end{array}$ & $\begin{array}{c}\text { May } 2011- \\
\text { April } 2012\end{array}$ \\
\hline Office-3 & 7,934 & $\begin{array}{c}\text { Burlington, } \\
\text { ON }\end{array}$ & $\begin{array}{l}\text { Jan } 2009- \\
\text { Mar } 2010\end{array}$ \\
\hline Office-4 & 1,777 & $\begin{array}{c}\text { Thornbury, } \\
\text { ON }\end{array}$ & $\begin{array}{l}\text { Oct } 2011- \\
\text { Sep } 2012\end{array}$ \\
\hline Office-5 & 4,148 & $\begin{array}{c}\text { Cambridge, } \\
\text { ON }\end{array}$ & $\begin{array}{c}\text { Jan } 2009- \\
\text { Dec } 2009\end{array}$ \\
\hline
\end{tabular}

\begin{tabular}{|c|c|c|c|}
\hline Office-6 & 416 & Toronto, ON & $\begin{array}{c}\text { Feb } 2008- \\
\text { Jan } 2009\end{array}$ \\
\hline Office-7 & 5,575 & $\begin{array}{c}\text { Owen } \\
\text { Sound, ON }\end{array}$ & $\begin{array}{l}\text { Oct } 2008- \\
\text { Sep } 2009\end{array}$ \\
\hline Office-8 & 114,141 & Toronto, ON & $\begin{array}{c}\operatorname{Jan} 2011- \\
\operatorname{Dec} 2011\end{array}$ \\
\hline Office-9 & 1,037 & Vaughn, ON & July 2007 - \\
\hline
\end{tabular}

Lighting, Receptacles,

Dehumidification, HVAC

Equipment, Ice Plant, Exterior

Lighting, Concession, Exhaust

Fans

Lighting, Receptacles, Misc Refrigeration, "Pumps and Fans"

"Stair and Corridor Lighting", "Lobby, Media, Amenity, and

Exterior Lighting", Garage, Suites, Elevators, Misc Equipment, "Garbage, Pool, and Receptacles", Make-up Air Units, Pumps

"Stair and Corridor Lighting", "Lobby, Amenity, Media and Exterior Lighting", "Garbage, Pool and Receptacles", Suites, Elevators, Pumps, Make-up Air Units, Garage $\mathrm{n} / \mathrm{a}$

"Lighting, Receptacles, Heating and Cooling", Exterior Lighting, Mechanical Equipment, Misc Panel $\mathrm{n} / \mathrm{a}$

Receptacles, "Chiller and MUA", Snow Melt, Emergency Panel, Parking, Suite Panel 1, Suite Panel 2

Lighting, Receptacles, Pumps, Exterior Lighting, Ramp Heater, Elevator, Pool, Fans $\mathrm{n} / \mathrm{a}$

Lighting, "Emergency and Exterior Lighting", Chiller, Mechanical, Tenant Loads Lighting, Receptacles, Exterior Lighting, Elevator, Emergency Panel, Space Heating, Space Cooling, HVAC Lighting, Receptacles, IT,

"Mechanical and Heating", ERV, Pumps

Lighting, "Receptacles and Lab Equipment", Mechanical

Equipment, HVAC Fans, Space Cooling $\mathrm{n} / \mathrm{a}$

Lighting, Receptacles, Space Cooling, Misc Mechanical, HVAC Lighting, Retail, UPS, Common Area, Tenants, Mechanical "Lighting and Receptacles", 
June 2008

\begin{tabular}{|c|c|c|}
\hline Office-10 & 79,000 & Toronto, ON \\
\hline ffic & 51,000 & Toronto, ON \\
\hline
\end{tabular}

\begin{tabular}{|c|c|c|c|}
\hline Office-12 & 14,547 & $\begin{array}{l}\text { Markham, } \\
\text { ON }\end{array}$ & $\begin{array}{c}\text { Sep } 2008- \\
\text { Aug } 2009\end{array}$ \\
\hline & & Vaughn, ON & $\begin{array}{c}\text { Mar } 2008- \\
\text { Feb } 2009\end{array}$ \\
\hline
\end{tabular}

Office-13 11,971

\begin{tabular}{|c|c|c|c|}
\hline Office-14 & 10,750 & e. & - \\
\hline Office-15 & 10,879 & $\begin{array}{c}\text { Kitchener, } \\
\text { ON }\end{array}$ & $\begin{array}{r}\text { Dec } 2007- \\
\quad \text { Nov } 2008\end{array}$ \\
\hline $\begin{array}{c}\text { Mail } \\
\text { Sorting-1 }\end{array}$ & 1,730 & Ancaster, ON & $\begin{array}{c}\text { Oct } 2009- \\
\text { Sep } 2010\end{array}$ \\
\hline $\begin{array}{c}\text { Mail } \\
\text { Sorting-2 }\end{array}$ & 6,380 & Toronto, ON & $\begin{array}{c}\text { Feb } 2011- \\
\text { Jan } 2012\end{array}$ \\
\hline $\begin{array}{c}\text { Mail } \\
\text { Sorting-3 }\end{array}$ & 1,147 & $\begin{array}{c}\text { Woodstock, } \\
\text { ON }\end{array}$ & $\begin{array}{c}\text { Oct } 2009- \\
\text { Sep } 2010\end{array}$ \\
\hline $\begin{array}{l}\text { Public } \\
\text { Assembly-1 }\end{array}$ & 1,185 & Jordan, ON & $\begin{array}{c}\text { Oct } 2008- \\
\text { Sep } 2009\end{array}$ \\
\hline Retail-1 & 13,000 & $\begin{array}{c}\text { East } \\
\text { Gwillimbury, } \\
\text { ON }\end{array}$ & $\begin{array}{l}\text { July } 2008- \\
\text { June } 2009\end{array}$ \\
\hline Retail-2 & 10,485 & Welland, ON & $\begin{array}{l}\text { July } 2009 \\
\text { Sep } 2009\end{array}$ \\
\hline
\end{tabular}

School-1 5,436 Windsor, ON Sep 2011 Aug 2012

School-2 4,792 Midland, ON Sep 2008 -
Emergency Panel, Space

Heating, Space Cooling, "HVAC, Pumps and Fans" $\mathrm{n} / \mathrm{a}$

"Tenant Lighting and Receptacles", Parking, Penthouse, Retail, Elevators, Emergency Panel, Technical Power, Mechanical, Tenant Mechanical

"Lighting and Receptacles", "Server, UPS and Emergency", "HVAC Pumps and Fans"

"Lighting and Receptacles", "Cooling and Ventilation", Exterior Lighting, Kitchen, Emergency Panel

Lighting, Exterior Lighting, Space Cooling, HVAC Fans, Elevators, Misc. Equipment, HVAC Pumps

Lighting, Space Cooling, HVAC Fans, PC's and Servers, Misc Equipment, HVAC Pumps, Exterior Lighting

Lighting, Receptacles, Exterior Lights, Misc, Mechanical

Lighting, Receptacles, Misc, HVAC

Lighting, Receptacles, Exterior Lights, Misc, HVAC Fans

Lighting, Receptacles, Exterior Lighting, HVAC

Lighting, "Mechanical and

Misc", Space Cooling, Battery Charging, DHW, Busway

Lights, Receptacles, Emergency

Lighting, Garage, "Coolers and Receptacles", Electro-strip,

"HVAC and Misc", "Pumps and Misc", "Mechanical"

Lighting, Receptacles, "Parking Lot, Lobby, and Corridor Lighting", "P1 and P2", Kitchen, Ancillary Equipment, Heat Pump, AHU, HVAC Pumps $\mathrm{n} / \mathrm{a}$

*composite meters are indicated in quotation marks 


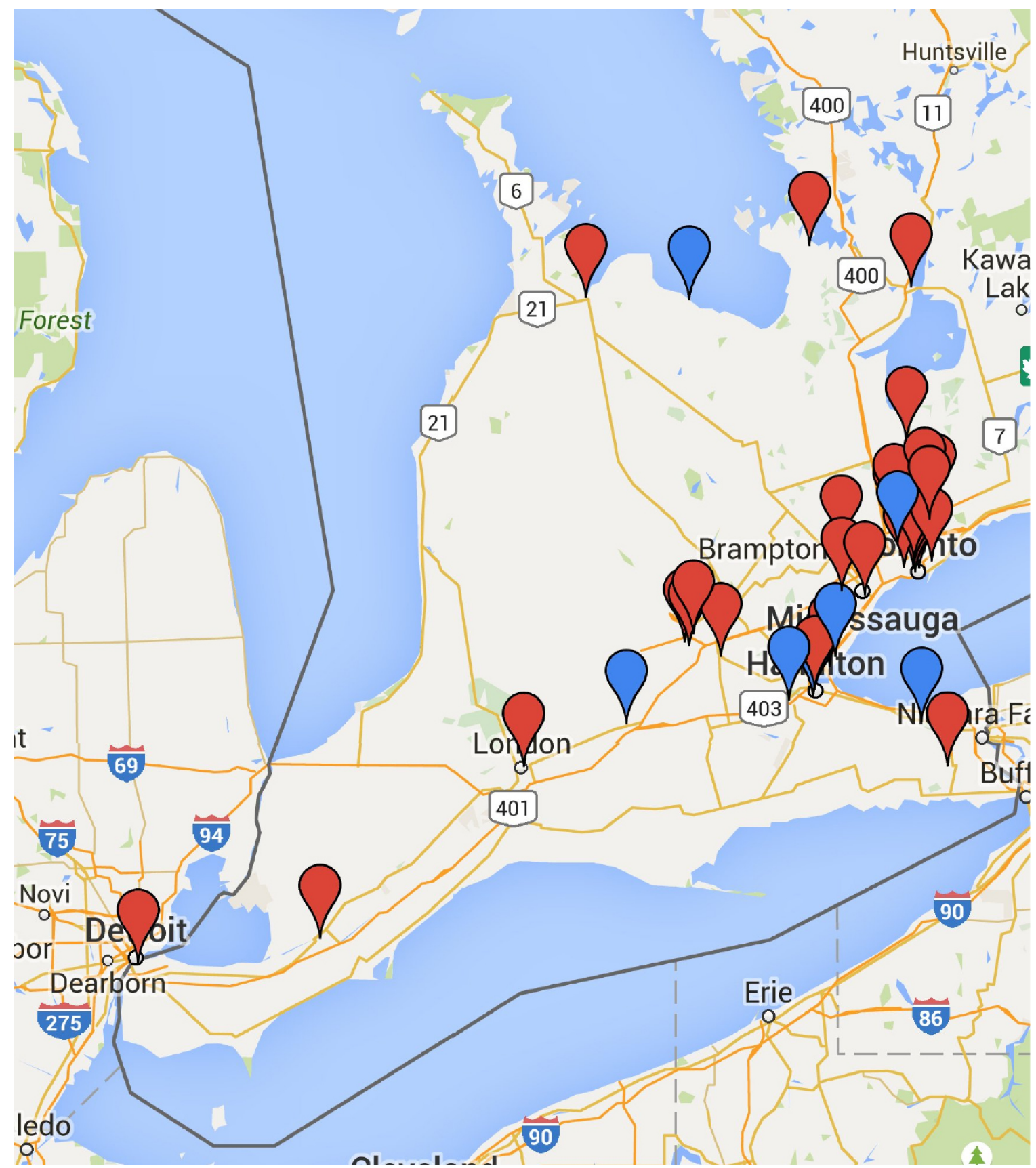

Figure 1. Locations of Buildings in Dataset (blue = buildings chosen for in-depth analysis)

\subsection{Data Sources and Availability}

All data in relation to the projects was made available through a variety of documents. The availability of each document varied from building to building. In general these documents included: 
- $M \& V$ reports made available quarterly to the clients to report on the performance of the building. These documents include energy consumption figures, as well as briefly discuss any issues that were occurring with building operations or building monitoring.

- Modeling reports were produced as part of the peer review process associated with LEED compliance energy modeling. The vast majority of these documents did not include the appendices, which contained the bulk of the relevant information describing the features of the buildings.

- $M \& V$ plans were documents provided to the clients describing the $M \& V$ procedure, these documents varied little from building to building.

- Modeling input and output files, including weather files. For the most part these files were well archived, clearly labeled, and fully available.

- Calibration documentation spreadsheets, these spreadsheets were for internal use to document the calibration process for each building. For the vast majority of buildings these documents these were complete enough to identify the correct calibrated modeling file.

- Shop drawings describing metering systems. These drawings occasionally described breakdowns of the electrical panels being metered, but more often than not only noted the location of the meters.

- Raw submetering files, the various submetering systems produced outputs in a variety of formats. Monitoring primarily took the form of 15-minute interval submetered data. Although 5-minute, daily, weekly, and monthly interval data also occurred within the dataset. The data and meters were from a variety of different systems, and known to have different levels of accuracy. In the case of the daily, weekly and monthly data, manual readings were primarily used. As such data data was not always logged at consistent intervals.

- Energy tabulation spreadsheets, for some buildings the raw submetering files had already been collated into a spreadsheet format.

This data allowed for all 36 buildings to be used in the overall comparison of metered data with calibrated energy models. However, a significant portion was found unsuitable for more detailed analysis of individual energy uses. Out of the 
total, 7 buildings were available for analysis with both lighting and receptacle energy use data. The exclusion of other buildings was due to the following factors.

- 8 were excluded due to undesirable building type. This primarily includes various types of multi-unit residential buildings, such as rental units, social housing and nursing homes.

- 11 buildings were excluded because of inadequate or missing data. This primarily includes buildings that 1) did not have separate submetering for the categories of heating, cooling, lighting or receptacles, 2) did not have calibration documentation required to determine and verify the identity of the correct version of the calibrated model, or 3) did not have any identifiable submetered energy data besides utility bills.

- 5 additional buildings have limited usefulness because the submetering data is only available for daily, weekly or monthly intervals.

- 4 buildings have 15 minute interval submetering which contained errors or abnormalities which prevented its inclusion.

- 2 Buildings exclusively have heating and cooling data.

It should be mentioned that within this subset there is variations in the quality of the data. For example some buildings have months of missing data that may prove to invalidate the usefulness of those buildings, and some buildings have submetering data that is not strictly segregated by end-use (such as some heating equipment being included with the receptacle loads for Mail Sorting-1 and Mail Sorting-3). A full breakdown for the availability of data for each building is as depicted in Figure 2 and Figure 3. Blank cells indicate the presence of that data was not verified to do prior exclusion from possible buildings. 


\begin{tabular}{|c|c|c|c|c|c|c|c|c|}
\hline Building Name & $\begin{array}{l}\text { Area } \\
{\left[\mathrm{m}^{2}\right]}\end{array}$ & 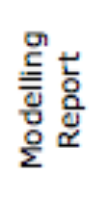 & $\begin{array}{l}\frac{c}{\frac{c}{\alpha}} \\
\frac{d}{2} \\
\frac{d}{\Sigma}\end{array}$ & 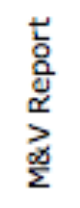 & 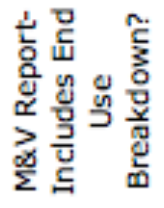 & 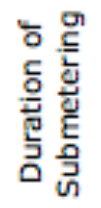 & 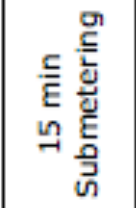 & 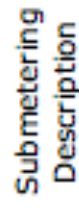 \\
\hline \multicolumn{9}{|c|}{ Lighting and Plugloads } \\
\hline Public Assembly-1 & 1,185 & NO & YES & YES & All & Year & $15 \mathrm{~min}$ & YES \\
\hline Public Admin-1 & 1,730 & YES & YES & YES & All & $4 \mathrm{mo}$ & $15 \mathrm{~min}$ & YES \\
\hline Public Admin-2 & 6,380 & YES & YES & YES & All & Year & $15 \mathrm{~min}$ & YES \\
\hline Public Admin-3 & 1,147 & YES & YES & YES & All & Year & $15 \mathrm{~min}$ & YES \\
\hline Office-2 & 27,000 & YES & $\mathrm{NO}$ & YES & Elec only & Year & $15 \mathrm{~min}$ & YES \\
\hline Academic-1 & 4,130 & YES & NO & YES & Elec only & Year & $15 \mathrm{~min}$ & YES \\
\hline Office-3 & 7,934 & NO & YES & YES & Elec only & $6 \mathrm{mo}$ & $15 \mathrm{~min}$ & YES \\
\hline Office-4 & 1,777 & YES & NO & YES & Elec only & $9 \mathrm{mo}$ & $15 \mathrm{~min}$ & YES \\
\hline \multicolumn{9}{|c|}{ Heating and Cooling Only } \\
\hline Academic- 2 & 7,063 & NO & YES & YES & Elec only & Year & $15 \mathrm{~min}$ & YES \\
\hline Office-6 & 416 & NO & YES & YES & NG only & $4 \mathrm{mo}$ & $15 \mathrm{~min}$ & YES \\
\hline \multicolumn{9}{|c|}{ Problems with Data } \\
\hline School-1 & 5,436 & YES & YES & YES & All & Year & $15 \mathrm{~min}$ & YES \\
\hline Office-1 & 2,154 & YES & NO & NO & $\mathrm{N} / \mathrm{A}$ & Year & $5.5 \mathrm{~min}$ & YES \\
\hline Office-5 & 4,148 & YES & NO & YES & All & Year & Hourly & NO \\
\hline \multicolumn{9}{|c|}{ Submetering Not Available at 15 Minute Intervals } \\
\hline Arena-2 & 7,300 & YES & YES & YES & Elec only & Year & Daily & YES \\
\hline Office-7 & 5,575 & YES & YES & YES & Elec only & $4 \mathrm{mo}$ & Weekly & $\mathrm{NO}$ \\
\hline Academic-3 & 9,624 & NO & YES & YES & Elec only & Year & Monthly & YES \\
\hline Office-8 & 114,141 & YES & YES & YES & Elec only & Year & Monthly & YES \\
\hline Office-9 & 1,037 & YES & YES & YES & Elec only & $9 \mathrm{mo}$ & Monthly & YES \\
\hline \multicolumn{9}{|c|}{ Inadequate or Missing Data } \\
\hline Office-10 & 79,000 & NO & YES & YES & $\mathrm{N} / \mathrm{A}$ & Year & Monthly & YES \\
\hline Office-11 & 51,000 & NO & YES & YES & Elec only & Year & Daily & YES \\
\hline Academic-4 & 12,600 & NO & YES & YES & NO & $8 \mathrm{mo}$ & YES & NO \\
\hline Office-12 & 14,547 & NO & YES & YES & Elec only & $4 \mathrm{mo}$ & YES & YES \\
\hline School-2 & 4,792 & $\mathrm{NO}$ & NO & YES & NO & NO & $\mathrm{NO}$ & NO \\
\hline Office-13 & 11,971 & $\mathrm{NO}$ & NO & YES & Elec only & NO & $\mathrm{NO}$ & $\mathrm{NO}$ \\
\hline Arena-1 & 10,250 & $\mathrm{NO}$ & NO & YES & Elec only & NO & $\mathrm{NO}$ & NO \\
\hline Office-14 & 10,750 & $\mathrm{NO}$ & NO & YES & All & NO & $\mathrm{NO}$ & $\mathrm{NO}$ \\
\hline Office-15 & 10,879 & $\mathrm{NO}$ & NO & YES & All & NO & $\mathrm{NO}$ & $\mathrm{NO}$ \\
\hline Retail-1 & 13,000 & NO & NO & YES & Elec only & NO & NO & NO \\
\hline Retail-2 & 10,485 & $\mathrm{NO}$ & YES & YES & All & Year & Dally & YES \\
\hline \multicolumn{9}{|c|}{ Undesirable Building Type } \\
\hline MURB-1 & 20,033 & NO & YES & YES & All & Year & YES & NO \\
\hline MURB-2 & 18,013 & $\mathrm{NO}$ & NO & YES & Elec only & $\mathrm{NO}$ & $\mathrm{NO}$ & NO \\
\hline MURB-3 & 11,971 & NO & NO & YES & NO & NO & NO & NO \\
\hline MURB-4 & 5,204 & NO & NO & YES & All & NO & NO & NO \\
\hline MURB-5 & 9,250 & NO & YES & YES & NG only & Year & Utility & NO \\
\hline MURB-6 & 16,568 & YES & NO & YES & All & $3 \mathrm{Yr}$ & YES & YES \\
\hline MURB-7 & 45,700 & YES & NO & YES & All & Year & YES & YES \\
\hline
\end{tabular}

Figure 2. Data Availability (part 1) 
Submetering as indicated

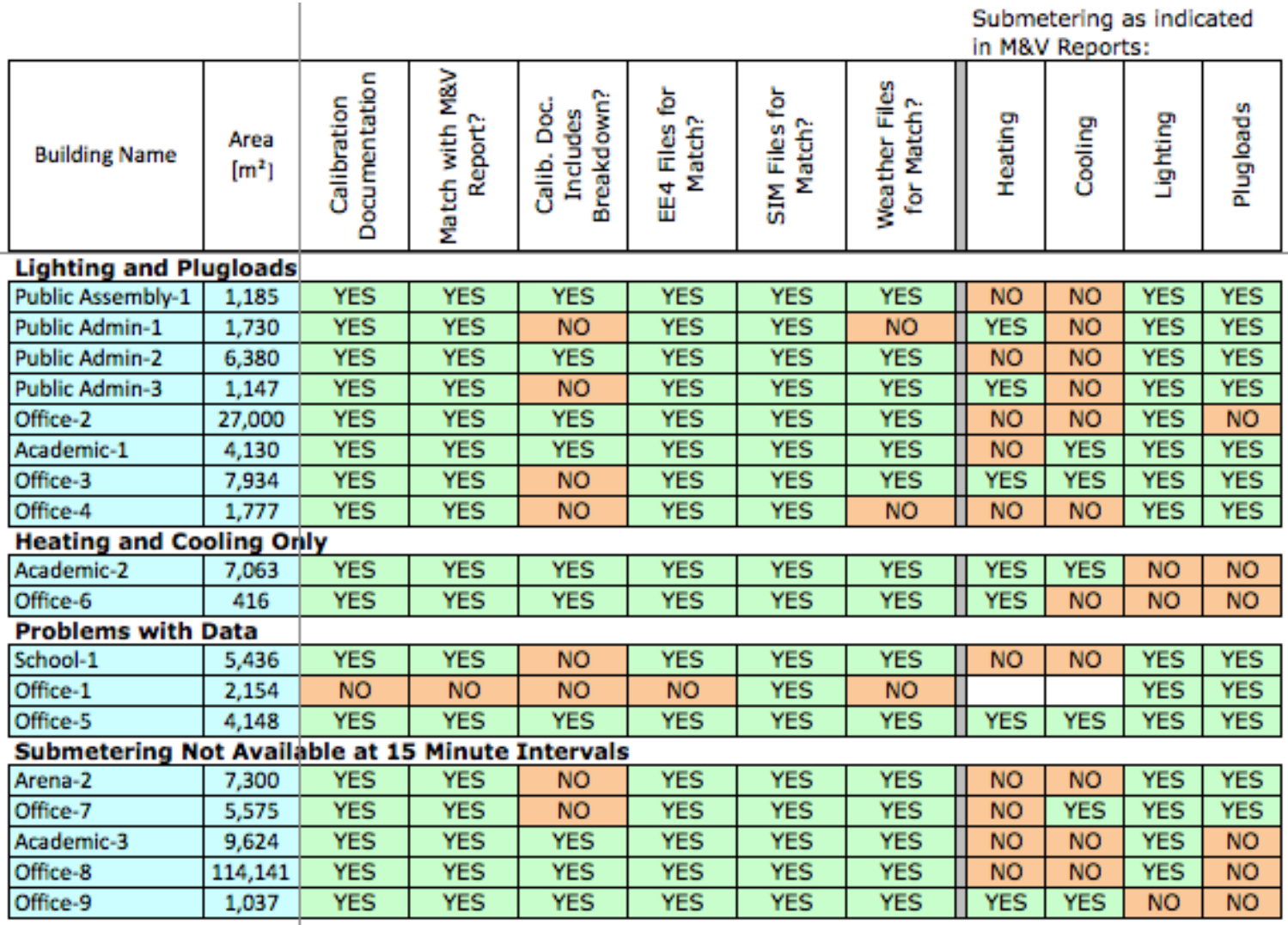

Inadequate or Missing Data

\begin{tabular}{|l|c|c|c|c|c|c|c||c|c|c|c|}
\hline Office-10 & 79,000 & YES & & & & & & NO & NO & NO & NO \\
\hline Office-11 & 51,000 & YES & & & & & & NO & NO & NO & NO \\
\hline Academic-4 & 12,600 & YES & & & & & & NO & NO & NO & NO \\
\hline Office-12 & 14,547 & NO & & & & & & NO & NO & NO & NO \\
\hline School-2 & 4,792 & YES & & & & & & NO & NO & NO & NO \\
\hline Office-13 & 11,971 & YES & & & & & & NO & NO & NO & NO \\
\hline Arena-1 & 10,250 & NO & & & & & & NO & NO & YES & YES \\
\hline Office-14 & 10,750 & YES & YES & NO & NO & NO & NO & YES & YES & YES & NO \\
\hline Office-15 & 10,879 & YES & YES & YES & YES & YES & YES & YES & YES & YES & NO \\
\hline Retail-1 & 13,000 & YES & YES & YES & YES & YES & YES & NO & YES & YES & NO \\
\hline Retail-2 & 10,485 & YES & NO & NO & N/A & N/A & N/A & NO & NO & YES & YES \\
\hline
\end{tabular}

Undesirable Building Type

\begin{tabular}{|l|c|c|c|c|c|c|c||c|c|c|c|}
\hline MURB-1 & 20,033 & NO & & & & & & NO & NO & NO & NO \\
\hline MURB-2 & 18,013 & NO & & & & & & NO & NO & NO & NO \\
\hline MURB-3 & 11,971 & YES & & & & & & NO & NO & NO & NO \\
\hline MURB-4 & 5,204 & YES & & & & & & NO & NO & NO & NO \\
\hline MURB-5 & 9,250 & NO & & & & & & YES & NO & NO & NO \\
\hline MURB-6 & 16,568 & YES & Elec Only & YES & YES & YES & YES & YES & NO & NO & NO \\
\hline MURB-7 & 45,700 & YES & YES & NO & YES & YES & YES & YES & NO & YES & YES \\
\hline
\end{tabular}

Figure 3. Data Availability (part 2) 


\subsection{Errors in Submetering Data}

A significant issue amongst the 7 buildings with adequate data to merit in depth analysis was the problem of errors or abnormalities in the submetering data. Addressing errors in submetering data required the use of significant personal judgment. Common errors can be separated into several categories:

- Duplication: In the case of duplication, the most continuous period of data was chosen the remaining duplicated data was deleted. An extreme example of this was found in Office-2, which was determined to be unusable. The following portion of the data was representative of the dates found through out the entire duration of metering for Office-2:

$$
\begin{aligned}
& \text { 12/19/2011 00:00 to } 01 / 14 / 2012 \text { 2:15 } \\
& \text { 12/05/2011 15:30 to } 12 / 10 / 2011 \text { 4:00 } \\
& \text { 12/14/2011 4:45 to } 12 / 27 / 2011 \text { 14:30 } \\
& \text { 12/10/2011 4:15 to } 12 / 16 / 2011 \text { 17:30 } \\
& \text { 12/27/2011 14:45 to } 01 / 06 / 2012 ~ 19: 45 \\
& \text { 12/16/2011 17:45 to } 12 / 24 / 20112: 15 \\
& \text { 01/06/2012 } 20: 15 \text { to } 01 / 13 / 20125: 45 \\
& 12 / 24 / 20112: 30 \text { to } 12 / 29 / 2011 \text { 7:30 }
\end{aligned}
$$

For the majority of buildings only very few periods of duplication like these occurred.

- Abnormal measurement periods: Measurements were rounded to the nearest 15-minute interval. For example:

$$
\begin{aligned}
& \text { 8/06/2010 1:02 -> 8/06/2010 1:00 } \\
& \text { 8/06/2010 1:17 -> 8/06/2010 1:15 } \\
& \text { 8/06/2010 1:32 -> 8/06/2010 1:30 } \\
& \text { 8/06/2010 1:47 -> 8/06/2010 1:45 } \\
& \text { 8/06/2010 2:02 -> 8/06/2010 2:00 } \\
& \text { 8/06/2010 2:17 -> 8/06/2010 2:15 }
\end{aligned}
$$


- Incorrect or inconsistent date format: As in the case of Academic-1 dates would switch back and forth between $\mathrm{mm} / \mathrm{dd} / \mathrm{yy}$ and dd/mm/yy. Data was adjusted to correct dates as best as possible.

- Incorrect dates: Occasionally reconciling dates was not possible (such as in the case of Mail Sorting-3) and analysis was conducted without a definite understanding of the time of year in which the energy use occurred. As mentioned for Mail Sorting-3 submetering is listed in the $M \& V$ report as occurring February 2011 to January 2012, and the dates from the metering file are from July 2010 to November 2011. No further information is available to determine which dates the metering file is referring too.

- Flatlines: Data was deemed incorrect when meters read identical loads consecutively over an extended period ("flatlining"). Data of this variety was deleted. This can be observed in the unmodified data for the receptacle load meters for Academic- 1 as is demonstrated in Figure 4.

Legend:

Jan Feb Mar Apr May Jun Jul Aug Sep Oct Nov Dec Jan

- c - Predicted Load Profile

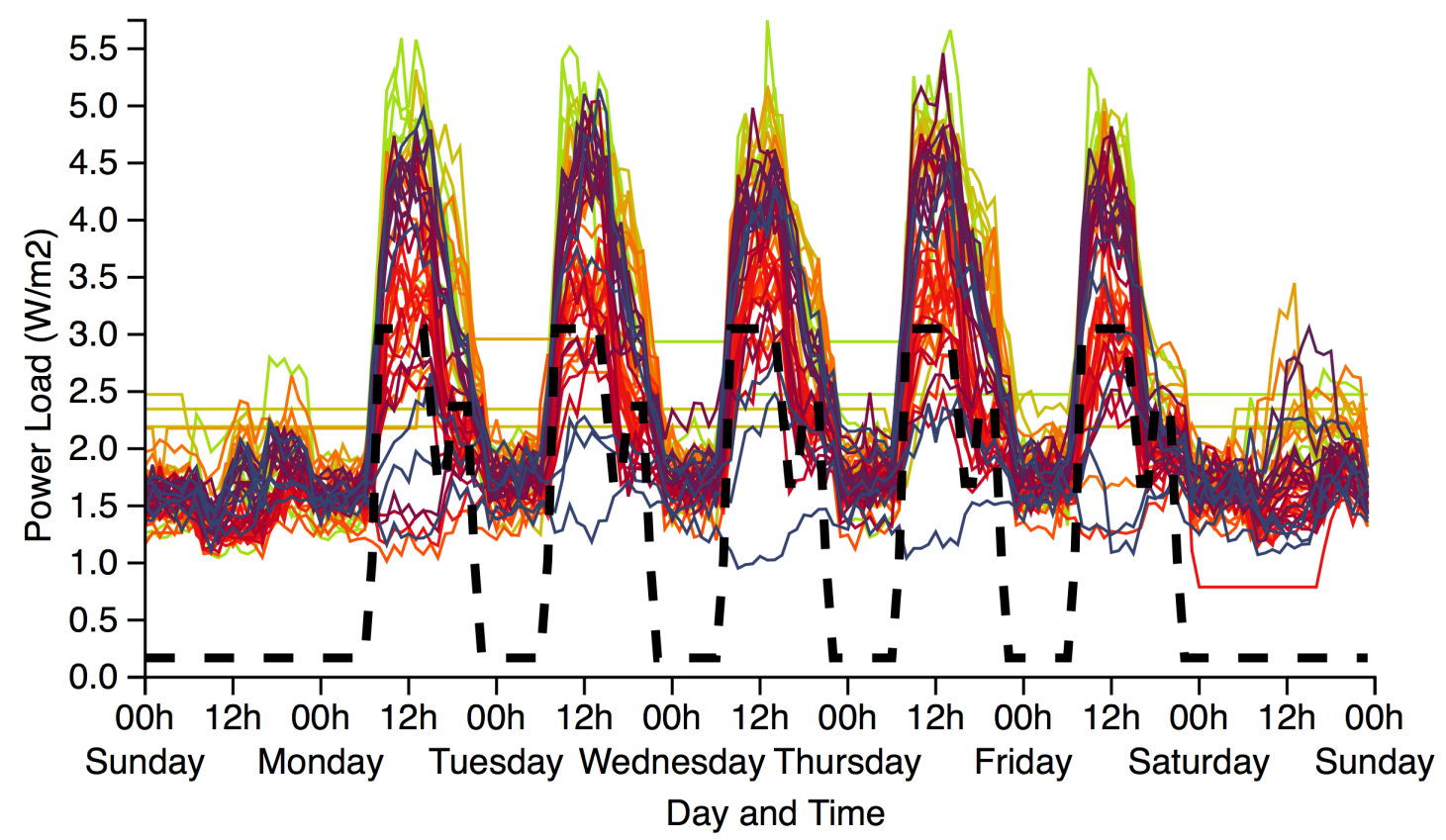

Figure 4. "Flatlines" in Unmodified Data from Academic-1 
- Incorrect Meter Values: Various buildings had data that was known to be inconsistent in comparison to utility bills. In these cases data was simply truncated, and the period of analysis was shortened. A likely cause of these issues was improperly commissioned meters. This measure was only taken in the case that MMM staff decided that the data was insufficiently accurate to include in the $\mathrm{M} \& \mathrm{~V}$ report. These metering issues were only encountered for lighting and receptacle data for Mail Sorting-3 and for receptacle data for Mail Sorting-1. An example of this can be seen in Figure 5. In this graph the data for July and August was obviously anomalous and was truncated for further analysis.

Legend:

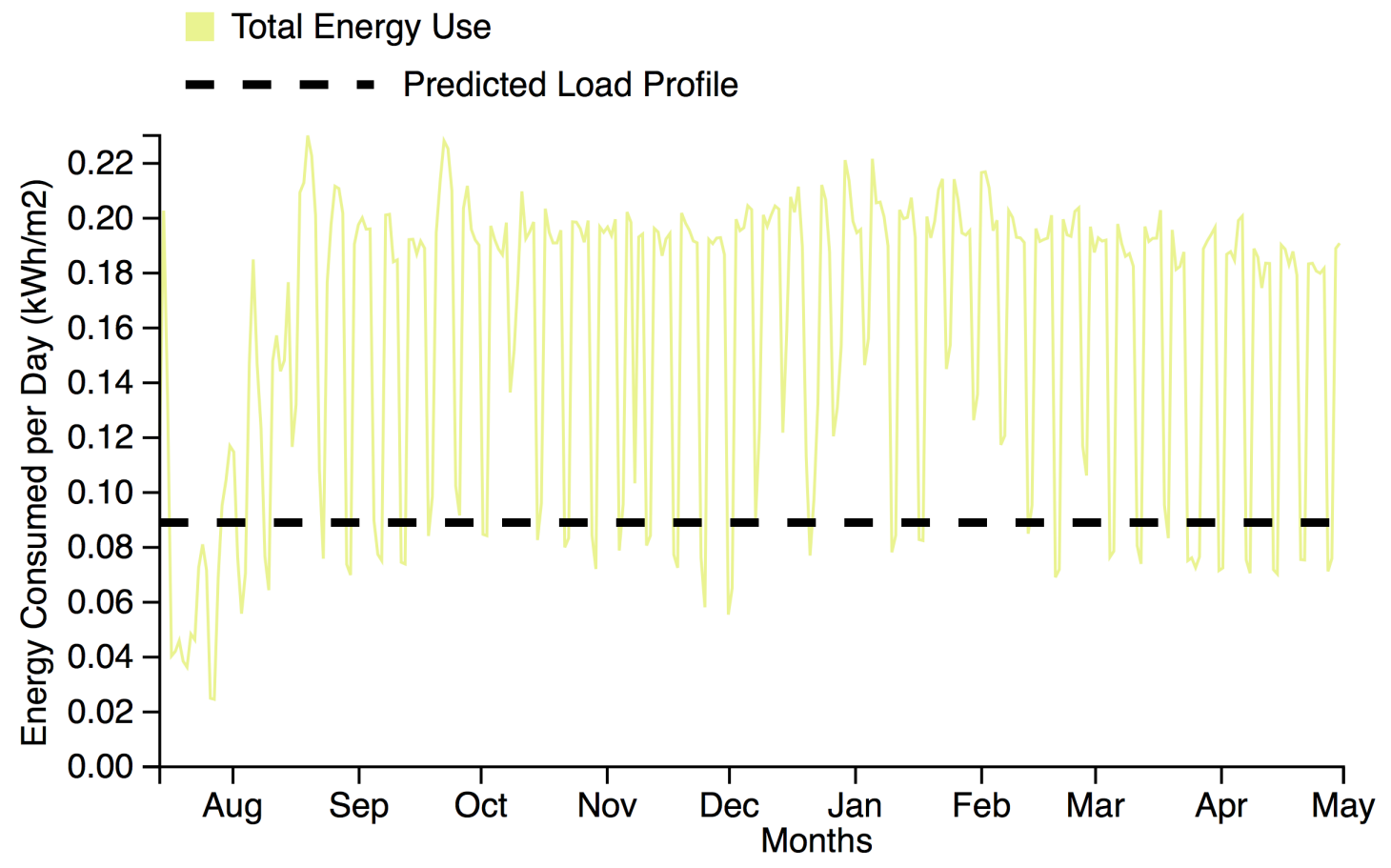

Figure 5. Metering issues in Unmodified Lighting Data from Mail Sorting-3

- Mixed Meters: For the buildings that had documentation showing the breakdown of loads included in each meter it was observed that often a variety of miscellaneous loads were mixed in with the predominant end-use. In the majority of cases it was not possible to disaggregate the data. In fact in the majority of cases documentation was not available to determine the exact extent that mixing was occurring. The three levels of documentation that 
were available were firstly column labels in excel spreadsheets (for example heating and cooling), secondly generic descriptions for meter numbers (for example heating and cooling), and full electrical panel shop drawings (both with and without individual wattages). The information about mixing is summarized in Table 2.

Table 2. Meter Mixing Summary.

\begin{tabular}{|c|c|c|c|}
\hline & $\begin{array}{c}\text { Meter } \\
\text { Composition }\end{array}$ & Known Mixing & $\begin{array}{c}\text { Suspected Mixing } \\
\text { (Post-processing } \\
\text { Predictions) }\end{array}$ \\
\hline Academic-1 & $\begin{array}{l}\text { Column } \\
\text { labelling }\end{array}$ & & $\begin{array}{l}\text { Exterior lighting } \\
\text { with lighting }\end{array}$ \\
\hline Office-3 & $\begin{array}{l}\text { Column } \\
\text { labelling }\end{array}$ & & \\
\hline Office-4 & $\begin{array}{l}\text { Generic } \\
\text { descriptions }\end{array}$ & & \\
\hline Mail Sorting-1 & $\begin{array}{l}\text { Detailed panel } \\
\text { breakdowns }\end{array}$ & $\begin{array}{l}1 \mathrm{~kW} \text { of other (exterior } \\
\text { lighting) out of } 21 \mathrm{~kW} \\
\text { lighting; } 21 \mathrm{~kW} \text { of other } \\
\text { (HVAC, heating) out of } 38 \\
\text { receptacles }\end{array}$ & \\
\hline Mail Sorting-2 & $\begin{array}{l}\text { Column } \\
\text { labelling }\end{array}$ & & $\begin{array}{l}\text { Exterior lighting } \\
\text { with lighting }\end{array}$ \\
\hline Mail Sorting-3 & $\begin{array}{l}\text { Detailed panel } \\
\text { breakdowns }\end{array}$ & $\begin{array}{l}1 \mathrm{~kW} \text { of other (exterior } \\
\text { lighting) out of } 13.7 \\
\text { lighting; } 14.9 \mathrm{~kW} \text { of other } \\
\text { (HVAC, heating) out of } 30.5 \\
\mathrm{~kW} \text { receptacles }\end{array}$ & \\
\hline $\begin{array}{c}\text { Public } \\
\text { Assembley-1 }\end{array}$ & $\begin{array}{l}\text { Detailed panel } \\
\text { breakdowns, no } \\
\text { wattage }\end{array}$ & $\begin{array}{l}3.6 \mathrm{~kW} \text { of other (exterior } \\
\text { lighting) out of } 20.9 \mathrm{~kW} \\
\text { lighting; } 18 \mathrm{~kW} \text { of other } \\
\text { (HVAC, heating) out of } 26.6 \\
\mathrm{~kW} \text { receptacles }\end{array}$ & \\
\hline
\end{tabular}

In the case of Mail Sorting-3, heating loads mixed with the building's receptacle loads produced a very definitive heating peak, as seen in Figure 6. In this instance the data for the period was removed in favour of estimated data. In all other cases meter mixing was taken into consideration upon evaluation of the building. 


\section{Legend:}

Total Energy Use

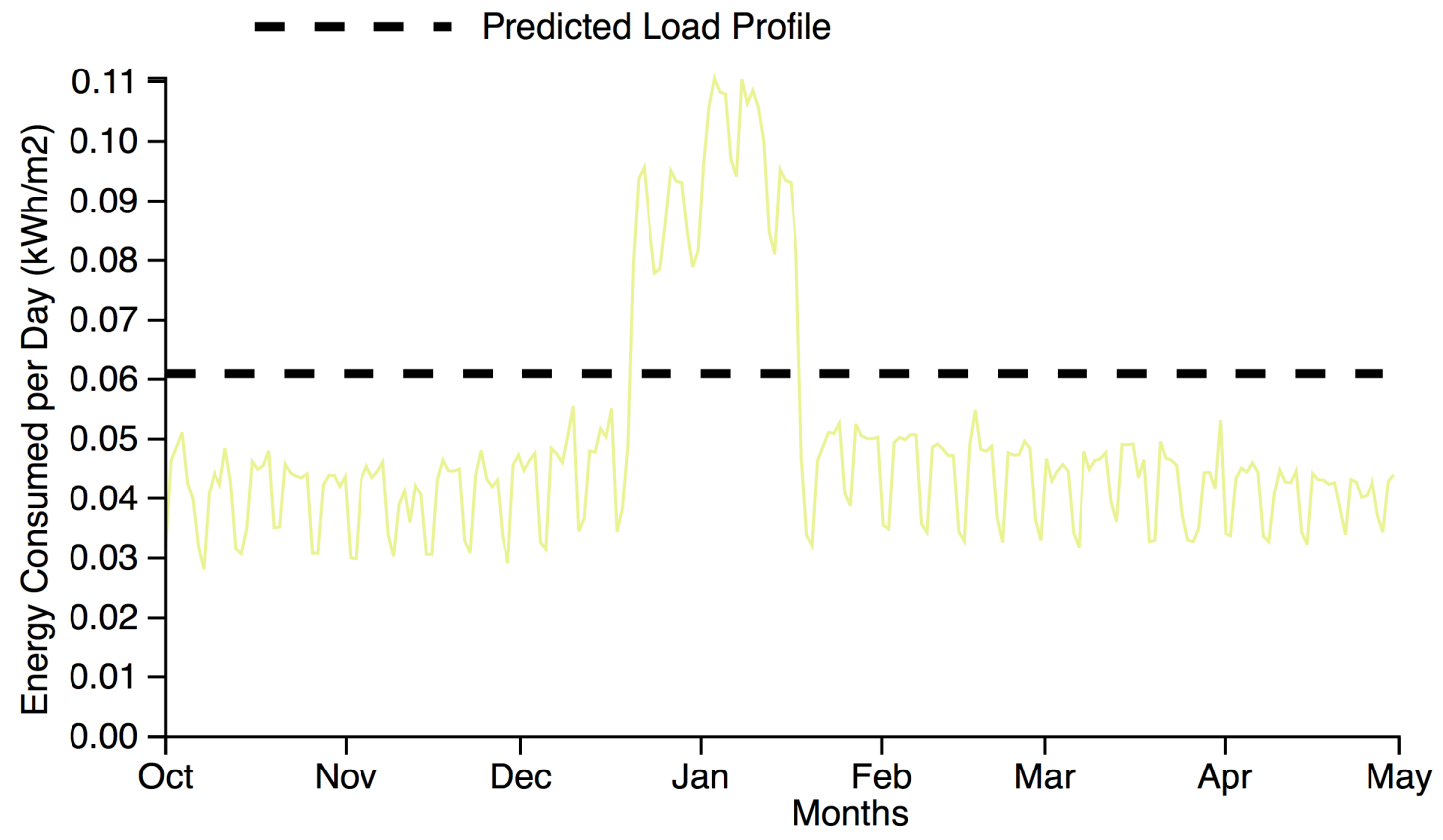

Figure 6. Heating Loads in Unmodified Receptacle Data from Mail Sorting-3

\subsection{Estimated Data}

Missing data (either missing altogether or due to the dates removal because of other issues as discussed in Section 3.3) was furthermore a challenge when working with the submetering data. This was specifically a problem when aggregating the data to produce values at intervals greater than the base 15-minute interval. As such missing data intervals were replaced with values from the yearly most typical week. The yearly most typical week is the week that was determined to have the most common values in comparison to all other weeks in a building's dataset. The calculation of the yearly most typical week will be discussed in further detail in Section 3.5.

Missing data was only estimated for the 7 buildings that underwent detailed analysis. Of those 7 buildings only 3 building had > 5\% missing data between both lighting and receptacle loads. The full breakdown is available in Table 3. 


\begin{tabular}{ccccc}
\hline & $\begin{array}{c}\text { Percent } \\
\text { Estimated } \\
\text { Data- } \\
\text { Lighting }\end{array}$ & $\begin{array}{c}\text { Duration- } \\
\text { Lighting } \\
\text { (months) }\end{array}$ & $\begin{array}{c}\text { Percent } \\
\text { Estimated } \\
\text { Data- } \\
\text { Receptacles }\end{array}$ & $\begin{array}{c}\text { Durations- } \\
\text { Receptacles } \\
\text { (months) }\end{array}$ \\
\hline Academic-1 & $7.2 \%$ & 13 & $7.3 \%$ & 13 \\
Office-3 & $2.2 \%$ & 6 & $2.2 \%$ & 6 \\
Office-4 & $23 \%$ & 10 & $0.6 \%$ & 8 \\
Mail Sorting-1 & $2.5 \%$ & 8 & $2.3 \%$ & 7.5 \\
Mail Sorting-2 & $4.1 \%$ & 12 & $4.3 \%$ & 12 \\
Mail Sorting-3 & $1.8 \%$ & 7.5 & $15.2 \%$ & 7.5 \\
Public & $1.8 \%$ & 13 & $1.8 \%$ & 13 \\
Assembly-1 & & & & \\
\hline
\end{tabular}

\subsection{Graphical Analysis}

The first step towards understanding the performance gap amongst the buildings is to compare the metered energy use data graphically to the predicted energy use. Graphic analysis of this sort provides an initial high level overview at the patterns of energy use consumption exhibited by the buildings. This is primarily a descriptive technique that allows for the identification of possible lines of investigation as well as check on data quality issues. It was only possible to identify the majority of the issues discussed in Section 3.3 through the use of these graphs, as each building has up to 40,000 data points. This analysis was only conducted for the 7 buildings with lighting and receptacle load data available.

Five graphs will be used to depict each energy load (for both lighting and receptacle loads) for each building. This will include a graph depicting the full data set of weekly profiles; the average, median, and most typical weekly load profile; the average median and most typical weekday load profile; the average, median and most typical weekend day load profile; and the daily total energy use for each day in the data set. All graphs present the data at an hourly resolution, with exception of the last, which presents the data at daily resolution.

The first variety of graph is depicted in Figure 7, which also happens to be representing the lighting load profile for Office-4. The figure displays the duration of energy use data separated into weeks and superimposed. Each week of data is 
coloured according to the time of year in which it occurs, the colour code is described in the legend. As can be read from the X-axis the week extends from 00:00 hours Sunday to 23:59 hours on Saturday evening. As can be read from the Y-axis the electricity use is depicted in $\mathrm{W} / \mathrm{m}^{2}$. The thick dashed line represents the energy use predicted by the calibrated energy models. A graph of this nature provides immediate insight into the general trend of the data, and how they compare to the predictions of the energy models. The shear number of data series (weeks) however obscure to a certain extent the average behaviour of the building, as well as certain patterns occurring week-to-week or month-to-month.

Legend:

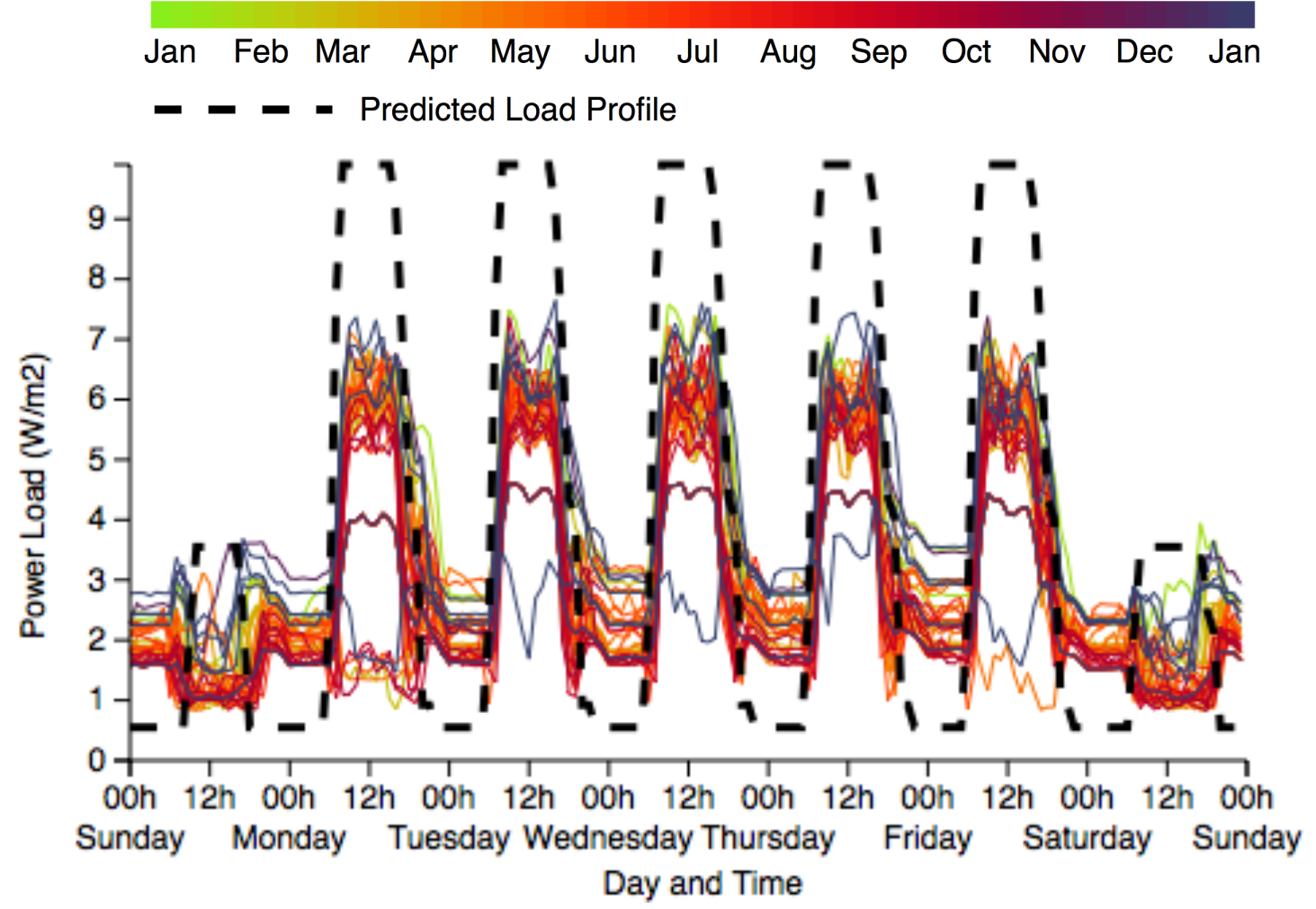

Figure 7. Weekly Load Profile for Full Data Set (Office-4, Lighting)

The second variety, as demonstrated in Figure 8, hopes to address some of these issues to a certain extent. The same building and end-use is depicted as Figure 7. The graph presents a radically slimmed down version of the time series in Figure 7. The graph depicts the yearly average, yearly median, and yearly typical week. The yearly average week is calculated by taking the average of all the values available in the data for a given time interval (for example Wednesday 19:15 hours). Similarly 
the yearly median week is calculated by taking the median of all the values for a given time interval. The yearly most typical week is chosen by determining which week is on average most similar to all the other weeks. This is done by calculating a CVRMSE between all possible combinations of weeks (see Section 3.10 for details on CVRMSE) and then averaging the value for the week in question to produce a coefficient of similarity for the week in question. The week with the lowest coefficient is considered the most "typical". It was decided that all three profiles (average, median, and typical) would be depicted as it was not possible to identify a best practice through the literature review. As can be seen in Figure 8, and elsewhere in this study, the three profiles are very similar, the "typical" series tends to be the most irregular, while average and median tend to be much more smooth. As above the time series starts Sunday morning and ends Saturday evening. Again the solid dashed line represents the energy use predicted by the model. The benefit of this graph is to immediately give a sense of the most common load profile without the clutter of all the deviations from the norm. Of course what is lost is a sense of how the load profile might vary over the year.

Legend:

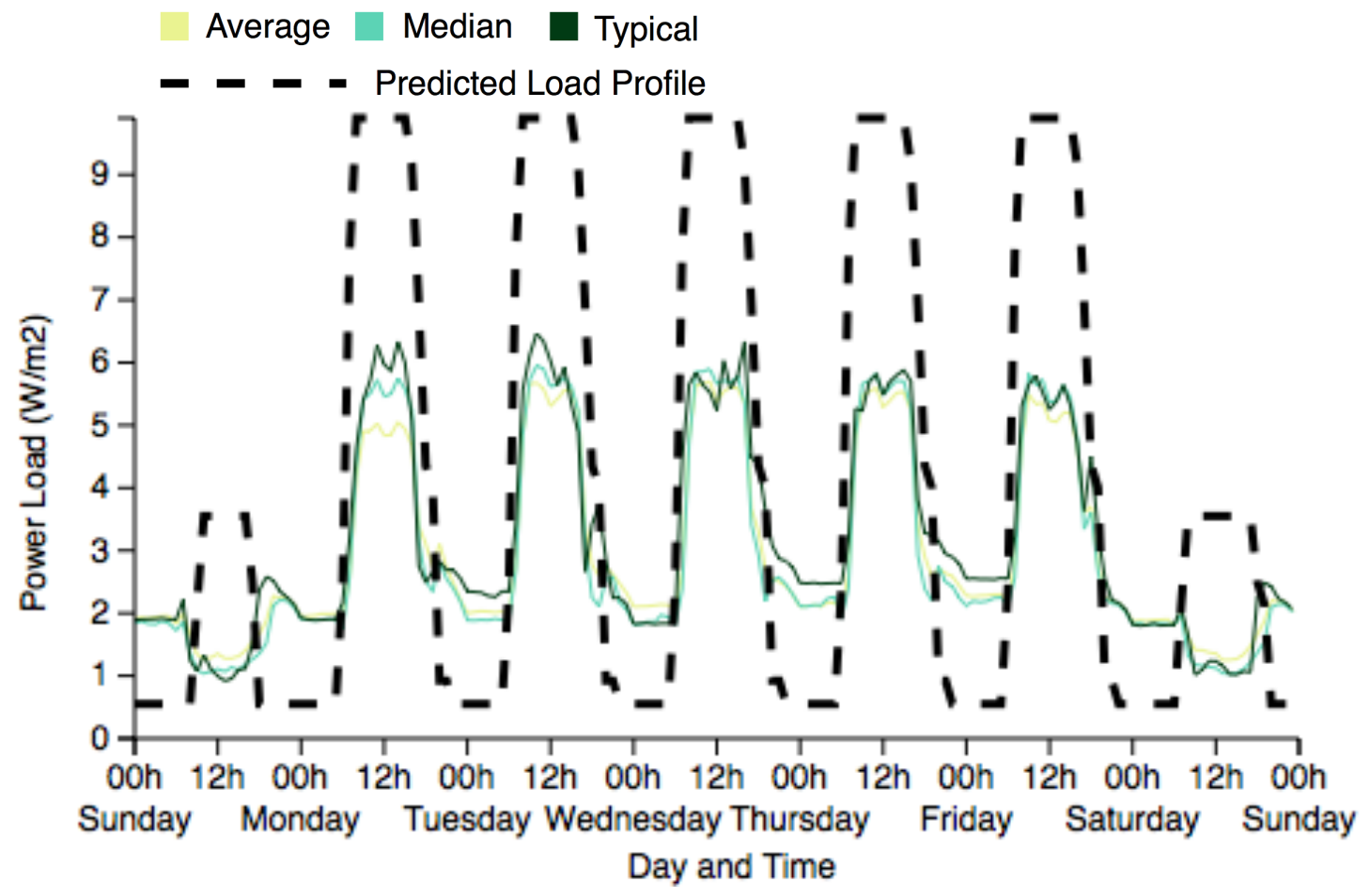

Figure 8. Average, Median and Typical Energy Use Profiles (Office-4, Lighting) 
The third and fourth graphs (Figure 9 and Figure 10) provide a similar feature. However instead of weekly time series, the graphs represent the average, median, and typical time series for weekdays, and weekend days, respectively. The various series are calculated in a fashion similar to as addressed above, however the days are first separated into a pool of weekdays and also weekend days. In many cases it is observable that the typical series draws closer to the median and average series as the duration of period in question shortens and the pool of possible series increases. Yet typical remains more irregular than its smoother counterparts, average and median. These graphs are able to demonstrate at a finer grain the way the daily profile conforms to predictions, this includes details such as whether the building is ramping up or powering down as anticipated. Again a weakness of only showing average, median, and typical is the lack of information about how the trends may be changing over the year.

Legend:

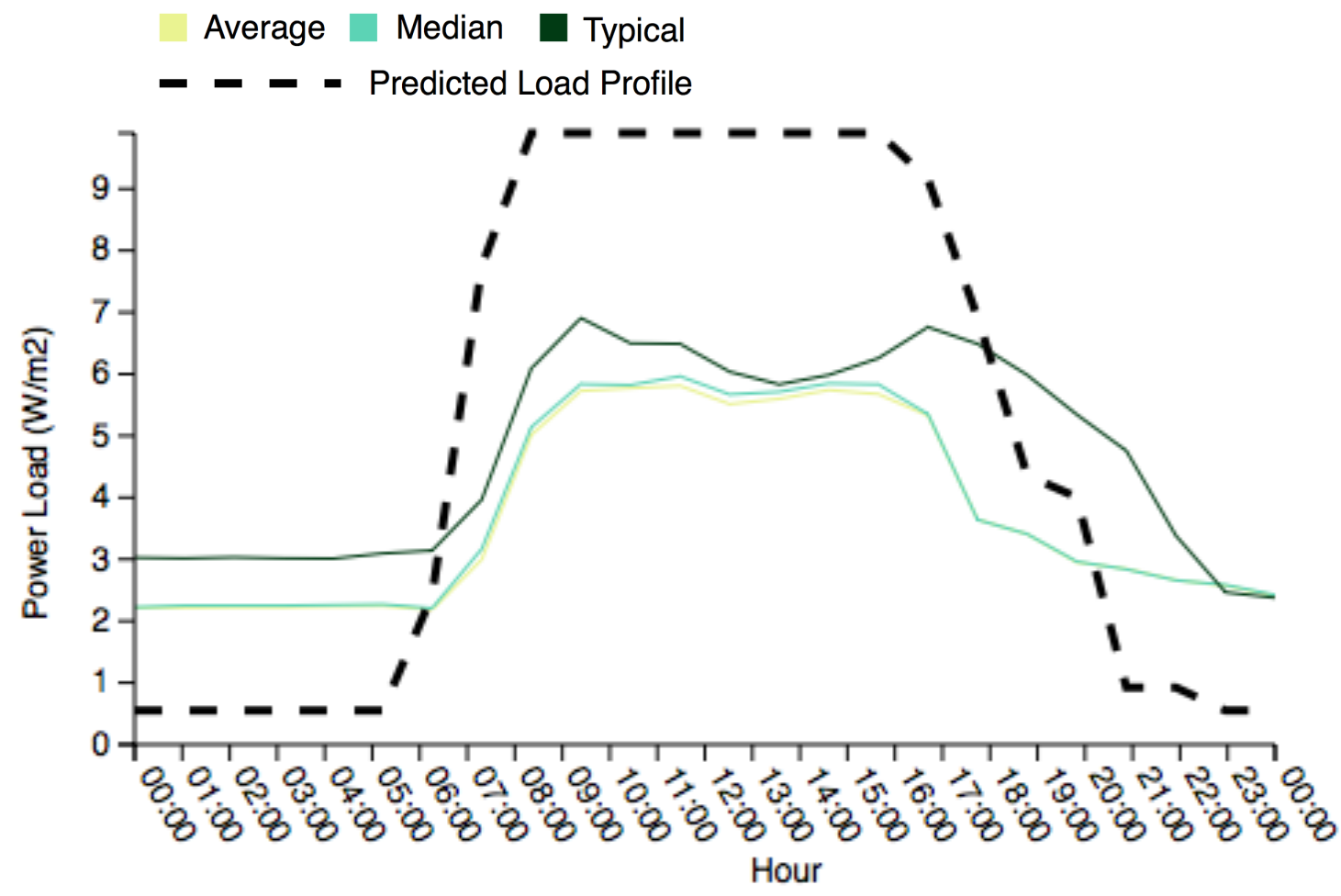

Figure 9. Weekday Energy Load Profile (Office-4, Lighting) 
Legend:

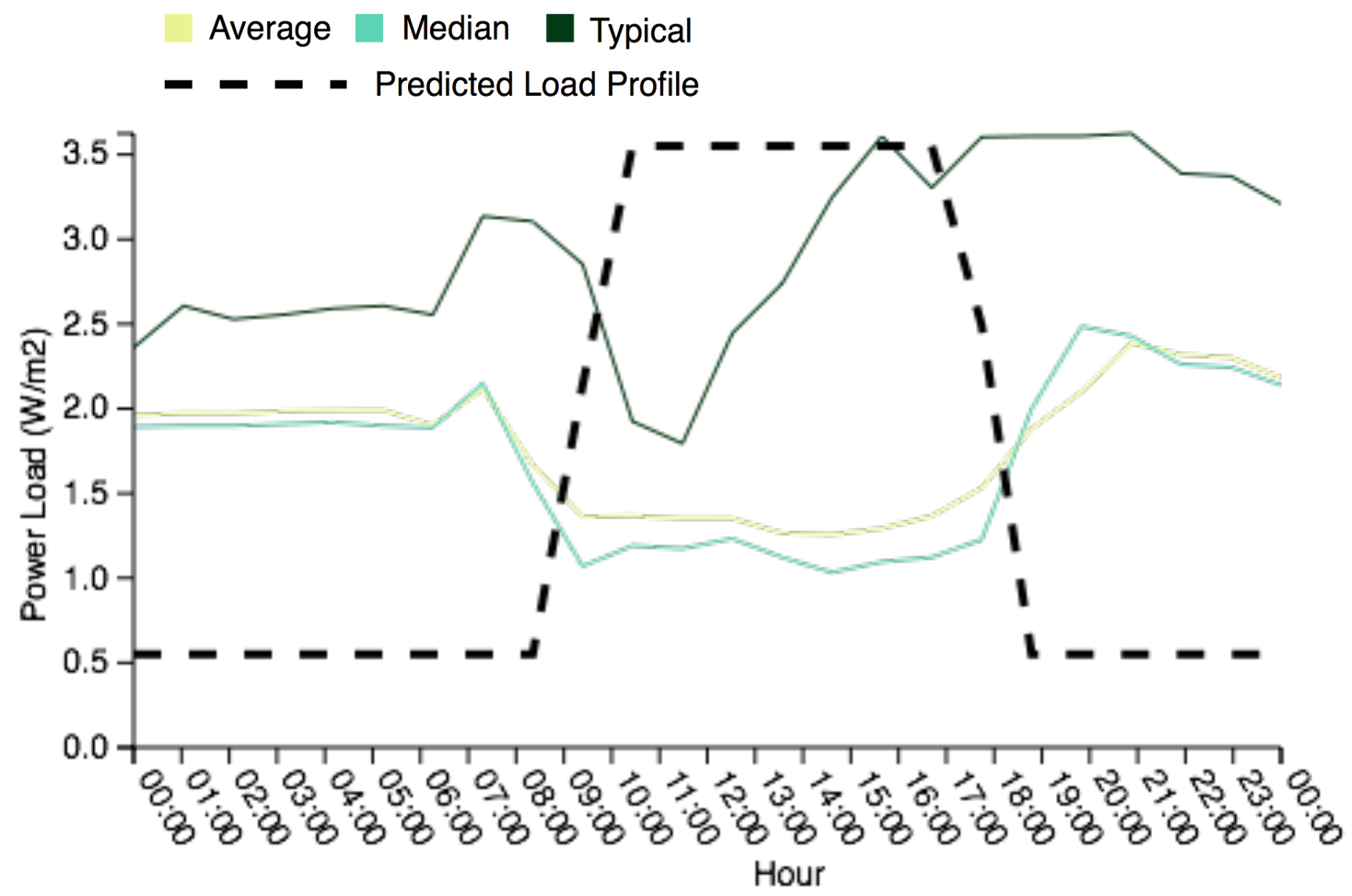

Figure 10. Weekend Day Energy Load Profile (Office-4, Lighting)

Lastly the graphs include the daily total energy use values, as depicted in Figure 11. As opposed to power load presented in the previous four graphs, this graphs displays energy use as an energy use intensity (EUI) in $\mathrm{kWh} / \mathrm{m}^{2}$. Again the black dashed line represents the predicted energy use by the calibrated energy models, however in this case it is the average daily energy use. The energy model does differentiate between weekday and weekend energy usage levels (which are readily apparent in this resolution of graph as an oscillation in the energy use data) however it was found to be graphical more legible to plot average predicted daily use rather than predicted daily use vary between weekends and weekdays. This graph provides a quick overview of the way that energy use varies across the year, as well as the difference between weekday energy use versus weekend use. The durations of perfectly regular energy use in this case are due to missing data being replaced by data from the median week. 
Legend:

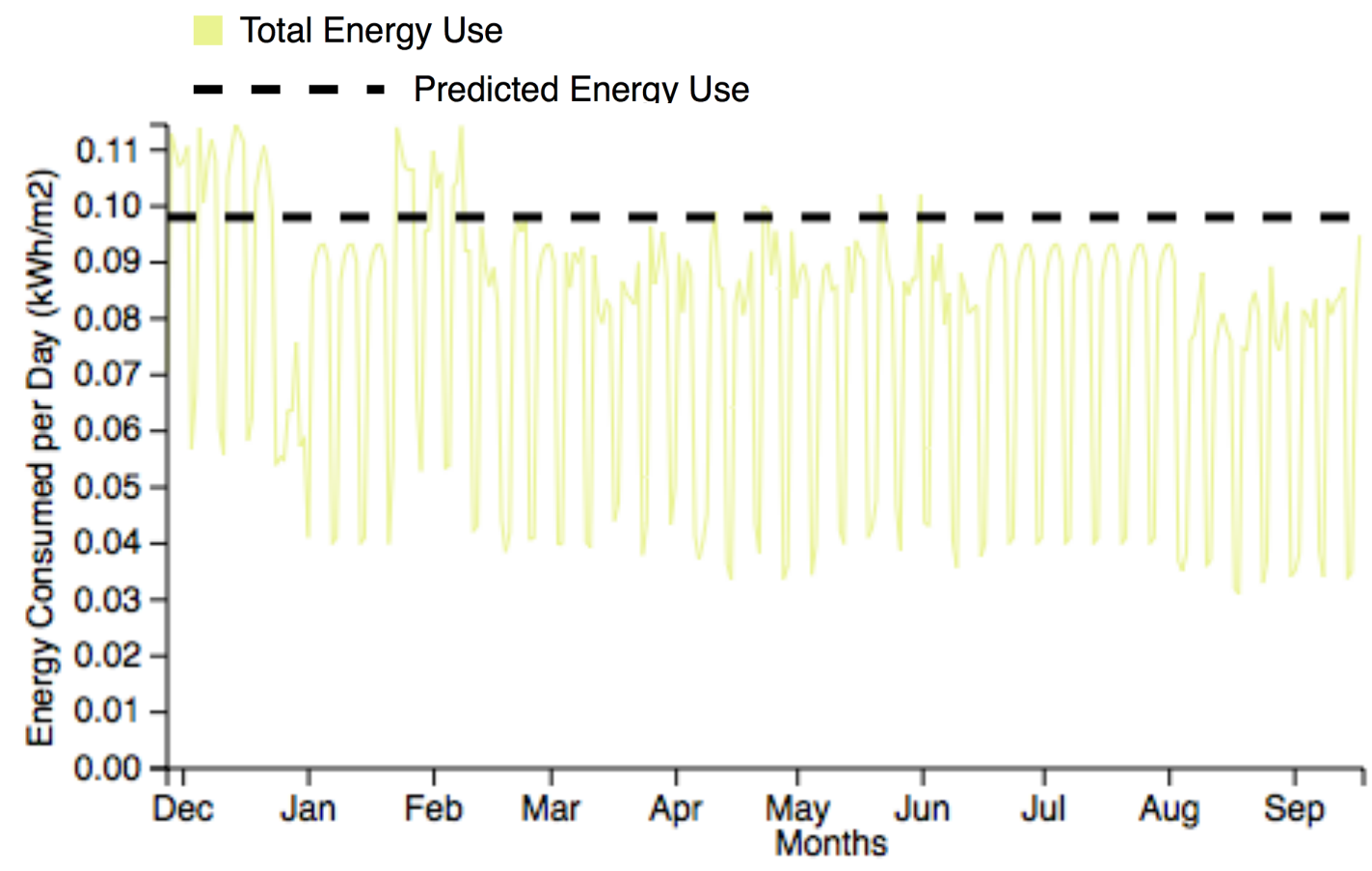

Figure 11. Total Daily Energy Use (Office-4, Lighting)

\subsection{Curve Characteristics}

Providing a more quantitative snapshot of the buildings undergoing in depth analysis curve characteristics were calculated, including daily peak and base energy loads, as well as total energy day time and nighttime energy use. These figures were calculated both for actual and predicted energy use.

The calculation of peak and base energy loads was suggested Price, Mathieu, Kilicote, and Piette (2011) as way to more precisely characterize a load profile. There definition, as seen in Table 4, suggested that near-peak and near-base values be used to limit the extreme outliers that might occur in the data. These definitions were adopted. However in addition, in order to also produce individual peak and base load terms for extended periods the near peak and base load were calculated for each day in the period then median value of these loads was taken. For example the median near peak load for a month is the median value of the near peak loads for each day in the month. This will be called the median near peak (MNP) load and median near base load (MNB) throughout the work. 
Table 4. Load Profile Characteristics

\begin{tabular}{cc}
\hline Parameter & Definition \\
\hline Near-peak load & 96.5 percentile daily load \\
Near-base load & 3.5 percentile daily load \\
\hline
\end{tabular}

The calculation of daily and nightly total energy use figures was slightly less complicated. Energy use was summed over the relevant periods in order to obtain a total. Daytime was assumed to last from between 7:00 hours and 18:59 hours, and nighttime, the inverse, 00:00 hours until 6:59 and 19:00 hours to 23:59 hours. For extended durations a similar sum was completed, with the total prorated for the number of days in the period.

These values were used to inform the interpretation of the aforementioned graphs, as well as in the case of daily total energy use in the daylight regression analysis.

\subsection{Alternative Benchmarking}

ASHRAE error tolerances defined in Guideline 14 have come to define to a certain extent the process of evaluating energy model calibration. ASHRAE suggests that models can be considered adequately calibrated when they meet the prescribed error tolerances listed in Table 5 (p.15, 2002). As such these terms are used in energy model calibration literature as the baselines for comparison.

Table 5. Error Tolerance Limits for Calibrated Energy Model Simulations

\begin{tabular}{ccc}
\hline Time-step & CVRMSE & NMBE \\
\hline Monthly & $<15 \%$ & $<+/-5 \%$ \\
Hourly & $<30 \%$ & $<+/-10 \%$ \\
\hline
\end{tabular}

In a not dissimilar manner, research in the field of the building performance gaps also compares actual and predicted energy use. Their goal of course is to determine the performance of the building. These comparisons are often made in the form of percent difference between predicted and actual energy use intensities 
(EUIs). Percent difference is a less rigorous metric of comparison, in relation to CVRMSE and NMBE. This is due in part to the fact that percent difference only compares the total figures, whereas CVRMSE and NMBE compare the constituent parts in order to judge the difference of the whole. This is equivalent to the difference comparing predicted and actual total yearly energy use, and comparing predicted and actual values for each month to determine the difference between the years as a whole.

Due to this, developing a reference point to compare the CVRMSE and NMBE results for individual buildings or groups of buildings, and eventually for individual end-uses in buildings, is crucial. This is essentially similar to the process of benchmarking, or developing databases on the magnitude of the performance gap in buildings. However this not only extends to the level of total building energy use (or

energy use by energy source) and not only using EUIs or percent difference between EUIs as a metric.

ASHRAE provides an initial baseline for which to compare CVRMSE and NMBE, outlined in Table 5. Unfortunately many of the data sets undergoing analysis within this study do not fit nicely into the categories provided by ASHRAE. Their error terms apply principally to energy use for all end uses for monthly and hourly breakdowns.

As a result it will be a goal of this study to produce a guideline in the form of error tolerances for CVRMSE and NMBE, for the energy use categories of hourly and monthly energy use for end-uses. Unfortunately it was not possible to determine how ASHRAE determined the values for the error tolerance it has defined. And due to this fact the error tolerance values will have to be based off of simple observed relationships.

\subsection{Error Regression Analysis}

Of the various phenomenon observed and measured through the graphical analysis, calculation of curve characteristics, and through the alternative 
benchmarking, it was decided to produce a regression model in order to attempt an initial explanation of the performance gap as witnessed for each building.

This regression model will test the correlation of the performance gap with the variability of management and occupancy of the building, as represented by peaks and bases in the data that did not follow typical load profiles. The model will combine both results for lighting and receptacle end-uses in order to have a larger sample size.

In these regression analyses the performance gap will be represented by the error term the coefficient of variation of root mean square error (CVRMSE) in lieu of percent difference in total EUI as is typically the case, as was discussed in Section 3.7.

Data variability will be represented in these regression analyses through the help of a variability coefficient. This statistical term will describe the normalized amount of variation that occurs between weeks within the hourly data for each building. This formula makes a comparison using CVRMSE of each week of data to each other week, takes a sum of all the resulting error terms, and then normalizes it by the number of terms in the summation. Thus demonstrating the average amount of difference between the weeks for each building.

\subsection{Daylight Regression Analysis}

As the graphs produced for the buildings clearly demonstrated seasonal variations in lighting energy use it was decided that a regression analysis would be completed to determine if lighting energy use could be correlated with daylighting availability, as many of the buildings included daylighting features in their designs. This will primarily consist of attempting to correlate total lighting energy consumption with bright sunshine hours. The will suggest the extent to which daylight influenced the amount of artificial lighting used. The regression model was developed on a monthly resolution. Total lighting energy consumption figures were calculated by summing the energy consumption for each month of the year for which full data was available. Day light hours were estimated with monthly bright 
sunshine hours provided by Environment Canada's monthly 1981-2010 “Monthly Climate Normals" (Environment Canada, 2015). Unfortunately bright sunshine hours were only available for Toronto. In this model a negative correlation would indicate that daylight energy use was decreasing as daylighting hours increased.

\subsection{Assorted Equations}

\section{CVRMSE}

The coefficient of variation of the root mean squared error is a derivative of a common statistical term used to measure the difference between two sets of values, often a set of predicted values compared against a set of measured values. In this work it is used as defined by ASHRAE in guideline 14 to measure the difference between actual and predicted energy use, as well as to indicate the amount of variation occurring between weeks within a data set. This term is well suited for representing the total error (or difference) between the two sets of values.

$$
\operatorname{CVRMSE}\left(\mathrm{y}, \mathrm{y}^{\urcorner}\right)=100 \times \sum_{i=0}^{n} \frac{\sqrt{\frac{\left(y_{i}-y_{i}^{l}\right)^{2}}{n-1}}}{\frac{y_{i}}{n}}
$$

Where:

- $y_{i}^{\prime}$ is the $i^{\text {th }}$ element of the predicted data

- $y_{i}$ is the $i^{\text {th }}$ element of the measured date

- $\mathrm{n}$ is the length of the sets $\mathrm{y}^{\prime}$ and $\mathrm{y}$

\section{NMBE}

Normalized mean bias error (NMBE) is a more uncommon statistical term also used to measure difference between two sets of values. In this work it is used as defined by ASHRAE in guideline 14 to measure the difference between actual and 
predicted energy use. Unlike CVRMSE, the NMBE doesn't take the power of the residuals $\left(\mathrm{y}_{\mathrm{i}}-\mathrm{y}_{\mathrm{i}}{ }^{\prime}\right)$, so negative and positive values cancel out, meaning the NMBE will indicate the amount of skew in the data.

$$
\operatorname{NMBE}\left(\mathrm{y}, \mathrm{y}^{\prime}\right)=100 \times \frac{n}{n-1} \sum_{i=0}^{n} \frac{\left(y_{i}-y_{i}^{\prime}\right)}{y_{i}}
$$

Where:

- $y_{i}^{\prime}$ is the $i^{\text {th }}$ element of the predicted data

- $y_{\mathrm{i}}$ is the $\mathrm{i}^{\text {th }}$ element of the measured date

- $\mathrm{n}$ is the length of the sets $\mathrm{y}^{\prime}$ and $\mathrm{y}$

\section{Percent Difference}

Percent difference is a less rigorous statistical term, one that however enjoys considerable use due to its intuitive meaning. It is occasionally used through out this study when a less rigorous term will suffice or where it is used as comparison to CVRMSE and NMBE.

Percent Difference $=100 \times \frac{\left|Y_{\text {teta }}-Y_{\text {tata }}\right|}{W_{\text {tata }} \mathcal{L}}$

Where:

- $\mathrm{y}_{\text {total }}$ is the total measured energy consumption for a given period

- $y^{\prime}$ total is the total predicted energy consumption for a given period

\section{Data Variability Coefficient}

The data variability coefficient is a term that measures the variation between a number of sets of values (for example weeks in a year). The term relies on a similar premise as CVRMSE to calculate the difference between each set of values, and a combinatorial sum (in other words a sum that takes into account all possible 
combination of sets) to find the total difference amongst the sets. This term is then normalized by the total number of comparisons made.

$$
=\frac{1}{\left(\begin{array}{l}
n \\
2
\end{array}\right)} \sum_{1 \leq j<k<n}^{m} \sum_{i=0}^{n} \frac{\sqrt{\frac{\left(y_{j, i}-y_{k, i}^{p}\right)^{2}}{n-1}}}{\frac{y_{j, i}}{n}}
$$

Where:

- $\mathrm{y}_{\mathrm{k}, \mathrm{i}^{\prime}}$ is the $\mathrm{i}^{\text {th }}$ element of the $\mathrm{k}^{\text {th }}$ week of predicted data

- $y_{j, I}$ is the $i^{\text {th }}$ element of the $j^{\text {th }}$ week of measured data

- $\mathrm{n}$ is the length of the sets $\mathrm{y}^{\prime}$ and $\mathrm{y}$, ie. the number of data points per week

- $\mathrm{m}$ is the number of weeks in the period of analysis

\section{Single Variable Linear Regression}

This study also relies on a common statistical technique known as single variable linear regression to determine correlations between various sets of data, and to determine the line of best fit that passes through them. The three main terms relevant to this study are the coefficient of determination $\left(r^{2}\right)$, as well as the slope and intercept of the line of best fit $(y=m x+b$, where the slope is $m$, and the intercept is $\mathrm{b}$ ). The $\mathrm{r}^{2}$ represent the amount of variation in the data explained by the line of best fit, and the line of best fit is described by $m$ and $b$. Values of $r^{2}$ range from 0 to 1.

$$
\begin{aligned}
r^{2} & =1-\frac{\sum_{i}^{n}\left(y_{i}-x_{i}\right)^{2}}{\sum_{i}^{n}\left(y_{i}-y_{a v g}\right)^{2}} \\
m & =\frac{n \sum_{i}^{n} x_{i} y_{i}-\sum_{i}^{n} x_{i} \sum_{i}^{n} y_{i}}{n \sum_{i}^{n} x_{i} x_{i}-\sum_{i}^{n} x_{i} \sum_{i}^{n} x_{i}}
\end{aligned}
$$


$b=\frac{\sum_{i}^{n} y_{i}-m \sum_{i}^{n} x_{i}}{n}$

Where:

- $y_{i}$ is the $i^{\text {th }}$ element of the dependent variable

- $y_{\text {avg }}$ is the arithmetic mean of $y$

- $\mathrm{X}_{\mathrm{i}}$ is the $\mathrm{i}^{\text {th }}$ element of the independent variable

- $\mathrm{n}$ is the length of the sets $\mathrm{y}^{\prime}$ and $\mathrm{y}$, ie. the number of data points per week 


\subsection{Results and Discussion}

\subsection{In-depth Building Profiles}

The following section will provide an in depth description of each of seven buildings for which detailed information is available on the building's energy use.

\subsubsection{Calibration Impact Summary}

The impact of MMM engineering's calibration process was studied for a similar set of buildings, and discussed in Samuelson, Ghorayshi and Reinhart's 2015 paper. The full results are described in the Table 6. The process of updating the energy use through the calibration process is completed using two techniques, revising the modeling inputs files, or through adding energy use onto the totals through stand-alone calculations (post-processing). The difference between these two methods is important because when post-processing the update energy consumption values are only available at a monthly resolution.

Table 6 should provide a sense of the extent that calibration has revised the energy use for the seven buildings in question. In fact the data set used by Samuelson, Ghorayshi, and Reinhart includes 5 of the 7 buildings that will be undergoing more detailed analysis, although it is not possible to know which of the buildings contributed the data to which of the calibration steps. The paper indicated that they found updating receptacle loads to have the highest impact on calibration at a mean increase of EUI of $32 \%$, while they found revising infiltration rates to increase mean EUI by 58\%, it is likely that they determined the sample size was too small to be indicative of larger trends. It should also be mentioned however that if 4 outlier buildings (none of which are included in the 7 buildings under in-depth investigation in this study) be excluded from the calculation of the impacts of revising receptacle loads, the mean change in EUI is reduced to 5\%. This lower figure is likely more representative about the buildings in question. 
Table 6. Impact of Energy Model Calibration (Samuelson, Ghorayshi, Reinhart, 2015)

\begin{tabular}{|c|c|c|c|c|c|}
\hline \multicolumn{3}{|c|}{ Through Revising Modeling Inputs } & \multicolumn{3}{|c|}{ Through Post-processing } \\
\hline Step & $\begin{array}{c}\text { \# of } \\
\text { Bldgs. }\end{array}$ & $\begin{array}{l}\text { Mean } \\
\text { Change } \\
\text { in EUI }\end{array}$ & Step & $\begin{array}{l}\text { \# of } \\
\text { Bldgs. }\end{array}$ & $\begin{array}{c}\text { Mean } \\
\text { Change } \\
\text { in EUI }\end{array}$ \\
\hline $\begin{array}{l}\text { Replacing Weather } \\
\text { Files }\end{array}$ & 18 & $-2 \%$ & $\begin{array}{c}\text { Revising Receptacle } \\
\text { Loads }\end{array}$ & 14 & $32 \%$ \\
\hline $\begin{array}{l}\text { Revising Occupant } \\
\text { Schedules and } \\
\text { Densities }\end{array}$ & 4 & $-4 \%$ & $\begin{array}{c}\text { Revising Receptacle } \\
\text { Loads (excluding } \\
\text { outliers) }\end{array}$ & 10 & $5 \%$ \\
\hline $\begin{array}{l}\text { Revising Lighting } \\
\text { Schedules and } \\
\text { Densities }\end{array}$ & 3 & $11 \%$ & $\begin{array}{c}\text { Adding Unregulated } \\
\text { Loads }\end{array}$ & 15 & $7 \%$ \\
\hline $\begin{array}{l}\text { Revising HVAC } \\
\text { Schedules }\end{array}$ & 1 & $20 \%$ & Revising DHW & 1 & $-6 \%$ \\
\hline $\begin{array}{l}\text { Revising HVAC } \\
\text { Characteristics }\end{array}$ & 3 & $9 \%$ & & & \\
\hline $\begin{array}{l}\text { Revising Infiltration } \\
\text { Rates }\end{array}$ & 2 & $58 \%$ & & & \\
\hline
\end{tabular}

The importance then of the calibration steps using post-processing techniques poses a challenge in comparing actual metered energy use to energy use predicted by calibrated energy models at an hourly resolution. In the case of lighting loads post-processing often includes additional exterior lighting loads. In the case of receptacles post-processing includes a variety of loads from different sources.

Table 7 list all of the lighting and receptacle energy use predicted strictly by the revised energy model files as well as the energy use predicted through postprocessing. In the table the post-processing energy consumption predictions that are highlighted green indicate loads that are accounted for through separate submeters. In these cases we do not have to worry about post-processing energy predictions interfering with the comparison of actual energy use and energy use predicted from the modeling outputs. For example in Office-3, when we are comparing the lighting submeter with the lighting predictions made through the energy model, we do not have to consider the impact that exterior lighting would have on the actual metered energy use because we know it is metered separately.

This would however be a concern in the case of Academic- 1 because we know there is no submeter covering exterior lighting, and thus the lighting submeter 
may include exterior lighting. These unaccounted for post-processing energy predictions are highlighted red in the table. However in the case that a prediction is unaccounted for doesn't necessarily mean that it will impede the comparison between calibrated energy model results and actual energy use. For example in the case of Mail Sorting-3 the post-processing addition "doors" was definitely not present in the receptacle meter panel descriptions that were available. This still however leaves a number of situations to consider.

In these cases with accounted for post-processing energy use predictions, it will be necessary to take these values into consideration when evaluating the buildings.

Table 7. Calibrated Energy Model Predictions and Post-processing Energy Consumption Additions

\begin{tabular}{|c|c|c|c|c|c|}
\hline & \multicolumn{2}{|c|}{$\begin{array}{l}\text { Approx. Calibrated } \\
\text { Energy Model } \\
\text { Predictions }\end{array}$} & \multicolumn{3}{|c|}{$\begin{array}{l}\text { Approx. Post-processing Energy } \\
\text { Consumption Additions }\end{array}$} \\
\hline & $\begin{array}{l}\text { Lighting } \\
\text { (MWh) }\end{array}$ & $\begin{array}{l}\text { Receptacles } \\
\text { (MWh) }\end{array}$ & $\begin{array}{l}\text { Exterior } \\
\text { Lighting } \\
\text { (MWh) }\end{array}$ & Receptacl & (MWh) \\
\hline Academic-1 & 100 & 42.5 & 11 & Recep. - 19 & \\
\hline Office-3 & 88.5 & 81 & 20 & Server - 66 & \\
\hline Office-4 & 63.5 & 29.5 & & IT - 29 & Misc. -63 \\
\hline $\begin{array}{c}\text { Mail } \\
\text { Sorting-1 }\end{array}$ & 48 & 57 & 29 & & \\
\hline $\begin{array}{c}\text { Mail } \\
\text { Sorting-2 }\end{array}$ & 319 & 99 & 29 & & \\
\hline $\begin{array}{c}\text { Mail } \\
\text { Sorting-3 }\end{array}$ & 37 & 25 & 9 & Doors - 14 & \\
\hline $\begin{array}{c}\text { Public } \\
\text { Assembley-1 }\end{array}$ & 45 & 20 & 20 & & \\
\hline
\end{tabular}

\subsubsection{Academic-1}

The building is a midsize academic building $\left(4,130 \mathrm{~m}^{2}\right)$ that includes classrooms, computer labs, a café and study areas.

Energy use data was collected between February 2011 and January 2012.

The building includes efficient lighting design as well as extensive use of occupancy sensors. No meter attribution documentation was available beyond column labeling in metering data. Due to this fact exact composition of the metered energy use is unavailable. Lighting energy use may include exterior or emergency lighting. Lighting schedules used in energy modeling do not include exterior lighting. 
Overall lighting is consuming more energy use than expected due to higher than expected overnight base loads. Receptacle loads are consuming more energy than expected due to higher daytime peak loads, as well as higher overnight and weekend base loads. Higher receptacle loads can in part be attributed to more computer and a/v equipment being installed than had been considered in the modeling schedules.

Table 8. Academic-1: Building Summary

\begin{tabular}{|c|c|}
\hline $\begin{array}{c}\text { Location } \\
\text { Floor Area } \\
\text { Submetering Categories }\end{array}$ & $\begin{array}{c}\text { Hamilton, Ontario } \\
4,130 \mathrm{~m}^{2} \\
\text { Lighting, } \\
\text { Receptacles, HVAC, } \\
\text { DHW, Café, Cooling }\end{array}$ \\
\hline Reporting Period & Feb 2011 - Jan 2012 \\
\hline Data Availability & Feb 2011 - Jan 2012 \\
\hline Metering Attribution & Limited \\
\hline $\begin{array}{l}\text { Lighting: } \\
\text { Installed LPD }\end{array}$ & $8.0 \mathrm{~W} / \mathrm{m}^{2}$ \\
\hline $\begin{array}{l}\text { Receptacles: } \\
\text { Installed Receptacles PD }\end{array}$ & $3.4 \mathrm{~W} / \mathrm{m}^{2}$ \\
\hline
\end{tabular}

Table 9 lists the key values for predicted and actual energy use of the building. Fairly substantial differences are visible between the various values. Adequate documentation to determine energy use from the uncalibrated energy models was not available.

Table 9. Academic-1: Predicted and Actual Energy Use

\begin{tabular}{cccc}
\hline & $\begin{array}{c}\text { Uncalibrated- } \\
\text { Predicted } \\
\left(\mathbf{k W h} / \mathbf{m}^{\mathbf{2}}\right)\end{array}$ & $\begin{array}{c}\text { Calibrated- } \\
\text { Predicted } \\
\mathbf{( k W h / \mathbf { m } ^ { 2 } )}\end{array}$ & $\begin{array}{c}\text { Actual } \\
\left(\mathbf{k W h} / \mathbf{m}^{\mathbf{2}}\right)\end{array}$ \\
\hline Total Energy Use & - & 87.4 & 99.6 \\
\hline $\begin{array}{c}\text { Total Electricity } \\
\text { Energy Use }\end{array}$ & - & 83.9 & 98.1 \\
Total Natural Gas & - & 3.6 & 1.5 \\
\hline Heating & - & - & - \\
Cooling & - & 21.3 & 11.8 \\
Lighting & - & 24.3 & 41.2 \\
Receptacles & - & 10.3 & 13.7 \\
\hline
\end{tabular}




\section{Lighting}

Table 10. Academic-1: Building Lighting Summary

\begin{tabular}{cccc}
\hline $\begin{array}{c}\text { As Modeled: } \\
\text { Installed LPD } \\
\left(\mathbf{W} / \mathbf{m}^{2}\right)\end{array}$ & $\begin{array}{c}\text { MNP Lighting } \\
\text { Load }\left(\mathbf{W} / \mathbf{m}^{2}\right)\end{array}$ & $\begin{array}{c}\text { MNB Lighting } \\
\text { Load }\left(\mathbf{W} / \mathbf{m}^{2}\right)\end{array}$ & $\begin{array}{c}\text { Base as \% } \\
\text { of Peak }\end{array}$ \\
\hline 8.0 & 7.2 & 0.4 & $6 \%$ \\
\hline $\begin{array}{c}\text { As Measured: } \\
\text { Installed LPD } \\
\left(\mathbf{W} / \mathbf{m}^{2}\right)\end{array}$ & $\begin{array}{c}\text { MNP Lighting } \\
\text { Load }\left(\mathbf{W} / \mathbf{m}^{2}\right)\end{array}$ & $\begin{array}{c}\text { MNB Lighting } \\
\text { Load }\left(\mathbf{W} / \mathbf{m}^{2}\right)\end{array}$ & $\begin{array}{c}\text { Base as \% } \\
\text { of Peak }\end{array}$ \\
\hline- & 6.9 & 3.9 & $56 \%$ \\
\hline
\end{tabular}

Table 10 summarizes the median near peak (MNP) lighting loads and median near base (MNB) lighting loads as modeled and as measured. Comparison between the lighting profile and lighting schedules show a significantly under-predicted nighttime and weekend base-load (Figure 12 and Figure 13). Calculated from measured data, MNP and MNB values are $6.9 \mathrm{~W} / \mathrm{m}^{2}$ and $3.9 \mathrm{~W} / \mathrm{m}^{2}$ respectively. Thus the off-hours MNB is approximately $56 \%$ of the MNP load, as opposed to the estimated 6\% assumed in the models. This indicates a high level of lighting being used at times when this was not expected, some of this may be contributed by exterior lighting not included in the model, but which may be included in the metering, although this should not contribute more than $0.9 \mathrm{~W} / \mathrm{m}^{2}$ (assuming exterior lights operated for on average approximately $8 \mathrm{hrs} /$ day at max power). On the other hand a measured MNP load of $6.9 \mathrm{~W} / \mathrm{m}^{2}$ matches closely the estimated peak load $(7.2 \mathrm{~W} / \mathrm{m} 2)$ for the building suggesting that when the lights are fully in use they are functioning as expected. Thus the variations between expected and actual lighting energy appear to be due to controls and building management issues, or a significant difference in occupancy compared to modeling assumptions. Site visits subsequent to building occupancy indicated that occupancy sensors could result in the lighting for an entire room being turned on even if an individual student was present. This was noticed as a particular issue during the night when 
lighting schedules predict low energy use. This is likely a contributing factor to the above-expected base load.

Legend:

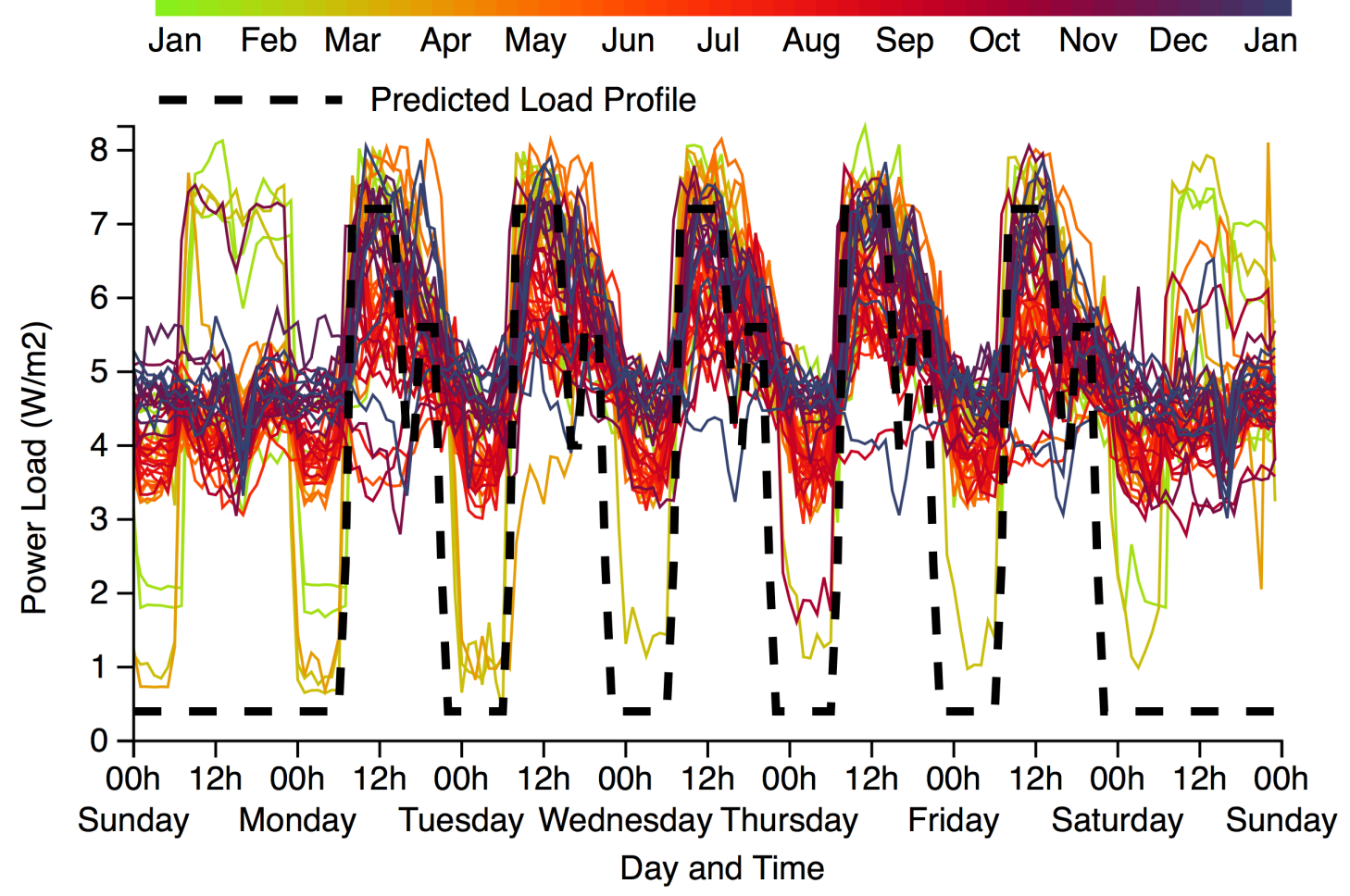

Figure 12. Academic-1: Full-year 60-Minute Interval Lighting Energy Use Data

Average, median and typical lighting loads are shown for weeks, weekdays and weekend, respectively, in Figure 13, Figure 14 and Figure 15. The values calculated for the MNP and MNB are more clearly represented in these figures. In Figure 14 the morning ramp up appears to occur 5:00 hours, earlier than expected, no real dip appears to occur around 17:30, and energy levels don't return to base levels until 00:00. In regards to the weekend load profile (Figure 15) very little variation occurs through out the day. Only small dips are visible for off-peak periods such as 00:00 until 5:00, and for breaks in occupancy such as between 17:00 and 18:00. 
Legend:

Average Median $\square$ Typical

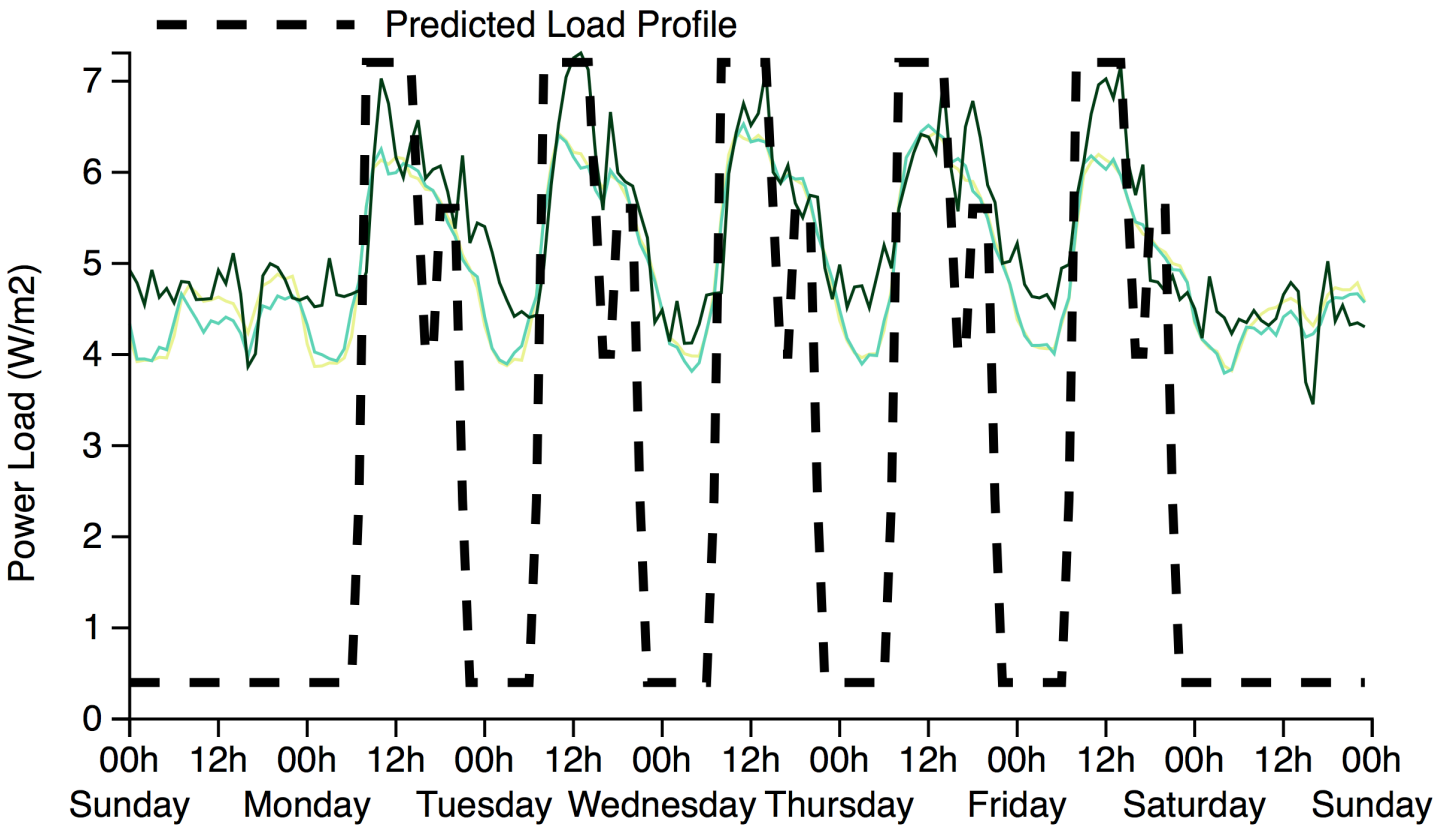

Day and Time

Figure 13. Academic-1: Average, Median, and Typical Weekly Profiles for Lighting Energy Use

Legend:

Average Median $\square$ Typical

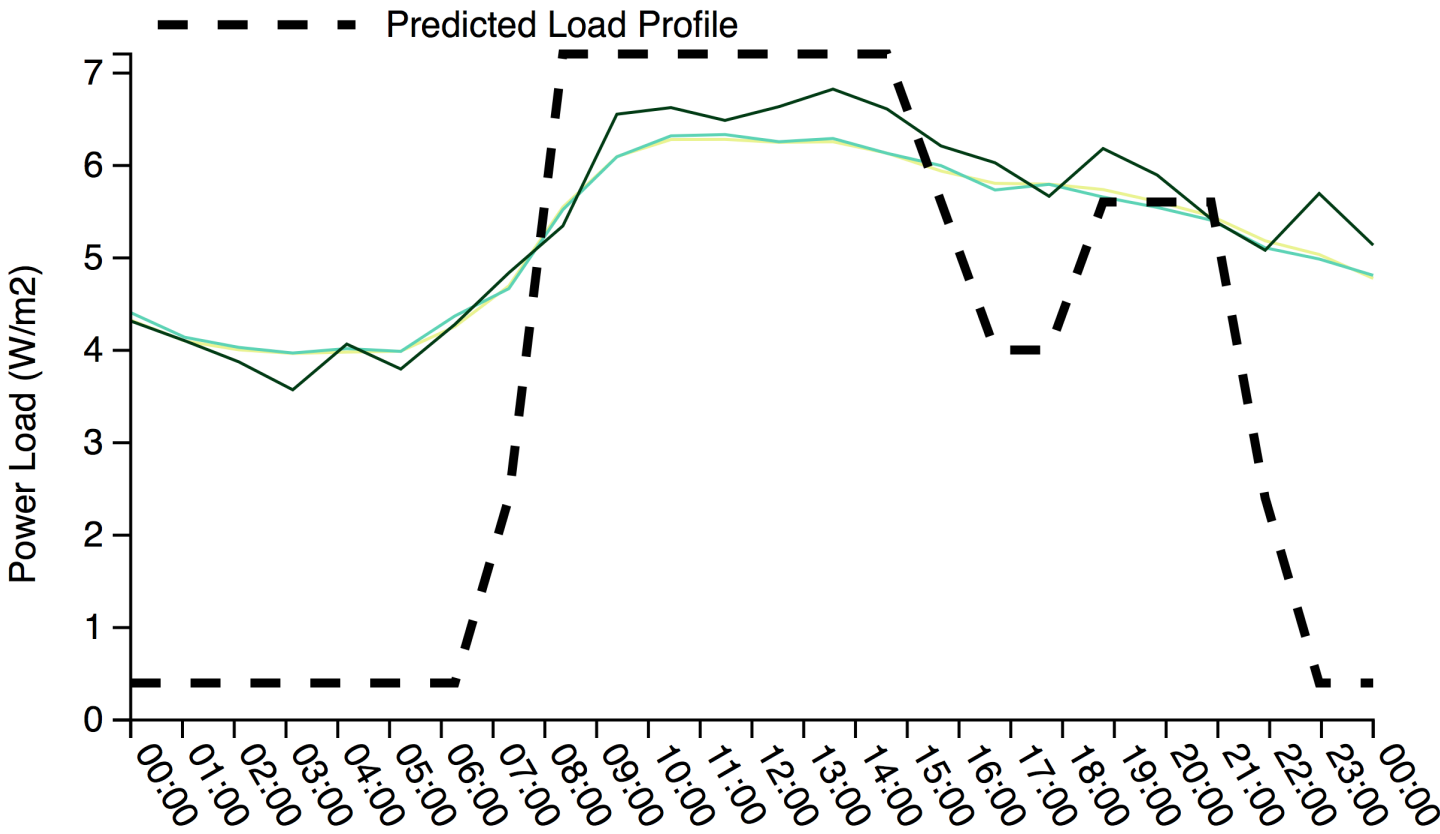

Hour

Figure 14. Academic-1: Average, Median, and Typical Weekday Lighting Profile

46 
Legend:

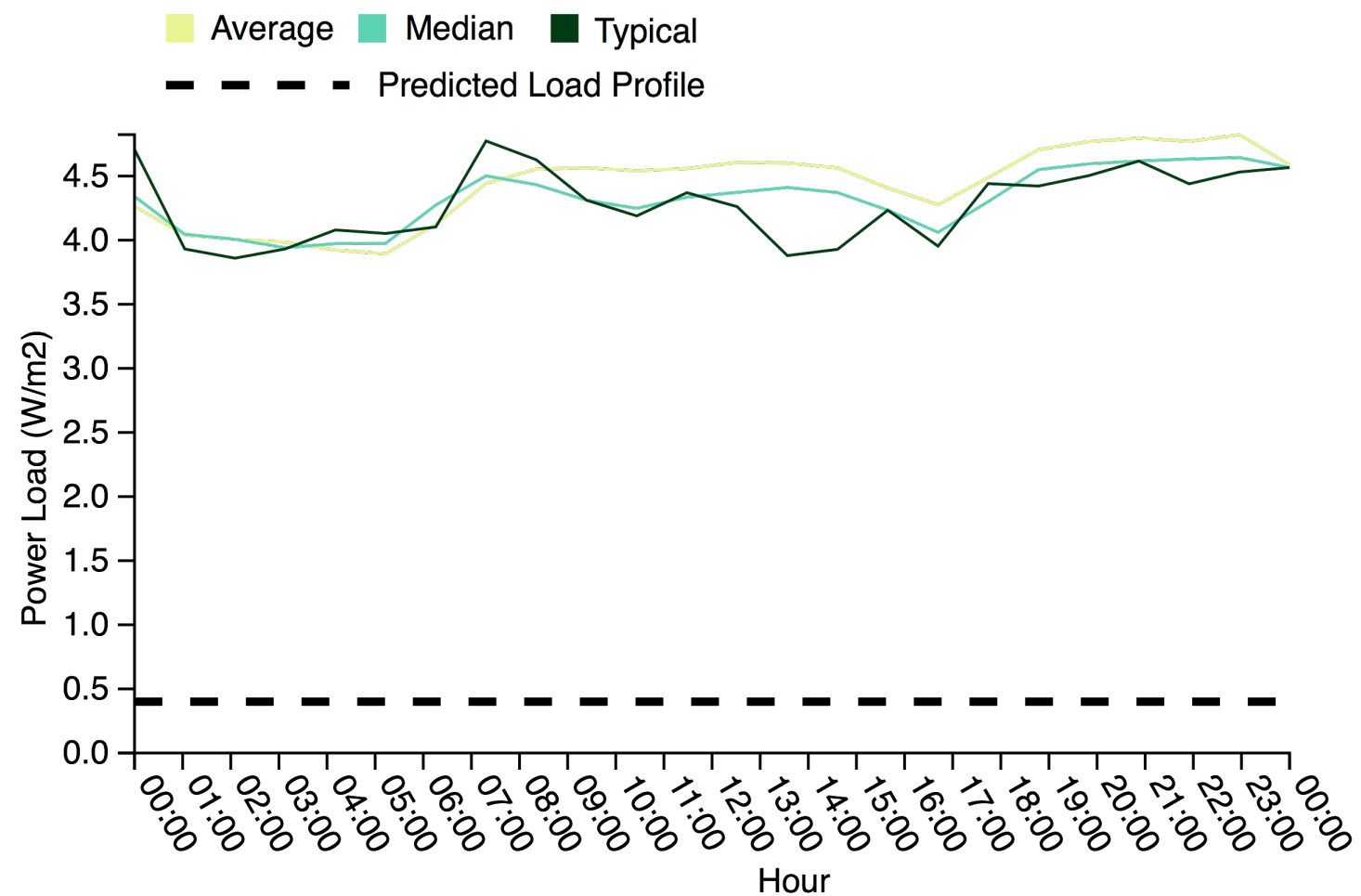

Figure 15. Academic-1: Average, Median, and Typical Weekend Day Lighting Profile

Considering Figure 16 it is possible to see the variation of the energy load profile throughout the year. As would be expected with an academic building higher energy use is witnessed during the academic year (September to April) with significantly lower energy use in December and June to August. Besides this it is interesting to note a fairly irregular daily load. Fluctuating weekday loads is likely due in part to a highly variable occupancy. It is also clear that even in the low occupancy months the building lighting use is well above the modeled assumptions for monthly load (the black dashed line). This may be due to the much higher than expected base load. 
Legend:

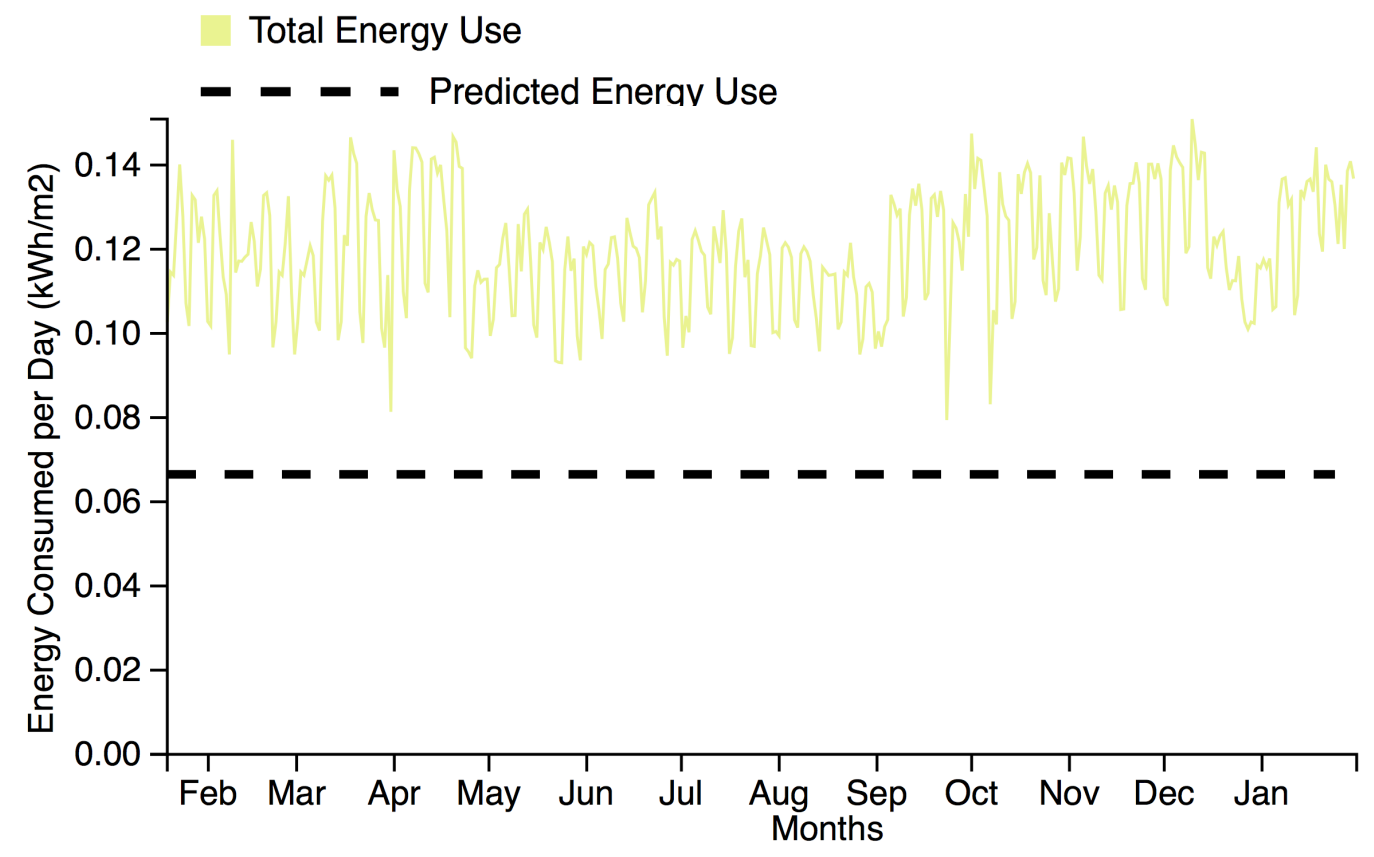

Figure 16. Academic-1: Full-year Total Daily Lighting Energy Use Data

Figure 17 shows a regression analysis correlating seasonal changes in lighting energy use with bright sunshine availability. The analysis demonstrates a fairly strong correlation $\left(\mathrm{r}^{2}=0.45\right)$ between lighting energy use and day light hours for this building. This indicates that on average light energy consumption does decrease as daylight availability increases. Although as mentioned above this will be affected by the occupancy dynamics of an academic building, where occupancy tends to be highest during the academic semester which coincides with the lowest light availability, and lowest in the summer which coincides with high light availability. Therefore it is possible that the observed affect is more a function of occupancy than it is of daylight availability. 


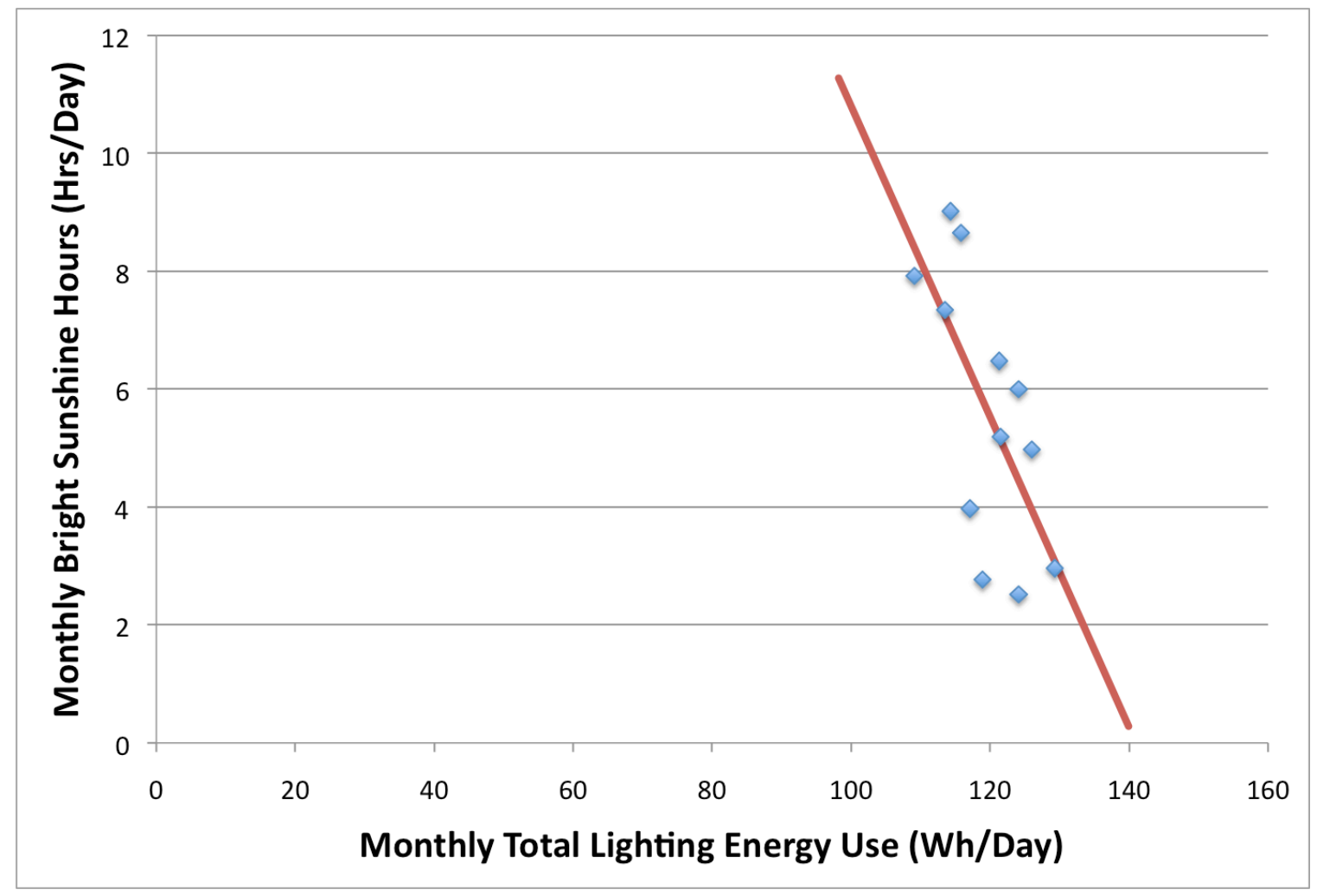

Figure 17. Academic-1: Lighting Energy Use Versus Sunshine Hours Regression $\left(r^{2}=0.45\right.$, slope $=-$ 0.26)

\section{Receptacles}

Table 11. Academic-1: Building Receptacles Summary

\begin{tabular}{cccc}
\hline $\begin{array}{c}\text { As Modeled: } \\
\text { Installed } \\
\text { Receptacles PD } \\
\left(\mathbf{W} / \mathbf{m}^{\mathbf{2}}\right)\end{array}$ & $\begin{array}{c}\text { MNP Receptacle } \\
\text { Loads } \\
\left(\mathbf{W} / \mathbf{m}^{\mathbf{2}}\right)\end{array}$ & $\begin{array}{c}\text { MNB Receptacle } \\
\text { Loads } \\
\left(\mathbf{W} / \mathbf{m}^{\mathbf{2}}\right)\end{array}$ & $\begin{array}{c}\text { Base as \% } \\
\text { of Peak }\end{array}$ \\
\hline 3.4 & 3.1 & 0.17 & $6 \%$ \\
\hline $\begin{array}{c}\text { As Measured: } \\
\text { Installed } \\
\begin{array}{c}\text { Receptacles PD } \\
\left(\mathbf{W} / \mathbf{m}^{\mathbf{2}}\right)\end{array}\end{array}$ & $\begin{array}{c}\text { MNP Receptacle } \\
\text { Loads }\left(\mathbf{W} / \mathbf{m}^{\mathbf{2}}\right)\end{array}$ & $\begin{array}{c}\text { MNB Receptacle } \\
\text { Loads } \\
\left(\mathbf{W} / \mathbf{m}^{\mathbf{2}}\right)\end{array}$ & $\begin{array}{c}\text { Base as \% } \\
\text { of Peak }\end{array}$ \\
\hline- & 4.2 & 1.4 & $43 \%$ \\
\hline
\end{tabular}

Table 11 summarizes the median near peak (MNP) receptacle loads and median near base (MNB) receptacle loads as modeled and as measured. Comparison between the receptacles load profile and the modeling schedules shows an under- 
prediction in daytime peak and nighttime base load (Figure 18 and Figure 19). Calculated from measured data, MNP and MNB loads have values of $4.2 \mathrm{~W} / \mathrm{m}^{2}$ and $1.4 \mathrm{~W} / \mathrm{m}^{2}$ respectively. Thus the off-hours base load is approximately $43 \%$ of the peak load, as opposed to the estimated $6 \%$ assumed in the models. This indicates that the building is not shutting off loads to the extent expected during off-hours. A MNP load of $4.2 \mathrm{~W} / \mathrm{m}^{2}$ is $135 \%$ of an estimated peak load of approximately 3.1 $\mathrm{W} / \mathrm{m}^{2}$. Indicating also that when the building is operating at max capacity that it is using more energy than expected. This quantity can be easily accounted for however. Post-processing calibration included a 19,000 kWh of additional receptacle loads that is not represented by the modeling schedules, assuming 12 hrs/day of operational time this receptacle energy use would amount to approximately $1.0 \mathrm{~W} / \mathrm{m}^{2}$ of receptacle loads bringing the estimated peak load of 3.1 $\mathrm{W} / \mathrm{m}^{2}$ up to approximately $4.1 \mathrm{~W} / \mathrm{m}^{2}$. As well through site visits conducted during the commissioning process it was observed that significant amounts of computer and $\mathrm{A} / \mathrm{V}$ equipment was present in the building that was unaccounted for in the modeling process. These kinds of additional loads can easily account for the additional energy use observable through the load profiles.

Figure 18 allows us to consider the day-to-day loads experienced by the building. It is demonstrated that for the most part these loads are fairly unstable, with both base and peak energy use and varying dramatically, this suggests exactly the extent to which occupancy affects the operation of the building. However it should also be noted that variations in receptacles energy use especially over weekends is markedly more stable than for lighting, perhaps due to the variation caused by systems such as occupancy sensors engaging the lighting systems even for low levels of occupancy over the weekends.

Average, median and typical receptacle loads are shown for weeks, weekdays and weekend, respectively, in Figure 19, Figure 20 and Figure 21. The values calculated for the MNP and MNB are more clearly represented in these figures. In Figure 20 the morning ramp up appears to begin when expected, and to begin ramping down around 17:30 as expected. Energy levels don't return to base levels until 00:00. In regards to the weekend load profile (Figure 21) very little meaningful 
variation occurs through out the day. Only small dips are visible around 9:00 and 17:00.

Legend:

Jan Feb Mar Apr May Jun Jul Aug Sep Oct Nov Dec Jan

- _ - Predicted Load Profile

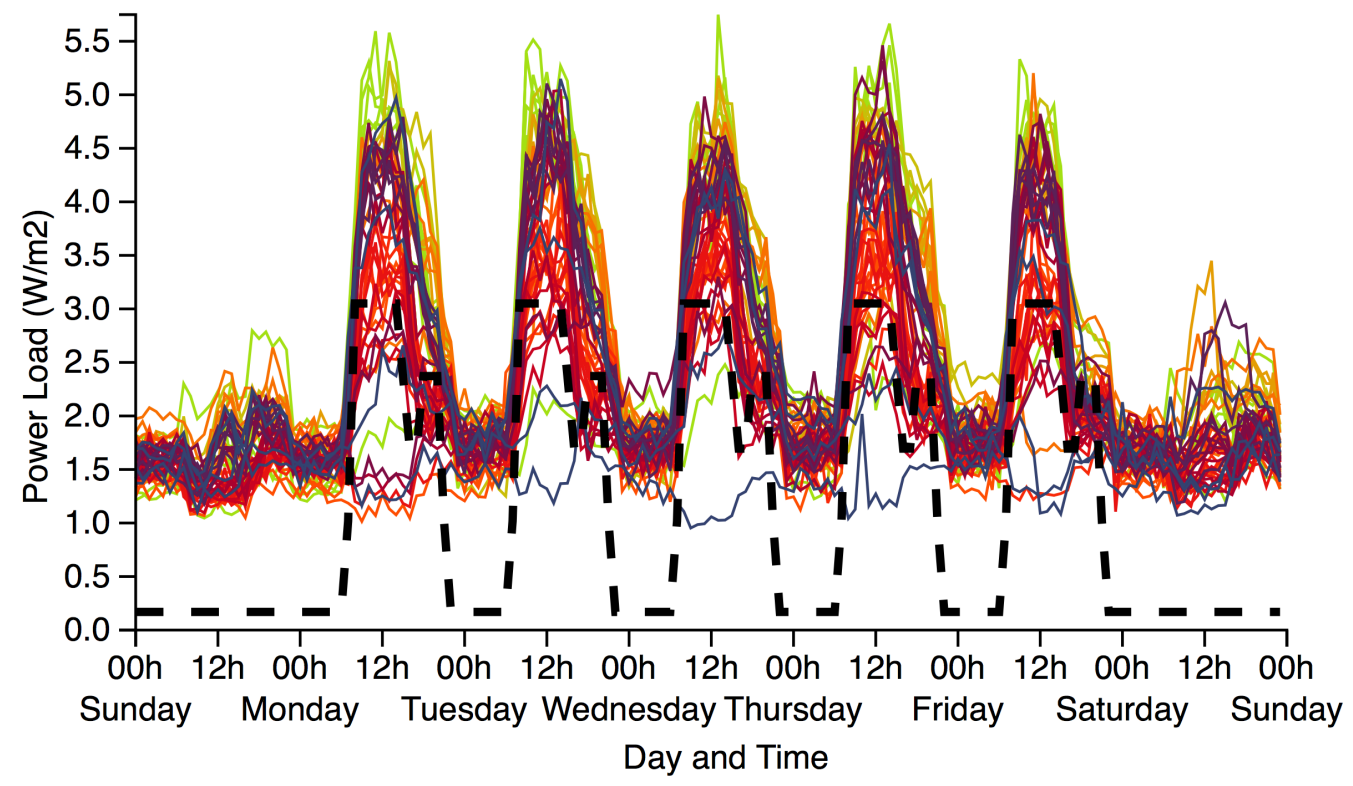

Figure 18. Academic-1: Full-year 60-Minute Interval Receptacles Energy Use Data

Legend:

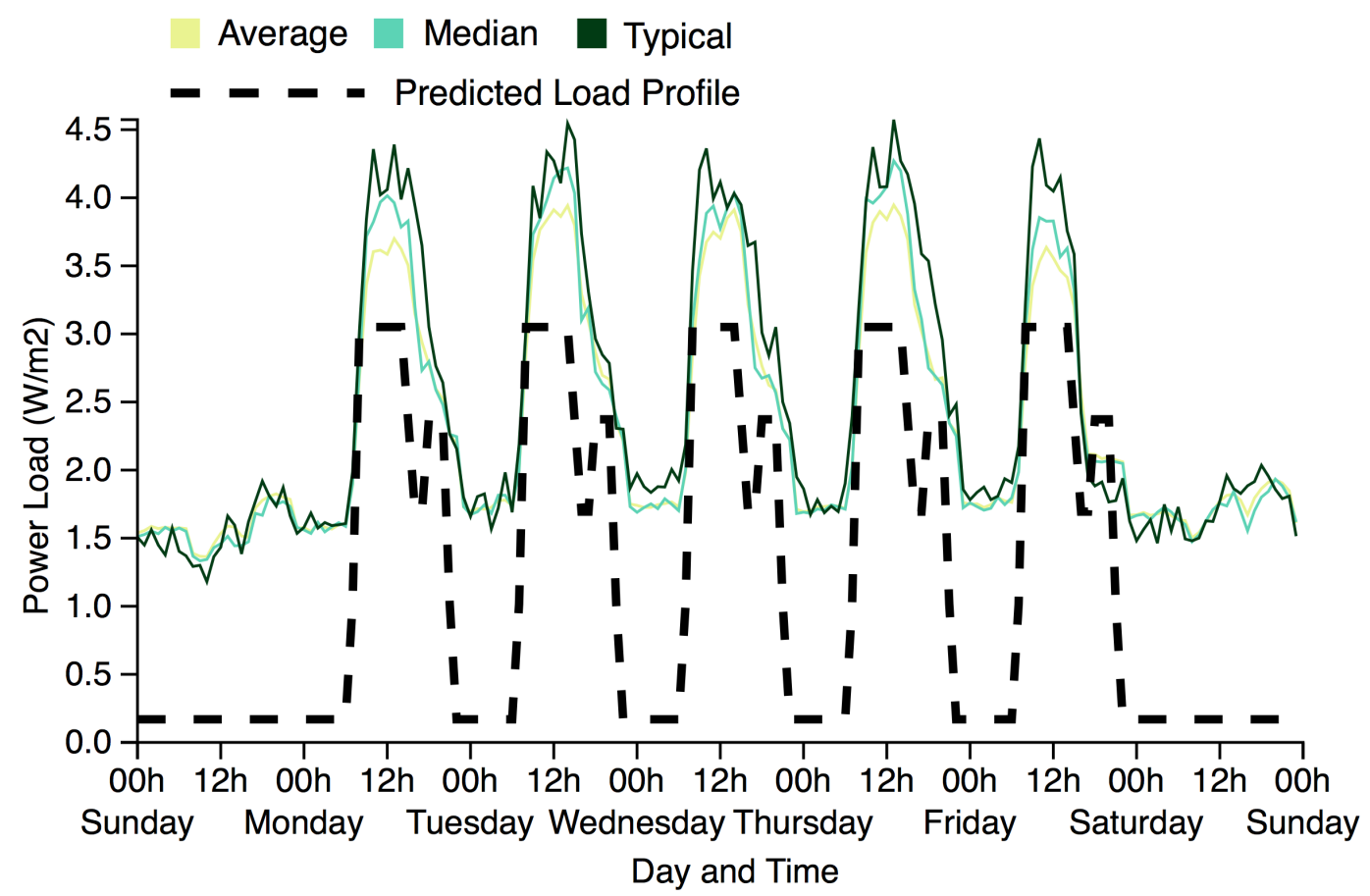

Figure 19. Academic-1: Average, Median, and Typical Weekly Profiles for Receptacles Energy Use 
Legend:

Average Median $\square$ Typical

- c - - Predicted Load Profile

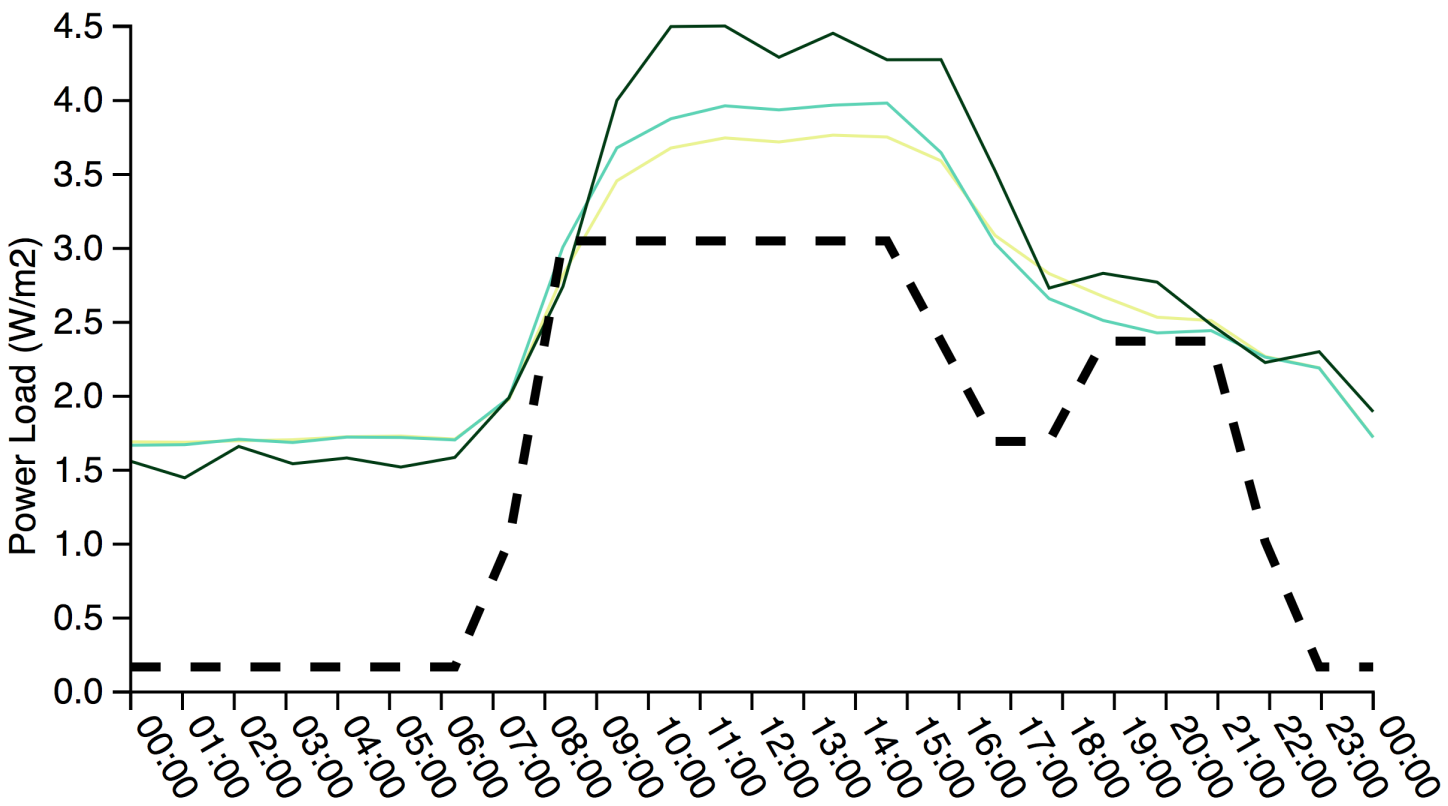

Hour

Figure 20. Academic-1: Average, Median, and Typical Weekday Receptacles Profile

Legend:

Average Median $\square$ Typical

- c - Predicted Load Profile

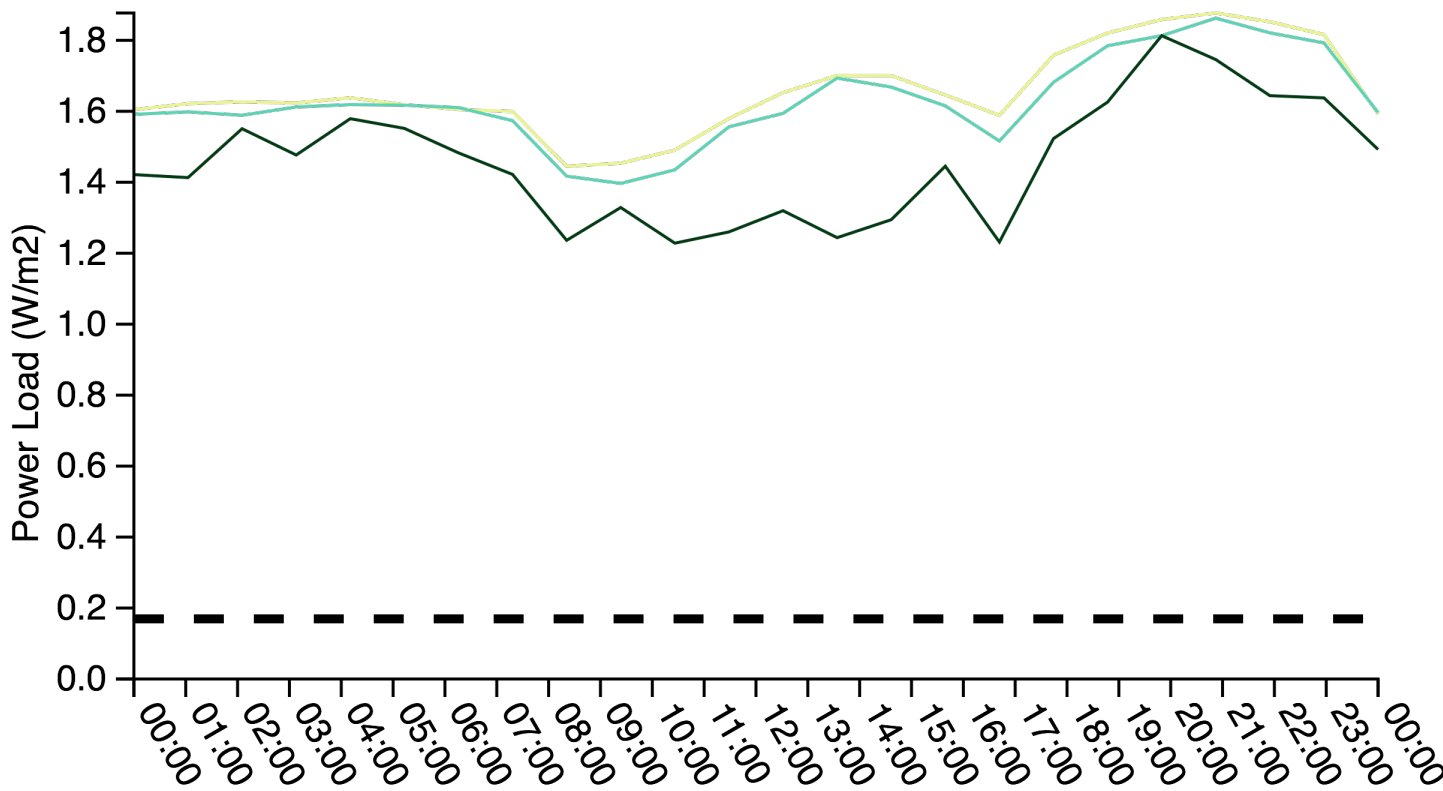

Hour

Figure 21. Academic-1: Average, Median, and Typical Weekend Day Receptacles Profile

52 
Considering Figure 22 it is possible to see the variations in receptacle energy use that occurs throughout the entire year. It is clear that the receptacle energy use is always above the average daily energy use assumed in the modeling (the dashed line). Energy use is reduced during summer and winter holidays (including May, June, and July but especially December and August) by about $0.01-0.015 \mathrm{kWh} / \mathrm{m}^{2}$ per day, but nevertheless remains consistently above the modeled values. These seasonal reductions in energy use are expected in academic buildings and are easily observable in the lighting load profiles visible above.

\section{Legend:}

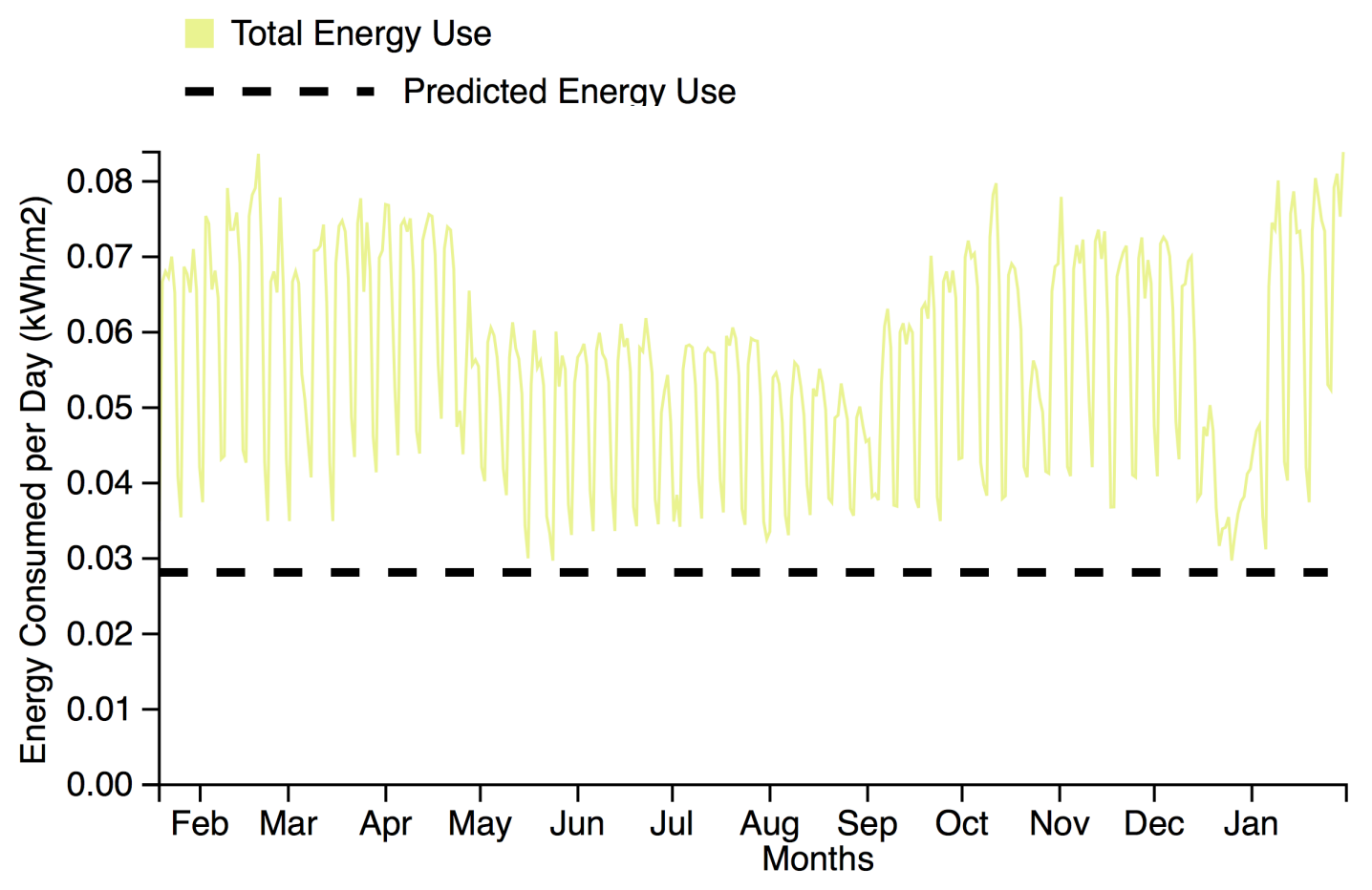

Figure 22. Academic-1: Full-year Total Daily Receptacles Energy Use Data

\subsubsection{Office-3}

The building is a 4-storey multi-tenant midsize office building $(7,934 \mathrm{~m} 2)$. Lighting Energy use data was available between October 2009 and April 2010. During the measurement period only the $1^{\text {st }}$ and $4^{\text {th }}$ floors were occupied. This is reflected in the schedules. As demonstrated in Figure 102 and Table 58 (Appendix B), Zones 6 through 10 (2nd and $3^{\text {rd }}$ floor) are scheduled to operate $24 / 7$ at $5 \%$ of 
their installed capacity. No meter attribution documentation was available beyond column labeling in metering data. Due to this fact exact composition of the metered energy use is unavailable. However exterior lighting and emergency lighting were metered separately. Lighting schedules used in energy modeling do not include exterior lighting. Overall lighting is consuming more energy than expected due to higher daytime peak loads, and higher overnight base loads. Receptacle loads are consuming more energy than expected due to higher daytime peak loads, as well as higher overnight and weekend base loads.

Table 12. Office-3: Building Summary

\begin{tabular}{cc}
\hline Location & $\begin{array}{c}\text { Toronto, Ontario } \\
\text { Floor Area }\end{array}$ \\
Submetering Categories & $\begin{array}{c}\text { Lighting, Receptacles, } \\
\text { Exterior Lighting, } \\
\text { Elevator, Emergency } \\
\text { Power, Heating, Cooling, } \\
\text { HVAC }\end{array}$ \\
$\begin{array}{c}\text { Reporting Period } \\
\text { Data Availability }\end{array}$ & $\begin{array}{c}\text { Jan } 2009-\text { Mar } 2010 \\
\text { Oct } 2009-\text { April } 2010 \\
\text { Limited }\end{array}$ \\
\hline $\begin{array}{c}\text { Lighting: } \\
\text { Installed LPD }\end{array}$ & $7.4 \mathrm{~W} / \mathrm{m}^{2}$ \\
\hline $\begin{array}{c}\text { Receptacles: } \\
\text { Installed Receptacles PD }\end{array}$ & $6.5 \mathrm{~W} / \mathrm{m}^{2}$ \\
\hline
\end{tabular}

Table 13 lists the key values for predicted and actual energy use of the building. Fairly substantial differences are visible between the various values.

Table 13. Office-3: Predicted and Actual Energy Use

\begin{tabular}{cccc}
\hline & $\begin{array}{c}\text { Uncalibrated- } \\
\text { Predicted } \\
\left(\mathbf{k W h} / \mathbf{m}^{\mathbf{2}}\right)\end{array}$ & $\begin{array}{c}\text { Calibrated- } \\
\text { Predicted } \\
\left(\mathbf{k W h} / \mathbf{m}^{\mathbf{2}}\right)\end{array}$ & $\begin{array}{c}\text { Actual } \\
\left(\mathbf{k W h} / \mathbf{m}^{\mathbf{2}}\right)\end{array}$ \\
\hline Total Energy Use & 99.1 & 109.8 & 133.7 \\
\hline $\begin{array}{c}\text { Total Electricity } \\
\text { Energy Use }\end{array}$ & 69.8 & 85.4 & 110.5 \\
Total Natural Gas & 29.3 & 24.0 & 22.9 \\
\hline Heating & 36.9 & 24.7 & - \\
Cooling & 9.7 & 10.0 & 11.0 \\
Lighting & 9.6 & 11.2 & 15.5 \\
Receptacles & 7.9 & 10.2 & 17.7 \\
\hline
\end{tabular}




\section{Lighting}

Table 14. Office-3: Building Lighting Summary

\begin{tabular}{cccc}
\hline $\begin{array}{c}\text { As Modeled: } \\
\text { Installed LPD } \\
\left(\mathbf{W} / \mathbf{m}^{2}\right)\end{array}$ & $\begin{array}{c}\text { MNP Lighting } \\
\text { Loads }\left(\mathbf{W} / \mathbf{m}^{2}\right)\end{array}$ & $\begin{array}{c}\text { MNB Lighting } \\
\text { Loads }\left(\mathbf{W} / \mathbf{m}^{2}\right)\end{array}$ & $\begin{array}{c}\text { Base as \% } \\
\text { of Peak }\end{array}$ \\
\hline 7.4 & 3.1 & 0.4 & $12 \%$ \\
\hline $\begin{array}{c}\text { As Measured: } \\
\text { Installed LPD } \\
\left(\mathbf{W} / \mathbf{m}^{2}\right)\end{array}$ & $\begin{array}{c}\text { MNP Lighting } \\
\text { Loads }\left(\mathbf{W} / \mathbf{m}^{2}\right)\end{array}$ & $\begin{array}{c}\text { MNB Lighting } \\
\text { Loads }\left(\mathbf{W} / \mathbf{m}^{2}\right)\end{array}$ & $\begin{array}{c}\text { Base as } \% \\
\text { of Peak }\end{array}$ \\
\hline- & 4.0 & 1.5 & $37 \%$ \\
\hline
\end{tabular}

Table 14 summarizes the median near peak (MNP) lighting loads and median near base (MNB) lighting loads as modeled and as measured. Comparison between the lighting energy use profile and lighting schedules show a significantly underpredicted nighttime and weekend base-load as well as an under-predicted daytime peak load (Figure 23 and Figure 24). Calculated from measured data, MNP and MNB load values are $4.0 \mathrm{~W} / \mathrm{m}^{2}$ and $1.5 \mathrm{~W} / \mathrm{m}^{2}$ respectively. Thus off-hours MNB is approximately $37 \%$ of the MNP load, as opposed to the estimated $12 \%$ as assumed in the modeling. This indicates higher levels of off-peak energy use than expected. The measured MNP load of $4.0 \mathrm{~W} / \mathrm{m}^{2}$ is $131 \%$ of an estimated peak load of approximately $3.1 \mathrm{~W} / \mathrm{m}^{2}$. This value is so low in comparison to the $7.4 \mathrm{~W} / \mathrm{m}^{2}$ of predicted installed lighting capacity, because only approximately half of the building is occupied, yet the loads are still normalized over the whole floor area.

Figure 23 demonstrates that very few weeks from the observed year varied outside of typical usage patterns, indicating similar usage patterns for each week throughout the year. Energy use peaks up to $5 \mathrm{~W} / \mathrm{m}^{2}$ may indicate a higher than anticipated level of lighting in the unoccupied areas. A base load of $1.5 \mathrm{~W} / \mathrm{m}^{2}$ (compared to the expected $0.4 \mathrm{~W} / \mathrm{m} 2$ ) during off-peak time likely indicates a greater number of lighting services are being left on overnight. A small weekend peak up to $2 \mathrm{~W} / \mathrm{m}^{2}$ likely demonstrates a greater level of use of the building over the weekends. 
Legend:

Jan Feb Mar Apr May Jun Jul Aug Sep Oct Nov Dec Jan

- _ - Predicted Load Profile

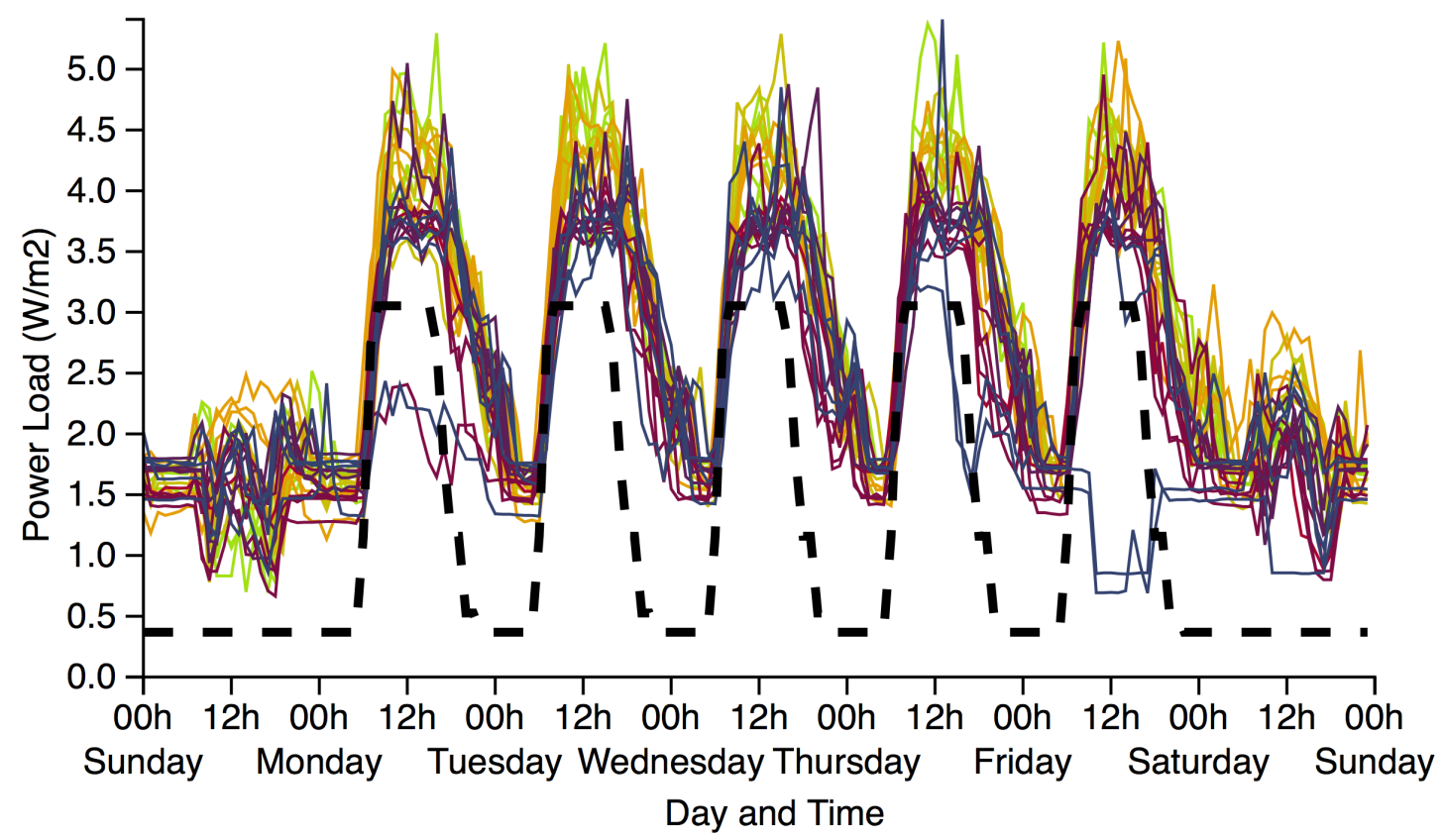

Figure 23. Office-3: Full-year 60-Minute Interval Lighting Energy Use Data

Average, median and typical lighting loads are shown for weeks, weekdays and weekend, respectively, in Figure 24, Figure 25 and Figure 26. The values calculated for the MNP and MNB are more clearly represented in these figures. In Figure 25 the weekday morning ramp up appears to begin when expected, and to begin ramping down around 17:30 as expected. The ramp down occurs much more slowly and energy levels don't return to base levels until 00:00. In regards to the weekend load profile (Figure 26) very little meaningful variation occurs through out the day. A small dip is visible around 9:00 with a larger dip around 17:00. A slight midday bulge is also visible. 
Legend:

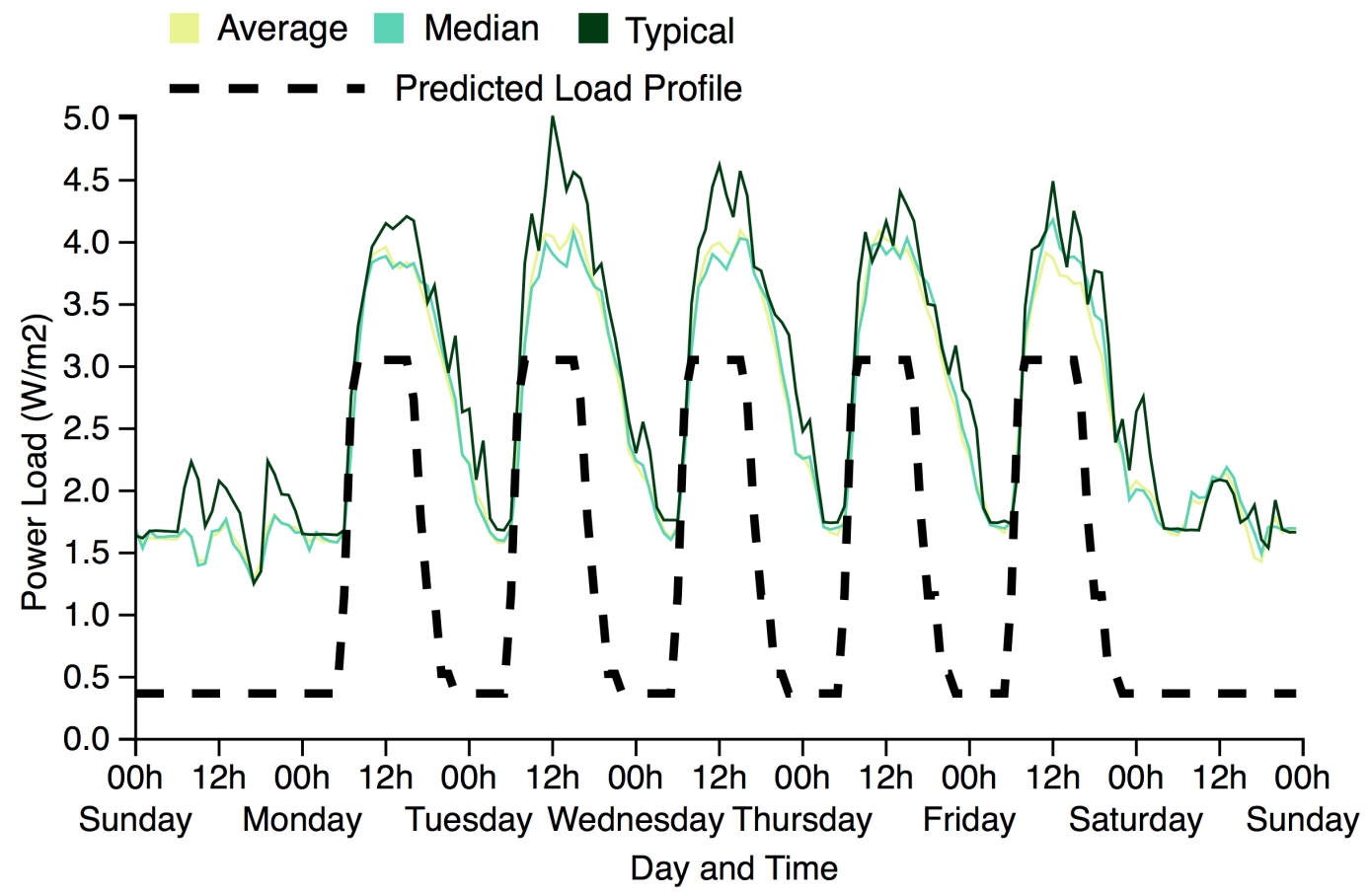

Figure 24. Office-3: Average, Median, and Typical Weekly Profiles for Lighting Energy Use

Legend:

Average Median $\square$ Typical

- c - a Predicted Load Profile

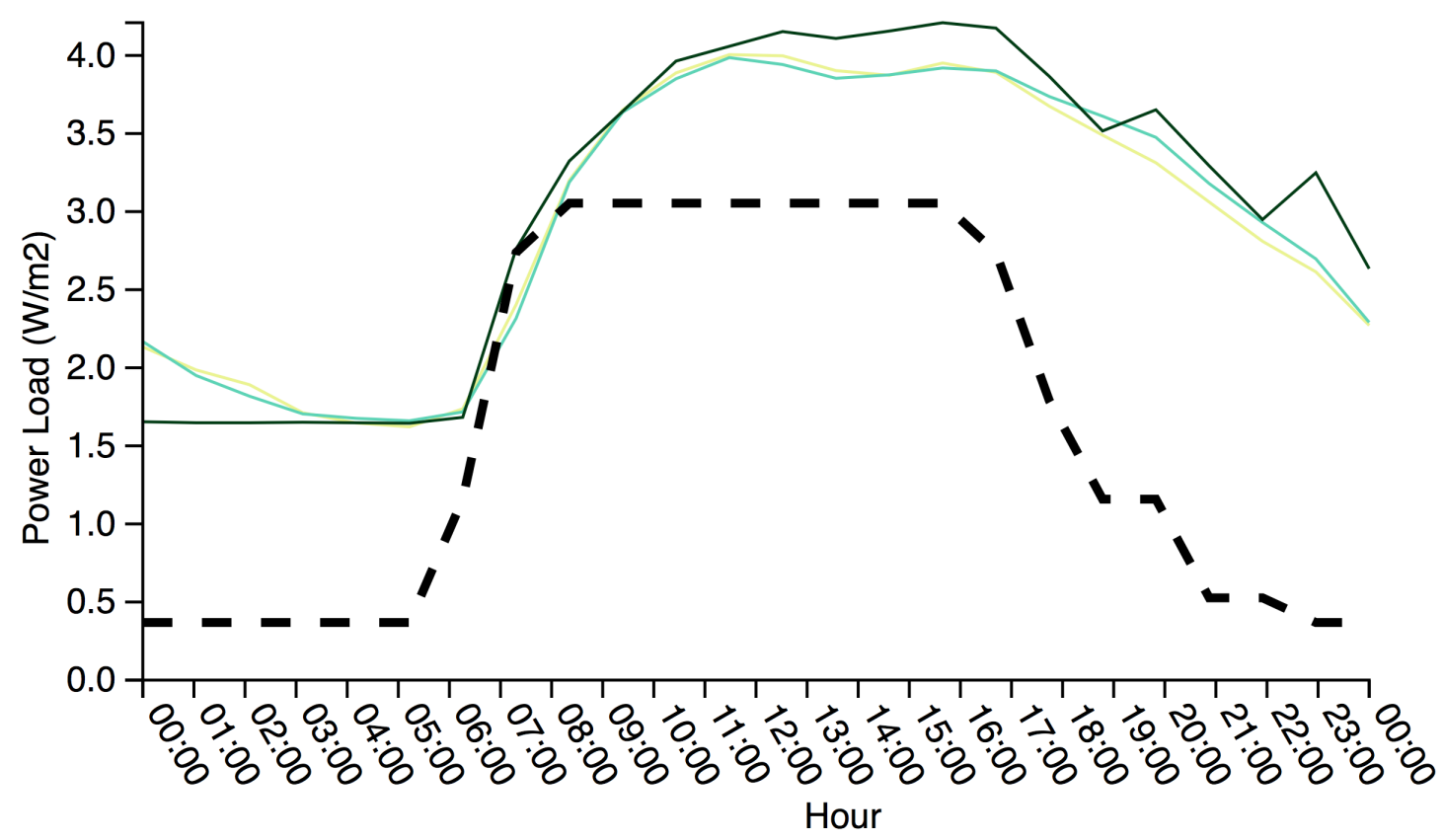

Figure 25. Office-3: Average, Median, and Typical Weekday Lighting Profile 
Legend:

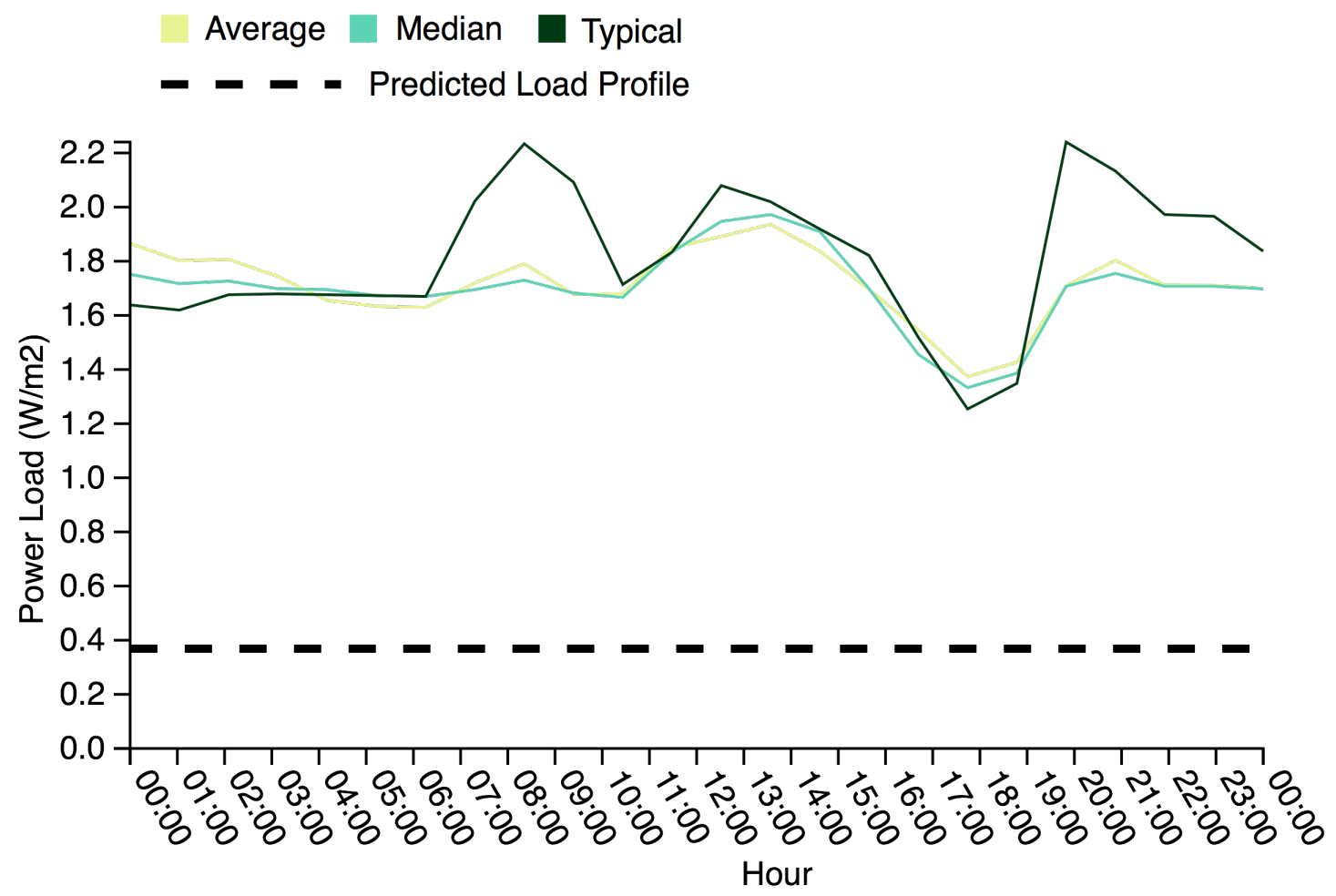

Figure 26. Office-3: Average, Median, and Typical Weekend Day Lighting Profile

Legend:

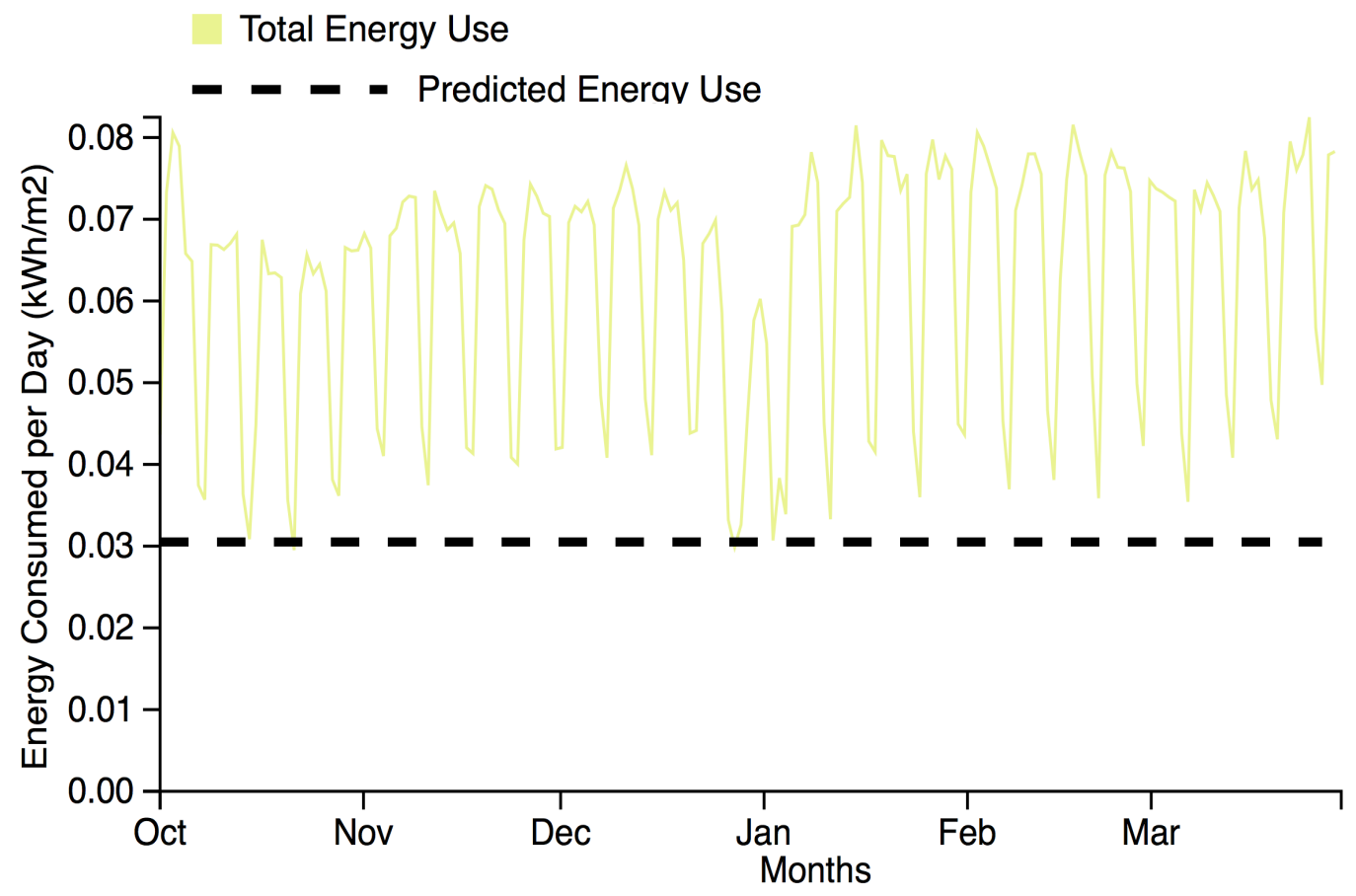

Figure 27. Office-3: Public Assembly-1: Full-year Total Daily Lighting Energy Use Data 
Considering Figure 27 it is possible to observe the lighting load profile over the course of the year. Energy consumption is noticeably lower in the fall months than in the winter months. Late December experiences a drop in energy use due to lower holiday occupancy. In general the load profile remains relatively stable, this is likely contributed to by a predictable and stable occupancy. However Figure 27 also shows that both the measured base and peak loads are considerably higher than the modeling assumptions. In fact the measurements suggest that the load never drops near to the assumed base load.

Figure 28 shows a regression analysis correlating seasonal changes in lighting energy use with bright sunshine availability. The analysis demonstrates a weak negative correlation $\left(r^{2}=0.01\right)$ between lighting energy use and daylight hours for this building. This indicates that on average light energy consumption does decrease as daylight availability increases. Out of the 7 buildings, Office-3 has the least data available with which to conduct the analysis due to the fact that metering is only available for the period of 7 months. This lessens the chance of observing a trend in the data.

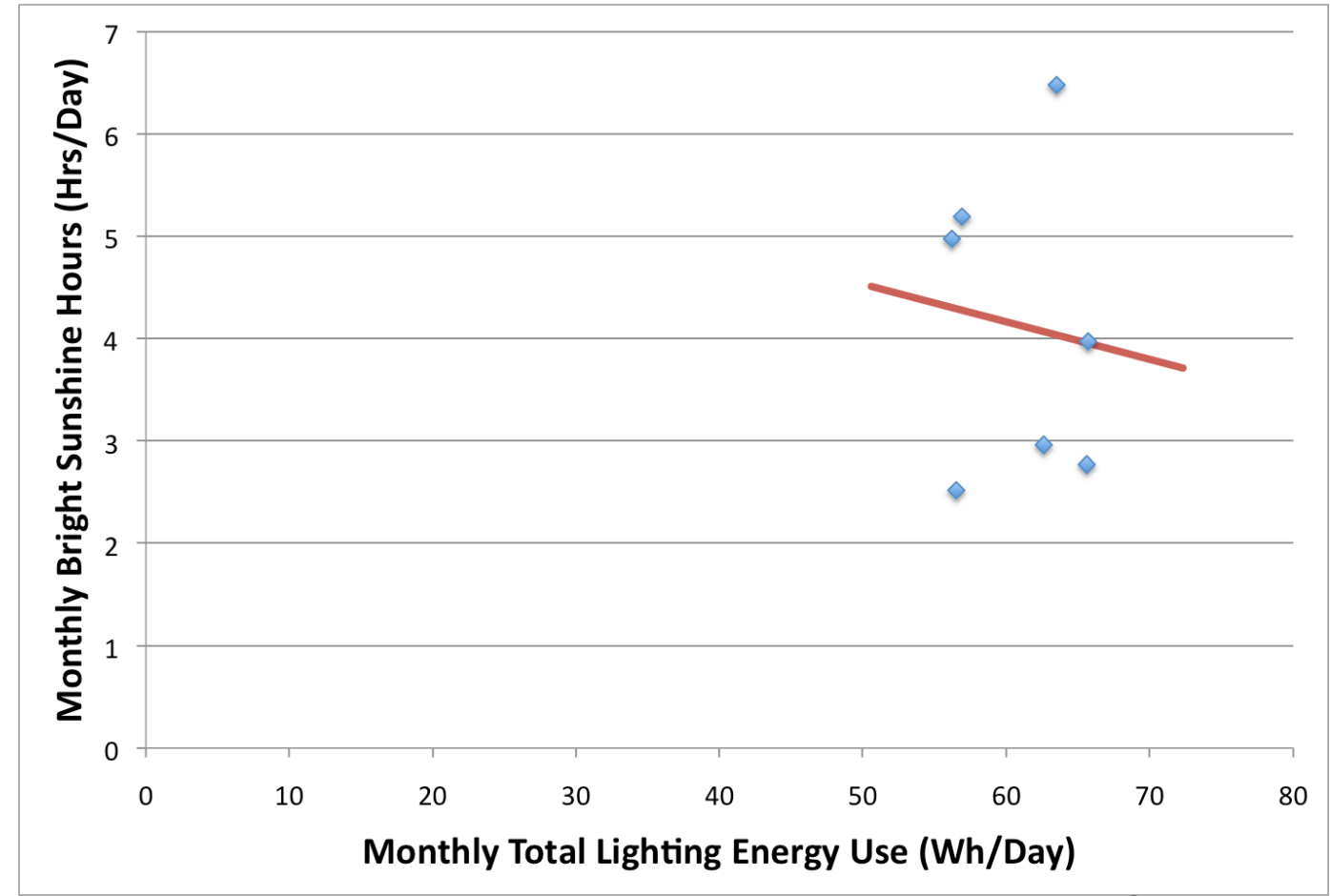

Figure 28. Office-3: Lighting Energy Use Versus Sunshine Hours Regression $\left(r^{2}=0.01\right.$, slope $\left.=-0.04\right)$ 


\section{Receptacles}

Table 15. Office-3: Building Receptacles Summary

\begin{tabular}{cccc}
\hline $\begin{array}{c}\text { As Modeled: } \\
\text { Installed } \\
\text { Receptacles PD } \\
\left(\mathbf{W} / \mathbf{m}^{\mathbf{2}}\right)\end{array}$ & $\begin{array}{c}\text { MNP Energy Use } \\
\left(\mathbf{W} / \mathbf{m}^{\mathbf{2}}\right)\end{array}$ & $\begin{array}{c}\text { MNB Energy Use } \\
\left(\mathbf{W} / \mathbf{m}^{\mathbf{2}}\right)\end{array}$ & $\begin{array}{c}\text { Base as \% } \\
\text { of Peak }\end{array}$ \\
\hline 6.5 & 2.5 & 0.55 & $22 \%$ \\
\hline $\begin{array}{c}\text { As Measured: } \\
\text { Installed } \\
\begin{array}{c}\text { Receptacles PD } \\
\left(\mathbf{W} / \mathbf{m}^{\mathbf{2}}\right)\end{array}\end{array}$ & $\begin{array}{c}\text { MNP Energy Use } \\
\left(\mathbf{W} / \mathbf{m}^{\mathbf{2}}\right)\end{array}$ & $\begin{array}{c}\text { MNB Energy Use } \\
\left(\mathbf{W} / \mathbf{m}^{\mathbf{2}}\right)\end{array}$ & $\begin{array}{c}\text { Base as \% } \\
\text { of Peak }\end{array}$ \\
\hline- & 3.0 & 1.5 & $49 \%$ \\
\hline
\end{tabular}

Table 15 summarizes the median near peak (MNP) receptacle loads and median near base (MNB) receptacle loads as modeled and as measured. Comparison between the receptacles profile and receptacles schedules shows a significantly under-predicted daytime peak load and nighttime base-load (Figure 29 and Figure 30). Calculated from measured data, MNP and MNP loads are $3.0 \mathrm{~W} / \mathrm{m}^{2}$ and 1.5 $\mathrm{W} / \mathrm{m}^{2}$ respectively. Thus the off-hours base load is approximately $49 \%$ of the peak load, as opposed to the estimated $22 \%$ as assumed by the models. This indicates higher levels of off-peak energy use than expected. A measured MNP load of 3.0 $\mathrm{W} / \mathrm{m}^{2}$ is $120 \%$ of estimated peak load of approximately $2.5 \mathrm{~W} / \mathrm{m}^{2}$. It may be possible to account for this difference however. Post-processing calibration included a $66,000 \mathrm{kWh}$ of additional server loads (that could be included on the receptacle meters) that is not represented by the modeling schedules. Assuming the servers operating at the equivalent of max power $18 \mathrm{hrs} /$ day this server energy use would amount to approximately $1.3 \mathrm{~W} / \mathrm{m}^{2}$ of receptacle loads bringing the estimated peak load of $2.5 \mathrm{~W} / \mathrm{m}^{2}$ up to approximately $3.8 \mathrm{~W} / \mathrm{m}^{2}$. Considering Figure 29 we can see that day-to-day loads are fairly stable, with occasional daytime loads in excess of the typical values, indicating fairly stable week-to-week operations. 


\section{Legend:}

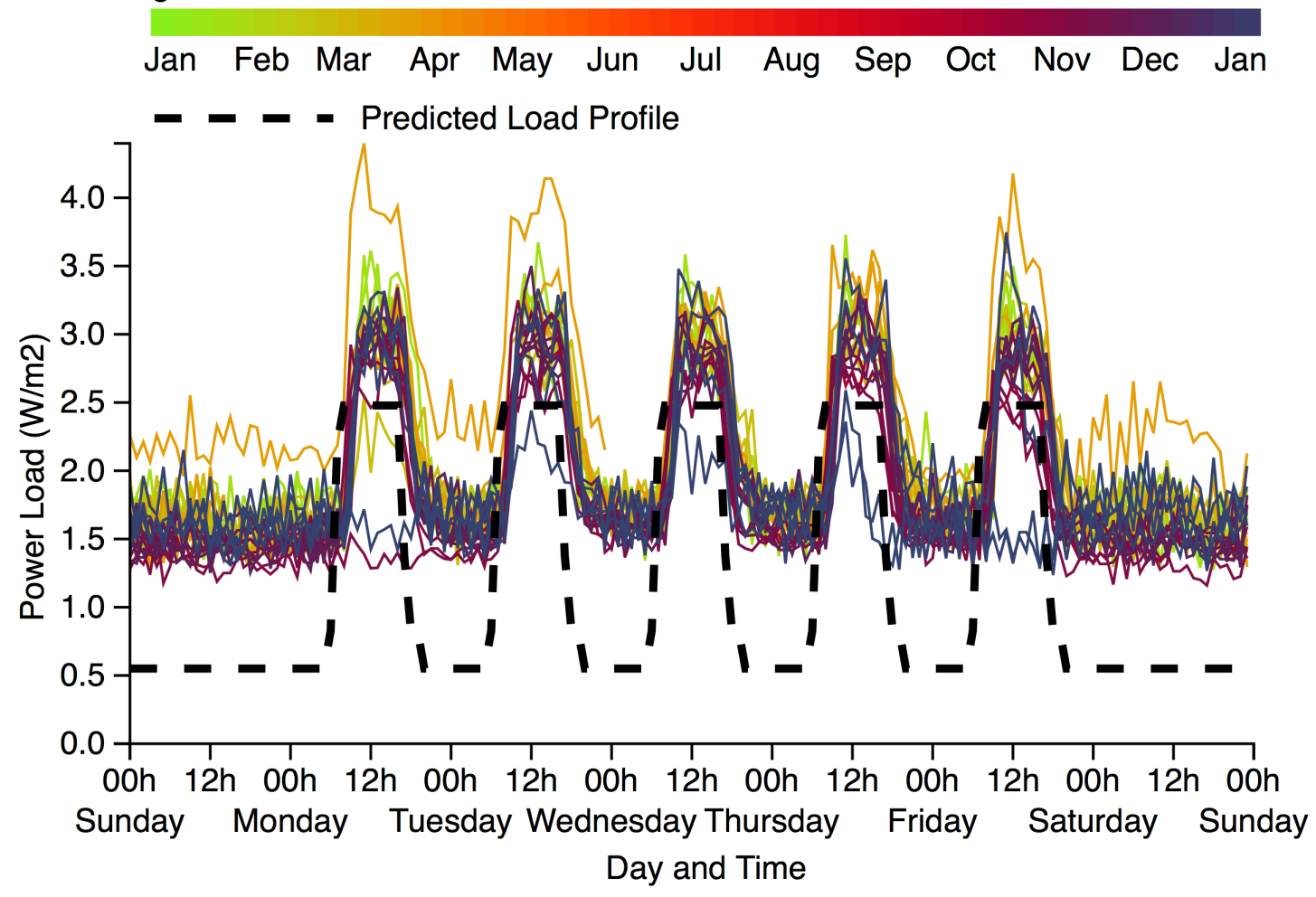

Figure 29. Office-3: Full-year 60-Minute Interval Receptacles Energy Use Data

Average, median and typical receptacle loads are shown for weeks, weekdays and weekend, respectively, in Figure 30, Figure 31 and Figure 32. The values calculated for the MNP and MNB are more clearly represented in these figures. In Figure 31 the weekday morning ramp up and evening ramp down appears to begin significantly later than expected. In fact the entire schedule appears to be shifted 2-3 hours later. The peak energy use period is approximately the same length as expected. The ramp down returns to base level around 21:00. In regards to the weekend load profile (Figure 32) nearly no variation occurs through out the day. However the various short spikes that occur in the typical profile indicate the cycling of various equipment on and off. 
Legend:

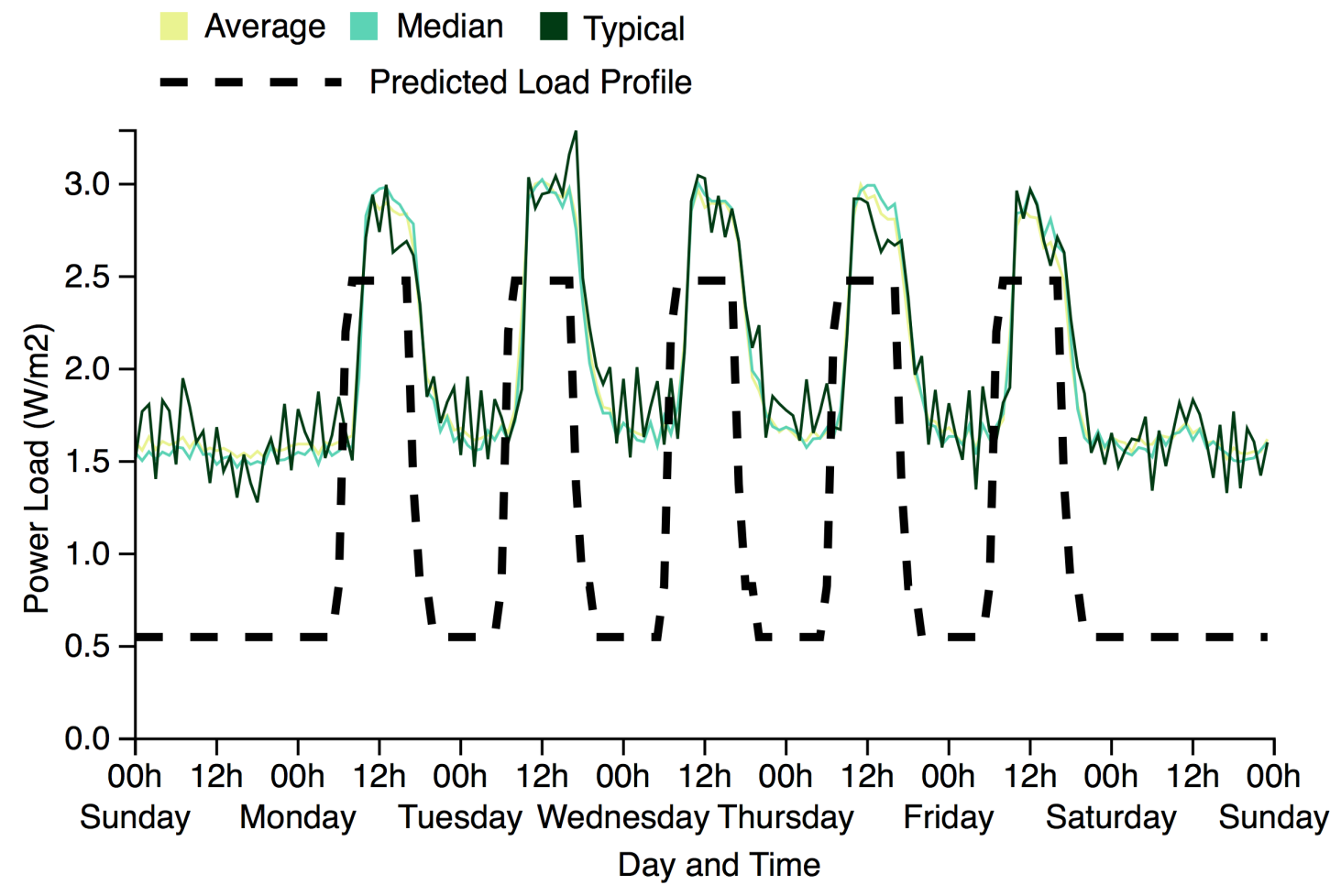

Figure 30. Office-3: Average, Median, and Typical Weekly Profiles for Receptacles Energy Use

Legend:

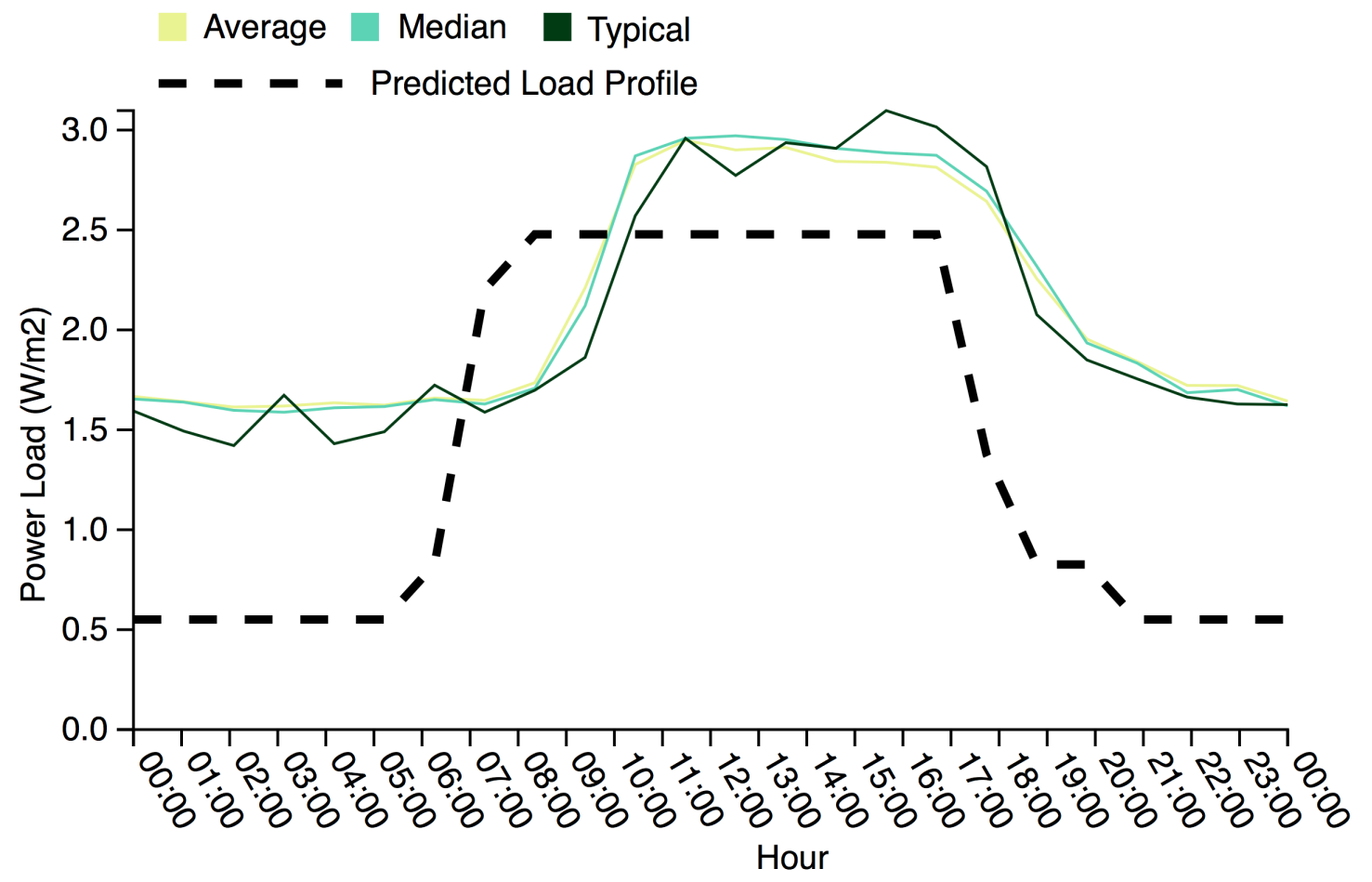

Figure 31. Office-3: Average, Median, and Typical Weekday Receptacles Profile 
Legend:

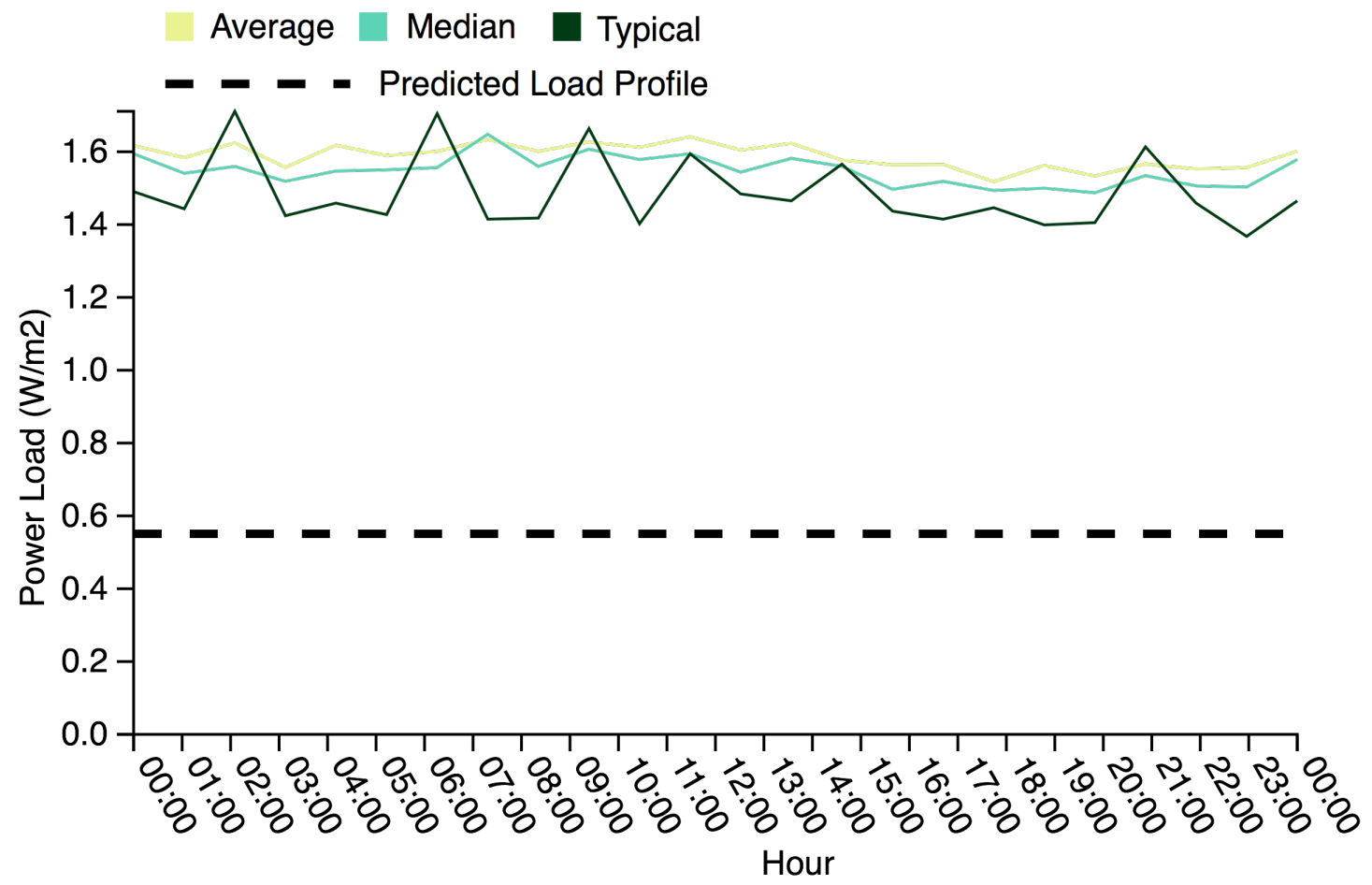

Figure 32. Office-3: Average, Median, and Typical Weekend Day Receptacles Profile

Considering Figure 33 it is possible to see the variations in energy use throughout the year. Energy use in general is fairly stable, but a slight increase does occur rising to a peak in March. No obvious explanation is available for this trend. It is possible that occupants were adding additional heating capacity however no direct evidence of this exists. A dip in energy use is visible in late December due to reduced holiday occupancy. Overall Figure 33 shows the building is consistently using more energy than anticipated. 


\section{Legend:}

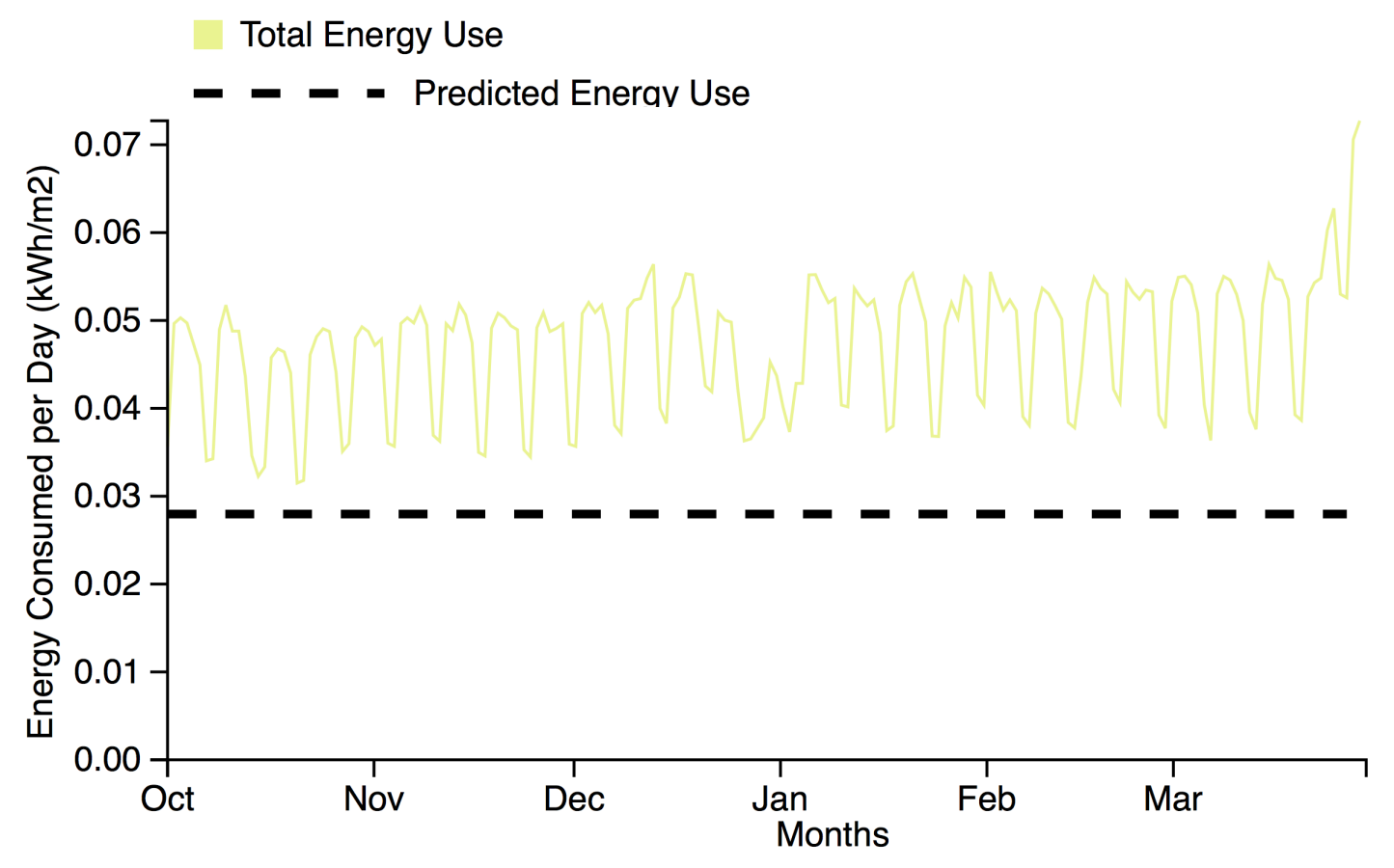

Figure 33. Office-3: Full-year Total Daily Receptacles Energy Use Data

\subsubsection{Office-4}

The building is a 2-storey small office building (1,777 m2). Energy use data was available between December 2011 and September 2012. A fairly extensive duration of data was unavailable between June and August 2012, estimated values have been substituted. The methodology for replacing missing data is discussed in Section 3.4.

Available meter attribution documentation consisted of generic meter designations (lights, receptacles, etc...). The full document is available in Appendix D. Due to this fact exact composition of the metered energy use is unavailable. Lighting energy use may include exterior or emergency lighting. Lighting schedules used in energy modeling do not include exterior lighting.

Overall lighting is consuming less energy than expected due to lower daytime peak loads, despite higher over night loads. Receptacle loads are consuming less energy than expected due to lower daytime peak loads. Weekend loads are generally lower than expected, while overnight base loads are generally as predicted. 
Table 16. Office-4: Building Summary

\begin{tabular}{|c|c|}
\hline $\begin{array}{c}\text { Location } \\
\text { Floor Area } \\
\text { Submetering Categories } \\
\text { Reporting Period } \\
\text { Data Availability } \\
\text { Metering Attribution } \\
\end{array}$ & $\begin{array}{c}\text { Thornbury, Ontario } \\
1,777 \text { m2 } \\
\text { Lighting, Receptacles, } \\
\text { IT, Mechanical/Heating, } \\
\text { ERV, Pumps } \\
\text { Oct } 2011 \text { - Sep } 2012 \\
\text { Dec } 2011 \text { - Sep } 2012 \\
\text { Limited } \\
\end{array}$ \\
\hline $\begin{array}{l}\text { Lighting: } \\
\text { Installed LPD } \\
\end{array}$ & $8.1 \mathrm{~W} / \mathrm{m}^{2}$ \\
\hline $\begin{array}{l}\text { Receptacles: } \\
\text { Installed Receptacles PD }\end{array}$ & $6.5 \mathrm{~W} / \mathrm{m}^{2}$ \\
\hline
\end{tabular}

Table 17 lists the key values for predicted and actual energy use of the building. Fairly substantial differences are visible between the various values.

Table 17. Office-4: Predicted and Actual Energy Use

\begin{tabular}{|c|c|c|c|}
\hline & $\begin{array}{c}\text { Uncalibrated- } \\
\text { Predicted } \\
\left(\mathbf{k W h} / \mathbf{m}^{2}\right)\end{array}$ & $\begin{array}{l}\text { Calibrated- } \\
\text { Predicted } \\
\left(\mathbf{k W h} / \mathbf{m}^{2}\right)\end{array}$ & $\begin{array}{c}\text { Actual } \\
\left(k W h / m^{2}\right)\end{array}$ \\
\hline Total Energy Use & 157.1 & 210.6 & 266.9 \\
\hline $\begin{array}{l}\text { Total Electricity } \\
\text { Energy Use }\end{array}$ & 157.1 & 238.8 & 258.2 \\
\hline Total Natural Gas & 0.0 & 0.0 & 8.6 \\
\hline Heating & 17.2 & 19.1 & - \\
\hline Cooling & 20.7 & 19.3 & - \\
\hline Lighting & 35.8 & 35.8 & 49.1 \\
\hline Receptacles & 16.6 & 16.6 & 15.5 \\
\hline
\end{tabular}

\section{Lighting}

Table 18. Office-4: Building Lighting Summary

\begin{tabular}{cccc}
\hline $\begin{array}{c}\text { As Modeled: } \\
\text { Installed LPD } \\
\left(\mathbf{W} / \mathbf{m}^{2}\right)\end{array}$ & $\begin{array}{c}\text { MNP Energy Use } \\
\left(\mathbf{W} / \mathbf{m}^{2}\right)\end{array}$ & $\begin{array}{c}\text { MNB Energy Use } \\
\left(\mathbf{W} / \mathbf{m}^{2}\right)\end{array}$ & $\begin{array}{c}\text { Base as \% } \\
\text { of Peak }\end{array}$ \\
\hline 8.1 & 9.9 & 0.6 & $6 \%$ \\
\hline $\begin{array}{c}\text { As Measured: } \\
\text { Installed LPD } \\
\left(\mathbf{W} / \mathbf{m}^{2}\right)\end{array}$ & $\begin{array}{c}\text { MNP Energy Use } \\
\left(\mathbf{W} / \mathbf{m}^{2}\right)\end{array}$ & $\begin{array}{c}\text { MNB Energy Use } \\
\left(\mathbf{W} / \mathbf{m}^{2}\right)\end{array}$ & $\begin{array}{c}\text { Base as } \% \\
\text { of Peak }\end{array}$ \\
\hline- & 6.2 & 1.1 & $18 \%$ \\
\hline
\end{tabular}

Table 18 summarizes the median near peak (MNP) lighting loads and median near base (MNB) lighting loads as modeled and as measured. Comparison between 
the lighting energy use profile and lighting schedules show a significantly underpredicted nighttime and weekend base-load as well as an over-predicted daytime peak load (Figure 34 and Figure 35). Calculated MNP and MNB values $\left(6.2 \mathrm{~W} / \mathrm{m}^{2}\right.$ and $1.1 \mathrm{~W} / \mathrm{m}^{2}$ respectively) the off hours base load is approximately $18 \%$ of the peak load, as opposed to the estimated 6\%. Even with lower than expected peak energy use, this indicates higher levels of off-peak energy use than expected. A MNP load of $6.2 \mathrm{~W} / \mathrm{m}^{2}$ is $63 \%$ of an estimated peak load of approximately $9.9 \mathrm{~W} / \mathrm{m}^{2}$ per hour. The building is not using as much energy as expected during peak periods. No obvious explanations for this observation are available.

Figure 34 demonstrates that very few weeks from the observed year varied outside of typical usage patterns. Attribution of the lower than expected peak energy load is not immediately obvious, it could be a result of lower than expected occupancy, or good building operations practices. Higher than expected base loads however do indicate that more lights are being left on overnight than expected.

Legend:

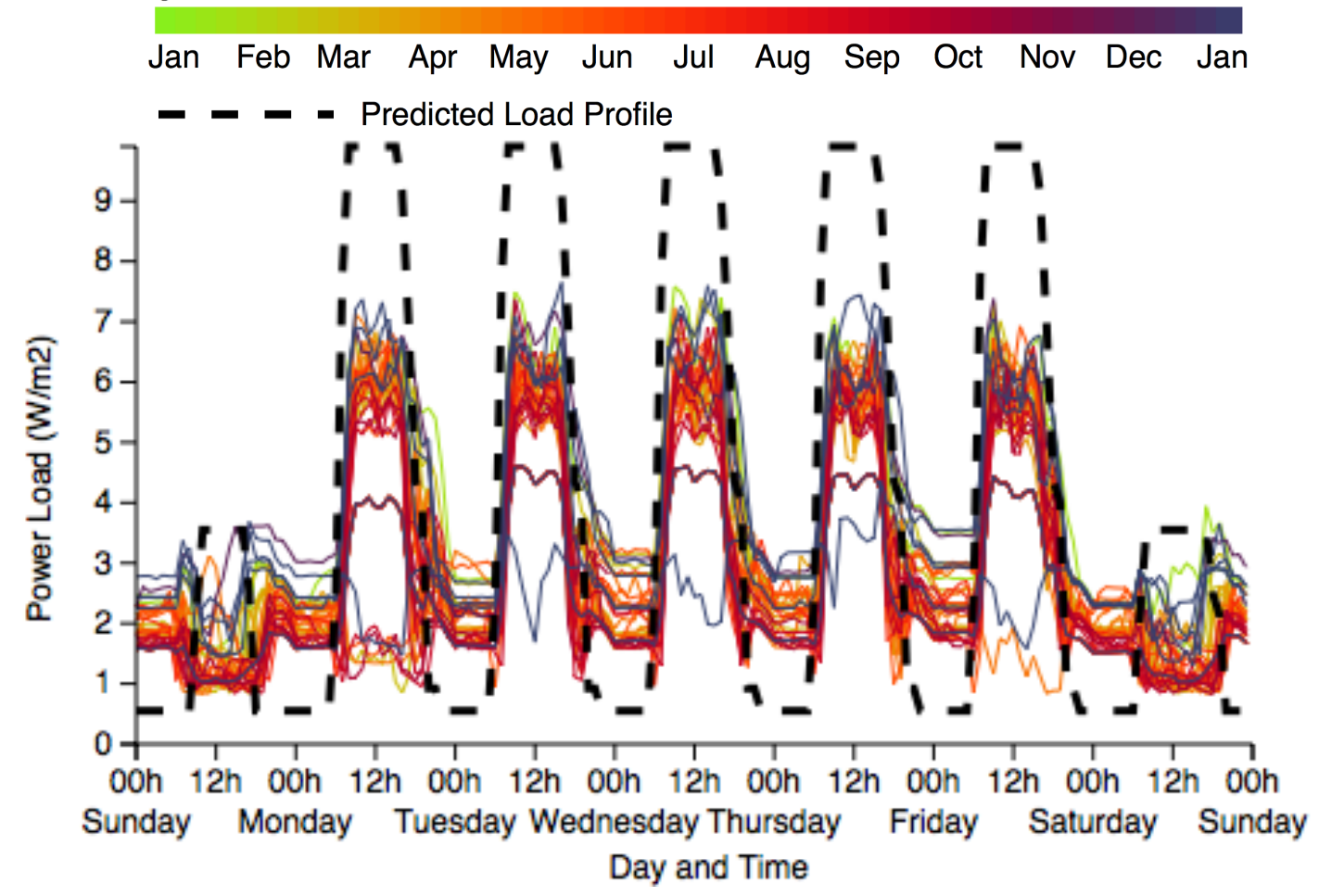

Figure 34. Office-4: Full-year 60-Minute Interval Lighting Energy Use Data 
Average, median and typical lighting loads are shown for weeks, weekdays and weekend, respectively, in Figure 35, Figure 36 and Figure 37. The values calculated for the MNP and MNB are more clearly represented in these figures. In Figure 36 the weekday morning ramp up appears to begin slightly later than expected, and ramping down begins around 17:00 as expected. Energy levels return to base levels until around 21:00. In regards to the weekend load profile (Figure 37) it is interesting to see an inverse relationship to the expected load profile. Schedules predict a midday peek load, similar to weekdays, however in actuality a midday trough is visible. This can only be attributed to lower weekend occupancies than expected.

Legend:

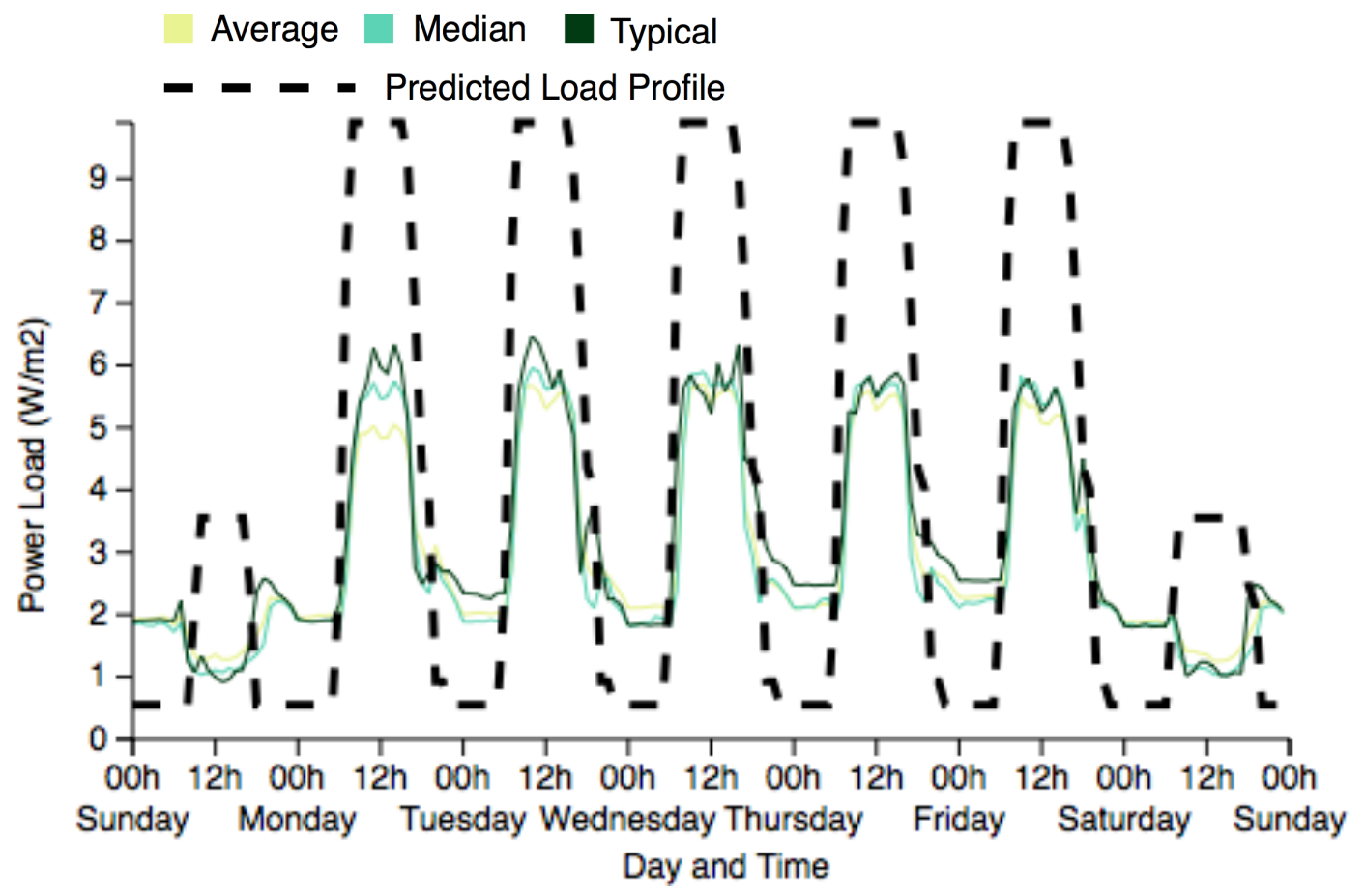

Figure 35. Office-4: Average, Median, and Typical Weekly Profiles for Lighting Energy Use 
Legend:

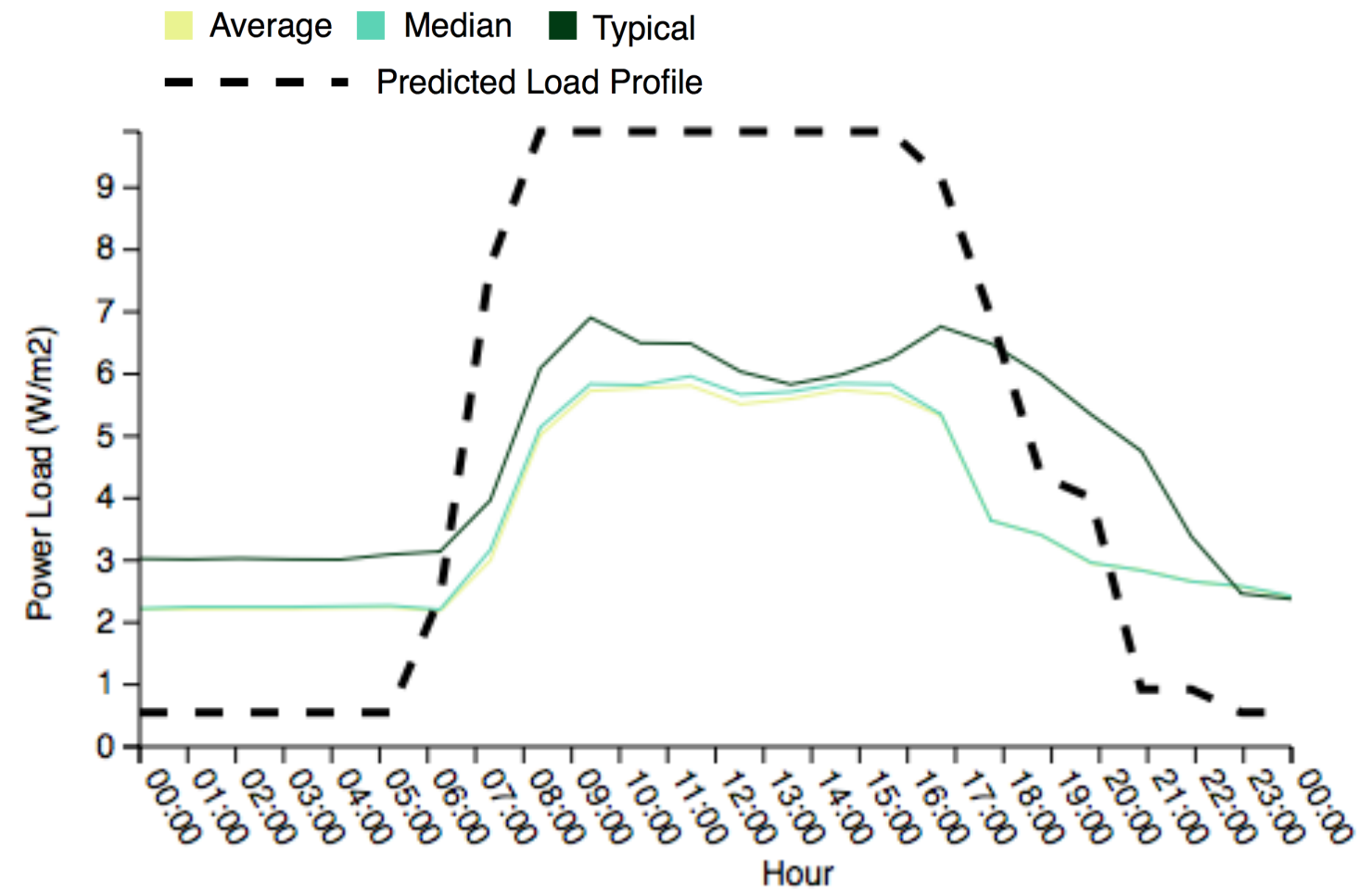

Figure 36. Office-4: Average, Median, and Typical Weekday Lighting Profile

Legend:

Average Median $\square$ Typical

- _ - a Predicted Load Profile

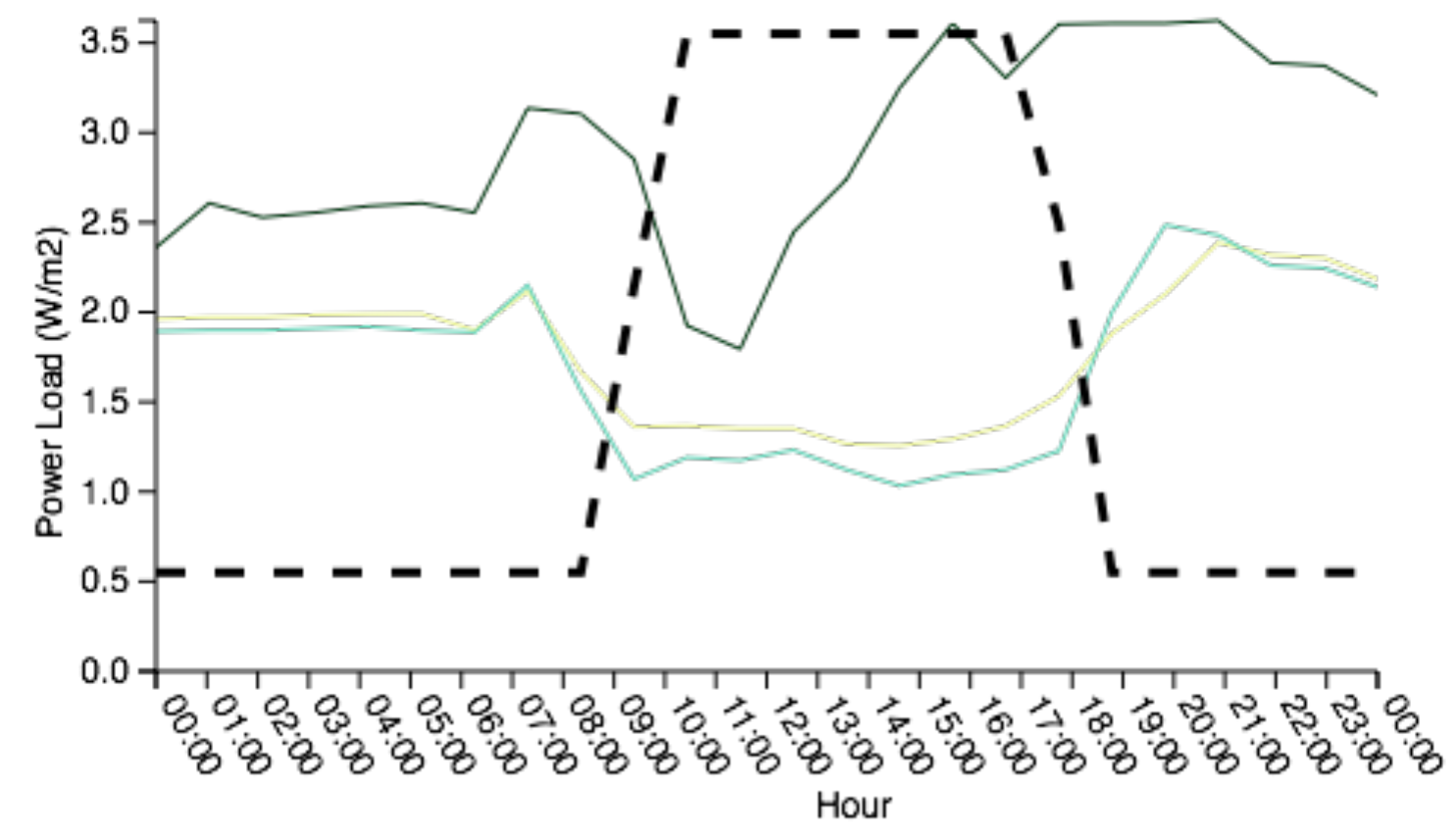

Figure 37. Office-4: Average, Median, and Typical Weekend Day Lighting Profile 
Considering Figure 38 it is possible to observe the lighting load profile over the course of the year. Energy consumption varies, markedly lower in the spring months than in the winter months. December experiences a drop in energy use due to lower holiday occupancy. The highly regular energy use in January, June and July reflects large durations of missing data being replaced by estimations. In general the load profile remains relatively stable, this is likely contributed to by a predictable and stable occupancy. Over all energy use is observed to stay consistently lower than predicted.

\section{Legend:}

Total Energy Use

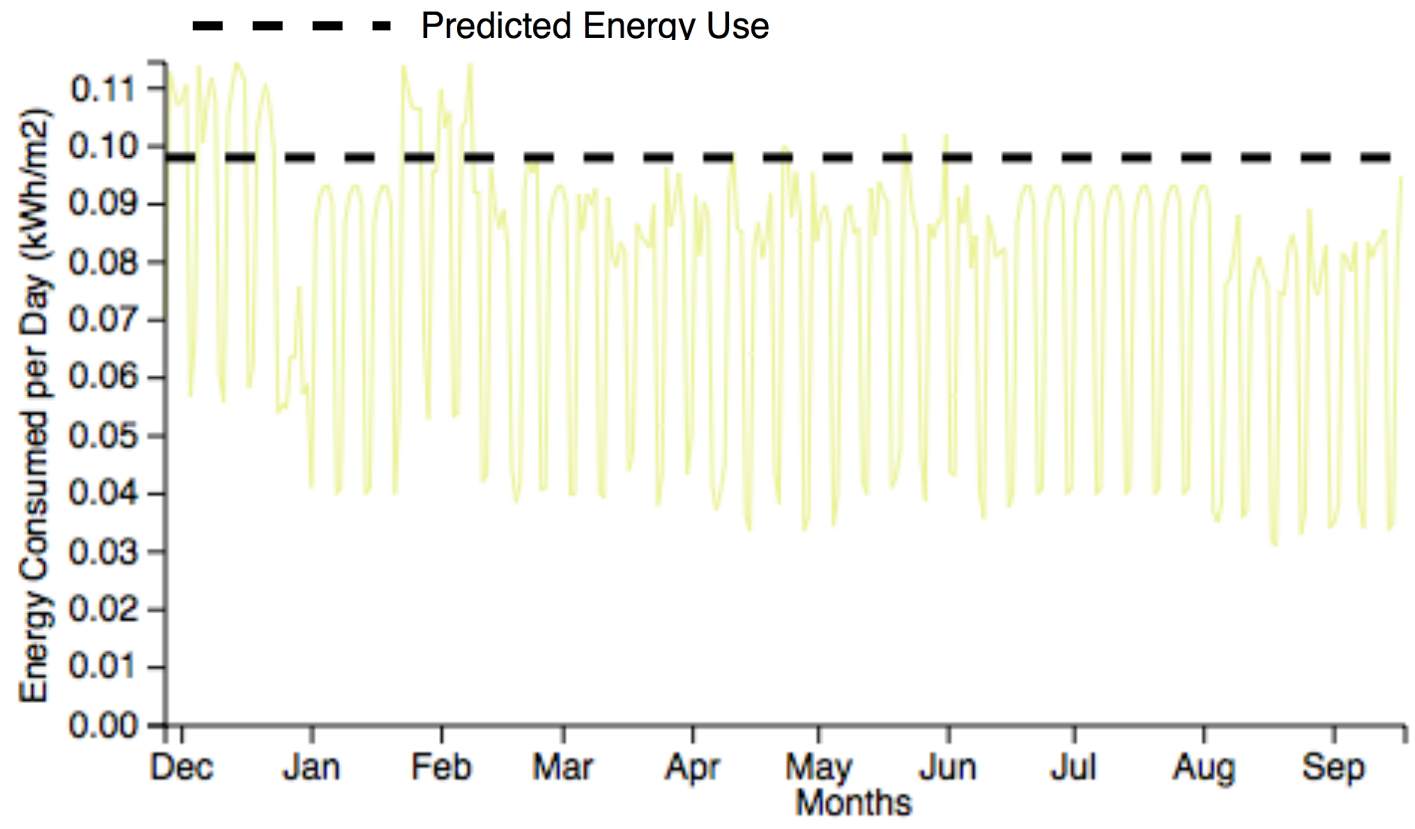

Figure 38. Office-4: Public Assembly-1: Full-year Total Daily Lighting Energy Use Data

Figure 39 shows a regression analysis correlating seasonal changes in lighting energy use with bright sunshine availability. The analysis demonstrates a fairly strong negative correlation $\left(\mathrm{r}^{2}=0.36\right)$ between lighting energy use and daylight hours for this building. This indicates that on average light energy consumption does decrease as daylight availability increases. Again this correlation could be influenced by occupancy issues as offices tend to have higher occupancy rates throughout the winter months than during the summer. 


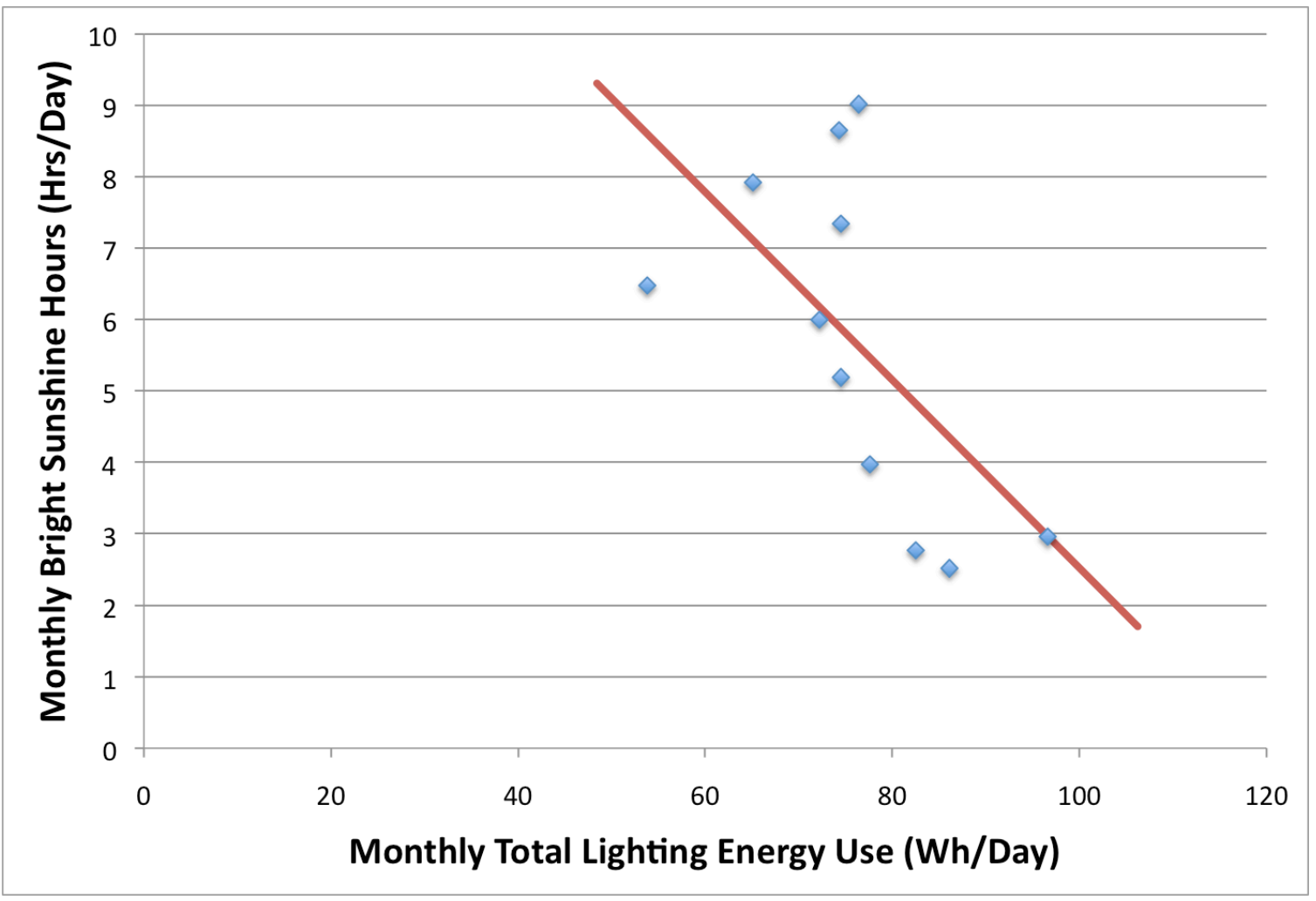

Figure 39. Office-4: Lighting Energy Use Versus Sunshine Hours Regression $\left(r^{2}=0.36\right.$, slope $\left.=-0.13\right)$

\section{Receptacles}

Table 19. Office-4: Building Receptacles Summary

\begin{tabular}{|c|c|c|c|}
\hline $\begin{array}{l}\text { As Modeled: } \\
\text { Installed } \\
\text { Receptacles PD } \\
\left(\mathrm{W} / \mathrm{m}^{2}\right) \\
\end{array}$ & $\begin{array}{c}\text { Median Near } \\
\text { Peak Energy Use } \\
\left(\mathrm{W} / \mathrm{m}^{2}\right)\end{array}$ & $\begin{array}{c}\text { Median Near } \\
\text { Base Energy Use } \\
\left(\mathrm{W} / \mathrm{m}^{2}\right)\end{array}$ & $\begin{array}{c}\text { Base as \% } \\
\text { of Peak }\end{array}$ \\
\hline 3.8 & 4.0 & 0.74 & $19 \%$ \\
\hline $\begin{array}{l}\text { As Measured: } \\
\text { Installed } \\
\text { Receptacles PD } \\
\left(\mathbf{W} / \mathbf{m}^{2}\right)\end{array}$ & $\begin{array}{c}\text { Median Near } \\
\text { Peak Energy Use } \\
\left(\mathbf{W} / \mathbf{m}^{2}\right)\end{array}$ & $\begin{array}{c}\text { Median Near } \\
\text { Base Energy Use } \\
\left(W / \mathbf{m}^{2}\right)\end{array}$ & $\begin{array}{c}\text { Base as } \% \\
\text { of Peak }\end{array}$ \\
\hline- & 2.8 & 0.57 & $21 \%$ \\
\hline
\end{tabular}

Table 19 summarizes the median near peak (MNP) receptacle loads and median near base (MNB) receptacle loads as modeled and as measured. Comparison between the receptacles profile and receptacles schedules show over predicted daytime and weekend peak-load (Figure 40 and Figure 41). Calculated with MNP and MNB values $\left(2.8 \mathrm{~W} / \mathrm{m}^{2}\right.$ and $0.57 \mathrm{~W} / \mathrm{m}^{2}$ respectively) the off-hours MNB is 
approximately $21 \%$ of the MNP load, as opposed to the estimated $19 \%$. In this case the base load is occurring very close to expectations. A near peak load of $2.8 \mathrm{~W} / \mathrm{m}^{2}$ is $74 \%$ of estimated peak load of approximately $3.8 \mathrm{~W} / \mathrm{m}^{2}$. Lower than expected energy use potentially indicates lower than expected occupancy, or in the case of receptacle loads, building usage patterns or programming. It could also be a case of only part of the receptacle loads being measured on this meter. A post-processing calibration step included the addition of $66,000 \mathrm{kWh}$ for miscellaneous, assuming these loads where running at max power for the equivalent of $12 \mathrm{hrs}$ a day this amounts to $8 \mathrm{~W} / \mathrm{m}^{2}$. Given the magnitude of the measured and predicted (via schedules and PD) peak, it seems unlikely that the receptacle meter in question includes those miscellaneous loads.

Figure 40 shows that throughout the year day to day load profiles have some significant variations, this include occasional weekend peeks matching daytime peeks, and occasional daytime energy use remaining as low as the base load.

Legend:

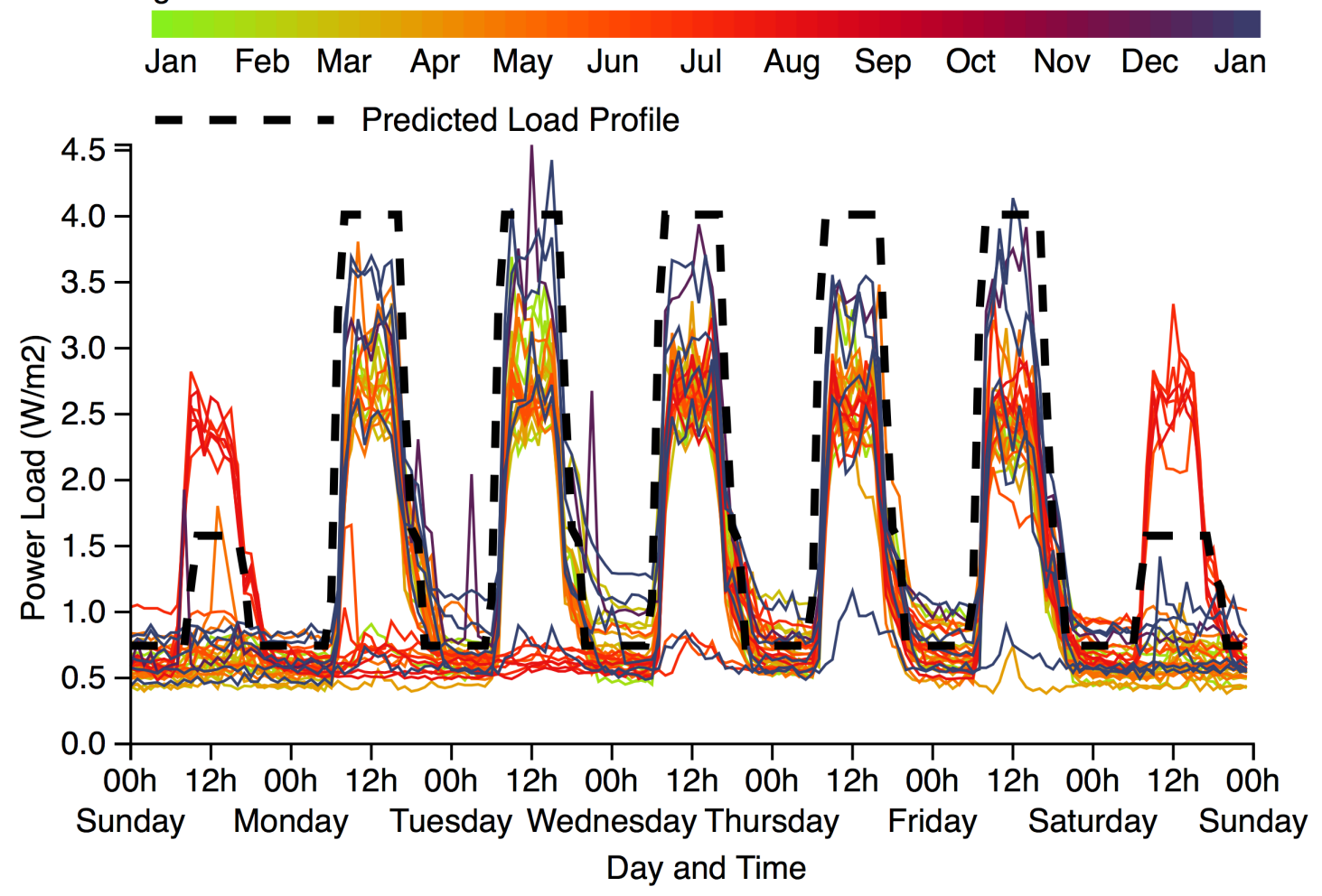

Figure 40. Office-4: Full-year 60-Minute Interval Receptacles Energy Use Data 
Average, median and typical receptacle loads are shown for weeks, weekdays and weekend, respectively, in Figure 41, Figure 42 and Figure 43. The values calculated for the MNP and MNB are more clearly represented in these figures. In Figure 42 the morning ramp up appears to begin slightly later than expected, and to begin ramping down around 17:00 as expected. Ramp down takes slightly longer than expected, and doesn't reach base levels until 21:00. In regards to the weekend load profile (Figure 43) very little meaningful variation occurs through out the day. The average load profile shows a midday rise in energy use as scheduled, however this is not represented in either median or typical, indicating that this trend is likely just a result of outliers (as can be supported by considering the occasional weekend midday peak hours visible in Figure 40). The sharp peak occurring in the typical load profile around 8:00 does not appear to bear any relation on the average and median profiles, and thus is likely just a quirky feature of the day in question.

Legend:

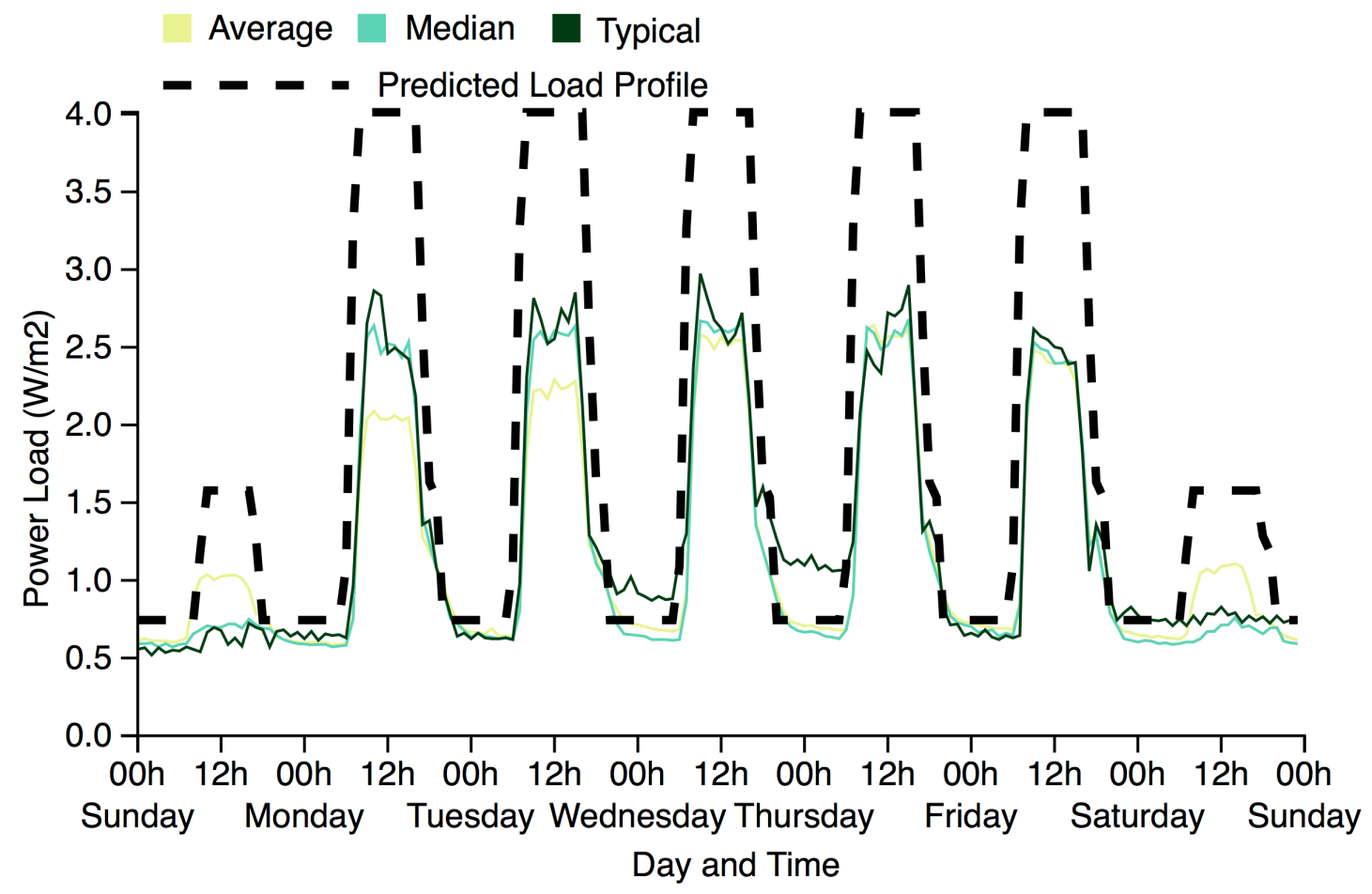

Figure 41. Office-4: Average, Median, and Typical Weekly Profiles for Receptacles Energy Use 
Legend:

Average Median $\square$ Typical

- - - Predicted Load Profile

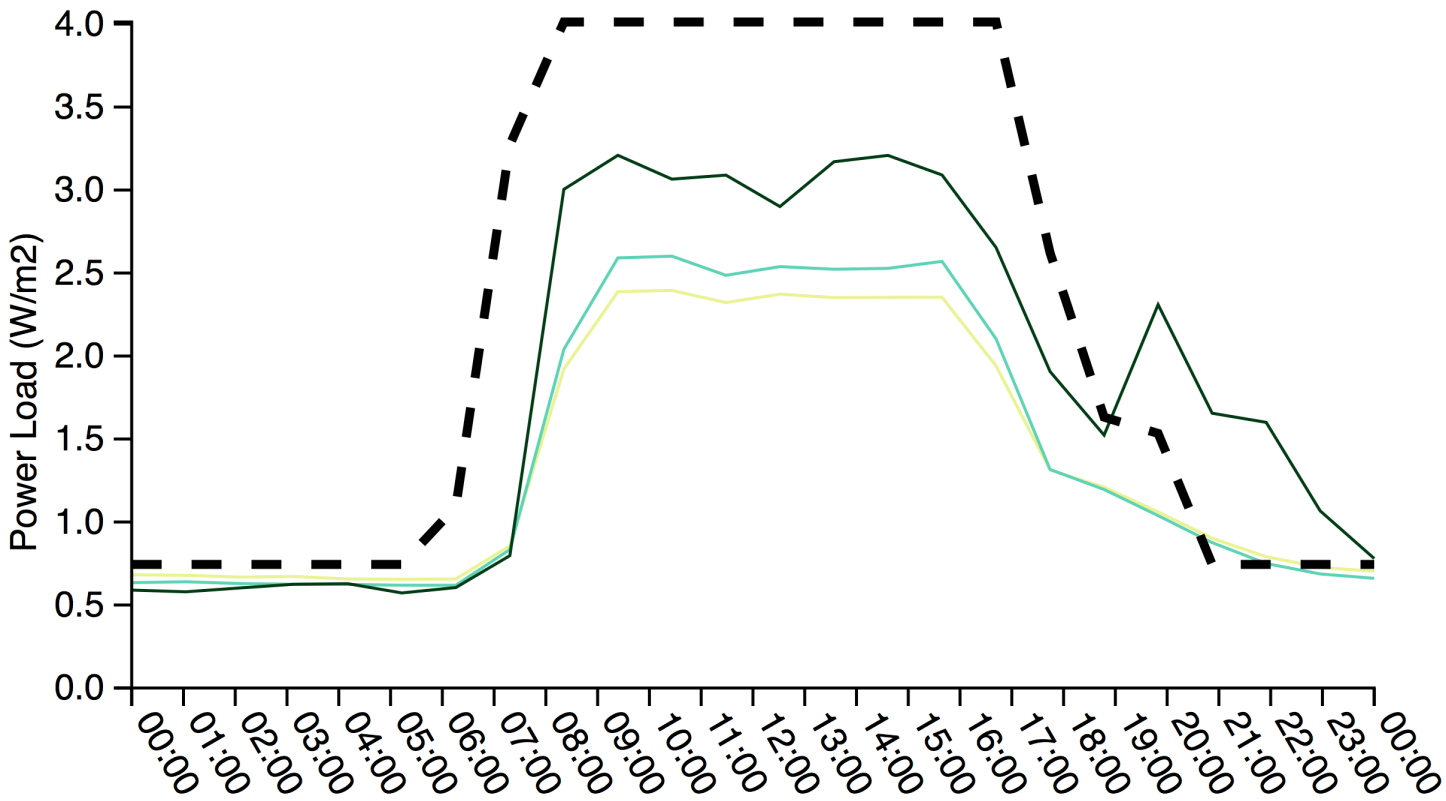

Hour

Figure 42. Office-4: Average, Median, and Typical Weekday Receptacles Profile

Legend:

Average Median Typical

- - a Predicted Load Profile

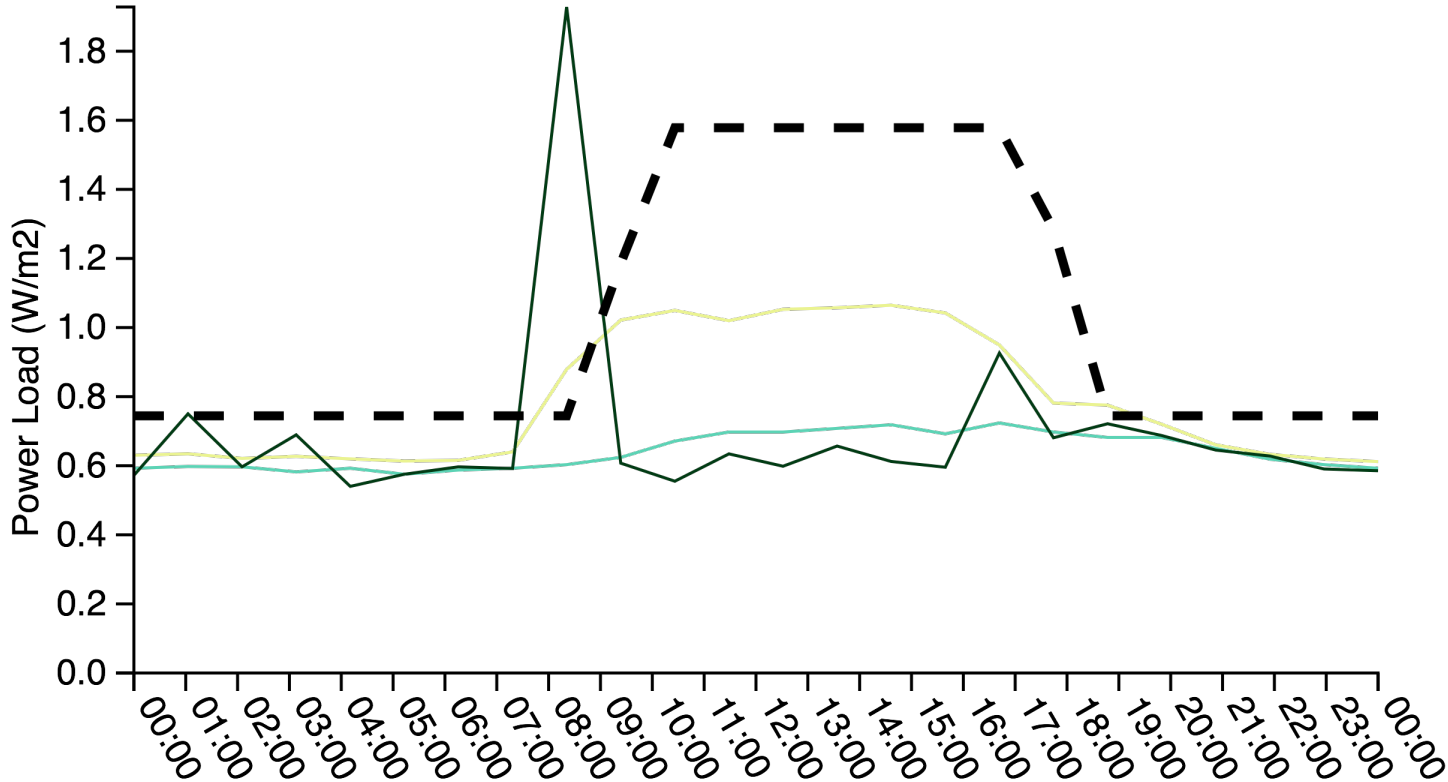

Hour

Figure 43. Office-4: Average, Median, and Typical Weekend Day Receptacles Profile

73 
Considering Figure 44 it is possible to observe energy use trends throughout the entire year. Relatively stable energy use is visible with the exception of considerably higher energy use in early December and a trough in late December easily associated with reduced holiday occupancy. The early December peak on the other hand has no readily available explanation, the addition of space heaters could explain some of this difference, however in this case there is no corresponding rise in the energy use of the subsequent months. Overall energy use remained wholly under expectations.

Legend:

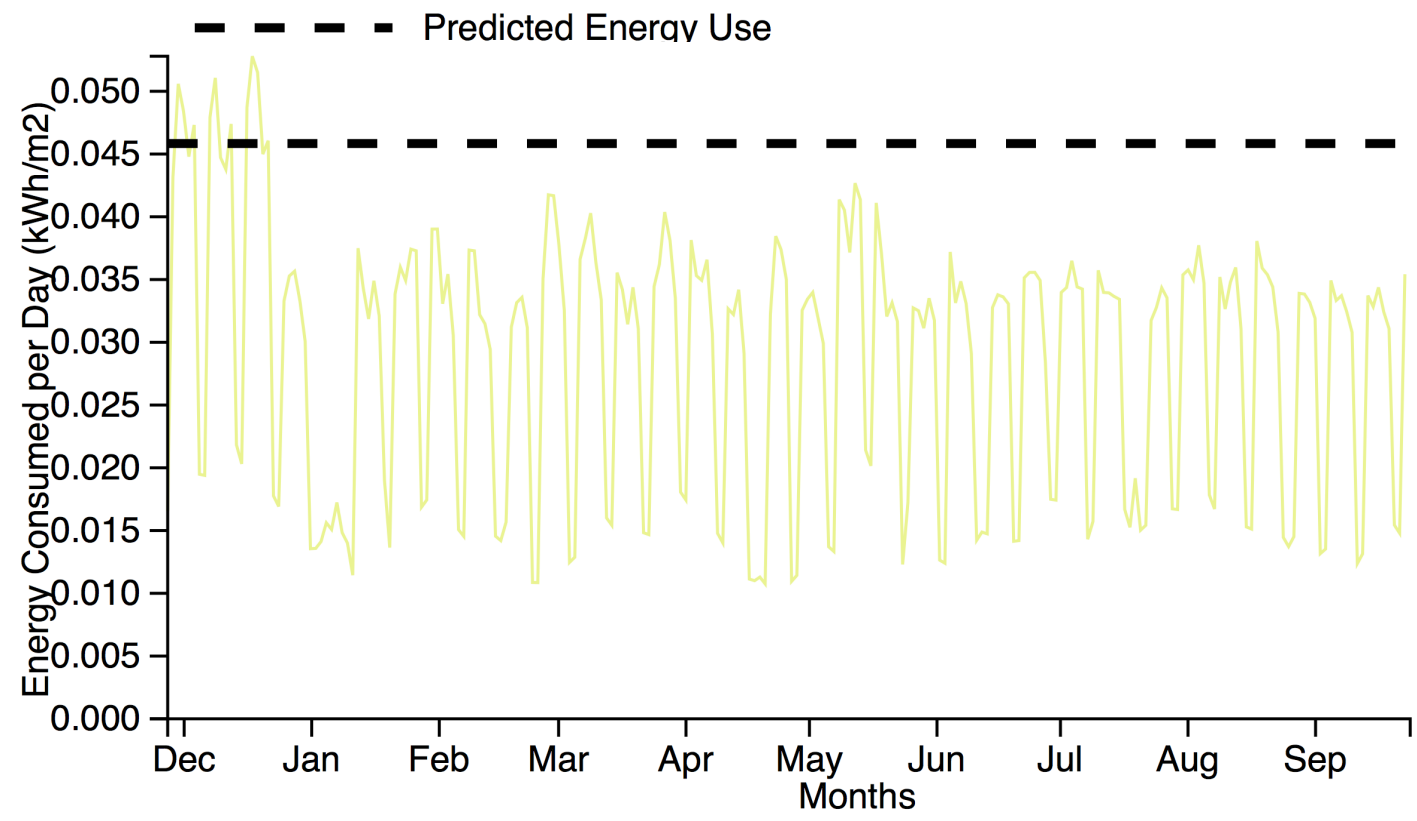

Figure 44. Office-4: Full-year Total Daily Receptacles Energy Use Data

\subsubsection{Mail Sorting-1}

The building is a small mail sorting facility $\left(1,730 \mathrm{~m}^{2}\right)$. Energy use data was available between April 2009 and November 2010. This building is very similar in design and construction to Mail Sorting-3.

Meter attribution documentation consisted of detailed electrical panel shop drawings (see Appendix D). As such it was possible to determine $1 \mathrm{~kW}$ out of $21 \mathrm{~kW}$ installed on the lighting panel was not lighting. Exterior lighting was metered 
separately. As well $21.4 \mathrm{~kW}$ out of $38.5 \mathrm{~kW}$ on the receptacles panel was not receptacles. This includes $8 \mathrm{~kW}$ of ACUs, $6.75 \mathrm{~kW}$ of heating, and $3 \mathrm{~kW}$ for a roof top unit.

Overall lighting is consuming more energy than expected due to much higher daytime peak loads and overnight loads. Receptacle loads are consuming less energy than expected due to much lower daytime peak loads, as well as lower overnight and weekend base loads. Evidence of highly mixed loads limits the amount of information on receptacle energy usage that is able to be drawn from this case.

Table 20. Mail Sorting-1: Building Summary

\begin{tabular}{cc}
\hline Location & Ancaster, Ontario \\
Floor Area & $1,730 \mathrm{~m} 2$ \\
Submetering Categories & Lighting, Receptacles, \\
& Heating, Mechanical, \\
DHW, Misc & Oct 2009 - Sep 2010 \\
Reporting Period & April 2009 - Nov 2010 \\
Data Availability & Detailed \\
Metering Attribution & $8.6 \mathrm{~W} / \mathrm{m}^{2}$ \\
\hline $\begin{array}{c}\text { Lighting: } \\
\text { Installed LPD }\end{array}$ & $10.0 \mathrm{~W} / \mathrm{m}^{2}$ \\
\hline $\begin{array}{c}\text { Receptacles: } \\
\text { Installed Receptacles PD }\end{array}$ & \\
\hline
\end{tabular}

Table 21 lists the key values for predicted and actual energy use of the building. Fairly substantial differences are visible between the various values.

Table 21. Mail Sorting-1: Predicted and Actual Energy Use

\begin{tabular}{|c|c|c|c|}
\hline & $\begin{array}{c}\text { Uncalibrated- } \\
\text { Predicted } \\
\left(\mathbf{k W h} / \mathbf{m}^{2}\right)\end{array}$ & $\begin{array}{l}\text { Calibrated- } \\
\text { Predicted } \\
\left(\mathrm{kWh} / \mathrm{m}^{2}\right)\end{array}$ & $\begin{array}{c}\text { Actual } \\
\left(\mathrm{kWh} / \mathrm{m}^{2}\right)\end{array}$ \\
\hline Total Energy Use & 150.8 & 201.6 & 219.0 \\
\hline $\begin{array}{c}\text { Total Electricity } \\
\text { Energy Use }\end{array}$ & 87.7 & 108.5 & 120.0 \\
\hline Total Natural Gas & 63.2 & 91.6 & 97.4 \\
\hline Heating & 52.6 & 40.3 & 96.4 \\
\hline Cooling & 3.7 & 5.3 & - \\
\hline Lighting & 28.1 & 28.1 & 50.4 \\
\hline Receptacles & 23.3 & 32.8 & 15.6 \\
\hline
\end{tabular}




\section{Lighting}

Table 22. Mail Sorting-1: Building Lighting Summary

\begin{tabular}{cccc}
\hline $\begin{array}{c}\text { As Modeled: } \\
\text { Installed LPD } \\
\left(\mathbf{W} / \mathbf{m}^{2}\right)\end{array}$ & $\begin{array}{c}\text { MNP Energy Use } \\
\left(\mathbf{W} / \mathbf{m}^{2}\right)\end{array}$ & $\begin{array}{c}\text { MNB Energy Use } \\
\left(\mathbf{W} / \mathbf{m}^{2}\right)\end{array}$ & $\begin{array}{c}\text { Base as \% } \\
\text { of Peak }\end{array}$ \\
\hline 8.6 & 7.5 & 0.4 & $6 \%$ \\
\hline $\begin{array}{c}\text { As Measured: } \\
\text { Installed LPD } \\
\left(\mathbf{W} / \mathbf{m}^{2}\right)\end{array}$ & $\begin{array}{c}\text { MNP Energy Use } \\
\left(\mathbf{W} / \mathbf{m}^{2}\right)\end{array}$ & $\begin{array}{c}\text { MNB Energy Use } \\
\left(\mathbf{W} / \mathbf{m}^{2}\right)\end{array}$ & $\begin{array}{c}\text { Base as } \% \\
\text { of Peak }\end{array}$ \\
\hline- & 11.2 & 2.8 & $25 \%$ \\
\hline
\end{tabular}

Table 22 summarizes the median near peak (MNP) lighting loads and median near base (MNB) lighting loads as modeled and as measured. Comparison between the lighting energy use profile and lighting schedules show a significantly underpredicted nighttime and weekend base-load as well as an under-predicted daytime peak load (Figure 45 and Figure 46). Calculated with MNP and MNB values (11.2 $\mathrm{W} / \mathrm{m}^{2}$ per hour and $2.8 \mathrm{~W} / \mathrm{m}^{2}$ respectively) the off-hours MNB is approximately $25 \%$ of the MNP, as opposed to the estimated $6 \%$. Suggesting higher levels of offhours energy use, especially due to the increased peak load. A MNP of $11.2 \mathrm{~W} / \mathrm{m}^{2}$ is $146 \%$ of an estimated peak load of approximately $7.5 \mathrm{~W} / \mathrm{m}^{2}$. Figure 45 demonstrates that very few weeks from the observed year varied outside of typical usage patterns.

This much higher than expected peak weekday load (of $11 \mathrm{~W} / \mathrm{m}^{2}$ ) is difficult to explain. Site visits during the calibration process also indicated furniture and equipment within the space may be blocking the proper functioning of the sensors, and thus likely also driving up peak energy consumption. However $11 \mathrm{~W} / \mathrm{m}^{2}$ surpasses the installed capacity of the system by $2 \mathrm{~W} / \mathrm{m}^{2}$, therefore the extra energy use cannot simply be a result of more intense use of the lighting system. In addition it is unlikely that the lighting power density in the models was incorrect as these values derived directly from the lighting shop drawings used in construction. As a result the metering system must have included some lighting use not included in the model. The electrical panel does show in fact that $2.9 \mathrm{~kW}$ of night lighting, $0.25 \mathrm{~kW}$ 
of exit lighting, $0.6 \mathrm{~kW}$ of dock lighting, and $1 \mathrm{~kW}$ of battery are included. These loads however would only account for approximately $1 \mathrm{~W} / \mathrm{m}^{2}$ additional energy use at peak, and are thus not large enough by themselves to account for the increased daily peak loads, as the largest amongst them night lighting is likely to only operate at night. This on the other hand does help to explain higher than expected nighttime base loads (adding approximately $1.7 \mathrm{~W} / \mathrm{m}^{2}$ ), however as witnessed in other buildings, an element of this is also likely a higher number of lights left on overnight than anticipated.

\section{Legend:}

Jan Feb Mar Apr May Jun Jul Aug Sep Oct Nov Dec Jan

- c - Predicted Load Profile

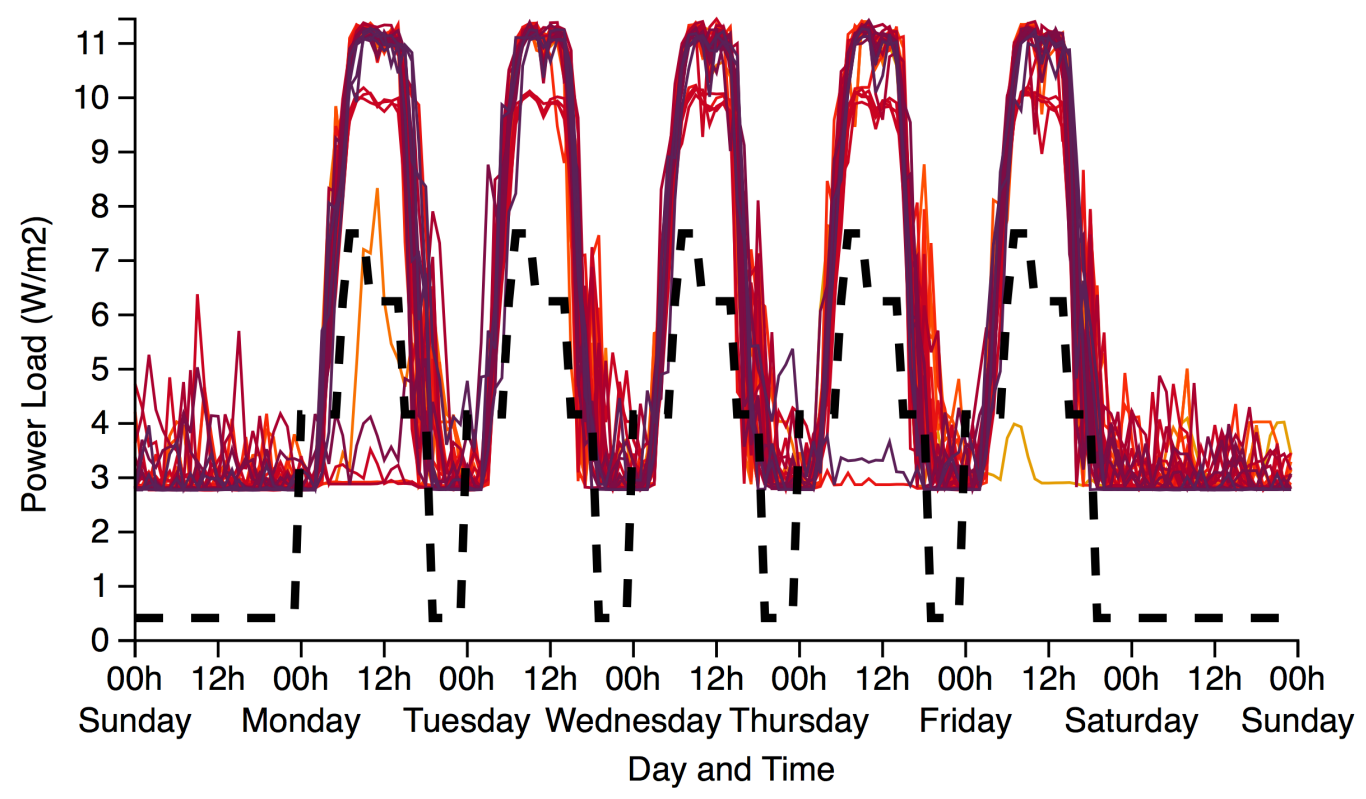

Figure 45. Mail Sorting-1: Full-year 60-Minute Interval Lighting Energy Use Data

Average, median and typical lighting loads are shown for weeks, weekdays and weekend, respectively, in Figure 46, Figure 47 and Figure 48. The values calculated for the MNP and MNB are more clearly represented in these figures. In Figure 47 the weekday morning ramp up appears to begin around 2:00, much earlier than expected. The ramp down begins as expected around 13:00. But occurs much more slowly with energy levels returning to base levels around 21:00. In regards to the weekend load profile (Figure 48) next to no meaningful variation occurs through out the day. 
Legend:

Average Median $\square$ Typical

- _ - - Predicted Load Profile

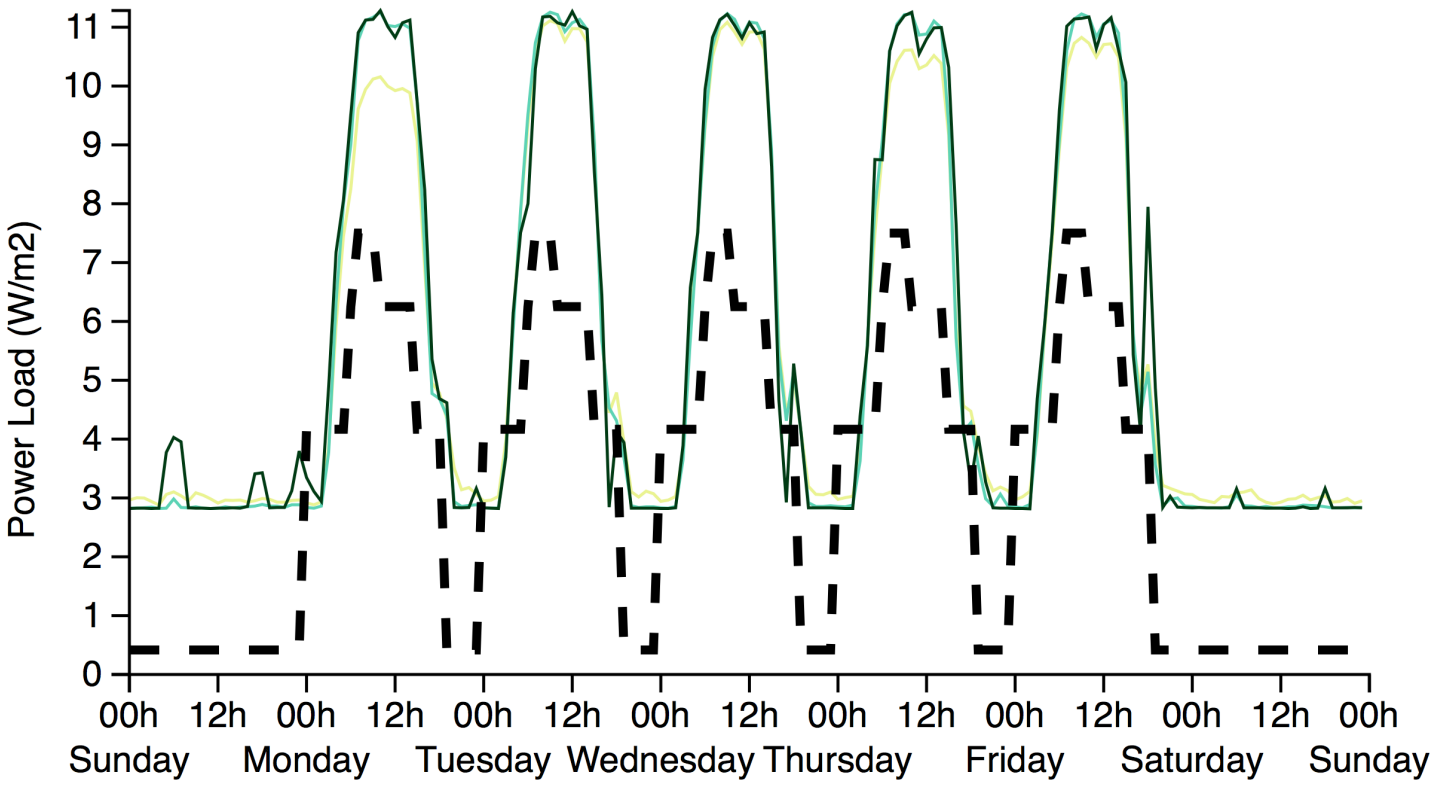

Day and Time

Figure 46. Mail Sorting-1: Average, Median, and Typical Weekly Profiles for Lighting Energy Use

Legend:

Average Median Typical

- _ - - Predicted Load Profile

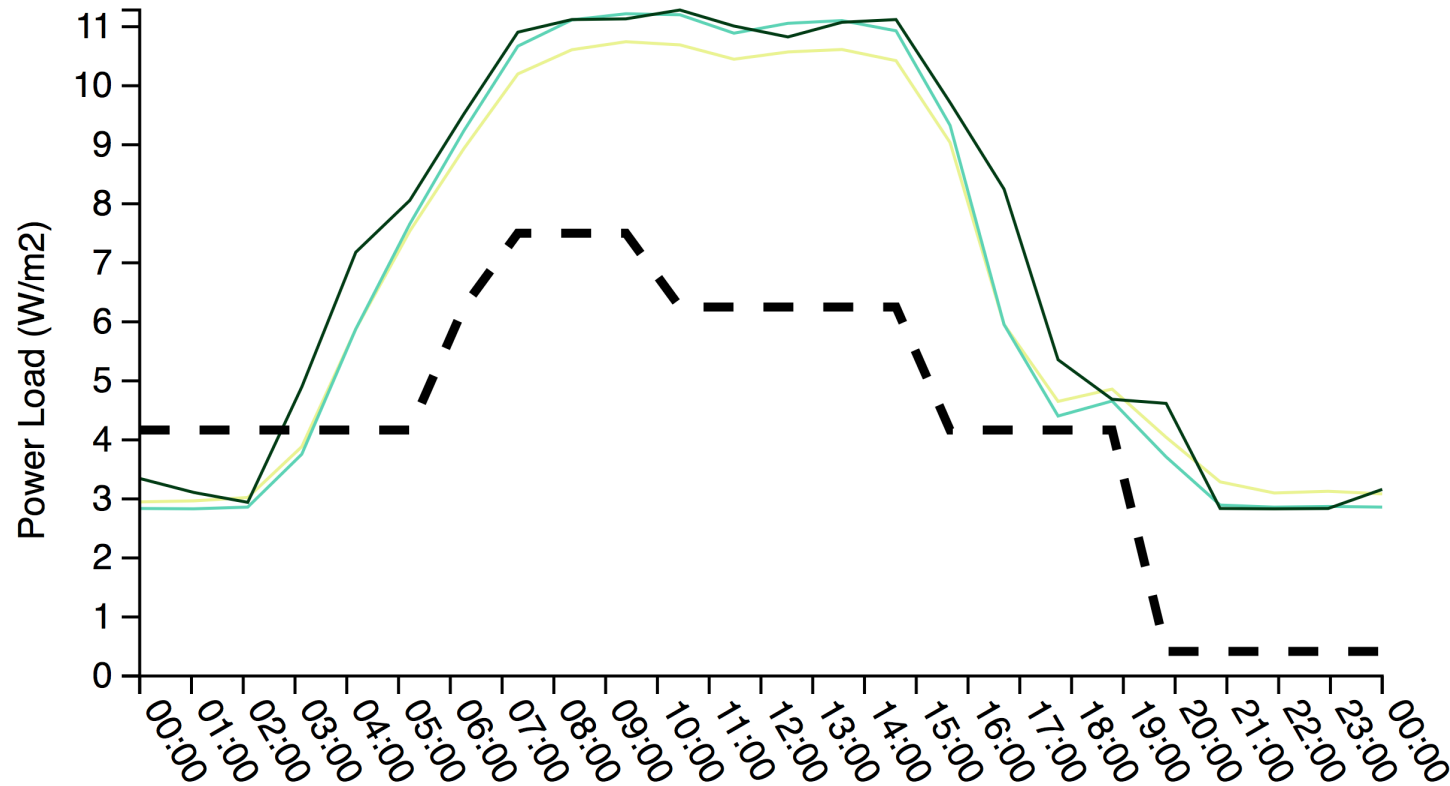

Hour

Figure 47. Mail Sorting-1: Average, Median, and Typical Weekday Lighting Profile

78 
Legend:

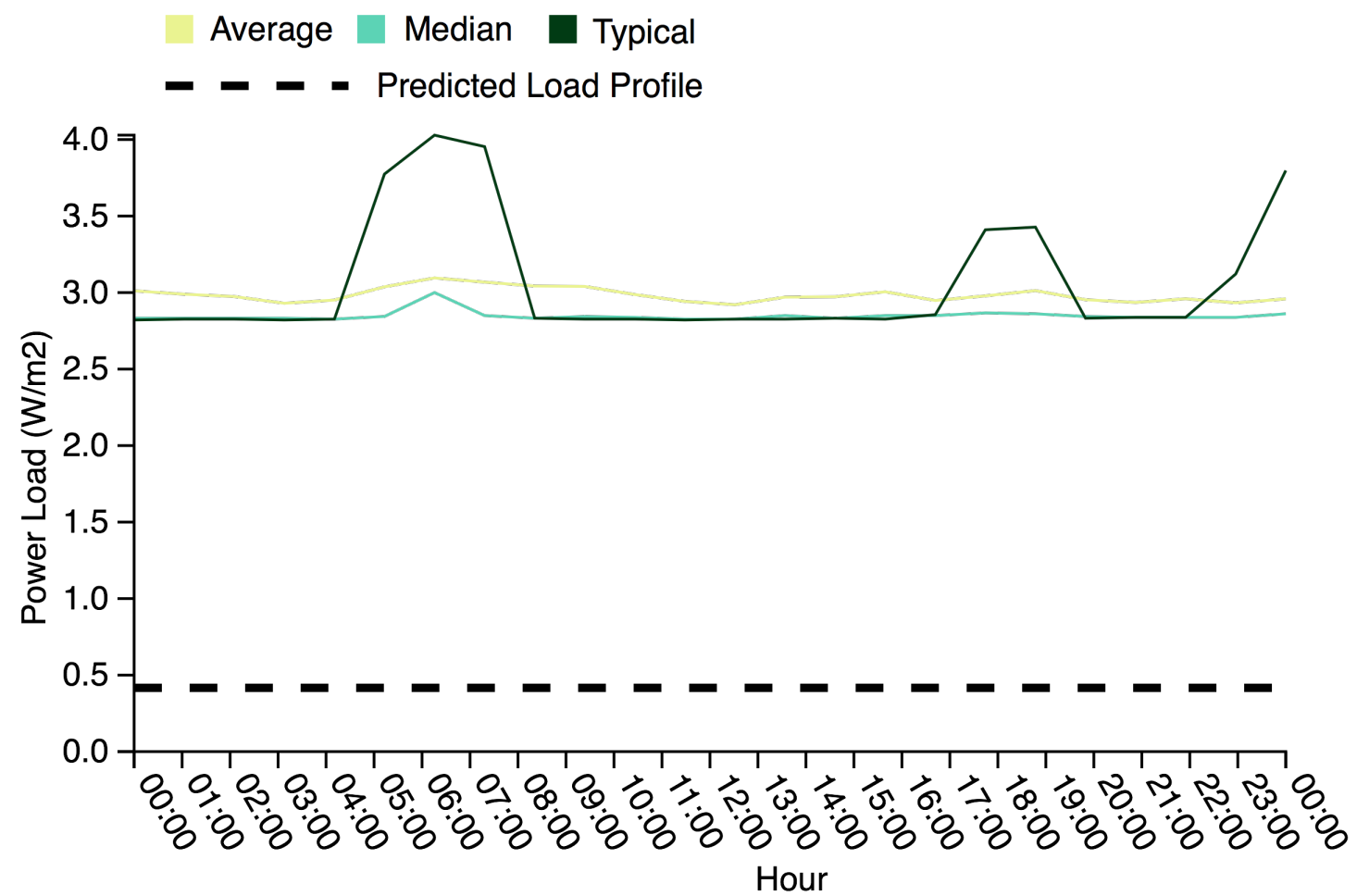

Figure 48. Mail Sorting-1: Average, Median, and Typical Weekend Day Lighting Profile

Legend:

Total Energy Use

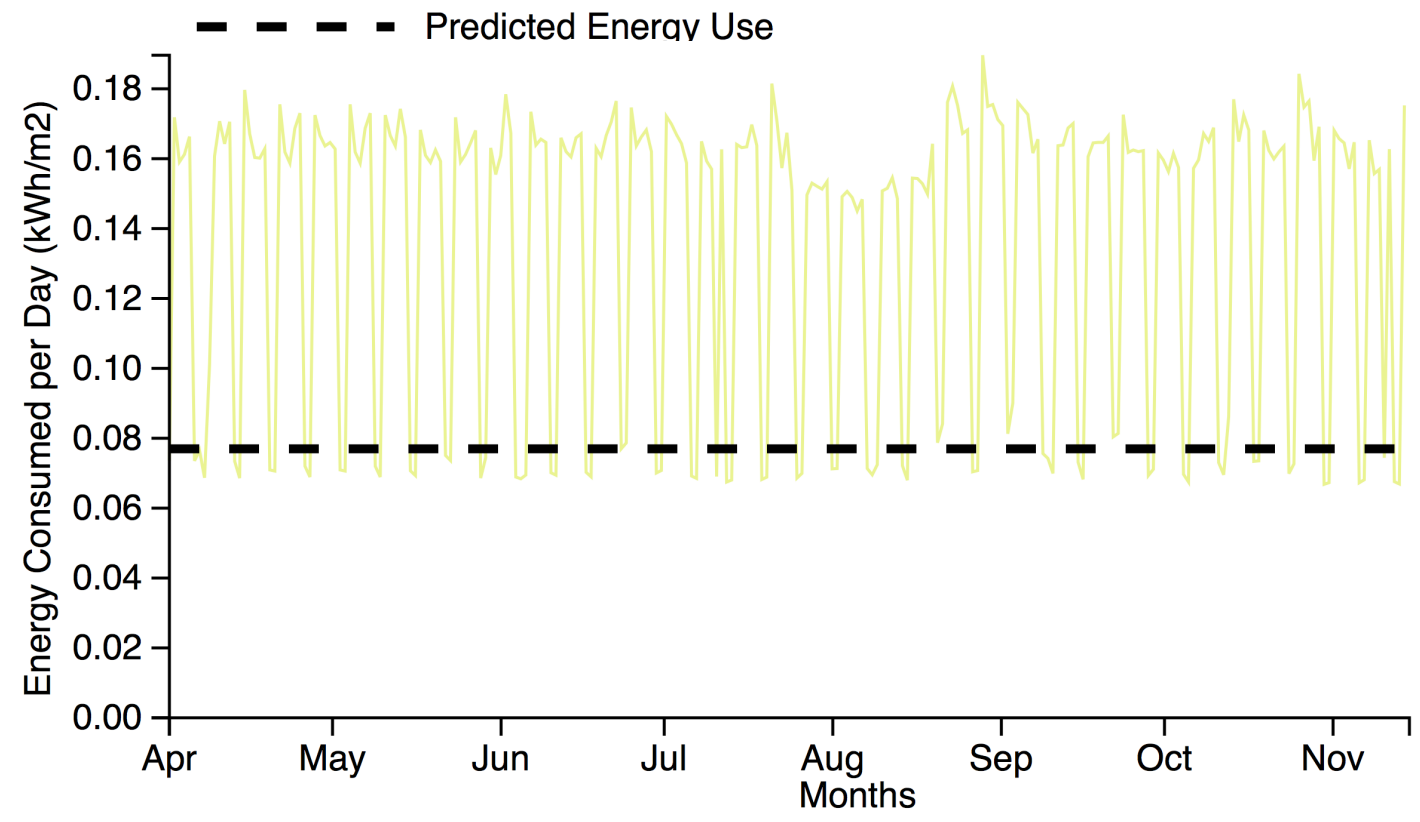

Figure 49. Mail Sorting-1: Full-year Total Daily Lighting Energy Use Data 
Considering Figure 49 it is possible to observe the lighting load profile over the course of the year. August experience a drop in energy consumption, likely due to reduced summer occupancy. In general the load profile remains relatively stable, this is likely contributed to by a predictable and stable occupancy. Overall energy use remains above expected.

Figure 50 shows a regression analysis correlating seasonal changes in lighting energy use with bright sunshine availability. The analysis appears to demonstrates a fairly strong positive correlation $\left(r^{2}=0.43\right)$ between lighting energy use and day light hours for this building. This indicates that on average light energy consumption increases as daylight availability increases. To a certain extent the strength of this regression seems somewhat suspect, removing the single outlier reduces the strength of the correlation to $\mathrm{r}^{2}=0.02$, indicating for all intents and purposes a poor correlation.

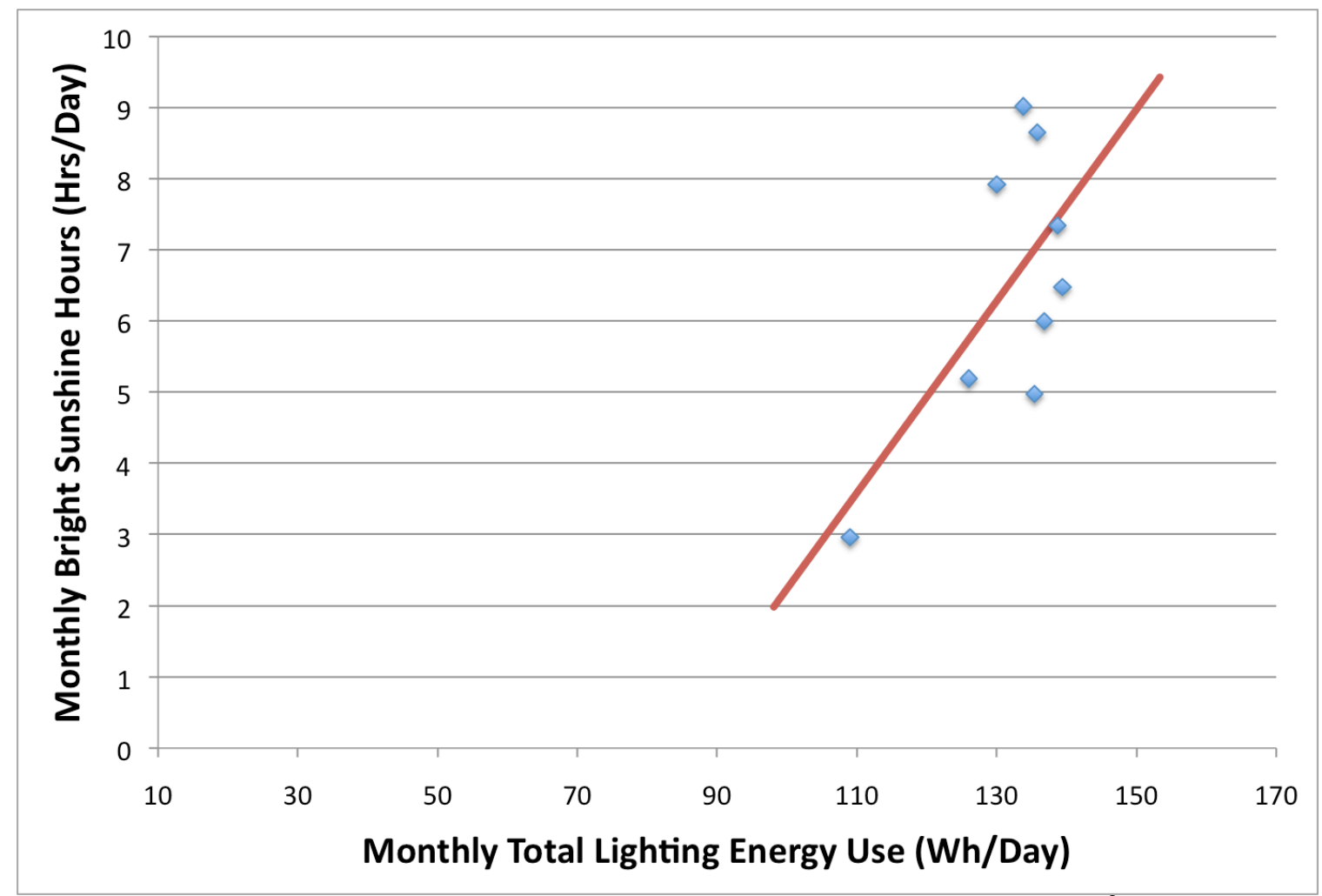

Figure 50. Mail Sorting-1: Lighting Energy Use Versus Sunshine Hours Regression $\left(r^{2}=0.43\right.$, slope $=$ 0.13) 
Receptacles

Table 23. Mail Sorting-1: Building Receptacles Summary

\begin{tabular}{cccc}
\hline $\begin{array}{c}\text { As Modeled: } \\
\text { Installed } \\
\text { Receptacles PD } \\
\left(\mathbf{W} / \mathbf{m}^{\mathbf{2}}\right)\end{array}$ & $\begin{array}{c}\text { MNP Energy Use } \\
\left(\mathbf{W} / \mathbf{m}^{2}\right)\end{array}$ & $\begin{array}{c}\text { MNB Energy Use } \\
\left(\mathbf{W} / \mathbf{m}^{\mathbf{2}}\right)\end{array}$ & $\begin{array}{c}\text { Base as \% } \\
\text { of Peak }\end{array}$ \\
\hline 10.0 & 7.5 & 2.1 & $28 \%$ \\
\hline $\begin{array}{c}\text { As Measured: } \\
\text { Installed } \\
\text { Receptacles PD } \\
\left(\mathbf{W} / \mathbf{m}^{2}\right)\end{array}$ & $\begin{array}{c}\text { MNP Energy Use } \\
\left(\mathbf{W} / \mathbf{m}^{\mathbf{2}}\right)\end{array}$ & $\begin{array}{c}\text { MNB Energy Use } \\
\left(\mathbf{W} / \mathbf{m}^{\mathbf{2}}\right)\end{array}$ & $\begin{array}{c}\text { Base as \% } \\
\text { of Peak }\end{array}$ \\
\hline- & 2.3 & 1.5 & $67 \%$ \\
\hline
\end{tabular}

Table 23 summarizes the median near peak (MNP) receptacle loads and median near base (MNB) receptacle loads as modeled and as measured. Comparison between the receptacles profile and receptacles schedules show a significantly overpredicted weekday and weekend peak-load (Figure 51 and Figure 52). Calculated with MNP and MNB values ( $2.3 \mathrm{~W} / \mathrm{m}^{2}$ and $1.5 \mathrm{~W} / \mathrm{m}^{2}$ respectively) the off-hours base load is approximately $67 \%$ of the peak load, as opposed to the estimated $28 \%$. In this case this is a result of a much lower than expected peak load, compensated also by a lower than expected base load. A near peak load of $2.3 \mathrm{~W} / \mathrm{m}^{2}$ is $31 \%$ of estimated peak load of approximately $7.5 \mathrm{~W} / \mathrm{m}^{2}$. This indicates that during peakhours the building is using much less electricity for receptacle loads than expected.

Figure 51 allows us to see energy use across the weeks of the year. It is interesting to see the entire load profile briefly shift downwards sometime in the late summer, this could be due to some of the HVAC loads on the panel, or alternatively due to some irregularities in operation. 


\section{Legend:}

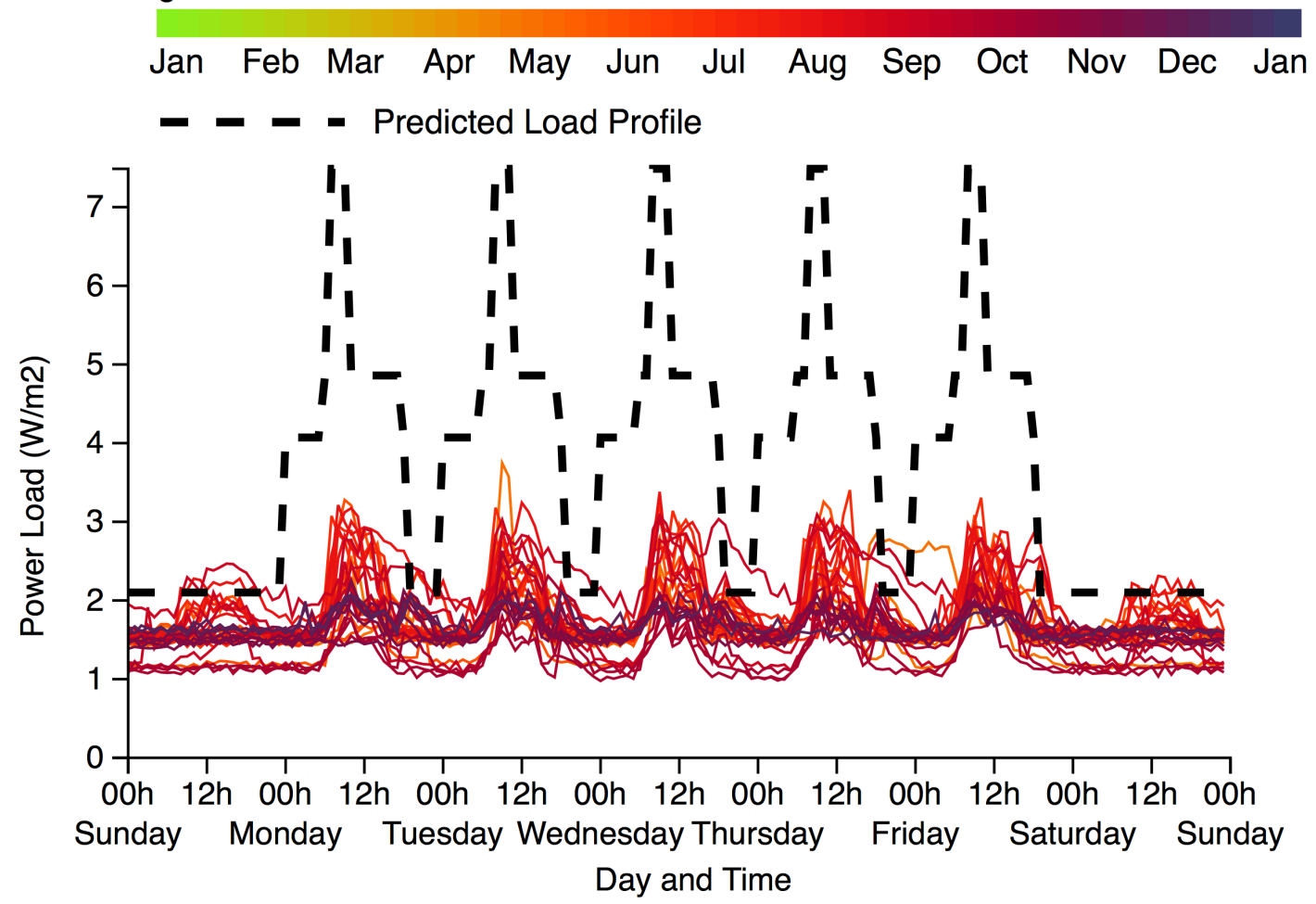

Figure 51. Mail Sorting-1: Full-year 60-Minute Interval Receptacles Energy Use Data

Average, median and typical lighting loads are shown for weeks, weekdays and weekend, respectively, in Figure 52, Figure 53 and Figure 54. The values calculated for the MNP and MNB are more clearly represented in these figures. In Figure 53 very little ramp up is observable, much less than is observed as fairly normal operations in Figure 51 or Figure 52. This is likely a case where significantly different operational modes produce load profiles that cancel each other out when calculating a median or average profile. Even when considering the most typical profile it would appear that a completely flat load profile slightly elevated from the base load is chosen as most representative. In regards to the weekend load profile (Figure 54) very little meaningful variation occurs through out the day. 
Legend:

Average Median $\square$ Typical

- - - - Predicted Load Profile

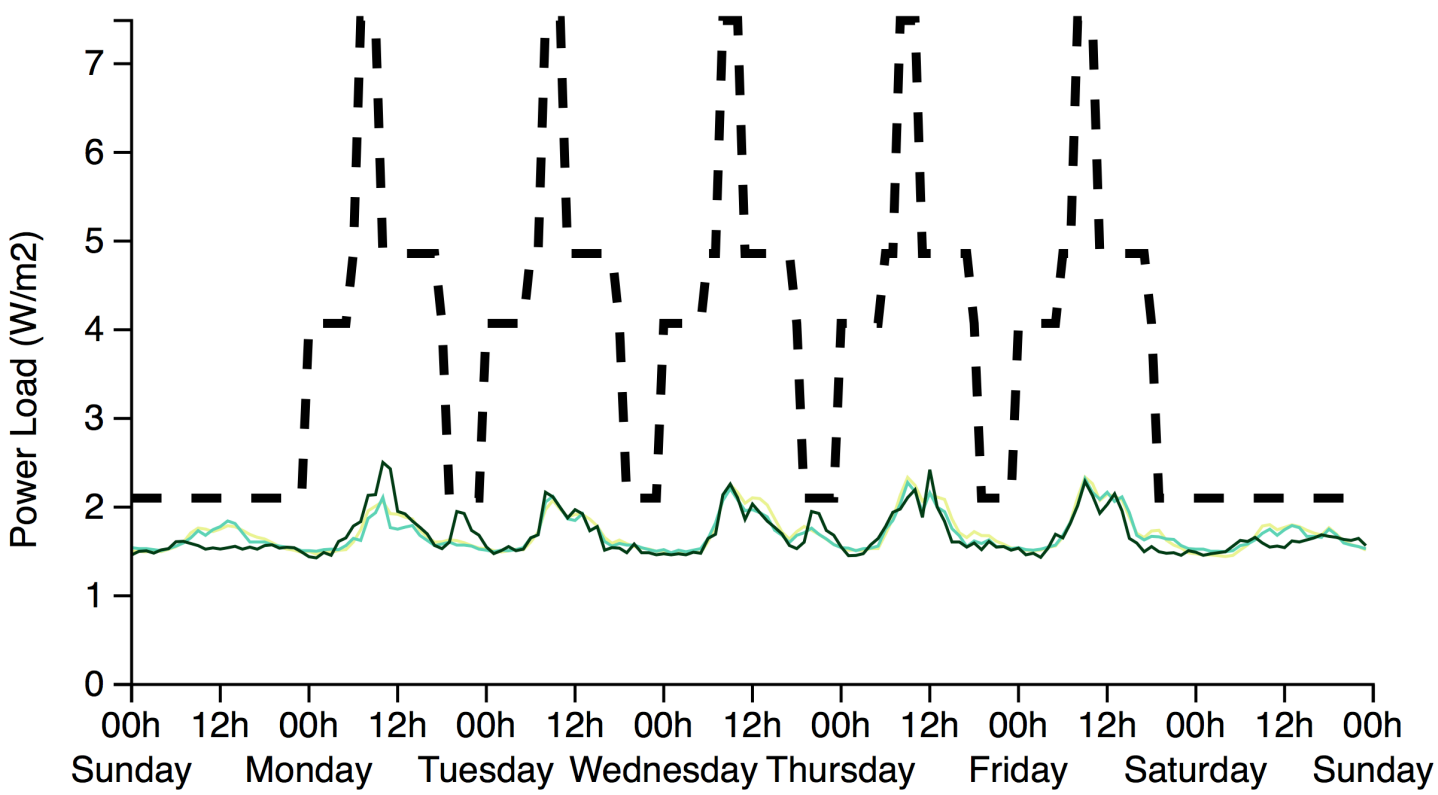

Day and Time

Figure 52. Mail Sorting-1: Average, Median, and Typical Weekly Profiles for Receptacles Energy Use

Legend:

Average $\square$ Median $\square$ Typical

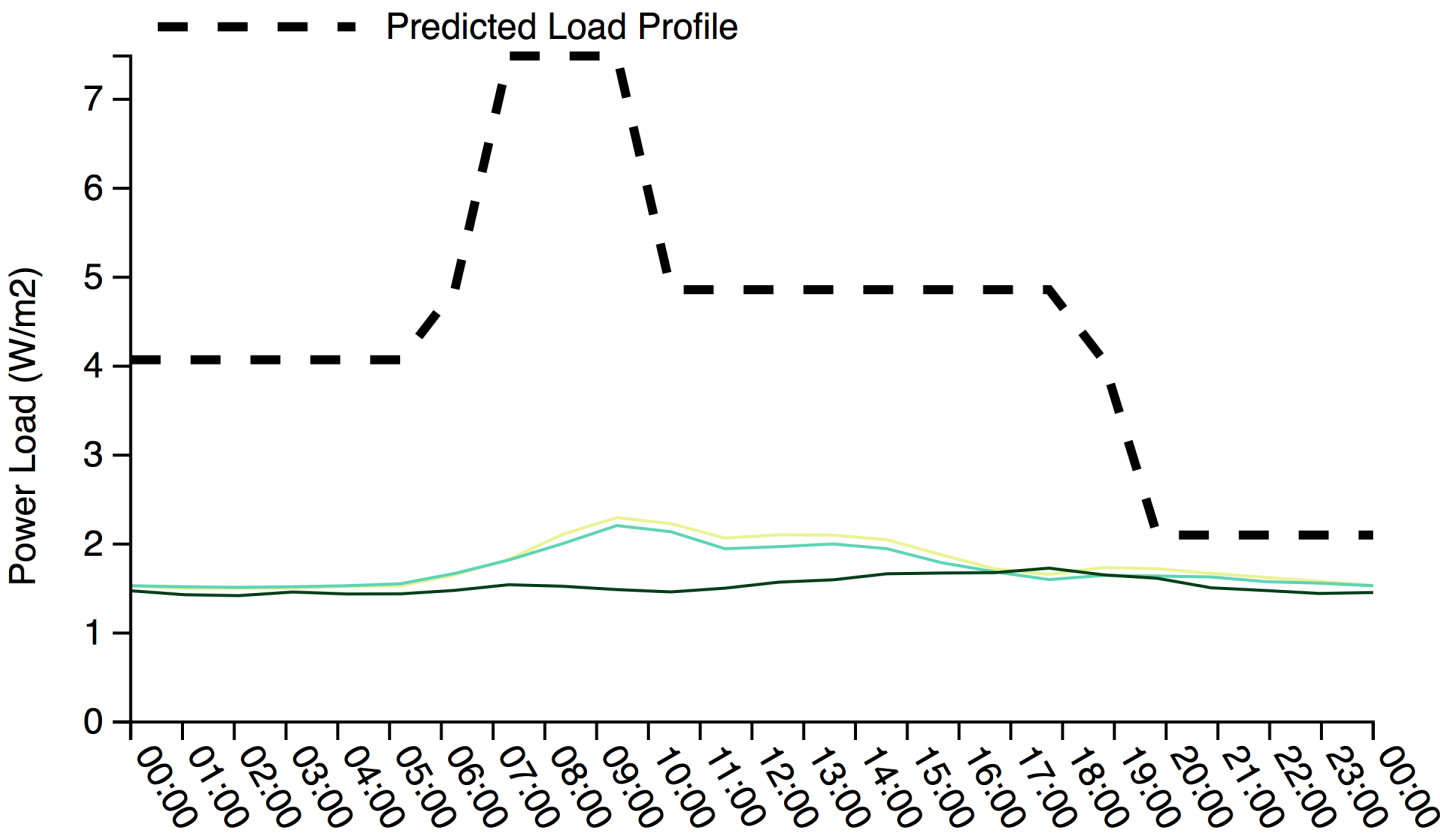

Hour

Figure 53. Mail Sorting-1: Average, Median, and Typical Weekday Receptacles Profile

83 


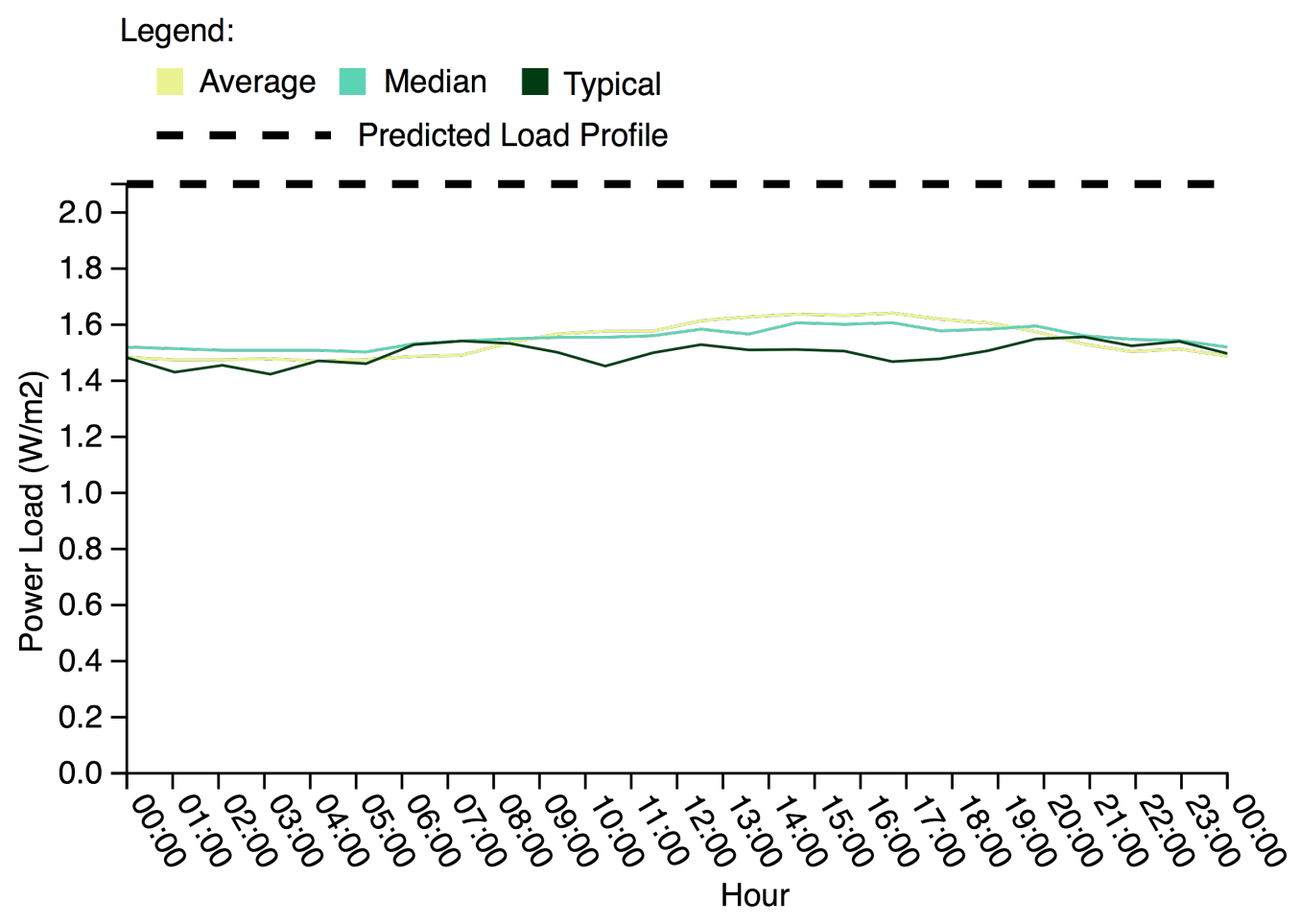

Figure 54. Mail Sorting-1: Average, Median, and Typical Weekend Day Receptacles Profile

Legend:

Total Energy Use

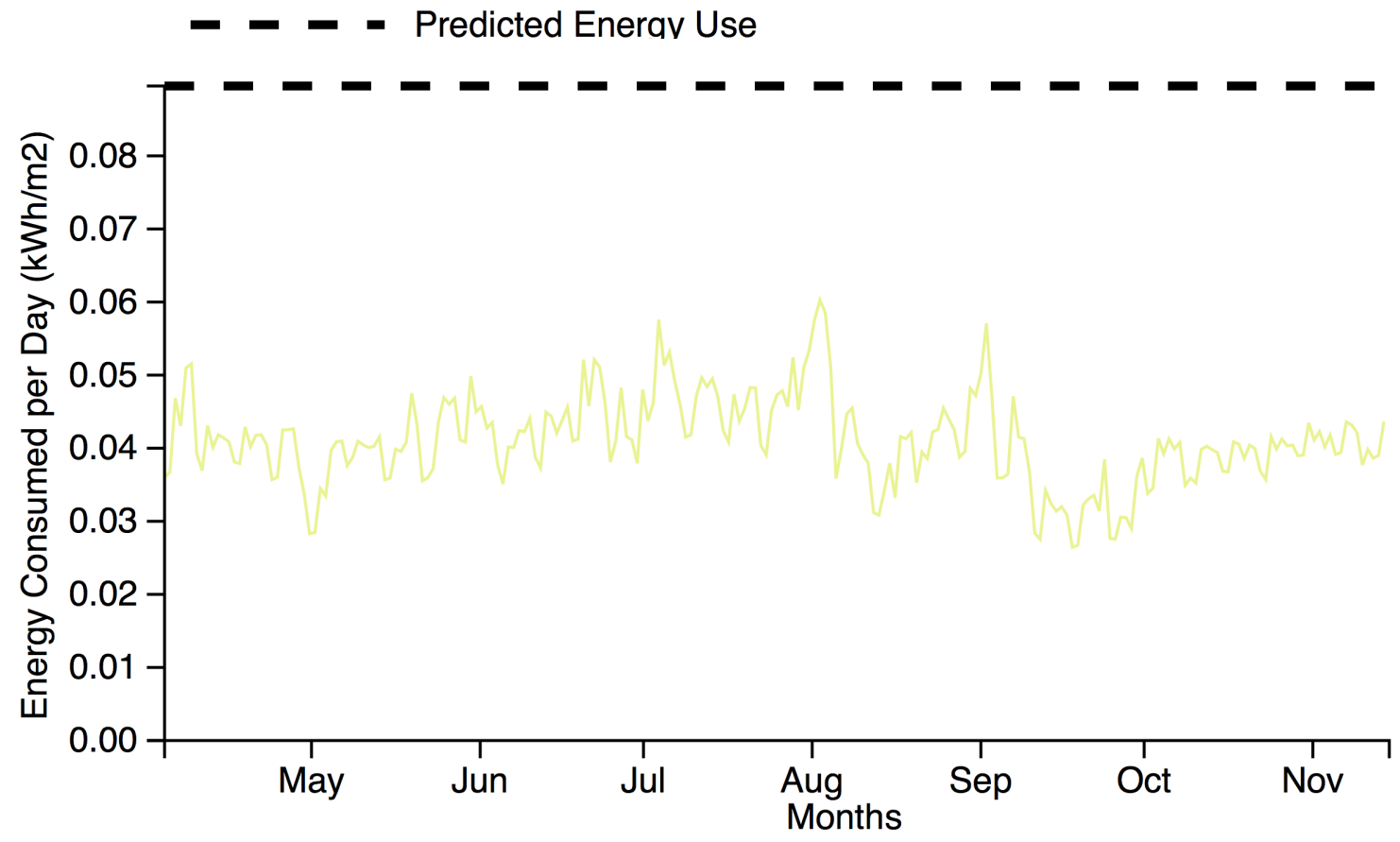

Figure 55. Mail Sorting-1: Full-year Total Daily Receptacles Energy Use Data 
Considering Figure 55 it is possible to see the variations in energy use throughout the year. A relatively unstable trend is observed, with no clear correlation between time of year or season. Overall energy use is consistently under-predicted.

\subsubsection{Mail Sorting-2}

The building is a midsize mail sorting facility $\left(6,380 \mathrm{~m}^{2}\right)$. Energy use data was collected between February 2011 and Jan 2012. However meter readings report dates between July 2010 and November 2011. It was not possible to determine the actual period the building data refers to. The dates from the metering data were maintained solely for ease of reference.

No meter attribution documentation was available beyond column labeling in metering data. Due to this fact exact composition of the metered energy use is unavailable. Lighting energy use may include exterior or emergency lighting. Lighting schedules used in energy modeling do not include exterior lighting.

Overall lighting is consuming more energy than expected due to much higher daytime and nighttime loads, as well as higher weekend lighting loads. Overnight lighting energy usage is certainly contributed by exterior lighting. Receptacle loads are consuming less energy than expected due to lower daytime peak loads, as well as lower overnight and weekend base loads.

Table 24. Mail Sorting-2: Building Summary

\begin{tabular}{|c|c|}
\hline $\begin{array}{c}\text { Location } \\
\text { Floor Area } \\
\text { Submetering Categories } \\
\text { Reporting Period } \\
\text { Data Availability } \\
\text { Metering Attribution }\end{array}$ & $\begin{array}{c}\text { Burlington, Ontario } \\
6,380 \text { m2 } \\
\text { Lighting, Receptacles, } \\
\text { Sortation, HVAC, Boiler } \\
\text { Feb } 2011 \text { - Jan } 2012 \\
\text { July } 2010 \text { - Nov } 2011 \\
\text { Limited } \\
\end{array}$ \\
\hline $\begin{array}{l}\text { Lighting: } \\
\text { Installed LPD }\end{array}$ & $9.6 \mathrm{~W} / \mathrm{m}^{2}$ \\
\hline $\begin{array}{l}\text { Receptacles: } \\
\text { Installed Receptacles PD }\end{array}$ & $2.1 \mathrm{~W} / \mathrm{m}^{2}$ \\
\hline
\end{tabular}

Table 25 lists the key values for predicted and actual energy use of the building. Fairly substantial differences are visible between the various values. 
Table 25. Mail Sorting-2: Predicted and Actual Energy Use

\begin{tabular}{|c|c|c|c|}
\hline & $\begin{array}{c}\text { Uncalibrated- } \\
\text { Predicted } \\
\left(\mathbf{k W h} / \mathbf{m}^{2}\right)\end{array}$ & $\begin{array}{l}\text { Calibrated- } \\
\text { Predicted } \\
\left(\mathbf{k W h} / \mathbf{m}^{2}\right)\end{array}$ & $\begin{array}{c}\text { Actual } \\
\left(\mathrm{kWh} / \mathrm{m}^{2}\right)\end{array}$ \\
\hline Total Energy Use & 137.1 & 137.7 & 169.3 \\
\hline $\begin{array}{l}\text { Total Electricity } \\
\text { Energy Use }\end{array}$ & 61.5 & 95.8 & 126.4 \\
\hline Total Natural Gas & 75.7 & 41.2 & 42.3 \\
\hline Heating & 74.1 & 54.8 & - \\
\hline Cooling & 3.1 & 7.2 & - \\
\hline Lighting & 35.2 & 50.0 & 74.6 \\
\hline Receptacles & 8.2 & 15.5 & 16.4 \\
\hline
\end{tabular}

\section{Lighting}

Table 26. Mail Sorting-2: Building Lighting Summary

\begin{tabular}{cccc}
\hline $\begin{array}{c}\text { As Modeled: } \\
\text { Installed LPD } \\
\left(\mathbf{W} / \mathbf{m}^{2}\right)\end{array}$ & $\begin{array}{c}\text { MNP Energy Use } \\
\left(\mathbf{W} / \mathbf{m}^{2}\right)\end{array}$ & $\begin{array}{c}\text { MNB Energy Use } \\
\left(\mathbf{W} / \mathbf{m}^{2}\right)\end{array}$ & $\begin{array}{c}\text { Base as \% } \\
\text { of Peak }\end{array}$ \\
\hline 9.6 & 9.5 & 0.5 & $5 \%$ \\
\hline $\begin{array}{c}\text { As Measured: } \\
\text { Installed LPD } \\
\left(\mathbf{W} / \mathbf{m}^{2}\right)\end{array}$ & $\begin{array}{c}\text { MNP Energy Use } \\
\left(\mathbf{W} / \mathbf{m}^{2}\right)\end{array}$ & $\begin{array}{c}\text { MNB Energy Use } \\
\left(\mathbf{W} / \mathbf{m}^{2}\right)\end{array}$ & $\begin{array}{c}\text { Base as \% } \\
\text { of Peak }\end{array}$ \\
\hline- & 12.5 & 1.4 & $11 \%$ \\
\hline
\end{tabular}

Table 26 summarizes the median near peak (MNP) lighting loads and median near base (MNB) lighting loads as modeled and as measured. Comparison between the lighting energy use profile and lighting schedules show an under-predicted nighttime and weekend base-load as well as an under-predicted daytime peak load (Figure 56 and Figure 57). It would also appear that the schedule under-predicts the building's weekly hours of operation, with a significant number of weekends appearing to operate at full capacity. Calculated with MNP and MNB values (12.5 $\mathrm{W} / \mathrm{m}^{2}$ and $1.4 \mathrm{~W} / \mathrm{m}^{2}$ respectively) the off hours base load is approximately $11 \%$ of the peak load, as opposed to the estimated 5\%. This indicates higher levels of offpeak energy use than expected. A MNP load of $12.5 \mathrm{~W} / \mathrm{m}^{2}$ is $132 \%$ of estimated peak load of approximately $9.5 \mathrm{~W} / \mathrm{m}^{2}$. Figure 56 shows fairly irregular patterns of energy use for lighting, it is unclear if this is due to occupancy or management issues. 
A much higher than expected peak weekday load of $12 \mathrm{~W} / \mathrm{m}^{2}$ is difficult to explain. This value surpasses the installed capacity of the system by $2.5 \mathrm{~W} / \mathrm{m}^{2}$, therefore the extra energy use cannot simply be a result of more intense use of the lighting system. In addition it is unlikely that the lighting power density in the models was incorrect as these values derived directly from the lighting shop drawings used in construction. As a result the metering system must have included some lighting use not included in the model as was seen in Mail Sorting-1, unfortunately fully meter attribution documentation is not available. However it is fairly likely that this meter could include exterior lighting, which is not included in the scheduled energy use, and thus would contribute to the observed "overcapacity".

Legend:

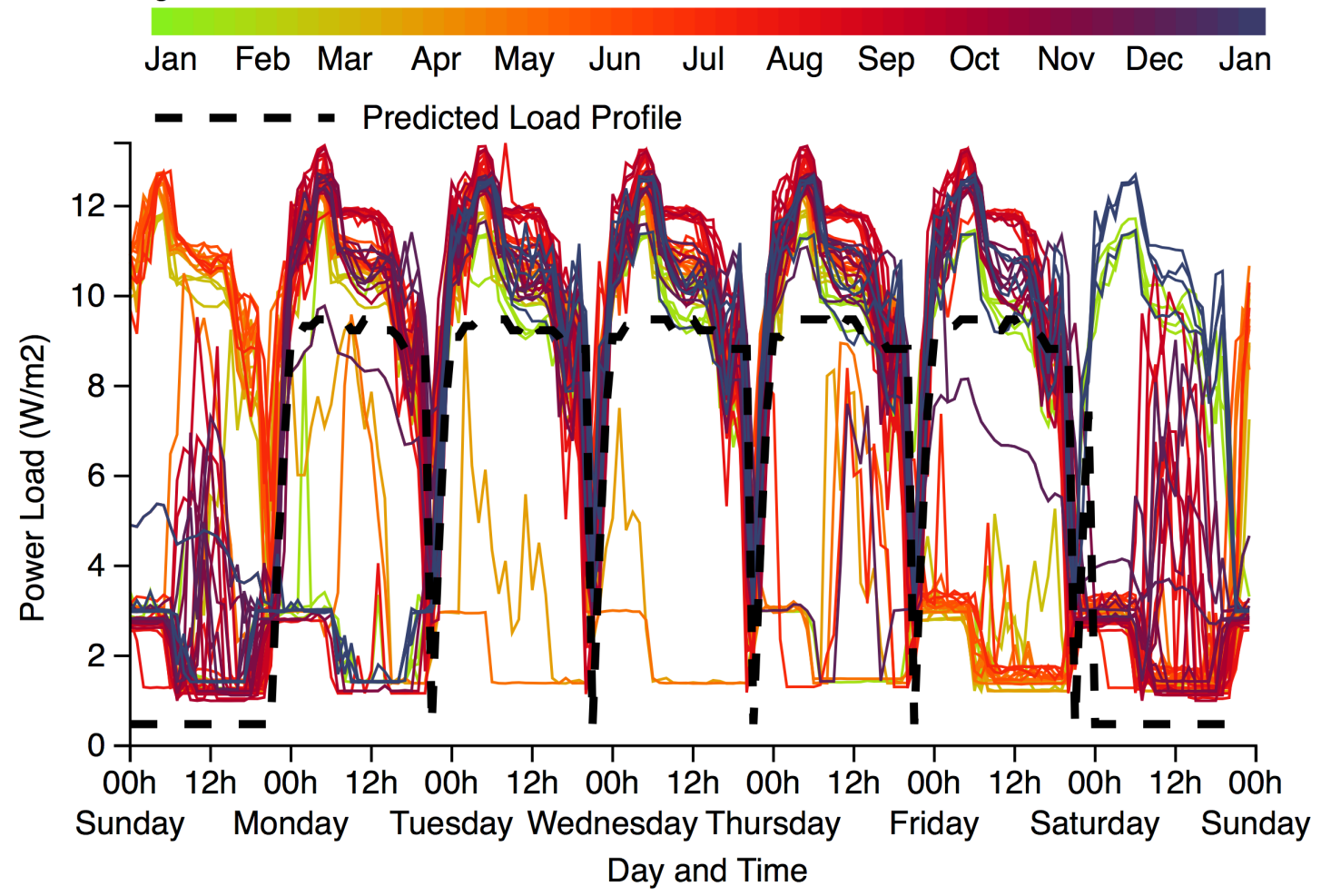

Figure 56. Mail Sorting-2: Full-year 60-Minute Interval Lighting Energy Use Data

Model calibration post-processing indicate that the building uses approximately 29,000 kWh per year of exterior lighting, assuming the equivalent of $8 \mathrm{hrs}$ per day of operation at maximum power, this would equate to approximately $1.6 \mathrm{~W} / \mathrm{m}^{2}$, and thus not accounting for the entirety of the $2.5 \mathrm{~W} / \mathrm{m}^{2}$ of unaccounted 
for lighting loads. Site visits to the building after occupancy concluded that lighting controls were either not functioning or were disabled resulting in an increase in energy use, however this should not be a factor in increasing energy use above the maximum installed capacity as is witnessed.

Average, median and typical lighting loads are shown for weeks, weekdays and weekend, respectively, in Figure 57, Figure 58 and Figure 59. The values calculated for the MNP and MNB are more clearly represented in these figures. In Figure 58 the weekday profile follows predictions generally as expected, although with slightly higher energy use through out the day, especially around 5:00. The sharp trough around 21:30 is matched quite closely with the actual energy use profile. The weekend load profile seen in Figure 59 proves to be somewhat of an enigma until Figure 56 is considered. The contradictory profiles in Figure 59 are produced due to the fact that both Saturday and Sunday function in either of three modes, which are more or less diametrically opposed, as can be seen in Figure 56. The first pattern is full energy use almost identical to a normal weekday schedule, the second is a lower base with a core peak during typical daytime hours, and the third is a core trough during typical business hours. These three patterns occur in large enough quantities that they cancel each other out for the most part when considering average, median, or typical. It is interesting to see in Figure 57 that the typical Sunday load profile ended up representing one of the patterns, while the typical Saturday load profile ended up representing of the other pattern, despite no clear connection with Saturday or Sunday any of the aforementioned patterns. In cases like these, the average, mean and typical can all become fairly unreliable. The average load profile in particular appears prone to effects from outlier data. This is seen in Figure 57, where average load profile is dragged up or down, whereas both the median and typical remain more or less similar. 


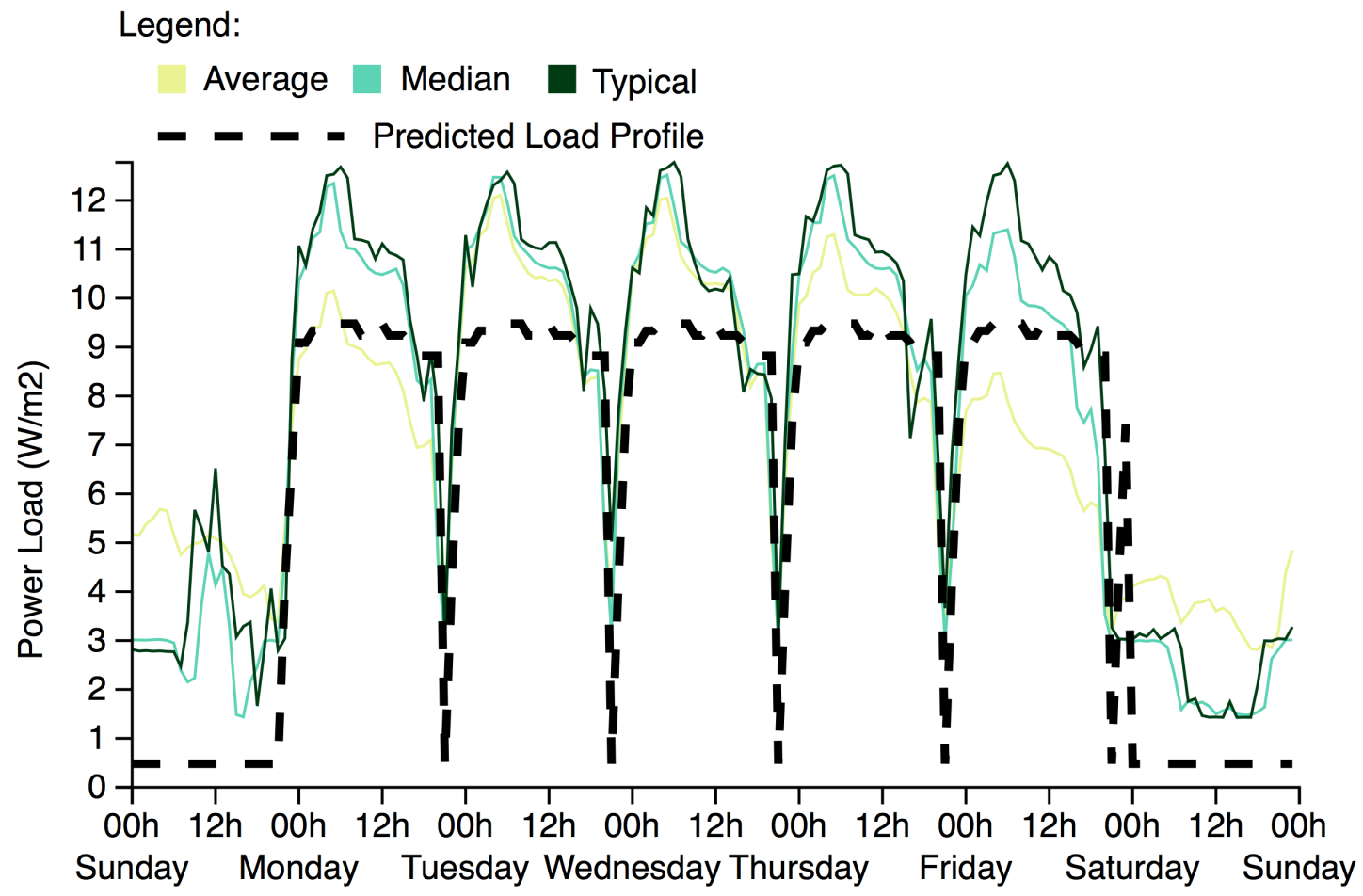

Day and Time

Figure 57. Mail Sorting-2: Average, Median, and Typical Weekly Profiles for Lighting Energy Use

Legend:

Average Median $\square$ Typical

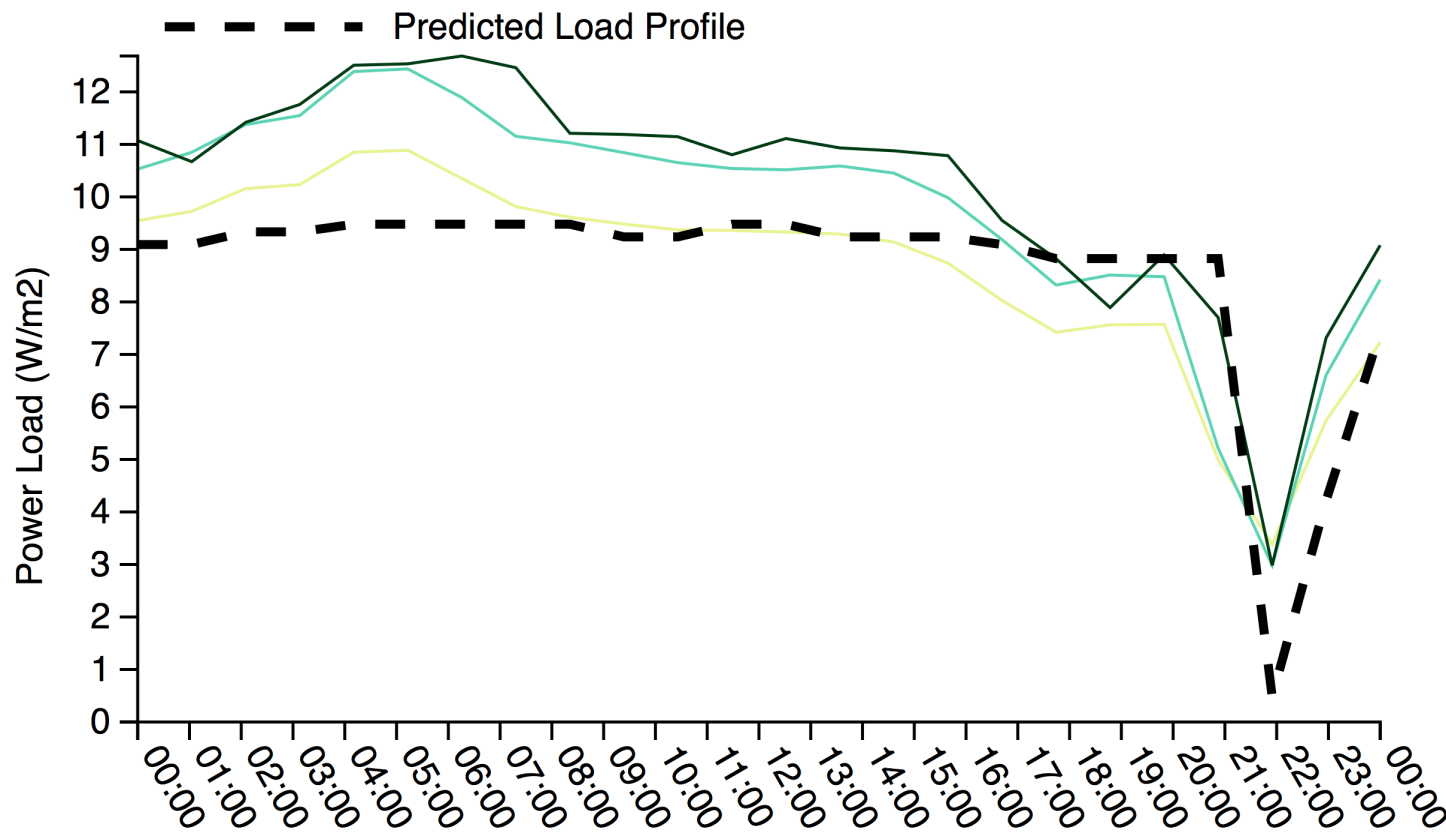

Hour

Figure 58. Mail Sorting-2: Average, Median, and Typical Weekday Lighting Profile

89 
Legend:

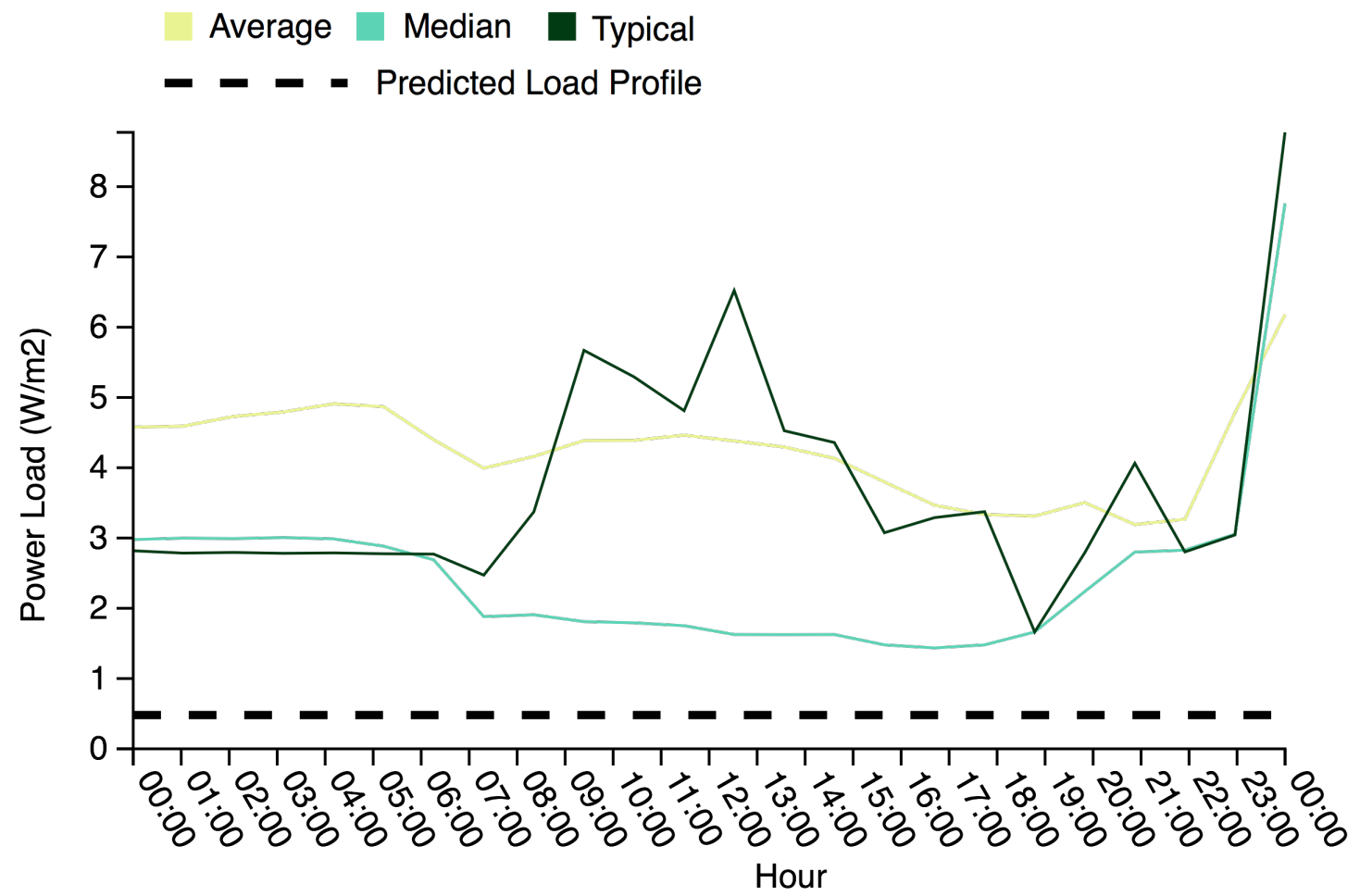

Figure 59. Mail Sorting-2: Average, Median, and Typical Weekend Day Lighting Profile

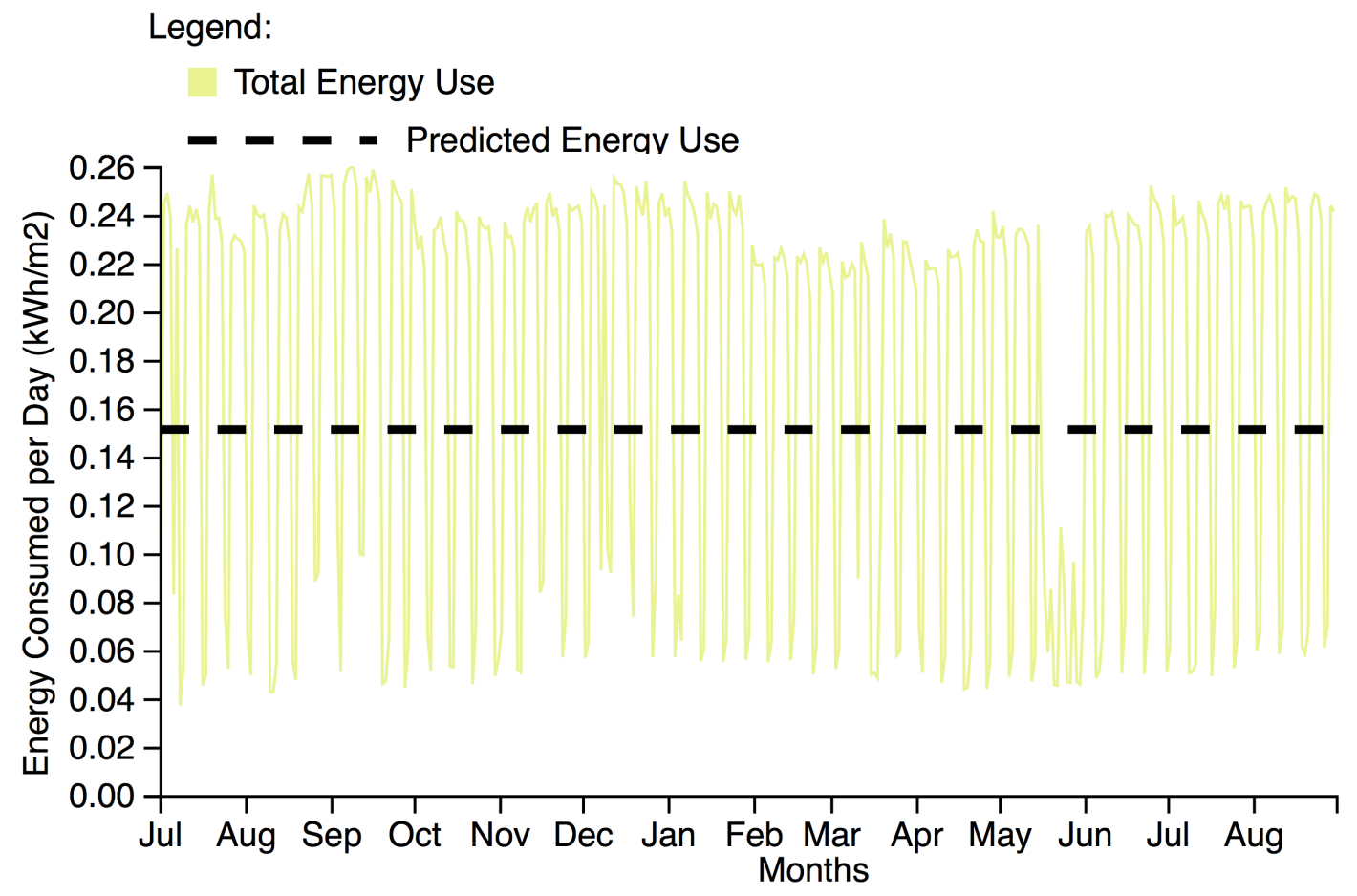

Figure 60. Mail Sorting-2: Full-year Total Daily Lighting Energy Use Data 
Considering Figure 60 it is possible to see the variation of the energy load profile throughout the year. Energy consumption varies through out the year, however any clear seasonal trends are difficult to establish. December experiences a drop in energy use due to lower holiday occupancy. A significant trough in energy use occurring in May is likely due to unusual circumstances. Overall energy use falls fairly close to the average predicted values.

Figure 61 shows a regression analysis correlating seasonal changes in lighting energy use with bright sunshine availability. The analysis demonstrates a weak negative correlation $\left(r^{2}=0.00\right)$ between lighting energy use and day light hours for this building. Nevertheless the negative slope does indicate that on average light energy consumption does decrease as daylight availability increases.

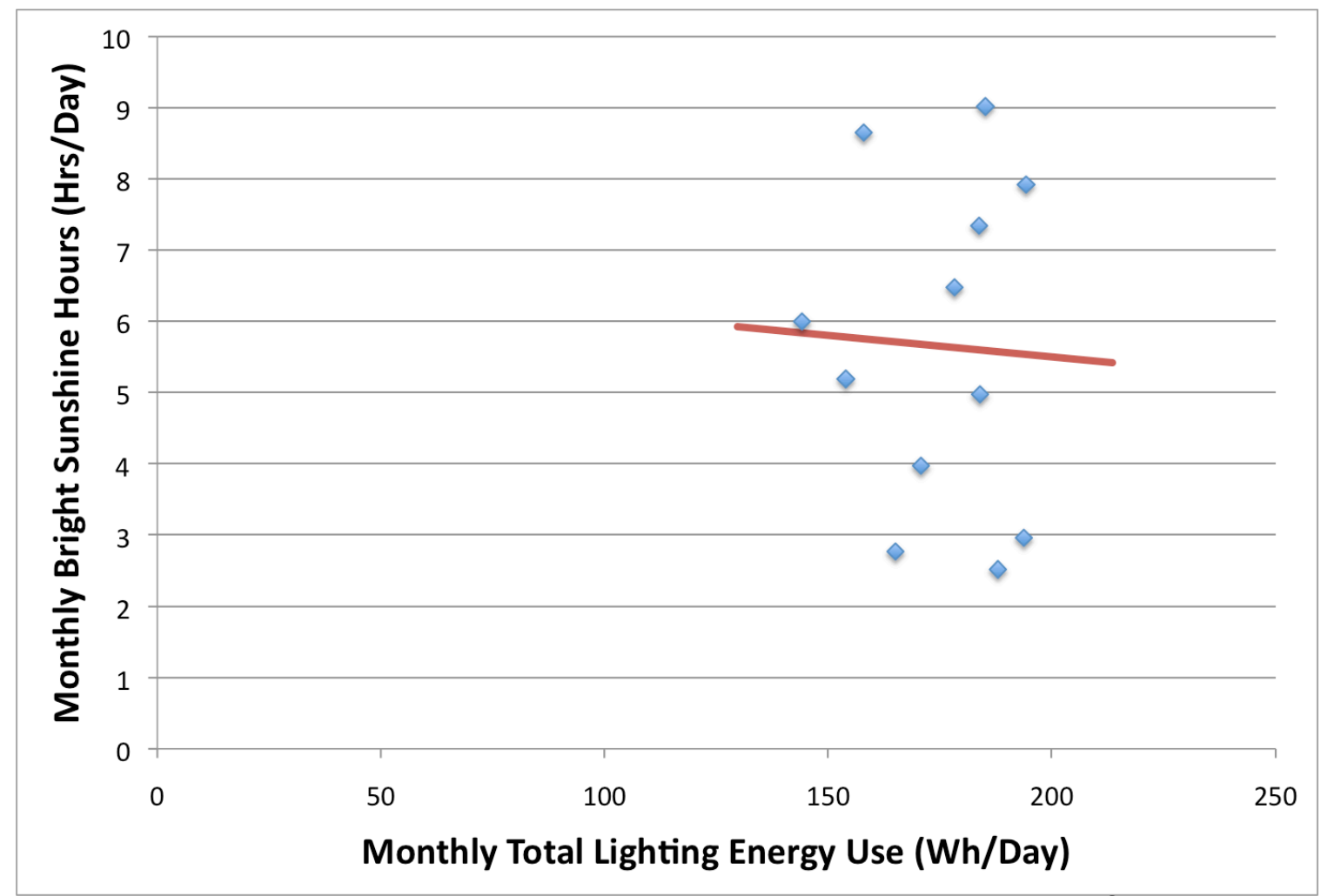

Figure 61. Mail Sorting-2: Lighting Energy Use Versus Sunshine Hours Regression $\left(r^{2}=0.00\right.$, slope = $-0.01)$ 


\section{Receptacles}

Table 27. Mail Sorting-2: Building Receptacles Summary

\begin{tabular}{cccc}
\hline $\begin{array}{c}\text { As Modeled: } \\
\text { Installed } \\
\text { Receptacles PD } \\
\left(\mathbf{W} / \mathbf{m}^{\mathbf{2}}\right)\end{array}$ & $\begin{array}{c}\text { MNP Energy Use } \\
\left(\mathbf{W} / \mathbf{m}^{\mathbf{2}}\right)\end{array}$ & $\begin{array}{c}\text { MNB Energy Use } \\
\left(\mathbf{W} / \mathbf{m}^{\mathbf{2}}\right)\end{array}$ & $\begin{array}{c}\text { Base as \% } \\
\text { of Peak }\end{array}$ \\
\hline 2.1 & 2.1 & 1.5 & $71 \%$ \\
\hline $\begin{array}{c}\text { As Measured: } \\
\text { Installed } \\
\text { Receptacles PD } \\
\left(\mathbf{W} / \mathbf{m}^{2}\right)\end{array}$ & $\begin{array}{c}\text { MNP Energy Use } \\
\left(\mathbf{W} / \mathbf{m}^{\mathbf{2}}\right)\end{array}$ & $\begin{array}{c}\text { MNB Energy Use } \\
\left(\mathbf{W} / \mathbf{m}^{\mathbf{2}}\right)\end{array}$ & $\begin{array}{c}\text { Base as \% } \\
\text { of Peak }\end{array}$ \\
\hline- & 1.8 & 1.2 & $65 \%$ \\
\hline
\end{tabular}

Table 27 summarizes the median near peak (MNP) receptacle loads and median near base (MNB) receptacle loads as modeled and as measured. Comparison between the receptacles profile and receptacles schedules shows an over-predicted base and peak-load (Figure 62 and Figure 63).

Legend:

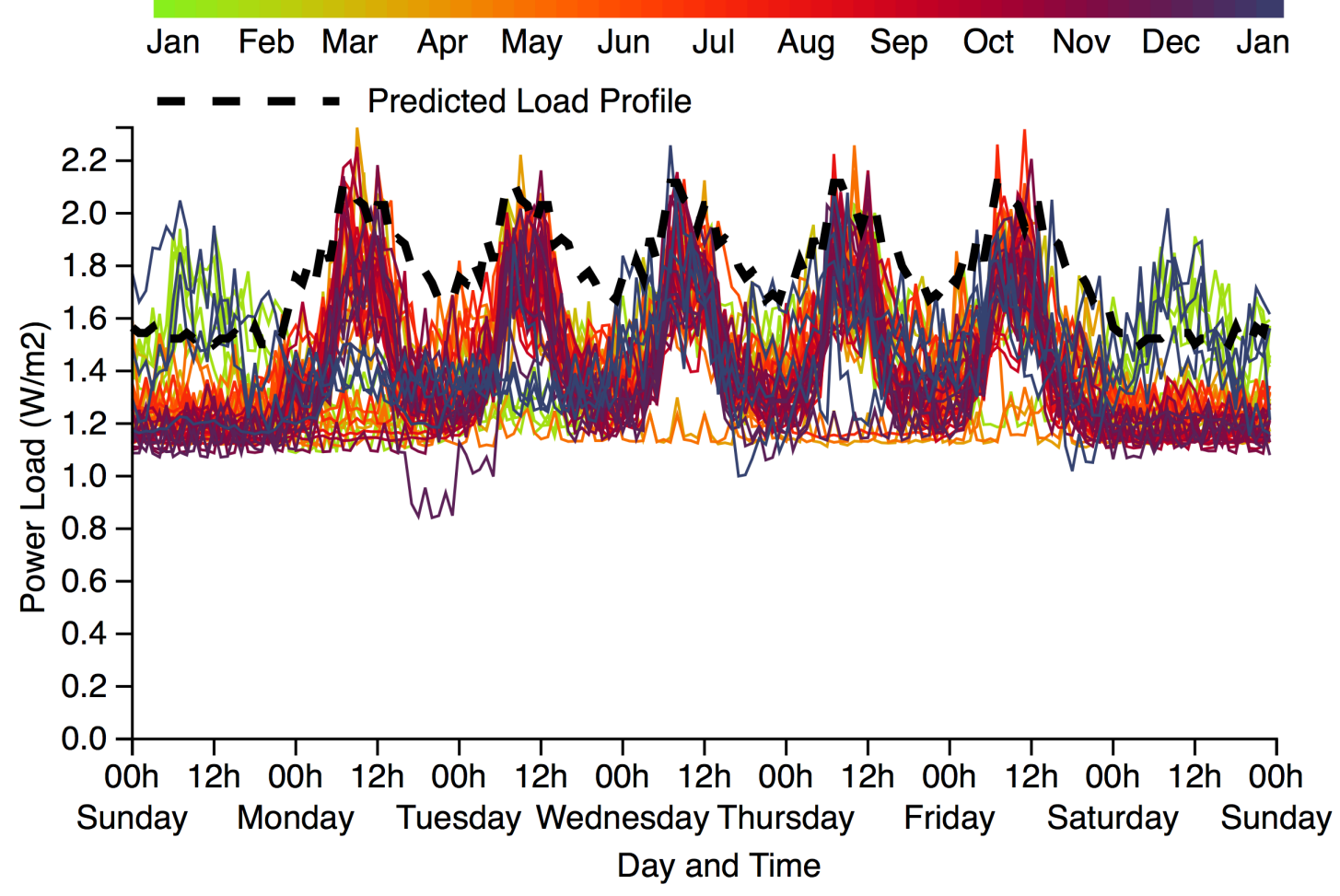

Figure 62. Mail Sorting-2: Full-year 60-Minute Interval Receptacles Energy Use Data

Calculated with MNP and MNB values $\left(1.8 \mathrm{~W} / \mathrm{m}^{2}\right.$ and $1.2 \mathrm{~W} / \mathrm{m}^{2}$ respectively) the off-hours baseload is approximately $65 \%$ of the peak load, as opposed to the 
estimated $71 \%$. A near peak load of $1.8 \mathrm{~W} / \mathrm{m}^{2}$ is $86 \%$ of the estimated peak load of approximately $2.1 \mathrm{~W} / \mathrm{m}^{2}$. Considering these two sets of values, as well as Figure 62 it is clear that proportionally the base and peak loads are occurring about as expected albeit slightly lower. The energy use profile is highly stable. This is supported by Figure 62, although it does show occasional increased energy use on weekends.

Average, median and typical receptacle loads are shown for weeks, weekdays and weekend, respectively, in Figure 63, Figure 64 and Figure 65. The values calculated for the MNP and MNB are more clearly represented in these figures. In both Figure 64 and Figure 65 it is observable that the predicted weekday and weekend load profiles are matched very closely with actual, except as mentioned above the actual load profiles remain slightly lower than expected.

\section{Legend:}

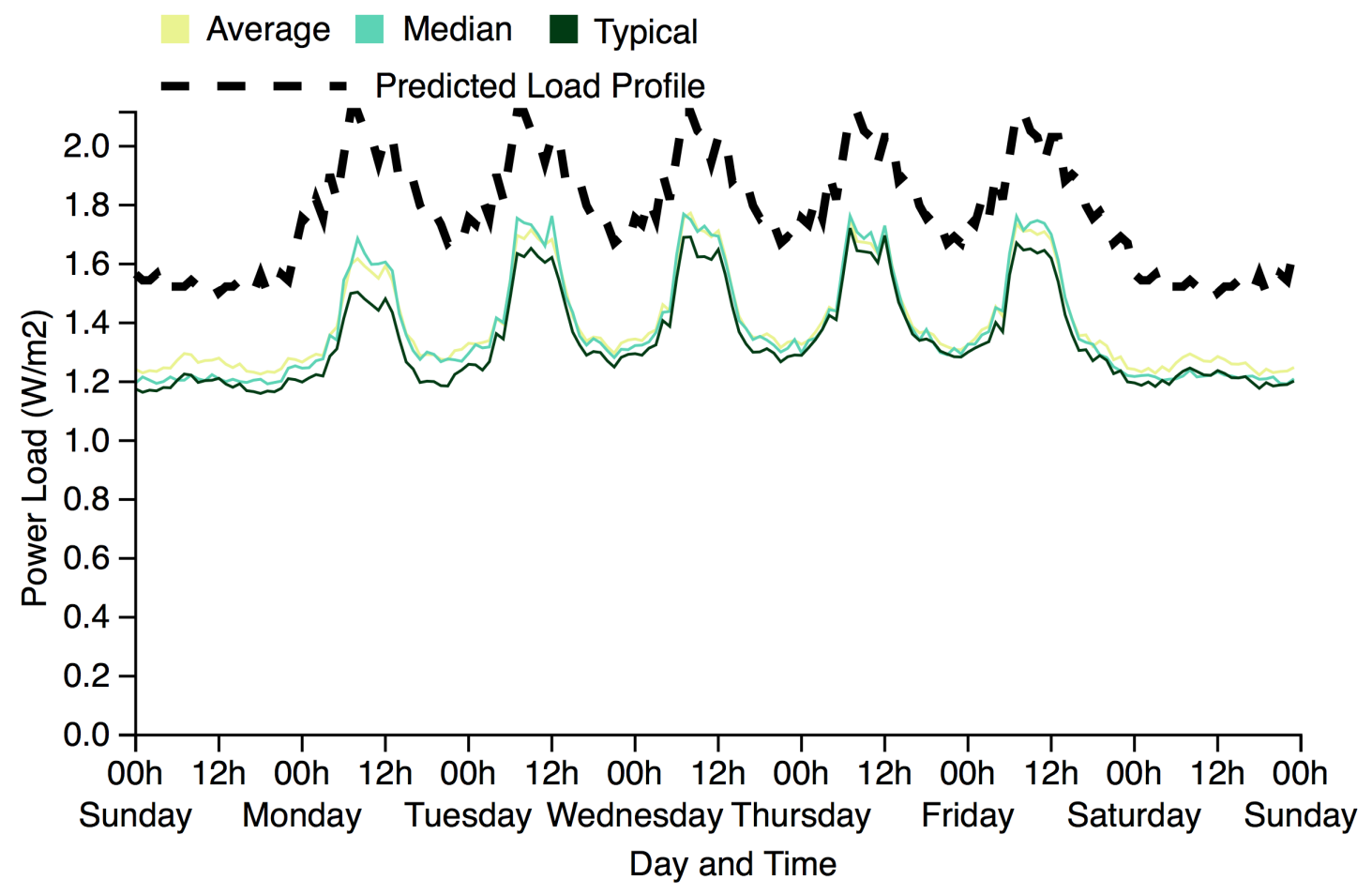

Figure 63. Mail Sorting-2: Average, Median, and Typical Weekly Profiles for Receptacles Energy Use 
Legend:

Average Median

Typical

- _ - - Predicted Load Profile

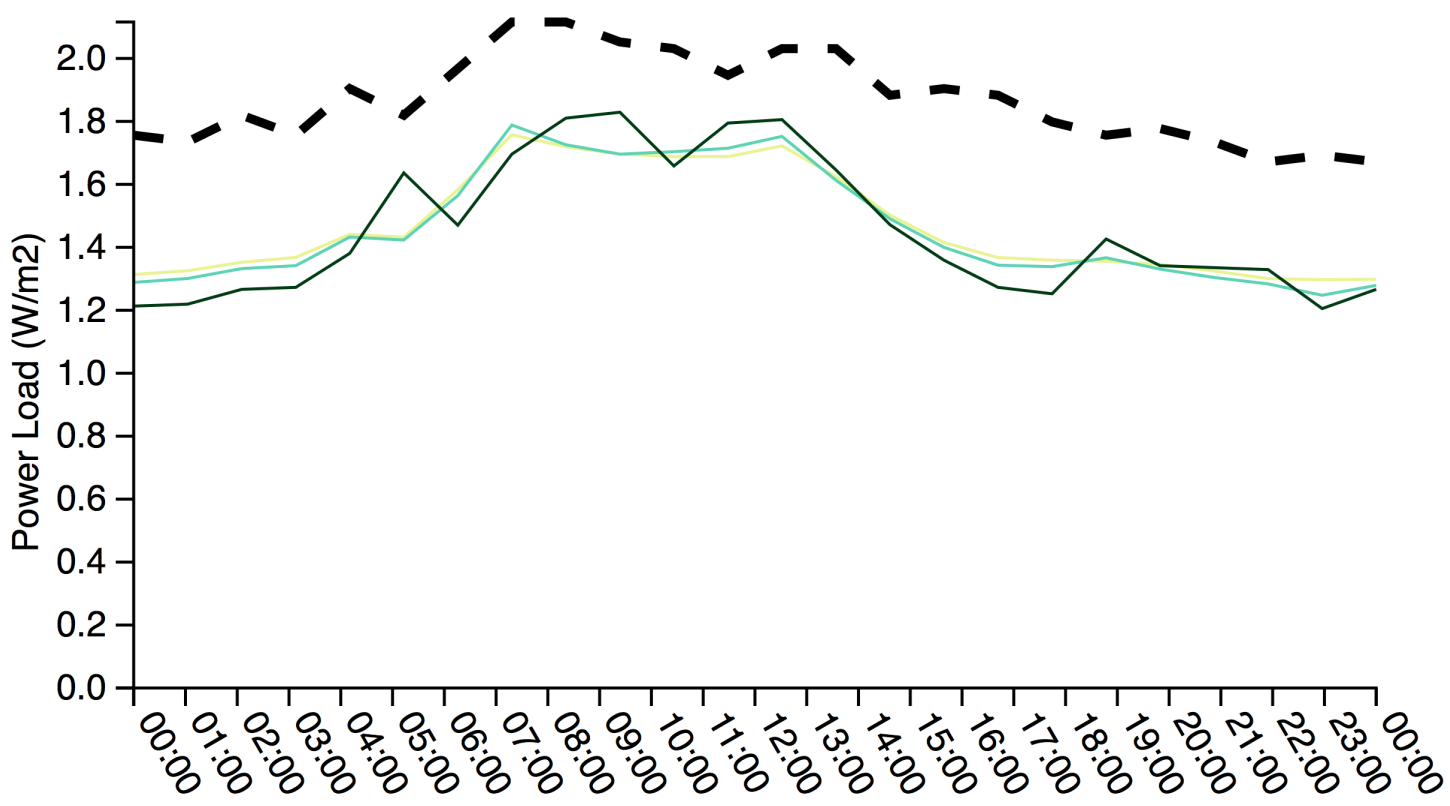

Hour

Figure 64. Mail Sorting-2: Average, Median, and Typical Weekday Receptacles Profile

Legend:

Average Median $\square$ Typical

- _ - - Predicted Load Profile

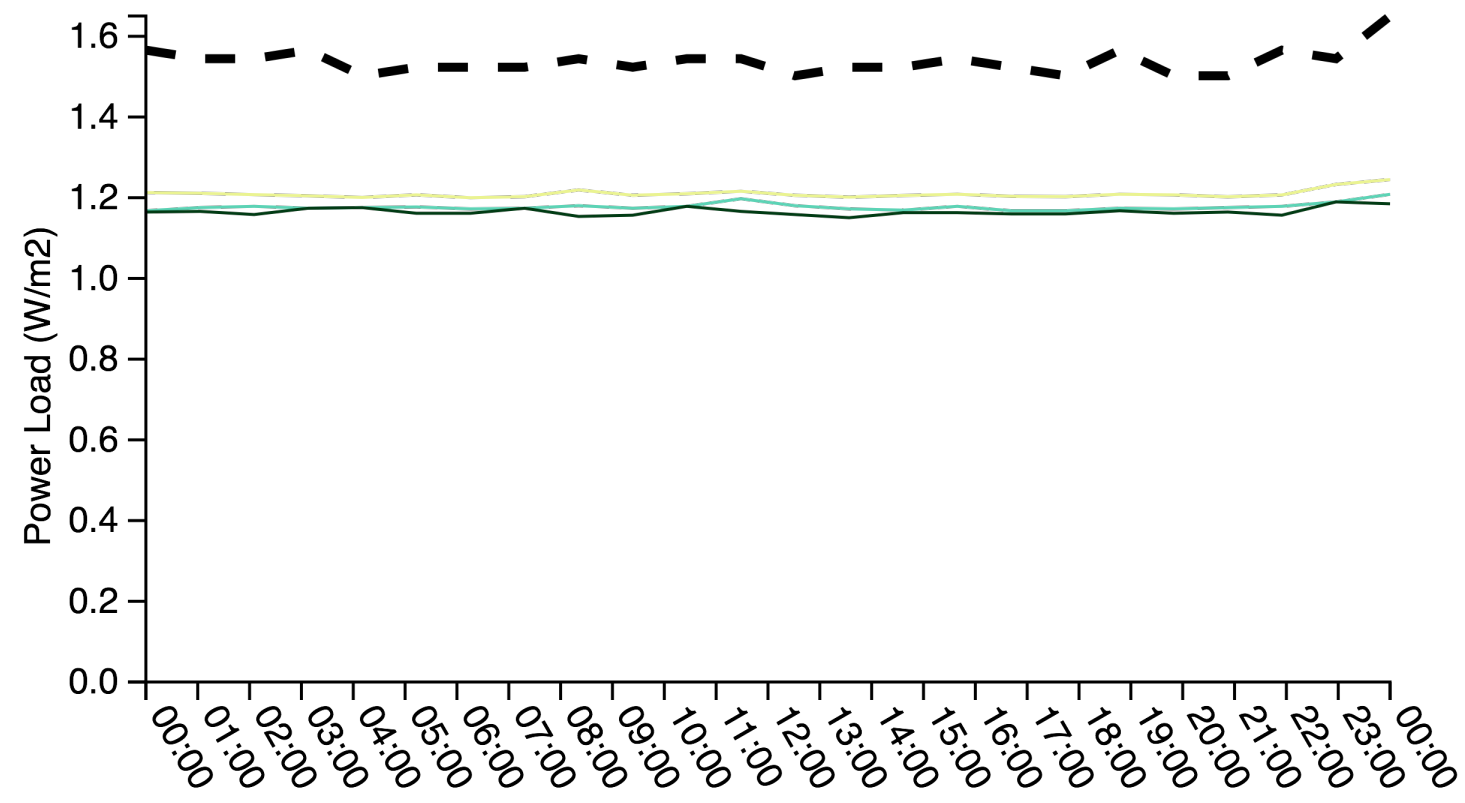

Hour

Figure 65. Mail Sorting-2: Average, Median, and Typical Weekend Day Receptacles Profile

94 
Considering Figure 66 it is possible to see variations in energy use throughout the year. One significant dip does occur in May, which does not have any obvious explanation. Over all energy use is wholly below predictions.

Legend:

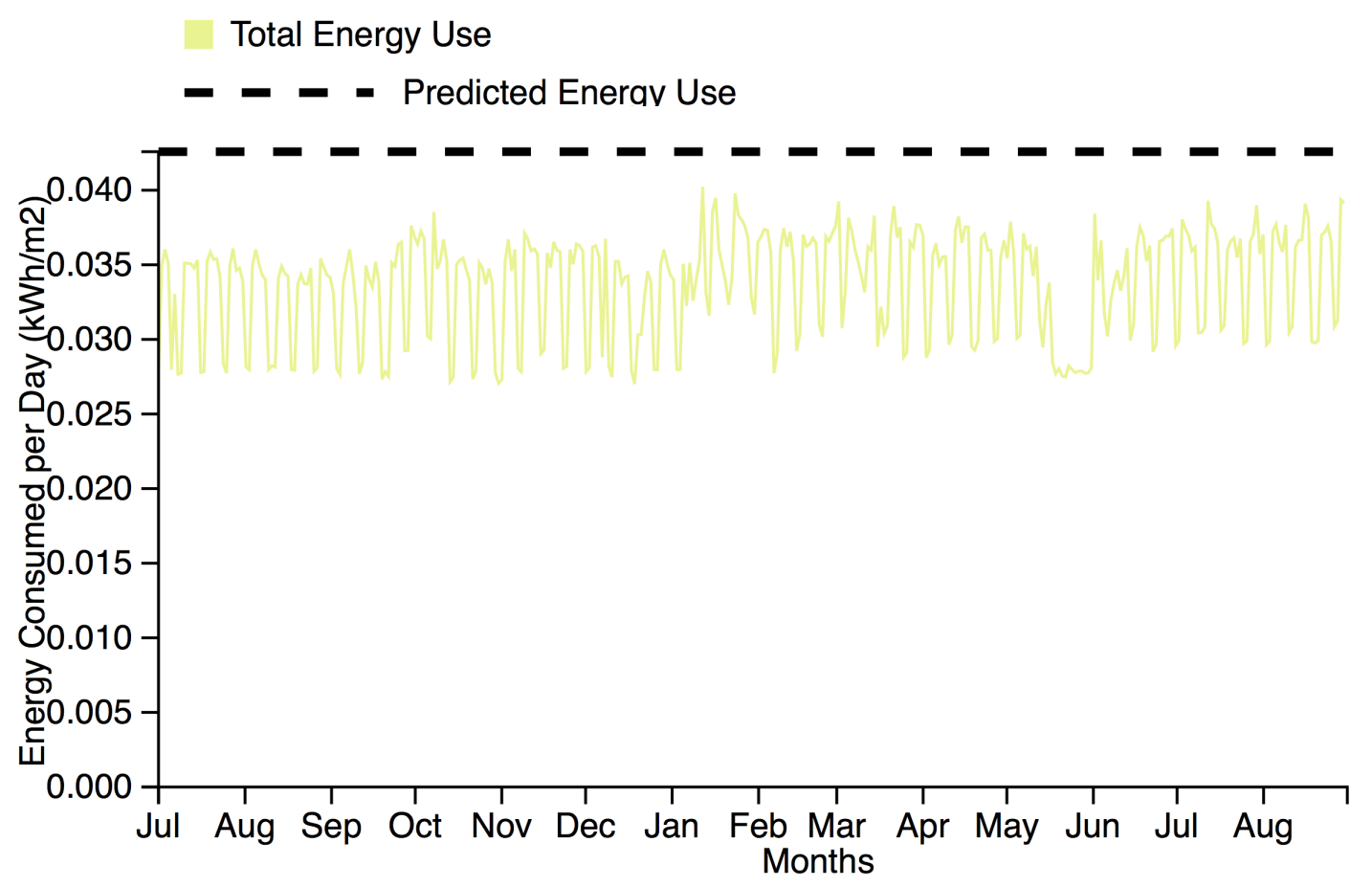

Figure 66. Mail Sorting-2: Full-year Total Daily Receptacles Energy Use Data

\subsubsection{Mail Sorting-3}

The building is a small mail sorting facility $\left(1,147 \mathrm{~m}^{2}\right)$. Energy use data was collected between October 2009 and April 2010. This building is very similar to Mail Sorting-1 in terms of design and construction.

Meter attribution documentation consisted of detailed Electrical panel shop drawings. As such it was possible to determine $1 \mathrm{~kW}$ out of $13.7 \mathrm{~kW}$ installed on the lighting panel was not lighting. Exterior lighting was metered separately. As well it was possible to determine that $14.9 \mathrm{~kW}$ out of $30.5 \mathrm{~kW}$ was not receptacles. This includes $2 \mathrm{~kW}$ of ACUs, $6.75 \mathrm{~kW}$ of heating, and $3 \mathrm{~kW}$ for a roof top unit.

Overall lighting is consuming more energy than expected due to much higher daytime peak loads, overnight, and weekend loads. Receptacle loads are consuming 
less energy than expected due to much lower daytime peak loads, as well as lower overnight and weekend base loads. Evidence of highly mixed loads limits the amount of information on receptacle energy usage that is able to be drawn from this case.

Table 28. Mail Sorting-3: Building Summary

\begin{tabular}{cc}
\hline Location & Woodstock, Ontario \\
Floor Area & $1,147 \mathrm{~m} 2$ \\
Submetering Categories & $\begin{array}{c}\text { Lighting, Receptacles, } \\
\text { Exterior Lighting, } \\
\text { Heating, Fans, DHW, } \\
\text { Misc }\end{array}$ \\
$\begin{array}{c}\text { Reporting Period } \\
\text { Data Availability }\end{array}$ & $\begin{array}{c}\text { Oct } 2009-\text { Sep } 2010 \\
\text { Oct 2009 - May 2010 } \\
\text { Detering Attribution }\end{array}$ \\
\hline $\begin{array}{c}\text { Lighting: } \\
\text { Installed LPD }\end{array}$ & $10.9 \mathrm{~W} / \mathrm{m}^{2}$ \\
\hline $\begin{array}{c}\text { Receptacles: } \\
\text { Installed Receptacles PD }\end{array}$ & $6.4 \mathrm{~W} / \mathrm{m}^{2}$ \\
\hline
\end{tabular}

Table 29 lists the key values for predicted and actual energy use of the building. Fairly substantial differences are visible between the various values.

Table 29. Mail Sorting-3: Predicted and Actual Energy Use

\begin{tabular}{|c|c|c|c|}
\hline & $\begin{array}{c}\text { Uncalibrated- } \\
\text { Predicted } \\
\left(\mathbf{k W h} / \mathbf{m}^{2}\right)\end{array}$ & $\begin{array}{l}\text { Calibrated- } \\
\text { Predicted } \\
\left(\mathrm{kWh} / \mathrm{m}^{2}\right)\end{array}$ & $\begin{array}{c}\text { Actual } \\
\left(\mathrm{kWh} / \mathrm{m}^{2}\right)\end{array}$ \\
\hline Total Energy Use & 146.4 & 206.9 & 232.8 \\
\hline $\begin{array}{c}\text { Total Electricity } \\
\text { Energy Use }\end{array}$ & 75.3 & 107.1 & 128.8 \\
\hline Total Natural Gas & 71.1 & 98.2 & 103.4 \\
\hline Heating & 74.1 & 52.3 & 101.3 \\
\hline Cooling & 3.1 & 6.3 & - \\
\hline Lighting & 25.6 & 32.6 & 61.8 \\
\hline Receptacles & 22.3 & 22.3 & 18.0 \\
\hline
\end{tabular}




\section{Lighting}

Table 30. Mail Sorting-3: Building Lighting Summary

\begin{tabular}{cccc}
\hline $\begin{array}{c}\text { As Modeled: } \\
\text { Installed LPD } \\
\left(\mathbf{W} / \mathbf{m}^{2}\right)\end{array}$ & $\begin{array}{c}\text { MNP Energy Use } \\
\left(\mathbf{W} / \mathbf{m}^{2}\right)\end{array}$ & $\begin{array}{c}\text { MNB Energy Use } \\
\left(\mathbf{W} / \mathbf{m}^{2}\right)\end{array}$ & $\begin{array}{c}\text { Base as \% } \\
\text { of Peak }\end{array}$ \\
\hline 10.9 & 9.4 & 0.5 & $6 \%$ \\
\hline $\begin{array}{c}\text { As Measured: } \\
\text { Installed LPD } \\
\left(\mathbf{W} / \mathbf{m}^{2}\right)\end{array}$ & $\begin{array}{c}\text { MNP Energy Use } \\
\left(\mathbf{W} / \mathbf{m}^{2}\right)\end{array}$ & $\begin{array}{c}\text { MNB Energy Use } \\
\left(\mathbf{W} / \mathbf{m}^{2}\right)\end{array}$ & $\begin{array}{c}\text { Base as } \% \\
\text { of Peak }\end{array}$ \\
\hline- & 10.9 & 2.9 & $26 \%$ \\
\hline
\end{tabular}

Table 30 summarizes the median near peak (MNP) lighting loads and median near base (MNB) lighting loads as modeled and as measured. Comparison between the lighting energy use profile and lighting schedules show an under-predicted nighttime and weekend base-load as well as an under-predicted daytime peak load (Figure 67 and Figure 68). Calculated with MNP and MNB values $\left(10.9 \mathrm{~W} / \mathrm{m}^{2}\right.$ and $2.9 \mathrm{~W} / \mathrm{m}^{2}$ respectively) the off hours baseload is approximately $25 \%$ of the peak load, as opposed to the estimated 6\%. This indicates higher levels of off-peak energy use than expected, especially consider a higher peak load than expected. A MNP load of $10.9 \mathrm{~W} / \mathrm{m}^{2}$ is $116 \%$ of estimated peak load of approximately $9.4 \mathrm{~W} / \mathrm{m}^{2}$. Figure 67 shows fairly irregular patterns of energy use for lighting, it is unclear if this is due to occupancy or management issues.

A higher than expected peak weekday load of $11 \mathrm{~W} / \mathrm{m}^{2}$ is difficult to explain. This value surpasses the installed capacity of the system by $1 \mathrm{~W} / \mathrm{m}^{2}$. A large portion of this energy use may simply be a result of more intense use of the lighting system. However it is likely that the metering setup must have included some lighting use not included in the model as was seen in Mail Sorting-1. Documentation shows us that the system includes $1.4 \mathrm{~kW}$ of nightlights, $0.25 \mathrm{~kW}$ of exit lights, $1 \mathrm{~kW}$ of battery, and $0.15 \mathrm{~kW}$ of dock lights. The $1.4 \mathrm{~kW}\left(1.2 \mathrm{~W} / \mathrm{m}^{2}\right)$ of night lights helps to explain the additional energy use observed during nighttime periods, however the load is not large enough to explain all the difference. Therefore building management practices must also play a role in the additional energy use. Site visits 
during the commissioning process, subsequent to project completion, indicated furniture and equipment within the space may be blocking the proper functioning of the sensors, however this would not contribute to lighting energy use exceeding the installed capacity.

Legend:

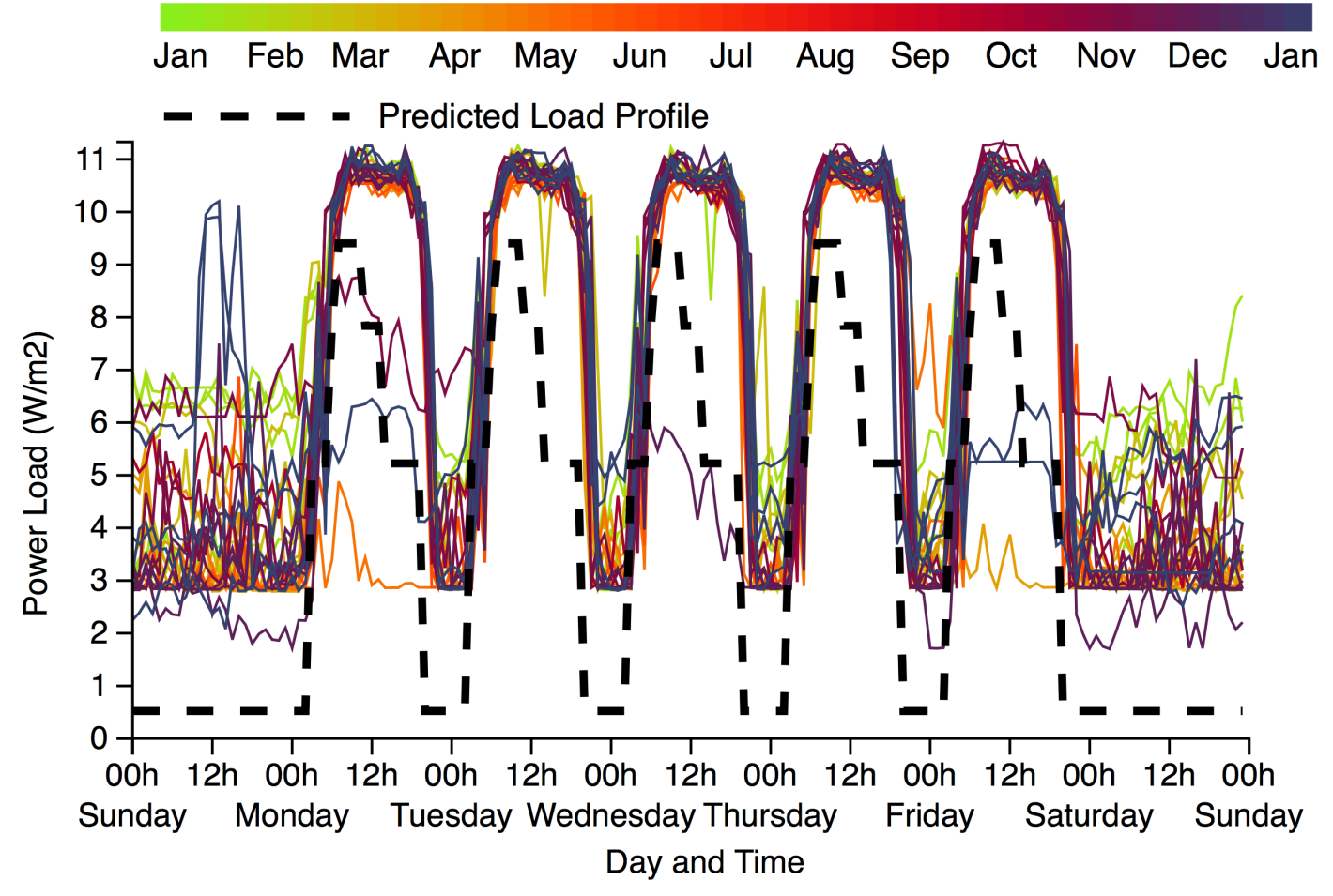

Figure 67. Mail Sorting-3: Full-year 60-Minute Interval Lighting Energy Use Data

Average, median and typical lighting loads are shown for weeks, weekdays and weekend, respectively, in Figure 68, Figure 69 and Figure 70. The values calculated for the MNP and MNB are more clearly represented in these figures. In Figure 69 the weekday morning ramp up appears to begin when expected. However the daytime peak continues much longer and higher than expected, only to ramp down faster around 22:00. In regards to the weekend load profile (Figure 70) very little meaningful variation occurs through out the day. 
Legend:

Average Median $\square$ Typical

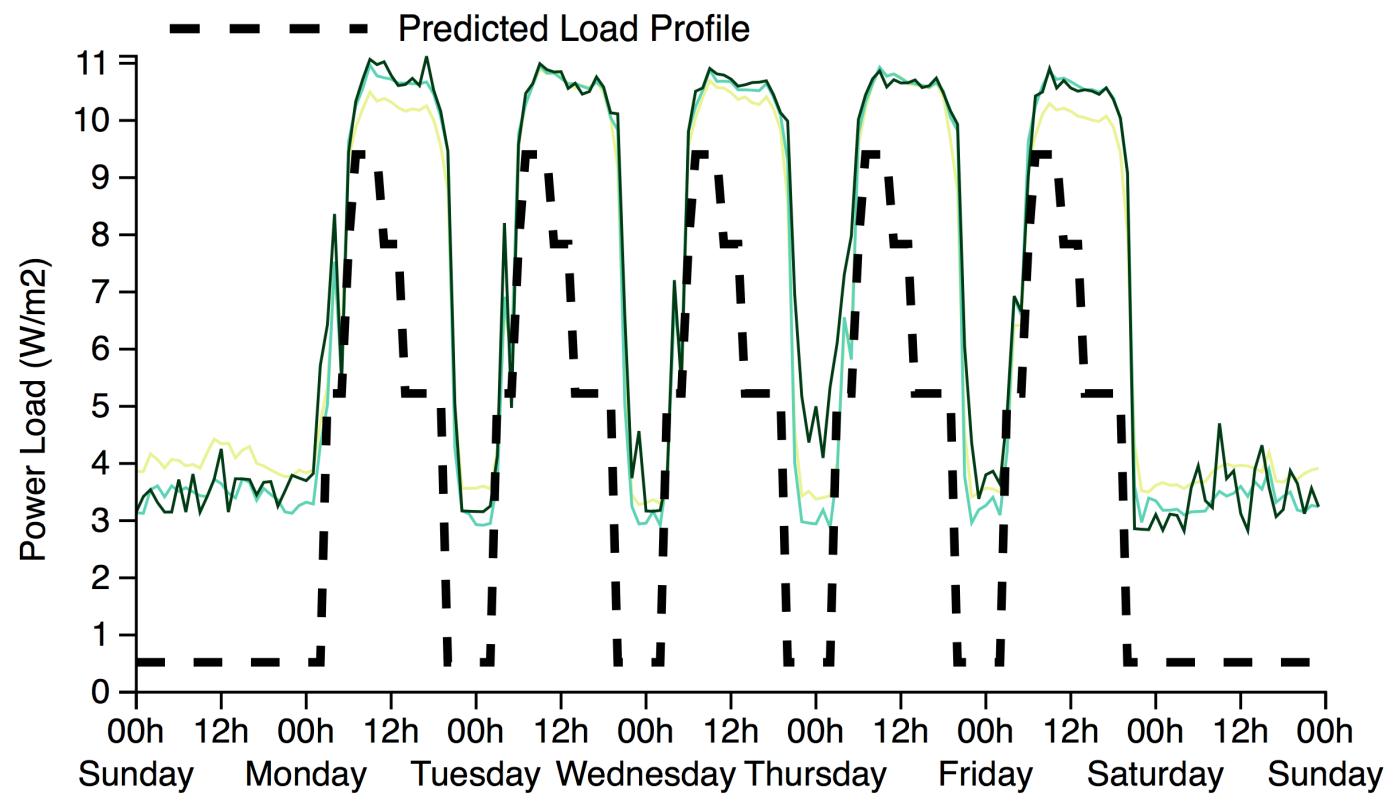

Day and Time

Figure 68. Mail Sorting-3: Average, Median, and Typical Weekly Profiles for Lighting Energy Use Legend:

Average Median $\square$ Typical

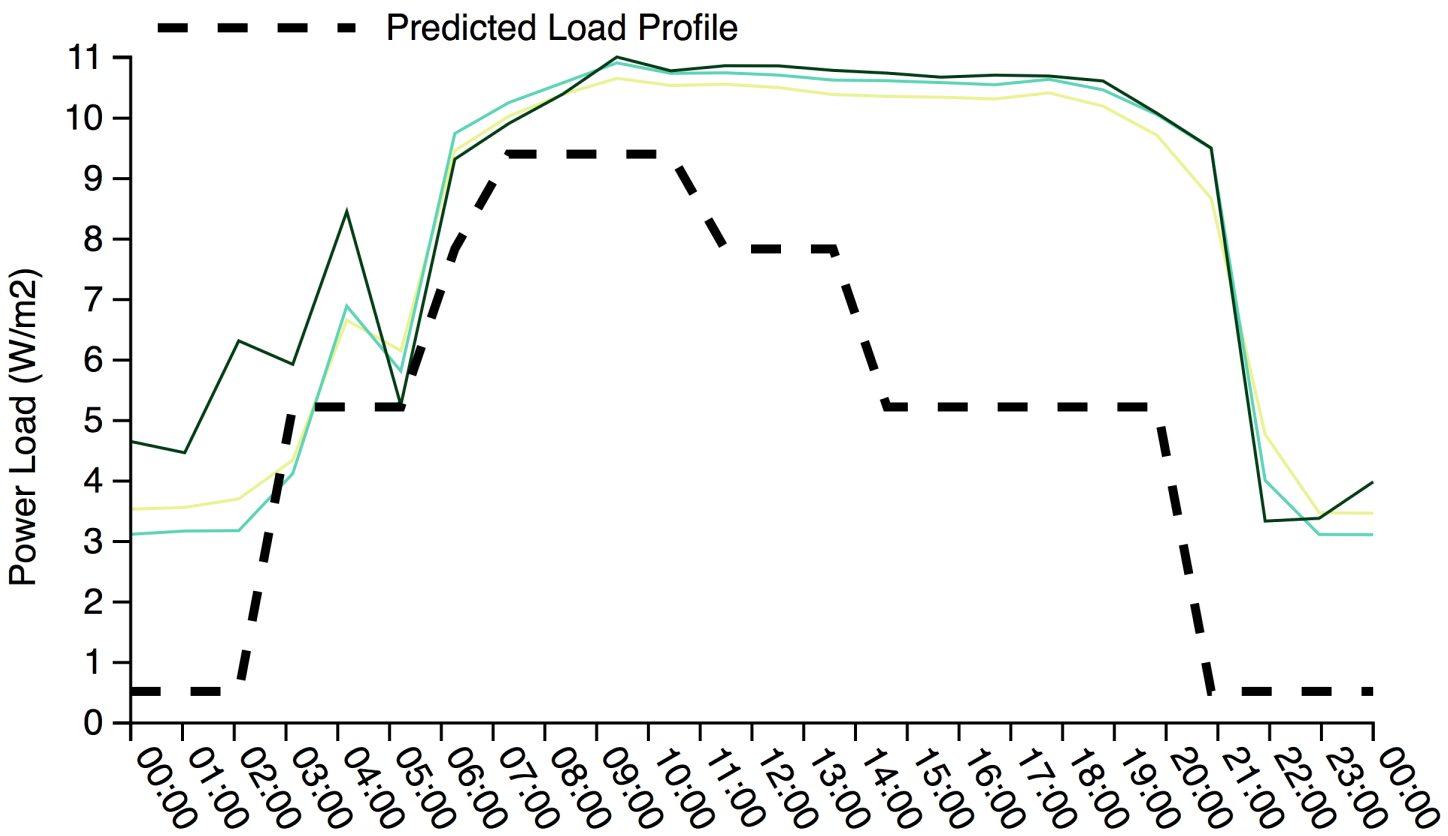

Hour

Figure 69. Mail Sorting-3: Average, Median, and Typical Weekday Lighting Profile

99 
Legend:

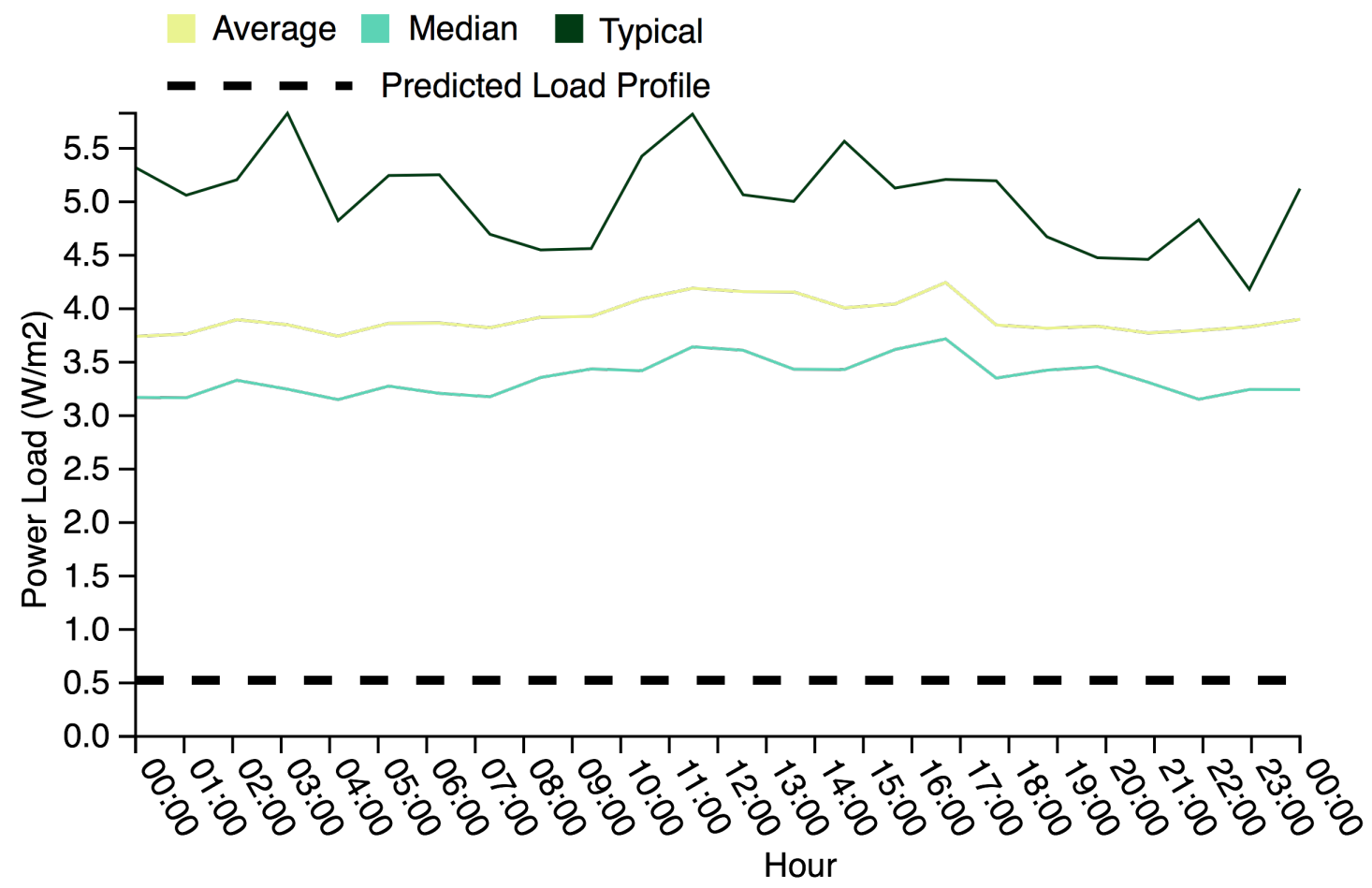

Figure 70. Mail Sorting-3: Average, Median, and Typical Weekend Day Lighting Profile

Legend:

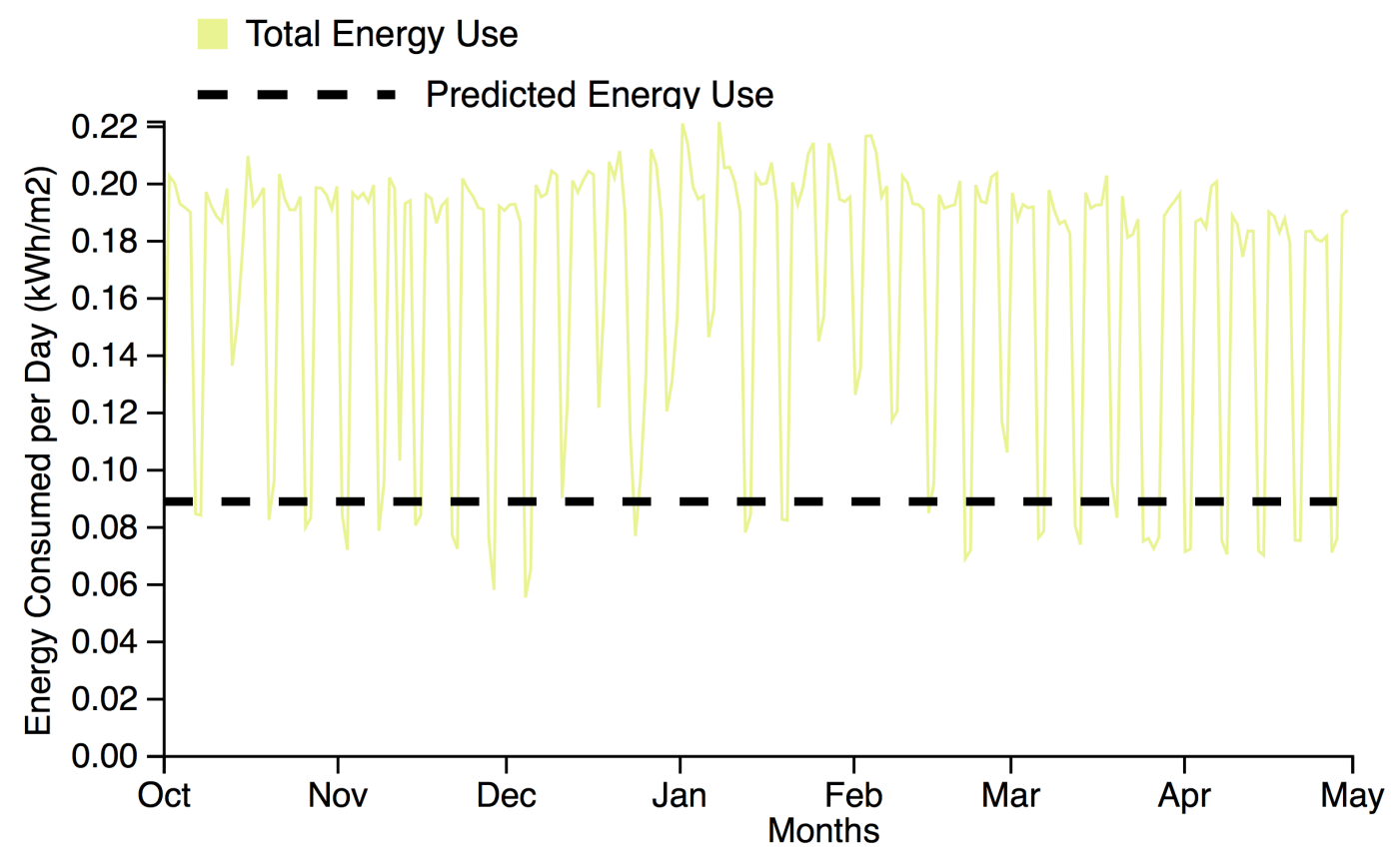

Figure 71. Mail Sorting-3: Full-year Total Daily Lighting Energy Use Data 
Considering Figure 71 it is possible to see the variation of the energy load profile throughout the year. Winter months appear to have relatively larger magnitudes of energy use. The significant trough in energy use occurring in the first several months of operation is likely due to management issues. Overall building's energy use remains above expected.

Figure 72 shows a regression analysis correlating seasonal changes in lighting energy use with bright sunshine availability. The analysis appears to demonstrate a fairly strong negative correlation $\left(r^{2}=0.42\right)$ between lighting energy use and daylight hours for this building. This indicates that on average light energy consumption does decrease as daylight availability increases. However removing the single outlier reduces the strength of the regression to $r^{2}=0.14$, and thus the correlation is actually much weaker than it would at first appear.

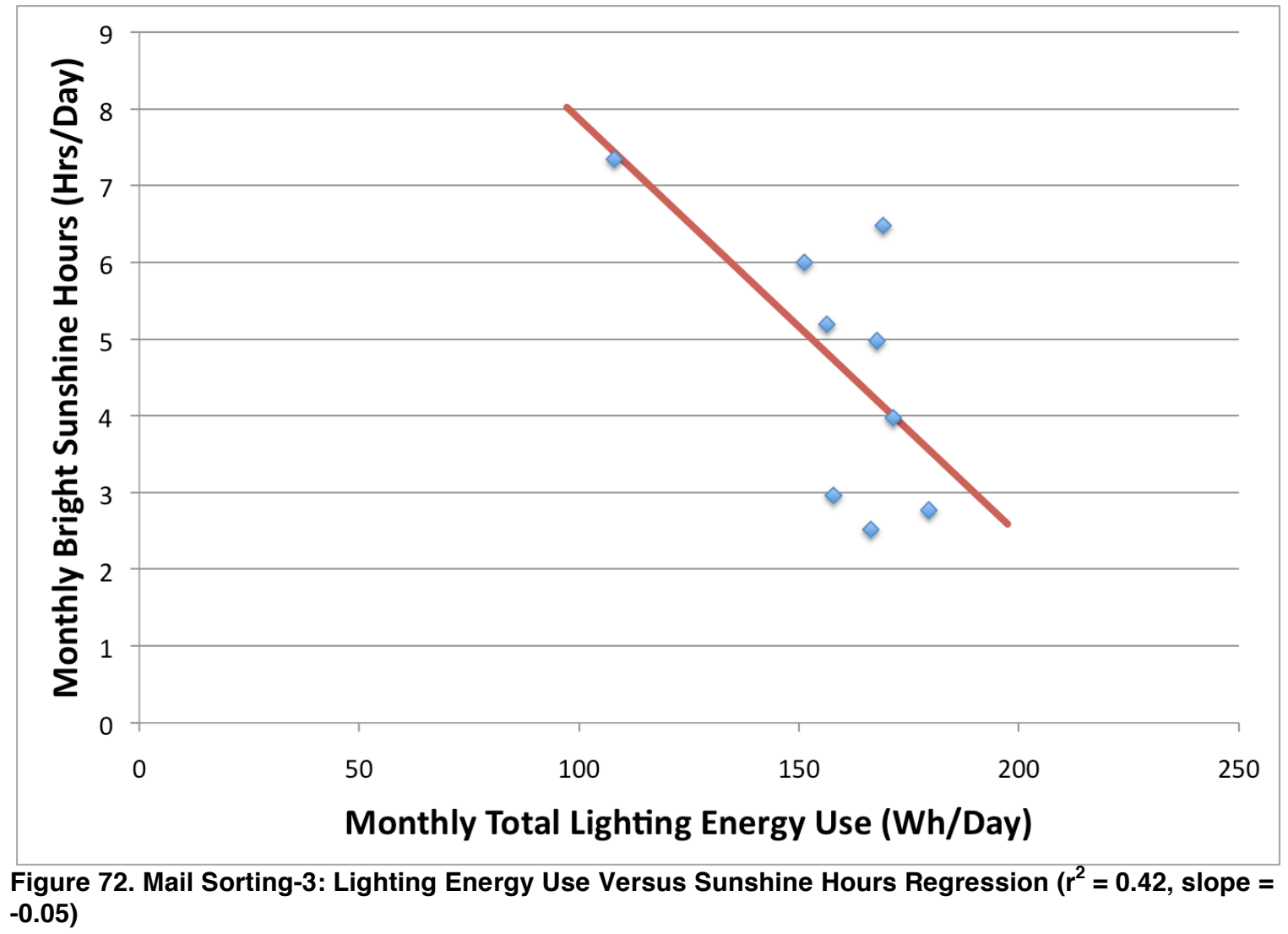


Receptacles

Table 31. Mail Sorting-3: Building Receptacles Summary

\begin{tabular}{cccc}
\hline $\begin{array}{c}\text { As Modeled: } \\
\text { Installed } \\
\begin{array}{c}\text { Receptacles PD } \\
\left(\mathbf{W} / \mathbf{m}^{2}\right)\end{array}\end{array}$ & $\begin{array}{c}\text { Median Near } \\
\text { Peak Energy Use } \\
\left(\mathbf{W} / \mathbf{m}^{2}\right)\end{array}$ & $\begin{array}{c}\text { Median Near } \\
\text { Base Energy Use } \\
\left(\mathbf{W} / \mathbf{m}^{2}\right)\end{array}$ & $\begin{array}{c}\text { Base as } \% \\
\text { of Peak }\end{array}$ \\
\hline 6.4 & 5.6 & 1.2 & $22 \%$ \\
\hline $\begin{array}{c}\text { As Measured: } \\
\text { Installed }\end{array}$ & $\begin{array}{c}\text { Median Near } \\
\text { Peak Energy Use } \\
\left(\mathbf{W} / \mathbf{m}^{2}\right)\end{array}$ & $\begin{array}{c}\text { Median Near } \\
\text { Base Energy Use } \\
\left(\mathbf{W} / \mathbf{m}^{2}\right)\end{array}$ & $\begin{array}{c}\text { Base as } \% \\
\text { of Peak }\end{array}$ \\
\hline $\begin{array}{c}\left.\mathbf{W} / \mathbf{m}^{2}\right) \\
-\end{array}$ & 2.5 & 1.3 & $54 \%$ \\
\hline
\end{tabular}

Table 31 summarizes the median near peak (MNP) receptacle loads and median near base (MNB) receptacle loads as modeled and as measured. Comparison between the receptacles profile and receptacles schedules show an under-predicted daytime peak load (Figure 73 and Figure 74).

Legend:

Jan Feb Mar Apr May Jun Jul Aug Sep Oct Nov Dec Jan

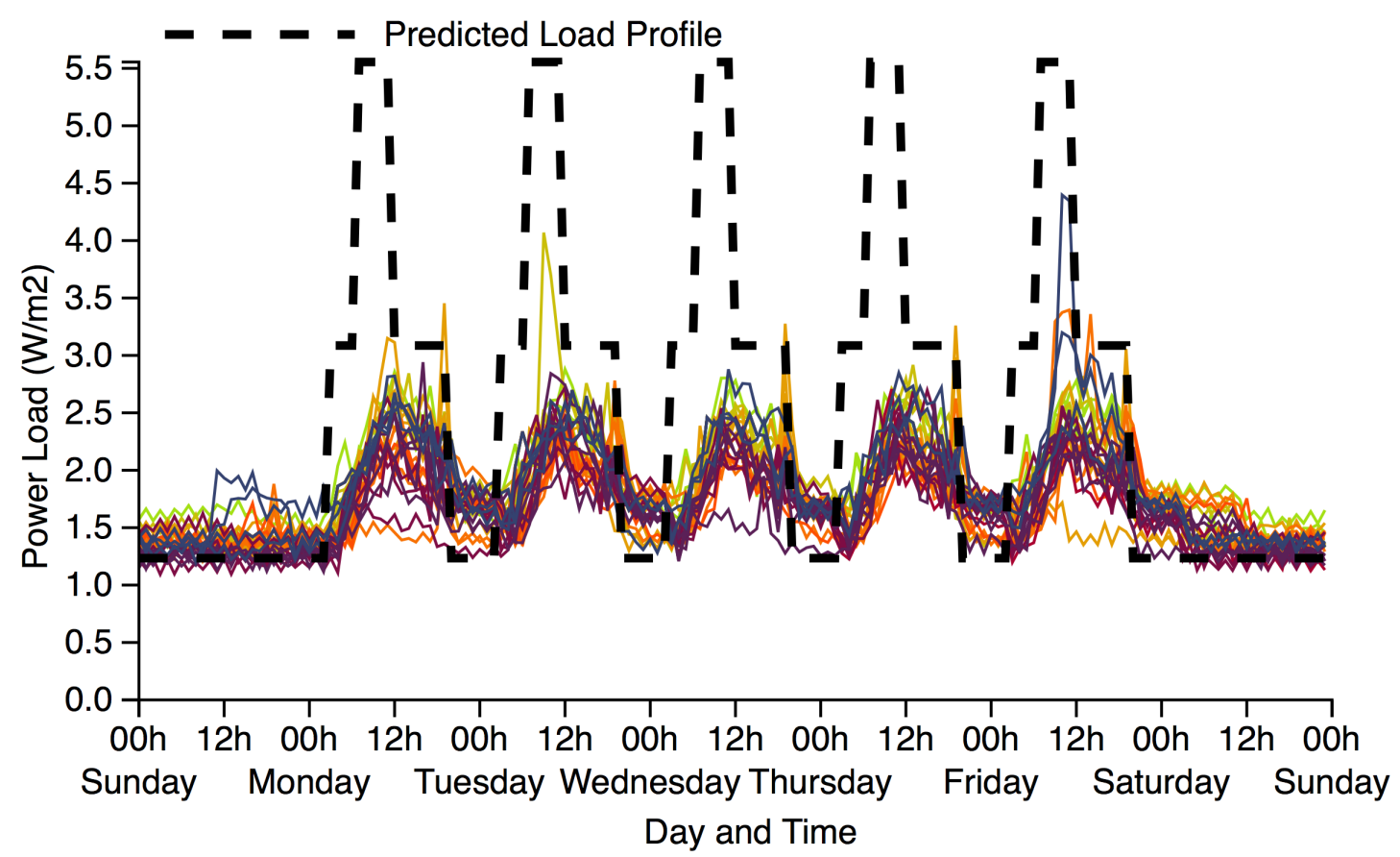

Figure 73. Mail Sorting-3: Full-year 60-Minute Interval Receptacles Energy Use Data 
Calculated with MNP and MNB values $\left(2.5 \mathrm{~W} / \mathrm{m}^{2}\right.$ and $1.3 \mathrm{~W} / \mathrm{m}^{2}$ respectively) the off-hours base load is approximately $54 \%$ of the peak load, as opposed to the estimated 22\%. In this case this does not indicate a higher than expected base load only a lower than expected peak load. A near peak load of $2.5 \mathrm{~W} / \mathrm{m}^{2}$ is $45 \%$ of estimated peak load of approximately $5.6 \mathrm{~W} / \mathrm{m}^{2}$. This indicates that at full operation that the building uses much less electricity for receptacle loads than originally expected.

Average, median and typical lighting loads are shown for weeks, weekdays and weekend, respectively, in Figure 74, Figure 75 and Figure 76. The values calculated for the MNP and MNB are more clearly represented in these figures. In Figure 75 the weekday morning ramp up and afternoon ramp down appear to begin when expected, however the daytime peak is much lower. In regards to the weekend load profile (Figure 76) very little meaningful variation occurs through out the day. However the very sharp localized peaks indicates equipment cycling on and off, perhaps a reflection of some of the non-receptacle loads included on the meter.

Legend:

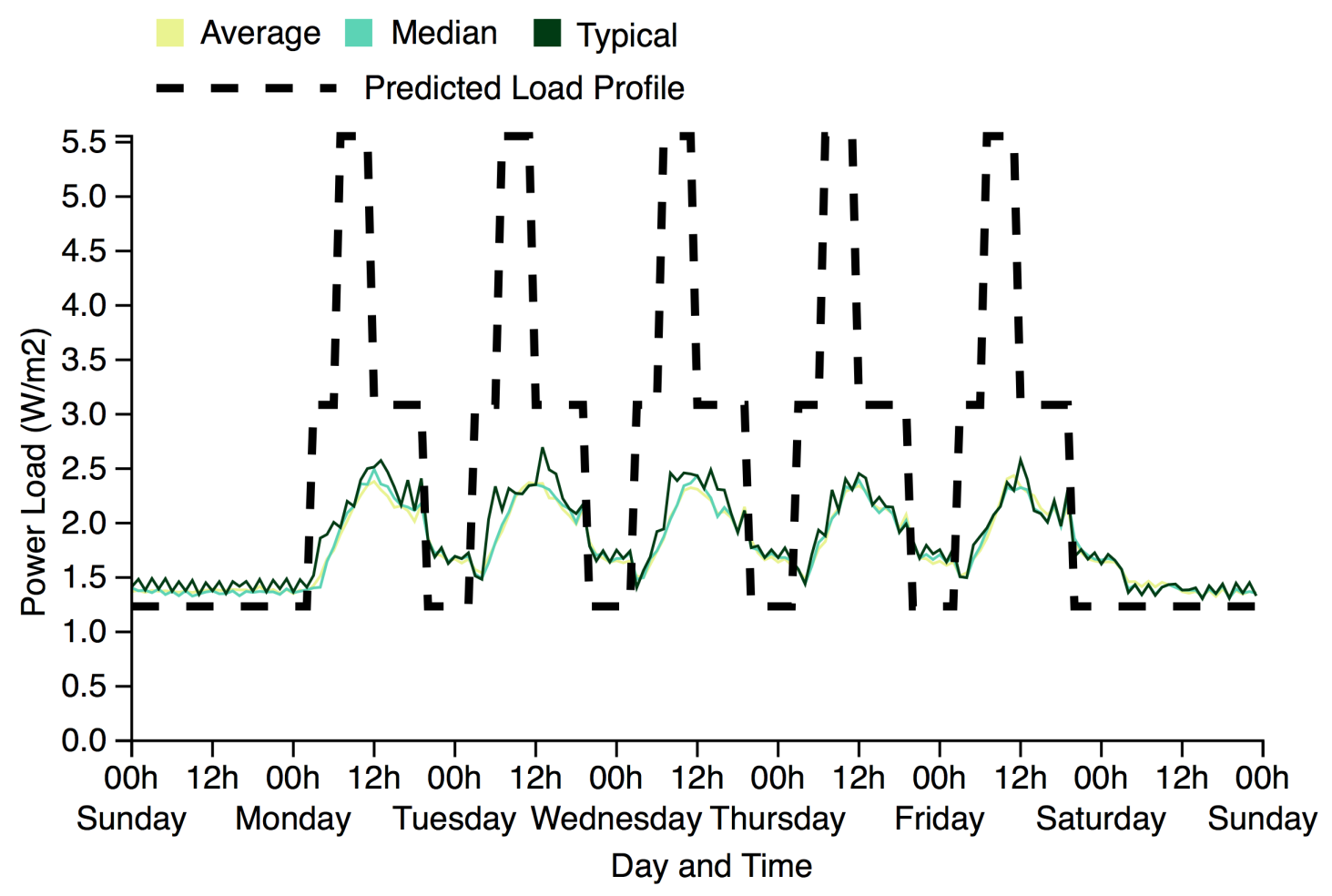

Figure 74. Mail Sorting-3: Average, Median, and Typical Weekly Profiles for Receptacles Energy Use 
Legend:

Average Median $\square$ Typical

- - - Predicted Load Profile

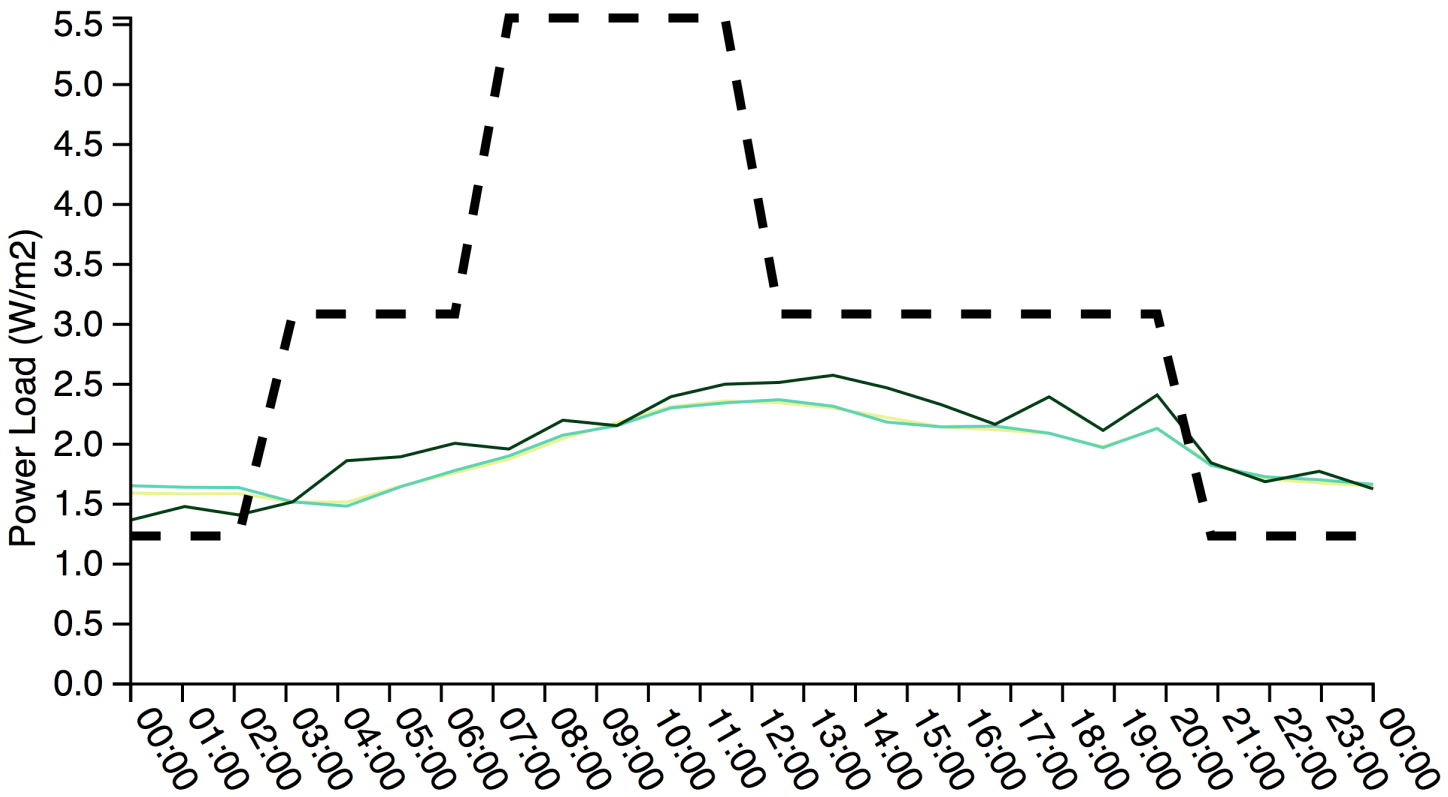

Hour

Figure 75. Mail Sorting-3: Average, Median, and Typical Weekday Receptacles Profile

Legend:

Average Median $\square$ Typical

- - a Predicted Load Profile

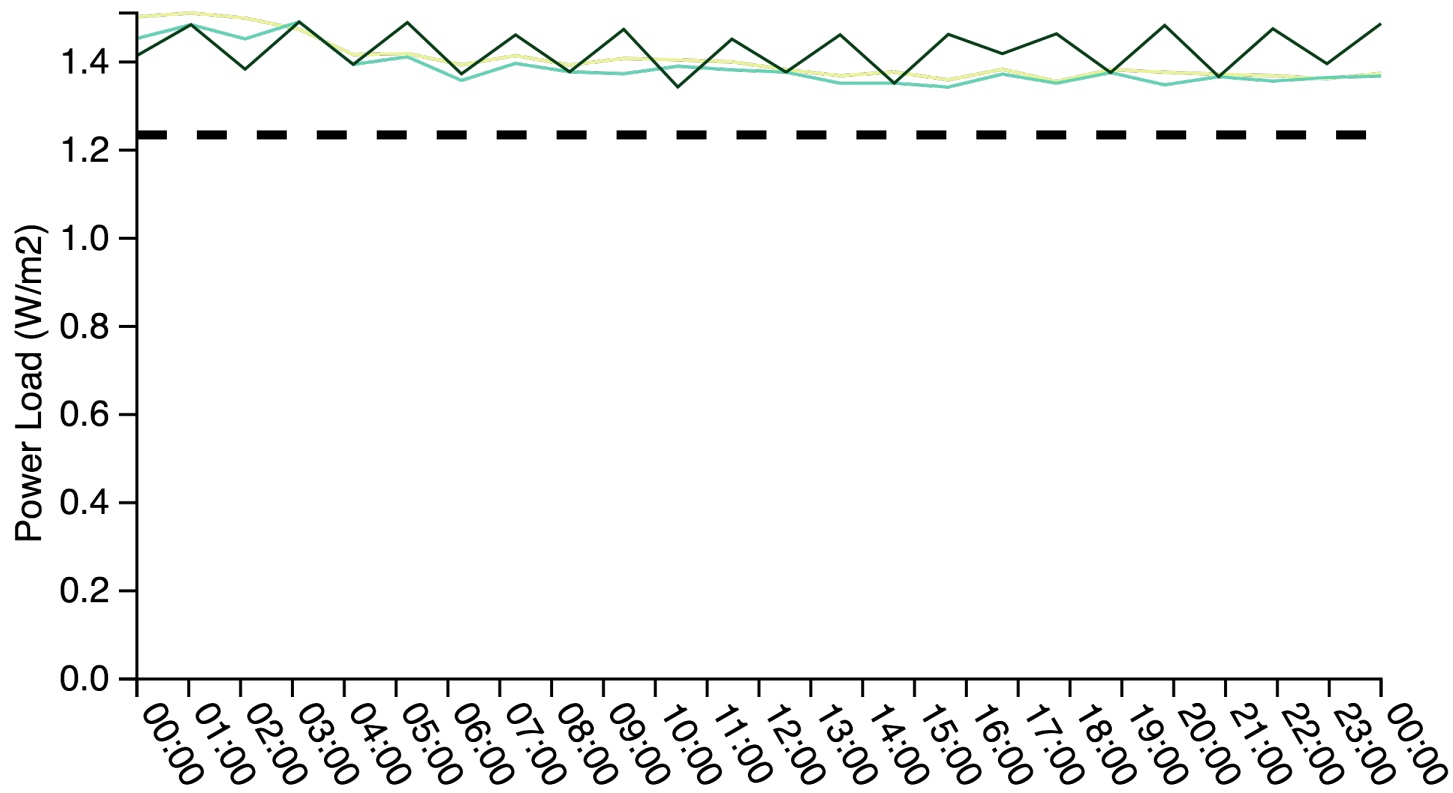

Hour

Figure 76. Mail Sorting-3: Average, Median, and Typical Weekend Day Receptacles Profile

104 
Considering Figure 77 it is possible to see variations in energy use throughout the year. Energy use appears relatively stable, this is supported by Figure 73, with a slight increase over the winter months compared to the spring and fall. A peak in December could likely be attributed to higher occupancy intensity around Christmas. As a whole energy use is consistently under-predicted due to a lower than expected peak load.

Legend:

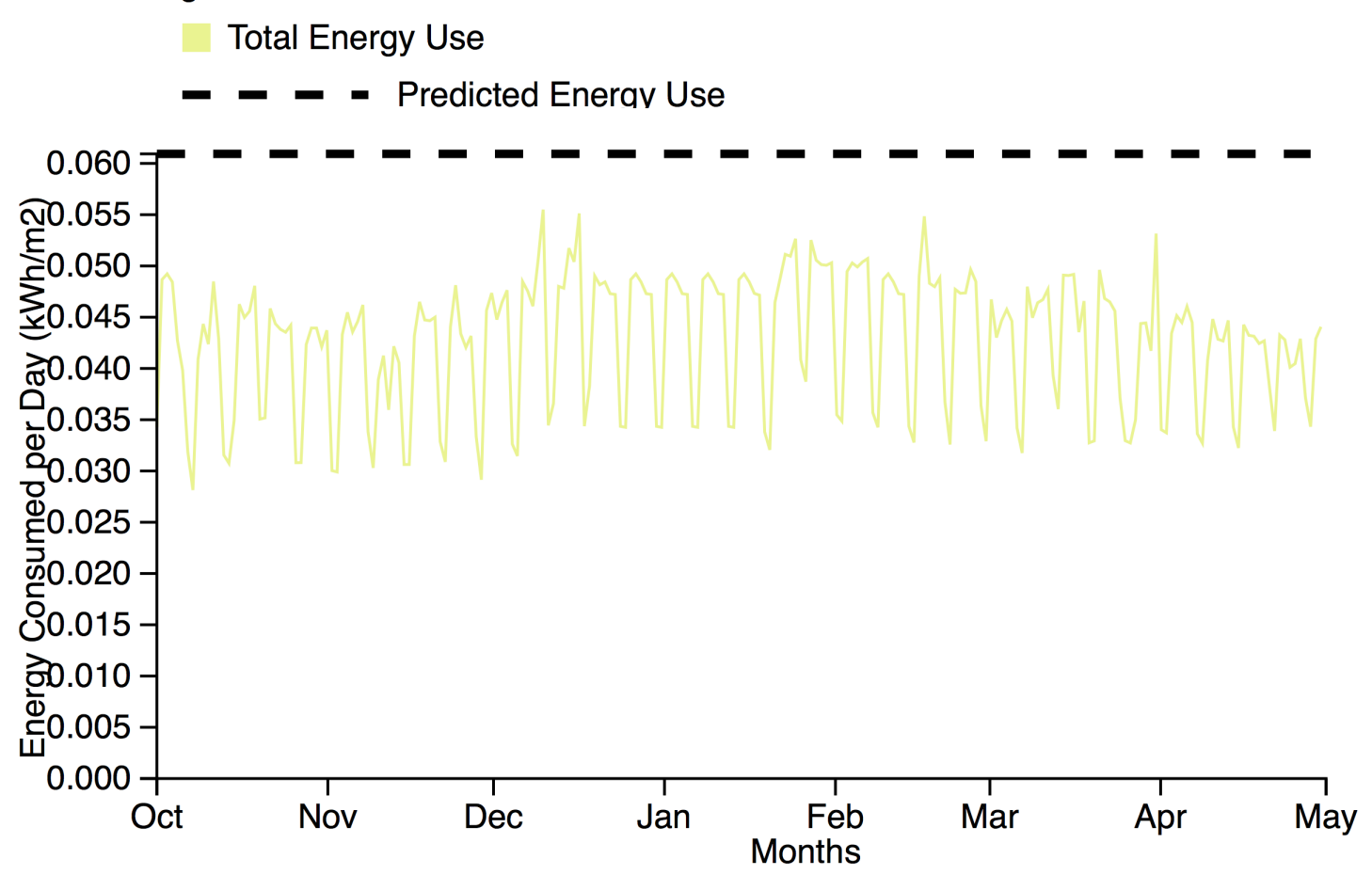

Figure 77. Mail Sorting-3: Full-year Total Daily Receptacles Energy Use Data

\subsubsection{Public Assembly-1}

The building is a small public education facility $\left(1,185 \mathrm{~m}^{2}\right)$ used to demonstrate energy and water conservation initiatives. Energy use data was collected between October 2008 and October 2009. Meter attribution documentation consisted of detailed Electrical panel shop drawings (see Appendix D). As such it was possible to determine the meter data represents exclusively interior lighting. Exterior lighting was (primarily) metered separately. As well possible it was observed that receptacles fell over two panels. Panel "P3" which was metered included $12 \mathrm{~kW}$ out of $20 \mathrm{~kW}$ that were not receptacles loads. Panel "P2", 
which was not metered, included $9 \mathrm{~kW}$ out of $23 \mathrm{~kW}$ that were not receptacle loads. It may seem incongruous for the meter to be monitoring $\sim 20 \mathrm{~kW}\left(16.8 \mathrm{~W} / \mathrm{m}^{2}\right)$ of load but only measuring a max load of around $5-7 \mathrm{~W} / \mathrm{m}^{2}$, however it is not untypical for a building to have a load factor of between $30-70 \%$. Overall lighting is consuming less energy than expected due to lower daytime peak loads, despite higher overnight loads. Extreme variation and high overnight peaks are related to space programming and occupancy issues. Overnight energy usage is certainly contributed by exterior lighting. Receptacle loads are consuming more energy than expected due to higher daytime peak loads, as well as higher overnight and weekend base loads. Evidence of highly mixed loads limits the amount of information on receptacle energy usage that is able to be drawn from this case.

Table 32. Public Assembley-1: Building Summary

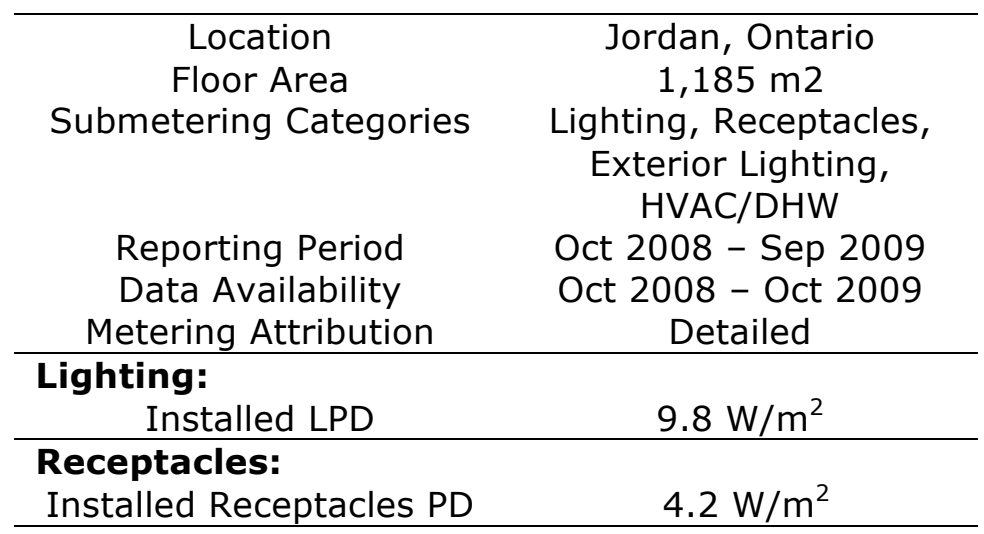

Table 33 lists the key values for predicted and actual energy use of the building. Fairly substantial differences are visible between the various values.

Table 33. Public Assembley-1: Predicted and Actual Energy Use

\begin{tabular}{|c|c|c|c|}
\hline & $\begin{array}{c}\text { Uncalibrated- } \\
\text { Predicted } \\
\left(\mathbf{k W h} / \mathbf{m}^{\mathbf{2}}\right)\end{array}$ & $\begin{array}{l}\text { Calibrated- } \\
\text { Predicted } \\
\left(\mathbf{k W h} / \mathbf{m}^{2}\right)\end{array}$ & $\begin{array}{c}\text { Actual } \\
\left(\mathrm{kWh} / \mathrm{m}^{2}\right)\end{array}$ \\
\hline Total Energy Use & 125.7 & 134.4 & 100.3 \\
\hline $\begin{array}{l}\text { Total Electricity } \\
\text { Energy Use }\end{array}$ & 125.7 & 134.4 & 100.3 \\
\hline Total Natural Gas & 0.0 & 0.0 & 0.0 \\
\hline Heating & 21.8 & 21.2 & - \\
\hline Cooling & 13.1 & 9.4 & - \\
\hline Lighting & 37.5 & 38.0 & 17.1 \\
\hline Receptacles & 9.2 & 17.0 & 18.1 \\
\hline
\end{tabular}




\section{Lighting}

Table 34. Public Assembly-1: Building Lighting Summary

\begin{tabular}{cccc}
\hline $\begin{array}{c}\text { As Modeled: } \\
\text { Installed LPD } \\
\left(\mathbf{W} / \mathbf{m}^{2}\right)\end{array}$ & $\begin{array}{c}\text { MNP Energy Use } \\
\left(\mathbf{W} / \mathbf{m}^{2}\right)\end{array}$ & $\begin{array}{c}\text { MNB Energy Use } \\
\left(\mathbf{W} / \mathbf{m}^{2}\right)\end{array}$ & $\begin{array}{c}\text { Base as \% } \\
\text { of Peak }\end{array}$ \\
\hline 9.8 & 8.7 & 0.5 & $6 \%$ \\
\hline $\begin{array}{c}\text { As Measured: } \\
\text { Installed LPD } \\
\left(\mathbf{W} / \mathbf{m}^{2}\right)\end{array}$ & $\begin{array}{c}\text { MNP Energy Use } \\
\left(\mathbf{W} / \mathbf{m}^{2}\right)\end{array}$ & $\begin{array}{c}\text { MNB Energy Use } \\
\left(\mathbf{W} / \mathbf{m}^{2}\right)\end{array}$ & $\begin{array}{c}\text { Base as \% } \\
\text { of Peak }\end{array}$ \\
\hline- & 4.5 & 0.15 & $3 \%$ \\
\hline
\end{tabular}

Table 33 summarizes the median near peak (MNP) lighting loads and median near base (MNB) lighting loads as modeled and as measured. Comparison between the lighting energy use profile and lighting schedules show an under-predicted nighttime and weekend base-load as well as an under-predicted daytime peak load (Figure 79 and Figure 80). Calculated with MNP and MNB values (4.5 W/m² and $0.15 \mathrm{~W} / \mathrm{m}^{2}$ respectively) the off-hours base load is approximately $3 \%$ of the peak load, as opposed to the estimated $6 \%$. A near peak load of $4.5 \mathrm{~W} / \mathrm{m}^{2}$ is $52 \%$ of estimated peak load of approximately $8.7 \mathrm{~W} / \mathrm{m}^{2}$. Combined these values indicate lower loads than expected, however while the base load reaches values as low as expected, there is also an overnight peak which regularly occurs that was not included in predictions.

Figure 79 shows various overnight peaks occurring through out the year (primarily summer months) that exceeds typical daytime peaks by nearly double. Discussion with the building management indicate that this additional energy use is in part due to the building's frequent hosting of wedding receptions throughout the summer months. Exterior lighting is for the most part metered separately, however documentation does show that $3.6 \mathrm{~kW}$ of exterior lighting are include on this meter or approximately $3 \mathrm{~W} / \mathrm{m}^{2}$. These additional loads will help account for the higher than expected overnight base load. 
Legend:

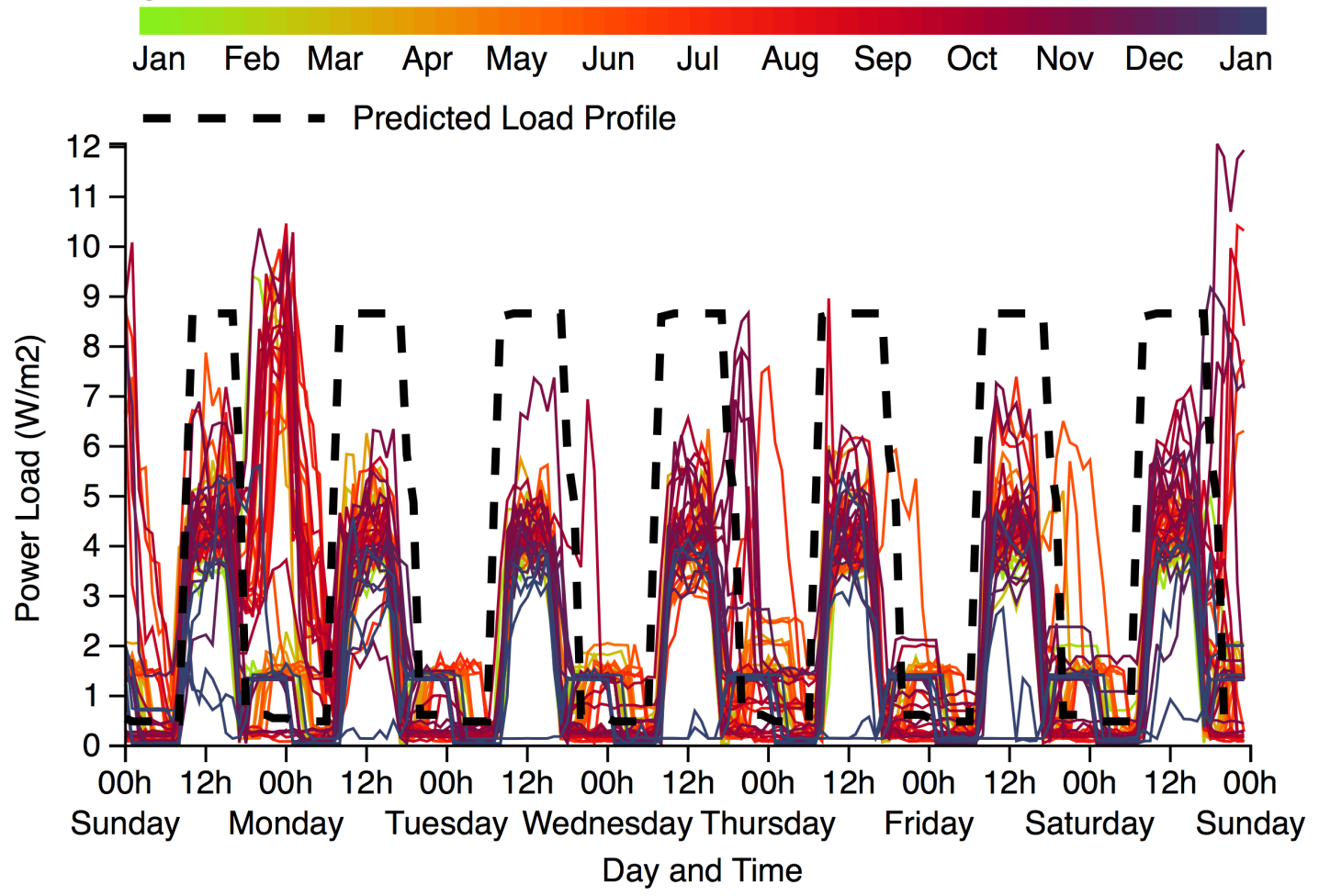

Figure 78. Public Assembly-1: Full-year 60-Minute Interval Lighting Energy Use Data

Average, median and typical receptacle loads are shown for weeks, weekdays and weekend, respectively, in Figure 79, Figure 80 and Figure 81. The values calculated for the MNP and MNB are more clearly represented in these figures. The load profile for the weekend and the weekday are seen to be very similar in all three figures. In Figure 80 and Figure 81 the morning ramp up begins around 8:00 several hours later than expected, and the afternoon ramp down begins several hours earlier than expected around 18:00, however in general the predicated and actual load profiles' general contours match. This might indicate a relatively less intense use of the building. However there is also a nighttime peak that starts around 21:00 and appears to last to around 2:00, which as mentioned might be associated with late night events. 
Legend:

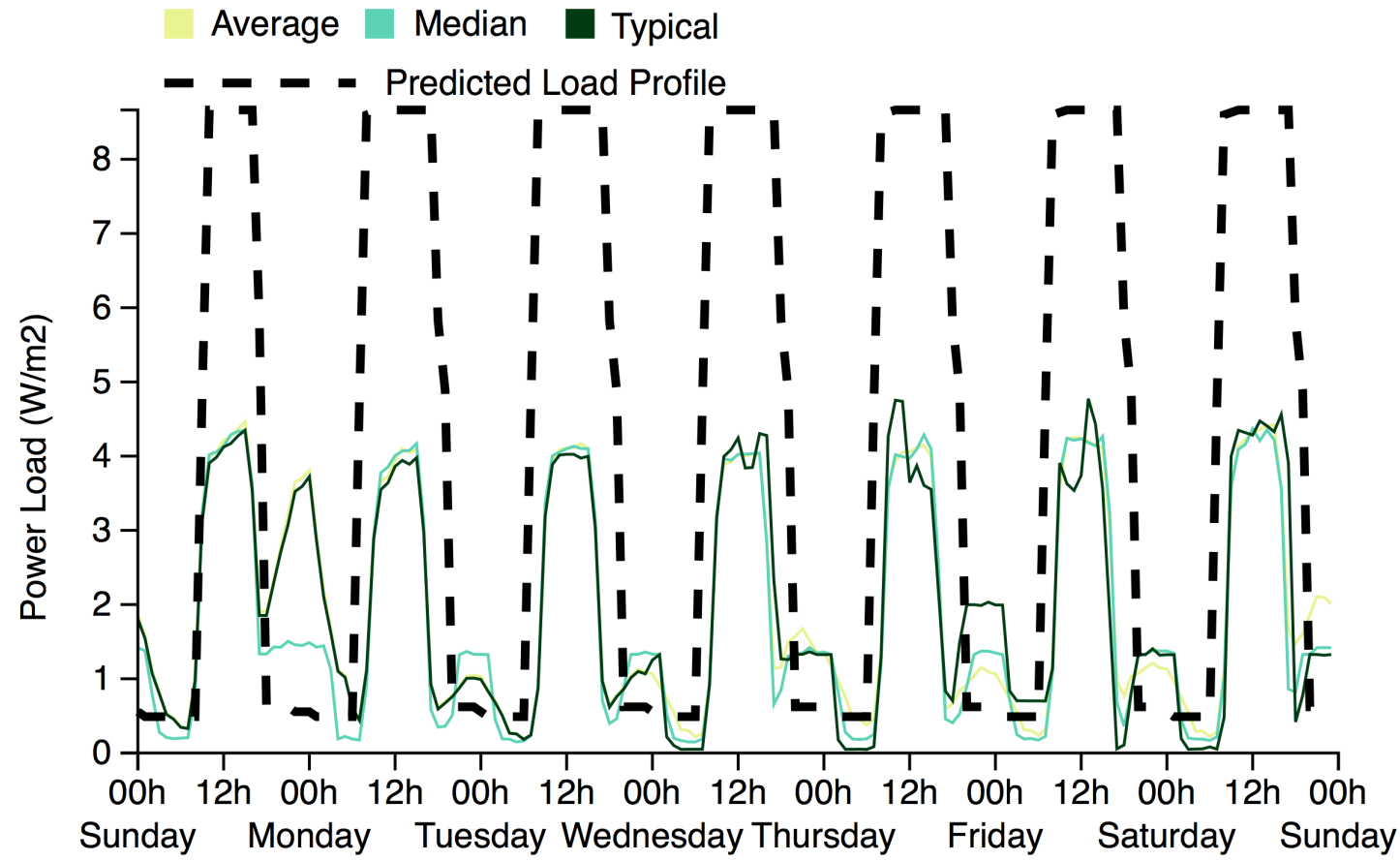

Day and Time

Figure 79. Public Assembly-1: Average, Median, and Typical Weekly Profiles for Lighting Energy Use

Legend:

Average Median $\square$ Typical

- c - Predicted Load Profile

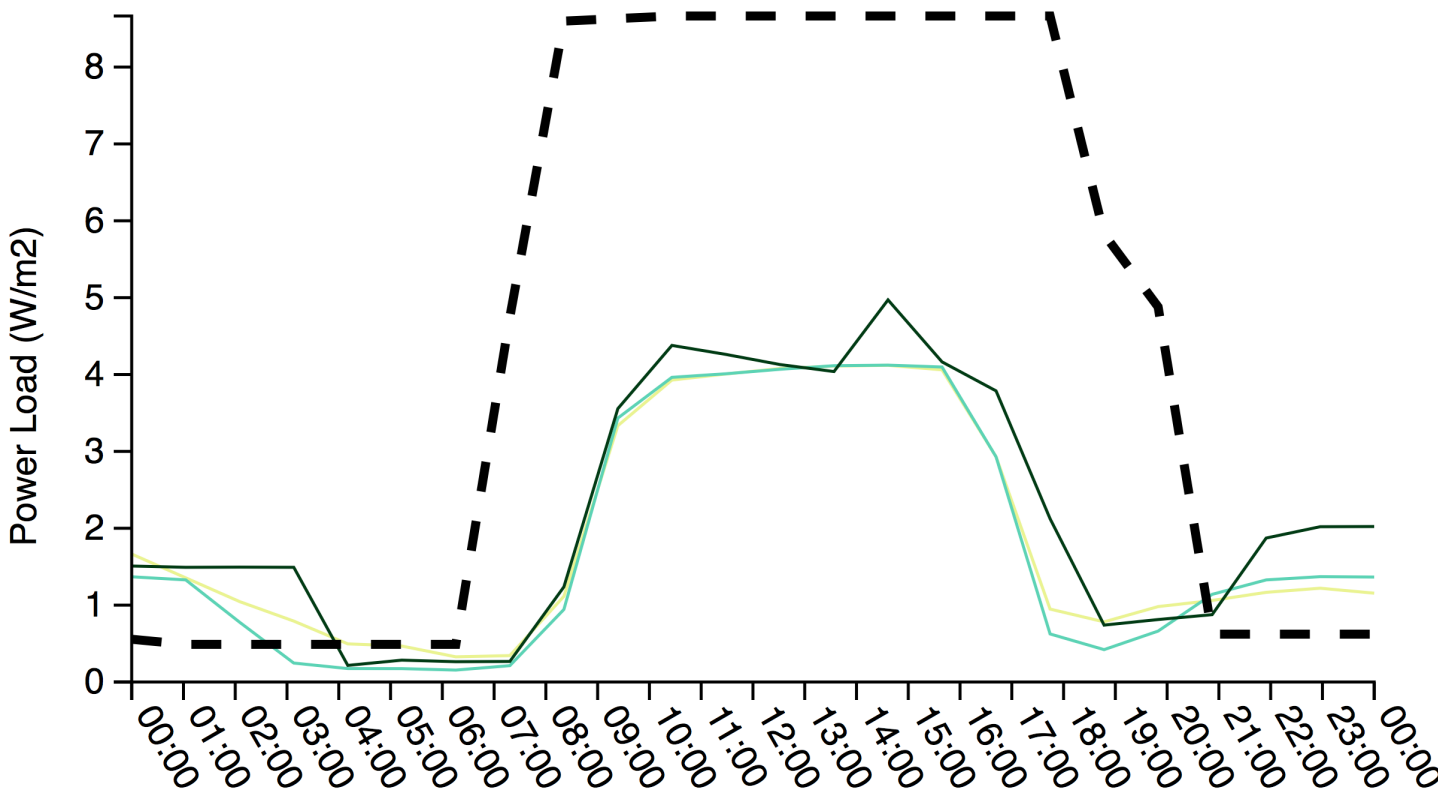

Hour

Figure 80. Public Assembley-1: Average, Median, and Typical Weekday Lighting Profile

109 


\section{Legend:}

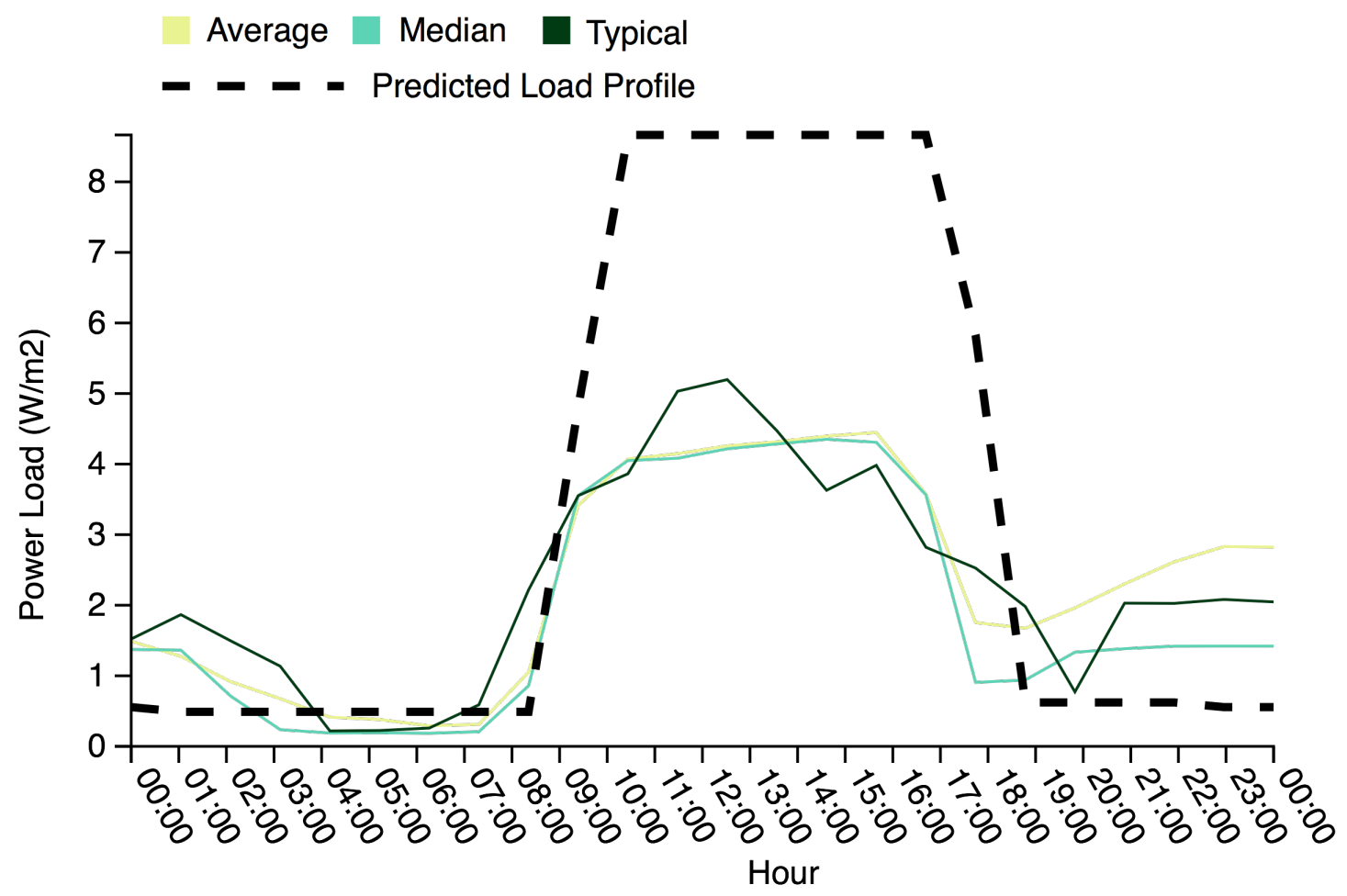

Figure 81. Public Assembley-1: Average, Median, and Typical Weekend Day Lighting Profile Legend:

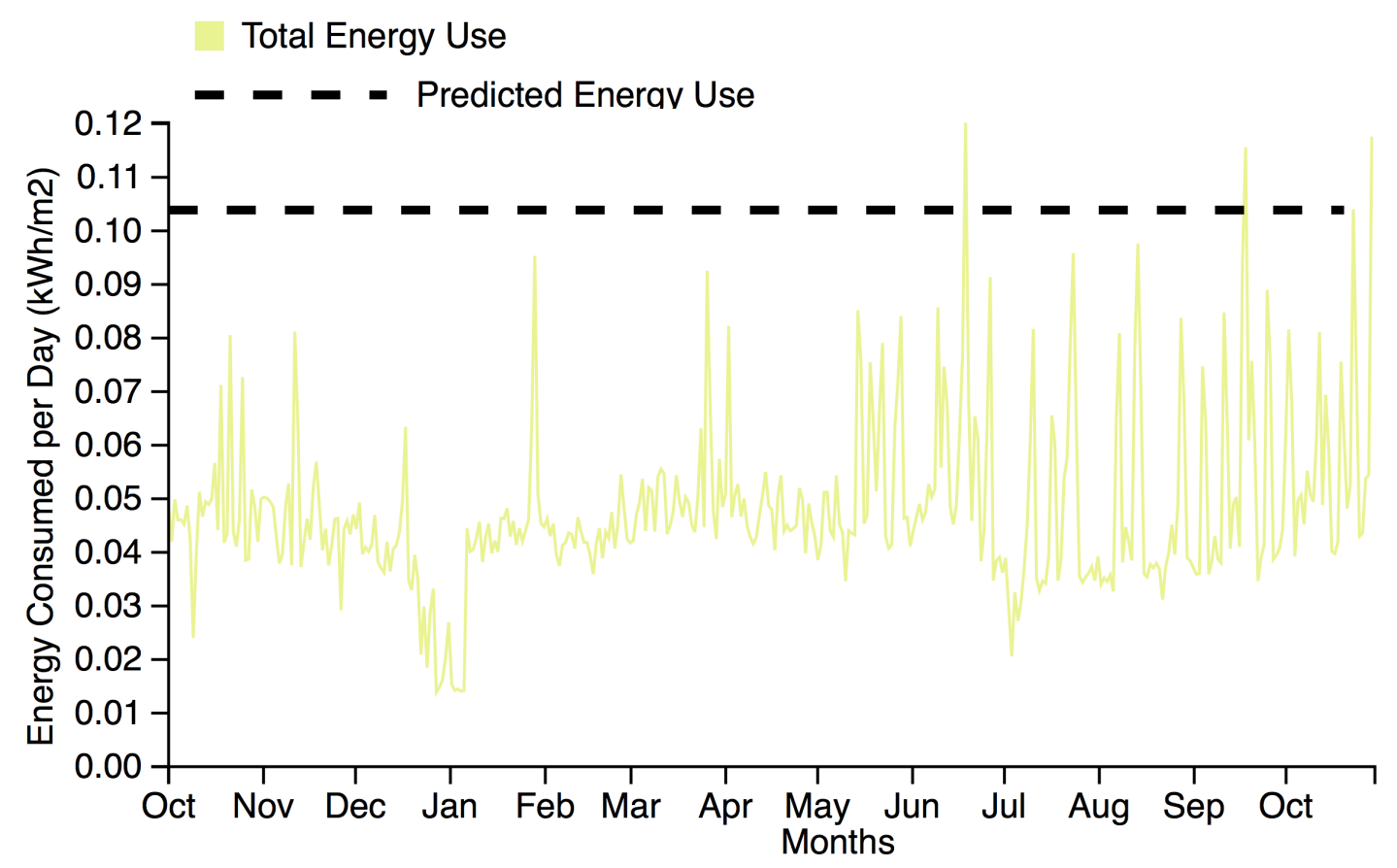

Figure 82. Public Assembly-1: Full-year Total Daily Lighting Energy Use Data 
Considering Figure 82 it is possible to see the variation of the energy load profile throughout the year. Extreme variation in daily energy consumption values is visible, they become more predominant in the second half of the 12 months under observation (May to October).

Figure 83 shows a regression analysis correlating seasonal changes in lighting energy use with bright sunshine availability. The analysis demonstrates a positive correlation $\left(\mathrm{r}^{2}=0.28\right)$ between lighting energy use and day light hours for this building. This indicates that on average light energy consumption increases as daylight availability increases. However removing the one outlier reduces the correlation to $\mathrm{r}^{2}=0.11$, indicating that the correlation is in fact much weaker than it would initially appear.

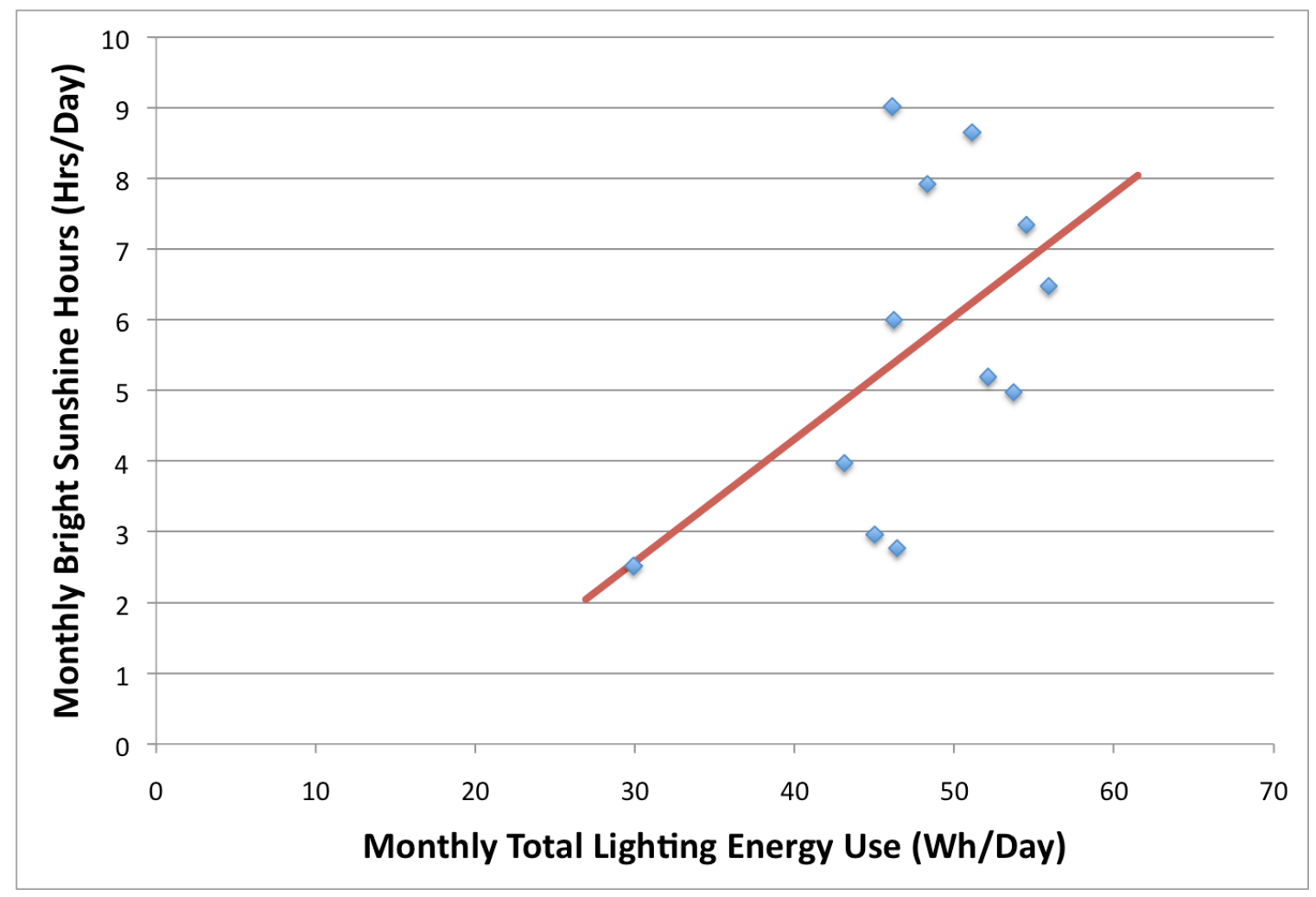

Figure 83. Lighting Energy Use Versus Sunshine Hours Regression $\left(r^{2}=0.28\right.$, slope $\left.=0.17\right)$ 
Receptacles

Table 35. Public Assembly-1: Building Receptacles Summary

\begin{tabular}{cccc}
\hline $\begin{array}{c}\text { As Modeled: } \\
\text { Installed } \\
\text { Receptacles PD } \\
\left(\mathbf{W} / \mathbf{m}^{\mathbf{2}}\right)\end{array}$ & $\begin{array}{c}\text { MNP Energy Use } \\
\left(\mathbf{W} / \mathbf{m}^{\mathbf{2}}\right)\end{array}$ & $\begin{array}{c}\text { MNB Energy Use } \\
\left(\mathbf{W} / \mathbf{m}^{\mathbf{2}}\right)\end{array}$ & $\begin{array}{c}\text { Base as \% } \\
\text { of Peak }\end{array}$ \\
\hline 4.2 & 3.8 & 0.23 & $6 \%$ \\
\hline $\begin{array}{c}\text { As Measured: } \\
\text { Installed } \\
\text { Receptacles PD } \\
\left(\mathbf{W} / \mathbf{m}^{\mathbf{2}}\right)\end{array}$ & $\begin{array}{c}\text { MNP Energy Use } \\
\left(\mathbf{W} / \mathbf{m}^{\mathbf{2}}\right)\end{array}$ & $\begin{array}{c}\text { MNB Energy Use } \\
\left(\mathbf{W} / \mathbf{m}^{\mathbf{2}}\right)\end{array}$ & $\begin{array}{c}\text { Base as \% } \\
\text { of Peak }\end{array}$ \\
\hline- & 3.9 & 0.92 & $23 \%$ \\
\hline
\end{tabular}

Table 34 summarizes the median near peak (MNP) receptacle loads and median near base (MNB) receptacle loads as modeled and as measured. Comparison between the receptacles profile and receptacles schedules show an under-predicted nighttime base-load (Figure 84 and Figure 85). Calculated with MNP and MNB values ( $3.9 \mathrm{~W} / \mathrm{m}^{2}$ and $0.92 \mathrm{~W} / \mathrm{m}^{2}$ respectively) the off-hours base load is approximately $23 \%$ of the peak load, as opposed to the estimated $6 \%$. This indicates higher levels of off-peak energy use than expected. A MNP load of $3.9 \mathrm{~W} / \mathrm{m}^{2}$ is $102 \%$ of estimated peak load of approximately $3.8 \mathrm{~W} / \mathrm{m}^{2}$. Showing a close match between predicted and actual peak loads.

Figure 84 allows us to see that the overnight load use clearly changed through out the year. Between January and May the overnight load was distinctly higher than between May and October. It is not clear the direct significance of this, as it is actually the inverse of the trend that was observed in regards to lighting. Again it may be a result of programming or occupancy changes. 
Legend:

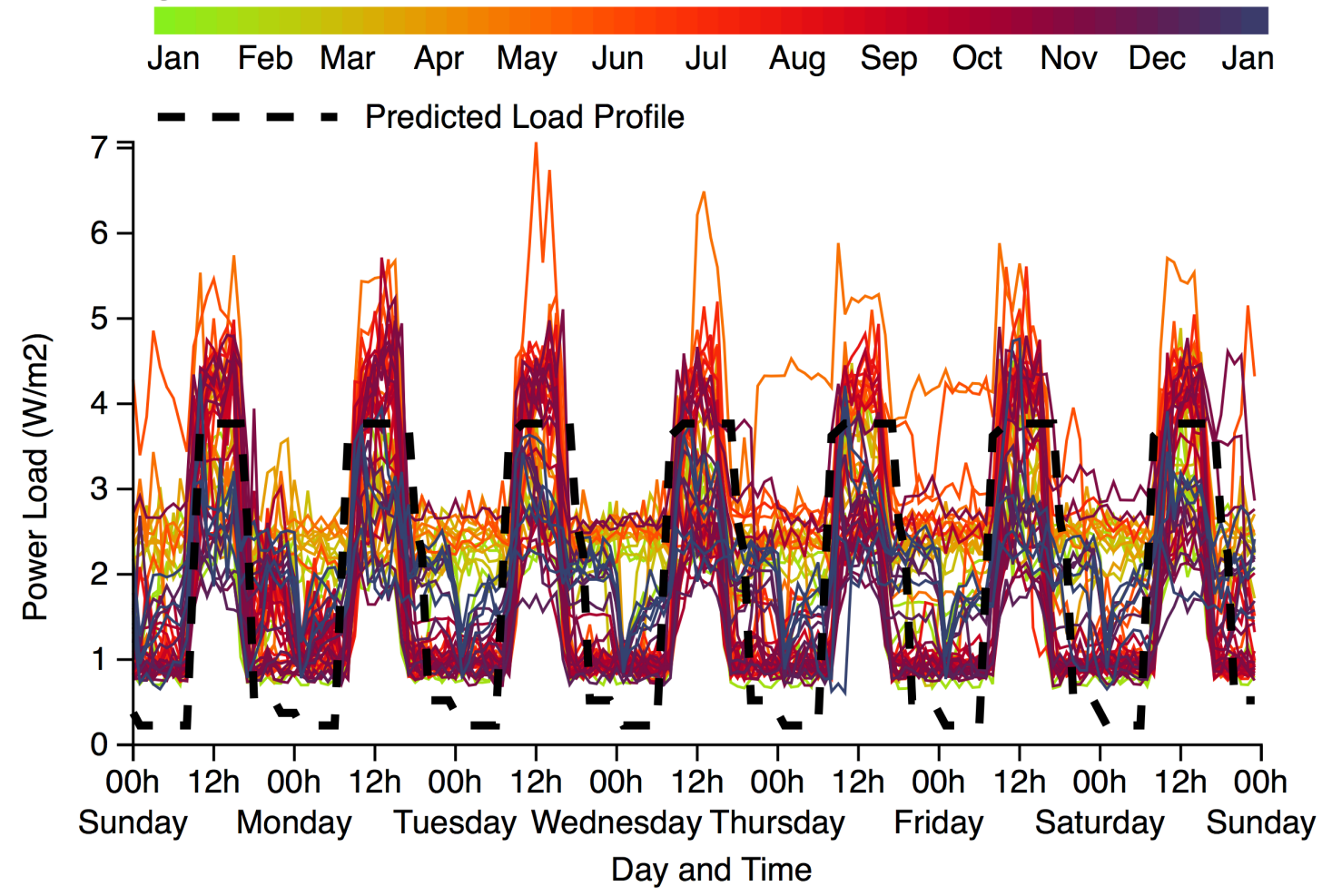

Figure 84. Public Assembly-1: Full-year 60-Minute Interval Receptacles Energy Use Data

Average, median and typical receptacle loads are shown for weeks, weekdays and weekend, respectively, in Figure 85, Figure 86 and Figure 87. The values calculated for the MNP and MNB are more clearly represented in these figures. In Figure 86 the morning ramp up appears to begin slightly later than expected around 8:00, and to begin ramping down slightly earlier than expected around 16:00. In regards to the weekend load profile (Figure 87) appears generally as expected however with ramp up and ramp down occurring approximately 1 hour earlier on both counts. 
Legend:

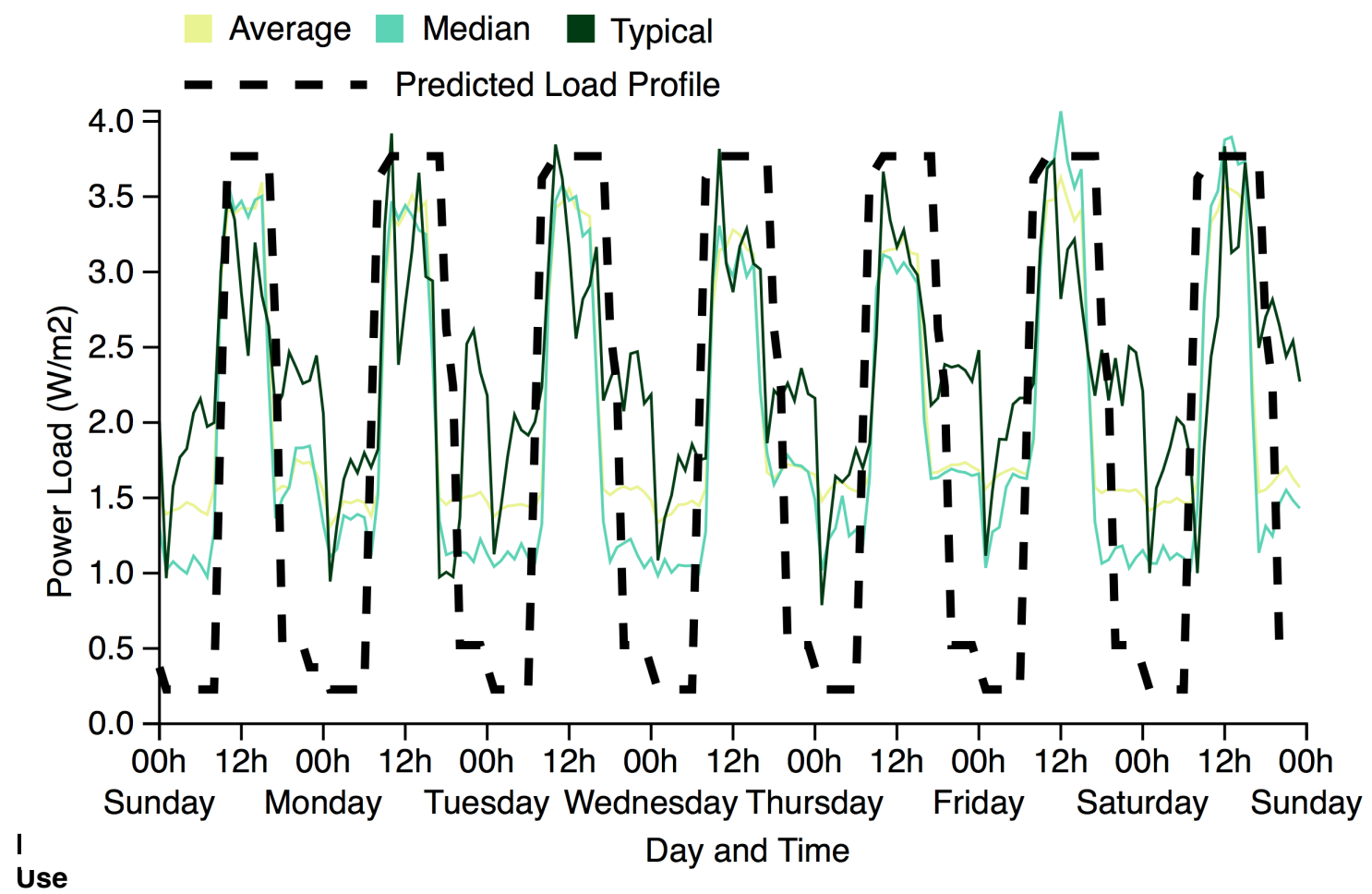

Legend:

Average Median $\square$ Typical

- c - o Predicted Load Profile

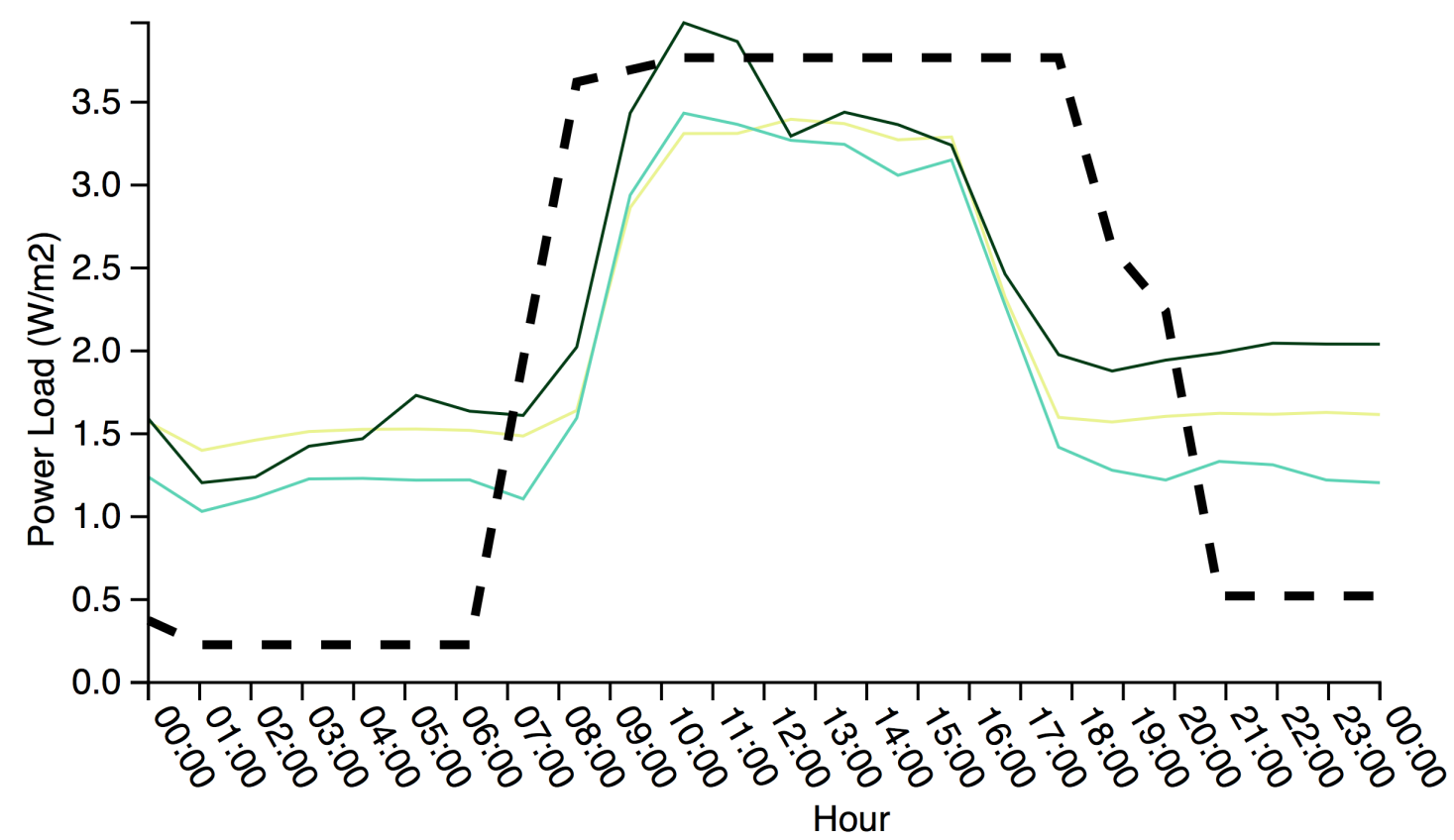

Figure 86. Public Assembley-1: Average, Median, and Typical Weekday Receptacles Profile 
Legend:

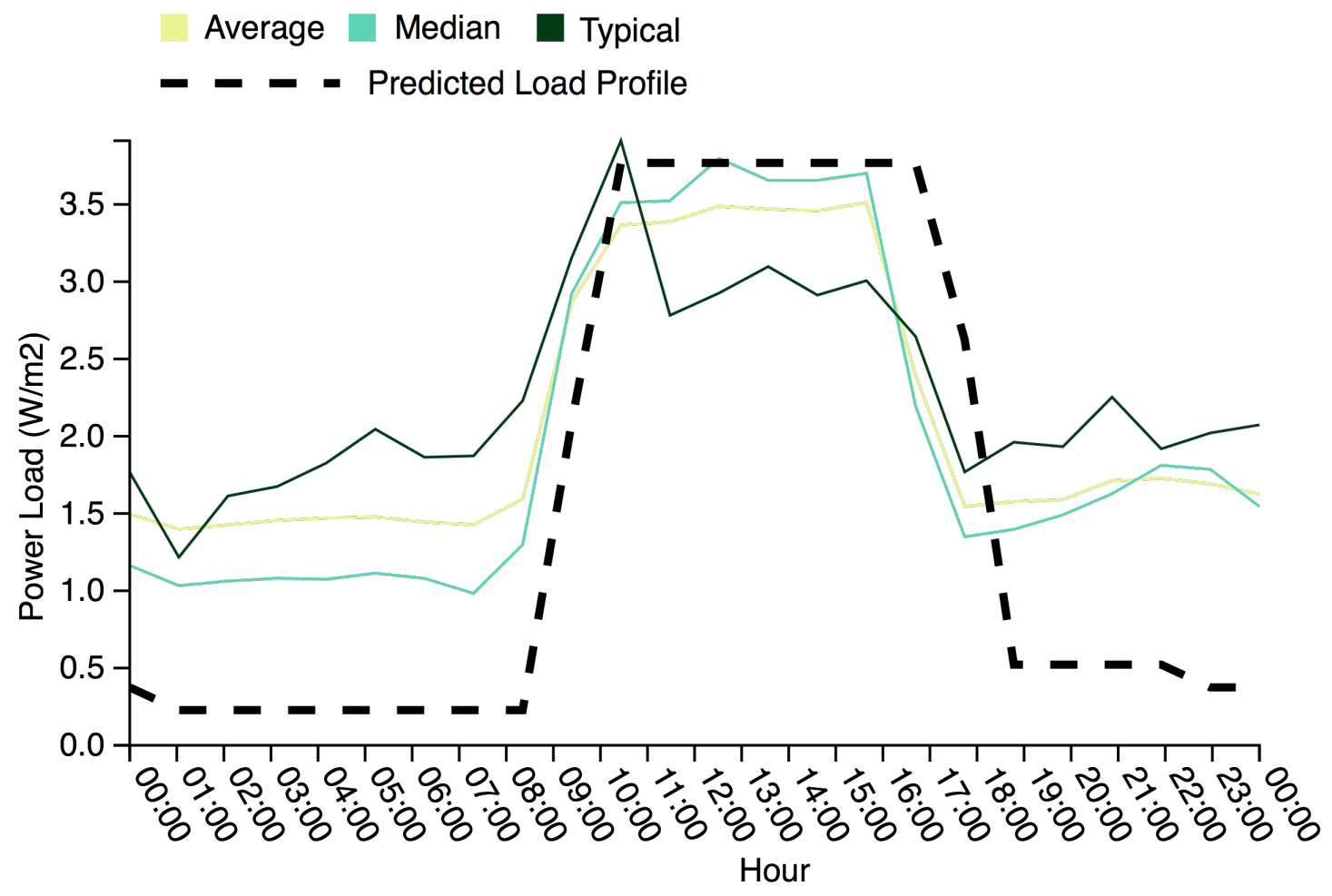

Figure 87. Public Assembley-1: Average, Median, and Typical Weekend Day Receptacles Profile

Considering Figure 88 it is possible to see variations in energy use throughout the year. Energy use trends appear highly unstable. This building is known to host a significant number of wedding receptions throughout the summer. Interestingly the variability of the data does not easily match with the variability seen in the lighting energy use plots. The same extreme overnight peak that are witnessed overnight for lighting energy use are not present for receptacles. As well the highest receptacles occur between February and May, whereas the highest lighting loads occur between May and October. This would perhaps indicate that different times of the year had different occupancies that had their own particular types of energy demands. Overall energy use is approximately as expected. 
Legend:

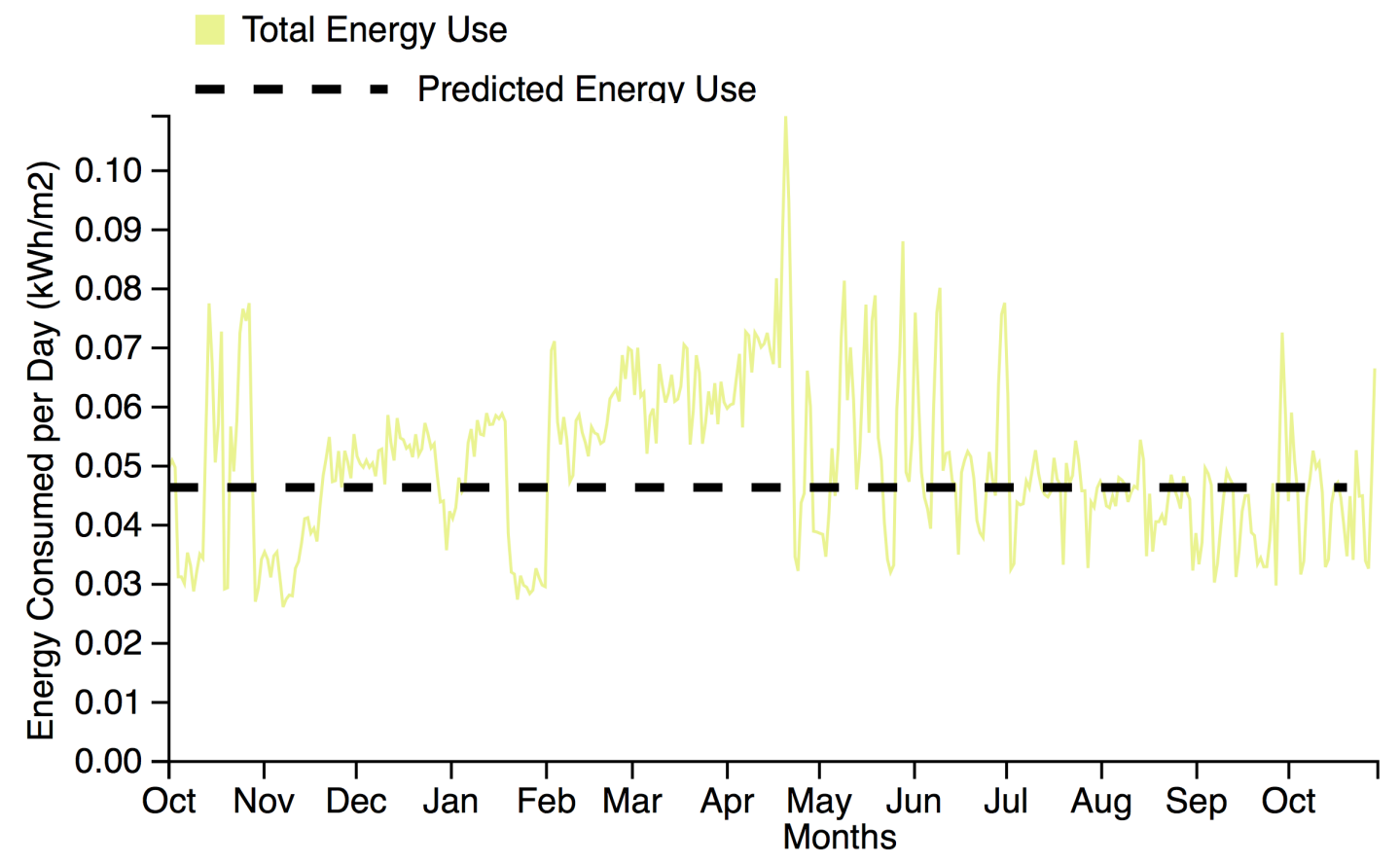

Figure 88. Public Assembly-1: Full-year Total Daily Receptacles Energy Use Data

\subsection{Alternative Benchmarking}

Conducting analysis at the level of the full dataset allows for this study to develop references for which to compare in depth analyses for the specific buildings. This is especially relevant because as we discussed in Section 3.7 ASHRAE has not developed standards for which to judge error between submetered data and energy models beyond, their standards only cover whole building energy use data.

Firstly comparison will be made between yearly predicted and actual energy use at the whole building level, at the level of energy sources (natural gas and electricity), and lastly at the end use level (heating, cooling, lighting, and receptacles). It is clear that more error (in comparing predicted and actual energy use) is likely present at the end use level in comparison to the buildings' total energy use, simply due to the fact that when summing up the components of the buildings' energy use under and over predictions will tend to cancel out. What is not clear is to what extent the error will be different at these different levels of measurement. 
Determining these values will provide a basic framework for assessing the error measured for the monthly and hourly-metered end uses in comparison to the available ASHRAE values.

The comparisons mentioned above, between predictions from calibrated energy models and metered data, are depicted in the following three graphs (Figure 89, Figure 90 and Figure 91). In these graphs each point represents a building. The dashed line represents the function where predicted values are equal to actual values (line of equivalence). Points above the line represent over predictions, and points under the line represent under predictions. The data is these graphs collected from the client reports produced for each building and includes data for 34 buildings. An investigation of the impact of calibration process on the modeling results is discussed in Appendix A.

Figure 89 shows a comparison for total energy use for each building. The majority of points remain fairly tightly clustered around the line of equivalence. Despite larger absolute differences for building's with larger energy use, percent difference remains relatively constant around $+/-15 \%$, as shown in Table 35. A larger number of points fall below the line of equivalence suggesting a tendency of the models to under predict usage values.

Table 36. Average Percent Difference in Relation to Actual Energy Consumption

\begin{tabular}{cc}
\hline $\begin{array}{c}\text { Actual } \\
\text { (kWh/m2) }\end{array}$ & $\begin{array}{c}\text { Average \% } \\
\text { Difference }\end{array}$ \\
\hline Total & $15 \%$ \\
$0-99$ & $13 \%$ \\
$100-199$ & $17 \%$ \\
$200-299$ & $14 \%$ \\
$300-399$ & $14 \%$ \\
$400-499$ & $15 \%$ \\
\hline
\end{tabular}




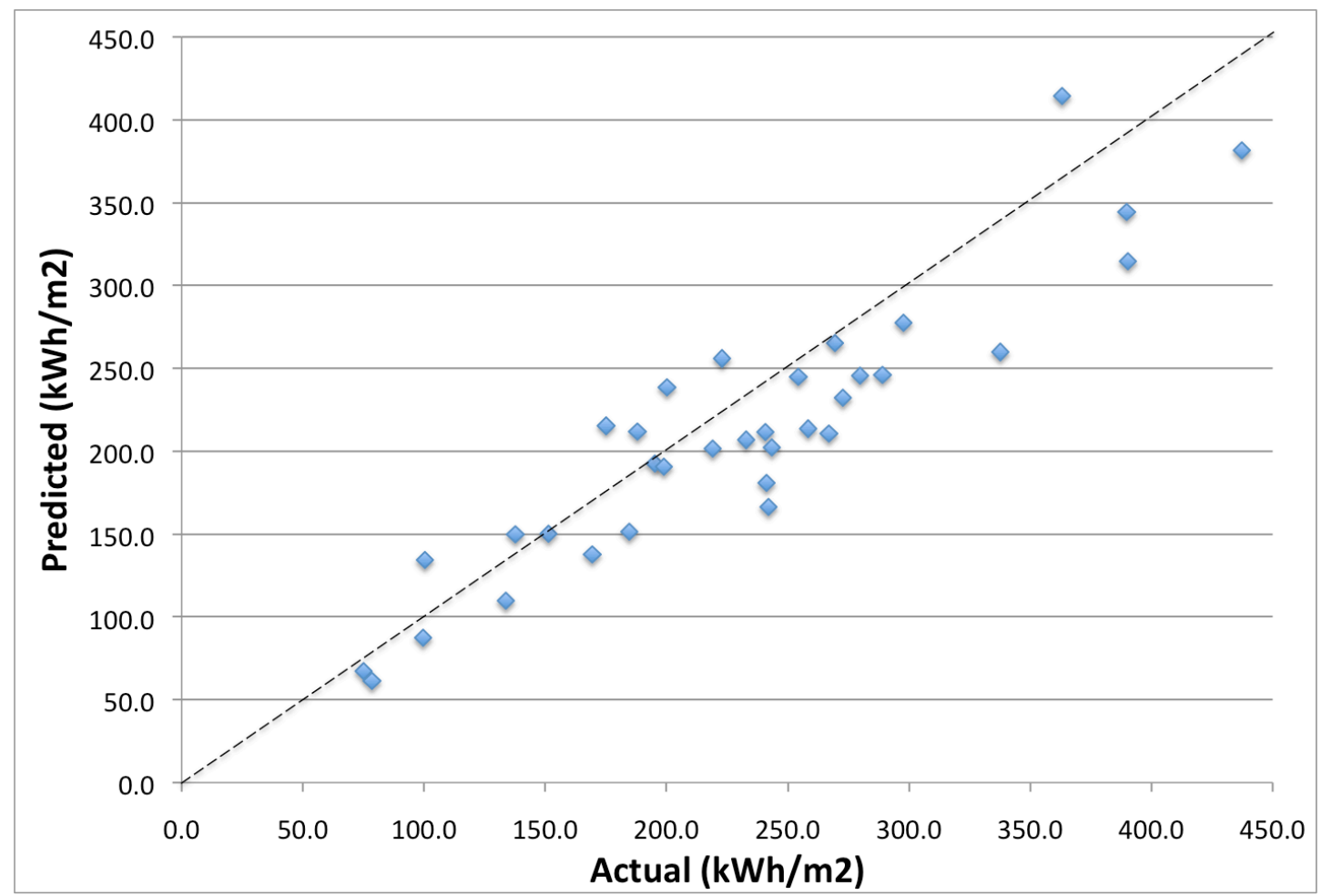

Figure 89. Actual Versus Predicted (calibrated) Total Building Energy Use $(n=34)$

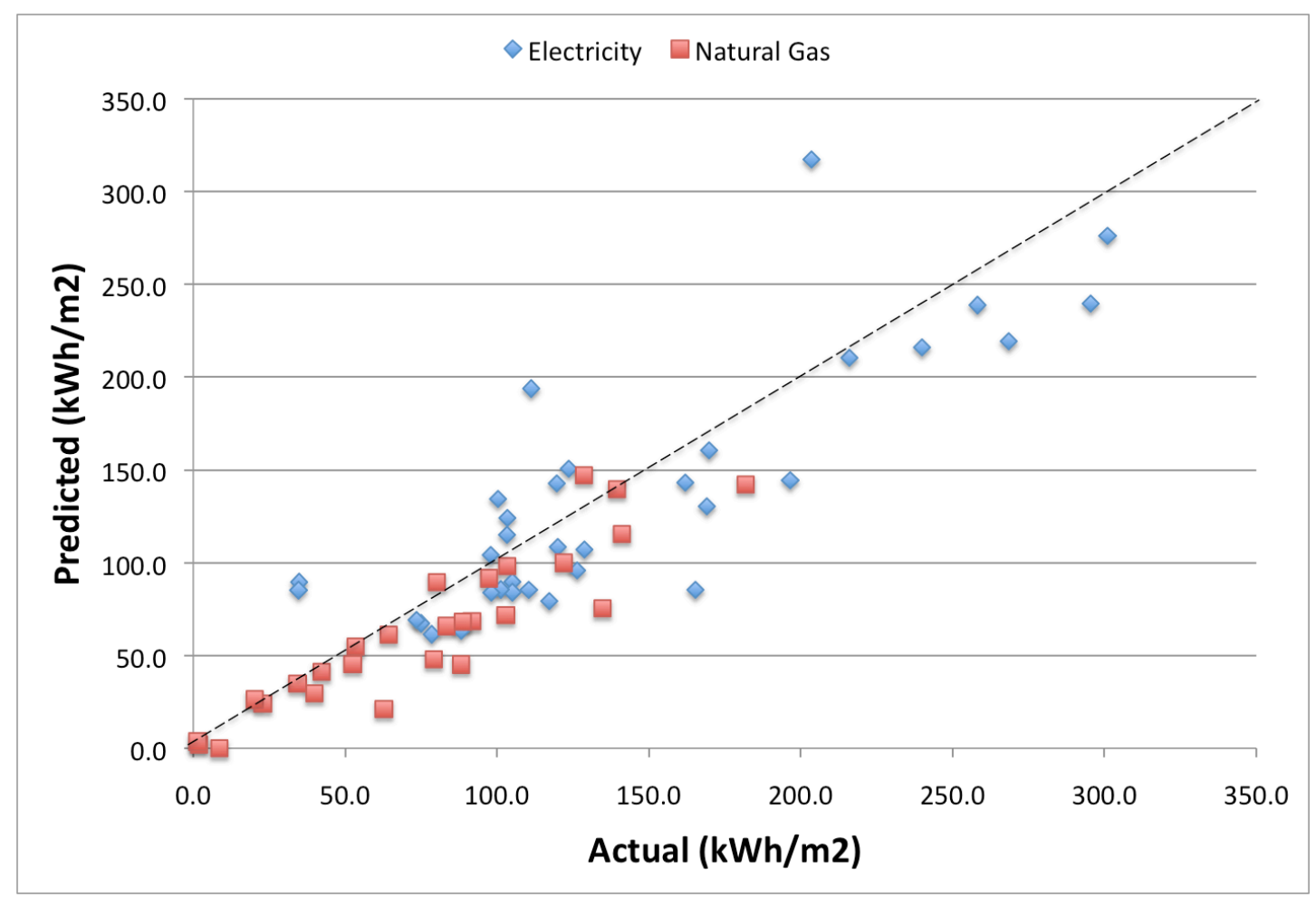

Figure 90. Actual Versus Predicted (calibrated) Total Energy Use by Type (natural gas- $\mathbf{n}=27$, electricity- $n=34$ ) 
A similar trend is seen in Figure 90, in this graph total energy consumption is divided based on fuel type. Natural gas is more tightly clustered around the line of equivalence than electricity in absolute terms, and also represents the lesser of the two in regards to quantity. Again percent difference appears to remain relatively stable amongst buildings of differing energy intensities at around $+/-25 \%$.

Finally Figure 91 shows the energy consumption separated down to end uses. Larger amounts of error are visible at this granularity of data. The extent to which the level of error of each end use differs is somewhat obscured by the different magnitudes of the energy consumption values. Taking a look at the statistical terms in Table 36, allows for a better understanding of the relationship between the end uses.

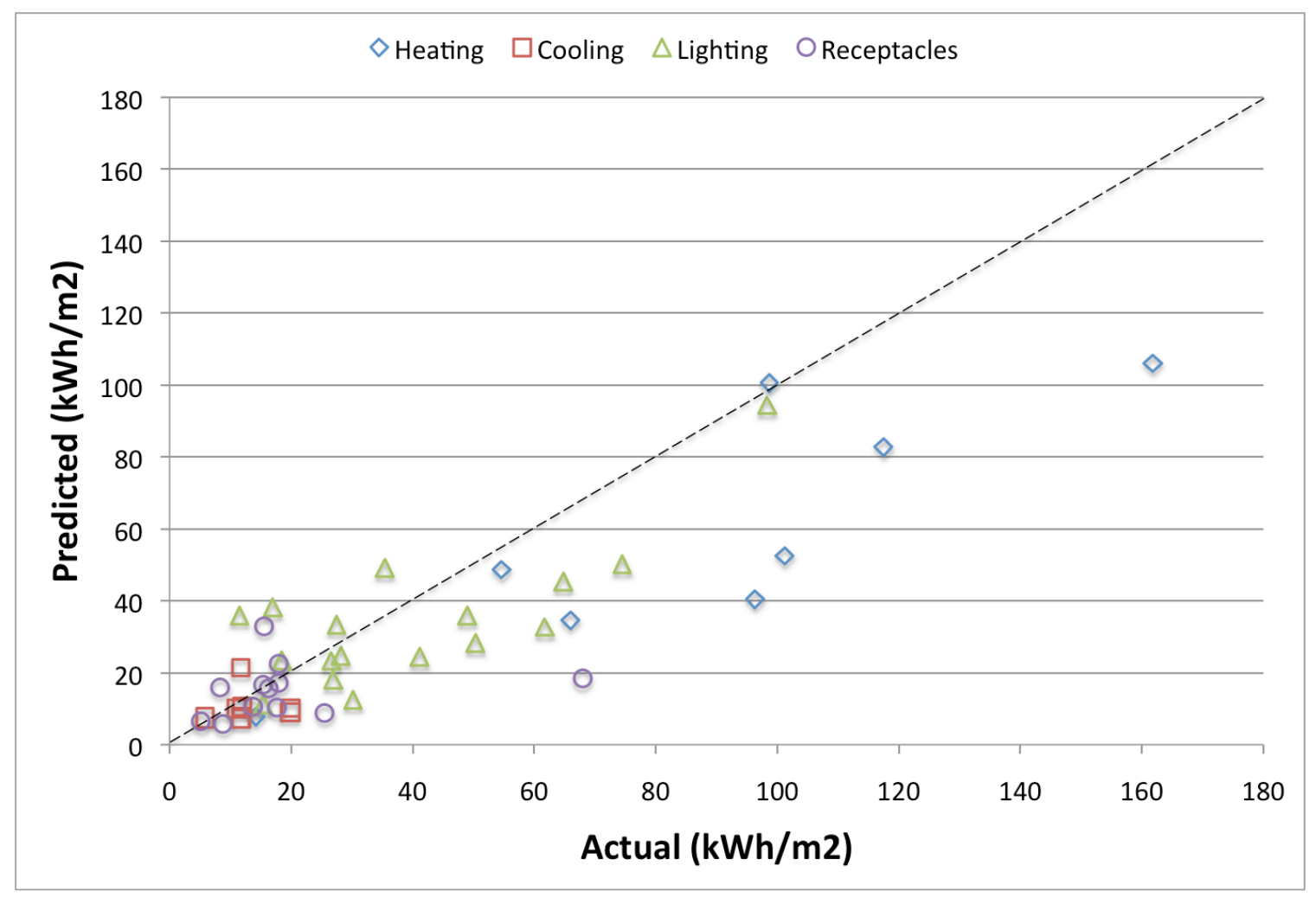

Figure 91. Actual Versus Predicted Total Energy Use Per End-use Breakdown (heating- $\mathbf{n}=8$, cooling- $n=8$, lighting $n=17$, receptacles- $n=12$ ) 
Table 37. Error Evaluation of Total Yearly Building Energy Use

\begin{tabular}{ccccc}
\hline CVRMSE & NMBE & $\begin{array}{c}\text { Average \% } \\
\text { Difference }\end{array}$ & $\begin{array}{c}\text { Average EUI } \\
\text { (ekWh/m2) }\end{array}$ \\
\hline All & $17 \%$ & $16 \%$ & $15 \%$ & 230.5 \\
\hline Electricity & $28 \%$ & $8 \%$ & $28 \%$ & 141.2 \\
Natural Gas & $26 \%$ & $26 \%$ & $27 \%$ & 76.6 \\
\hline Lighting & $50 \%$ & $18 \%$ & $52 \%$ & 33.9 \\
Receptacles & $55 \%$ & $-7 \%$ & $35 \%$ & 17.8 \\
Heating & $48 \%$ & $33 \%$ & $33 \%$ & 71.1 \\
Cooling & $61 \%$ & $19 \%$ & $44 \%$ & 8.7 \\
\hline
\end{tabular}

Out of these three terms (CVRMSE, NMBE, and average \% difference) percent difference is most affected by long tails in the data (because it lacks any term normalizing the value), and net mean bias error is most affected by skew (because subsequent negative and positive values cancel out). Due to this CVRMSE is the best to describe total error in the data sets. Relying on CVRMSE indicates that predictions regarding cooling and receptacles energy use are the least accurate followed by lighting and heating. NMBE, indicating the amount of skew in the error, shows us that receptacles are the only category that is over predicted, the rest are solidly under predicted. It is also interesting to note that receptacles and electricity are the two areas where the values are the most evenly balanced between under and over prediction.

One underlying observation that can be made is that within energy consumption of the buildings, the accuracy of the models tend to increase as the aspects of energy consumption are compounded into a single figure. On the other hand as end uses are examined individually the accuracy tends to decrease. As mentioned previously this is likely a reflection of the tendency for over predictions and under predictions to occur commonly, and as with NMBE over and under predictions will cancel out. However additionally it also appears that the size of the CVRMSE term is inversely proportional to the size of the load. Reflecting perhaps that in small loads (for example like cooling) a small deviation from the norm, in terms of typical usage patterns, has a larger percent impact on the difference between actual and predicted values. 
For a certain subset of these buildings (totaling 19 buildings) it is also possible to calculate CVRMSE and NMBE values in order to compare monthly total predicted energy use (from calibrated models) to actual monthly energy use values. In this case ASHRAES error tolerances (see Section 3.7) are directly applicable. The results are shown in Table 37. Of the 19 buildings, 7 (37\%) adhere to the error tolerance of $<15 \%$ CVRMSE and 4 (21\%) adhere to the error tolerance $<+/-5 \%$ NMBE. This relatively low adherence indicates that to a significant extent there are processes that are factors driving energy use in the buildings that are not well understood.

Table 38. CVRMSE and NMBE for Monthly Total Building Energy Use.

\begin{tabular}{ccc}
\hline Building & CVRMSE & NMBE \\
\hline Academic-1 & $20 \%$ & $7 \%$ \\
Academic-3 & $35 \%$ & $25 \%$ \\
Arena-2 & $12 \%$ & $-6 \%$ \\
Office-2 & $7 \%$ & $-2 \%$ \\
Office-3 & $23 \%$ & $-18 \%$ \\
Office-4 & $18 \%$ & $-11 \%$ \\
Office-5 & $15 \%$ & $13 \%$ \\
Office-6 & $25 \%$ & $-18 \%$ \\
Office-7 & $31 \%$ & $-27 \%$ \\
Office-8 & $12 \%$ & $-3 \%$ \\
Office-9 & $79 \%$ & $-1 \%$ \\
Office-15 & $24 \%$ & $-22 \%$ \\
Mail Sorting-1 & $26 \%$ & $-9 \%$ \\
Mail Sorting-2 & $15 \%$ & $-5 \%$ \\
Mail Sorting-3 & $24 \%$ & $-10 \%$ \\
Public Assembly-1 & $39 \%$ & $33 \%$ \\
Retail-1 & $13 \%$ & $-12 \%$ \\
Retail-2 & $14 \%$ & $-7 \%$ \\
School-1 & $35 \%$ & $31 \%$ \\
\hline Average & $25 \%$ & $14 \% *$ \\
\hline
\end{tabular}

*Figure is the average of the absolute values of the NMBES

A summary of the previous analysis, the average error terms for the entire data set, is presented in the following two tables (Table 38 and Table 39). 
Table 39. Overview Average CVRMSE

\begin{tabular}{cccc}
\hline $\begin{array}{c}\text { Level of } \\
\text { Energy } \\
\text { Breakdown }\end{array}$ & $\begin{array}{c}\text { Average } \\
\text { Yearly } \\
\text { CVRMSE }\end{array}$ & $\begin{array}{c}\text { Average } \\
\text { Monthly } \\
\text { CVRMSE }\end{array}$ & $\begin{array}{c}\text { Average } \\
\text { Hourly } \\
\text { CVRMSE }\end{array}$ \\
\hline Total & $17 \%$ & $25 \%$ & - \\
Energy Type & $27 \%$ & - & - \\
End Use & $53.5 \%$ & $56 \%$ & $79 \%$ \\
\hline
\end{tabular}

Table 40. Overview Average NMBE (calculated with absolute values)

\begin{tabular}{cccc}
\hline $\begin{array}{c}\text { Level of } \\
\text { Energy } \\
\text { Breakdown }\end{array}$ & $\begin{array}{c}\text { Average } \\
\text { Yearly } \\
\text { NMBE }\end{array}$ & $\begin{array}{c}\text { Average } \\
\text { Monthly } \\
\text { NMBE }\end{array}$ & $\begin{array}{c}\text { Average } \\
\text { Hourly } \\
\text { NMBE }\end{array}$ \\
\hline Total & $16 \%$ & $14 \%$ & - \\
Energy Type & $17 \%$ & - & - \\
End Use & $19.25 \%$ & $49 \%$ & $49 \%$ \\
\hline
\end{tabular}

This is followed by Table 40 and Table 41, which using the same table format outlines the ASHRAE error tolerances (mentioned above), that are applicable to this type of investigation. As should be immediately obvious no values are suggested for the relevant categories of investigation, especially for the comparison between predicted (calibrated) and actual energy use for end uses at monthly and hourly intervals.

Table 41. Overview ASHRAE Suggested Error Tolerances (CVRMSE)

\begin{tabular}{cccc}
\hline $\begin{array}{c}\text { Level of } \\
\text { Energy } \\
\text { Breakdown }\end{array}$ & $\begin{array}{c}\text { Allowable } \\
\text { Yearly } \\
\text { CVRMSE }\end{array}$ & $\begin{array}{c}\text { Allowable } \\
\text { Monthly } \\
\text { CVRMSE }\end{array}$ & $\begin{array}{c}\text { Allowable } \\
\text { Hourly } \\
\text { CVRMSE }\end{array}$ \\
\hline Total & - & $<15 \%$ & $<30 \%$ \\
Energy Type & - & - & - \\
End Use & - & - & - \\
\hline
\end{tabular}

Table 42. Overview ASHRAE Suggested Error Tolerances (NMBE)

\begin{tabular}{cccc}
\hline $\begin{array}{c}\text { Level of } \\
\text { Energy } \\
\text { Breakdown }\end{array}$ & $\begin{array}{c}\text { Allowable } \\
\text { Yearly } \\
\text { NMBE }\end{array}$ & $\begin{array}{c}\text { Allowable } \\
\text { Monthly } \\
\text { NMBE }\end{array}$ & $\begin{array}{c}\text { Allowable } \\
\text { Hourly } \\
\text { NMBE }\end{array}$ \\
\hline Total & - & $<+/-5 \%$ & $<+/-10 \%$ \\
Energy Type & - & - & - \\
End Use & - & - & - \\
\hline
\end{tabular}

As a result it will be necessary to suggest provisionary values for error tolerances for the categories of CVRMSE and NMBE for hourly and monthly end use 
comparisons. These provisionary error tolerances will serve as an indicator for which to judge further analysis of energy use consumption at the building level. Unfortunately it has not been possible to identify how ASHRAE developed the error tolerances listed in Table 40 and Table 41, and therefore the provisionary error tolerances will be based solely off of the approximate observed ratio between monthly total and end use error values observed in the data set. In this case the absolute value was used for the NMBE figures as we are primarily concerned with their magnitude not their sign.

Table 43. Total to End use Ratio for CVRMSE and NMBE.

\begin{tabular}{ccccccc}
\hline Building & $\begin{array}{c}\text { Monthly } \\
\text { CVRMSE- } \\
\text { Total }\end{array}$ & $\begin{array}{c}\text { Monthly } \\
\text { CVRMSE- } \\
\text { End Uses }\end{array}$ & $\begin{array}{c}\text { Ratio- } \\
\text { End Use } \\
\text { to Total }\end{array}$ & $\begin{array}{c}\text { Monthly } \\
\text { NMBE- } \\
\text { Total } \\
\text { (Abs) }\end{array}$ & $\begin{array}{c}\text { Monthly } \\
\text { NMBE- } \\
\text { End Uses } \\
\text { (Abs) }\end{array}$ & $\begin{array}{c}\text { Ratio- } \\
\text { End Use } \\
\text { to Total }\end{array}$ \\
\hline Academic-1 & $20 \%$ & $49 \%$ & 2.5 & $7 \%$ & $49 \%$ & 6.9 \\
Office-3 & $23 \%$ & $61 \%$ & 2.7 & $18 \%$ & $46 \%$ & 2.6 \\
$\begin{array}{c}\text { Office-4 } \\
\text { Public }\end{array}$ & $18 \%$ & $43 \%$ & 2.4 & $11 \%$ & $37 \%$ & 3.3 \\
$\begin{array}{c}\text { Admin-1 } \\
\text { Public } \\
\text { Admin-2 }\end{array}$ & $26 \%$ & $97 \%$ & 3.7 & $9 \%$ & $80 \%$ & 8.9 \\
$\begin{array}{c}\text { Public } \\
\text { Admin-3 } \\
\text { Public }\end{array}$ & $24 \%$ & $22 \%$ & 1.5 & $5 \%$ & $22 \%$ & 4.3 \\
Assembley-1 & $29 \%$ & $51 \%$ & 2.1 & $10 \%$ & $45 \%$ & 4.5 \\
\hline Average & $72 \%$ & 1.8 & $33 \%$ & $67 \%$ & 2.0 \\
\hline
\end{tabular}

The analysis conducted in Table 42 suggests that the CVRMSE error tolerances for end uses energy consumption should be 2.5 times greater than for total building energy consumption. Table 42 also suggests that NMBE error tolerances for end uses energy consumption should be 4.5 times greater than for total building energy consumption. Based on these figures it is possible suggest provisionary error tolerance values for comparing end use energy consumption predictions to actual metered data. These values are listed in Table 43 and Table 44 alongside ASHRAE error tolerances for total building energy use. 
Table 44. Overview ASHRAE Suggested Error Tolerances (CVRMSE) with Suggested Values for End Use Error Terms

\begin{tabular}{cccc}
\hline $\begin{array}{c}\text { Level of } \\
\text { Energy } \\
\text { Breakdown }\end{array}$ & $\begin{array}{c}\text { Allowable } \\
\text { Yearly } \\
\text { CVRMSE }\end{array}$ & $\begin{array}{c}\text { Allowable } \\
\text { Monthly } \\
\text { CVRMSE }\end{array}$ & $\begin{array}{c}\text { Allowable } \\
\text { Hourly } \\
\text { CVRMSE }\end{array}$ \\
\hline Total & - & $<15 \%$ & $<30 \%$ \\
Energy Type & - & - & - \\
End Use & - & $<37.5 \%$ & $<75 \%$ \\
\hline
\end{tabular}

Table 45. Overview ASHRAE Suggested Error Tolerances (NMBE) with Suggested Values for End Use Error Terms

\begin{tabular}{cccc}
\hline $\begin{array}{c}\text { Level of } \\
\text { Energy } \\
\text { Breakdown }\end{array}$ & $\begin{array}{c}\text { Allowable } \\
\text { Yearly } \\
\text { NMBE }\end{array}$ & $\begin{array}{c}\text { Allowable } \\
\text { Monthly } \\
\text { NMBE }\end{array}$ & $\begin{array}{c}\text { Allowable } \\
\text { Hourly } \\
\text { NMBE }\end{array}$ \\
\hline Total & - & $<+/-5 \%$ & $<+/-10 \%$ \\
Energy Type & - & - & - \\
End Use & - & $<+/-22.5 \%$ & $<+/-45 \%$ \\
\hline
\end{tabular}

In order to calculate CVRMSE and NMBE error tolerances at the end-use level for buildings, submetered energy consumption is compared with a combination of zone lighting schedules and lighting power densities employed in the modeling. CVRMSE and NMBE were calculated for the comparison between actual and predicted data for both hourly and monthly intervals. For the case of hourly intervals the terms were calculated using the full duration of data available matched to the corresponding duration of predicted data. For the case of monthly intervals, actual monthly energy use was calculated with monthly medians.

The results for these calculations are listed in Table 43 and Table 44. It is readily apparent that the CVRMSE and NMBE for lighting and receptacles end-uses do not fall within the error tolerance levels defined by ASHRAE for hourly and monthly intervals. However as discussed in Section 3.7 it is inappropriate to compare end-uses and total building energy use by the same standards. Considering however the error tolerance suggested in Table 43 and Table 44 for CVRMSE and NMBE for monthly and hourly end-uses (as opposed to totals) we can compare the values in Table 41 to a relevant standard. As we can see many of the buildings still fail to meet the prescribed level of error. 
The results for the following error tolerance calculations for lighting and receptacles energy use (discussed below) are only useful in as much as they give an indication of how well the models are predicting energy use in buildings. For those building's that do not adhere to the error tolerance prescribed by ASHRAE (and for those categories suggested in Table 44) it will indicate to what extent further scrutiny is required in the analysis of the buildings' energy consumption patterns.

It is interesting to also note that relatively similar results were found for both the receptacles and lighting energy use. This would perhaps lend support to the notion that a common set of error tolerance levels are appropriate across various end-uses. On the other hand it seems strange that the percent adherance is much higher for hourly data than it is for monthly data. This would suggest that the suggested value end-use error tolerances for hourly and monthly data should be readjusted once more information is available.

Table 46. Statistical Results for Submetering Comparison to Lighting Schedules

\begin{tabular}{ccccc}
\hline & $\begin{array}{c}\text { CVRMSE } \\
\text { Hourly } \\
\text { (full) }\end{array}$ & $\begin{array}{c}\text { CVRMSE } \\
\text { Monthly } \\
\text { (median) }\end{array}$ & $\begin{array}{c}\text { NMBE } \\
\text { Hourly } \\
\text { (full) }\end{array}$ & $\begin{array}{c}\text { NMBE } \\
\text { Monthly } \\
\text { (median) }\end{array}$ \\
\hline Academic-1 & $63 \%$ & $46 \%$ & $-44 \%$ & $-46 \%$ \\
Office-3 & $59 \%$ & $68 \%$ & $-51 \%$ & $-51 \%$ \\
$\begin{array}{c}\text { Office-4 } \\
\text { Mail }\end{array}$ & $90 \%$ & $27 \%$ & $29 \%$ & $23 \%$ \\
$\begin{array}{c}\text { Sorting-1 } \\
\text { Mail }\end{array}$ & $54 \%$ & $50 \%$ & $-43 \%$ & $-43 \%$ \\
$\begin{array}{c}\text { Sorting-2 } \\
\text { Mail }\end{array}$ & $53 \%$ & $19 \%$ & $-14 \%$ & $-19 \%$ \\
$\begin{array}{c}\text { Sorting-3 } \\
\text { Public }\end{array}$ & $197 \%$ & $124 \%$ & $114 \%$ & $123 \%$ \\
Assembly-1 & & $54 \%$ & $-45 \%$ & $-47 \%$ \\
\hline $\begin{array}{c}\text { Percent } \\
\text { Adherence* }\end{array}$ & $71 \%(5 / 7)$ & $29 \%(2 / 7)$ & $14 \%(1 / 7)$ & $43 \%(3 / 7)$ \\
\hline
\end{tabular}

*Percent adherence to suggested error tolerances for end-use energy consumption as suggested in Table 43 and Table 44. 
Table 47. Statistical Results for Submetering Comparison to Receptacles Schedules

\begin{tabular}{|c|c|c|c|c|}
\hline & $\begin{array}{l}\text { CVRMSE } \\
\text { Hourly } \\
\text { (full) }\end{array}$ & $\begin{array}{l}\text { CVRMSE } \\
\text { Monthly } \\
\text { (median) }\end{array}$ & $\begin{array}{l}\text { NMBE } \\
\text { Hourly } \\
\text { (full) }\end{array}$ & $\begin{array}{l}\text { NMBE } \\
\text { Monthly } \\
\text { (median) }\end{array}$ \\
\hline $\begin{array}{l}\text { Academic-1 } \\
\text { Office-3 }\end{array}$ & $\begin{array}{l}59 \% \\
51 \%\end{array}$ & $\begin{array}{l}52 \% \\
54 \%\end{array}$ & $\begin{array}{l}-49 \% \\
-40 \%\end{array}$ & $\begin{array}{l}-51 \% \\
-41 \%\end{array}$ \\
\hline Office-4 & $97 \%$ & $59 \%$ & $59 \%$ & $50 \%$ \\
\hline $\begin{array}{l}\text { Mail } \\
\text { Sorting-1 }\end{array}$ & $149 \%$ & $144 \%$ & $118 \%$ & $117 \%$ \\
\hline $\begin{array}{l}\text { Mail } \\
\text { Sorting-2 }\end{array}$ & $30 \%$ & $25 \%$ & $26 \%$ & $24 \%$ \\
\hline $\begin{array}{l}\text { Mail } \\
\text { Sorting-3 }\end{array}$ & $85 \%$ & $48 \%$ & $42 \%$ & $42 \%$ \\
\hline $\begin{array}{l}\text { Public } \\
\text { Assembly-1 }\end{array}$ & $64 \%$ & $19 \%$ & $-7 \%$ & $-10 \%$ \\
\hline $\begin{array}{l}\text { Percent } \\
\text { Adherence* }\end{array}$ & $57 \%(4 / 7)$ & $29 \%(2 / 7)$ & $57 \%(4 / 7)$ & $14 \%(1 / 7)$ \\
\hline
\end{tabular}

\subsection{Energy Usage Patterns}

Further understanding the energy usage patterns identified in Section 4.1 is key to understanding the performance gap as identified for these buildings. The analysis in this section will take the form of characterizing the key components of the load profiles as well as quantifying the difference between the actual and predicted energy consumption values.

The building load profiles are characterized in the following six tables. Table 47, Table 48, and Table 49 relate to the lighting load profile. Table 50, Table 51, and Table 52 relate to the receptacle load profile. Respectively these tables show the MNB and MNP in relation to predictions, amount of energy consumed over daytime hours versus nighttime hours, and the percent of the time that the building operates as percent of its max load. 


\section{Lighting}

Two key observations can be taken from Table 47. Firstly that the vast majority of buildings (6/7) have a base load that is much higher than expected. The predicted base load was on average $5 \%$ of the peak, on the other hand the predicted MNB was on average $26 \%$ of the MNP. And secondly the majority of buildings (4/7) have an actual MNP greater than expected. Although over all seven buildings the MNP is on average $104 \%$ of the predicted peak, indicating over and underprediction are occurring approximately in the same quantity. This indicates that buildings do not shutdown to as full an extent as predicted by the models. The second observation allows us to make some slightly more interesting conclusions. While an over-prediction can be easily associated with an over-predicted occupancy in the building, and under-prediction is a bit more puzzling. The max load is determined in the energy model by the maximum installed capacity of lighting fixtures as taken from shop drawings. Energy use above the max load indicates perhaps either meters that are including energy consumption not associated with lighting energy consumption as defined by the model, or fairly drastic as-built differences between what was designed and what was actually constructed.

Considering Table 48 one important result should be observed. In every single building, nighttime energy use was greater than predicted in an absolute sense (the shear amount of electricity consumed was greater), and in a relative sense (the amount of energy as a proportion of the building's energy use) was greater. On average this amounted to actual daytime energy use $16 \%$ greater than predicted and nighttime energy use $153 \%$ greater than predicted. This finding is elucidated when considering that (6/7) buildings experienced higher base loads than predicted and that base loads occur primarily overnight.

Table 49 allows us to further examine how the breakdown of energy is organized. In a number of buildings it is easy to see the previous result that much more energy consumption is occurring during building operations at a base load that is higher than predicted (especially Academic-1, Office-3, Office-4, and Mail 
Sorting-1). However other than that it is not clear that there is a strong pattern. In very few cases do the predicted levels of energy use match with those actually occurring. This indicates that not only are the magnitudes of loads predicted incorrectly, but the general distribution of loads across time periods are also predicted incorrectly.

From these observations two conclusions can be made. Firstly that the load profile for lighting energy consumption in buildings is poorly understood. It has been difficult for modelers to accurately predict energy consumption, both magnitude of total load and the percent of total load that occurs throughout the day with varying buildings occupancy. Secondly the increase in energy use overnight points to management decisions driving increases in energy use consumption, as occupancy issues would for the most part not effect the buildings during nighttime hours. Although for two buildings Academic-1, and Public Assembly-1 we know that overnight occupancy is part of the explanation. In the case of the Academic-1 site visits and discussion with building management (conducted as part of the commissioning process) points to students using the facilities over longer hours than was anticipated, in the case of the Public Assembley-1 site visits and discussion with building management (conducted as part of the commissioning process) points to more late night events booked for the space than originally anticipated.

Table 48. Median Near Base Lighting Loads as Percent of Median Near Peak Lighting Loads

\begin{tabular}{cccc}
\hline & $\begin{array}{c}\text { Predicted } \\
\text { MNB (as \% } \\
\text { of MNP) }\end{array}$ & $\begin{array}{c}\text { Actual } \\
\text { MNB (as \% } \\
\text { of MNP) }\end{array}$ & $\begin{array}{c}\text { Actual MNP (as } \\
\text { \% of Predicted } \\
\text { Peak) }\end{array}$ \\
\hline Academic-1 & $5 \%$ & $59 \%$ & $93 \%$ \\
Office-3 & $5 \%$ & $35 \%$ & $131 \%$ \\
Office-4 & $5 \%$ & $21 \%$ & $63 \%$ \\
$\quad \begin{array}{l}\text { Mail } \\
\text { Sorting-1 } \\
\text { Mail }\end{array}$ & $5 \%$ & $26 \%$ & $146 \%$ \\
$\begin{array}{c}\text { Sorting-2 } \\
\text { Mail }\end{array}$ & $5 \%$ & $12 \%$ & $132 \%$ \\
Sorting-3 & $5 \%$ & $27 \%$ & $116 \%$ \\
Public & $5 \%$ & $3 \%$ & $50 \%$ \\
Assembly-1 & & & \\
\hline Average & $5 \%$ & $26 \%$ & $104 \%$ \\
\hline
\end{tabular}


Table 49. Actual Total Lighting Energy Use for Daytime and Nighttime Hours (Predicted Values in Parenthesis)

\begin{tabular}{|c|c|c|c|c|c|}
\hline & 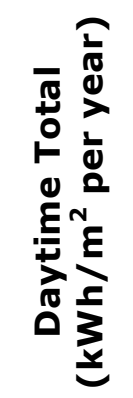 & 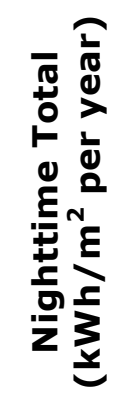 & 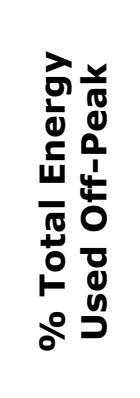 & 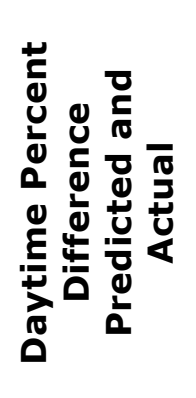 & 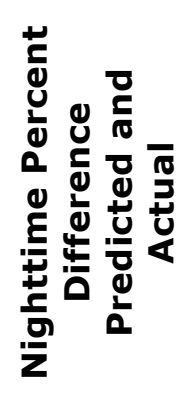 \\
\hline Academic-1 & $\begin{array}{c}24.2 \\
(19.3)\end{array}$ & $\begin{array}{l}19.8 \\
(5.0)\end{array}$ & $\begin{array}{c}45 \% \\
(21 \%)\end{array}$ & $26 \%$ & $297 \%$ \\
\hline Office-3 & $\begin{array}{l}13.6 \\
(9.0)\end{array}$ & $\begin{array}{c}9.2 \\
(2.1)\end{array}$ & $\begin{array}{c}36 \% \\
(18 \%)\end{array}$ & $50 \%$ & $333 \%$ \\
\hline Office-4 & $\begin{array}{l}17.6 \\
(31.7)\end{array}$ & $\begin{array}{l}10.2 \\
(4.1)\end{array}$ & $\begin{array}{l}36 \% \\
(11 \%)\end{array}$ & $-45 \%$ & $149 \%$ \\
\hline $\begin{array}{l}\text { Mail } \\
\text { Sorting-1 }\end{array}$ & $\begin{array}{c}32.1 \\
(18.9)\end{array}$ & $\begin{array}{l}17.2 \\
(9.2)\end{array}$ & $\begin{array}{c}35 \% \\
(32 \%)\end{array}$ & $70 \%$ & $87 \%$ \\
\hline Mail & 32.9 & 31.8 & $49 \%$ & $12 \%$ & $23 \%$ \\
\hline Sorting-2 & (29.5) & (25.9) & $(46 \%)$ & & \\
\hline $\begin{array}{l}\text { Mail } \\
\text { Sorting-3 }\end{array}$ & $\begin{array}{c}37.5 \\
(23.4)\end{array}$ & $\begin{array}{l}22.4 \\
(9.1)\end{array}$ & $\begin{array}{c}37 \% \\
(28 \%)\end{array}$ & $60 \%$ & $146 \%$ \\
\hline $\begin{array}{l}\text { Public } \\
\text { Assembly-1 }\end{array}$ & $\begin{array}{c}12.7 \\
(34.2)\end{array}$ & $\begin{array}{c}4.9 \\
(3.7) \\
\end{array}$ & $\begin{array}{l}28 \% \\
(11 \%) \\
\end{array}$ & $-63 \%$ & $32 \%$ \\
\hline Average & & & & $16 \%$ & $153 \%$ \\
\hline
\end{tabular}

Table 50. Actual Percent Time Building Operates as Percent of Max Lighting Load (Predicted Values in Parenthesis).

\begin{tabular}{llllll}
\hline \multicolumn{5}{c}{ Percent Time at Percent of Max Load } \\
\hline & $\mathbf{0 \% - 2 0 \%}$ & $\mathbf{2 1 \% - 4 0 \%}$ & $\mathbf{4 1 \% - 6 0 \%}$ & $\mathbf{6 1 \% - 8 0 \%}$ & $\mathbf{8 1 - 1 0 0 \%}$ \\
\hline Academic-1 & $1.7 \%$ & $\mathbf{4 4 . 5 \%}$ & $21.1 \%$ & $27.2 \%$ & $5.5 \%$ \\
& $(\mathbf{5 5 . 4 \% )}$ & $(6.0 \%)$ & $(6.0 \%)$ & $(11.9 \%)$ & $(20.8 \%)$ \\
Office-3 & $0.9 \%$ & $2.1 \%$ & $\mathbf{5 3 . 7 \%}$ & $33.2 \%$ & $10.2 \%$ \\
& $\mathbf{( 5 8 . 3 \% )}$ & $(8.9 \%)$ & $(3.0 \%)$ & $(0 \%)$ & $(29.8 \%)$ \\
Office-4 & $11.4 \%$ & $\mathbf{5 2 . 6 \%}$ & $10.2 \%$ & $19.7 \%$ & $6.2 \%$ \\
& $\mathbf{( 4 5 . 2 \% )}$ & $(16.1 \%)$ & $(6.0 \%)$ & $(6.0 \%)$ & $(26.8 \%)$ \\
Mail & $0 \%$ & $\mathbf{5 8 . 8} \%$ & $8.3 \%$ & $6.8 \%$ & $26.1 \%$ \\
Sorting-1 & $\mathbf{( 4 3 . 5 \% )}$ & $(0 \%)$ & $(29.8 \%)$ & $(0 \%)$ & $(26.8 \%)$ \\
Mail & $18.7 \%$ & $18.1 \%$ & $7.1 \%$ & $26.5 \%$ & $\mathbf{2 9 . 6 \%}$ \\
Sorting-2 & $(30.4 \%)$ & $(0 \%)$ & $(3.6 \%)$ & $(3.6 \%)$ & $\mathbf{( 6 2 . 5 \% )}$ \\
Mail & $0.6 \%$ & $37.9 \%$ & $14.8 \%$ & $4.8 \%$ & $\mathbf{4 1 . 9 \%}$ \\
Sorting-3 & $(\mathbf{4 9 . 4 \% )}$ & $(0 \%)$ & $(26.8 \%)$ & $(0 \%)$ & $(23.8 \%)$ \\
Public & $\mathbf{6 4 . 1 \%}$ & $29.4 \%$ & $5.3 \%$ & $1.1 \%$ & $0.2 \%$ \\
Assembly-1 & $(48.2 \%)$ & $(0 \%)$ & $(7.7 \%)$ & $(4.2 \%)$ & $\mathbf{( 4 9 . 9 \% )}$ \\
\hline
\end{tabular}




\section{Receptacles}

Similar to Lighting, Table 50, demonstrates that the vast majority of buildings (6/7) have a base load that is higher than expected. The predicted base load was on average $25 \%$ of the peak, while the predicted MNB was on average $46 \%$ of the MNP. On the other hand, for receptacle loads, a minority of buildings (3/7) have an actual MNP greater than expected. Again this initial observation indicates that buildings do not shutdown to as full an extent as predicted by the models.

Over all seven buildings the MNP is on average $85 \%$ of the predicted peak, indicating over-prediction are occurring more than under-predictions, especially Mail Sorting-1 and Mail Sorting-2. Through access to shop drawings and panel descriptions we know both these buildings receptacle meters include large amounts of non-receptacle loads, however it is difficult to determine what role this could have had in the large over-prediction energy consumption. On the other hand overpredictions can also occur due to an over-predicted occupancy load. In the case of the under-predictions, and as stated above, the situation is a bit more puzzling. The max load is determined in the energy model by the maximum installed capacity of receptacles as taken from shop drawings. Energy use above the max load indicates perhaps either meters that are including energy consumption not associated with lighting energy consumption as defined by the model (as we know is the case in several buildings), or fairly drastic as-built differences between what was designed and what was actually constructed.

Considering Table 51 in comparison to Table 48, overnight energy for receptacles loads does not experience nearly as drastic an increase as was observed in the case of lighting. For a minority of buildings (3/7) nighttime energy use was greater than predicted in an absolute sense (the shear amount of electricity consumed was greater), even while it was greater in a relative sense for all buildings (the amount of energy as a proportion of the building's energy use). And so although the phenomenon was less extreme it was still present, with on average actual daytime energy use 15\% less than predicted and nighttime energy use $85 \%$ greater 
than predicted. And again this finding is supported considering that $(6 / 7)$ buildings experienced higher base loads than predicted and that base loads occur primarily overnight.

Table 52 allows us to further examine how the breakdown of energy is organized. The previous result that much more energy consumption is occurring during building operations at a base load that is higher than predicted, is not as clear cut as it was in the case of lighting. While our other investigation do indicate that base load energy consumption is higher, at the resolution available in Table 52, energy use at base load appears at approximately the same magnitude for predicted and actual for a number of the buildings. Other than that it is not clear that there is a strong pattern. Although predicted levels of energy use match with those actually occurring far more often than in the case of lighting.

From these observations two conclusions can be made. Firstly that the load profile for receptacles energy consumption is less poorly understood than lighting. Or (considering that the evidence still points to management issues driving up overnight energy use) that the receptacle load profile is not as sensitive to impact by the aforementioned management issues.

Secondly the increase in energy use overnight (although not as extreme as for lighting) still points to management decisions driving increases in energy use consumption, as occupancy issues would for the most part not effect the buildings during nighttime hours. Although, again, for two buildings Academic-1, and Public Assembly-1 we know that overnight occupancy is part of the explanation. In the case of the Academic-1 site visits and discussion with building management (conducted as part of the commissioning process) points to students using the facilities over longer hours than was anticipated, in the case of the Public Assembley-1 site visits and discussion with building management (conducted as part of the commissioning process) points to more late night events booked for the space than originally anticipated. 
Table 51. Median Near Base Lighting Loads as Percent of Median Near Peak Lighting Loads

\begin{tabular}{cccc}
\hline & $\begin{array}{c}\text { Predicted } \\
\text { MNB (as \% } \\
\text { of MNP) }\end{array}$ & $\begin{array}{c}\text { Actual } \\
\text { MNB (as \% } \\
\text { of MNP) }\end{array}$ & $\begin{array}{c}\text { Actual MNP (as } \\
\text { \% of Predicted } \\
\text { Peak) }\end{array}$ \\
\hline Academic-1 & $6 \%$ & $43 \%$ & $135 \%$ \\
Office-3 & $22 \%$ & $49 \%$ & $123 \%$ \\
Office-4 & $19 \%$ & $21 \%$ & $69 \%$ \\
$\quad \begin{array}{c}\text { Mail } \\
\text { Sorting-1 } \\
\text { Mail }\end{array}$ & $28 \%$ & $67 \%$ & $31 \%$ \\
$\begin{array}{c}\text { Sorting-2 } \\
\text { Mail }\end{array}$ & $71 \%$ & $65 \%$ & $86 \%$ \\
$\begin{array}{c}\text { Sorting-3 } \\
\text { Public }\end{array}$ & $22 \%$ & $54 \%$ & $45 \%$ \\
Assembly-1 & $6 \%$ & $23 \%$ & $105 \%$ \\
\hline Average & $25 \%$ & $46 \%$ & $85 \%$ \\
\hline
\end{tabular}

Table 52. Actual Total Receptacle Energy Use During Daytime and Nightime Hours (Predicted Values in Parenthesis)

\begin{tabular}{|c|c|c|c|c|c|}
\hline & 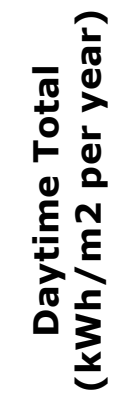 & 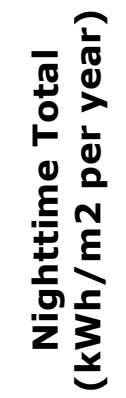 & 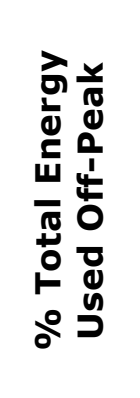 & 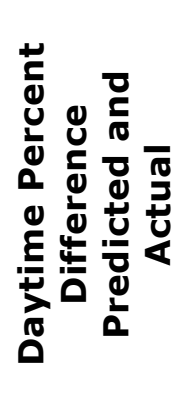 & 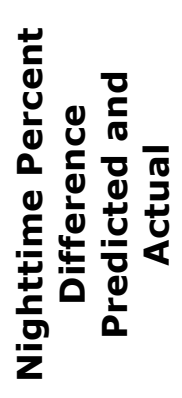 \\
\hline Academic-1 & $\begin{array}{l}12.2 \\
(8.2)\end{array}$ & $\begin{array}{c}8.2 \\
(2.1)\end{array}$ & $\begin{array}{c}40 \% \\
(20 \%)\end{array}$ & $50 \%$ & $287 \%$ \\
\hline Office-3 & $\begin{array}{l}10.0 \\
(7.7)\end{array}$ & $\begin{array}{c}7.3 \\
(2.6)\end{array}$ & $\begin{array}{c}41 \% \\
(27 \%)\end{array}$ & $30 \%$ & $185 \%$ \\
\hline Office-4 & $\begin{array}{c}7.4 \\
(13.2)\end{array}$ & $\begin{array}{c}3.1 \\
(3.6)\end{array}$ & $\begin{array}{c}30 \% \\
(24 \%)\end{array}$ & $-44 \%$ & $-13 \%$ \\
\hline Mail & 8.2 & 6.8 & $47 \%$ & $-58 \%$ & $-48 \%$ \\
\hline Sorting-1 & (19.7) & $(13.0)$ & $(39 \%)$ & & \\
\hline Mail & 6.5 & 5.8 & $50 \%$ & $-20 \%$ & $-22 \%$ \\
\hline Sorting-2 & $(8.1)$ & $(7.5)$ & $(47 \%)$ & & \\
\hline Mail & 8.5 & 7.1 & $44 \%$ & $-41 \%$ & $-10 \%$ \\
\hline Sorting-3 & (14.4) & $(7.8)$ & $(36 \%)$ & & \\
\hline Public & 11.5 & 6.8 & $37 \%$ & $-22 \%$ & $221 \%$ \\
\hline Assembly-1 & $(14.8)$ & $(2.1)$ & $(12 \%)$ & & \\
\hline Average & & & & $-15 \%$ & $86 \%$ \\
\hline
\end{tabular}


Table 53. Actual Percent Time Building Operates as Percent of Max Receptacle Load (Predicted Values in Parenthesis)

\begin{tabular}{llllll}
\hline \multicolumn{5}{c}{ Percent Time at Percent of Max Load } \\
\hline & $\mathbf{0 \% - 2 0 \%}$ & $\mathbf{2 1 \% - 4 0 \%}$ & $\mathbf{4 1 \% - 6 0 \%}$ & $\mathbf{6 1 \% - 8 0}$ & $\mathbf{8 1 - 1 0 0 \%}$ \\
\hline Academic-1 & $0.5 \%$ & $\mathbf{6 4 . 2 \%}$ & $18.9 \%$ & $13.7 \%$ & $2.7 \%$ \\
& $\mathbf{( 5 5 . 4 \% )}$ & $(6.0 \%)$ & $(6.0 \%)$ & $(11.9 \%)$ & $(20.8 \%)$ \\
Office-3 & $0 \%$ & $\mathbf{5 2 . 3 \%}$ & $27.6 \%$ & $19.3 \%$ & $0.7 \%$ \\
& $(0 \%)$ & $\mathbf{( 6 7 . 3 \% )}$ & $(3.0 \%)$ & $(0 \%)$ & $(29.8 \%)$ \\
Office-4 & $\mathbf{6 1 . 4 \%}$ & $14.1 \%$ & $18.3 \%$ & $5.8 \%$ & $0.3 \%$ \\
& $\mathbf{( 4 5 . 2 \% )}$ & $(19.0 \%)$ & $(3.0 \%)$ & $(3.0 \%)$ & $(29.8 \%)$ \\
Mail & $0 \%$ & $24.1 \%$ & $\mathbf{6 6 . 6 \%}$ & $8.7 \%$ & $0.6 \%$ \\
Sorting-1 & $(0 \%)$ & $\mathbf{( 4 3 . 5 \% )}$ & $(20.8 \%)$ & $(26.8 \%)$ & $(8.9 \%)$ \\
Mail & $0 \%$ & $0 \%$ & $\mathbf{5 9 . 3 \%}$ & $36.9 \%$ & $3.8 \%$ \\
Sorting-2 & $(0 \%)$ & $(0 \%)$ & $(0 \%)$ & $(37.5 \%)$ & $(\mathbf{6 2 . 5 \% )}$ \\
Mail & $0 \%$ & $\mathbf{5 5 . 5 \%}$ & $42.7 \%$ & $1.7 \%$ & $0.1 \%$ \\
Sorting-3 & $(0 \%)$ & $\mathbf{( 4 9 . 4 \% )}$ & $(35.7 \%)$ & $(0 \%)$ & $(14.9 \%)$ \\
Public & $37 \%$ & $\mathbf{4 1 . 4 \%}$ & $16 \%$ & $5.4 \%$ & $0.2 \%$ \\
Assembly-1 & $\mathbf{( 4 8 . 2 \% )}$ & $(0 \%)$ & $(7.7 \%)$ & $(4.2 \%)$ & $(39.9 \%)$ \\
\hline
\end{tabular}

\section{Data Variability- Regression Analysis}

While the previous section investigates to what extent the schedule for the load profile was accurately predicted, it should also be considered that error measured between predicted and actual energy use may not be due to the fact that a building's schedule was not accurately predicted, but instead that the building may not have been able to follow any schedule. Buildings with unpredictable occupancy or management issues can appear to have wide week-to-week variations in energy use that does not follow any regular patterns. Consider the two following figures, Figure 92 is the lighting load profile for Public Assembley-1, the profile varies significantly from week-to-week, and was determined to have the highest variability within the data (the details of this will be discussed below). Figure 93 is the receptacle profile for Mail Sorting-2, there is much less variation from week-toweek, and was determined to have the lowest variability within the data (again the details of this will be discussed below). Understanding the error between predicted and actual energy consumption values in these two cases should be approached differently, as they are contributed to by different factors (in one case variability, and in the other inaccurate schedules). 
Legend:

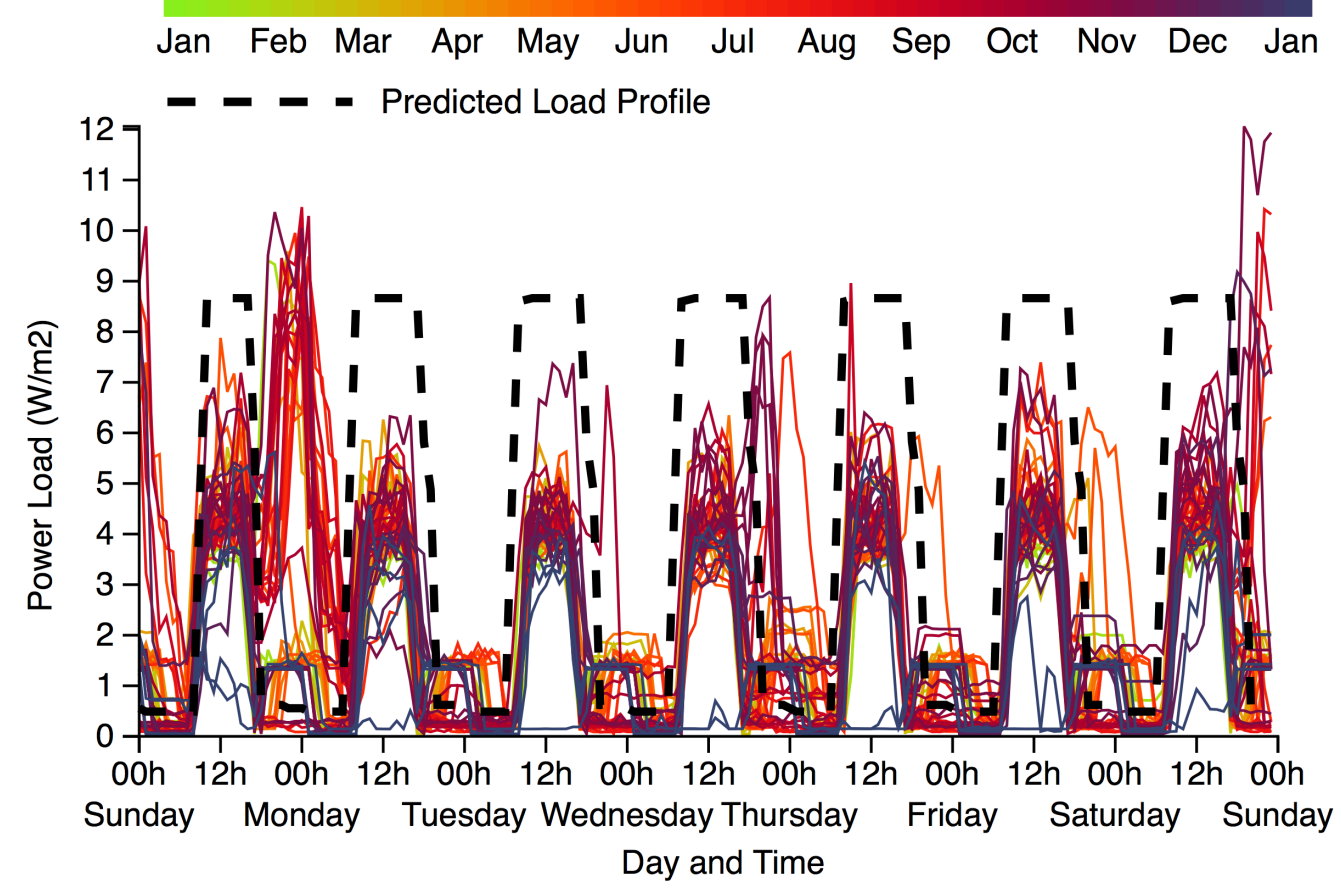

Figure 92. High Data Variability Seen in Lighting Load Profile for Public Assembley-1

Legend:

Jan Feb Mar Apr May Jun Jul Aug Sep Oct Nov Dec Jan

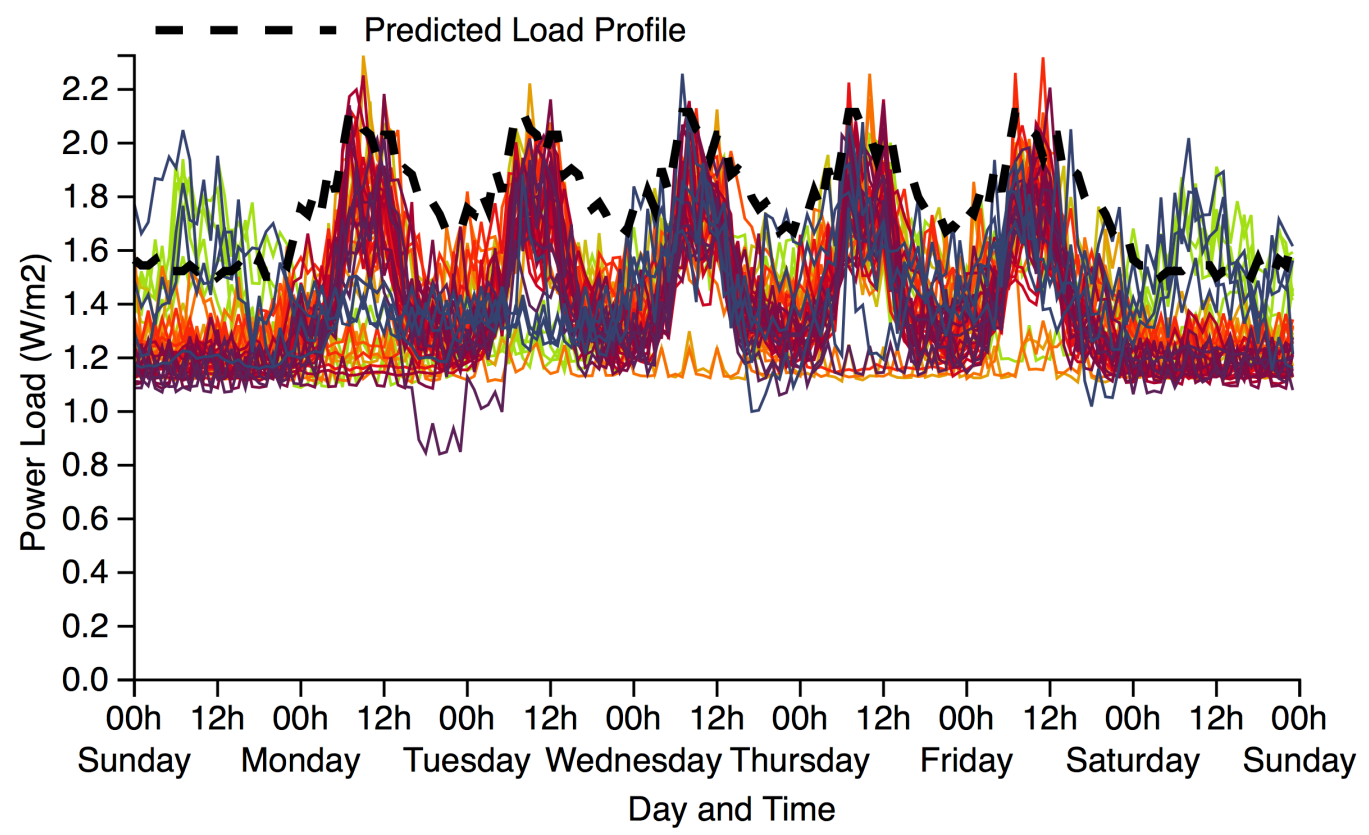

Figure 93. Low Data Variability Seen in Receptacle Load Profile for Mail Sorting-2

In order to determine the overall affect of data variability as a driver behind the error observed between actual and predicted energy use, a regression analysis 
was developed to correlate these two qualities of the data. The data variability was represented with a variability coefficient, this value was calculated by taking the sum of the CVRMSE between every possible combination of weeks in the data set for one building and dividing the number by the possible number of combinations. What is measured is the regularity of the data, and by extension the regularity of the building's actual operations.

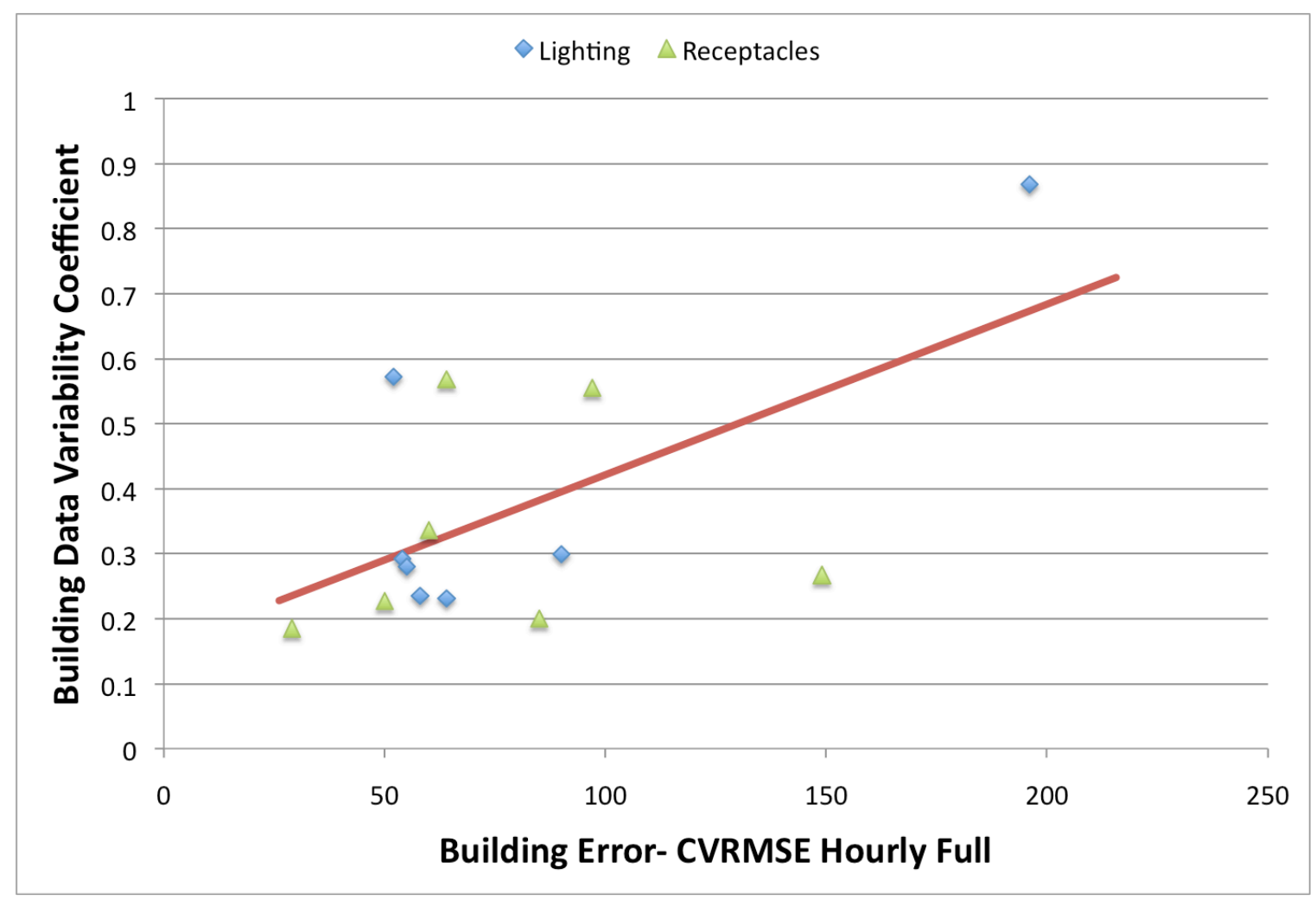

Figure 94. Regression Analysis- Error vs. Data Variability $\left(r^{2}=0.34\right.$, slope $\left.=0.002\right)$

The regression then correlates this values with the error measured between actual and predicted building energy use. The dataset for this regression included both the error and variability for lighting and receptacles, therefore each building appears in the regression as two separate points. Further details on the calculation of the data variability coefficient is available in Section 3.10. The results of the correlation are presented in Figure 94.

The regression found an $r^{2}$ value of 0.34 , suggesting that variation in the data was partially correlated to error levels between actual and predicted energy use. This aspect of the performance gap is especially out of the hands of the modelers, 
and could only be rectified by changes to building operations. It should be said however that data variability doesn't necessarily imply poor operations, simply irregular operations (including variable occupancy and usage patterns). This is anecdotally supported by this research. The lighting load profile for Public Assembly-1, the building with the highest error and the highest data variability, is subject to variable occupancies which are an integral part of the building's programming, thus out of control of the management team of the building and not necessarily something that can be "corrected".

The regression analysis also allows us to split the buildings (and end-uses) into three categories, those with disproportionately high data variability in relation to building error (as represented by the buildings that place above the regression line), those with proportionate data variability in relation to building error (as represented by the buildings that fall on or near the regression line), and those with disproportionately low data variability in relation to building error (as represented by the buildings that place below the regression line). These groups are as follows:

1. Mail Sorting-2 lighting, Public Assembley-1 lighting, Office-4 receptacles, and Public Assembley-1 receptacles

2. Mail Sorting-1 lighting, Mail Sorting-3 lighting, Academic-1 receptacles, Office-3 receptacles, and Mail Sorting-2 receptacles

3. Academic-1 lighting, Office-3 lighting, Office-4 lighting, Mail Sorting-1 receptacles, and Mail Sorting-3 receptacles

It is interesting to note that both receptacles and lighting end uses are divided amongst the three groups quite evenly, indicating that neither lighting nor receptacles loads are more prone to variability. And with the exception of Public Assembly-1 (which has already been highlighted as the building exhibiting the most variability) the buildings are scattered across the 3 groups with no clear pattern.

\subsection{Daylight Regression}

Considering that the majority of buildings were designed with a consideration towards natural daylighting, and that significant seasonal variation 
was present within the data, it was determined that it would be relevant to consider whether lighting availability contributed to the variation in lighting energy consumption.

It is understood that this type of analysis is complicated by a host of issues, such as the fact that certain daylighting design features may be more effective in the winter months despite less daylight hours, shading on the building may influence what time of year daylighting is most effective, or certain occupancy patterns may skew the energy use towards certain times of year. Therefore without further information on the particularities of each building, this will remain a somewhat cursory, exploratory investigation of lighting energy use as it relates to daylight availability. The analysis will take the form of a regression model correlating monthly total energy use per day with monthly bright sunshine hours per day. Table 53 lists the results, which all in all, paint a fairly inconclusive picture.

Green cells highlight buildings with fairly strong correlation of increased daylight hours to reduced lighting energy consumption, red cells highlight buildings with correlation of increased daylight hours to increased lighting energy consumption. It is interesting to see strong negative correlation almost as often as strong positive correlations.

A strong positive correlation indicates increased lighting energy use as daylight hours increase, which suggests poor use of natural daylight. These positive correlations could also be explained by seasonal changes in the use of the building. For example heavier occupancy in the summer could drive up lighting energy use, while low winter occupancy would artificially depress lighting energy use. This type of scenario may take place in building's hosting events, for example Public Assembly-1.

On the other hand a strong negative correlation could indicate that as daylight hours increase, daylighting strategies in a building reduce the need for electric lighting. However it should also be noted that trends in energy use that correspond with a negative correlation may also be affected by building usage patterns. For example in academic buildings, the school semester coincides with periods of the year with less daylight, and therefore any simple single variable 
regression analysis may exaggerate the correlation between daylight hours and energy use.

Table 54. Results of Daylighting Regression Analysis

\begin{tabular}{|c|c|c|}
\hline & \multicolumn{2}{|c|}{ Median Peak Load } \\
\hline & $r^{2}$ & Slope \\
\hline Academic-1 & 0.45 & -0.26 \\
\hline Office-3 & 0.01 & -0.04 \\
\hline Office-4 & 0.36 & -0.13 \\
\hline Mail Sorting-1 & 0.43 & 0.13 \\
\hline Mail Sorting-2 & 0.00 & -0.01 \\
\hline Mail Sorting-3 & 0.42 & -0.05 \\
\hline $\begin{array}{c}\text { Public } \\
\text { Assembly-1 }\end{array}$ & 0.28 & 0.17 \\
\hline
\end{tabular}




\subsection{Conclusion}

\subsection{Data Quality}

Data quality issues throughout this research prevented the data set from being used to its fullest potential. The majority of the buildings in the study demonstrated some data quality issues. These included missing data, incorrect measurements, and incorrect dates (wrong dates as well as repeating dates). These are likely a result of both issues with the physical meter installation and operations, as well as an issue with data formatting and storage. Unfortunately investigating the source and nature of the data quality issues was beyond the scope of this investigation.

It is not entirely surprising that issues of this sort have arisen in such a young field of study. At this point little best practice guidelines exist for collecting, managing or analyzing data of this nature. Further articulating the utility of this type of data will certainly provide a motivation for effective data management practices to be developed going forward. Towards these ends this study can suggest several recommendations:

- Standardized and robust data formats are required to facilitate proper storage, transfer and manipulation of the data. For example, while Excel is an extremely powerful tool, interoperability issues between versions affected date values while transferring data between files.

- Standardized procedures to deal with the data quality issues when they arise are required. This is linked to better understanding the nature of the issues, and therefore providing an avenue to properly address them when they arrive.

Further issues in regards to the data quality include reliance primarily on models produced with one modeling software and from one source. The modeling software used for these buildings, EE4, is a relatively simple software in comparison to eQuest or EnergyPlus, and thus comes with limitations to the number of 
situations it can accurately model commensurate with its complexity. For some HVAC systems it requires some creativity on the part of the modeler. On the other hand as EE4 was commonly used in Canada until recently due to its adoption by the Commercial Buildings Incentive Program and as an approved method to demonstrate compliance with LEED energy credits, it is important to understand the limitations it imposes on modeling buildings and conducting research on those models. One example specific to this research was that it was found that EE4 would not produce hourly results for its heating or cooling predictions if any warnings were present in the models. Warnings of this kind were present nearly unanimously amongst all the models in question, as modelers override defaults in order to tailor the models to the buildings in question. Further research could be conducted into what exactly is the difference and limitations of each software in regards to the others.

In addition sourcing the models from one company should also be recognized as a limitation to the quality of the research. Every company will have a particular methodology and protocols towards completing models. These particularities will affect the models in ways that make the results from this research not necessarily generalizable to the industry as a whole. A solution to this would be to source models and data from a diverse number of companies. This will become more possible as similar types of measurement and verification services are offered by a greater number of firms. 


\subsection{Submetering Practices}

Submetering practices, circumscribed by client imposed budget constraints, significantly limited the number and type of buildings from the data set on which analysis was able to be performed. The majority of buildings that were included were amongst the smaller and less complex of the buildings in the data set. Furthermore data quality issues limited the ability to accurately associate electricity consumption with the respective end-use for buildings where analyses was conducted. This finding was supported in case of all of the buildings with documentation detailing the breakdown of loads installed on each panel (Appendix D). Unfortunately this was only the case for a limited number of buildings. This was a more significant issue with receptacles where up to half of the installed loads on a "receptacles" meter where associated with other end-uses. This was significantly less of a problem with lighting (often only $10 \%$ of the installed load was incorrectly defined). However lighting meters also experienced the problem of exterior and interior lighting being metered together, whereas energy models only include interior lighting, and exterior lighting is considered unregulated.

These issues highlight the importance of carefully creating a submetering strategy at the design stage of the building. To achieve the maximum utility that can be made from metering data, certain improvements must be made in regards to the design and implementation of metering systems. Ultimately this will require developing a strong financial or environmental case for advanced metering practices and will require educating building owners and policy makers to the benefits of energy conservation measures. In part this will also occur as metering cost come down due to wider market acceptance and improved technology. Similar conclusions have been made by others such as Chisholm, "Making Sense of Metering" (2010), in which she notes that use of metering data is held back by client driven value engineering and data management issues. Discussion with industry professionals suggest that to move submetering practices forward designers must 
indicate to clients that submetering is not an optional feature, but instead a crucial part of the building management system.

An optimal submetering strategy would require the implementation of the following suggestions:

- Metering building energy end use must be more strictly separated. As we have seen, due to cost issues, different end uses can be included on the same electrical panels (such as receptacles and HVAC) which will then be measured by the same meter. This muddies any understanding that can be developed on either of the two uses.

- A regular and predictable set of metering categories should be available, such as those provided by energy models results (For example fans, heating, cooling or receptacles). This will allow for more widespread comparison between buildings with the same occupancies or sector (for example, residential, retail, office), and between model results and measurements. Ideally unregulated loads should be kept separate from other loads measured by models in order to allow for direct comparisons (for example separating exterior lighting from other lighting loads), this may not necessarily be a straight forward procedure because to a certain extent building codes are moving to include previously unregulated loads into the code.

- Metering categories in different types of building with different occupancies as well as for different sophistication and types of systems should be developed to account for particular challenges in metering various systems.

- For even further levels of details metering should closely match the zones as described in model. This is more possible for certain types of systems, or for certain end-uses. For example a forced air system will not be able to meter the amount of heating required for each space, however a zone by zone breakdown of lighting energy use may certainly be feasible.

- Long (multi-year) metering periods are required to understand building operations over time, and in order to better identify what are seasonal trends and what are short term phenomena. 
In a more realistic world, where the vast majority of projects will not have adequate financial resources to implement stringent submetering plans, it may be much more beneficial to only meter a limited area of the building that is representative of the whole. And thus provide a partial, but more accurate snapshot into the building's performance. Furthermore the results suggest that relatively speaking natural gas consumption is well understood compared to the welter of various electricity end-uses. In light of this, it may also be a relevant strategy to focus primarily on submetering electricity end-uses.

\subsection{Receptacles and Lighting Performance Gap}

The goal of this research was to investigate the nature of the performance gap using lighting and receptacle submetering data as well as whole building energy models. Several observations indicate that operations and occupancy issues are responsible for a significant portion of the gap in performance for lighting and receptacles. This is suggested primarily by a correlation between data variability (energy use/occupancy variability) and modeling error, a lack of correlation between lighting energy use and daylight availability, and a disproportionate increase in energy use primarily during non-peak hours.

As mentioned results from regression analysis suggests data variability, likely driven by occupancy and management irregularity in the buildings is correlated to the error calculated comparing metered and modeled energy use. The week-to-week variability in the data (the extent energy use varied from one week to the next) appeared to be a source $\left(\mathrm{r}^{2}=0.34\right)$ of the measured difference between energy predicted and actual data. This suggests that the assumptions made in the energy model did not match the actual energy use because the operation of the buildings were not regular, and that week-to-week changes in the building, likely related to occupancy or other relatively intransient management issues, were a source of the error. Any building of this sort that has irregular operations as an integral part of the buildings programming (such as occasional events) will appear to have a larger performance gap because variability in operations are incredibly 
difficult to model. This conclusion is also supported by the inability to find a strong correlation between lighting energy use and daylight availability, despite the fact that daylighting design was included into the buildings. The lack of evidence that energy use was determined by design features, further bolster the notion that occupancy and management are responsibility for increased energy use.

These two lines of argumentation should only be taken as an initial investigation into the area. In order to provide more conclusive arguments in those regards further information and analysis would be necessary. In regards to data variability, further validation of the concept is required. In regards to evaluating daylighting effectiveness a number of crucial pieces of additional data would be required. This includes additional information on the building's characteristics (for example massing, orientation, and shading), daily occupancy patterns, as well as finer grain daylight availability data.

Further supporting this observation, for both lighting and receptacle loads the additional energy use that was present in the buildings primarily occurred overnight. Nighttime loads were observed to be on average $153 \%$ and $85 \%$ higher than prediction for lighting and receptacles, respectively. And daytime loads were on average $16 \%$ higher and 15\% lower for lighting and receptacles, respectively. This would suggest that management practices and occupancy to be at fault. While for the most part one would not expect to find occupancy issues affecting overnight loads, in several cases it was directly possible to attribute additional energy use to these types of issues, specifically in the case of late night occupancy of computer labs and other spaces in Academic-1 and unexpected additional late night events in Public Assembley-1.

On the other hand it was not found that the ASHRAE tolerance values provided for comparing actual and predicted energy use in Guideline 14, provided a useful framework for understanding the size of the performance gap amongst the buildings. Nor was there adequate information in order to develop values for enduses in a sufficiently robust manner. This is partly due to the fact that no documentation was available to determine how the original ASHRAE error tolerances were calculated. 
Other researchers have come to similar conclusions. Samuelson, Ghorayshi and Reinhart state that the main discrepancy between models and actual performance is a result of how the buildings are used and operated. They even go on to question the fundamental ability of the modeler to predict loads due to specificities of building operations (Samuelson, Ghorayshi and Reinhart, 2015). This echoes the discussion raised recently by Ted Kesik (2014). In his paper he argues that it is more important to understand the performance of the building's passive systems (daylighting potential and envelope thermal performance for example) rather than their active systems (mechanical and building occupancy for example). In the end it may turn out that understanding both active and passive building elements will be important especially in light of the fact that active system can drive energy use. However it does appear that Kesik has a strong point to be made that these aspects of a building should be understood separately, and that in fact it is how the passive and active systems interact that will be crucial to understand in the future. For example it is difficult to understand the performance of lighting energy use in a building without first understanding the fundamental daylighting potential of the building. In the end this can be summarized simply, judging the performance of the building in relation to a predicted energy model appears to be fundamentally misleading, with factors totally outside the control of the energy models ability to predict often driving energy use. While on the other hand physical characteristics of the building such as the thermal resistance of the envelope, and excluding issues of management and occupancy, can be reliably predicted.

While it is clear that end-uses driven by occupancy or management, such as lighting and receptacle loads, can be very difficult to predict, this research can suggest at least one practical improvement to energy modeling practices. Lighting and receptacle load profiles are not accurately predicting overnight energy use. From this data set, it is witnessed that much higher energy use is experienced, during overnight periods, than expected. Energy modeling practices should be changed to reflect this reality. 


\subsection{Future Work}

Despite the difficulties associated with large volumes of data and rough data sets, the line of investigation explored in this thesis holds promise as a research methodology to define the nature of the performance gap in buildings. As submetering becomes more sophisticated and widespread, this genre of data will become more readily available for researchers to use to investigate building performance issues. The use of this type of data has several benefits. The automated nature of the data collection systems, not without their own challenges, requires less labour by researchers to collect, and therefore will be easily collected for a large number of buildings. The more standardized this process becomes the larger the data sets will be able to be. On the other hand this type of submetering data is also directly useful to building owners in regards to building operations and management. This overlap in interest between researchers and building owners, will also lighten the burden on researchers conducting this genre of work.

Further research along these lines has two options, firstly to continue and develop a broad understanding of specific aspects of building operations amongst a large number of buildings, and secondly to develop a more in depth description of particular buildings.

The first approach has the merits of large sample sizes from which generalizations about a certain region or sector's building stock can be made. This approach largely follows the path of building benchmarking studies that examine energy use at the building level. This kind of benchmarking could be expanded to also include benchmarking particularly for specific energy end-uses, such as lighting or receptacle loads as was the focus of this work. Figures of this sort would have the ability to allow building owners to pin point the source of unnecessary energy consumption or other performance issues in their buildings, whereas traditional benchmarking only has the ability to suggest whether or not a building has performance issues at all. This kind of research would allow researchers to highlight buildings for further examination. Pursuing this line of research will continue to be difficult while high quality submetering is not standard practice for the industry. 
The second option has the merits of providing more detailed information about an individual building. This approach is more in line with certain "case-study" oriented building performance evaluations as well as the techniques of building commissioning. Ultimately submetering data could be compared and used to enhance data from other sources such as measurements of environmental conditions (for example temperature and relative humidity) or surveys addressing occupant satisfaction. This type of research is ideally suited for exploring specific design strategies in depth, perhaps in the case of particularly innovative buildings, and evaluating their performance. This research will continue to be more achievable while high quality submetering systems remain less common. 


\section{Appendix A- MMM's Modeling and Calibration Process}

Samuelson, Arayshi and Reinhart found that the calibration process reduced under prediction of energy consumption from $36 \%$ to $7 \%$. This research (with a more limited) found that the calibration process on average reduced the under prediction of energy consumption from $23 \%$ to $4 \%$ for total energy, that it on average reduced the under prediction of energy consumption for natural gas energy use from $22 \%$ to $14 \%$, and that it on average reduced the under prediction of energy consumption for lighting energy use from $22 \%$ to $0 \%$. The results of this calibration process are visible in the following four figures. Figure 95 and Figure 96 show the full comparison between actual and predicted total energy consumption, including calibration impacts, using a data set of 34 buildings. While Figure 97 and Figure 98 show the comparison between actual and predicted energy consumption per energy source, including the calibration impacts, for a data set of approximately 19 buildings. 


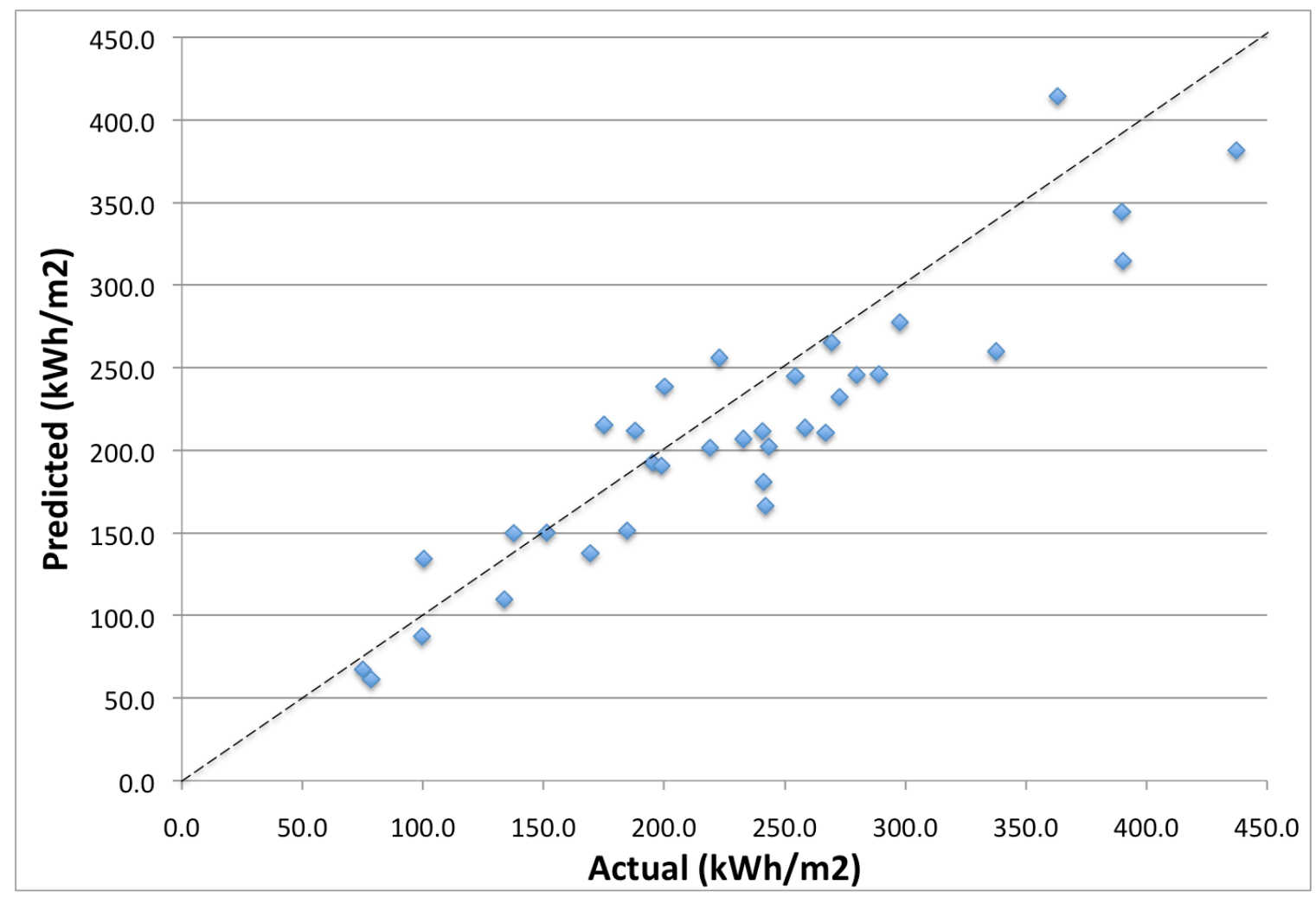

Figure 95. Actual Versus Predicted (calibrated) Total Building Energy Use.

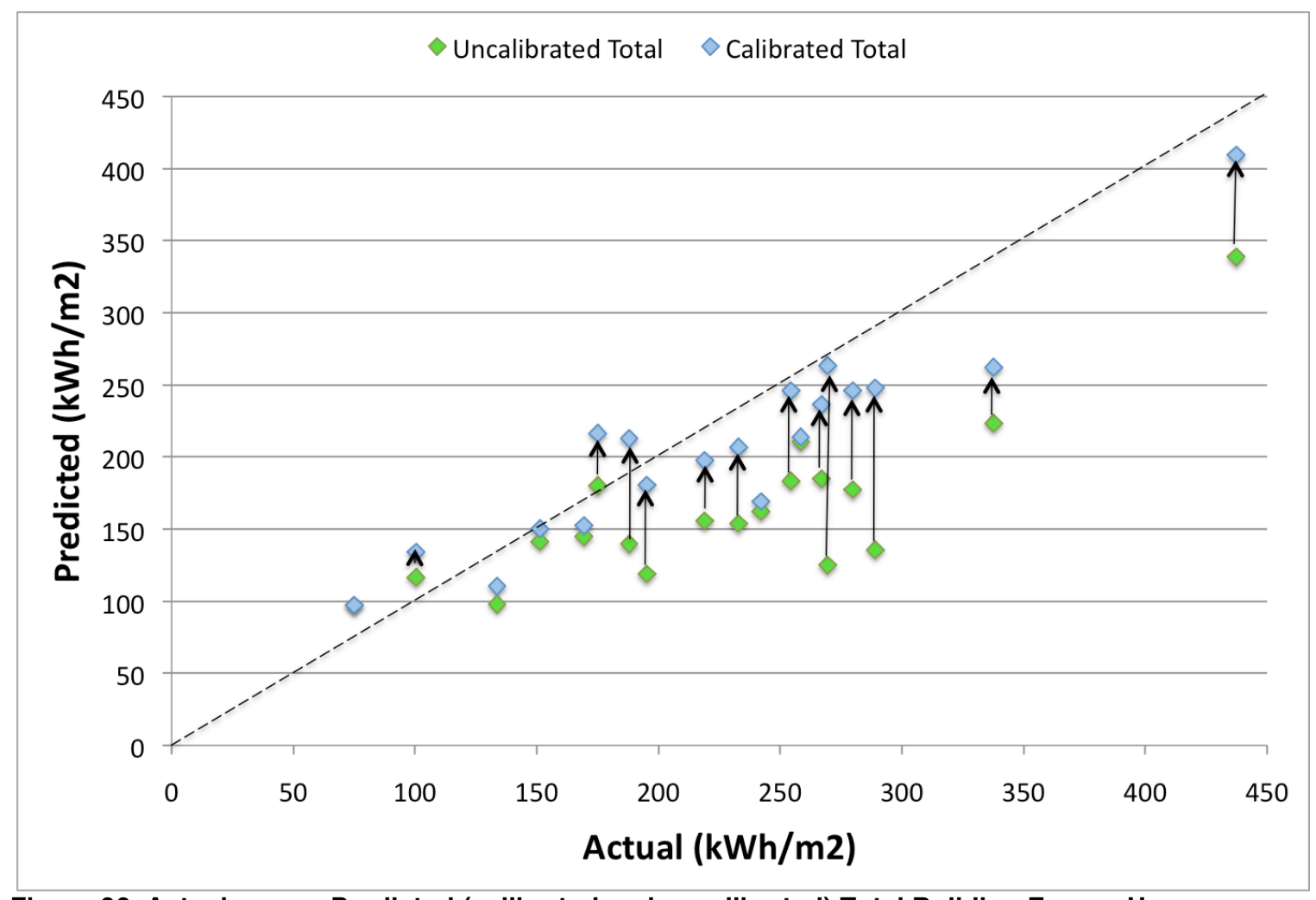

Figure 96. Actual versus Predicted (calibrated and uncalibrated) Total Building Energy Use. 


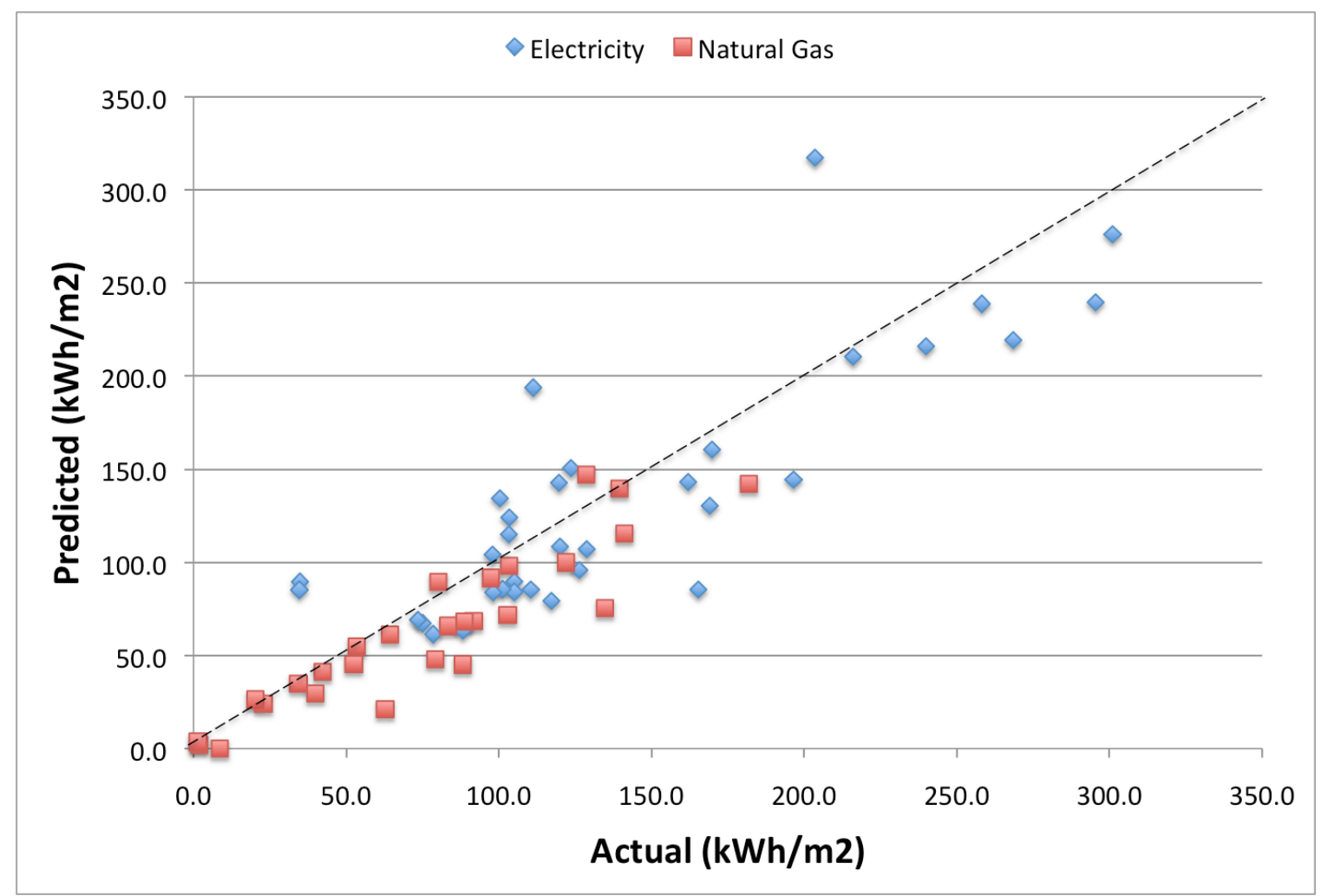

Figure 97. Actual Versus Predicted (calibrated) Total Energy Use by Type.

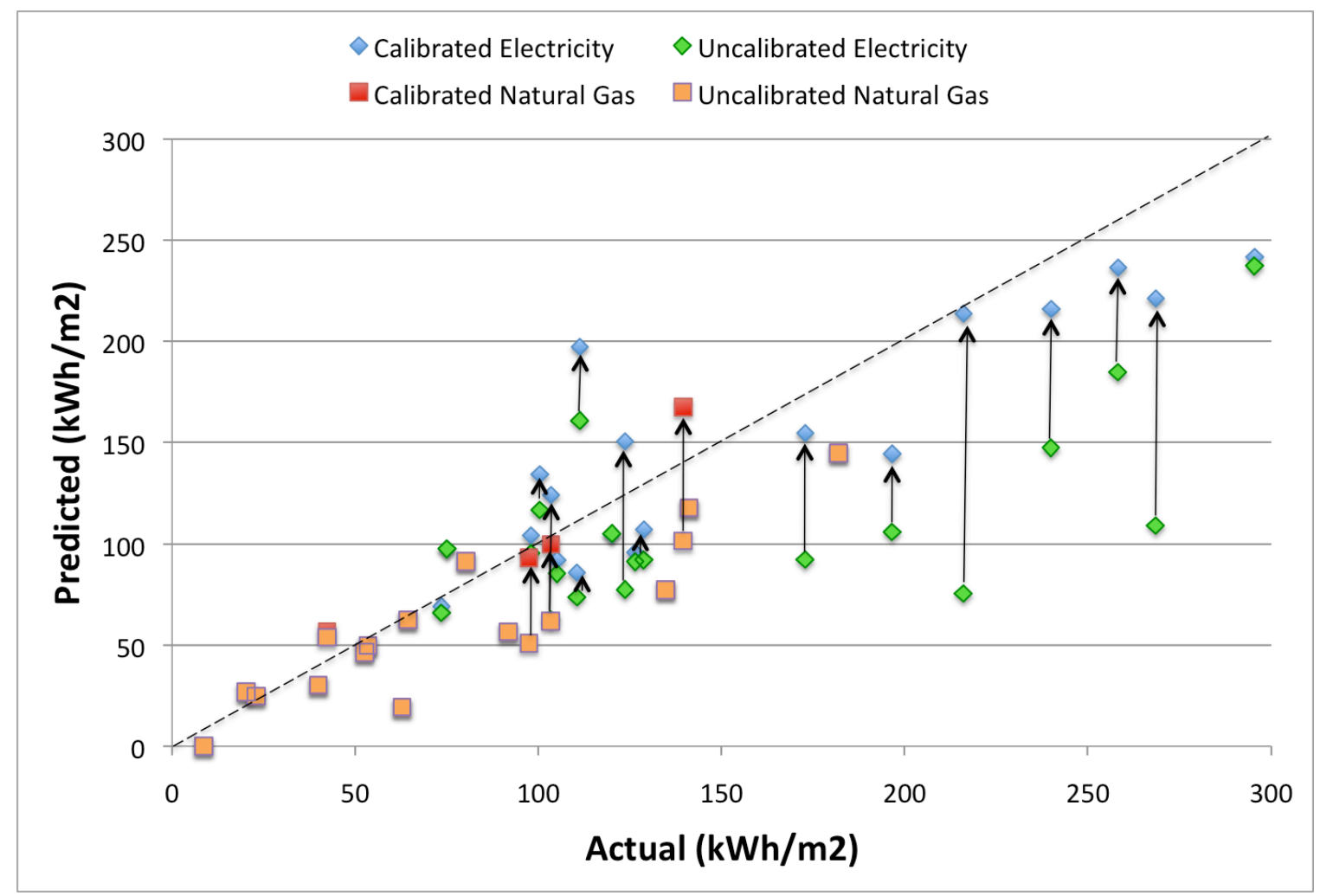

Figure 98. Actual Versus Predicted (calibrated and uncalibrated) Total Energy Use by Type. 


\section{Appendix B- Lighting Modeling Schedules}

The following appendix details the zone characteristics used in the building energy modeling. Varying power density were attributed to the buildings' zones based on zone occupancy. The following table describes the area weighted average power density for each building.

Figure 99. Lighting Power Density Summary

\begin{tabular}{cc}
\hline Building & $\begin{array}{c}\text { Area Weighted } \\
\text { Average Power } \\
\text { Density }\left(\mathbf{W} / \mathbf{m}^{\mathbf{2}}\right)\end{array}$ \\
\hline Academic-1 & 7.4 \\
Office-3 & 8 \\
Office-4 & 8.1 \\
Mail Sorting-1 & 8.6 \\
Mail Sorting-2 & 9.6 \\
Mail Sorting-3 & 10.9 \\
Public Assembly-1 & 9.8 \\
\hline
\end{tabular}

\section{Academic-1}

The model only employs one schedule representing $100 \%$ of the installed lighting capacity, and $100 \%$ of the scheduled energy use.

Table 55. Academic-1: Zone and Schedule Description

\begin{tabular}{cccc}
\hline Name & Schedule & PD $\left(\mathbf{W} / \mathbf{m}^{\mathbf{2}}\right)$ & $\begin{array}{c}\text { Floor Area } \\
\left(\mathbf{m}^{\mathbf{2}}\right)\end{array}$ \\
\hline Zone 1 & SCHED-A & 6.4 & 801 \\
Zone 2 & SCHED-A & 8.8 & 584 \\
Zone 3 & SCHED-A & 8.5 & 118 \\
Zone 4 & SCHED-A & 9.1 & 327 \\
Zone 5 & SCHED-A & 9.6 & 483 \\
Zone 6 & SCHED-A & 9.2 & 438 \\
Zone 7 & SCHED-A & 6.8 & 463 \\
Zone 8 & SCHED-A & 12.4 & 247 \\
Zone 9 & SCHED-A & 5.5 & 670 \\
\hline
\end{tabular}




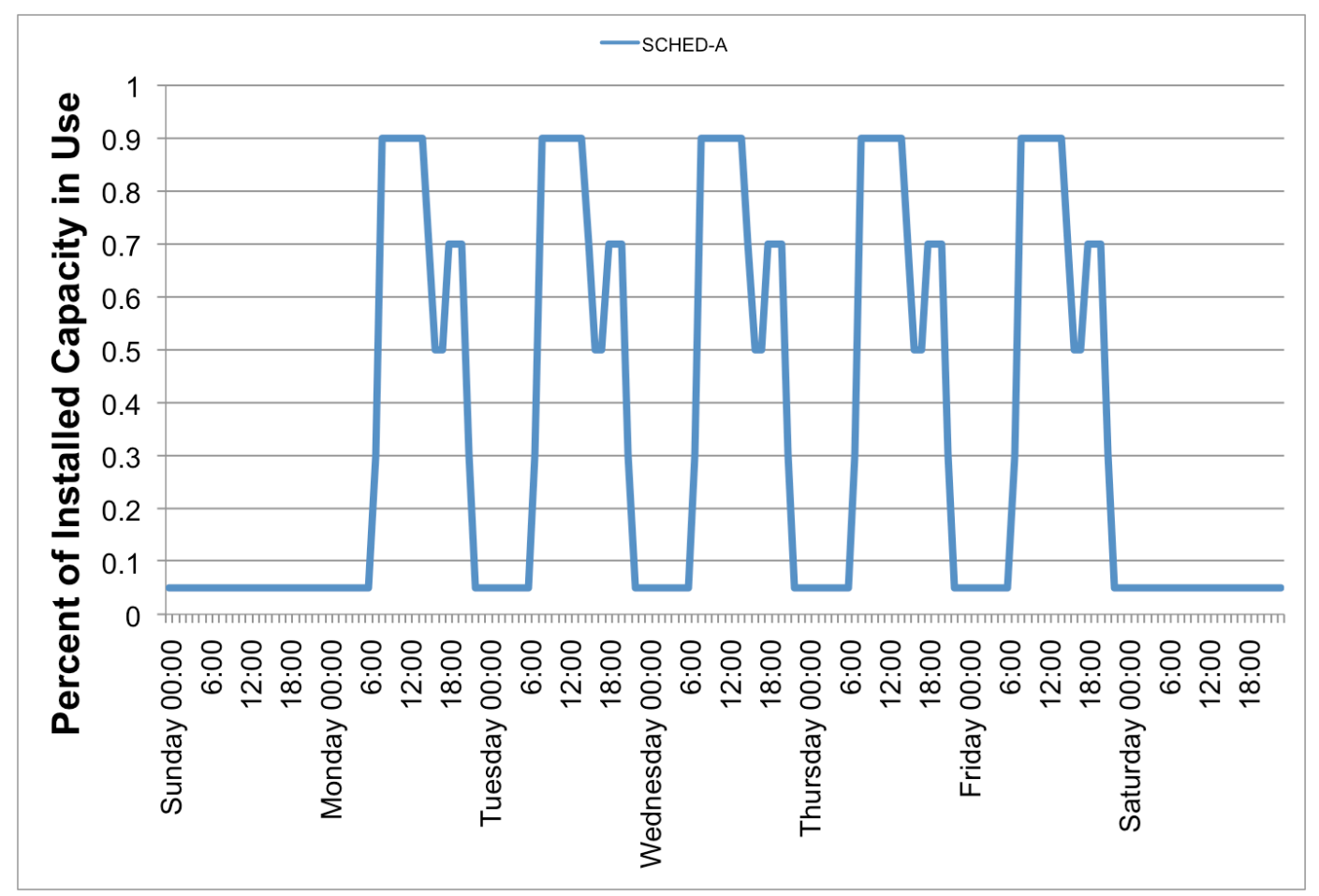

Figure 100. Academic-1: Hourly Breakdowns for Lighting Schedule A

\section{Office-3}

The model employs two schedules. Schedule A represents $43 \%$ of the installed lighting capacity, and $83 \%$ of the scheduled energy use. Schedule B represents $57 \%$ of the installed lighting capacity, and $17 \%$ of the scheduled energy use.

Table 56. Office-3: Zone and Schedule Description

\begin{tabular}{|c|c|c|c|}
\hline Name & Schedule & PD $\left(W / m^{2}\right)$ & $\begin{array}{c}\text { Floor Area } \\
\left(\mathrm{m}^{2}\right)\end{array}$ \\
\hline Zone 1 & SCHED-A & 29.1 & 80 \\
\hline Zone 2 & SCHED-A & 7.1 & 233 \\
\hline Zone 3 & SCHED-A & 8.7 & 135 \\
\hline Zone 4 & SCHED-A & 6.5 & 155 \\
\hline Zone 5 & SCHED-A & 8.1 & 135 \\
\hline Zone 6 & SCHED-B & 7.3 & 3,233 \\
\hline Zone 7 & SCHED-B & 8.1 & 413 \\
\hline Zone 8 & SCHED-B & 6.2 & 307 \\
\hline Zone 9 & SCHED-B & 7.0 & 368 \\
\hline
\end{tabular}




\begin{tabular}{cccc}
\hline Zone 10 & SCHED-B & 6.4 & 307 \\
Zone 11 & SCHED-A & 8.0 & 213 \\
Zone 12 & SCHED-A & 5.7 & 159 \\
Zone 13 & SCHED-A & 7.5 & 213 \\
Zone 14 & SCHED-A & 6.2 & 160 \\
Zone 15 & SCHED-A & 7.3 & 1,207 \\
Zone 16 & SCHED-A & 7.0 & 538 \\
\hline
\end{tabular}

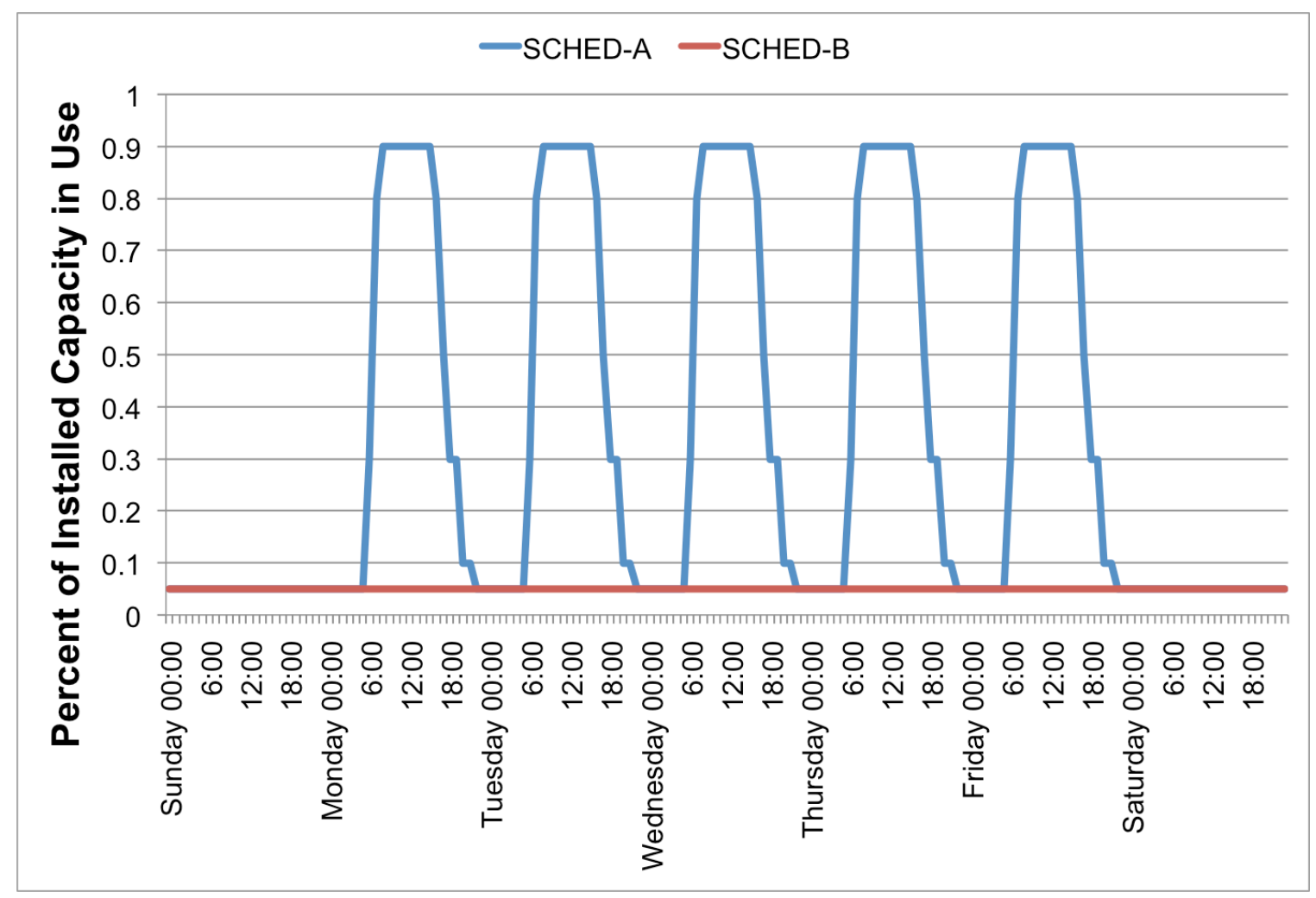

Figure 101. Office-3: Hourly Breakdowns for Lighting Schedules A and B

\section{Office-4}

The model employs two schedules. With Schedule A accounts for 35\% of the installed lighting capacity, and 39\% of the scheduled energy use. Schedule B accounts for the remaining $65 \%$ percent of the installed lighting capacity and $61 \%$ of the scheduled energy use. 
Table 57. Office-4: Zone and Schedule Description

\begin{tabular}{|c|c|c|c|}
\hline Name & Schedule & PD $\left(W / m^{2}\right)$ & $\begin{array}{c}\text { Floor Area } \\
\left(\mathrm{m}^{2}\right)\end{array}$ \\
\hline Zone 1 & SCHED-A & 14.9 & 31 \\
\hline Zone 2 & SCHED-A & 7.4 & 145 \\
\hline Zone 3 & SCHED-B & 3.8 & 19 \\
\hline Zone 4 & SCHED-B & 8.7 & 92 \\
\hline Zone 5 & SCHED-B & 6.2 & 11 \\
\hline Zone 6 & SCHED-B & 9.2 & 34 \\
\hline Zone 7 & SCHED-B & 6.0 & 19 \\
\hline Zone 8 & SCHED-B & 5.7 & 107 \\
\hline Zone 9 & SCHED-B & 5.6 & 14 \\
\hline Zone 10 & SCHED-B & 8.4 & 10 \\
\hline Zone 11 & SCHED-B & 7.2 & 224 \\
\hline Zone 12 & SCHED-B & 5.9 & 245 \\
\hline Zone 13 & SCHED-B & 5.4 & 90 \\
\hline Zone 14 & SCHED-B & 8.1 & 90 \\
\hline Zone 15 & SCHED-B & 3.9 & 56 \\
\hline Zone 16 & SCHED-B & 8.4 & 91 \\
\hline Zone 17 & SCHED-B & 11.5 & 38 \\
\hline Zone 18 & SCHED-B & 4.8 & 59 \\
\hline Zone 19 & SCHED-A & 11.4 & 162 \\
\hline Zone 20 & SCHED-A & 17.4 & 161 \\
\hline Zone 21 & SCHED-B & 9.3 & 195 \\
\hline Zone 22 & SCHED-B & 11.3 & 30 \\
\hline Zone 23 & SCHED-B & 3.9 & 57 \\
\hline Zone 24 & SCHED-B & 6.1 & 59 \\
\hline Zone 25 & SCHED-B & 6.0 & 130 \\
\hline Zone 26 & SCHED-A & 4.7 & 15 \\
\hline Zone 27 & SCHED-B & 7.1 & 31 \\
\hline Zone 28 & SCHED-B & 6.6 & 114 \\
\hline Zone 29 & SCHED-B & 4.8 & 14 \\
\hline Zone 30 & SCHED-B & 7.3 & 22 \\
\hline Zone 31 & SCHED-B & 12.7 & 34 \\
\hline
\end{tabular}




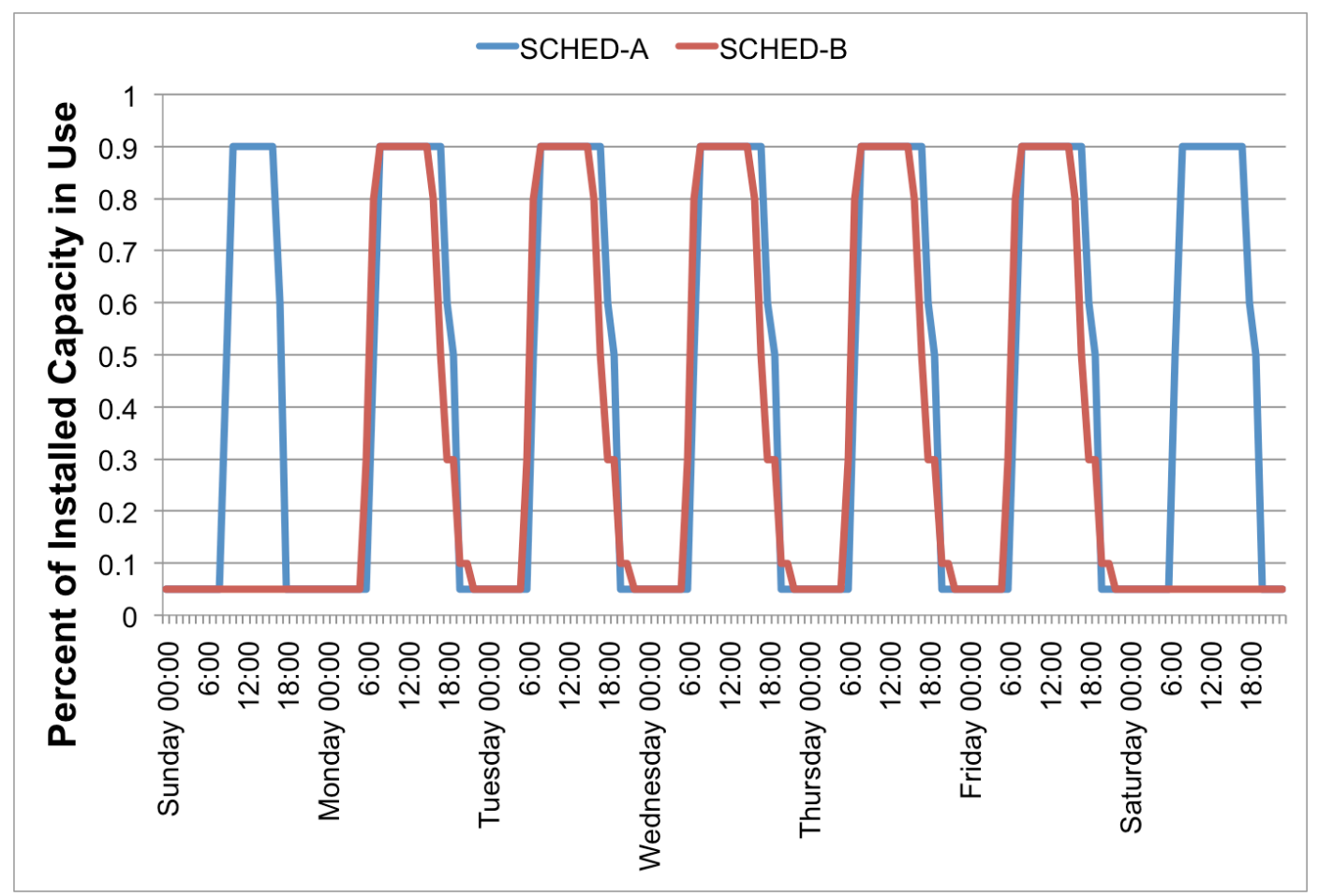

Figure 102. Office-4: Hourly Breakdowns for Lighting Schedules A and B

\section{Mail Sorting-1}

The model only employs one schedule representing $100 \%$ of the installed lighting capacity, and $100 \%$ of the scheduled energy use.

Table 58. Mail Sorting-1: Zone and Schedule Description

\begin{tabular}{|c|c|c|c|}
\hline Name & Schedule & $P D\left(W / m^{2}\right)$ & $\begin{array}{c}\text { Floor Area } \\
\left(\mathrm{m}^{2}\right)\end{array}$ \\
\hline Zone 1 & SCHED-A & 9.5 & 819 \\
\hline Zone 2 & SCHED-A & 7.6 & 720 \\
\hline Zone 3 & SCHED-A & 8.4 & 55 \\
\hline Zone 4 & SCHED-A & 7.9 & 89 \\
\hline
\end{tabular}




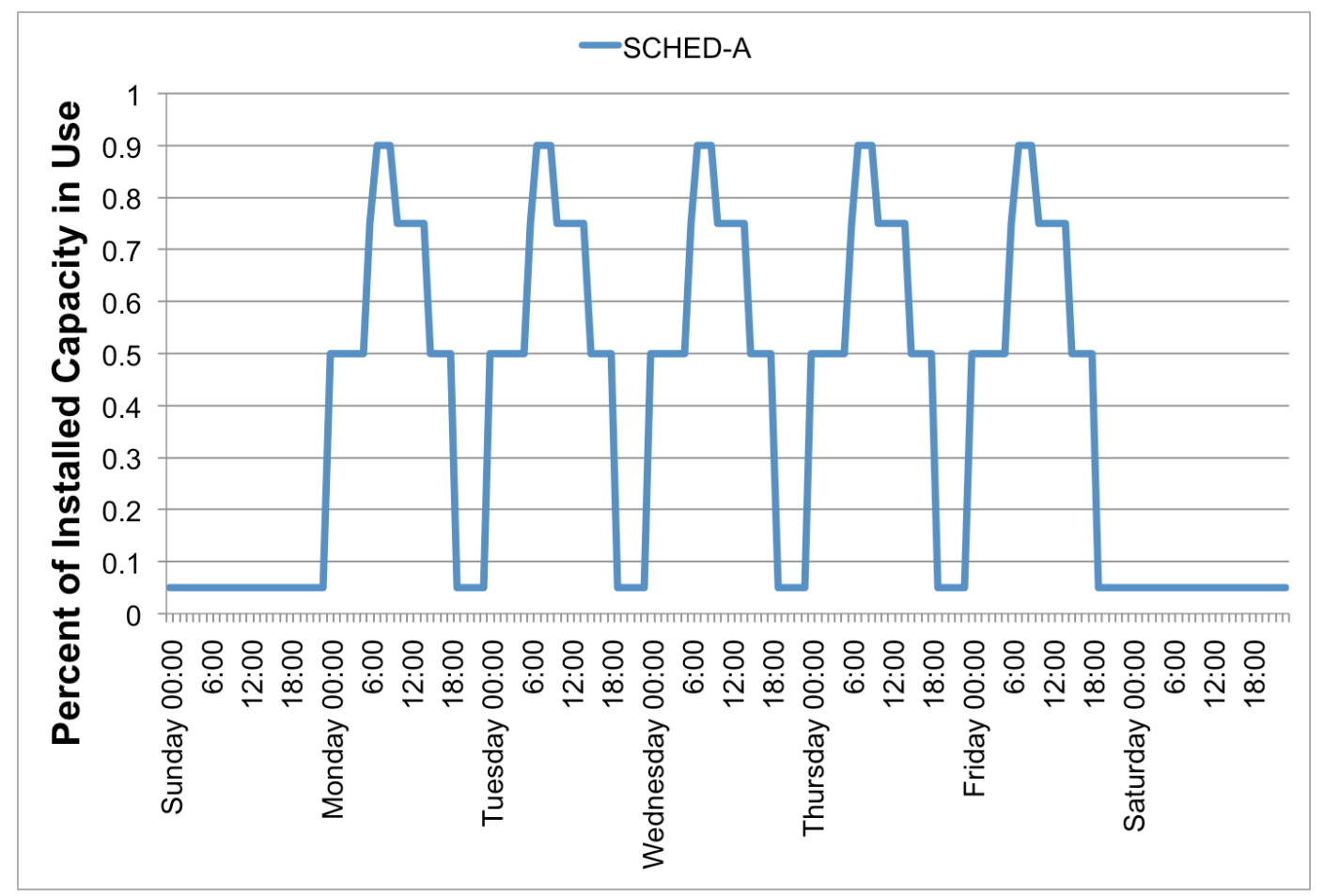

Figure 103. Mail Sorting-1: Hourly Breakdowns for Lighting Schedule A

\section{Mail Sorting-2}

The model employs 3 schedules. Schedule A (sortation area) represents $87 \%$ of the installed lighting capacity and represents $90 \%$ of the scheduled energy use. Schedule B (office) represents $5 \%$ of the installed lighting capacity and $3 \%$ of the scheduled energy use. Schedule C (other) represents $8 \%$ of the installed lighting capacity and $6 \%$ of the scheduled energy use.

Table 59. Mail Sorting-2: Zone and Schedule Description

\begin{tabular}{cccc}
\hline Name & Schedule & PD $\left(\mathbf{W} / \mathbf{m}^{\mathbf{2}}\right)$ & $\begin{array}{c}\text { Floor Area } \\
\left(\mathbf{m}^{\mathbf{2}}\right)\end{array}$ \\
\hline Zone 1 & SCHED-A & 10.1 & 1,370 \\
Zone 2 & SCHED-A & 8.9 & 213 \\
Zone 3 & SCHED-A & 10.2 & 1,320 \\
Zone 4 & SCHED-B & 10.3 & 202 \\
Zone 5 & SCHED-A & 10.1 & 1,145 \\
Zone 6 & SCHED-A & 10.1 & 1,210 \\
Zone 7 & SCHED-C & 4.5 & 281
\end{tabular}




\begin{tabular}{cccc} 
Zone 8 & SCHED-C & 7.1 & 379 \\
Zone 9 & SCHED-B & 8.0 & 24 \\
Zone 10 & SCHED-C & 8.3 & 142 \\
Zone 11 & SCHED-B & 8.1 & 41 \\
Zone 12 & SCHED-B & 9.5 & 40 \\
Zone 13 & SCHED-B & 9.0 & 13 \\
\hline
\end{tabular}

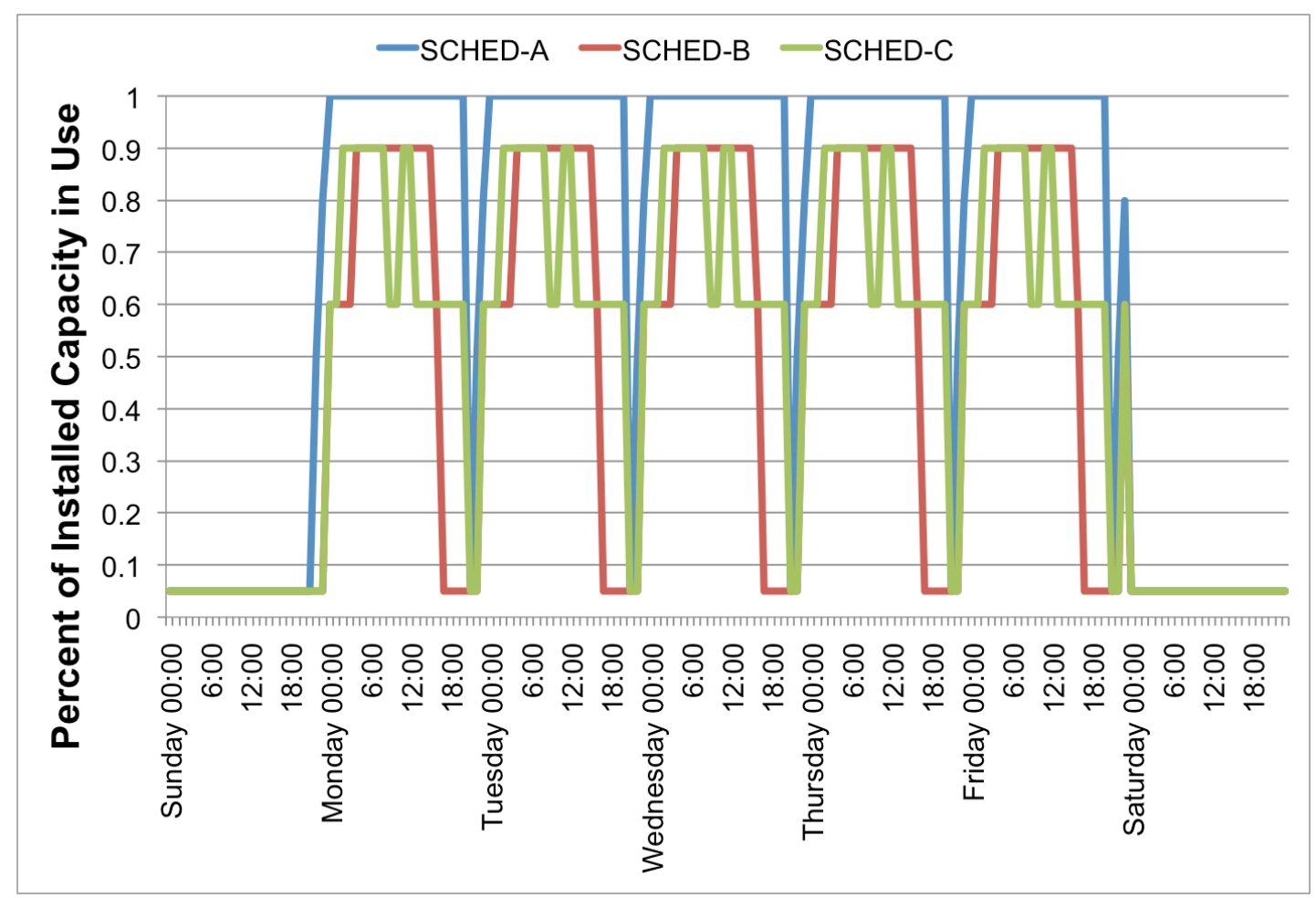

Figure 104. Mail Sorting-2: Hourly Breakdowns for Lighting Schedule A

\section{Mail Sorting-3}

The model only employs one schedule representing 100\% of lighting energy use and $100 \%$ of the scheduled energy use.

Table 60. Mail Sorting-3: Zone and Schedule Description

\begin{tabular}{cccc}
\hline Name & Schedule & PD $\left(\mathbf{W} / \mathbf{m}^{\mathbf{2}}\right)$ & $\begin{array}{c}\text { Floor Area } \\
\left(\mathbf{m}^{\mathbf{2}}\right)\end{array}$ \\
\hline Zone 1 & SCHED-A & 10.9 & 495 \\
Zone 2 & SCHED-A & 12.4 & 451 \\
Zone 3 & SCHED-A & 4.6 & 81 \\
Zone 4 & SCHED-A & 8.8 & 74 \\
\hline
\end{tabular}




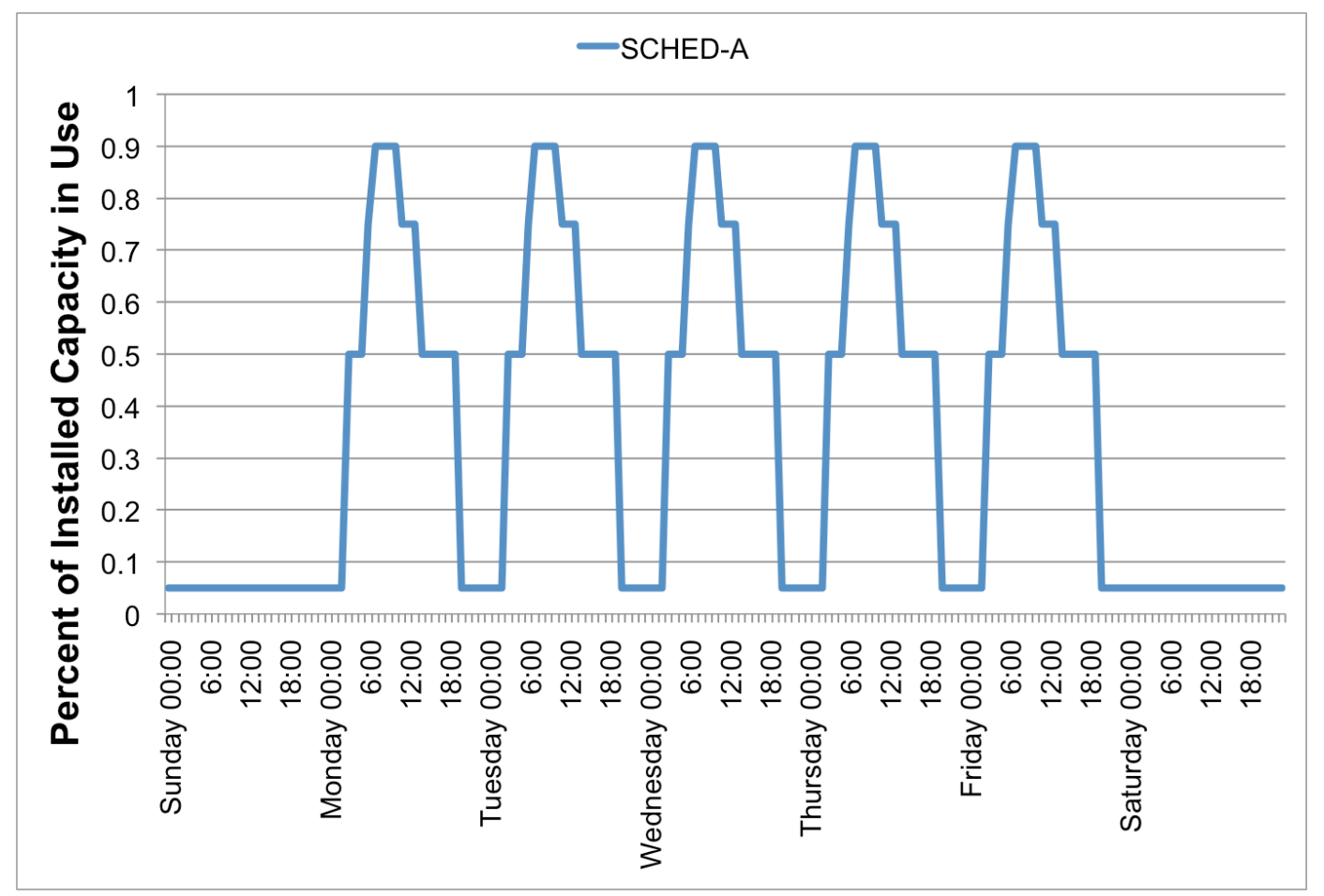

Figure 105. Mail Sorting-3: Hourly Breakdowns for Lighting Schedule A

\section{Public Assembly-1}

The model employs two schedules. With Schedule A accounting for $98 \%$ of installed lighting capacity and $98 \%$ of the scheduled energy use. Schedule B accounts for the remaining $2 \%$ percent of the installed lighting capacity and $2 \%$ of the scheduled energy use.

Table 61. Zone and Schedule Description.

\begin{tabular}{cccc}
\hline Name & Schedule & PD $\left(\mathbf{W} / \mathbf{m}^{\mathbf{2}}\right)$ & $\begin{array}{c}\text { Floor Area } \\
\left(\mathbf{m}^{\mathbf{2}}\right)\end{array}$ \\
\hline Zone 1 & SCHED-A & 7.0 & 245 \\
Zone 2 & SCHED-A & 8.6 & 170 \\
Zone 3 & SCHED-A & 6.8 & 201 \\
Zone 4 & SCHED-A & 7.4 & 214 \\
Zone 5 & SCHED-B & 7.6 & 26 \\
Zone 6 & SCHED-A & 16.2 & 312 \\
\hline
\end{tabular}




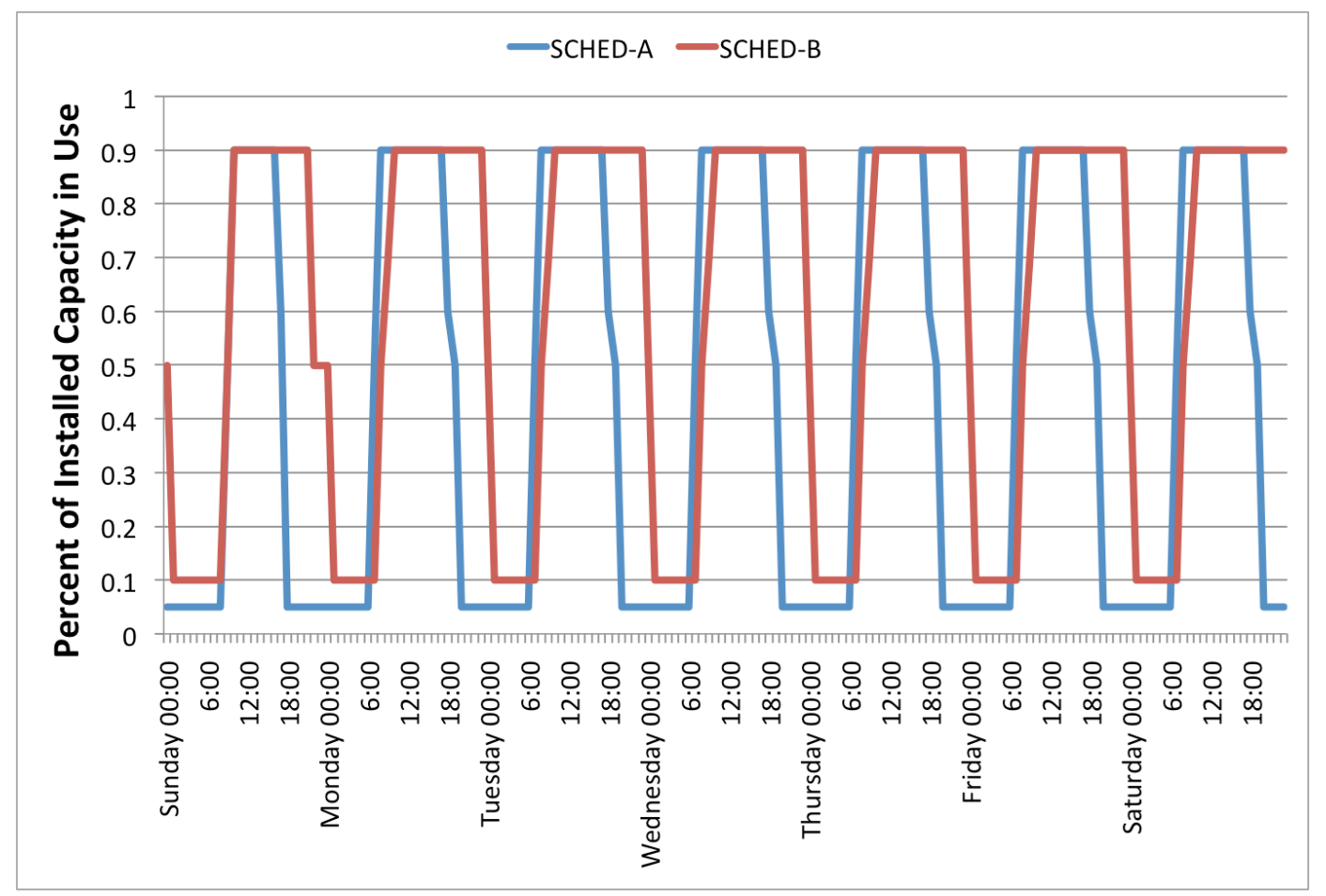

Figure 106. Hourly Breakdowns for Lighting Schedules A and B 


\section{Appendix C- Receptacles Modeling Schedules}

The following appendix details the zone characteristics used in the building energy modeling. Varying power density were attributed to the buildings' zones based on zone occupancy. The following table describes the area weighted average power density for each building.

Table 62. Receptacles Power Density Summary

\begin{tabular}{|c|c|}
\hline Building & $\begin{array}{l}\text { Area Weighted } \\
\text { Average Power } \\
\text { Density }\left(\mathrm{W} / \mathrm{m}^{2}\right)\end{array}$ \\
\hline Academic-1 & 3.4 \\
\hline Office-3 & 6.5 \\
\hline Office-4 & 3.8 \\
\hline Mail Sorting-1 & 10.0 \\
\hline Mail Sorting-2 & 2.1 \\
\hline Mail Sorting-3 & 6.4 \\
\hline Public Assembly-1 & 4.2 \\
\hline
\end{tabular}

\section{Academic-1}

The model only employs one schedule representing $100 \%$ of lighting energy use and $100 \%$ of the scheduled energy use.

Table 63. Academic-1: Zone and Schedule Description

\begin{tabular}{cccc}
\hline Name & Schedule & $\begin{array}{c}\text { PD } \\
(\mathbf{W} / \mathbf{m} \mathbf{2})\end{array}$ & $\begin{array}{c}\text { Floor Area } \\
\left(\mathbf{m}^{\mathbf{2}}\right)\end{array}$ \\
\hline Zone 1 & SCHED-A & 1.1 & 801 \\
Zone 2 & SCHED-A & 4.7 & 583 \\
Zone 3 & SCHED-A & 4.9 & 118 \\
Zone 4 & SCHED-A & 4.9 & 327 \\
Zone 5 & SCHED-A & 4.5 & 483 \\
Zone 6 & SCHED-A & 1.8 & 437 \\
Zone 7 & SCHED-A & 4.5 & 463 \\
Zone 8 & SCHED-A & 4.3 & 246 \\
Zone 9 & SCHED-A & 2.9 & 670 \\
\hline
\end{tabular}




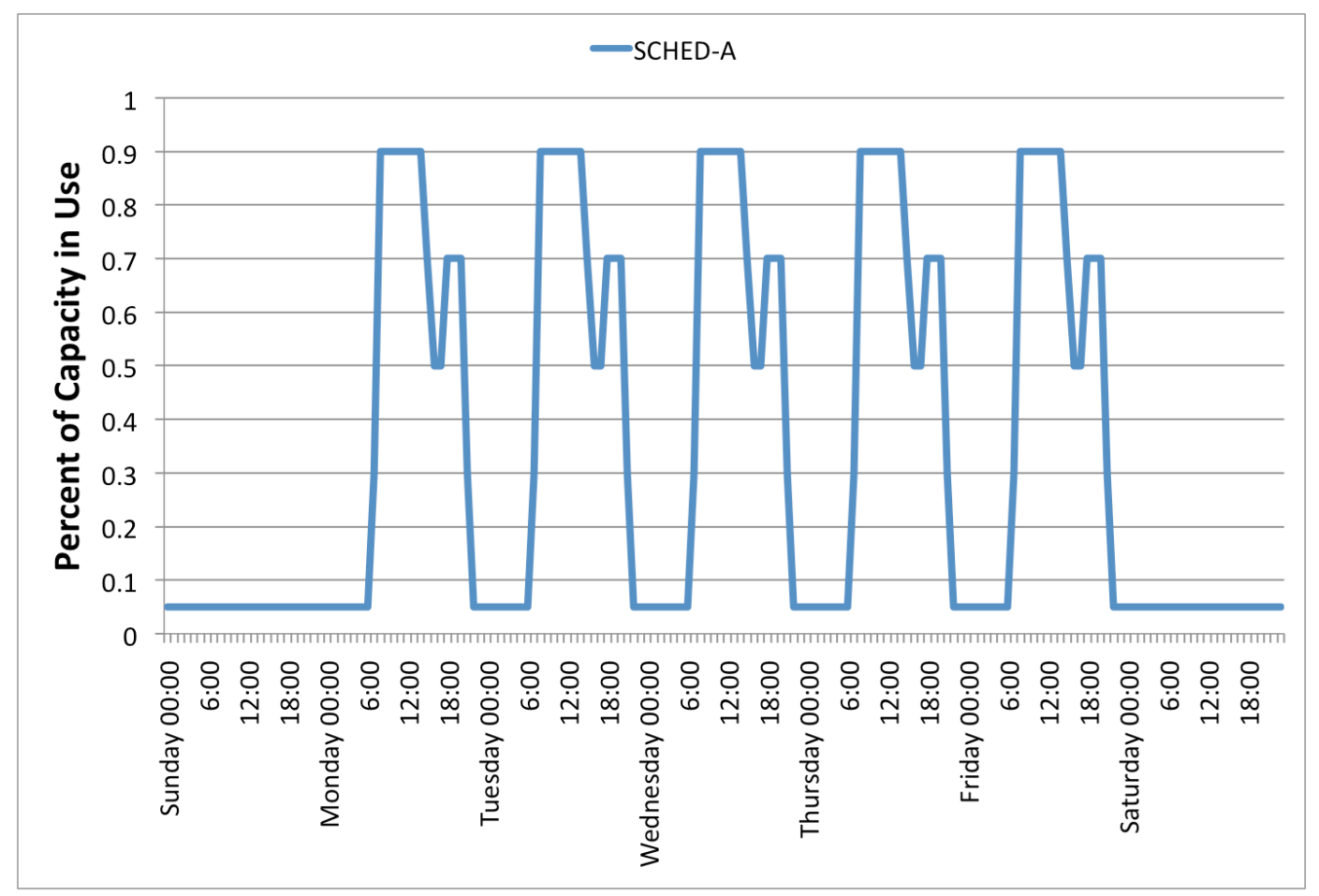

Figure 107. Hourly Breakdowns for Receptacles Schedule A

\section{Office-3}

The model employs two schedules. Schedule A represents $42 \%$ of the installed lighting capacity, and $100 \%$ of the scheduled energy use. Schedule B represents $58 \%$ of the installed lighting capacity, and $0 \%$ of the scheduled energy use. Schedule B accounts for $0 \%$ of the scheduled energy use due to the fact that a portion of the building was unoccupied at the time of metering.

Table 64. Office-3: Zone and Schedule Description

\begin{tabular}{cccc}
\hline Name & Schedule & $\begin{array}{c}\text { PD } \\
(\mathbf{W} / \mathbf{m} \mathbf{2})\end{array}$ & $\begin{array}{c}\text { Floor Area } \\
(\mathbf{m} \mathbf{2})\end{array}$ \\
\hline Zone 1 & SCHED-A & 1.0 & 80 \\
Zone 2 & SCHED-A & 7.5 & 233 \\
Zone 3 & SCHED-A & 7.5 & 135 \\
Zone 4 & SCHED-A & 7.5 & 155 \\
Zone 5 & SCHED-A & 7.5 & 135 \\
Zone 6 & SCHED-B & 5.9 & 3,233 \\
Zone 7 & SCHED-B & 7.5 & 413 \\
Zone 8 & SCHED-B & 7.5 & 307
\end{tabular}




\begin{tabular}{cccc} 
Zone 9 & SCHED-B & 7.5 & 368 \\
Zone 10 & SCHED-B & 7.5 & 307 \\
Zone 11 & SCHED-A & 7.5 & 213 \\
Zone 12 & SCHED-A & 7.5 & 159 \\
Zone 13 & SCHED-A & 7.5 & 213 \\
Zone 14 & SCHED-A & 7.5 & 160 \\
Zone 15 & SCHED-A & 5.9 & 1,207 \\
Zone 16 & SCHED-A & 7.5 & 538 \\
\hline
\end{tabular}

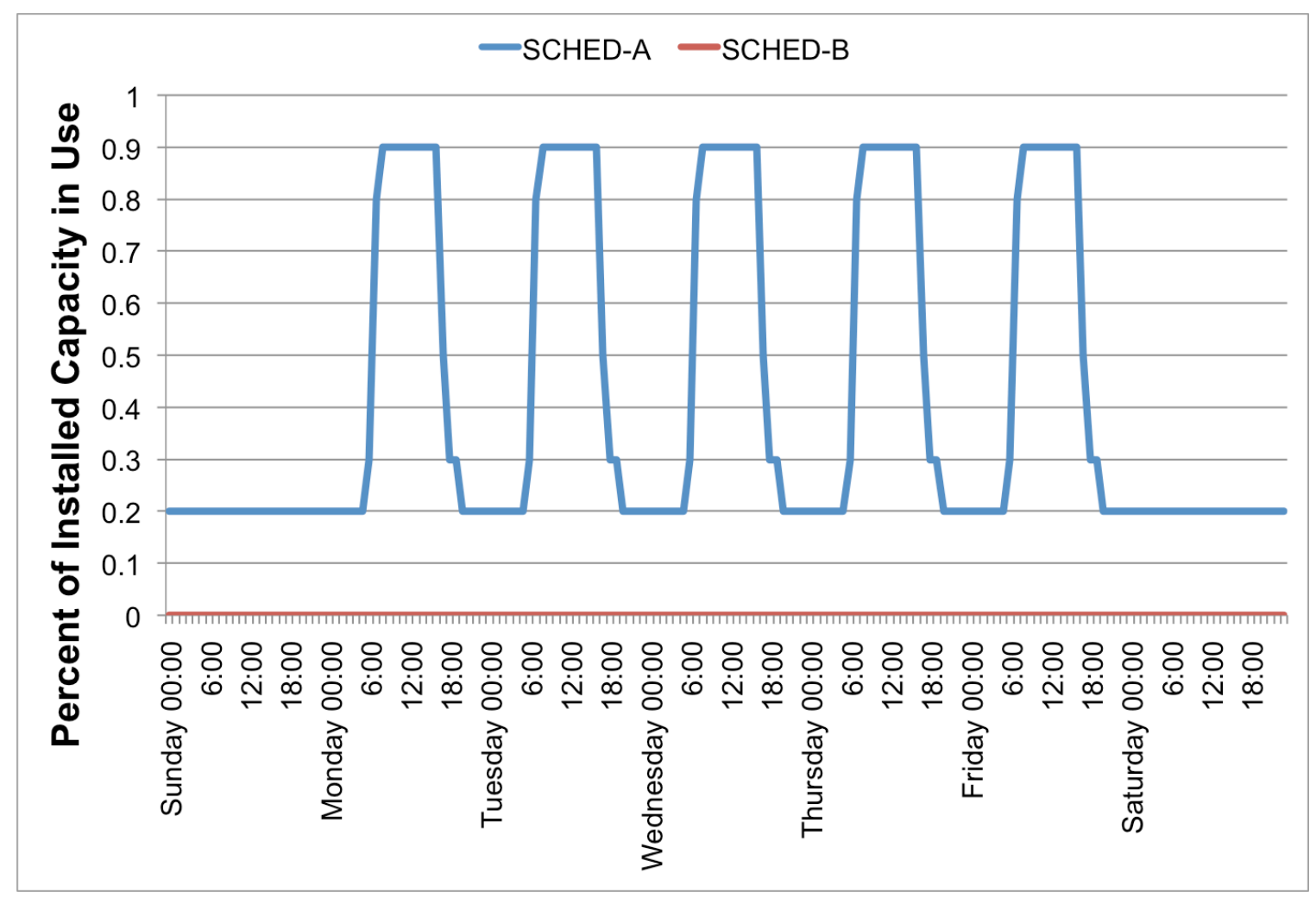

Figure 108. Office-3: Hourly Breakdowns for Receptacles Schedules A and B

\section{Office-4}

The model employs two schedules. Schedule A represents $18 \%$ of the installed lighting capacity, and 23\% of the scheduled energy use. Schedule B represents $82 \%$ of the installed lighting capacity, and $77 \%$ of the scheduled energy use. 
Table 65. Office-4: Zone and Schedule Description

\begin{tabular}{|c|c|c|c|}
\hline Name & Schedule & $\begin{array}{c}\text { PD } \\
(w / m 2)\end{array}$ & $\begin{array}{c}\text { Floor Area } \\
(\mathrm{m} 2)\end{array}$ \\
\hline Zone 1 & SCHED-A & 1.0 & 31 \\
\hline Zone 2 & SCHED-A & 1.4 & 145 \\
\hline Zone 3 & SCHED-B & 7.5 & 19 \\
\hline Zone 4 & SCHED-B & 4.2 & 92 \\
\hline Zone 5 & SCHED-B & 7.5 & 11 \\
\hline Zone 6 & SCHED-B & 6.8 & 34 \\
\hline Zone 7 & SCHED-B & 7.5 & 19 \\
\hline Zone 8 & SCHED-B & 7.5 & 107 \\
\hline Zone 9 & SCHED-B & 7.5 & 14 \\
\hline Zone 10 & SCHED-B & 7.5 & 10 \\
\hline Zone 12 & SCHED-B & 0.5 & 245 \\
\hline Zone 14 & SCHED-B & 2.8 & 90 \\
\hline Zone 15 & SCHED-B & 7.5 & 56 \\
\hline Zone 16 & SCHED-B & 4.3 & 91 \\
\hline Zone 17 & SCHED-A & 7.5 & 38 \\
\hline Zone 18 & SCHED-A & 7.5 & 59 \\
\hline Zone 19 & SCHED-B & 0.4 & 162 \\
\hline Zone 20 & SCHED-B & 0.7 & 161 \\
\hline Zone 21 & SCHED-B & 4.3 & 195 \\
\hline Zone 22 & SCHED-B & 4.6 & 30 \\
\hline Zone 23 & SCHED-B & 7.5 & 57 \\
\hline Zone 24 & SCHED-A & 7.5 & 59 \\
\hline Zone 25 & SCHED-B & 4.1 & 130 \\
\hline Zone 26 & SCHED-B & 1.0 & 15 \\
\hline Zone 27 & SCHED-B & 7.5 & 31 \\
\hline Zone 28 & SCHED-B & 4.6 & 115 \\
\hline Zone 29 & SCHED-B & 7.5 & 14 \\
\hline Zone 30 & SCHED-B & 7.5 & 22 \\
\hline Zone 31 & SCHED-B & 5.0 & 34 \\
\hline
\end{tabular}




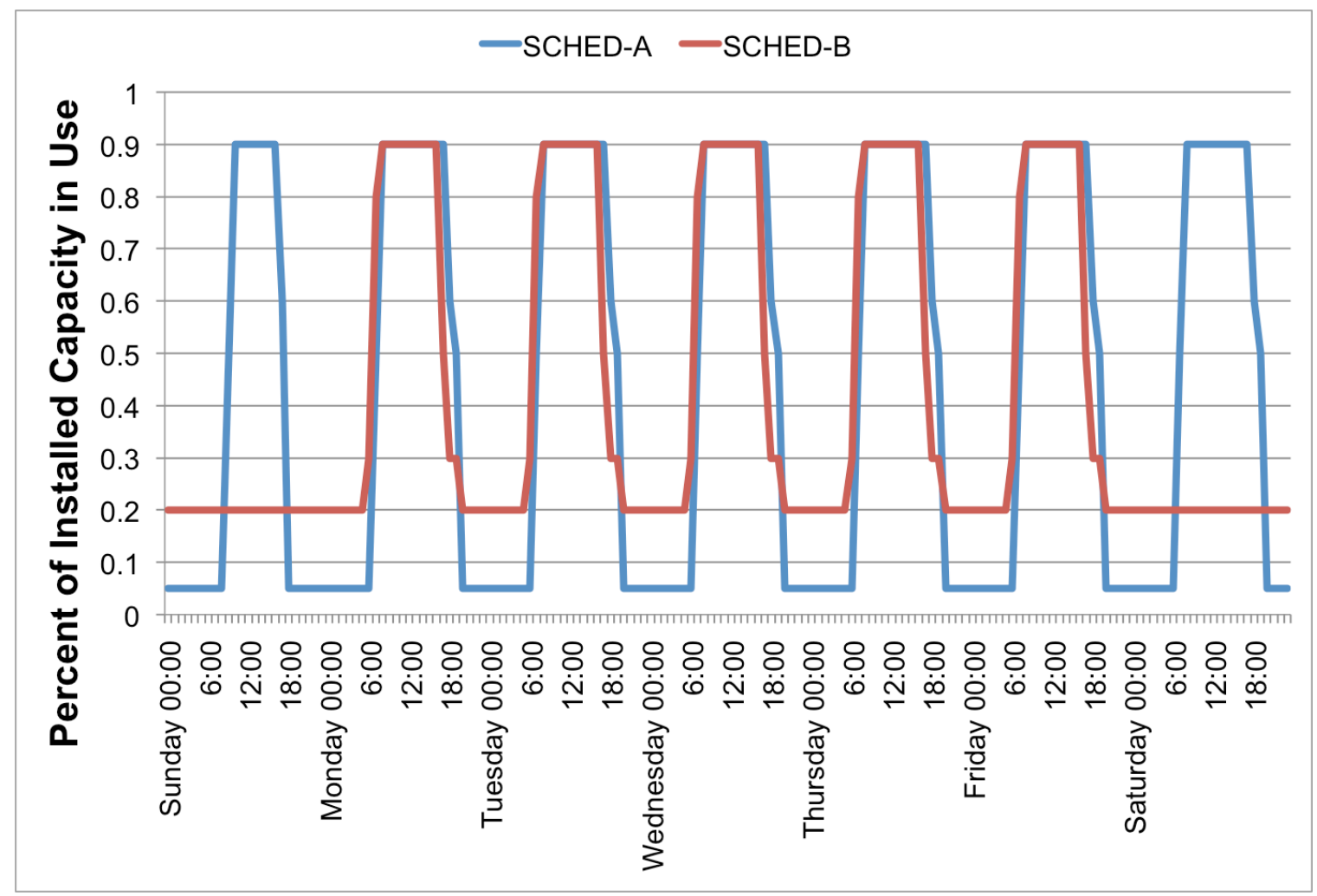

Figure 109. Office-4: Hourly Breakdowns for Receptacles Schedules A and B

\section{Mail Sorting-1}

The model employs two schedules. Schedule A represents $68 \%$ of the installed lighting capacity, and 71\% of the scheduled energy use. Schedule B represents $32 \%$ of the installed lighting capacity, and $29 \%$ of the scheduled energy use.

Table 66. Mail Sorting-1: Zone and Schedule Description

\begin{tabular}{cccccc}
\hline Name & Schedule & $\begin{array}{c}\text { PD } \\
(\mathbf{W} / \mathbf{m} \mathbf{2})\end{array}$ & $\begin{array}{c}\text { Floor Area } \\
\left(\mathbf{m}^{\mathbf{2}}\right)\end{array}$ & $\begin{array}{c}\text { Secondary } \\
\text { Schedule }\end{array}$ & $\begin{array}{c}\text { Equipment } \\
\text { (W) }\end{array}$ \\
\hline Zone 1 & SCHED-A & 7.5 & 819 & SCHED-B & 3,245 \\
Zone 2 & SCHED-A & 6.3 & 720 & SCHED-B & 2,210 \\
Zone 3 & SCHED-A & 0.9 & 54 & - & - \\
Zone 4 & SCHED-A & 6.3 & 89 & - & - \\
\hline
\end{tabular}




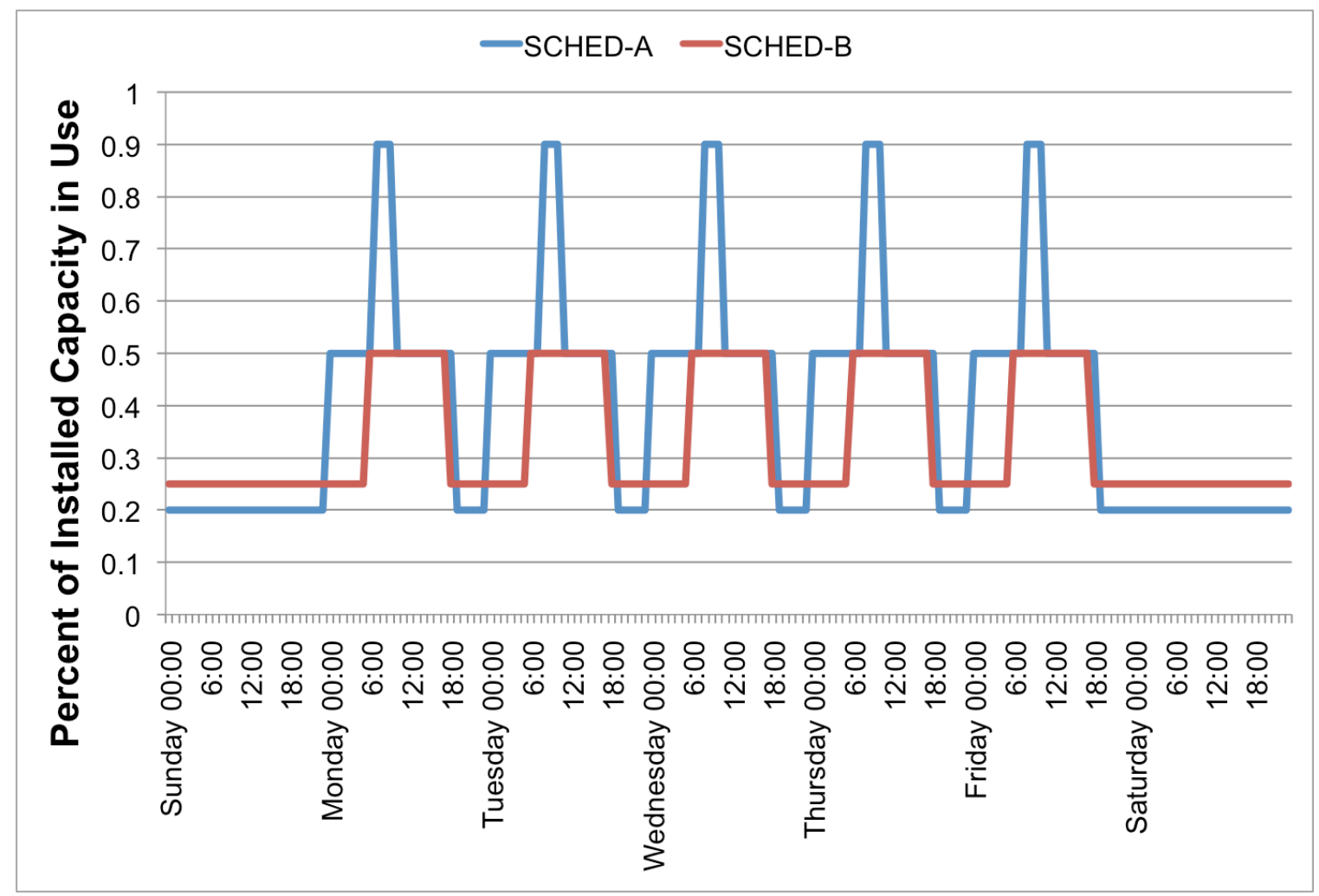

Figure 110. Mail Sorting-1: Hourly Breakdowns for Receptacles Schedules A and B

\section{Mail Sorting-2}

The model only employs one schedule representing 100\% of lighting energy use and $100 \%$ of the scheduled energy use.

Table 67. Zone and Schedule Description

\begin{tabular}{cccc}
\hline Name & Schedule & PD $\left(\mathbf{W} / \mathbf{m}^{\mathbf{2}}\right)$ & $\begin{array}{c}\text { Floor Area } \\
\left(\mathbf{m}^{\mathbf{2}}\right)\end{array}$ \\
\hline Zone 1 & SCHED-A & 2.2 & 1370.3 \\
Zone 2 & SCHED-A & 1.6 & 213.2 \\
Zone 3 & SCHED-A & 2.1 & 1320.4 \\
Zone 4 & SCHED-A & 2.4 & 202.1 \\
Zone 5 & SCHED-A & 2.1 & 1145 \\
Zone 6 & SCHED-A & 2.1 & 1210.4 \\
Zone 7 & SCHED-A & 0.9 & 281.1 \\
Zone 8 & SCHED-A & 1.8 & 379.1 \\
Zone 9 & SCHED-A & 8.2 & 23.5 \\
Zone 10 & SCHED-A & 0 & 142.1 \\
Zone 11 & SCHED-A & 1 & 40.6 \\
Zone 12 & SCHED-A & 8.2 & 40.1 \\
Zone 13 & SCHED-A & 8.2 & 13.2 \\
\hline
\end{tabular}




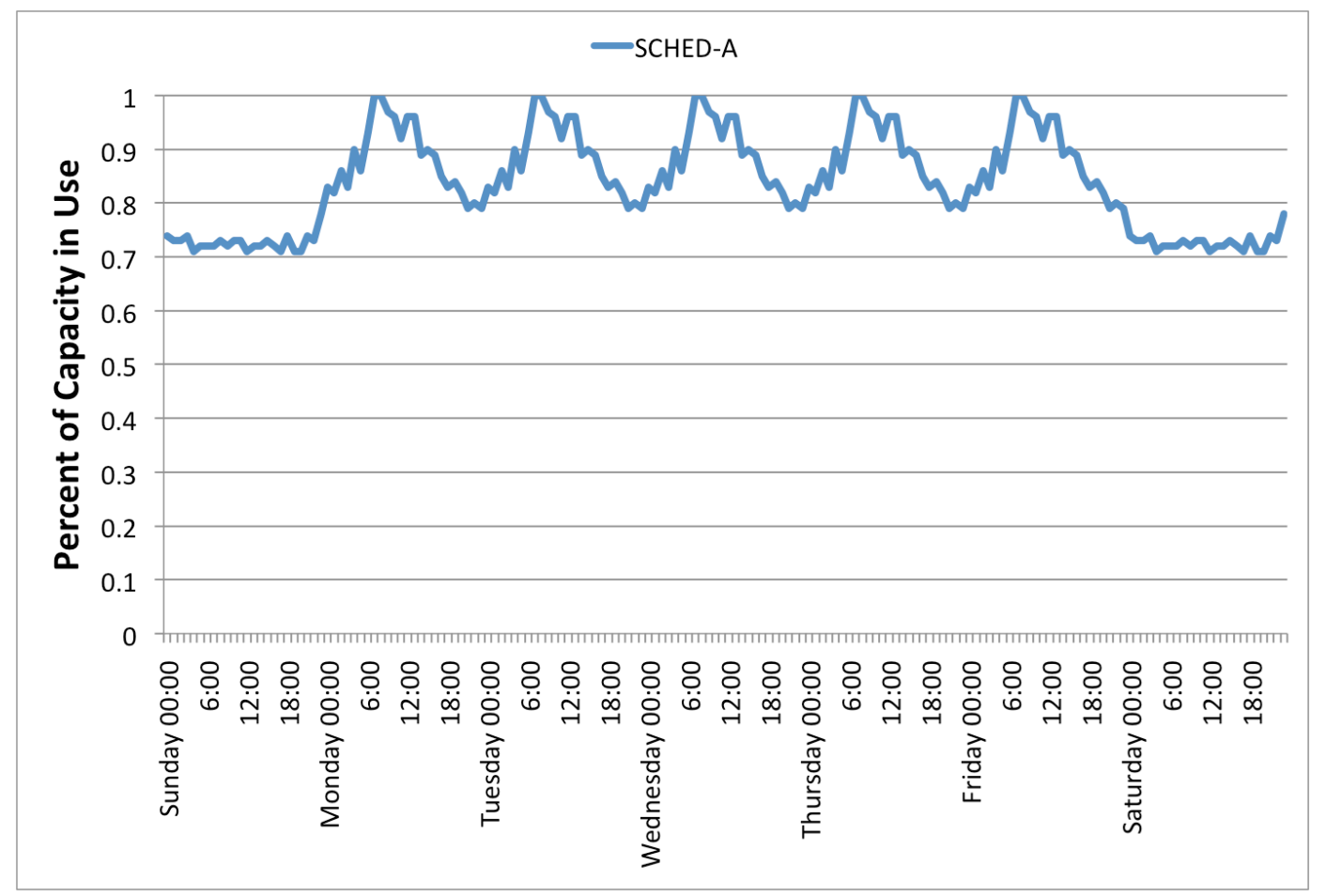

Figure 111. Hourly Breakdowns for Receptacles Schedule A

\section{Mail Sorting-3}

The model only employs one schedule representing $100 \%$ of lighting energy use and $100 \%$ of the scheduled energy use.

Table 68. Zone and Schedule Description

\begin{tabular}{cccc}
\hline Name & Schedule & $\begin{array}{c}\text { PD } \\
(\mathbf{W} / \mathbf{m} \mathbf{2})\end{array}$ & $\begin{array}{c}\text { Floor Area } \\
(\mathbf{m} 2)\end{array}$ \\
\hline Zone 1 & SCHED-A & 6.3 & 494.9 \\
Zone 2 & SCHED-A & 7.5 & 451.2 \\
Zone 3 & SCHED-A & 0.9 & 80.5 \\
Zone 4 & SCHED-A & 6.2 & 73.8 \\
\hline
\end{tabular}




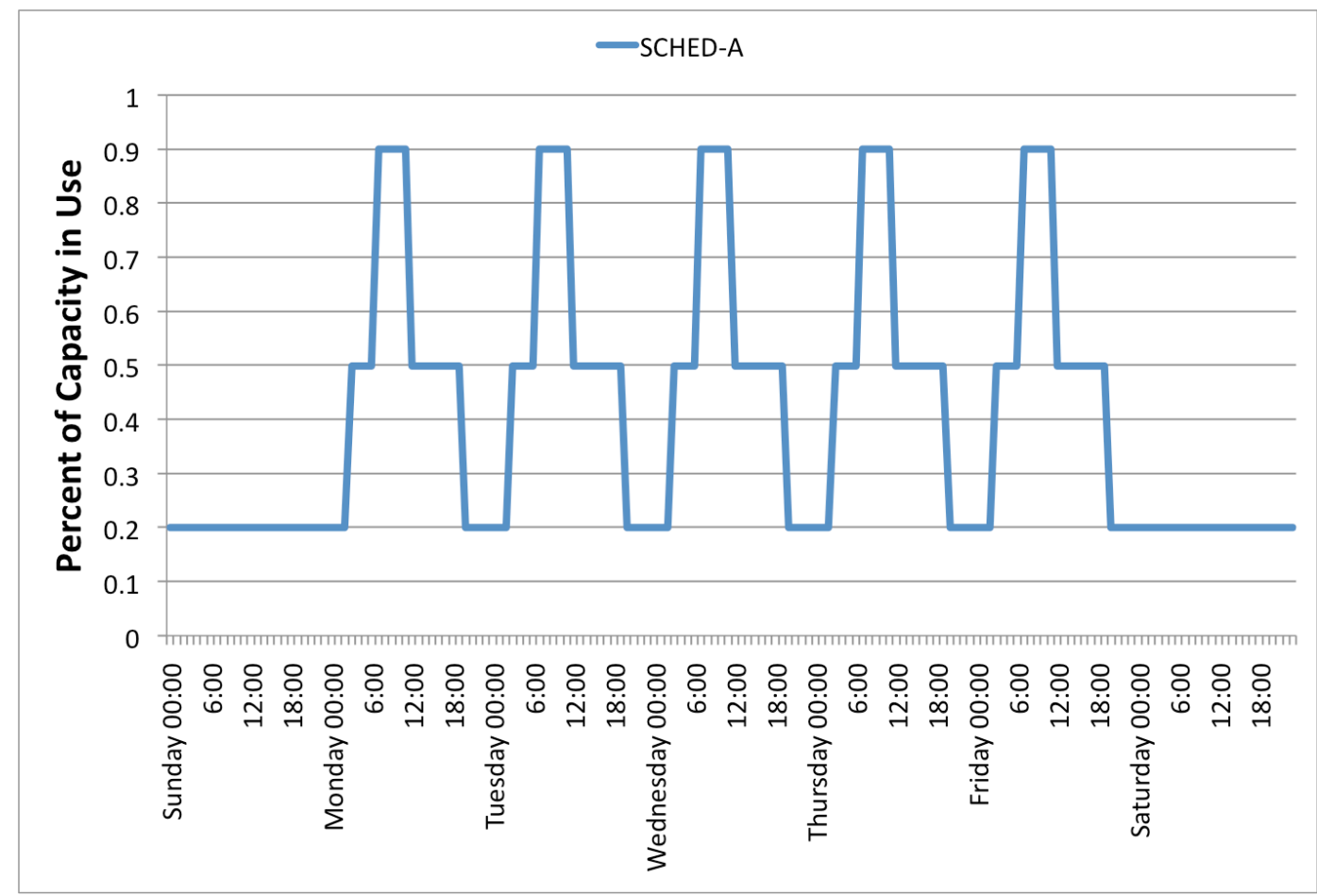

Figure 112. Hourly Breakdowns for Receptacles Schedule A

\section{Public Assembly-1}

The model employs two schedules. Schedule A represents $91 \%$ of the installed lighting capacity, and $88 \%$ of the scheduled energy use. Schedule B represents $9 \%$ of the installed lighting capacity, and $12 \%$ of the scheduled energy use.

Table 69. Public Assembly-1: Zone and Schedule Description

\begin{tabular}{|c|c|c|c|c|c|}
\hline Name & Schedule & $P D\left(W / m^{2}\right)$ & $\begin{array}{c}\text { Floor Area } \\
\left(\mathbf{m}^{2}\right)\end{array}$ & $\begin{array}{c}\text { Secondary } \\
\text { Schedule }\end{array}$ & $\begin{array}{l}\text { Equipment } \\
\text { (W) }\end{array}$ \\
\hline Zone 1 & SCHED-A & 3.1 & 245 & SCHED-A & 728 \\
\hline Zone 2 & SCHED-A & 1.8 & 170 & SCHED-A & 209 \\
\hline Zone 3 & SCHED-A & 1.3 & 201 & SCHED-A & 175 \\
\hline Zone 4 & SCHED-A & 1.9 & 214 & SCHED-A & 272 \\
\hline Zone 5 & SCHED-B & 10.0 & 26 & SCHED-B & 178 \\
\hline Zone 6 & SCHED-A & 2.5 & 312 & SCHED-A & 618 \\
\hline
\end{tabular}




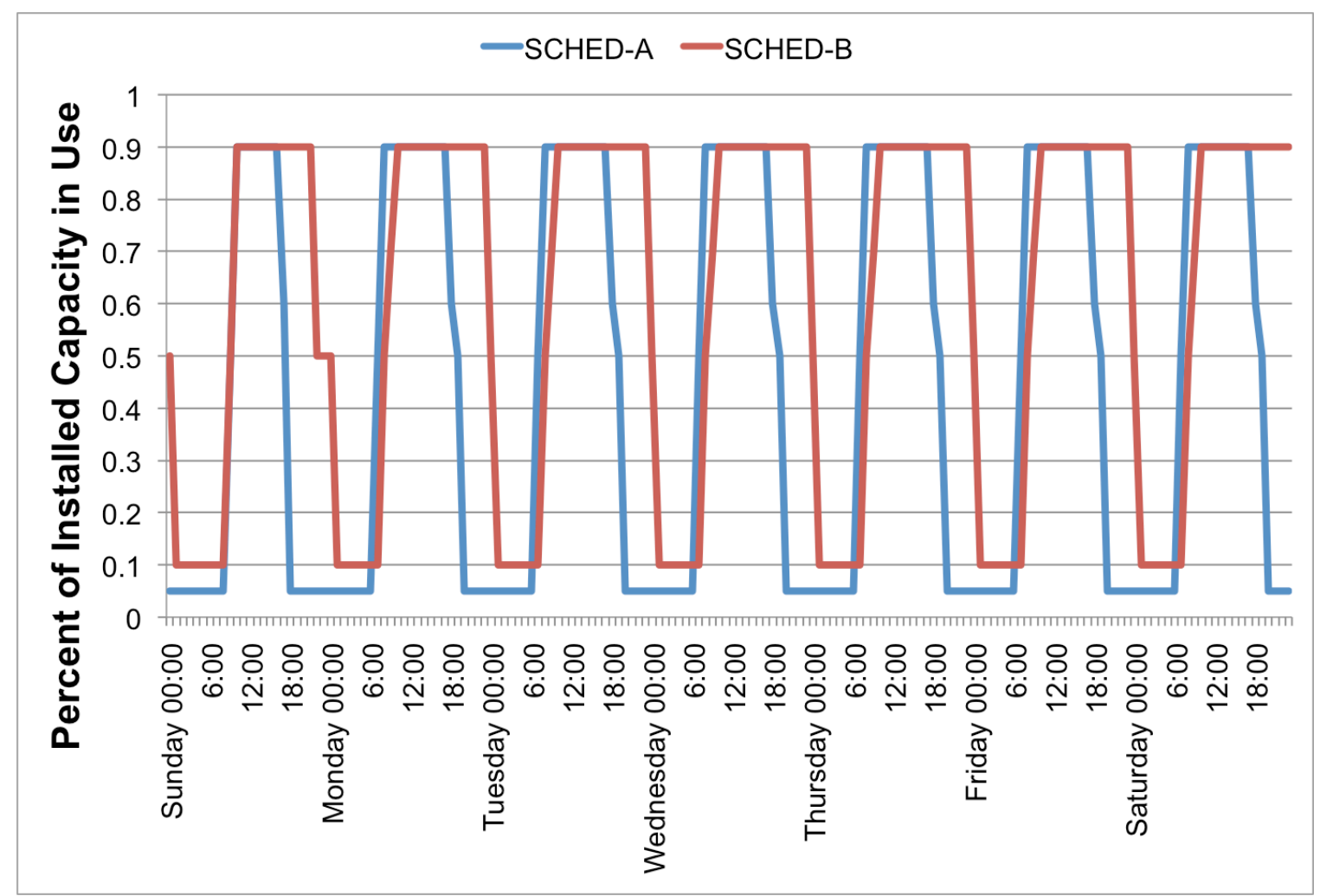

Figure 113. Public Assembly-1: Hourly Breakdowns for Receptacles Schedules A and B 


\section{Appendix D- Meter Attribution Documentation}

\section{Academic-1}

No meter attribution data was available except for labeled excel sheets.

\section{Office-3}

No meter attribution data was available except for labeled excel sheets.

\section{Office-4}

\begin{tabular}{|c|c|c|c|}
\hline Location & Panel & Service ID & Meter Serial \\
\hline Basement - Electrical Room & & EMP 1 & E001 \\
\hline Basement - Electrical Room & DP-PB & [5] 120V WYE - Internal PT & E001PTA \\
\hline Basement - Electrical Room & DP-PB & Receptacle Circuit \#1 & E001M01 \\
\hline Basement - Electrical Room & DP-PB & Receptacle Circuit \#2 & E001M02 \\
\hline Basement - Electrical Room & DP-PB & Lighting Circuit \#1 & E001M03 \\
\hline Basement - Electrical Room & DP-PB & Lighting Circuit \#2 & E001M04 \\
\hline Basement - Electrical Room & DP-PB & Mechancial Circuit \#1 & E001M05 \\
\hline Basement - Electrical Room & DP-PB & Mechancial Circuit \#2 & E001M06 \\
\hline Basement - Electrical Room & DP-P2 & Receptacle Circuit \#1 & E001M07 \\
\hline Basement - Electrical Room & DP-P2 & Receptacle Circuit \#2 & E001M08 \\
\hline Basement - Electrical Room & DP-P2 & Lighting Circuit \#1 & E001M09 \\
\hline Basement - Electrical Room & DP.P2 & Lighting Circuit \#2 & E001M10 \\
\hline Basement - Electrical Room & DP.P2 & Mechancial Circuit \#1 & E001M11 \\
\hline Basement - Electrical Room & DP-P2 & Mechancial Circuit \#2 & E001M12 \\
\hline Ground Floor - Electrical Room & & EMP 2 & E002 \\
\hline Ground Floor - Electrical Room & DP-P1 & [5] 120V WYE - Internal PT & E002PTA \\
\hline Ground Floor - Electrical Room & DP-P1 & Receptacle Circuit \#1 & E002M01 \\
\hline Ground Floor - Electrical Room & DP-P1 & Receptacle Circuit \#2 & E002M02 \\
\hline Ground Floor - Electrical Room & DP-P1 & Lighting Circuit \#1 & E002M03 \\
\hline Ground Floor - Electrical Room & DP-P1 & Lighting Circuit \#2 & Е002M04 \\
\hline Ground Floor - Electrical Room & DP-P1 & Mechancial Circuit \#1 & E002M05 \\
\hline Ground Floor - Electrical Room & DP-P1 & Mechancial Circuit \#2 & Е002M06 \\
\hline Ground Floor - Electrical Room & DP-1A & Receptacle Circuit \#1 & E002M07 \\
\hline Ground Floor - Electrical Room & DP-1A & Receptacle Circuit \#2 & E002M08 \\
\hline Ground Floor - Electrical Room & DP-1A & Lighting Circuit \#1 & E002M09 \\
\hline Ground Floor - Electrical Room & DP-1A & Lighting Circuit \#2 & E002M10 \\
\hline Ground Floor - Electrical Room & DP-1A & Mechancial Circuit \#1 & E002M11 \\
\hline Ground Floor - Electrical Room & DP-1A & Mechancial Circuit \#2 & E002M12 \\
\hline
\end{tabular}

Figure 114. Office-4: Meter Atribution 


\begin{tabular}{l|l|c|c}
\hline Location & Panel & Service ID & Meter Serial \\
\hline Basement - Electrical Room & & EMP 3 & E003 \\
\hline Basement - Electrical Room & DP-EA & {$[5] 120 \mathrm{~V}$ WYE - Internal PT } & E003PTA \\
\hline Basement - Electrical Room & DP-EA & Receptacle Circuit \#1 & E003M01 \\
\hline Basement - Electrical Room & DP-EA & Receptacle Circuit \#2 & E003M02 \\
\hline Basement - Electrical Room & DP-EA & Lighting Circuit \#1 & E003M03 \\
\hline Basement - Electrical Room & DP-EA & Lighting Circuit \#2 & E003M04 \\
\hline Basement - Electrical Room & DP-EA & Mechancial Circuit \#1 & E003M05 \\
\hline Basement - Electrical Room & DP-EA & Mechancial Circuit \#2 & E003M06 \\
\hline Basement - Electrical Room & DP-EB & Receptacle Circuit \#1 & E003M07 \\
\hline Basement - Electrical Room & DP-EB & Receptacle Circuit \#2 & E003M08 \\
\hline Basement - Electrical Room & DP-EB & Lighting Circuit \#1 & E003M09 \\
\hline Basement - Electrical Room & DP-EB & Lighting Circuit \#2 & E003M10 \\
\hline Basement - Electrical Room & DP-EB & Mechancial Circuit \#1 & E003M11 \\
\hline Basement - Electrical Room & DP-EB & Mechancial Circuit \#2 & E003M12 \\
\hline Basement - Electrical Room & & EMP 4 & E004 \\
\hline Basement - Electrical Room & MAIN SWBD "AA" & {$[5]$ 120V WYE - Internal PT } & E004PTA \\
\hline Basement - Electrical Room & MAIN SWBD "AA" & Main Meter & E004M01 \\
\hline Basement - Electrical Room & MAIN SWBD "AA" & Mechanical Service P1 & E004M02 \\
\hline Basement - Electrical Room & MAIN SWBD "AA" & Mechanical Service P2 & E004M03 \\
\hline Basement - Electrical Room & MAIN SWBD "AA" & Mechanical Service ERV-1 & E004M04 \\
\hline Basement - Electrical Room & MAIN SWBD "AA" & Mechanical Service ERV-2 & E004M05 \\
\hline Basement - Electrical Room & MAIN SWBD "AA" & Mechanical Service ERV-3 & E004M06
\end{tabular}

Figure 115. Office-4 Meter Attribution

\section{Mail Sorting-1}

\begin{tabular}{|c|c|c|c|c|c|c|c|c|}
\hline $\begin{array}{l}\square * \text { VIA TIME SWITCH } \\
\square \text { **PROVIDE BREAKER } \\
\text { LOCKING DEVICES }\end{array}$ & \multicolumn{4}{|c|}{ PANEL "A" } & \multicolumn{4}{|c|}{$\begin{array}{l}\square 225 \mathrm{~A}, 120 / 208 \mathrm{~V}, 3 \phi, 4 \mathrm{~W} \text {. MAINS } \\
\square 225 \mathrm{~A}, 347 / 600 \mathrm{~V}, 3 \phi, 4 \mathrm{~W} \text {. MAINS } \\
\square \text { RECESSED SURFACE MOUNTED } \\
\square \text { DOUBLE LUGS }\end{array}$} \\
\hline DESCRIPTION & LOAD & BKR & CCT & & CCT & BKR & LOAD & DESCRIPTION \\
\hline LIGHTING & 1640 & $20 \mathrm{~A}$ & 1 & $\phi 1$ & 2 & $20 \mathrm{~A}$ & 1290 & WASHROOM LIGHTING \\
\hline LIGHTING & 1722 & $20 \mathrm{~A}$ & 3 & $\phi$ & 4 & $20 \mathrm{~A}$ & 1558 & GEN AREA UGHTING \\
\hline LIGHTING & 1312 & $20 \mathrm{~A}$ & 5 & 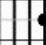 & 6 & $20 \mathrm{~A}$ & 1394 & NIGHT LIGHT ** \\
\hline LIGHTING & 1886 & $20 \mathrm{~A}$ & 7 & ? & 8 & $20 \mathrm{~A}$ & 1476 & NIGHT LIGHT ** \\
\hline LIGHTING & 1640 & $20 \mathrm{~A}$ & 9 & $\phi$ & 10 & $15 \mathrm{~A}$. & 250 & EXIT LIGHT ** \\
\hline LIGHTING & 1763 & $20 \mathrm{~A}$ & 11 & 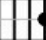 & 12 & $15 \mathrm{~A}$ & 1000 & BATTERY UNITS *** \\
\hline LIGHTING & 1886 & $20 \mathrm{~A}$ & 13 & 6. & 14 & $15 \mathrm{~A}$ & 600 & DOCK UGHTS \\
\hline LIGHTING & 1804 & $20 \mathrm{~A}$ & 15 & 10 & 16 & - & -- & SPACE \\
\hline SPARE & -- & $20 \mathrm{~A}$ & 17 & 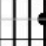 & 18 & - & -- & SPACE \\
\hline SPACE & -- & - & 19 & $\phi$ & 20 & - & -- & SPACE \\
\hline SPACE & -- & - & 21 & 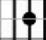 & 22 & - & -- & SPACE \\
\hline SPACE & -- & - & 23 & (8) & 24 & - & -- & SPACE \\
\hline
\end{tabular}

TOTAL CONNECTED LOAD: 21,221 WATT

Figure 116. Mail Sorting-1: Lighting Panel, "A" 


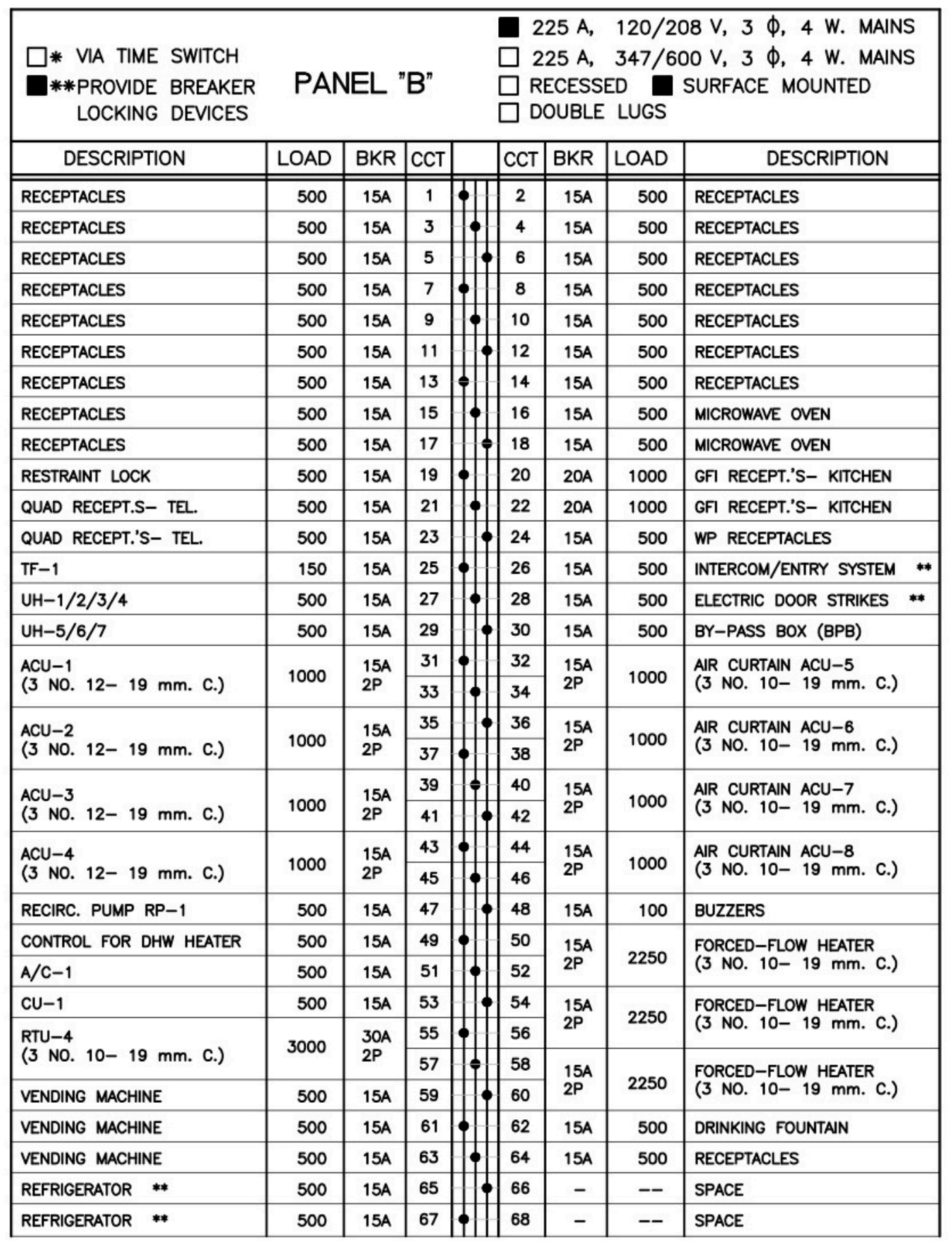

TOTAL CONNECTED LOAD: 38,500 WATT

Figure 117. Mail Sorting-1: Receptacles Panel, "B" 


\section{Mail Sorting-2}

No meter attribution data was available except for labeled excel sheets.

\section{Mail Sorting-3}

\begin{tabular}{|c|c|c|c|c|c|c|c|c|}
\hline $\begin{array}{l}\square * \text { VIA TIME SWITCH } \\
\square \text { **PROVIDE BREAKER } \\
\text { LOCKING DEVICES }\end{array}$ & \multicolumn{4}{|c|}{ PANEL "A" } & \multicolumn{4}{|c|}{$\begin{array}{l}225 \mathrm{~A}, 120 / 208 \mathrm{~V}, 3 \phi, 4 \mathrm{~W} . \text { MAINS } \\
225 \mathrm{~A}, 347 / 600 \mathrm{~V}, 3 \phi, 4 \mathrm{~W} . \text { MAINS } \\
\text { RECESSED } \square \text { SURFACE MOUNTED } \\
\text { DOUBLE LUGS }\end{array}$} \\
\hline DESCRIPTION & LOAD & BKR & CCT & & CCT & BKR & LOAD & DESCRIPTION \\
\hline UGHTING & 1355 & $20 \mathrm{~A}$ & 1 & $\phi$ & 2 & $20 \mathrm{~A}$ & 1098 & WASHROOM LIGHTING \\
\hline LIGHTING & 1148 & $20 \mathrm{~A}$ & 3 & 18 & 4 & $20 \mathrm{~A}$ & 1310 & GEN AREA LIGHTING \\
\hline LIGHTING & 1129 & $20 \mathrm{~A}$ & 5 & 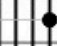 & 6 & $20 \mathrm{~A}$ & 820 & NIGHT LIGHT *** \\
\hline UGGTING & 1394 & $20 \mathrm{~A}$ & 7 & 6 & 8 & $20 \mathrm{~A}$ & 820 & NIGHT LIGHT *** \\
\hline UGHTING & 984 & $20 \mathrm{~A}$ & 9 & $\phi$ & 10 & 15A. & 250 & EXIT UGHT ** \\
\hline LGHTING & 1148 & $20 \mathrm{~A}$ & 11 & 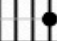 & 12 & $15 \mathrm{~A}$ & 1000 & BATTERY UNITS $\quad * *$ \\
\hline LGHTING & 1107 & $20 \mathrm{~A}$ & 13 & $\phi$ & 14 & $15 \mathrm{~A}$ & 150 & DOCK LIGHT \\
\hline SPARE & -- & $20 \mathrm{~A}$ & 15 & $\phi$ & 16 & - & -- & SPACE \\
\hline SPARE & -- & $20 \mathrm{~A}$ & 17 & t & 18 & - & -- & SPACE \\
\hline SPACE & -- & - & 19 & $\phi$ & 20 & - & -- & SPACE \\
\hline SPACE & -- & - & 21 & 0 & 22 & - & -- & SPACE \\
\hline SPACE & -- & - & 23 & & 24 & - & -- & SPACE \\
\hline
\end{tabular}

TOTAL CONNECTED LOAD: 13,713 WATT

Figure 118. Mail Sorting-3: Lighting Panel, "A" 


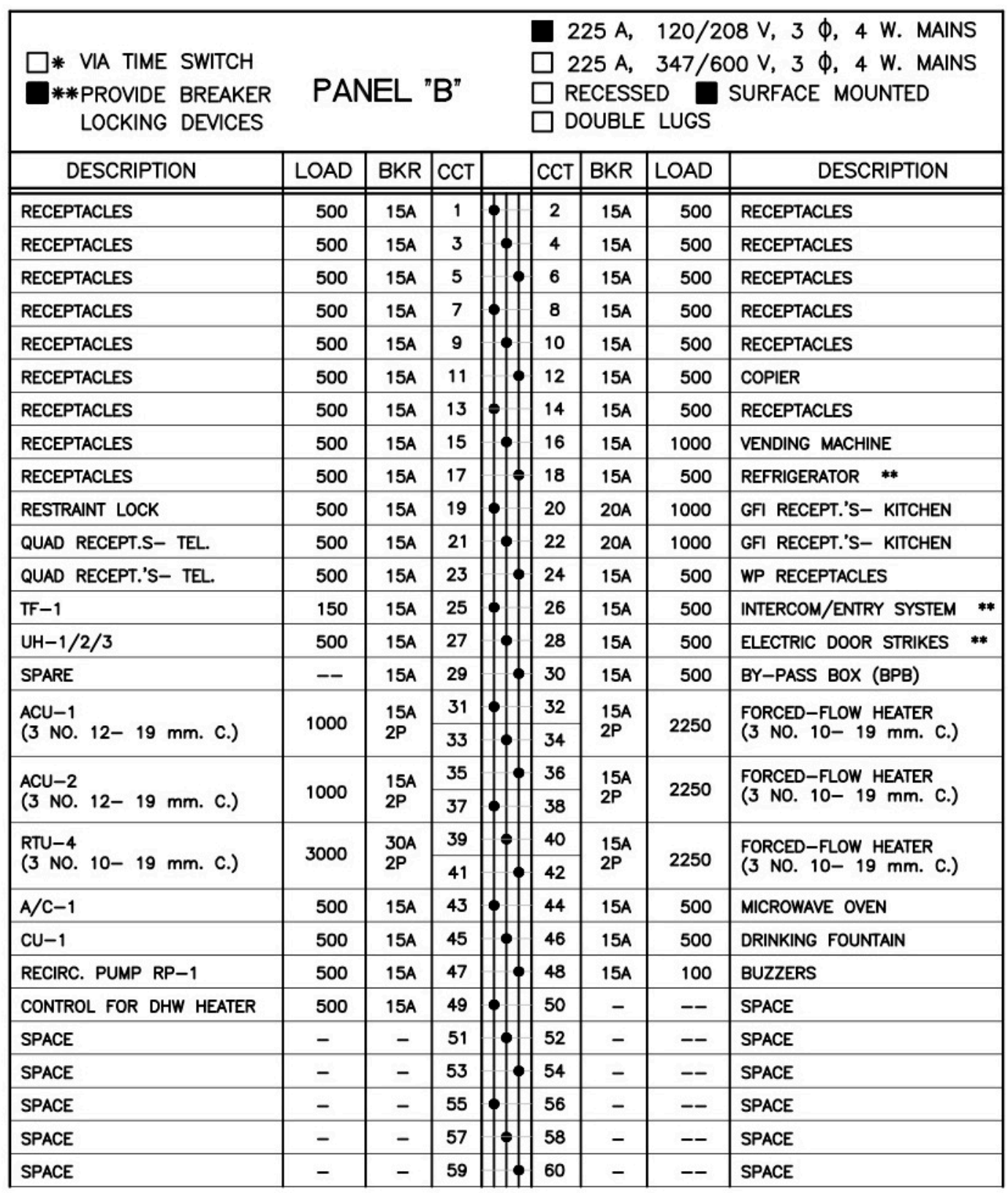

TOTAL CONNECTED LOAD: 30,500 WATT

Figure 119. Mail Sorting-3: Receptacles Panel, "B" 


\section{Public Assembly-1}

\begin{tabular}{|c|c|c|c|c|c|c|c|}
\hline \multicolumn{3}{|c|}{ PANEL: L1 } & FE BY: & \multirow{2}{*}{\multicolumn{4}{|c|}{$\begin{array}{l}\text { PANEL P1 } \\
\text { SURFACE } \\
\text { WAMN LUGS } \\
36,4 W\end{array}$}} \\
\hline \multicolumn{2}{|c|}{$\begin{array}{l}\text { VOLTAGE: } \\
\text { AMERAGE: }\end{array}$} & $\begin{array}{l}120 / 208 \\
100\end{array}$ & $\begin{array}{l}\text { MAIN BREAKER: } \\
\text { INTERIOR: }\end{array}$ & & & & \\
\hline & BKR & & RIPTION & $A B C$ & DESCRIPTION & EKR & \\
\hline 1 & 20 & & 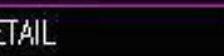 & & SPARE & 20 & 2 \\
\hline 3 & 20 & & FICE & & WASHROOWS & 20 & 4 \\
\hline 5 & 20 & & CE TRACK & & WEST ENIRY & 20 & 6 \\
\hline 7 & 20 & $\mathrm{COF}$ & R PENDENT & & STORAGE/GALLERY & 20 & 8 \\
\hline 9 & 20 & & OR TRACK & & GALLERY TRACK 1 & 20 & 10 \\
\hline 11 & 20 & STORAE & CYCLING ROOM & & GALLERY TRACK 2 & 20 & 12 \\
\hline 13 & 20 & & TRACK 4 & & GALLERY TRACK 3 & 20 & 14 \\
\hline 15 & 20 & EXTR & PARKING 1 & & BUILDING MOUNTED 1 & 20 & 16 \\
\hline 17 & 20 & EXTE & PARKING 2 & & BUILDING WOUNTED 2 & 20 & 18 \\
\hline 19 & 20 & EXTE & PARKING 3 & & BUILDING WOUNTED 3 & 20 & 20 \\
\hline 21 & 20 & EXTE & PARKINIG 4 & & SPARE & 20 & 22 \\
\hline 23 & 20 & EXTE & PARKING 5 & 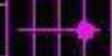 & SPARE & 20 & 24 \\
\hline
\end{tabular}

Figure 120. Public Assembly-1: Lighting Panel, "L1"

\begin{tabular}{|c|c|c|c|c|c|c|c|}
\hline \multicolumn{3}{|c|}{ PANEL: L2 } & & \multirow{2}{*}{$\begin{array}{l}\text { PANEL L1 } \\
\text { SURFACE } \\
\text { MAIN LUGS } \\
30,4 \mathrm{~W}\end{array}$} & & & \\
\hline \multicolumn{2}{|c|}{$\begin{array}{l}\text { VOLTAGE: } \\
\text { AMERAGE: }\end{array}$} & $\begin{array}{l}120 / 208 \\
100\end{array}$ & $\begin{array}{l}\text { MAIN BREAKER: } \\
\text { INTERIOR: }\end{array}$ & & & & \\
\hline & BKR & & RIPTION & A日C & DESCRIPTION & BKR & \\
\hline 1 & 20 & & /STORAGE & 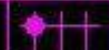 & EDUCATION ROOM & 20 & 2 \\
\hline 3 & 20 & & UPLIGHTS & & MULTIPURPOSE ROOM & 20 & 4 \\
\hline 5 & 20 & & DR TRACK & 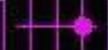 & MULTIPURPOSE ROOM & 20 & G \\
\hline 7 & 15 & & ARE & 4 & EDUCATION ROOM & 20 & 8 \\
\hline 9 & 15 & & ARE & & SPARE & 20 & 10 \\
\hline 11 & & & & 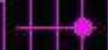 & 5PARE & 20 & 12 \\
\hline 13 & & & & 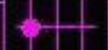 & SPARE & 20 & 14 \\
\hline
\end{tabular}

Figure 121. Public Assembly-1: Lighting Panel, "L2" 


\begin{tabular}{|c|c|c|c|c|c|c|c|}
\hline \multicolumn{3}{|c|}{ PANEL: $\mathrm{P2}$} & \multirow{3}{*}{$\begin{array}{l}\text { FED EY: } \\
\text { WOUNTING: } \\
\text { WLIN ERESKER: } \\
\text { INTERIOR: } \\
\text { RIPTON }\end{array}$} & \multirow{2}{*}{\multicolumn{4}{|c|}{$\begin{array}{l}\text { PANEL P1 } \\
\text { SURFACE } \\
\text { MAMIN LUGS } \\
3 \omega_{1} 4 W\end{array}$}} \\
\hline \multicolumn{2}{|c|}{$\begin{array}{l}\text { VOLTAGE } \\
\text { AMERAGE: }\end{array}$} & $\begin{array}{l}120 / 208 \\
225\end{array}$ & & & & & \\
\hline & BKR & & & $A B C$ & DESCRIPTION & BKR & \\
\hline 1 & 15 & UPPER & RECETTACLES & 4 & EXIT/EMERGENCY LIGHTING (LD) & 20 & 2 \\
\hline 3 & \multirow{3}{*}{$\begin{array}{l}45 \\
3 P\end{array}$} & \multirow{3}{*}{\multicolumn{2}{|c|}{ GSHP-5A }} & & DWH -1 & 20 & 4 \\
\hline 5 & & & & & INFLOOR RECEPTACLES & 15 & 6 \\
\hline 7 & & & & & REC, BF OPERATORS & 15 & 8 \\
\hline 9 & \multirow{3}{*}{$\begin{array}{l}45 \\
3 \mathrm{P}\end{array}$} & \multirow{3}{*}{\multicolumn{2}{|c|}{ CSHP-5日 }} & & KITCHEN REC. & 20 & 10 \\
\hline 11 & & & & & KITCHEN REC. & 20 & 12 \\
\hline 13 & & & & & KITCHEN REC. & 20 & 14 \\
\hline 15 & $\rightarrow$ & \multirow{2}{*}{\multicolumn{2}{|c|}{ GSHP-B }} & & KTCHEN FRIDGE & 15 & 16 \\
\hline$\frac{17}{19}$ & 3 & & & & STOVE & $\begin{array}{l}50 \\
2 P\end{array}$ & $\frac{18}{20}$ \\
\hline 21 & 15 & \multicolumn{2}{|c|}{ 5PARE } & & RECETARLLES & 15 & 27 \\
\hline 23 & 15 & REC. 0 & DOR [AUERY & & \multirow{2}{*}{$E V-2$} & \multirow{2}{*}{$\begin{array}{l}15 \\
2 \mathrm{P}\end{array}$} & 24 \\
\hline 25 & 15 & \multicolumn{2}{|c|}{ DISHWASHER } & & & & 26 \\
\hline 27 & 15 & \multicolumn{2}{|c|}{ (FREF ZER } & & MOTORIZED EUNDS & 15 & 28 \\
\hline 29 & 20 & \multicolumn{2}{|c|}{ SPARE } & 8 & & & 30 \\
\hline
\end{tabular}

Figure 122. Public Assembly-1: Plugoad Panel, "P2" 


\begin{tabular}{|c|c|c|c|c|c|c|c|}
\hline \multicolumn{3}{|c|}{ PANE- P3 } & \multirow{3}{*}{$\begin{array}{l}\text { FED BY: } \\
\text { WOUNTING: } \\
\text { WLIN BRESKER: } \\
\text { INTERIOR: } \\
\text { RIPTION }\end{array}$} & \multirow{2}{*}{\multicolumn{4}{|c|}{$\begin{array}{l}\text { PANE P1 } \\
\text { SURFACE } \\
\text { WBN WUGS } \\
3 \%, 4 W\end{array}$}} \\
\hline \multicolumn{2}{|c|}{$\begin{array}{l}\text { WOLTAGE: } \\
\text { AMERAGE: }\end{array}$} & $\begin{array}{l}120 / 208 \\
225\end{array}$ & & & & & \\
\hline & BKR & DESCRIPTION & & $\mathrm{ABC}$ & DESCRIPTION & BKR & \\
\hline 1 & 15 & \multicolumn{2}{|c|}{ REC. - STOR./EXHIEIT/ME } & & EXIT/EKERGENCY UGHTNG (D) & 20 & 2 \\
\hline 3 & 15 & \multicolumn{2}{|c|}{ RECEPTACLES - STOR./EXHIEIT } & & REC. WASHROONS & 15 & 4 \\
\hline 5 & 15 & \multicolumn{2}{|c|}{ RECEPTACLES - STOR./EXHIEIT } & & HAND DRYER & 20 & 6 \\
\hline 7 & 15 & \multicolumn{2}{|c|}{ RECETACLES - STOR./EXHIEIT } & & HAND [RYER & 20 & 8 \\
\hline 9 & 15 & \multicolumn{2}{|c|}{ RECEPTACLES - RESOURCE } & & HAND DRYER & 20 & 10 \\
\hline 11 & 15 & \multicolumn{2}{|c|}{ RECEPTACLES - EXHIIT FLOOR } & & HAND DRYER & 20 & 12 \\
\hline 13 & 15 & \multicolumn{2}{|c|}{ REC. - WULTIPURPOSE FLOOR } & & HAND DPFER & 20 & 14 \\
\hline 15 & 15 & \multicolumn{2}{|c|}{ RECEPTACLES - EXHIET WAШ } & & REC. MECH/ELEC & 15 & 16 \\
\hline 17 & 15 & \multicolumn{2}{|c|}{ REC. - OFFICE FRICEE } & & \multirow{2}{*}{ HUM } & \multirow{2}{*}{$\begin{array}{l}15 \\
2 P\end{array}$} & 18 \\
\hline 19 & 15 & \multicolumn{2}{|c|}{ REC. - RET创 } & & & & 20 \\
\hline 21 & 15 & \multicolumn{2}{|c|}{ REC. OFFICE } & & $E F-1, E F-2, E F-3$ & 15 & 22 \\
\hline 23 & 15 & \multicolumn{2}{|c|}{ REC, OFFICE } & & DFFCE COUNTER & 20 & 24 \\
\hline 25 & 15 & \multicolumn{2}{|c|}{ REC. OFFICE } & & DWH -2 & 20 & 28 \\
\hline 27 & 15 & \multicolumn{2}{|c|}{ REC. ENTRANLE, BF OPERATOR } & & D내 -3 & 20 & 28 \\
\hline 29 & 15 & \multicolumn{2}{|c|}{ REC - EXHIBT WHШ } & & OFFCE - WICROWHE & 20 & 30 \\
\hline 31 & 20 & \multicolumn{2}{|c|}{ SPARE } & & & 15 & 32 \\
\hline 33 & 20 & \multicolumn{2}{|c|}{ SPARE } & & $5 P-3$ & $2 \mathrm{P}$ & 34 \\
\hline $\begin{array}{l}35 \\
37\end{array}$ & $\begin{array}{l}20 \\
2 P\end{array}$ & \multicolumn{2}{|c|}{ SP1.1, SP1.2 } & & $W P-1$ & $\begin{array}{l}15 \\
2 P\end{array}$ & $\frac{38}{38}$ \\
\hline $\begin{array}{l}39 \\
41\end{array}$ & $\begin{array}{l}20 \\
2 P\end{array}$ & \multicolumn{2}{|c|}{ 5P2.1, SP2.2 } & & $W P-1, W P-2$ & $\begin{array}{l}20 \\
2 P\end{array}$ & \begin{tabular}{|l|}
40 \\
42
\end{tabular} \\
\hline
\end{tabular}

Figure 123. Public Assembly-1: Receptacles Panel, "P3" 


\section{Appendix E- Full-size Graphics}
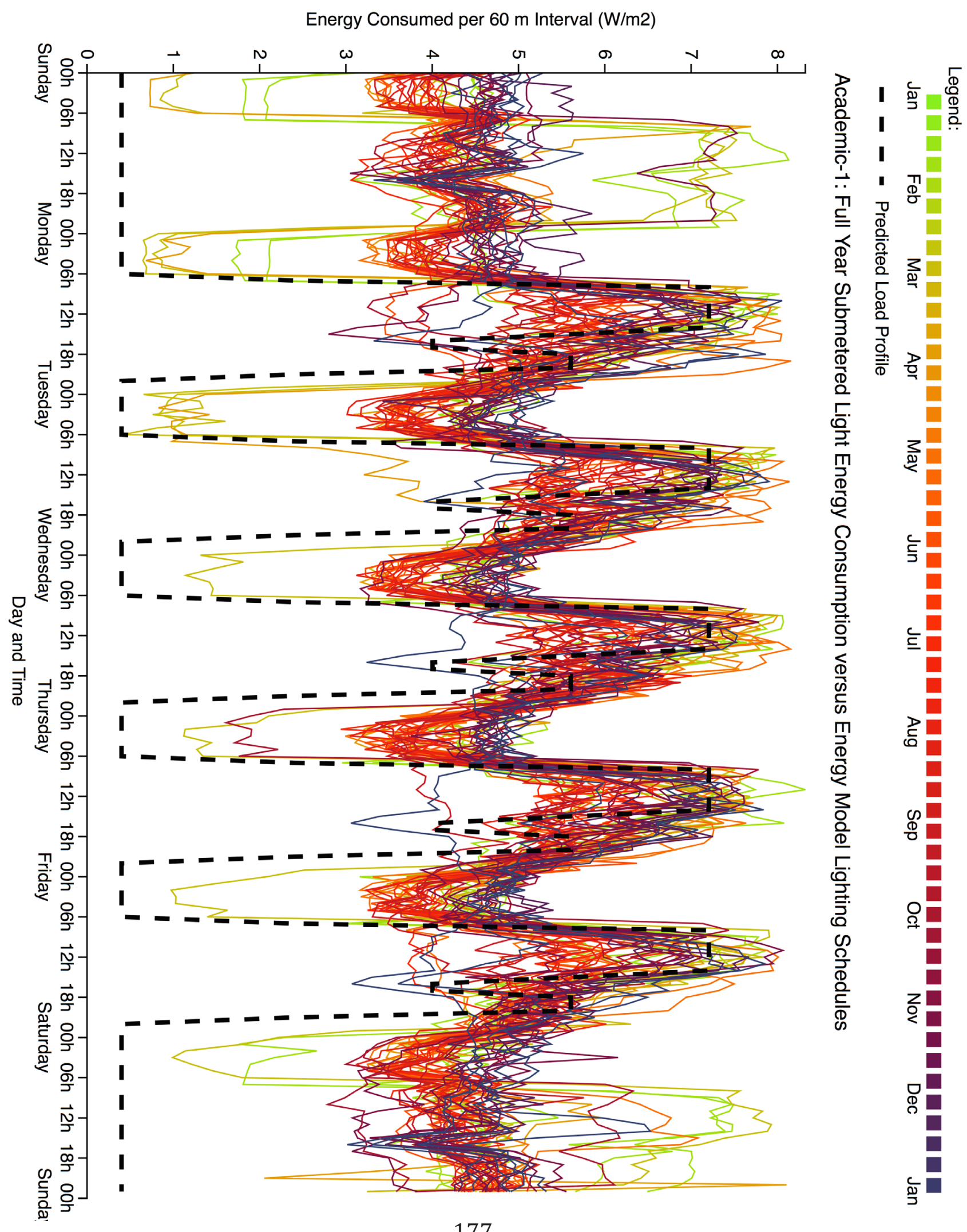
Energy Consumed per $60 \mathrm{~m}$ Interval (W/m2)

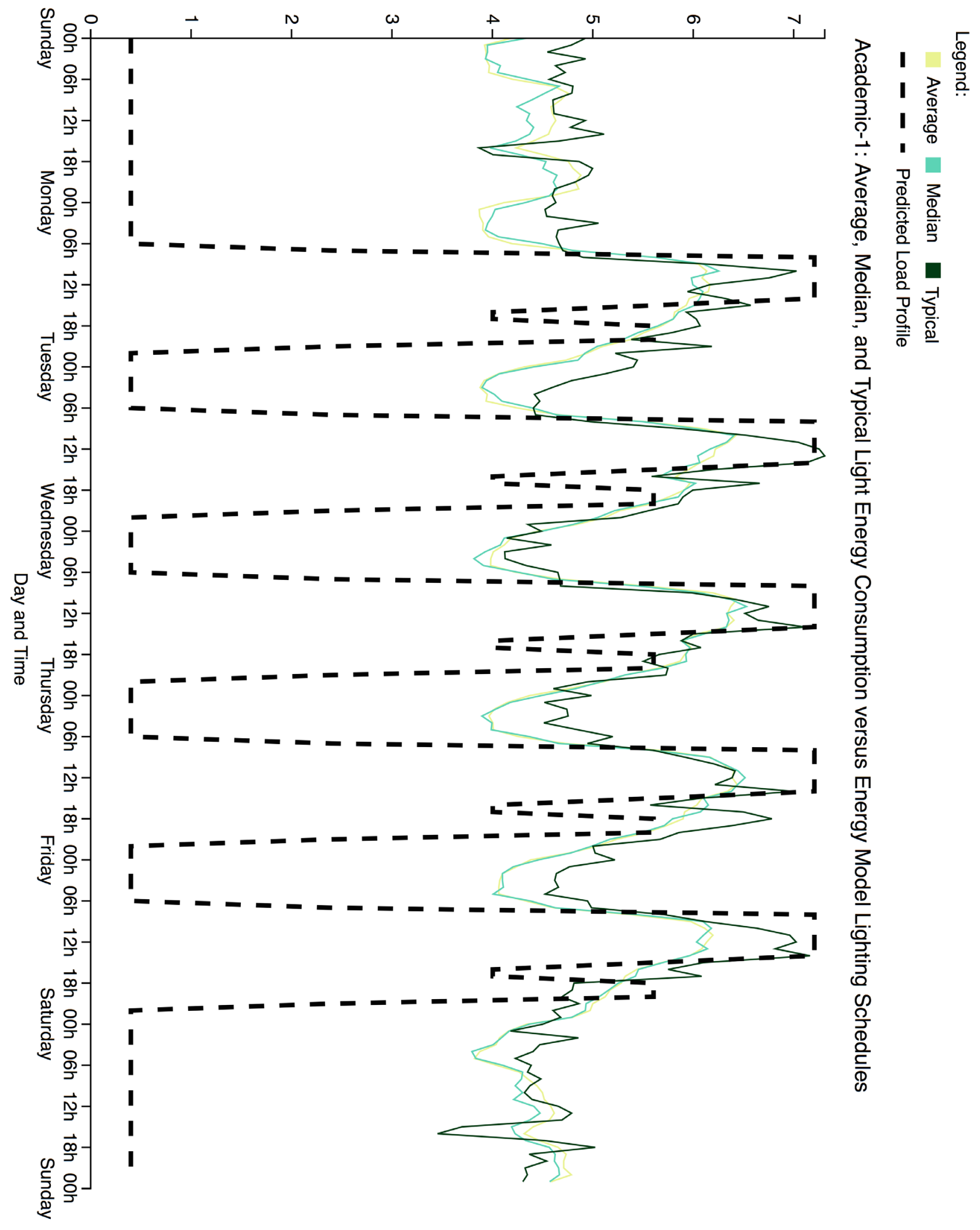




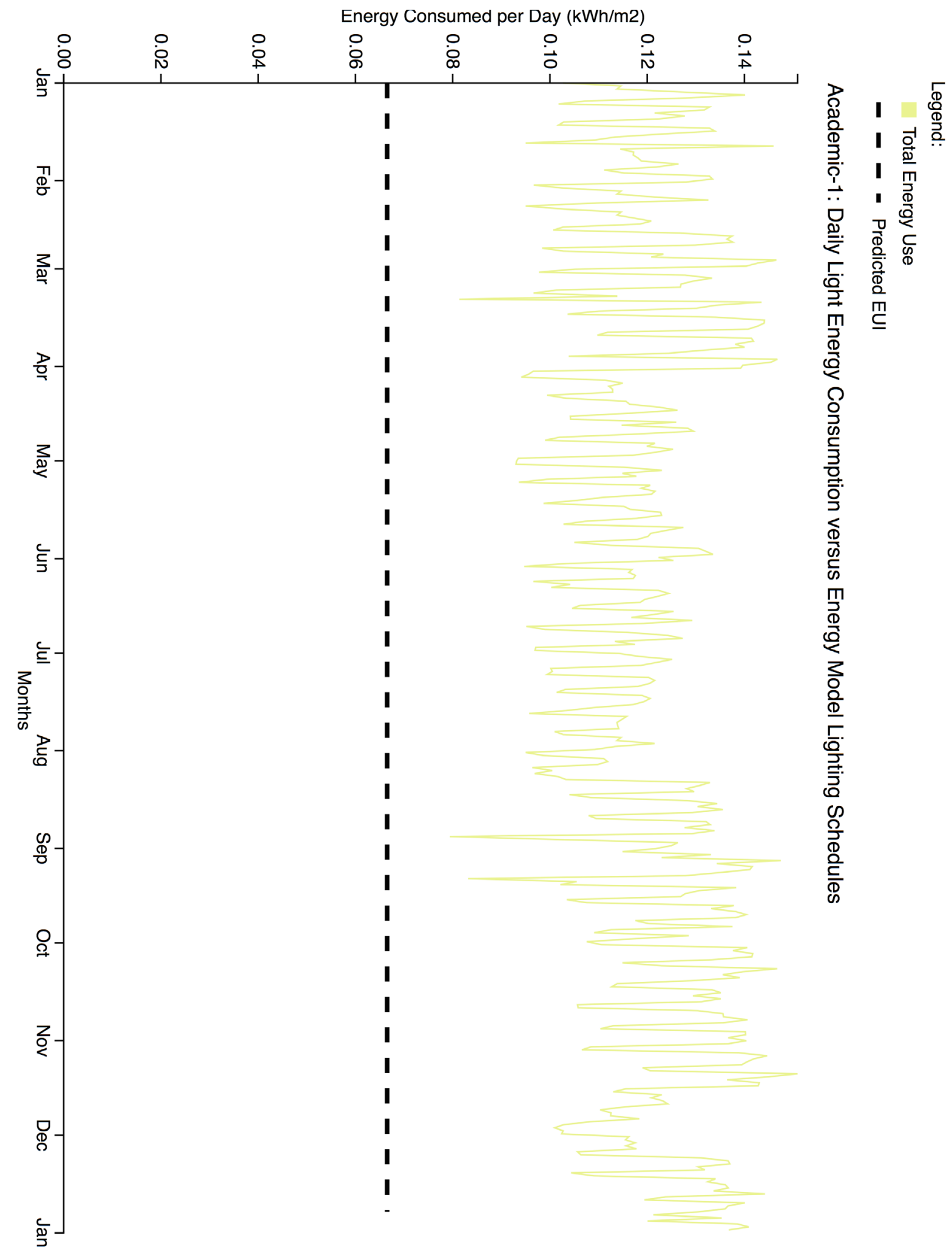



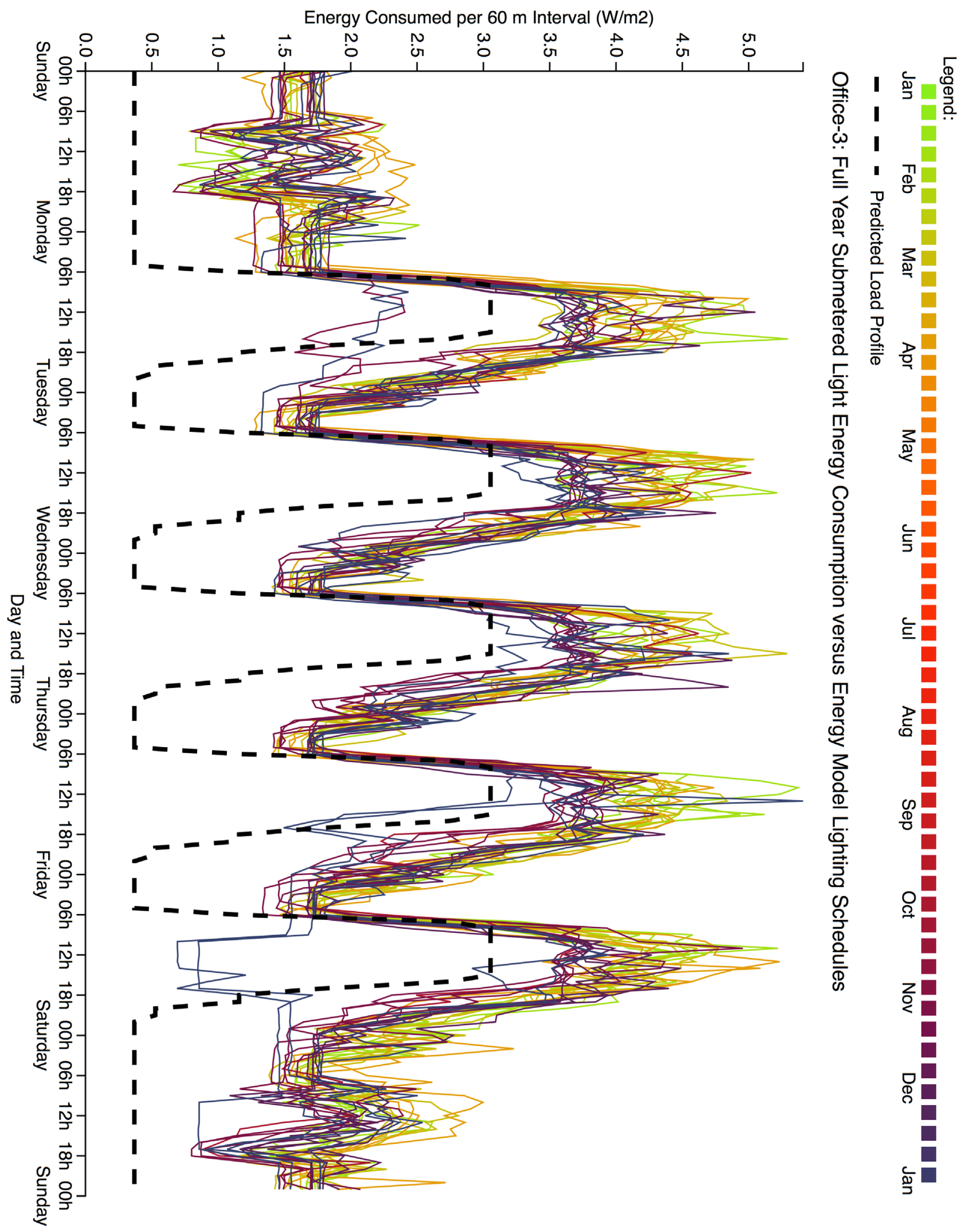


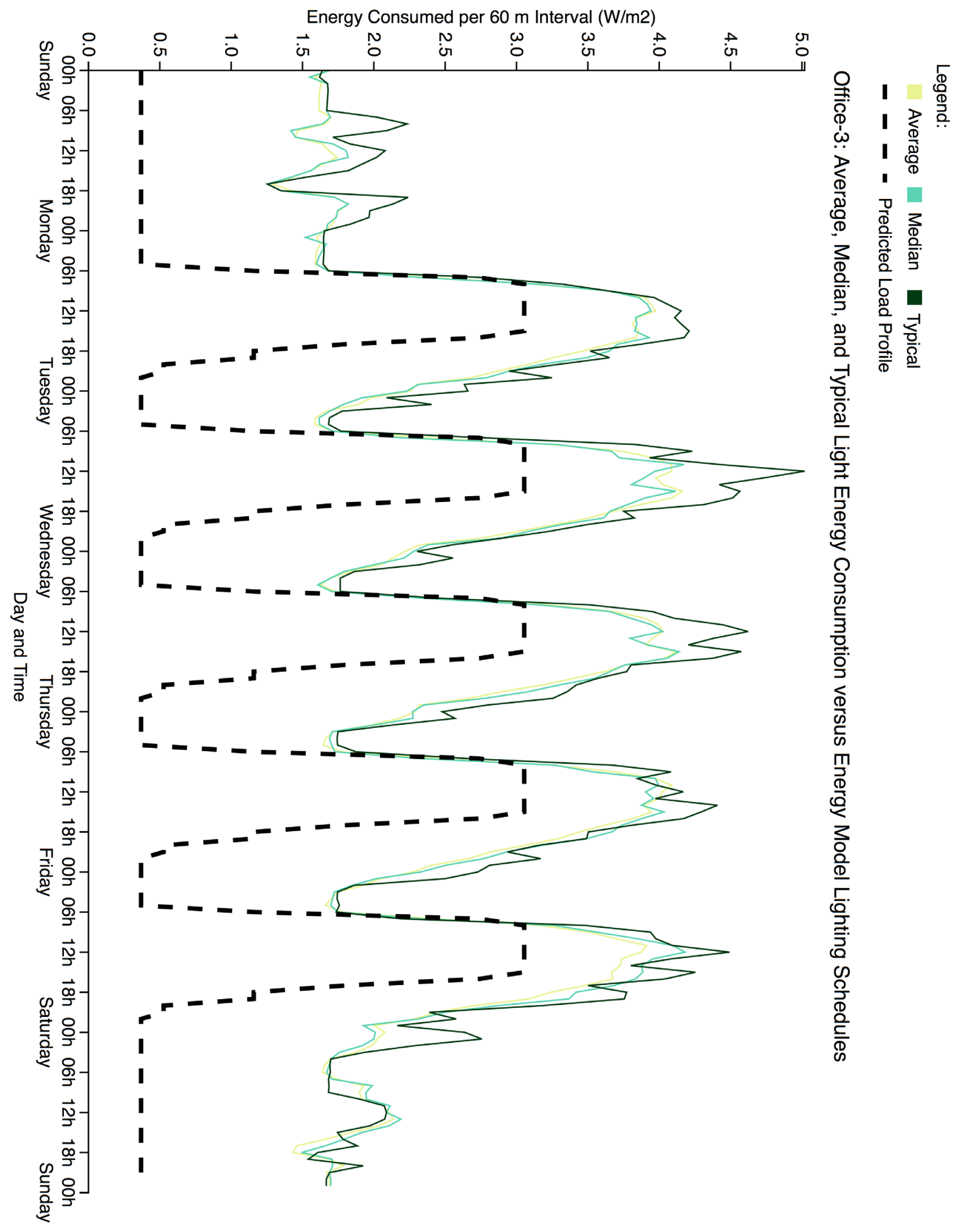




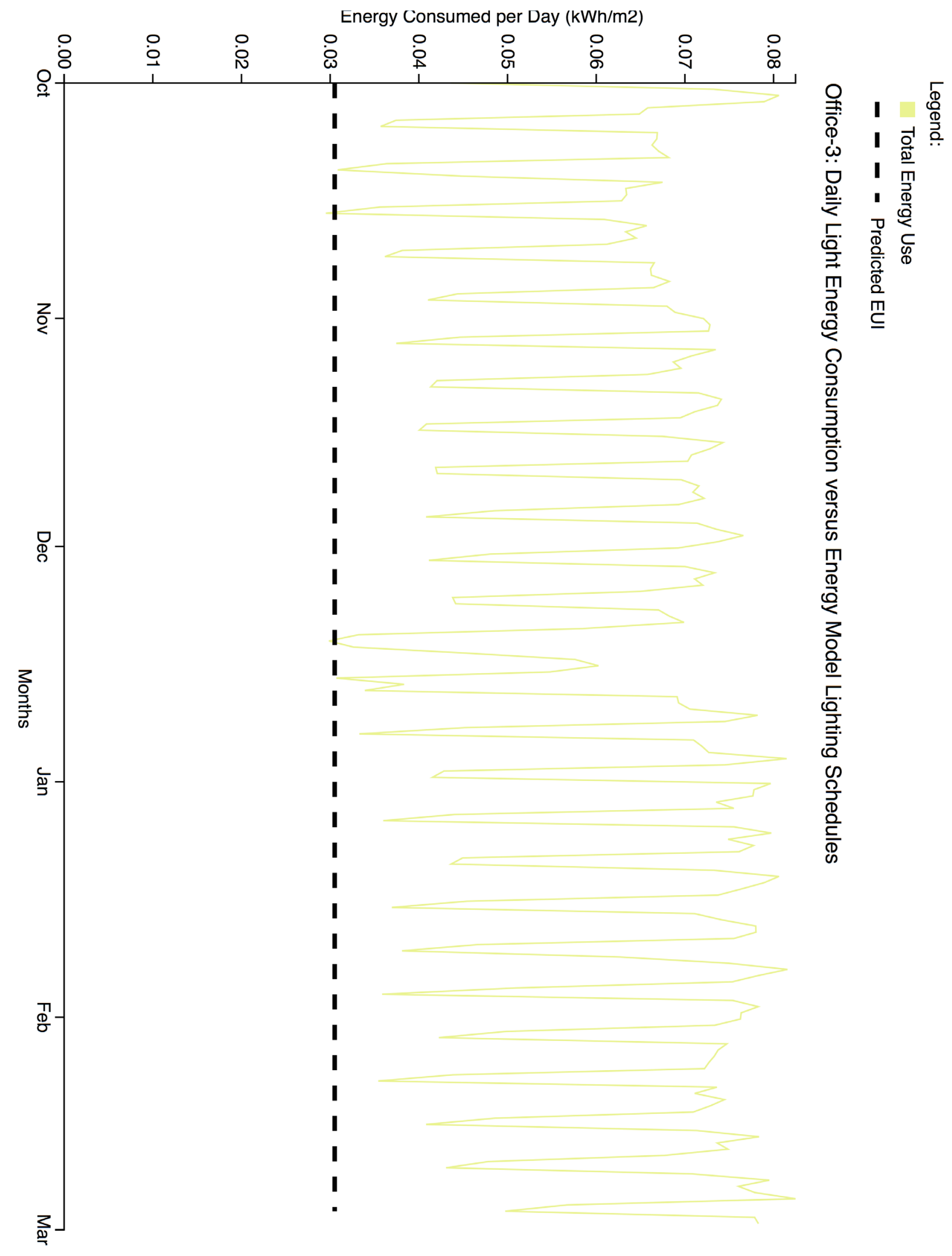




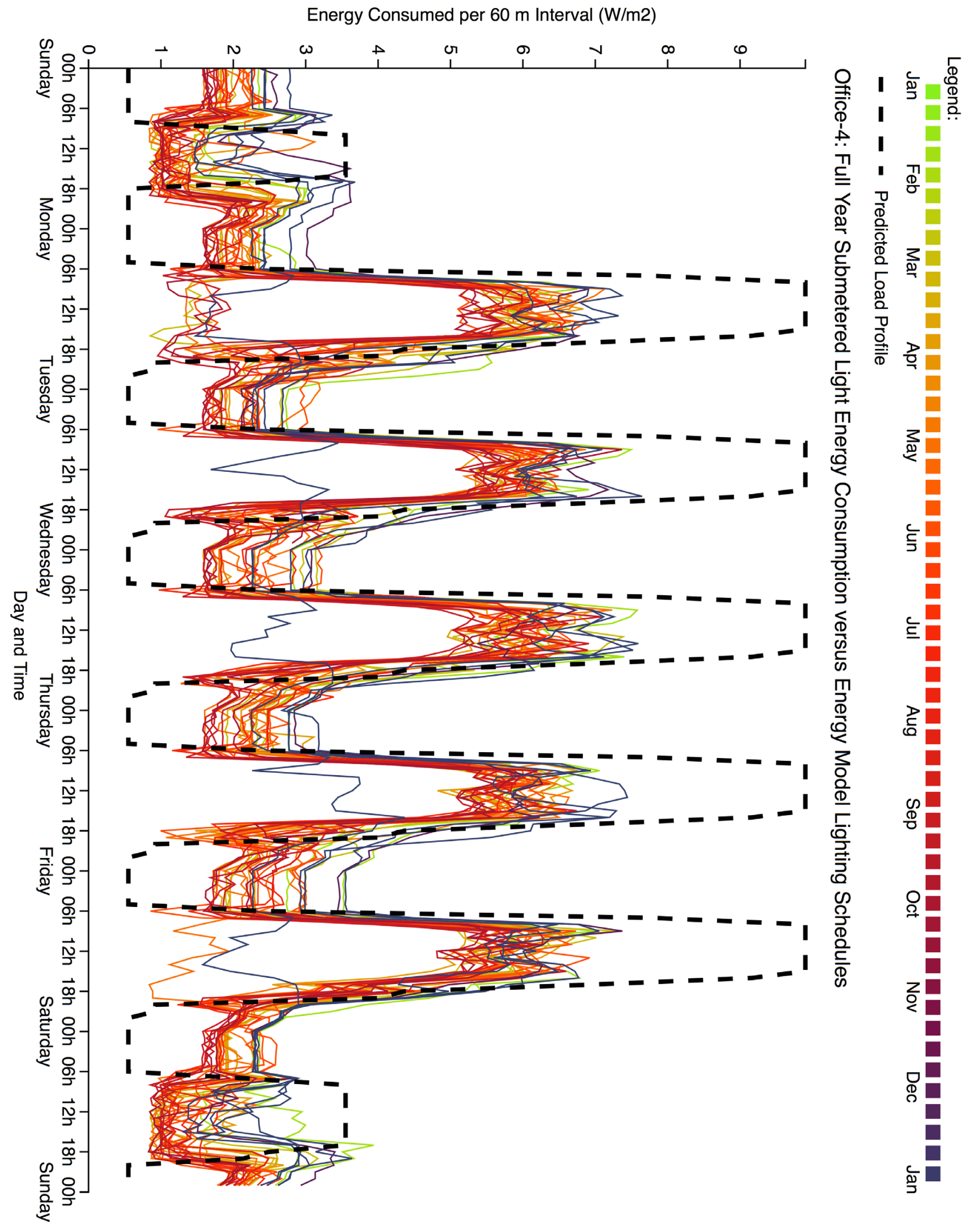




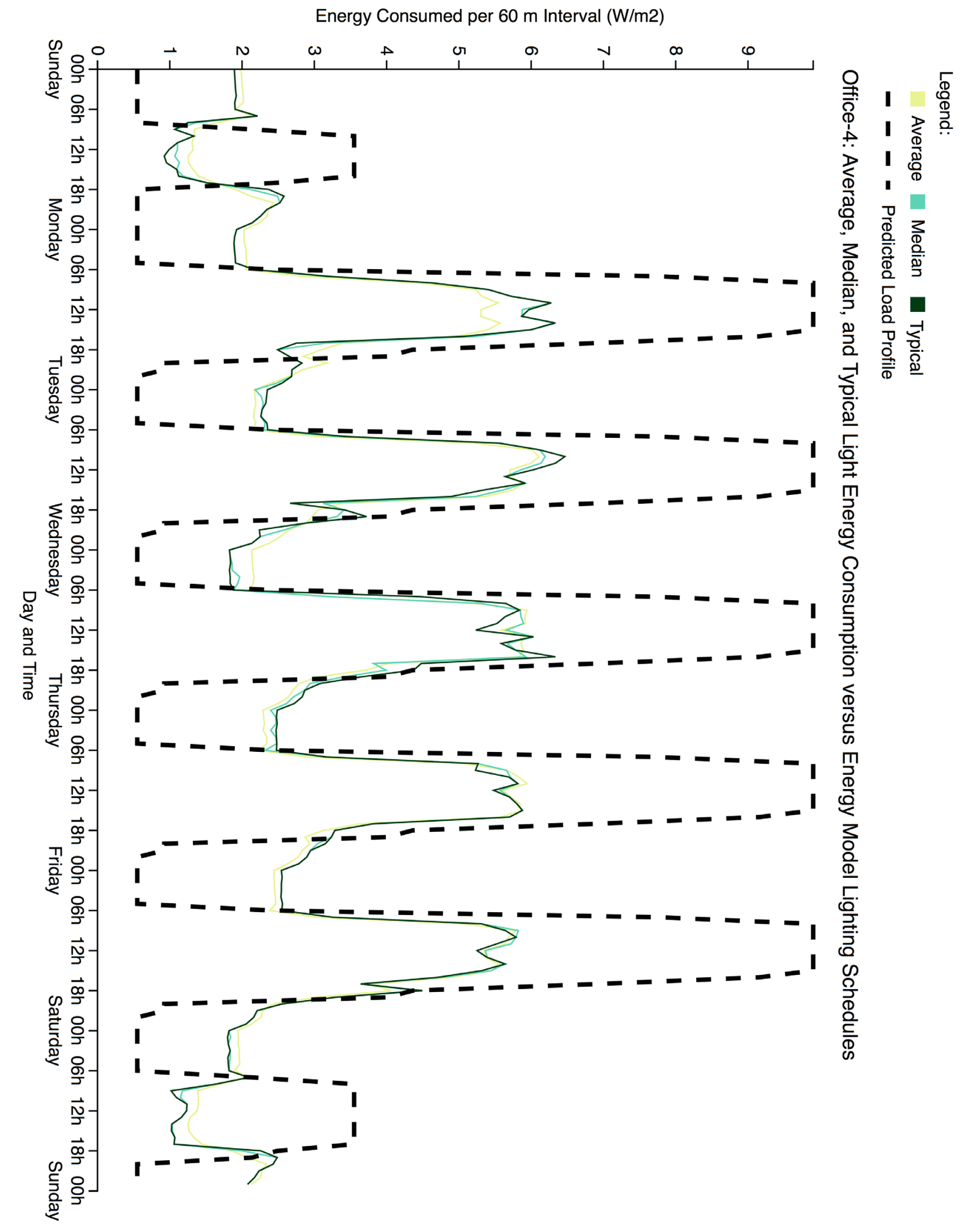




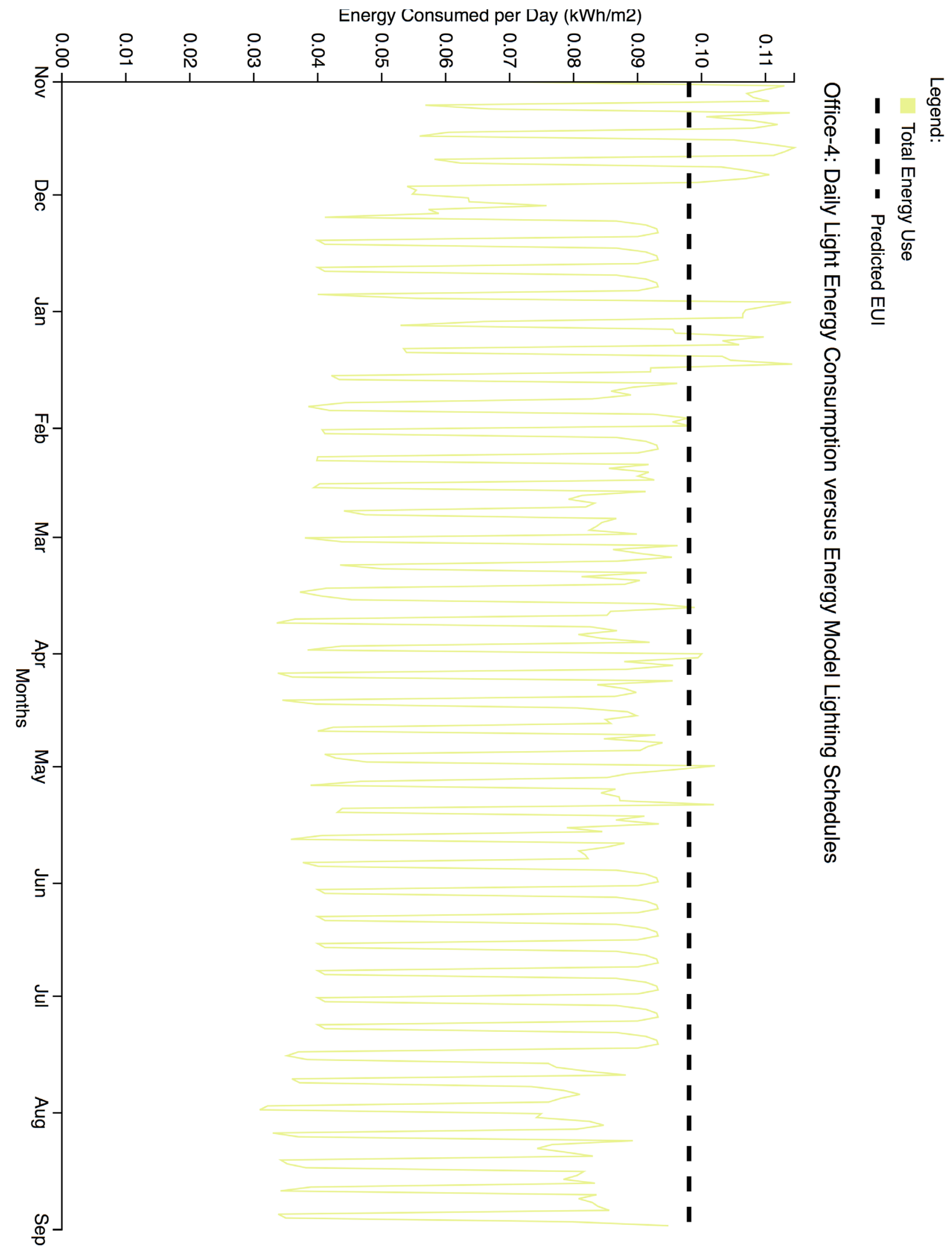



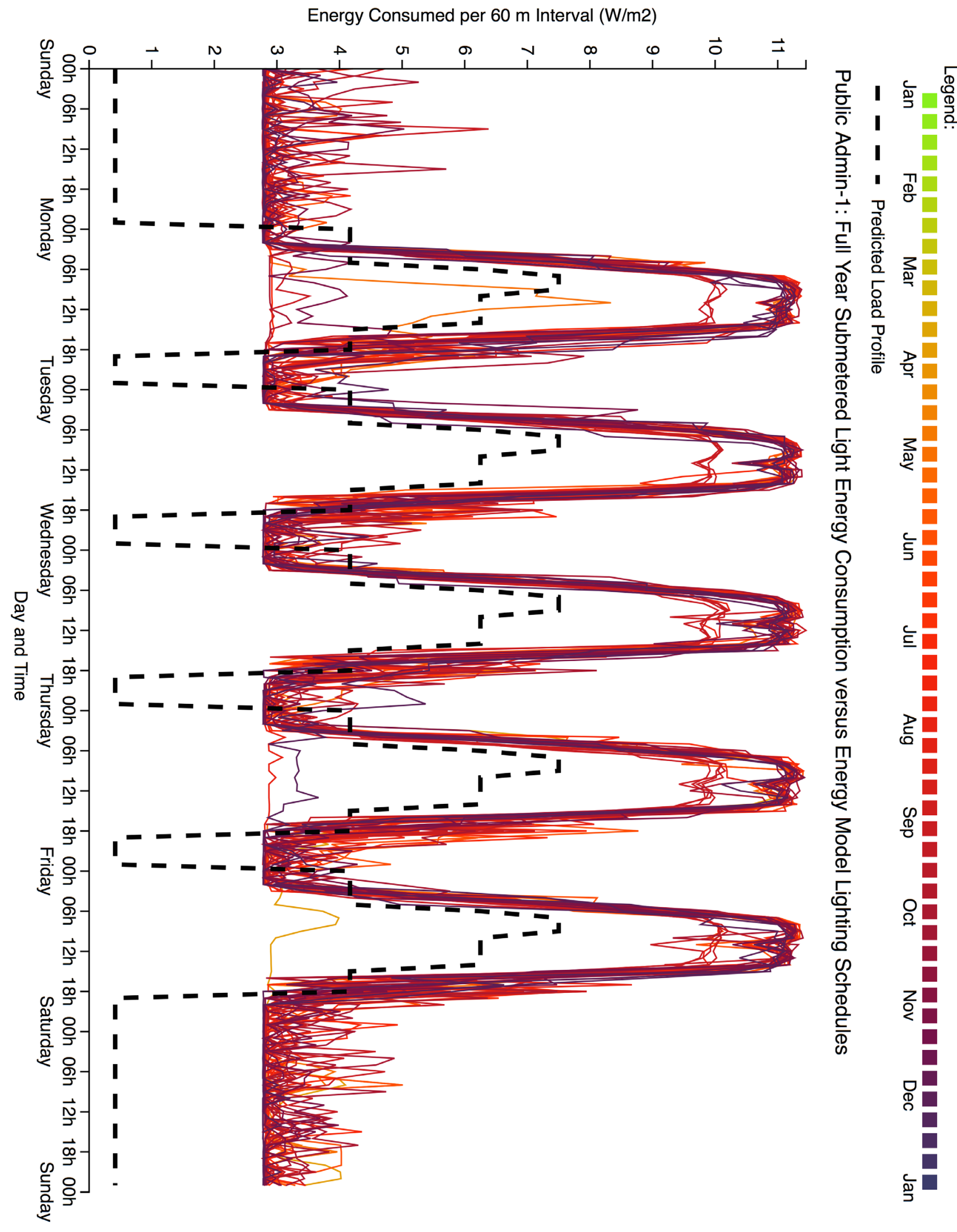


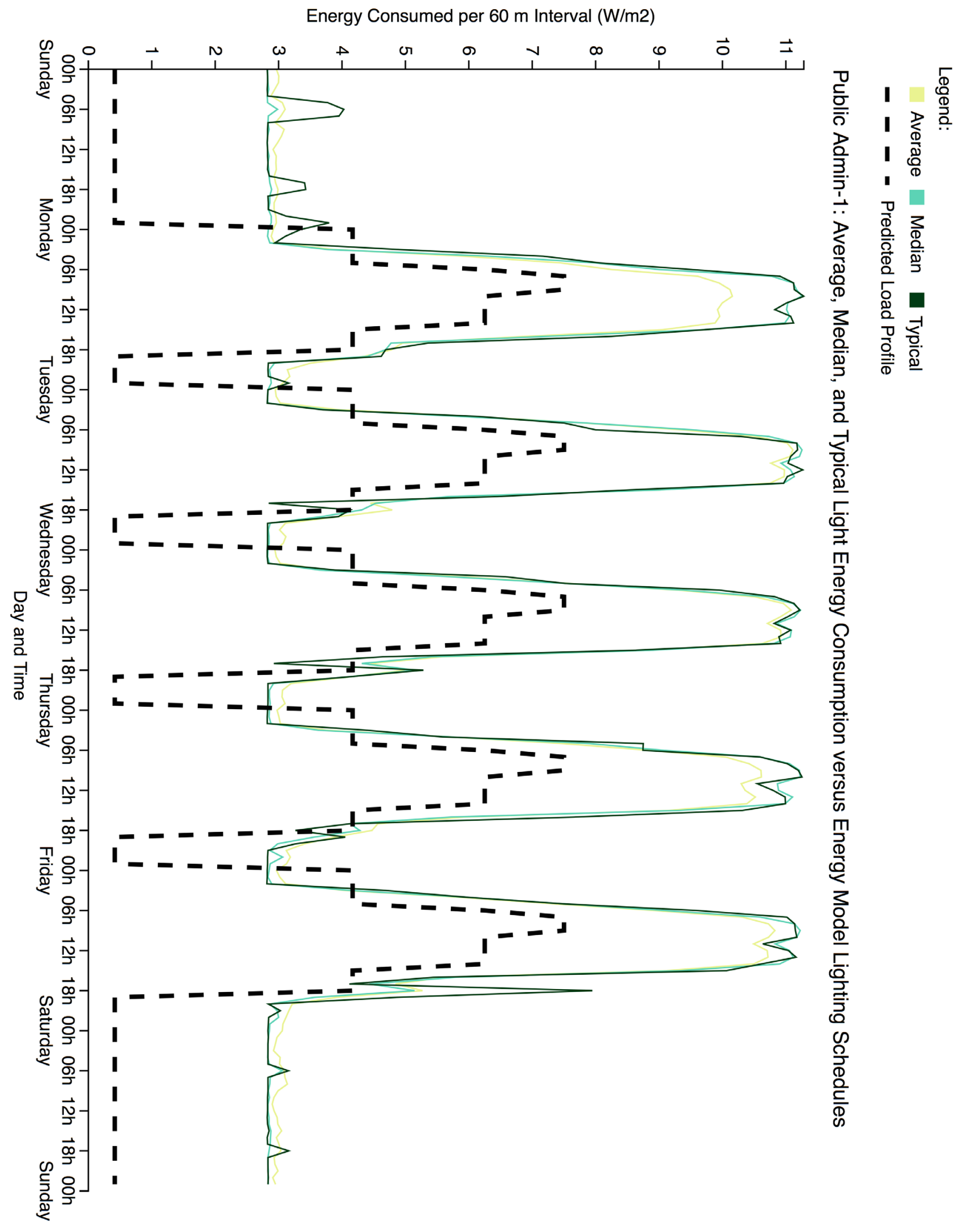




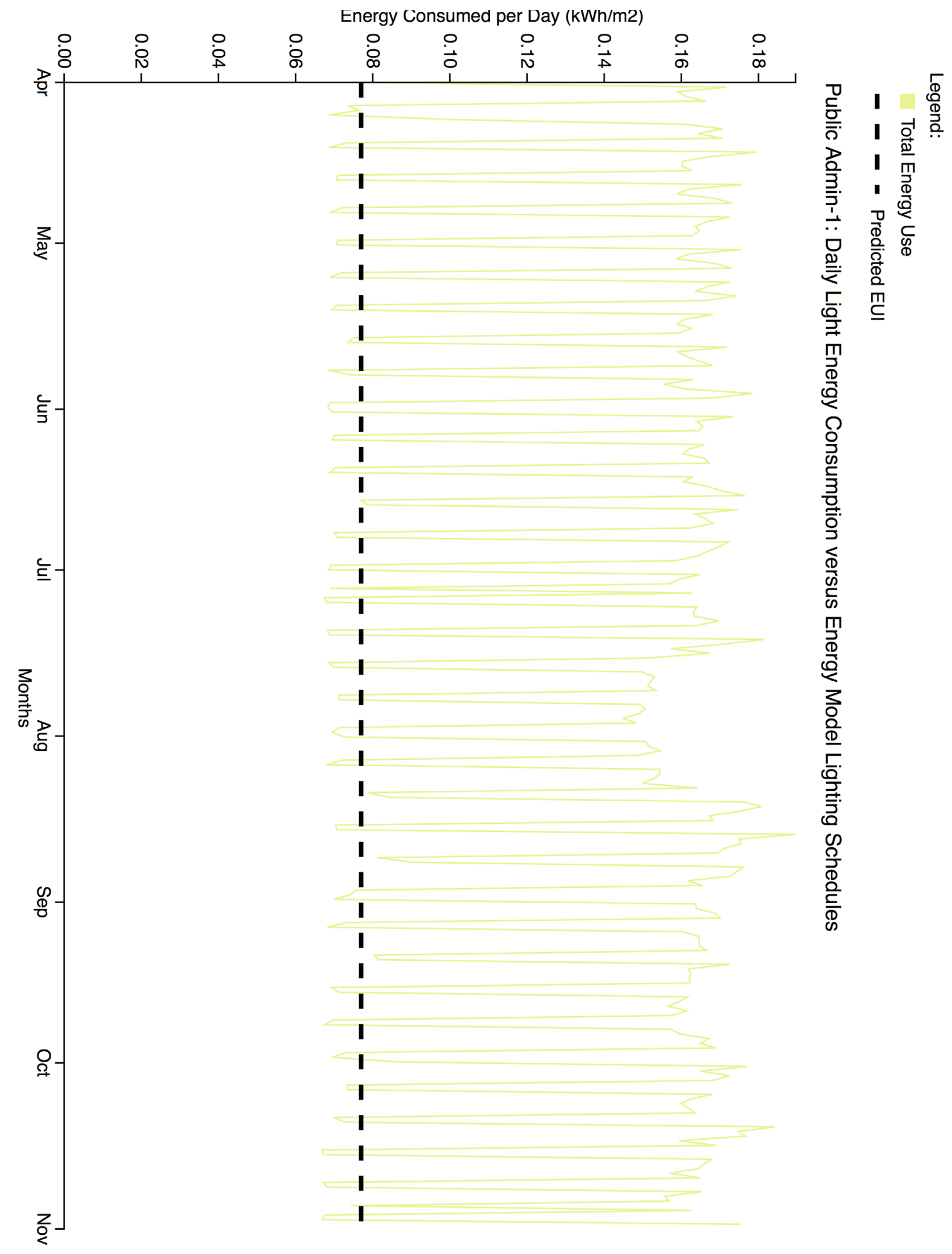



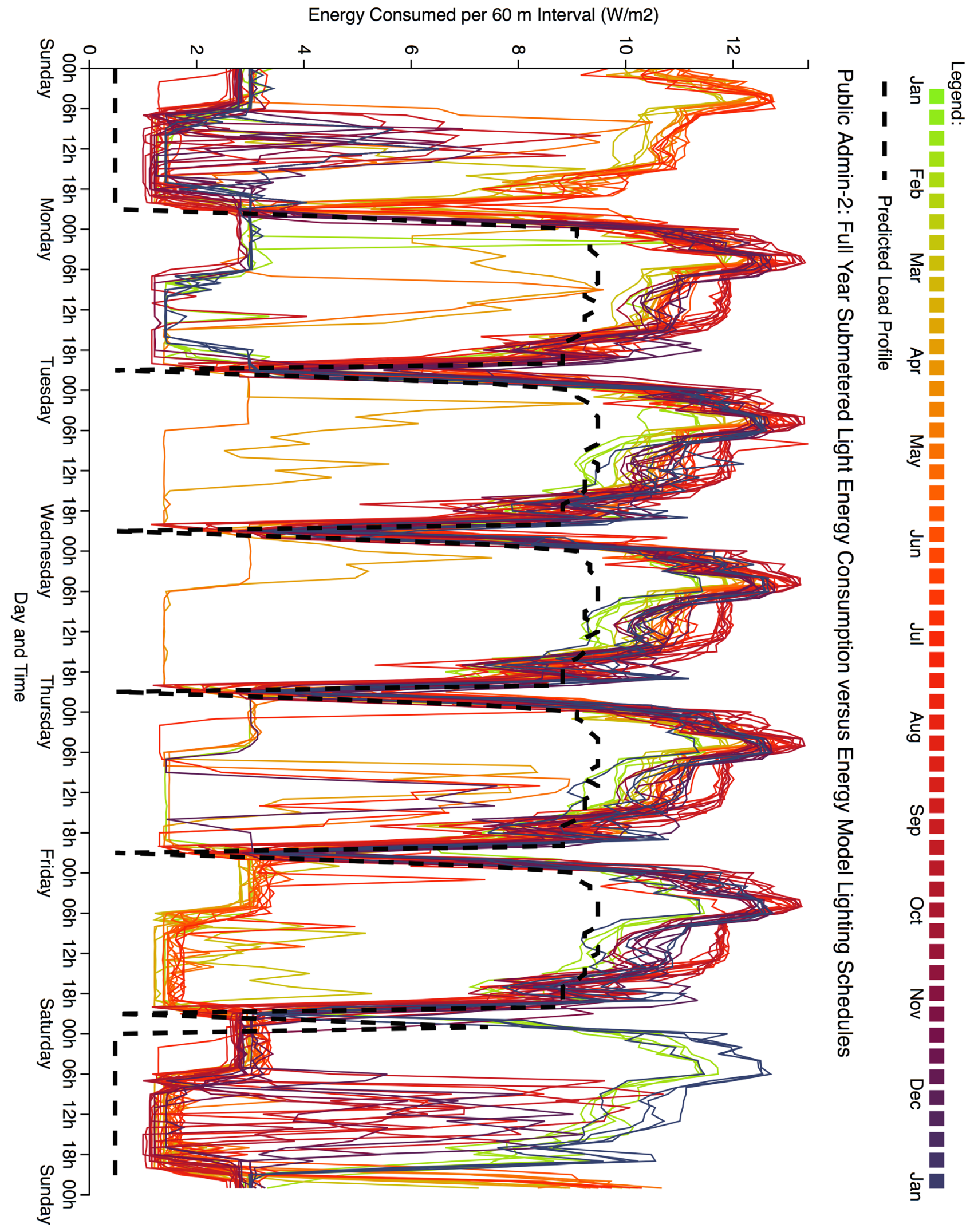

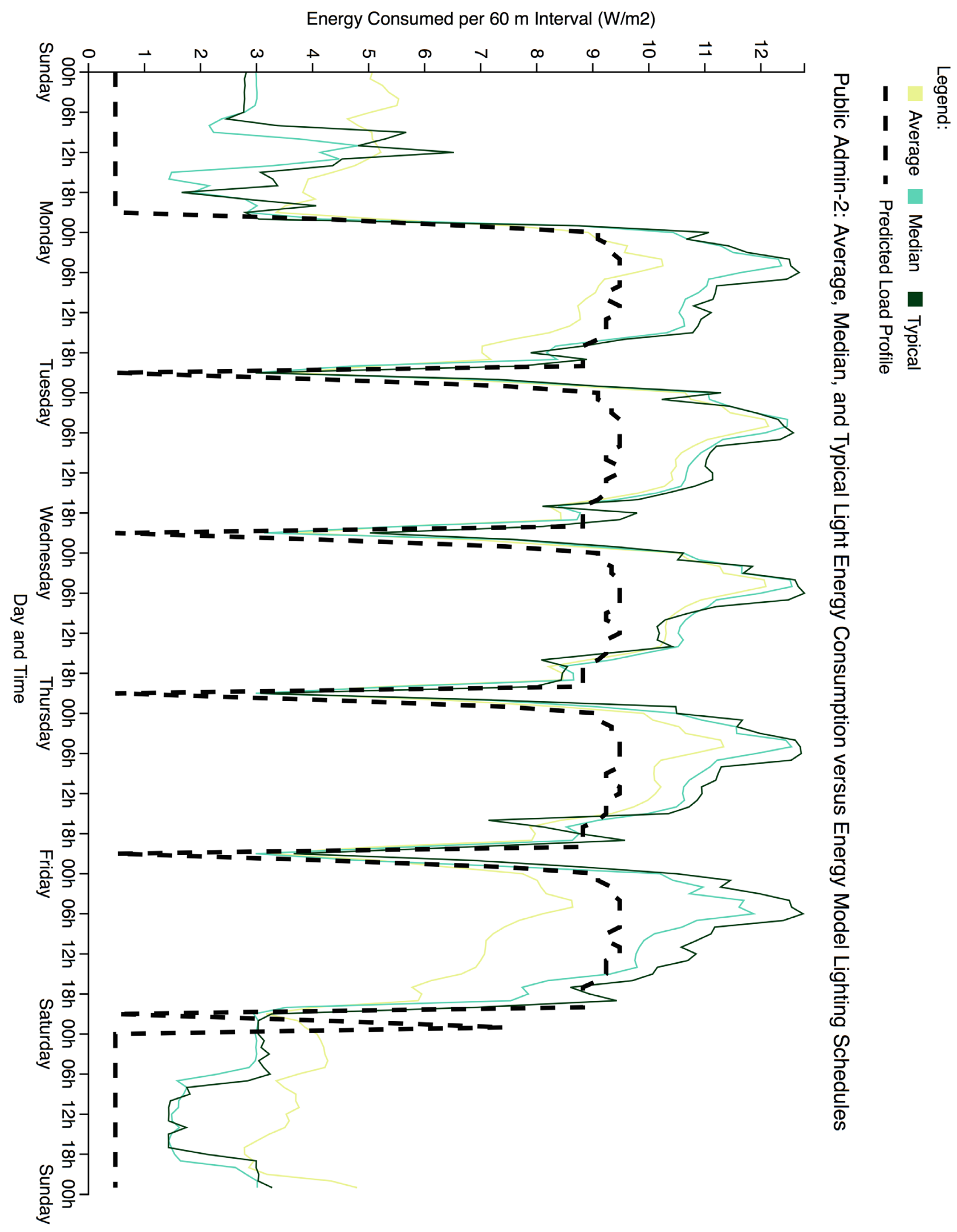


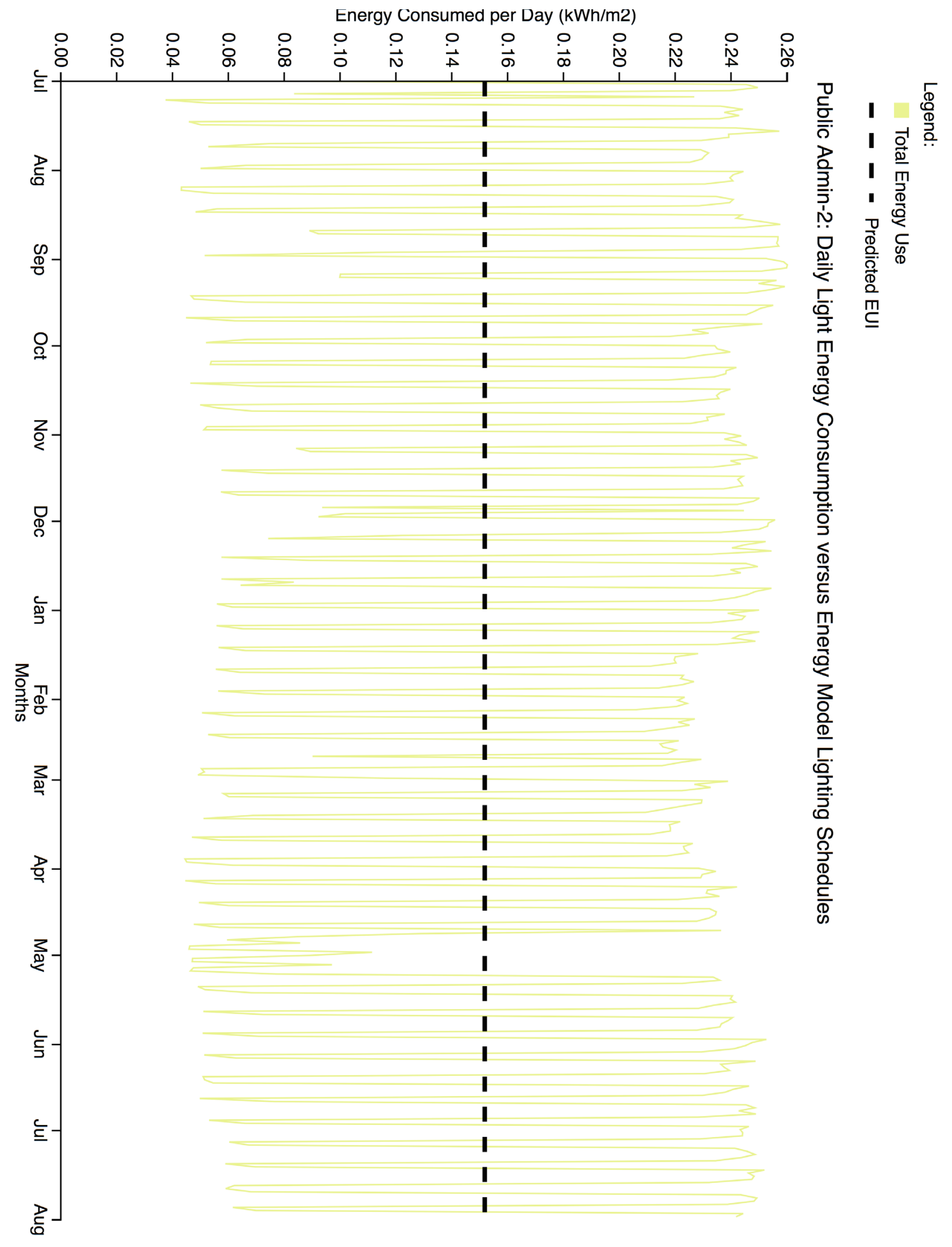


Energy Consumed per $60 \mathrm{~m}$ Interval (W/m2)
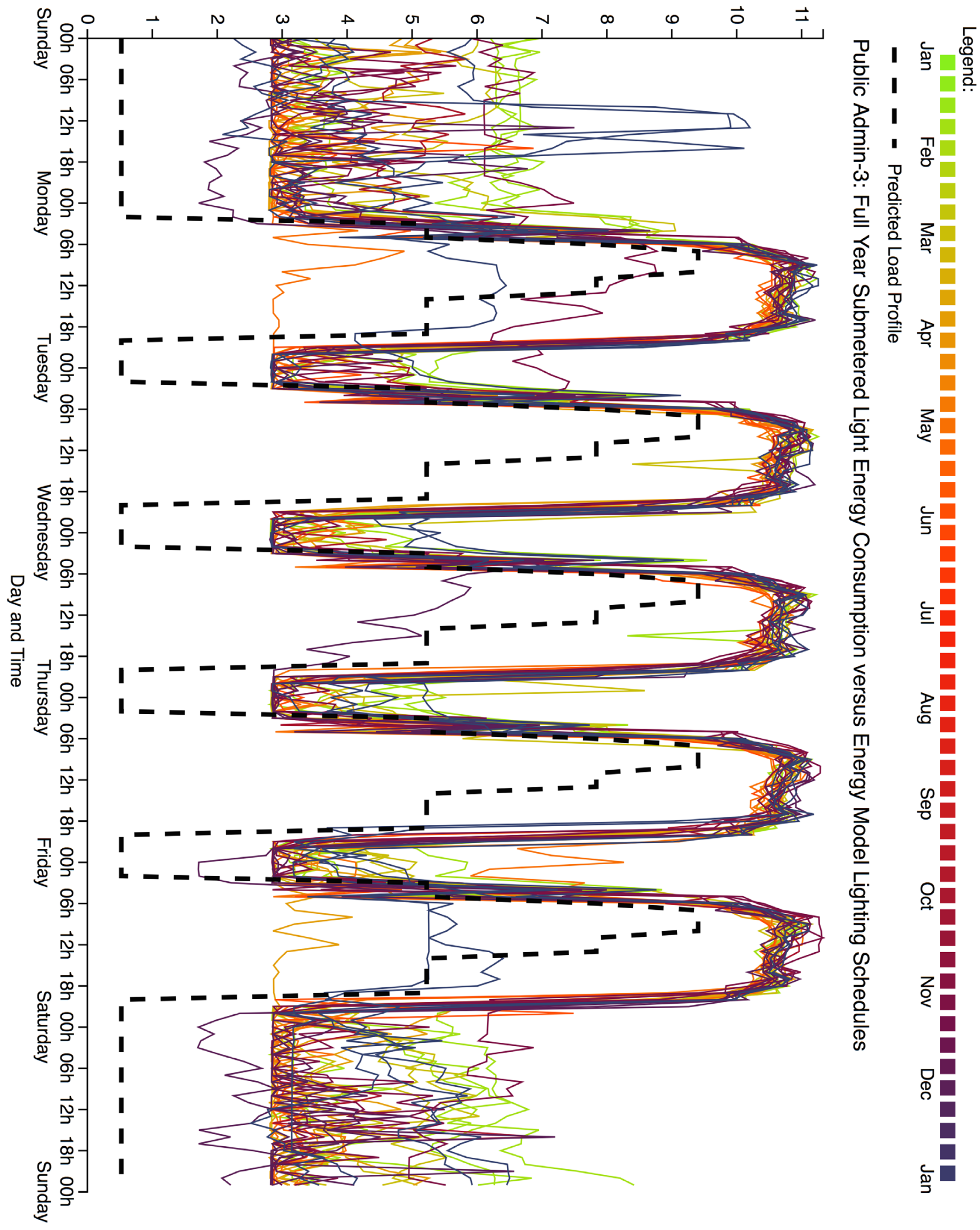


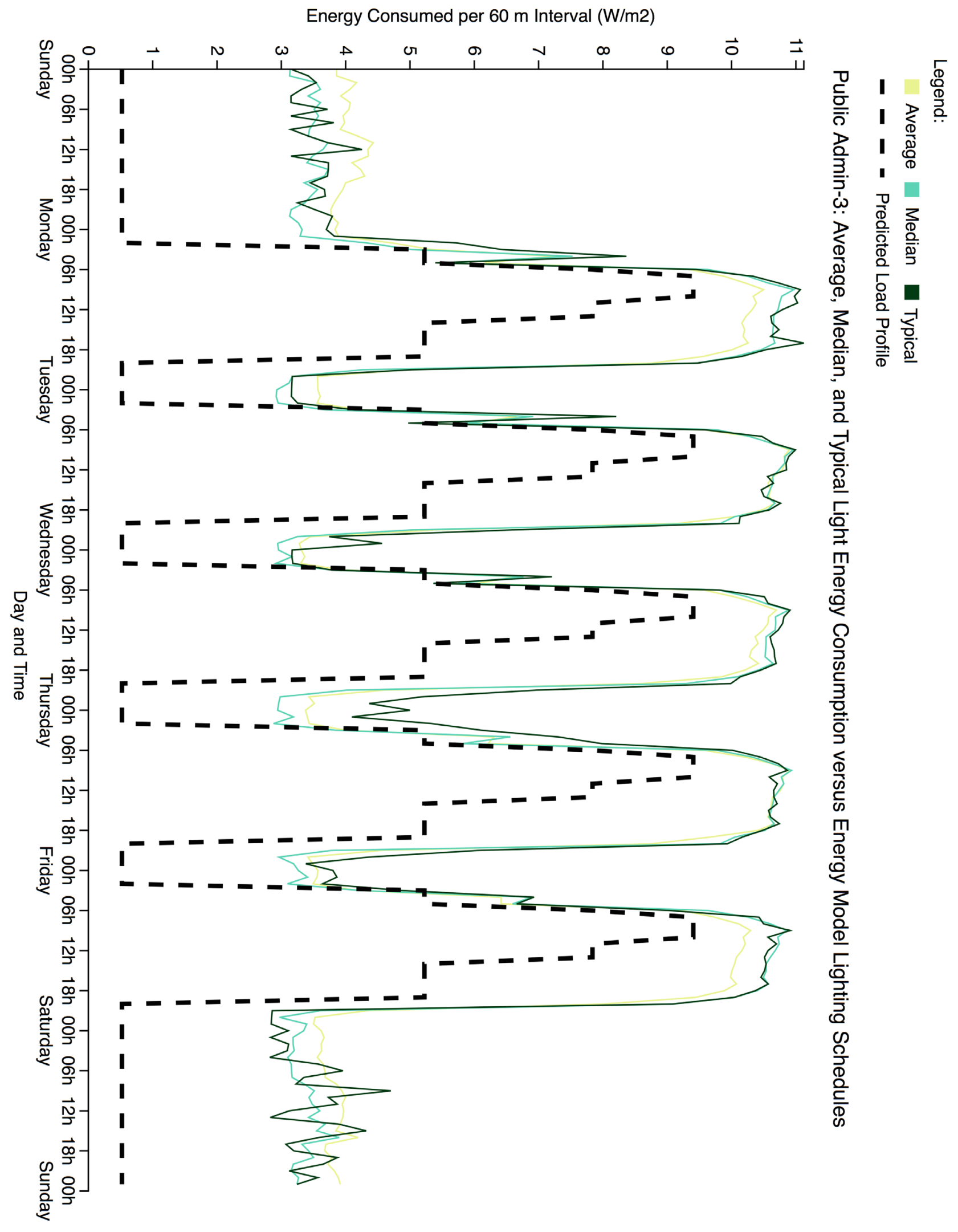




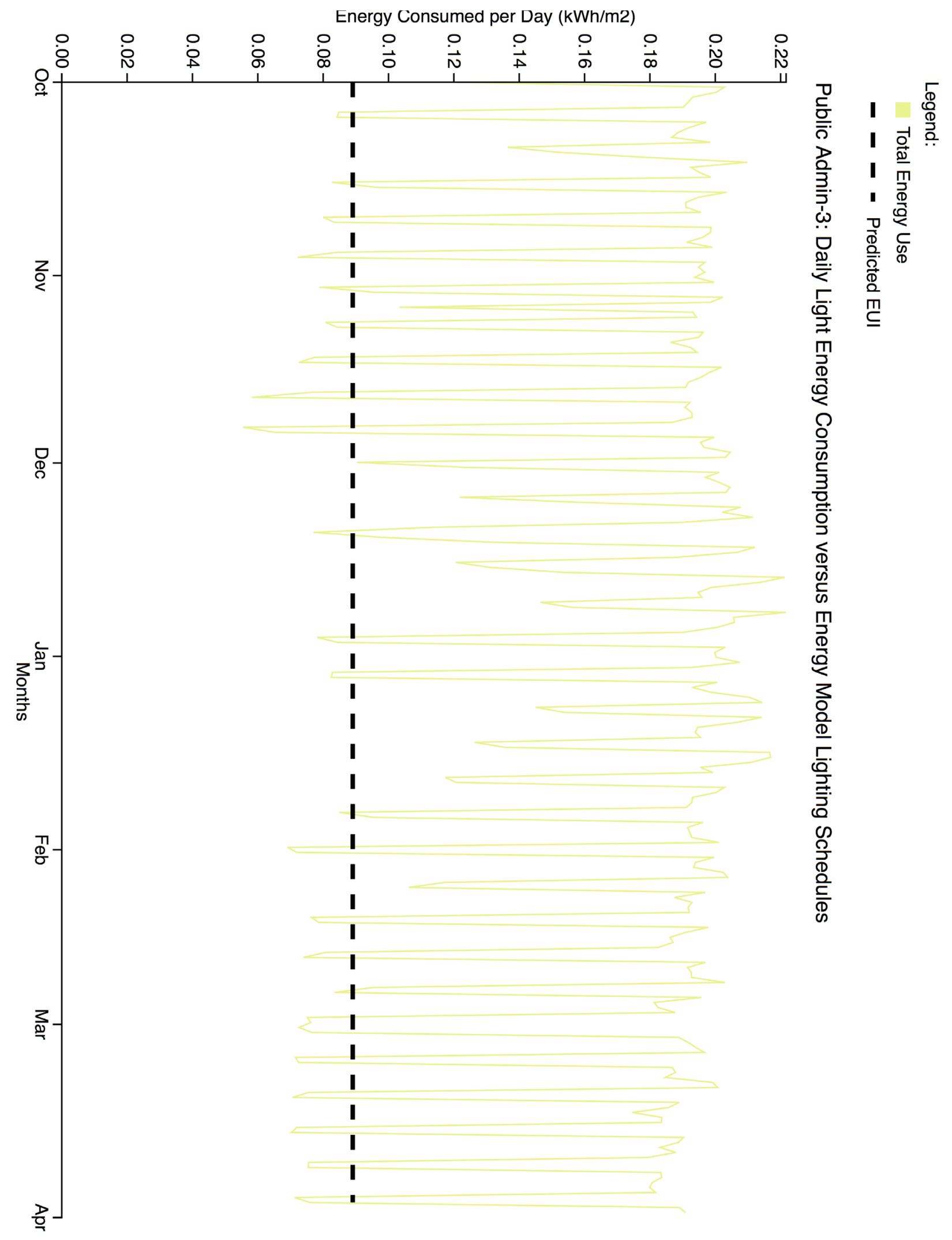


Energy Consumed per $60 \mathrm{~m}$ Interval (W/m2)

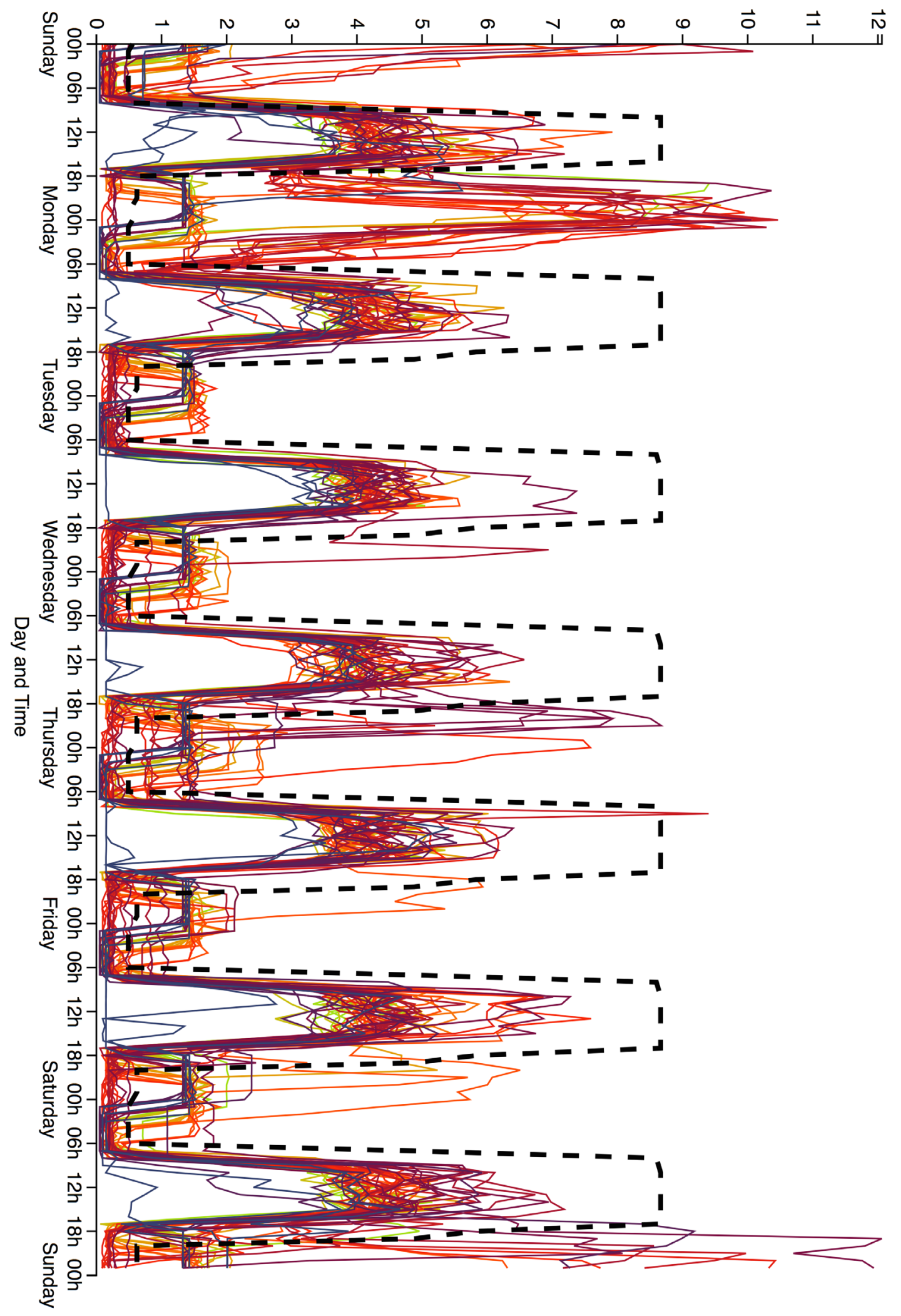

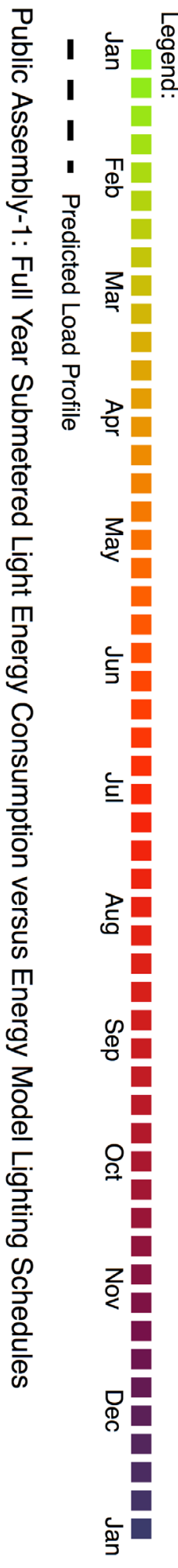




\section{Energy Consumed per $60 \mathrm{~m}$ Interval (W/m2)}

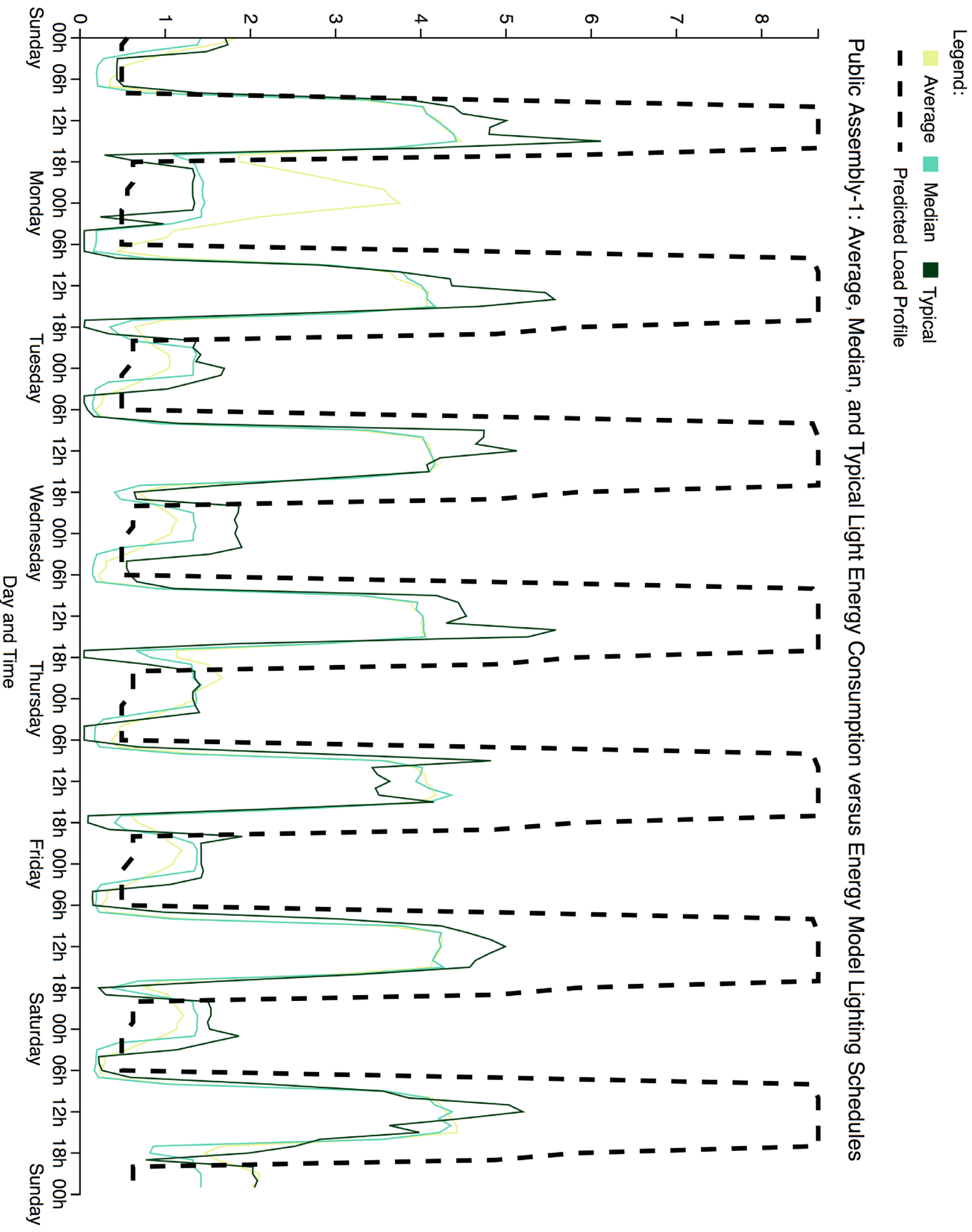




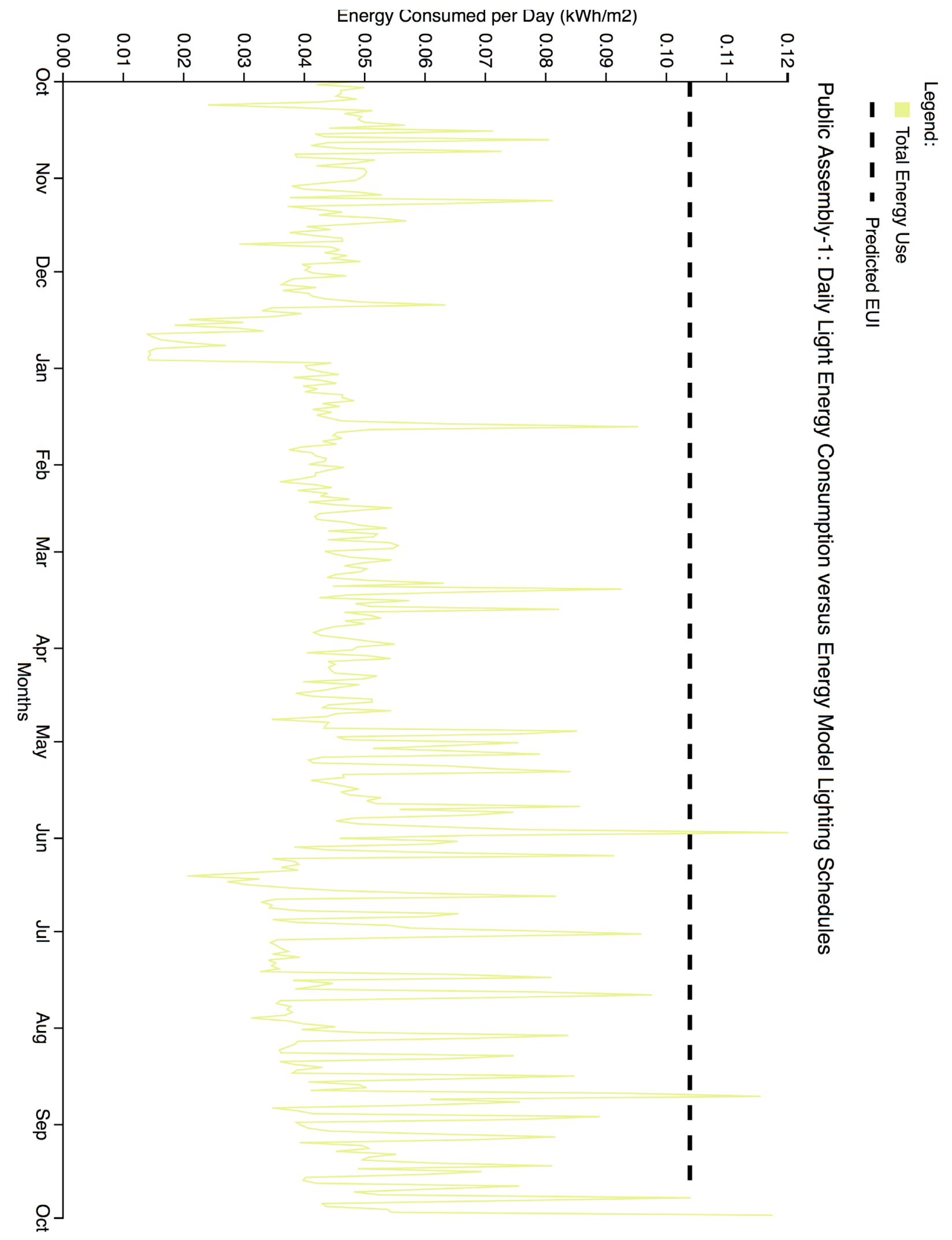



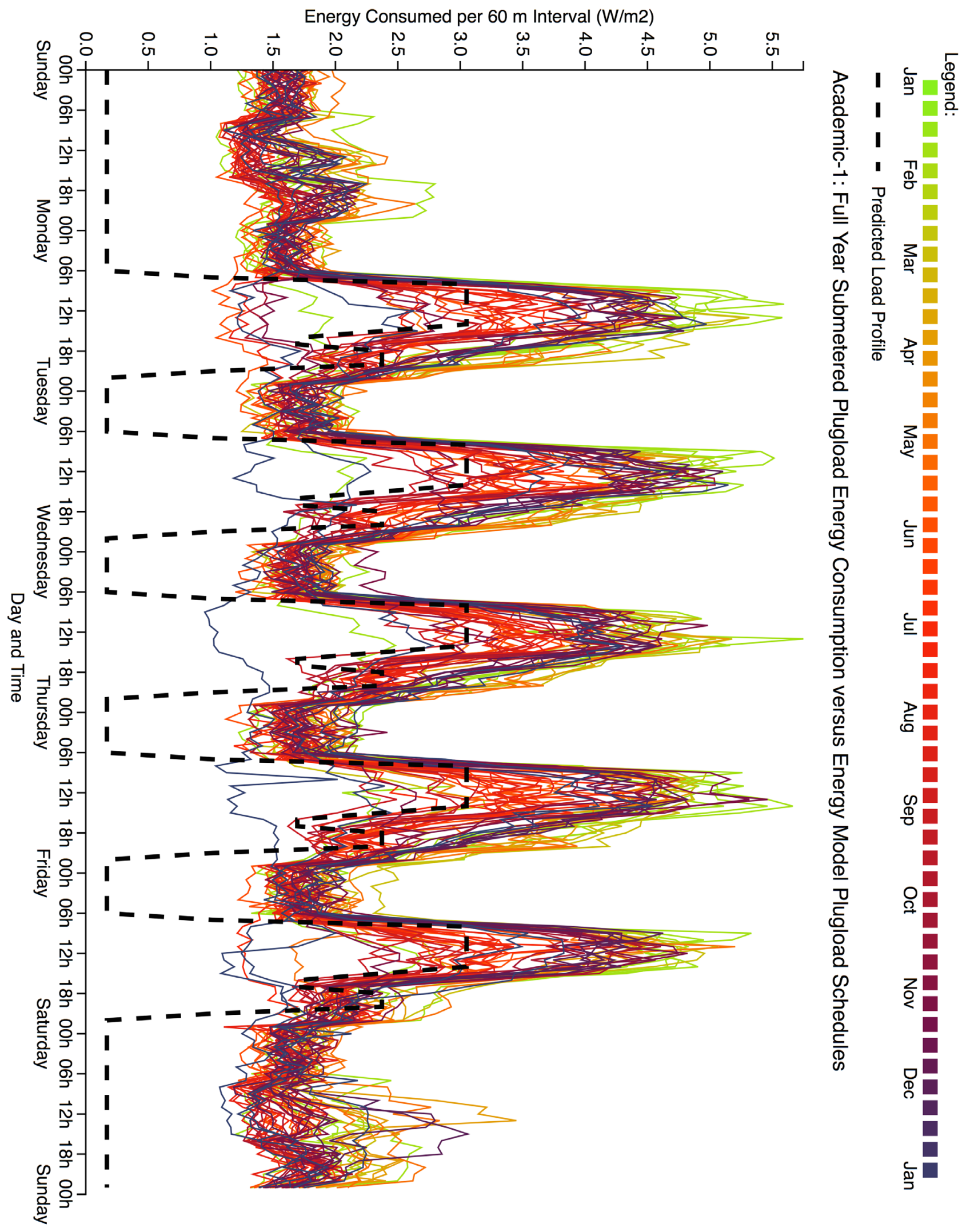


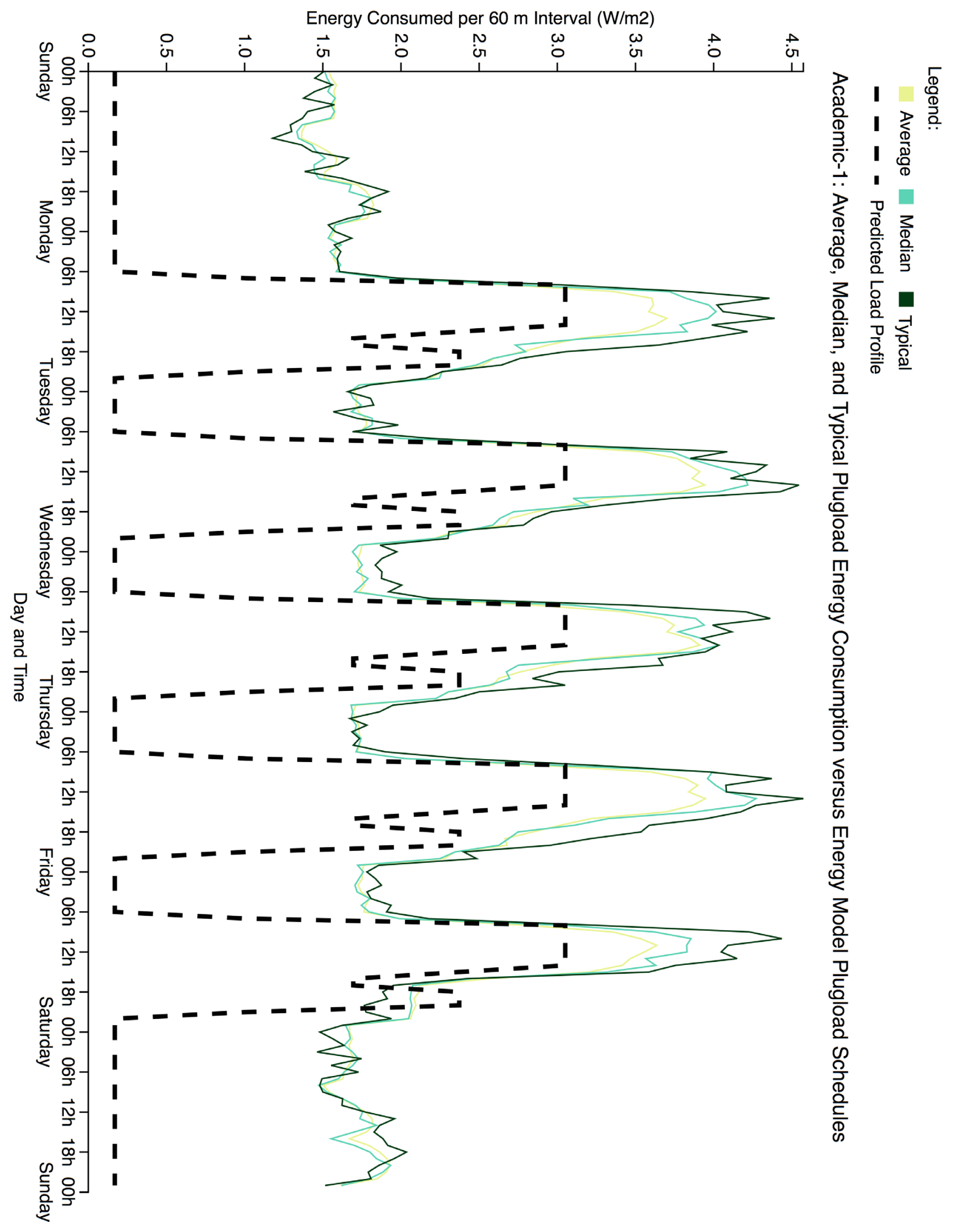




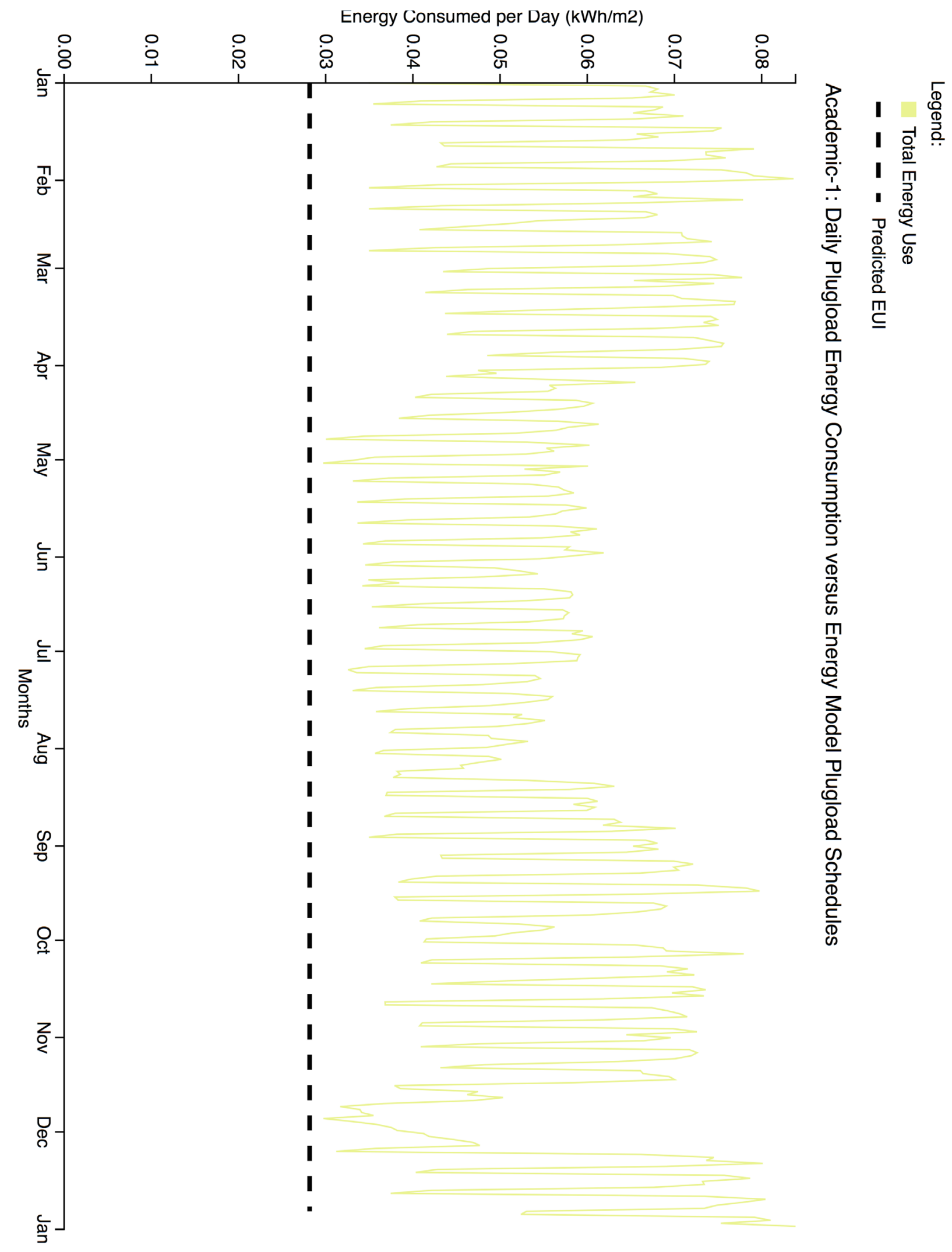




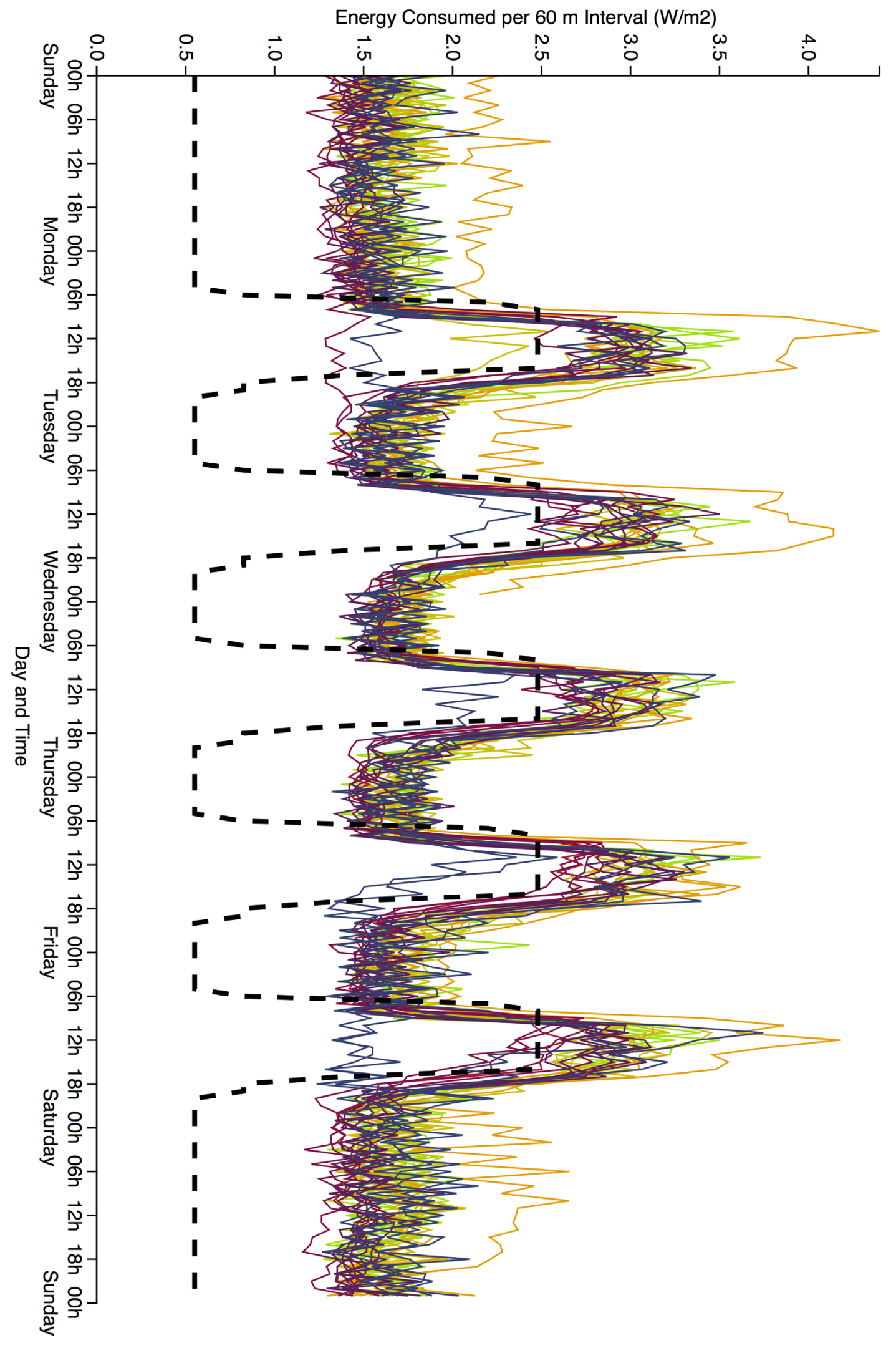

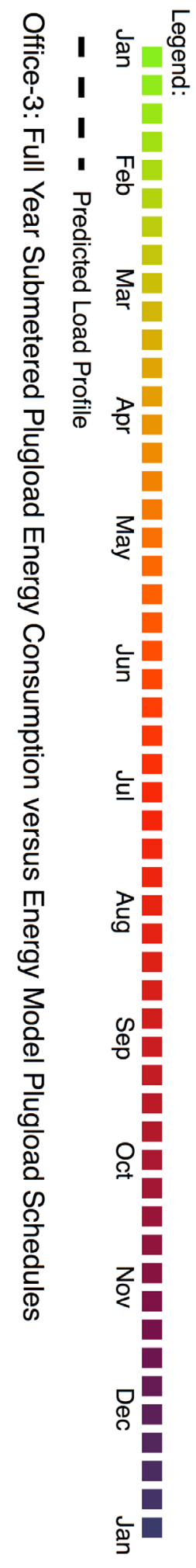




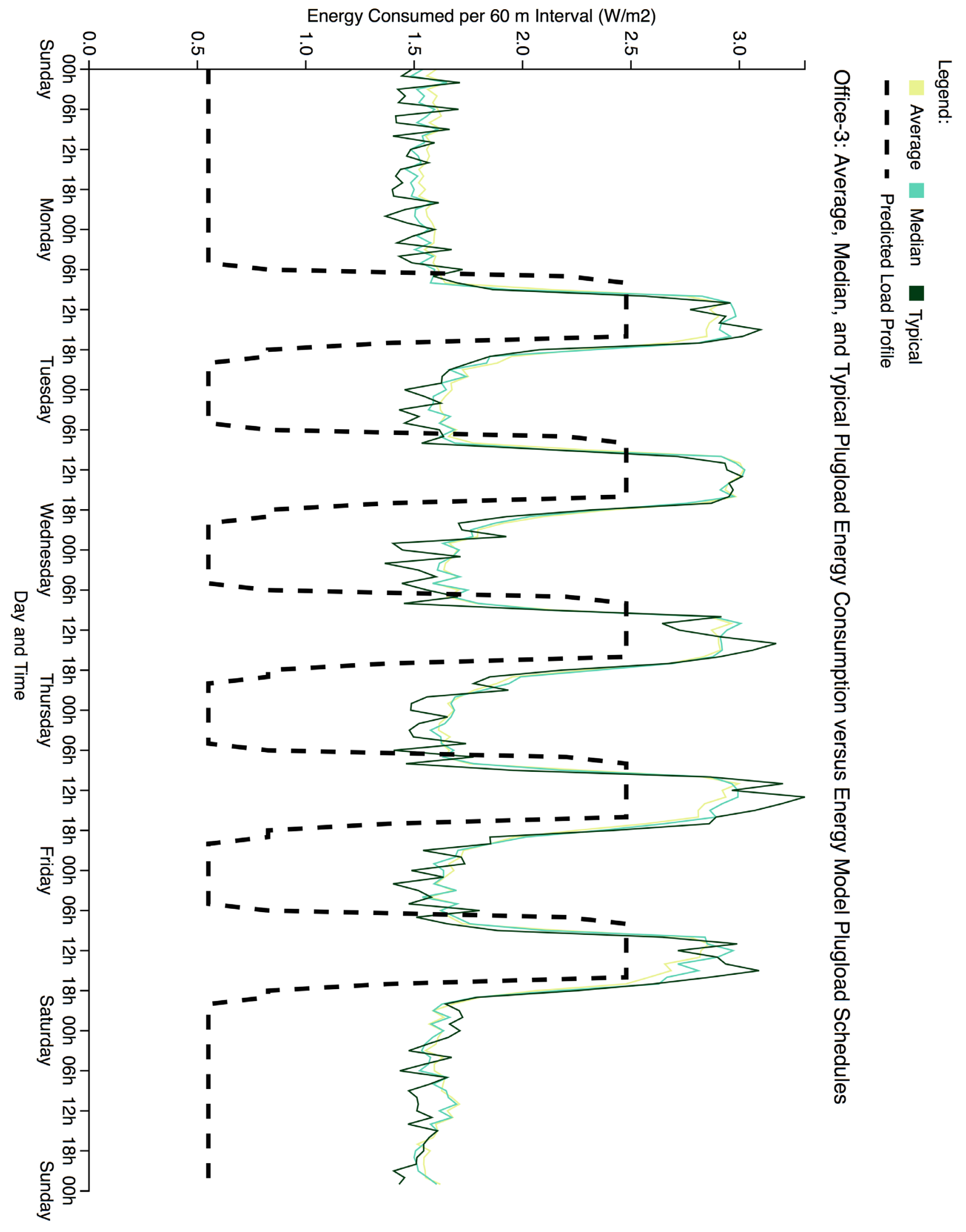




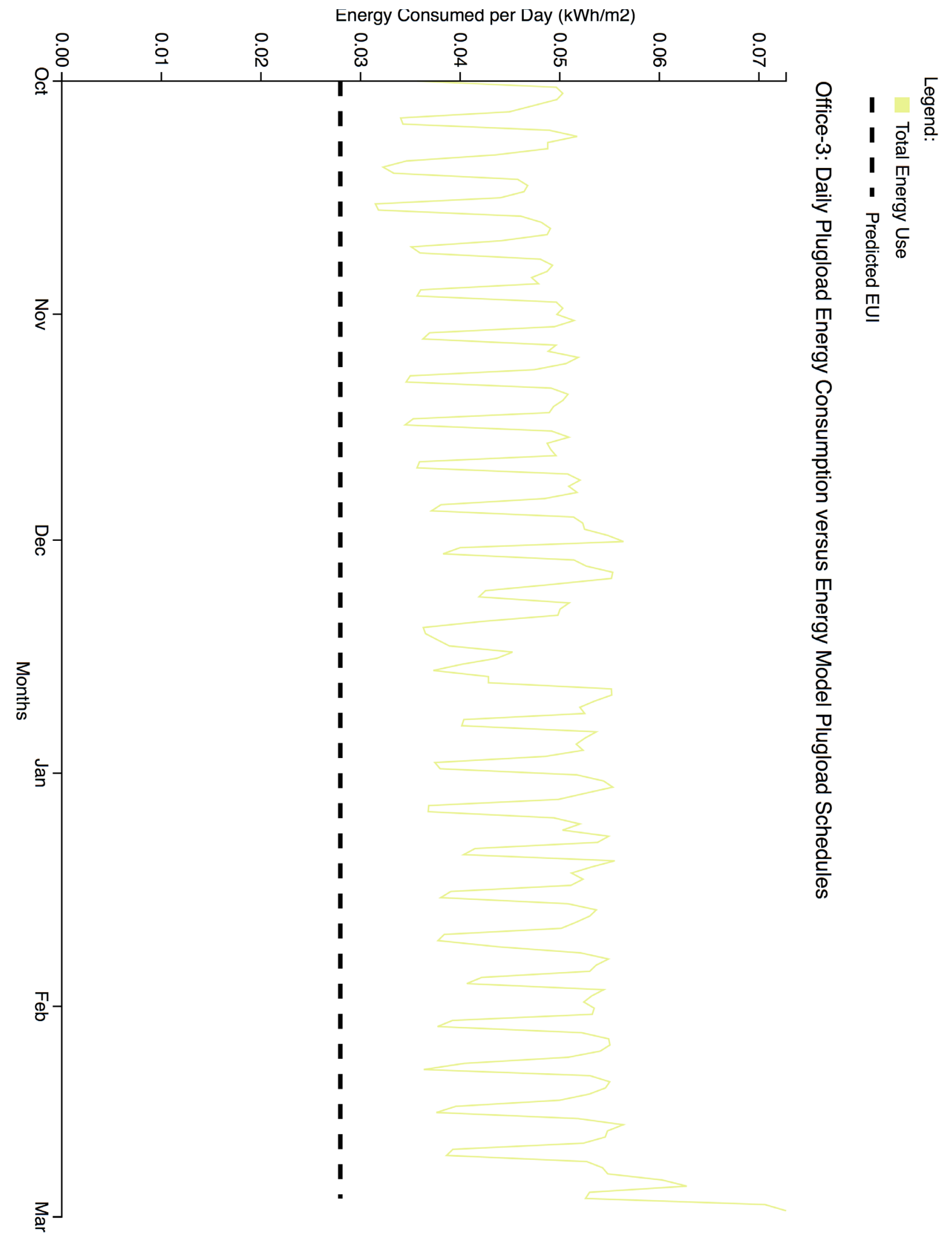




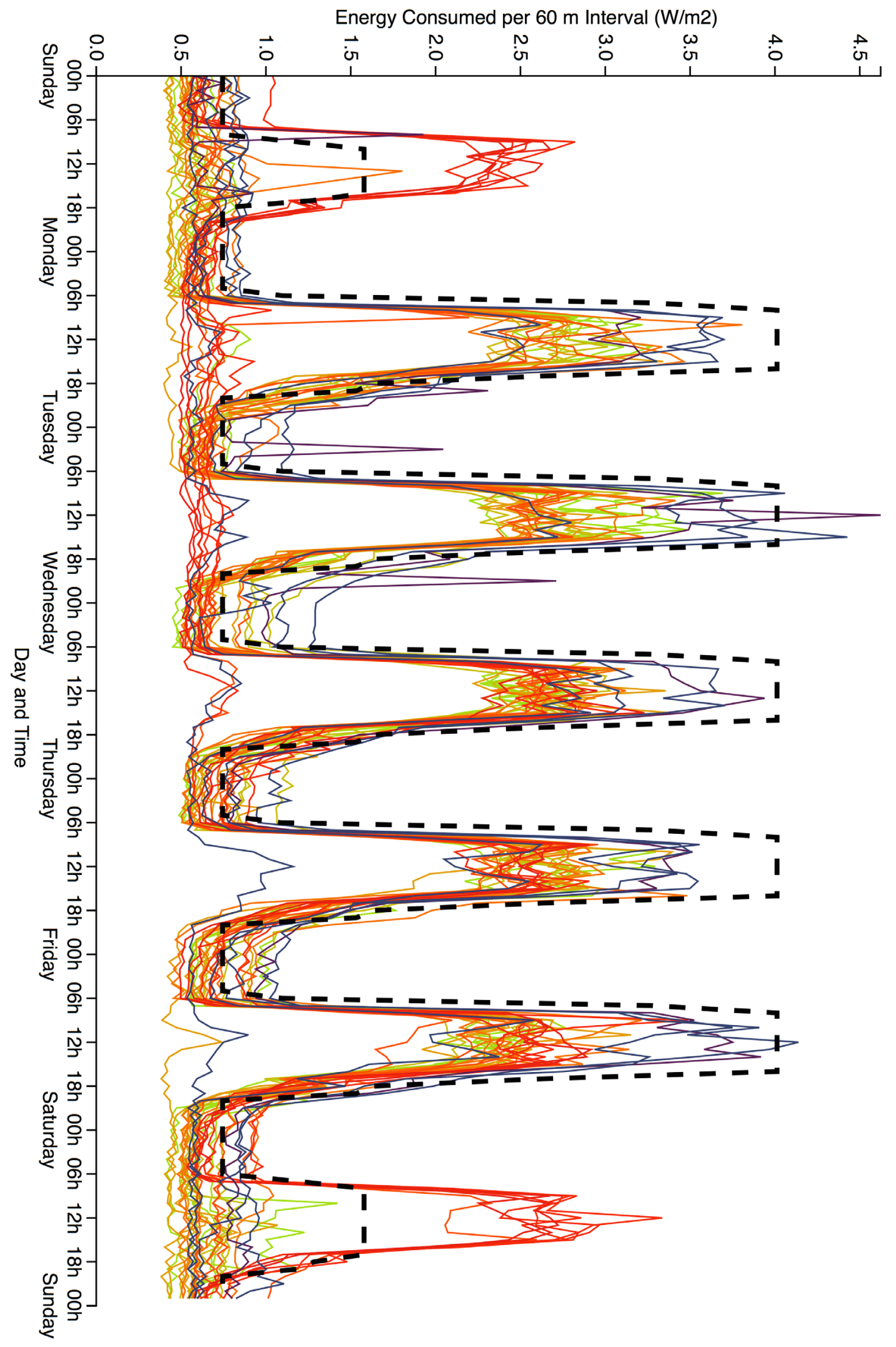

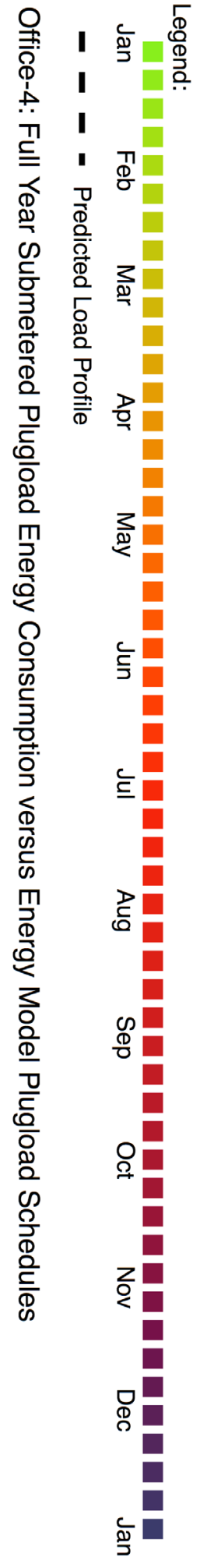




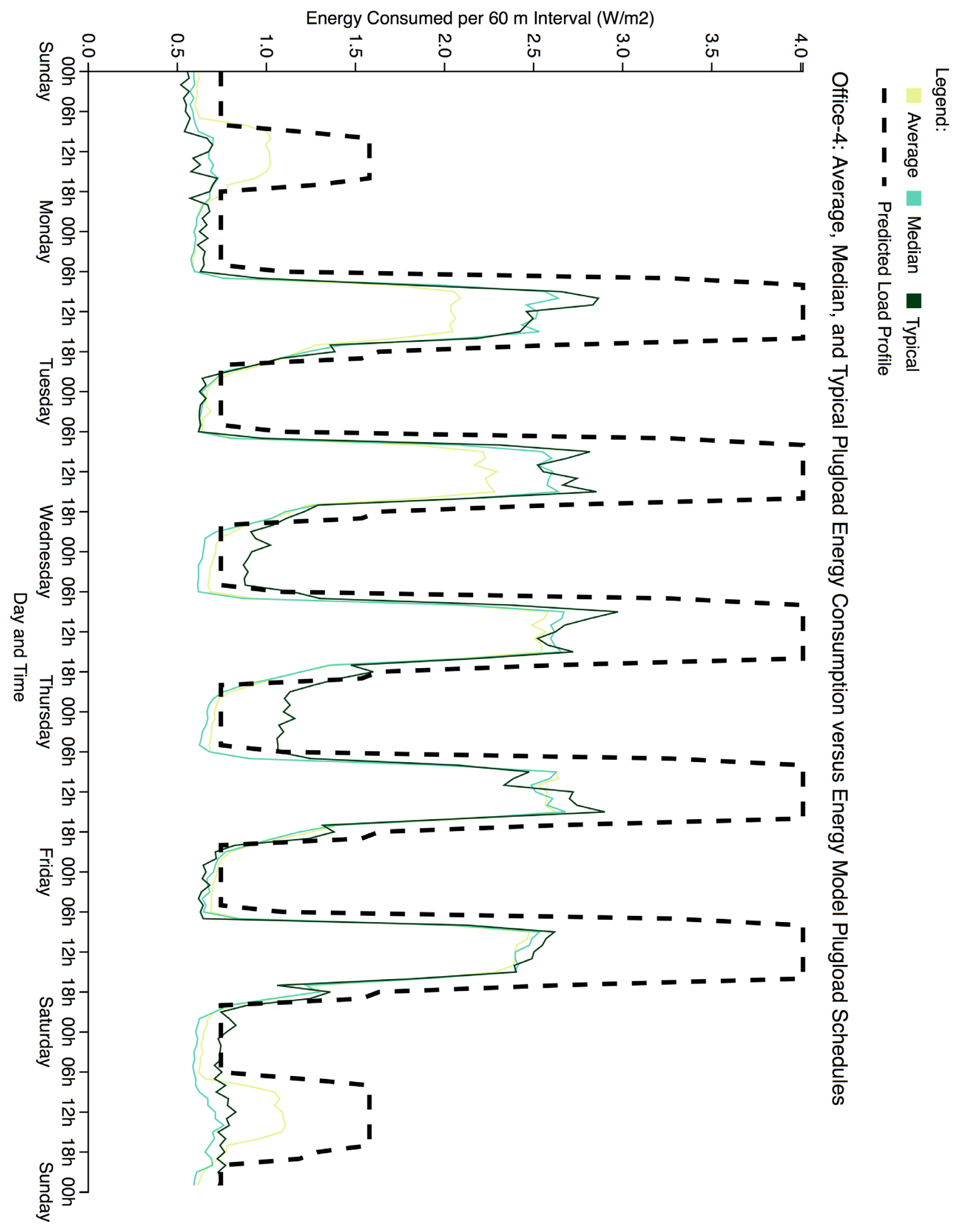




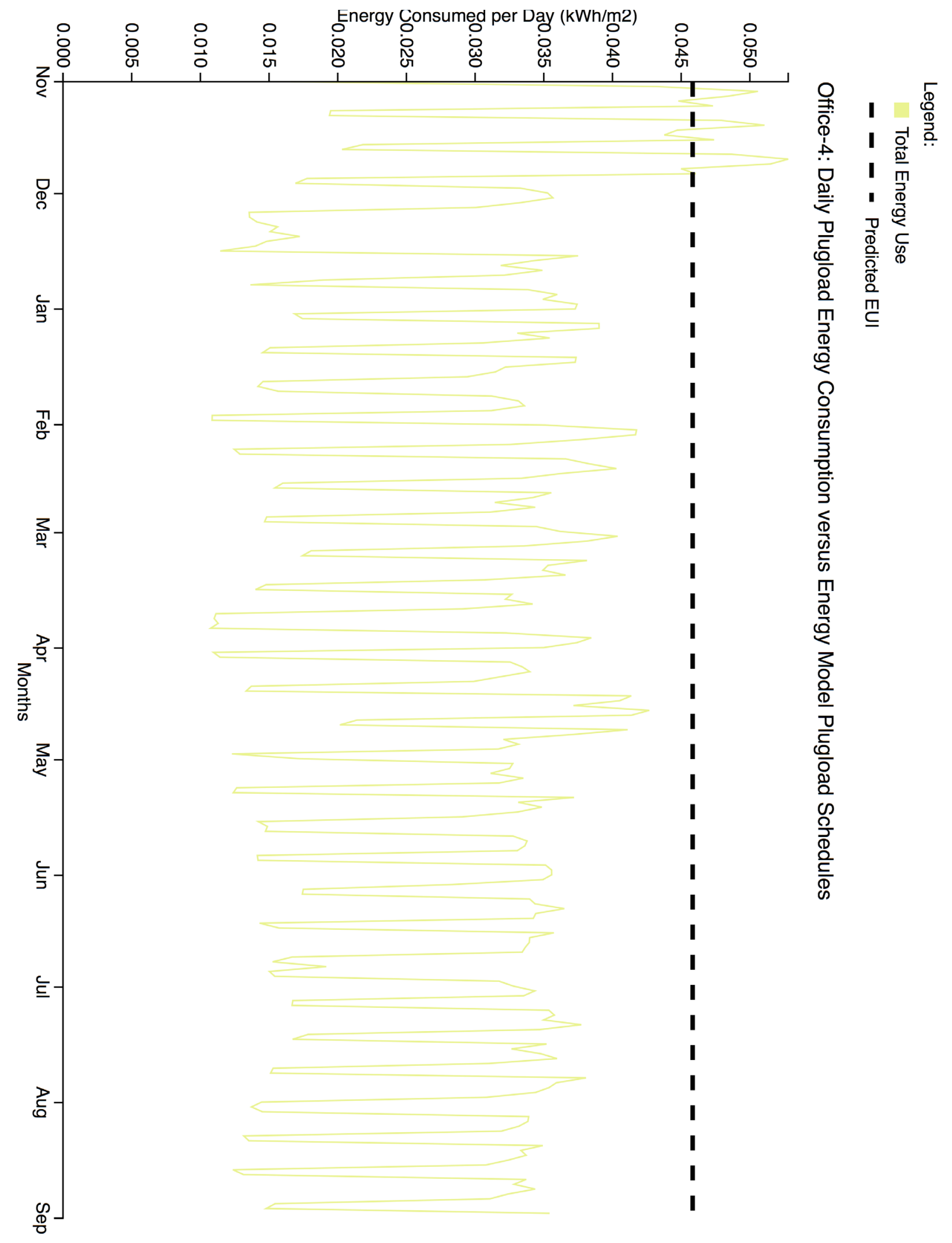



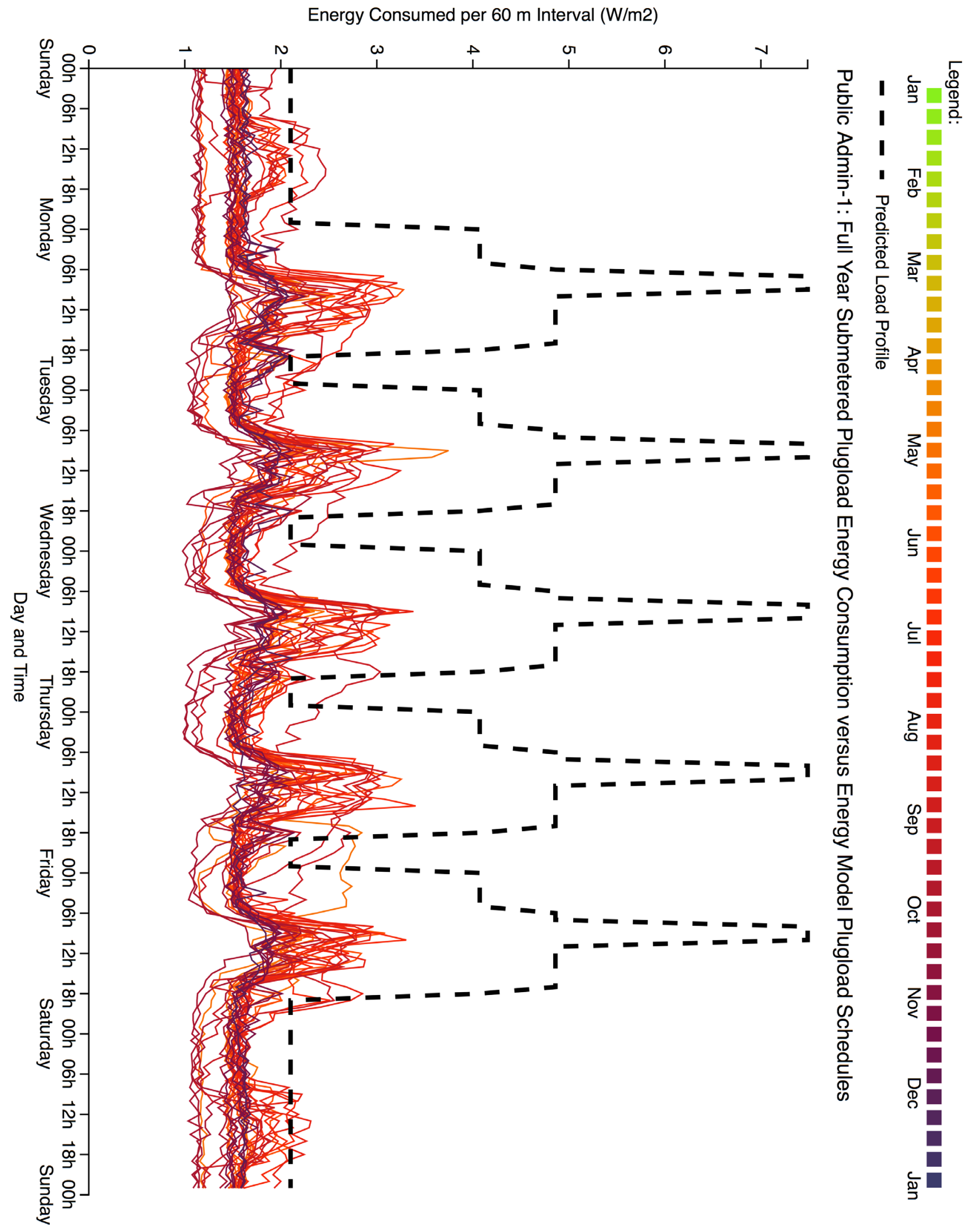


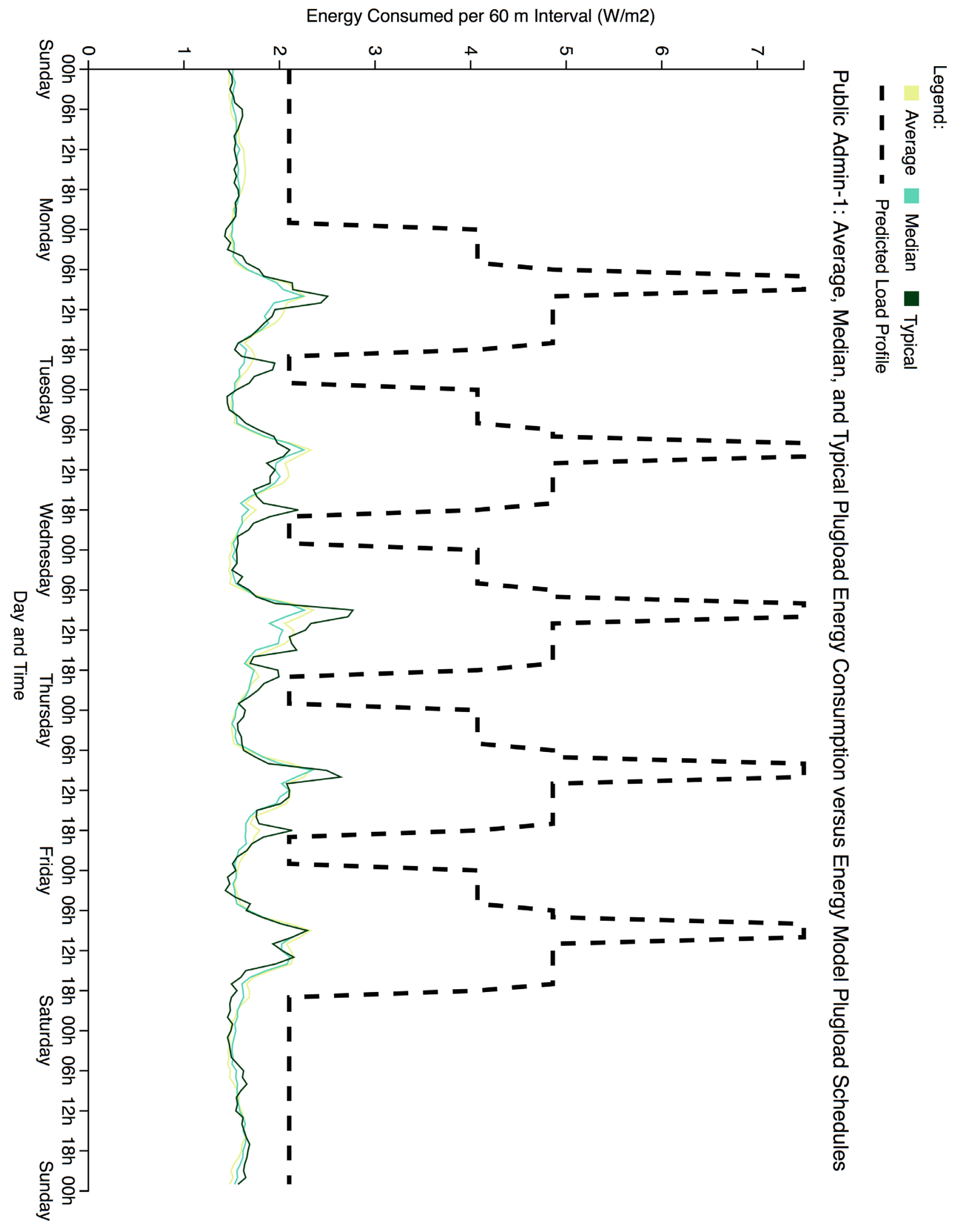




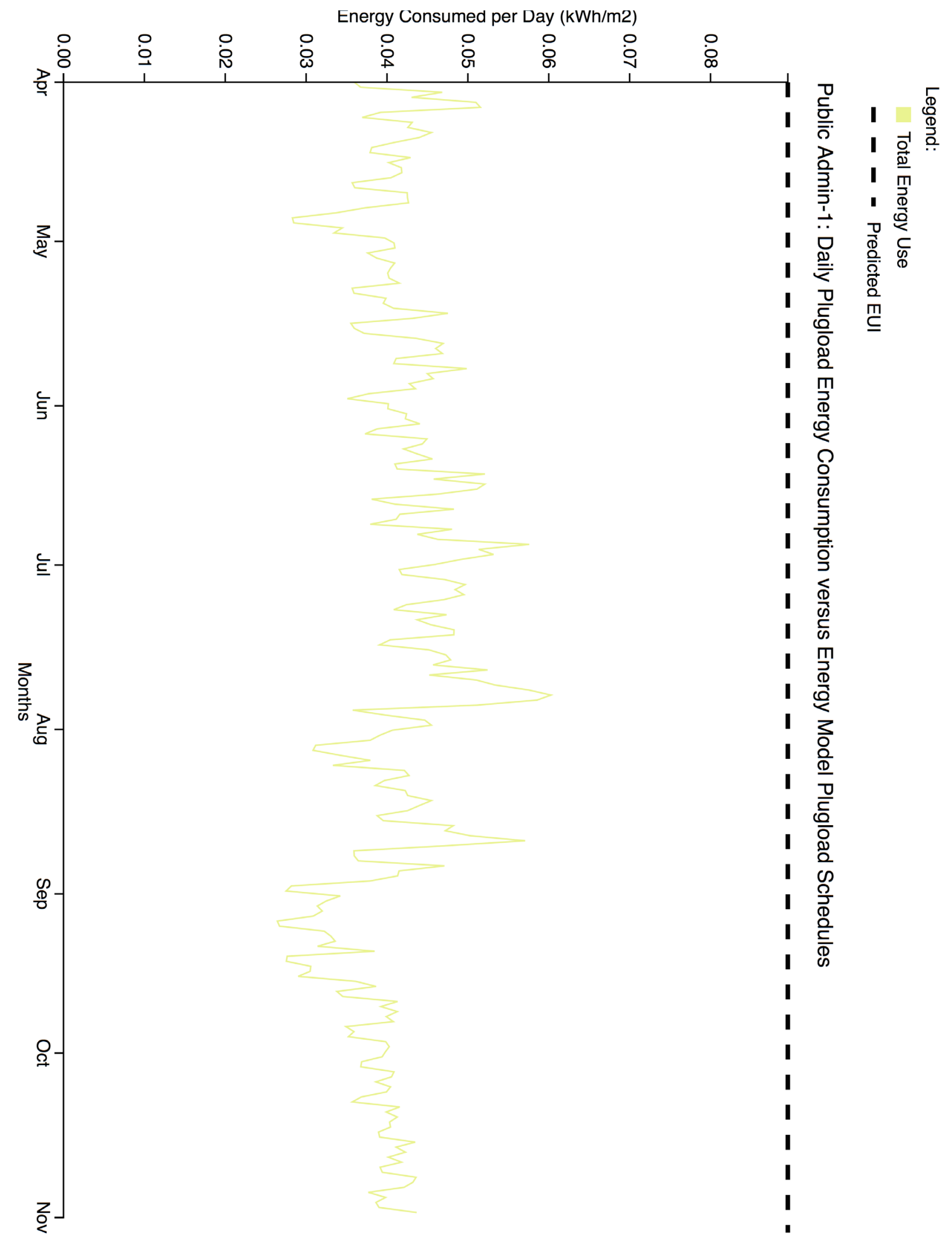



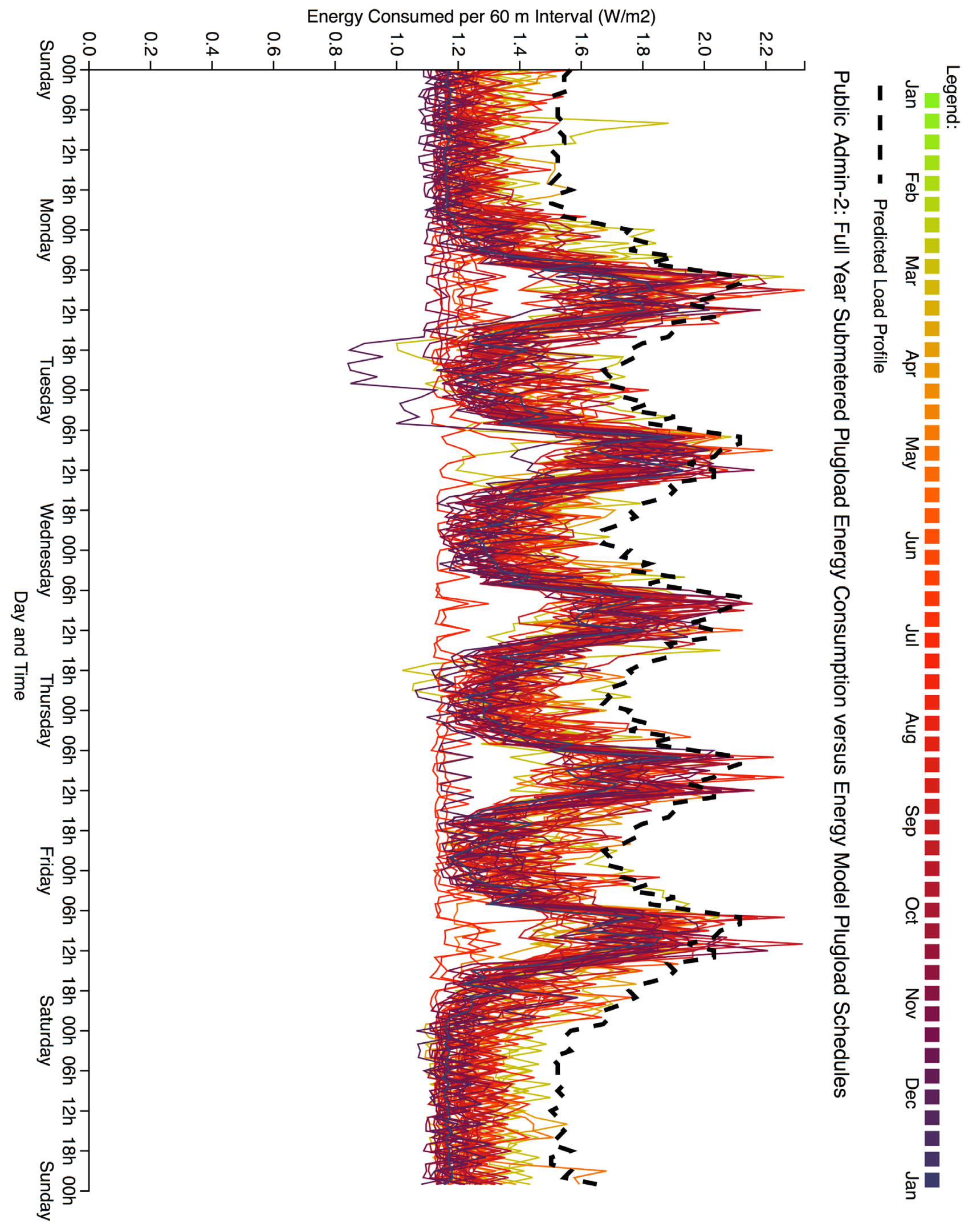


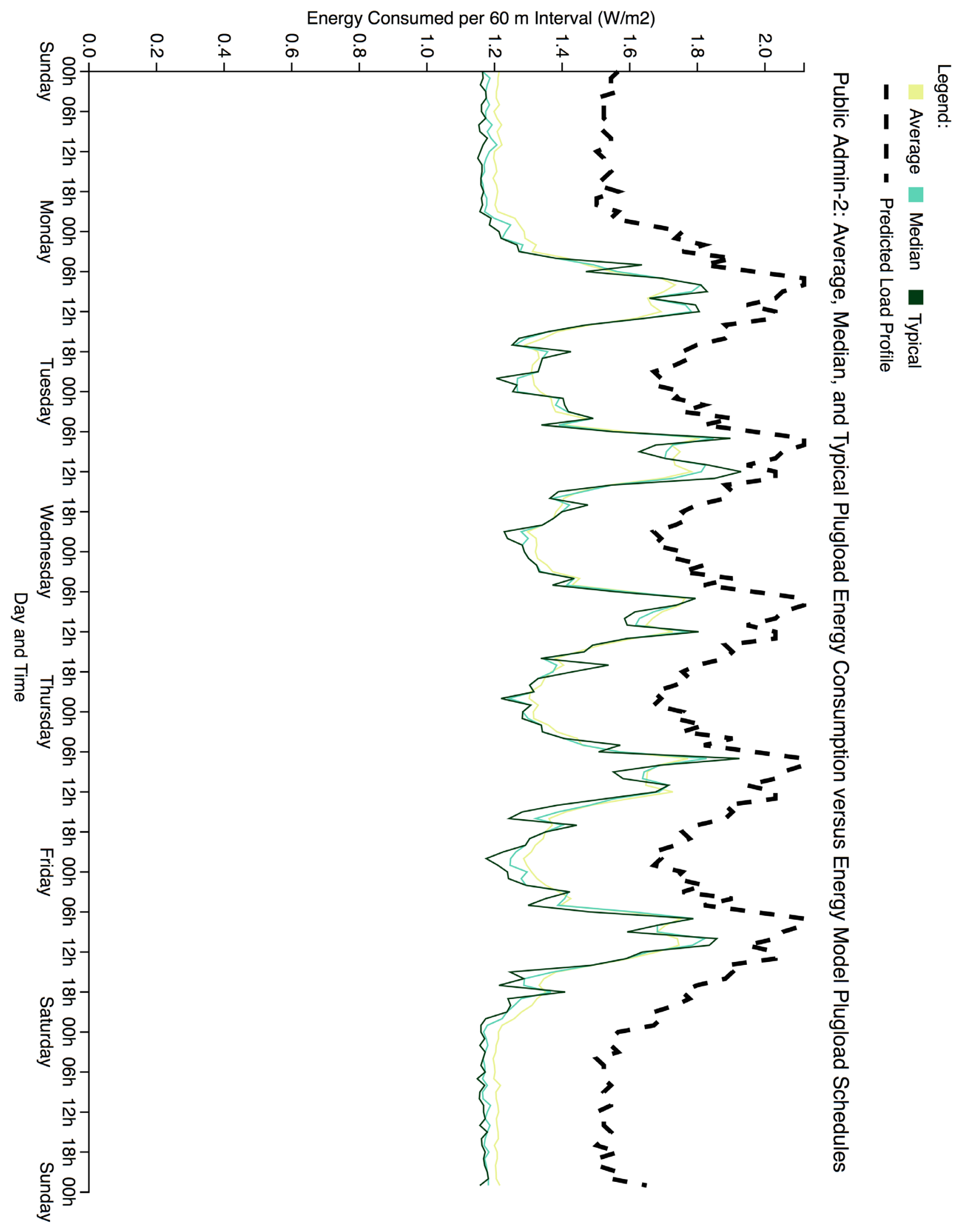




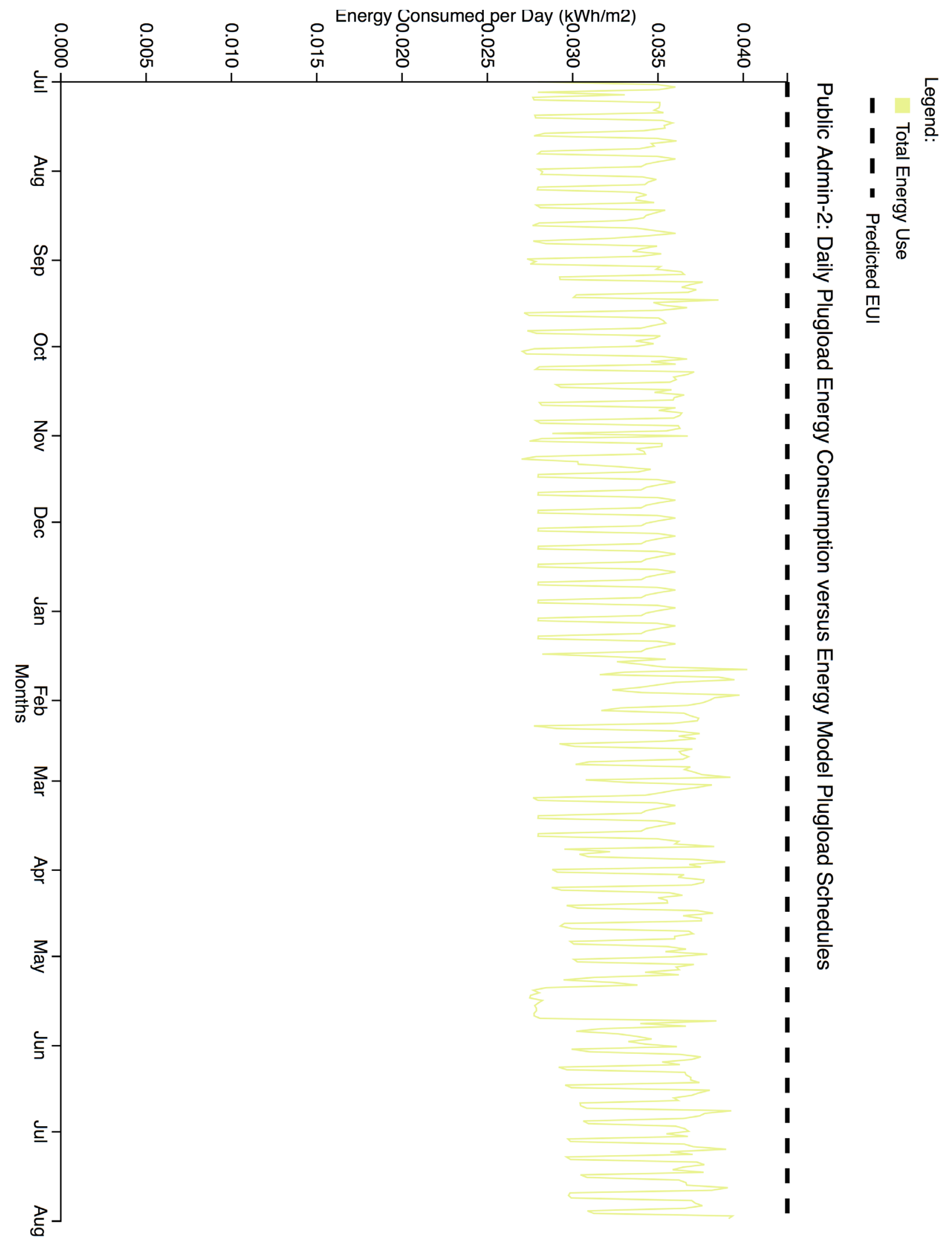




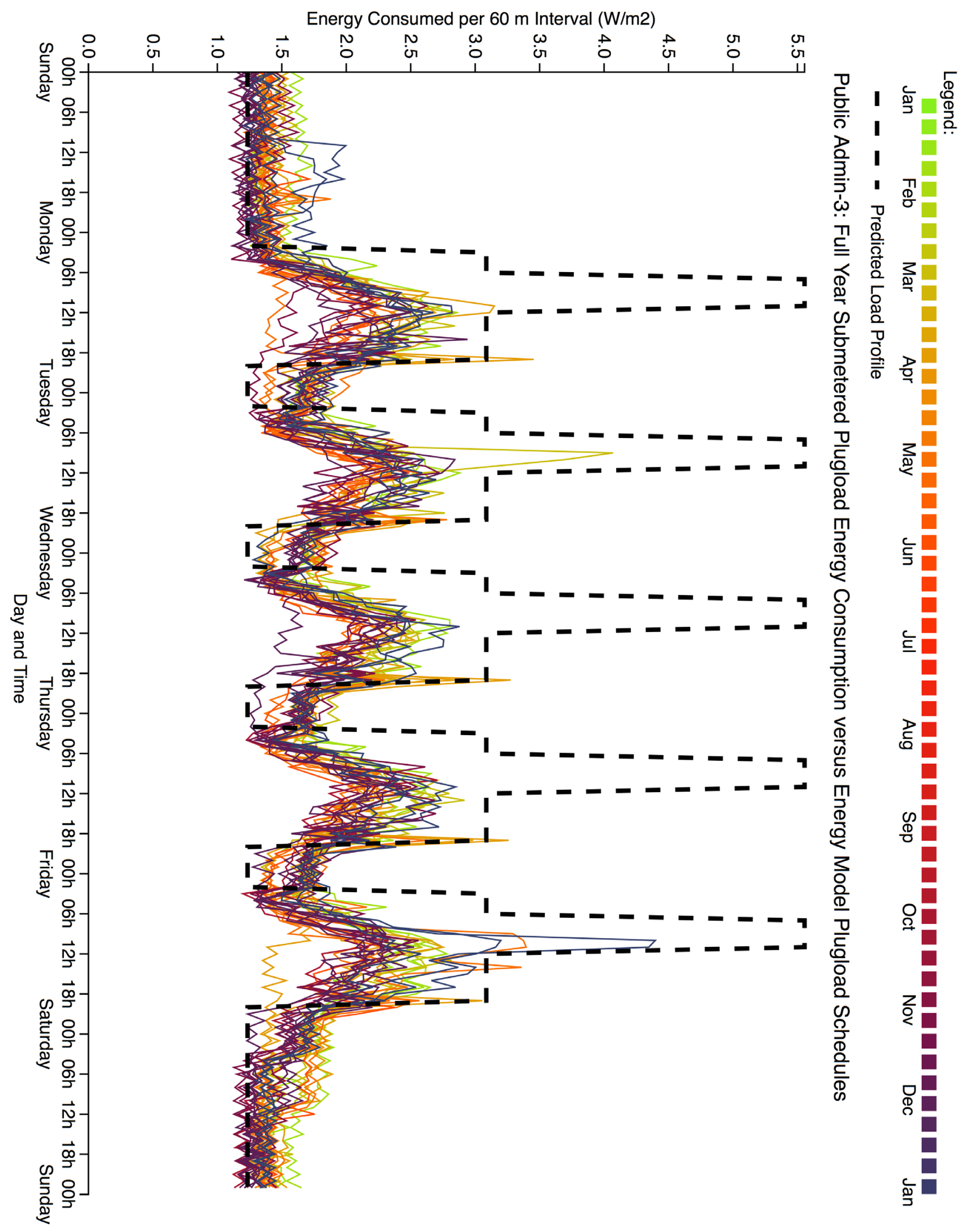




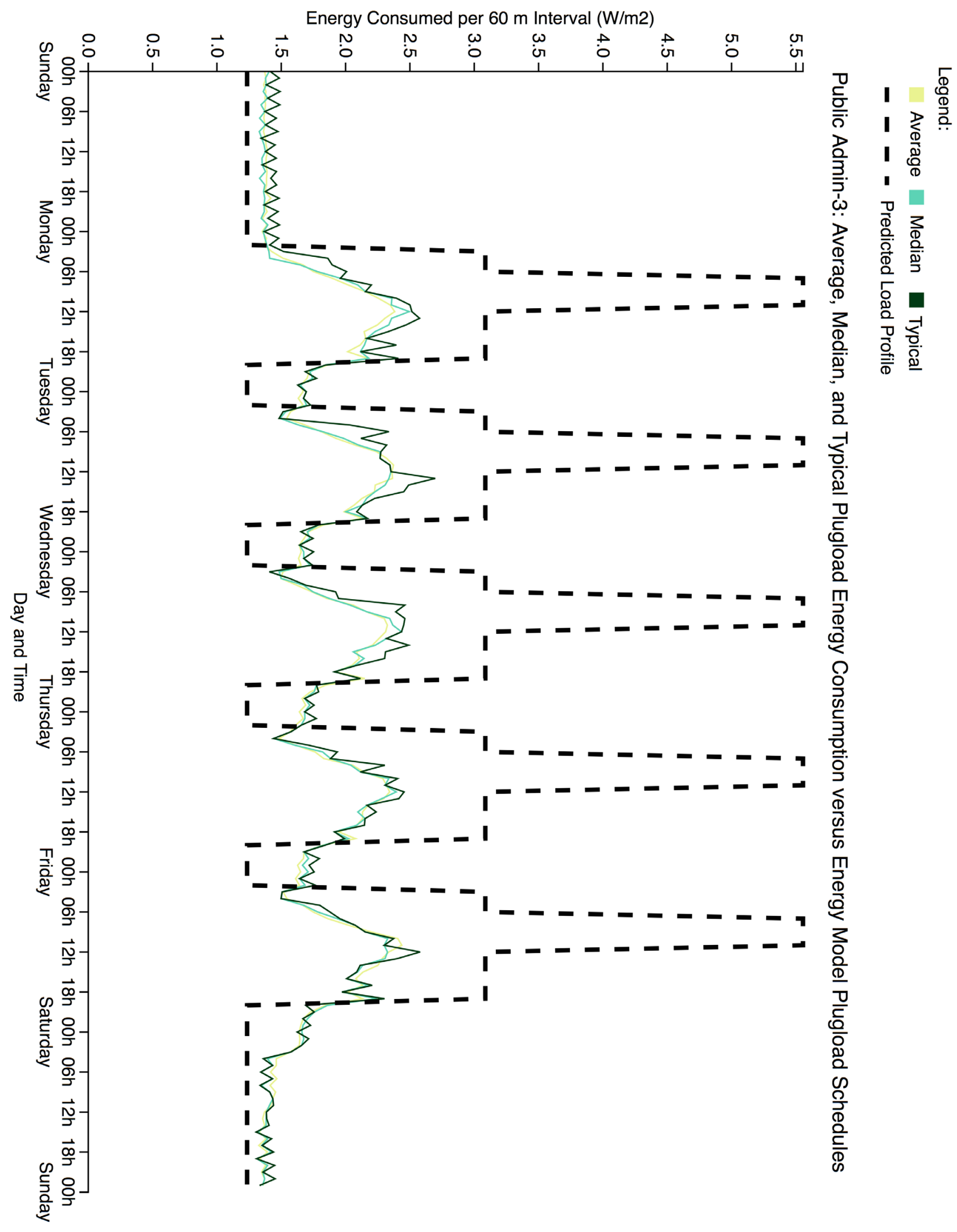




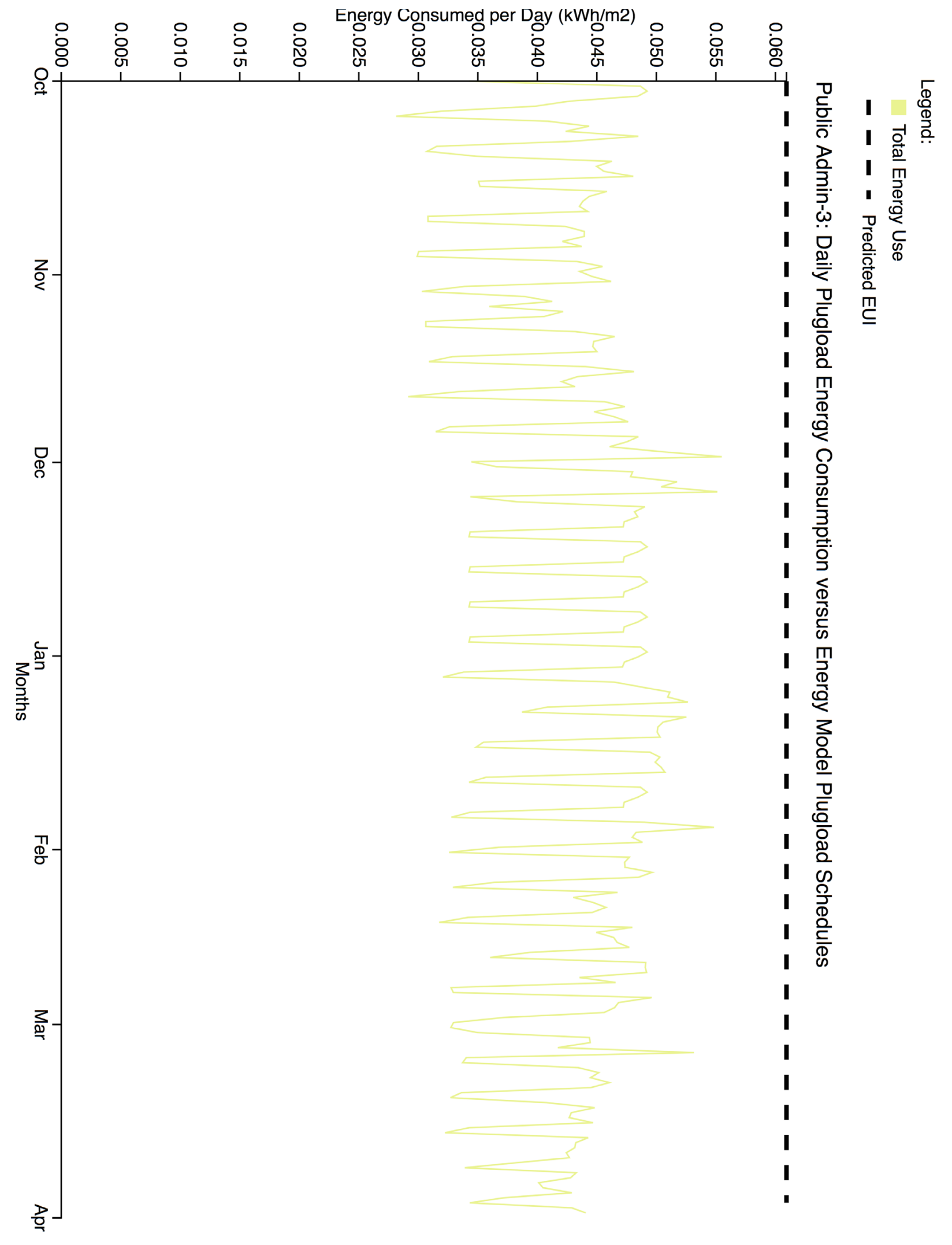


Energy Consumed per $60 \mathrm{~m}$ Interval (W/m2)
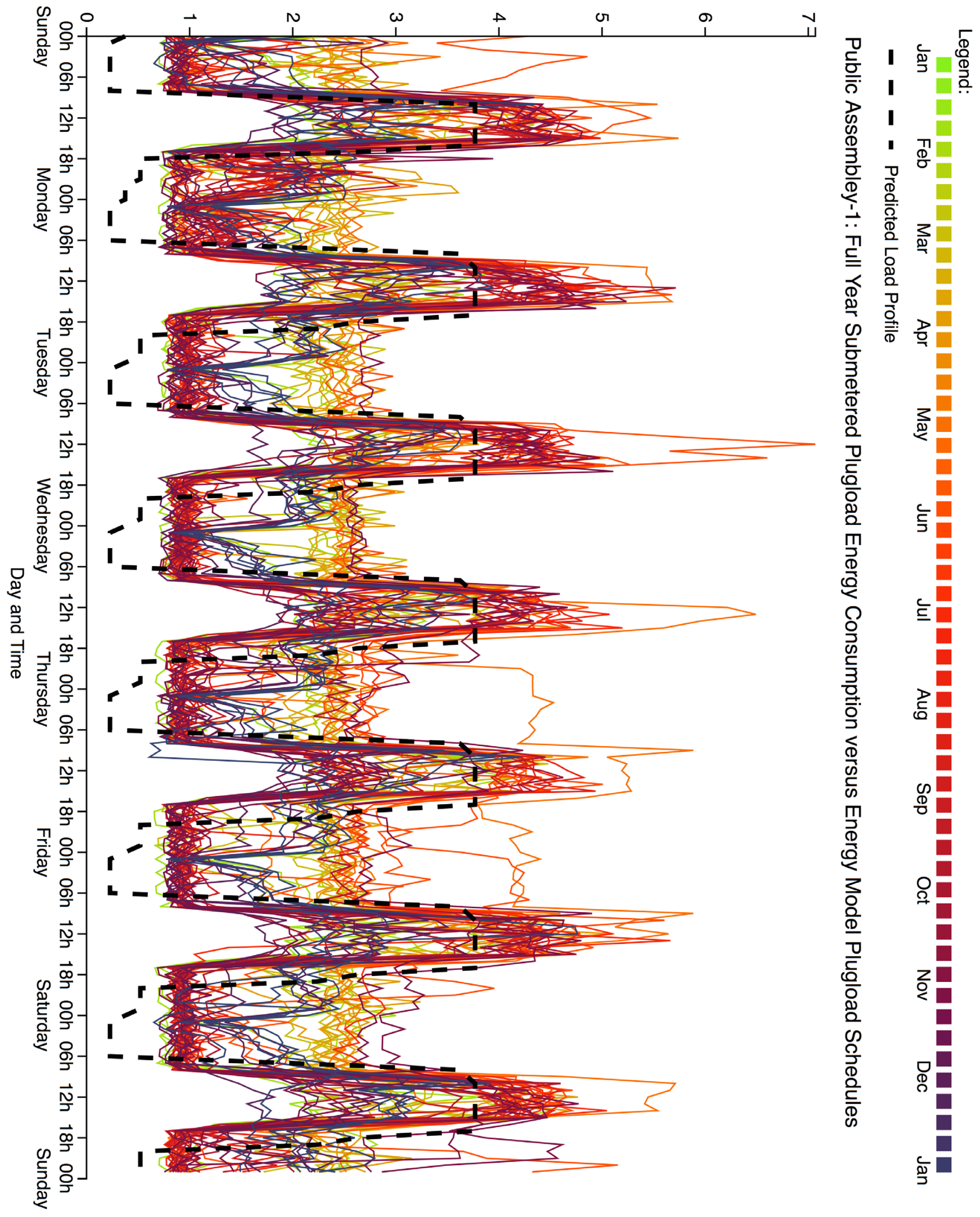


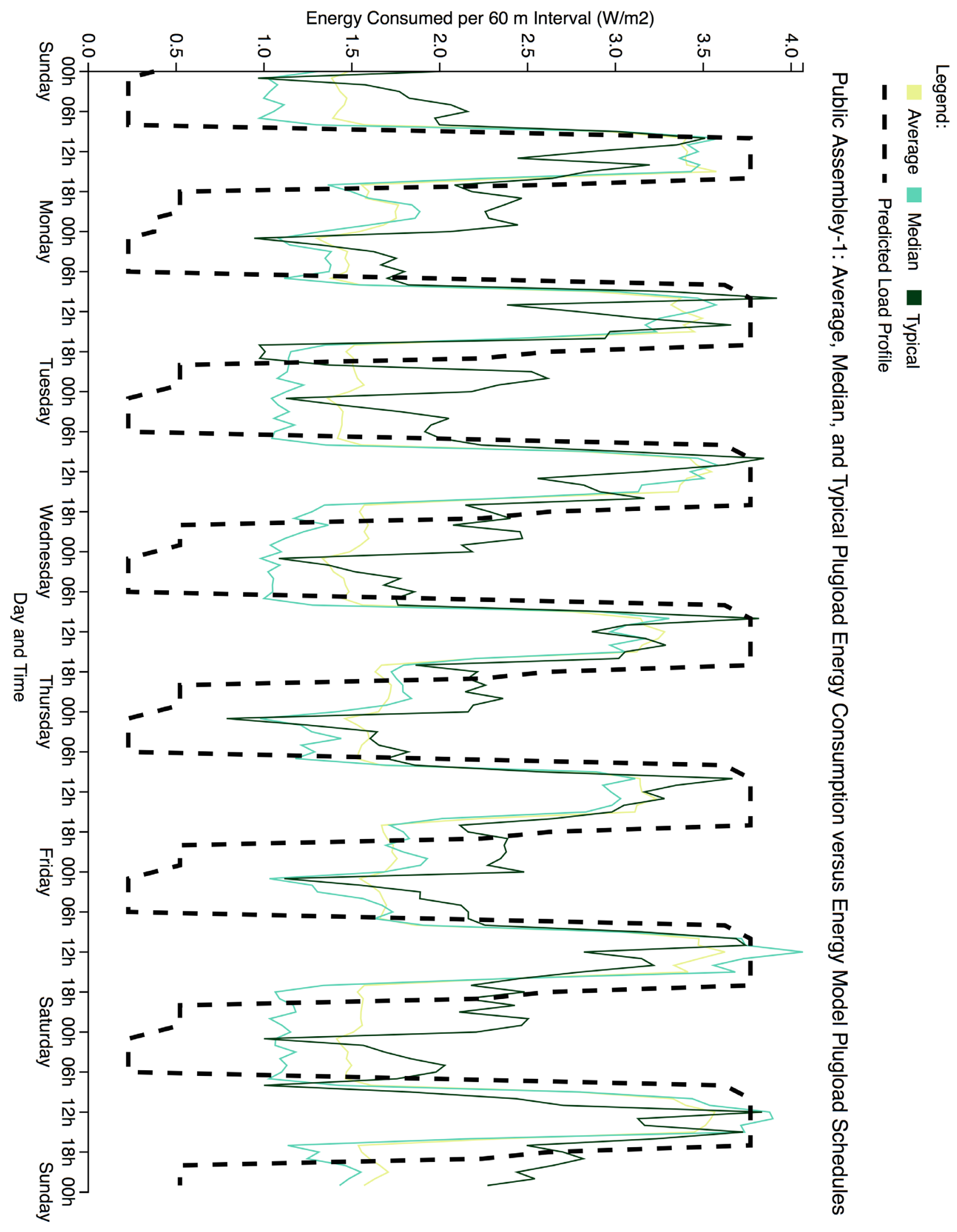




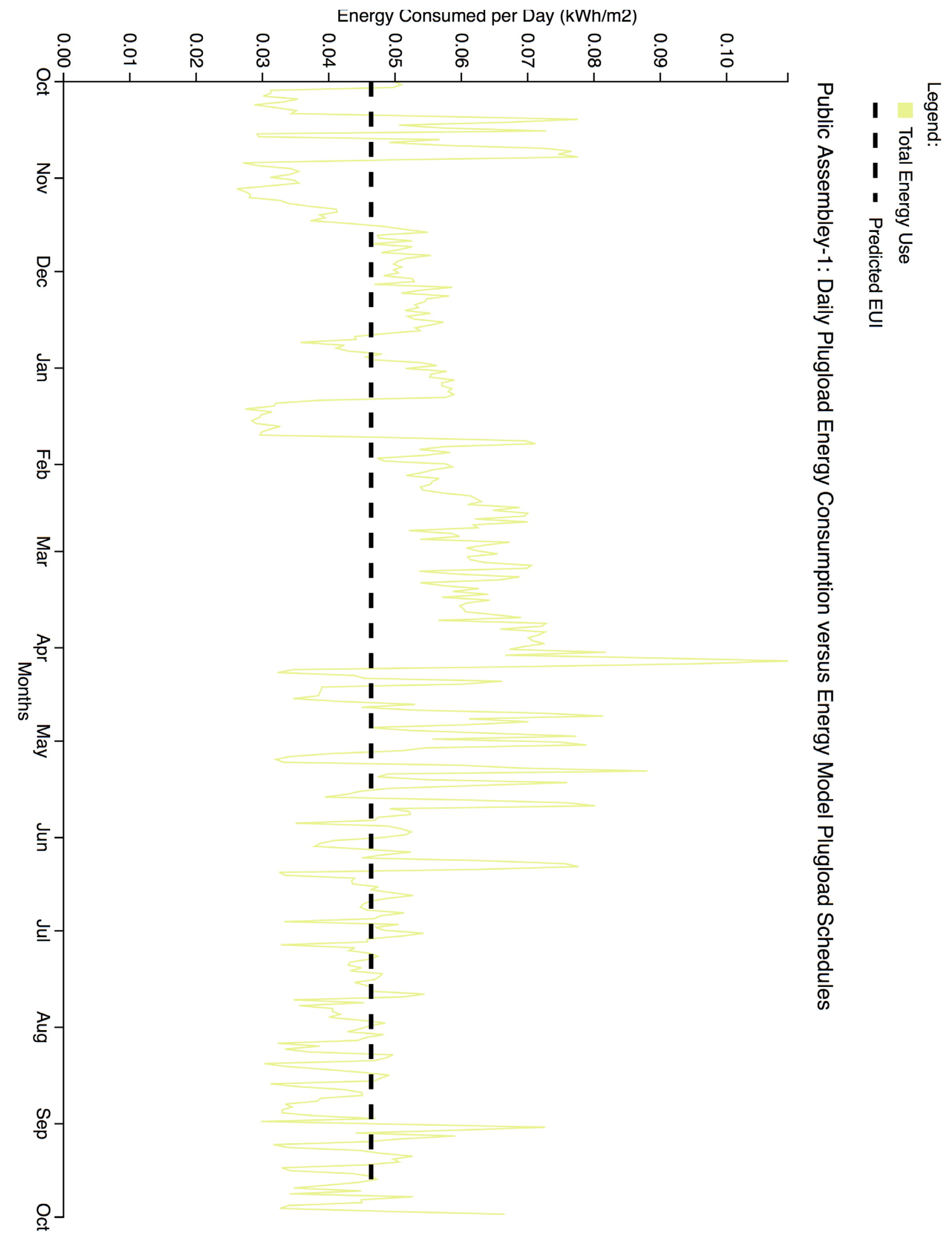




\section{References}

Ahmad, M., and Culp, C. (2006). Uncalibrated Building Energy Simulation Modeling Results. HVAC\&R Research, 12(4), 1141-1155.

ANSI, ASHRAE, USGBC, \& IES. (2010). 189.1 Standard for the Design of HighPerformance Green Buildings. Atlanta, GA, USA: ASHRAE.

Arup. (2013). The Performance Gap: Causes and Solutions - A report for the Green Construction Buildings Working Group. www.greenconstructionboard.org/index.php/resources/performance-gap. Accesed July 12, 2015.

ASHRAE. (2009). Indoor Air Quality Guide: Best Practices for Design, Construction, and Commissioning. ASHRAE: Atlanta, Georgia.

ASHRAE. (2002). Guideline 14-2002: Measurement of Energy and Demand Savings.

Bartlett, K.; Brown, C.; Chu, A.; Ebrahimi, G.; Gorgolewski, M.; Hodgson, M.; Issa, M.; Mallory-Hill, S.; Ouf, M.; Scannel, L.; and Turcato, M. (2014, October). “Do Our Green Buildings Perform as Intended?" Paper presented at World Sustainable Buildings 2014, Barcelona, Spain, 28-30 October.

Baylon, D., and Storm, P. (2008). Comparison of Commercial LEED Buildings and Non-LEED Buildings within the 2002-2004 Pacific Northwest Commercial Building Stock. Presented at the ACEEE Summer Study on Energy Efficiency in Buildings. aceee.org/files/proceedings/2008/data/papers/4_57.pdf. Accessed July 12, 2015.

Belfast, J. (2014). Fault Diagnostics Tools for Commercial Buildings: Applications, Algorithms, and Barriers. Energy Engineering, volume 111 issue 3, 57-66, 6978.

Brown, N., Wright, A. J., Shukla, A., and Stuart, G. (2010). Longitudinal Analysis of Energy Metering Data from Non-Domestic Buildings. Building Research \& Innovation, 38(1), 80-91.

Cardell-Oliver, C., and Peach, G. (2013, April). Making Sense of Smart Metering Data: A Data Mining Approach for Discovering Water Use Patterns. Water. watersensitivecities.org.au/resource-library/making-sense-of-smartmetering-data-a-data-mining-approach-for-discovering-water-use-patterns/. Accessed July 12, 2015.

Chisholm, S. (2010). Making Sense of Metering - How Do We Maximize Output Value? CBx White Papers. 
Coakley, D., Raftery, P., Molloy, P. (2012). Calibration of whole Building Energy Simulation Models: Detailed Case Study of a Naturally Ventilated Building Using Hourly Data. Presented at the First Building Simulation and Optimization Conference, September 10-11, 2012, Loughborough, UK.

Cory, S., Donn, M., and Pollard, A. (2015). Comparison of NZ's Energy Efficiency Regulation and Verification Assumptions to Real Building Loads and Operations. Buildings, 3, 116-129.

Diamond, R., Opitz, M., Neida, B., and Herrera, S. (2006). Evaluating the Energy Performance of the First Generation of LEED-Certified Commercial Buildings. www.southface.org/ez/media/lbnl59853-leed.pdf. Accessed July 12, 2015.

Environment Canada. (2015). Monthly Climate Normals. Available from climate.weather.gc.ca/climate_normals/index_e.html. Accessed July 15, 2015.

Fowler, K., and Rauch, E. (2008). A Post Occupancy Evaluation of 12 GSA Buildings. Pacfic Northwest National Laboratory. www.gsa.gov/portal/getMediaData?mediaId=214299. Accessed July 12, 2015.

Gul, M., and Patidar, S. (2015). Understanding the Energy Consumption and Occupancy of a Multi-purpose Academic Building. Energy and Buildings, 87, 155-165.

Im, P., and Bhandari, M. (2014). Is Monthly Whole Building Level Calibration Enough?: a Detailed Modeling and Calibration Study of an Ultra-efficient Occupancy Simulated House. Presented at 2014 ASHRAE/IBPSA-USA Building Simulation Conference, September 10-12, Atlanta, GA.

Innovate UK. (2014). Building Performance Evaluation Programme: Early Findings from Non-Domestic Projects.

Kesik, T. (2015, March). Vital Signs: Towards Meaningful Building Performance Indicators. University of Toronto Green Papers.

Khan, A. (2007). LEED Canada Energy Performance Modelling of a Medical Office Building Using Carrier HAP and NRCan EE4 (dissertation). Ryerson University, Toronto.

Lee, Y., An, L., Liu, F., Horesh, R., Chae, Y., and Zhang, R. (2013). Applying Science and Mathematics to Big Data for Smarter Buildings. Annals of the New York Academy of Sciences, 1295, 18-25.

Lindelof, D. and Morel, N. (2006). A Field Investigation of the Intermediate Light Switching By Users. Energy and Buildings, 38, 790-801.

Mahdavi, A., Mohammadi, A., Kabir, E. and Lambeva, L. (2008). Occupant's Operation of Lighting and Shading Sytems in Office Buildings. Journal of Building Performance Simulation, 1(1), 57-65. 
Masoso, O., and Grobler, L. (2010). The Dark Side of Occupants' Behaviour on Building Energy Use. Energy and Buildings, 42, 173-177.

Menezes, C., Cripps, A., Bouchlaghem, D., and Buswell, R. (2011, September). Analysis of electricity consumption for lighting and small power in office buildings. Presented at 2011 CIBSE Technical Symposium, September 6-7, Leicester, UK.

NASA. (2015). Prediction of Worldwide Energy Resource (POWER): Near Real-time Daily Global Radiation and Meteorology. Accessed July 12, 2015. power.larc.nasa.gov/cgibin/cgiwrap/solar/timeseries.cgi?email=daily@larc.nasa.gov.

Newsham, G., Mancini, S., Birt, B. (2009). Do LEED-certified Buildings Save Energy? Yes, but... . Energy and Buildings, 41, 897-905.

Nguyen, T., and Aiello, M. (2013). Energy Intelligent Buidlings Based on User Activity: a survey. Energy Build, 56, 244-257.

NRCan. (2014). EE4. Accessed December 9, 2014. www.nrcan.gc.ca/energy/software-tools/7453.

NRCan. (2006). National Energy Use Database. Accessed July 12, 2015. http://oee.nrcan.gc.ca/corporate/statistics/neud/dpa/menus/trends/compre hensive_tables/list.cfm.

O'Neill, Z., and Eisenhower, B. (2013). Leveraging the Analysis of Parametric Uncertainty for Building Energy Model Calibration. Build Simul, 6, 365-377.

Poirazis, H., Blomsterberg, A., and Wall, M. (2008). Energy Simulations for Glazed Office Buildings in Sweden. Energy Build, 40, 1161-1170.

Preiser, W., \& Vischer, J. (Eds.). (2005). Assessing Building Performance. Oxford, UK: Elsevier.

Price, P., Mathieu, J., Kiliccote, S., and Piette, M. (2011). Using Whole-Building Electric Load Data in Continuous or Retro-Commissioning. Presented at the National Conference on Building Comissioning, August 10-12.

Raferty, P., Keane, M., \& Costa, A. (2011). Calibrating whole Building Energy Models: Detailed Case Study Using Hourly Measured Data. Energy and Buildings, 43, 3666-3679.

Raferty, P., Keane, M., \& O’Donnell, J. (2011). Calibrating whole Building Energy Models: An Evidence-based Methodology. Energy and Buildings, 43, 23562364. 
Reddy, T. (2006). Literature Review on Calibration of Building Energy Simulation Programs: Uses, Problems, Procedures, Uncertainty, and Tools. ASHRAE Transactions, 112, 226-240.

Samuelson, H., Ghorayshi, A., \& Reinhart, C. (2014). Analysis of a Simplified Calibration Procedure for 18 Design-Phase Building Energy Models. Manuscript under review for publication in the Journal of Building Performance.

Scofield, J. (2009). Do LEED-certified buildings save energy? Not really... Energy and Buildings, 41, 1386-1390.

SINTEF. (2013, May 22). Big Data, for better or worse: 90\% of world's data generated over last two years. ScienceDaily. www.sciencedaily.com/releases/2013/05/130522085217.htm. Accessed December 5, 2014.

Staats, H., Van Leeuwen, E., and Wit, A. (2010). A Longitudinal Study of Information Interventions to Save Energy in an Office Building. Journal of Applied Behavioural Analysis, 33, 101-104.

Tian, Z., Love, J., and Tian, W. (2009). Applying Quality Control in Building Energy Modelling: comparative simulation of a high performance building. Journal of Building Performance Simulation ,Vol. 2 No. 3, 163-178.

Torcellini, P., Pless, S., Deru, M., Griffith, B., Long, N., and Judkoff, R. (2006). Lessons Learned from Case Studies of Six High-Performance Buildings. National Renewable Energy Laboratory. www.nrel.gov/docs/fy04osti/36290.pdf. Accessed July 12, 2015.

Turner, C., and Frankel, M. (2008). Energy Performance of LEED for New Construction. New Building Institute. www.usgbc.org/Docs/Archive/General/Docs3930.pdf. Accessed July 12, 2015.

Webber, C., Roberson, J., McWhinney, M., Brown, R., and Pinckard, M. (2006). Afterhours Power Status of Office Equipment in the USA. Energy, 31, 2823-2838.

Wright, A., and Brown, N. (2008). The Analysis and Interpretation of Half Hourly Utility Data in UK Buildings.

Yun, G., Kim, H., and Kim, J. (2012). Effects of Occupancy and Lighting use Patterns on Energy Consumption. Energy and Buildings, 46, 152-158. 


\section{Glossary}

BPE- Building Performance Evaluation

CVRMSE- Coefficient of Variation of the Root Mean Square Error

DHW- Domestic Hot Water

EUI- Energy Use Intensity $\left(\mathrm{kWh} / \mathrm{m}^{2}\right)$

HVAC- Heating, Ventilation and Air Conditioning

LPD- Lighting Power Density $\left(\mathrm{W} / \mathrm{m}^{2}\right)$

MND- Median Near Base

MNECB- Model National Energy Code for Buildings

MNP- Median Near Peak

NMBE- Normalized Mean Bias Error

NRCan- Natural Resources Canada

OBC- Ontario Building Code

PD- Power Density $\left(\mathrm{W} / \mathrm{m}^{2}\right)$

POE- Post-occupancy Evaluation

WWR- Window-to-wall Ratio

WFR- Window-to-floor-area Ratio 